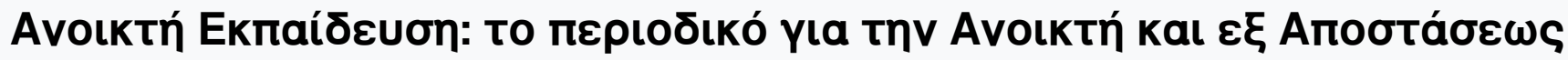

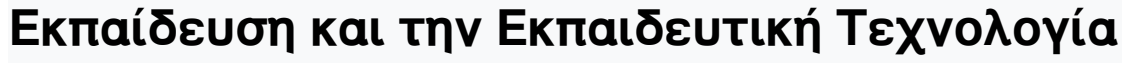

Tóp. 7, Ap. 1 (2011)

Open Education - The Journal for Open and Distance Education and Educational Technology

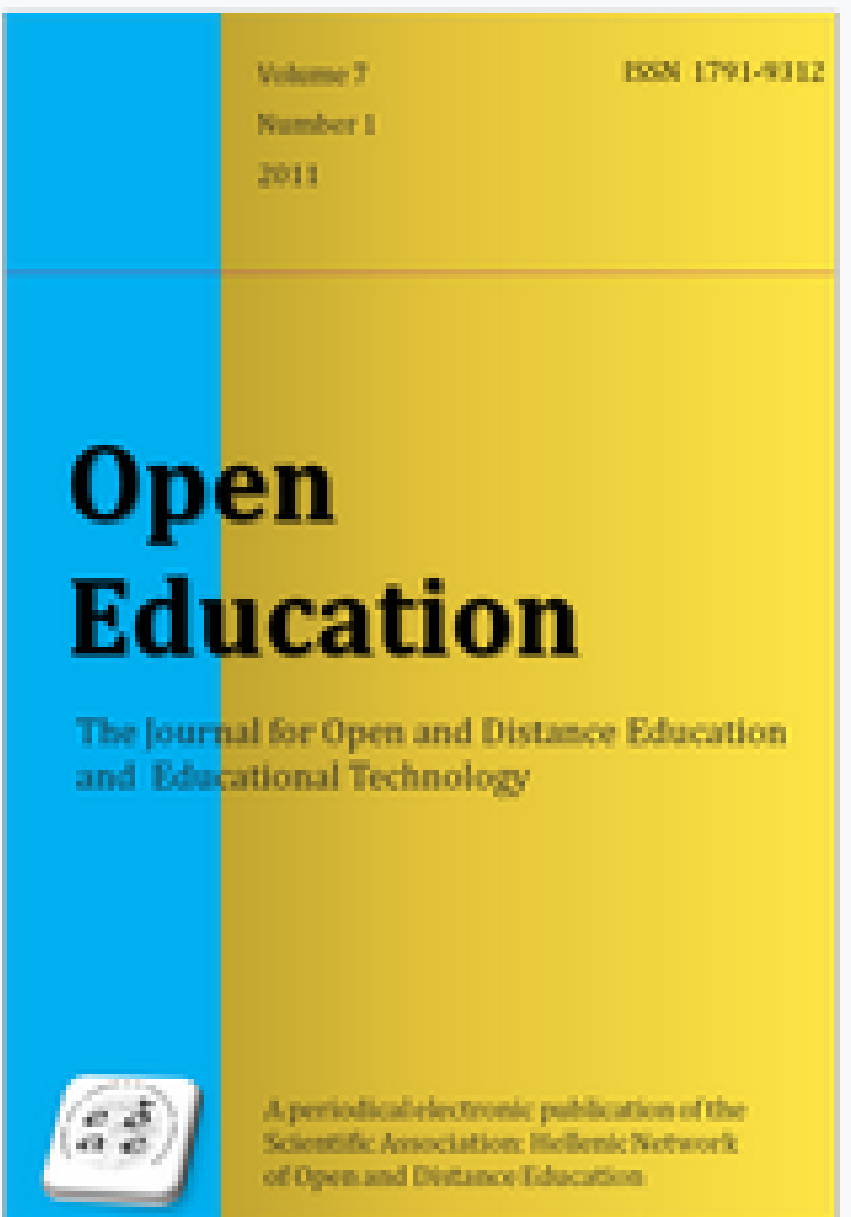

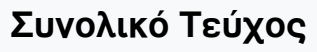

Full Issue

doi: $10.12681 /$ jode. 9848

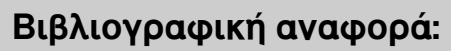


Volume 7

ISSN 1791-9312

Number 1

2011

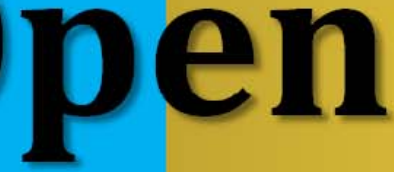

Education

The Journal for Open and Distance Education and Educational Technology

A periodical electronic publication of the Scientific Association: Hellenic Network of Open and Distance Education 


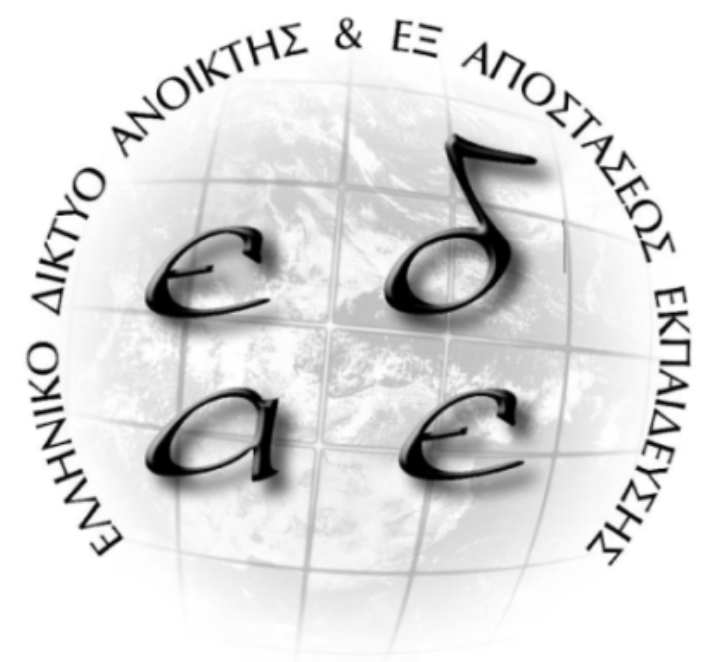

\section{Editorial Communication}

Antonis Lionarakis

Acssociate Professor

Hellenic Open Universirty \&

Hellenic Network of Open \& Distance Education

Sahtouri 23, 26222 Patra

Greece

E-mail : alionar@eap.gr

Web site : http://www.opennet.gr/

Gelly Manousou \& Antonia Maria Xartofylaka

Books for review should be addressed to the above postal address Hellenic Network of Open and Distance Education 2010 c OPEN EDUCATION ISSN: 1791-9312 
Open Education - The J oumal for Open and Distance Education and Educational Technology

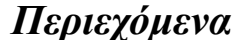

\begin{tabular}{|c|c|c|}
\hline & 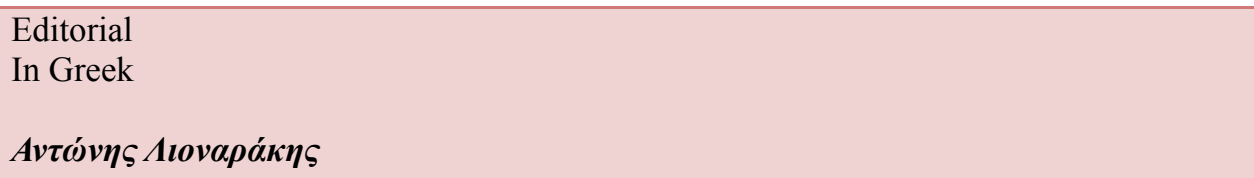 & 4 \\
\hline 1 & 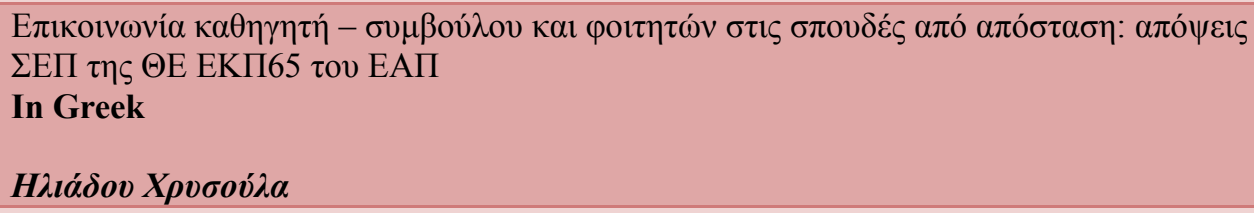 & 6 \\
\hline 2 & $\begin{array}{l}\text { A Proposed Sketch of a Distance Education Model for the Enrichment of Adult Literacy } \\
\text { in Pakistan } \\
\text { In English } \\
\text { Khalid Saleem, Mumtaz Ahmad }\end{array}$ & 21 \\
\hline 3 & 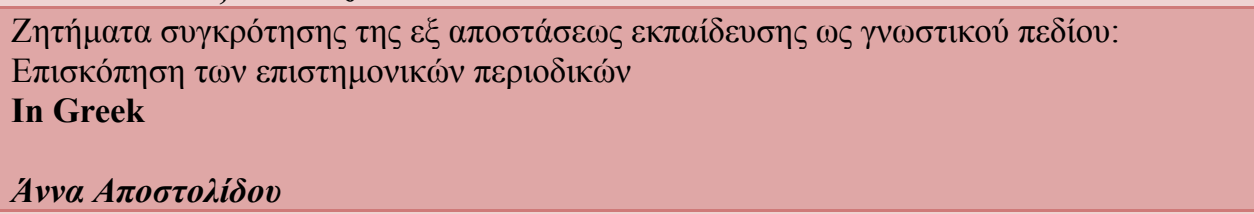 & 36 \\
\hline 4 & $\begin{array}{l}\text { What kind of Haptic devices and applications are needed in education? Requirements, } \\
\text { Specifications and hands-on experience derived from an IST project } \\
\text { In English } \\
\text { S. P. Christodoulou, D. M. Garyfallidou, G. S. Ioannidis, T. S. Papatheodorou, E. A. } \\
\text { Stathi }\end{array}$ & 54 \\
\hline 5 & 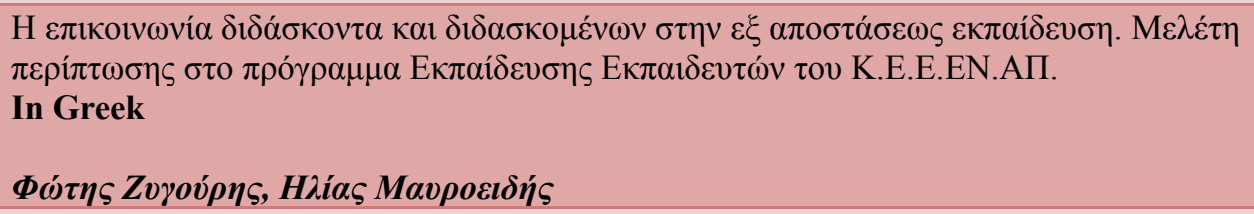 & 69 \\
\hline 6 & $\begin{array}{l}\text { Digital Radio broadcast: new technological resources to produce educational programs } \\
\text { online } \\
\text { In English } \\
\text { Teixeira, Marcelo Mendonça, Silva, Bento Duarte }\end{array}$ & 87 \\
\hline 7 & 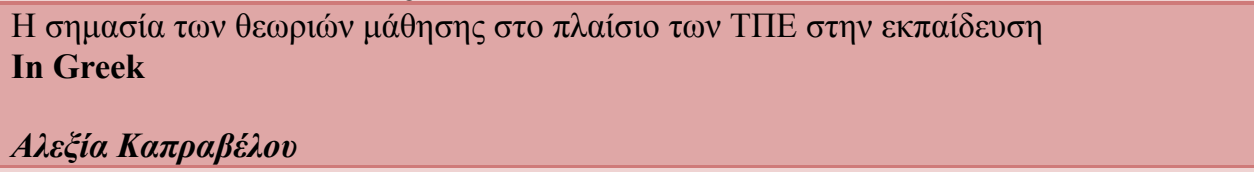 & 98 \\
\hline 8 & 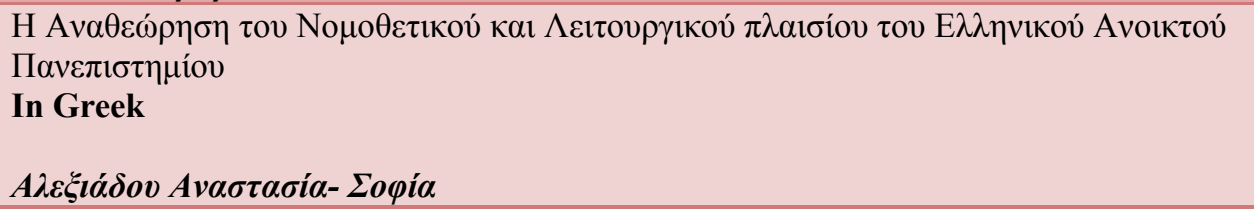 & 118 \\
\hline
\end{tabular}




\section{Editorial}

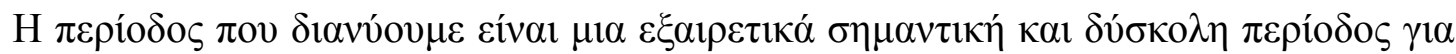

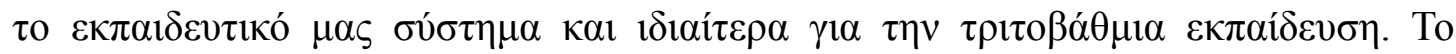

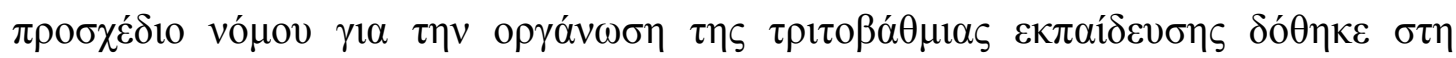

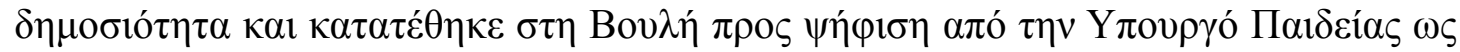

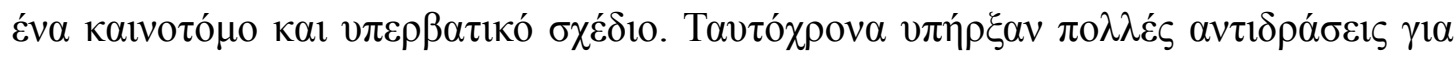

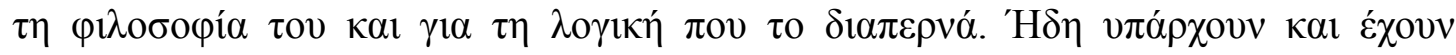

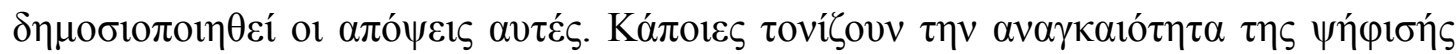

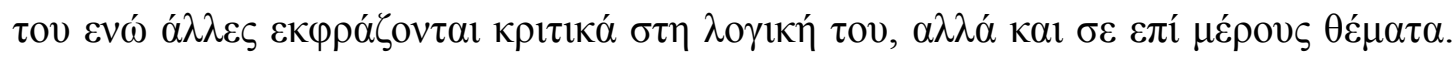

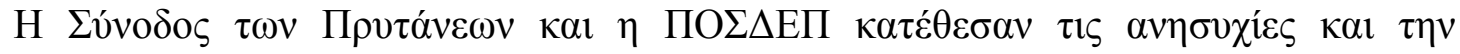

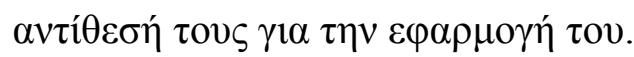

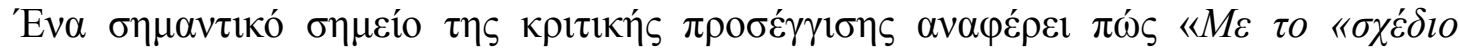
vó

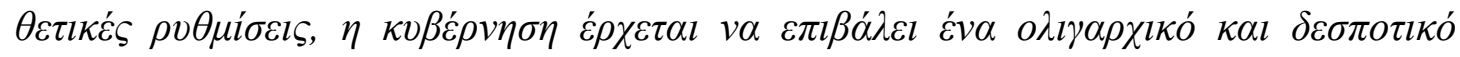

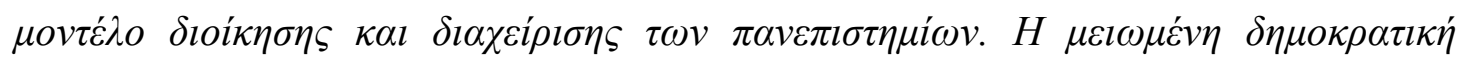

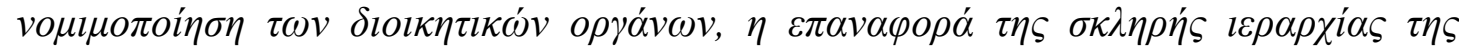

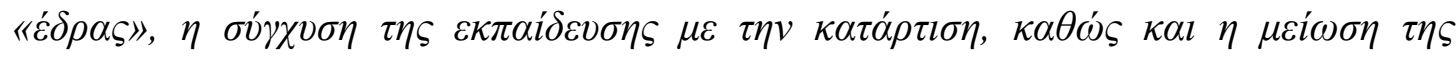

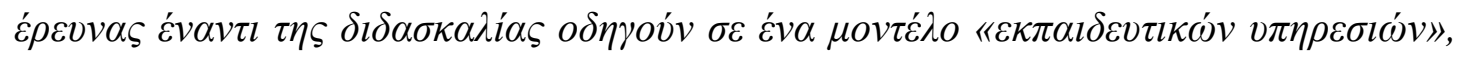

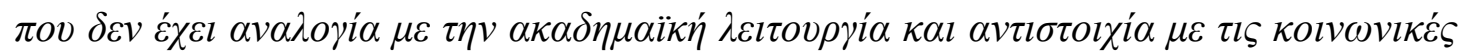

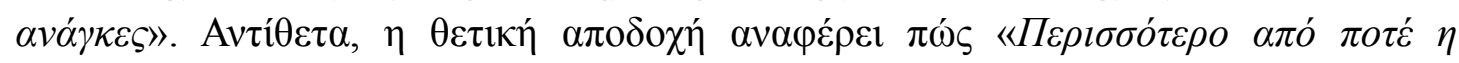

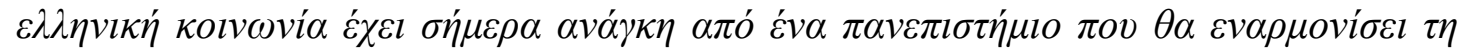

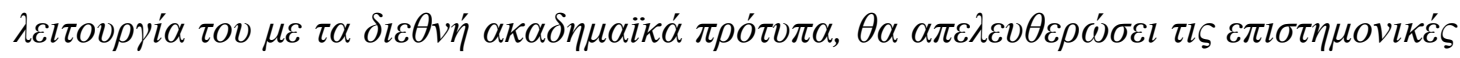

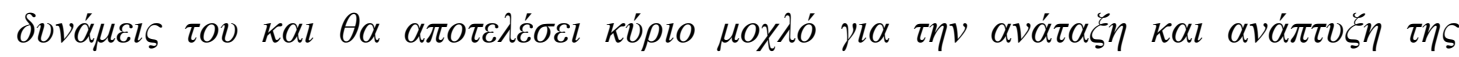

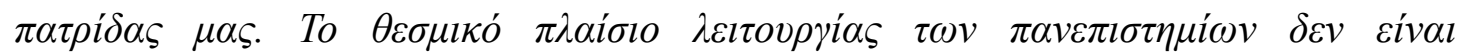

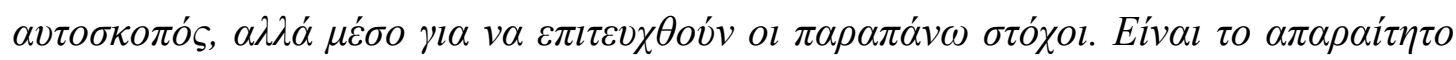

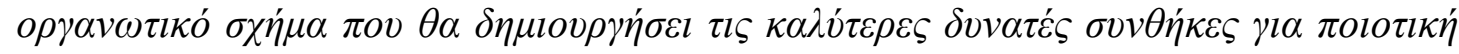

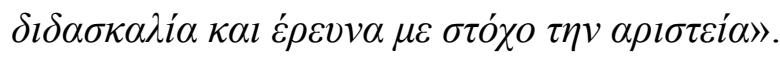

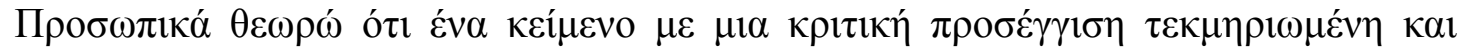

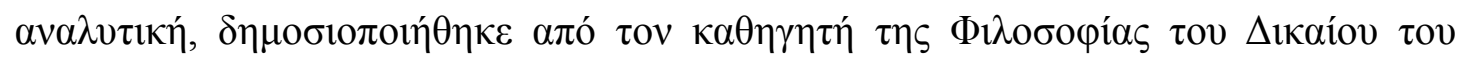

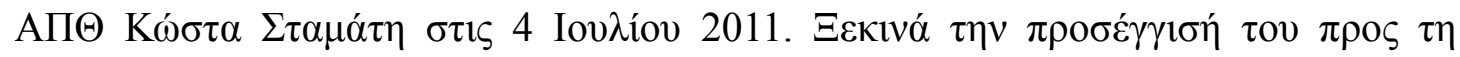

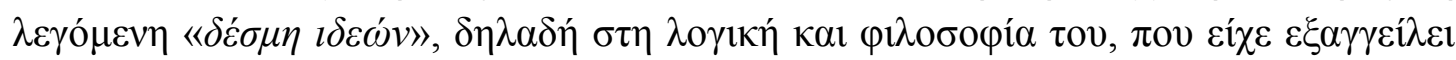

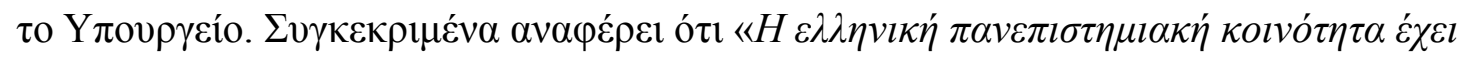




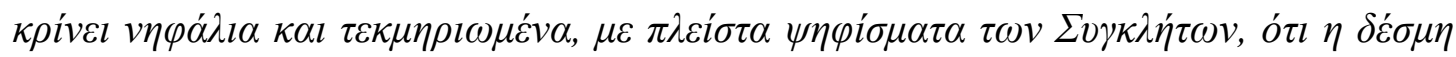

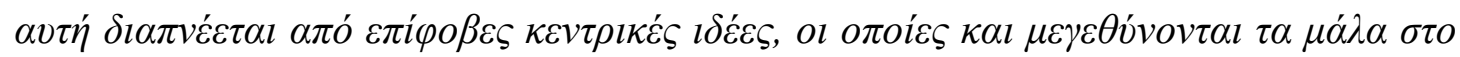

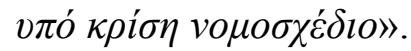

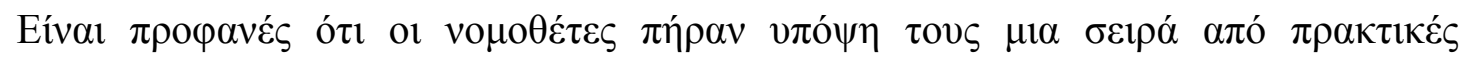

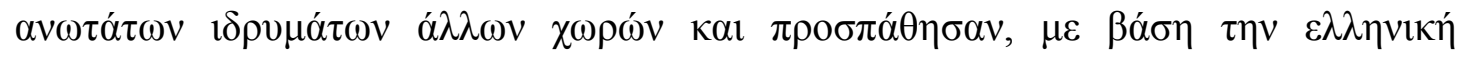

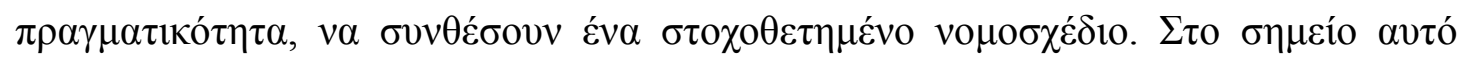

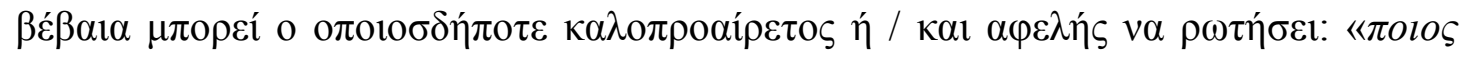

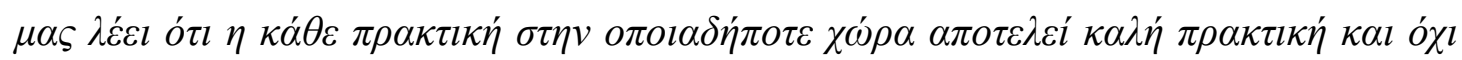

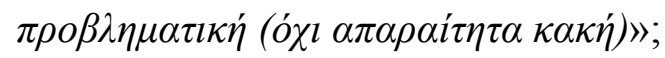

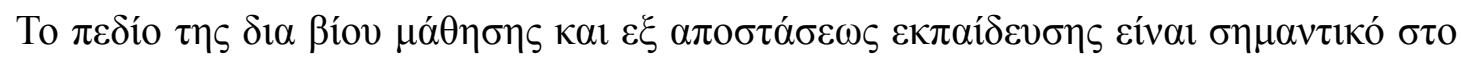

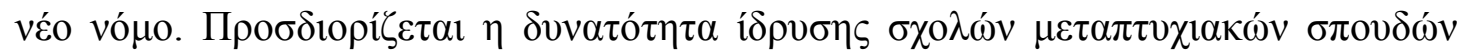

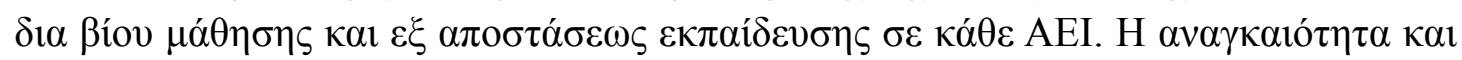

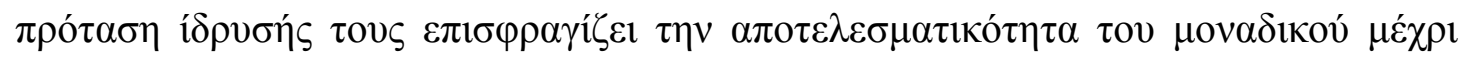

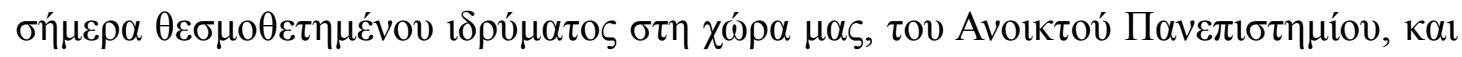

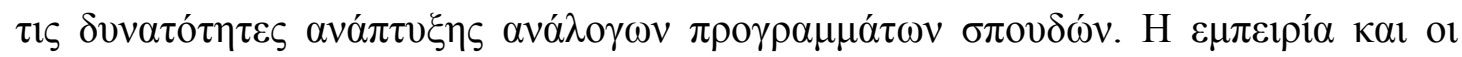

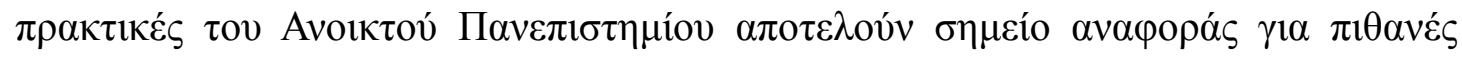

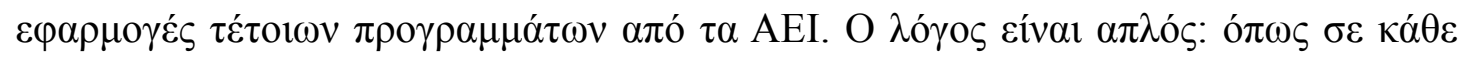

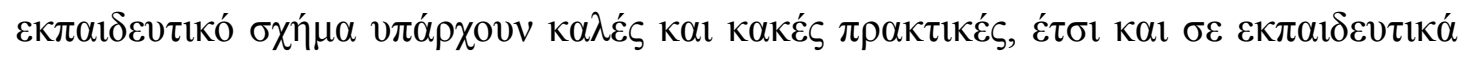

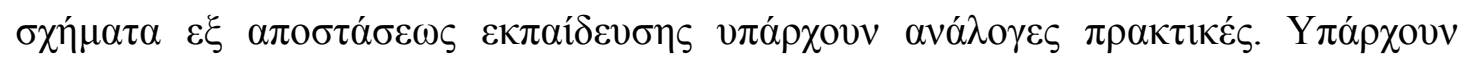

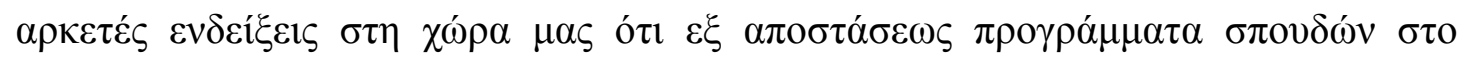

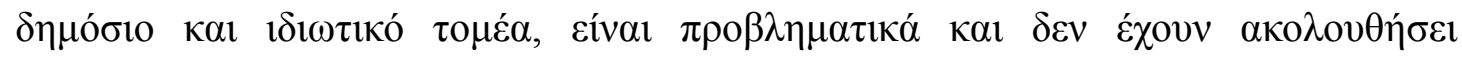

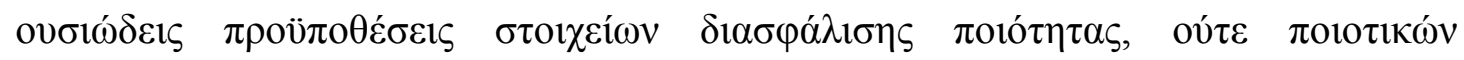

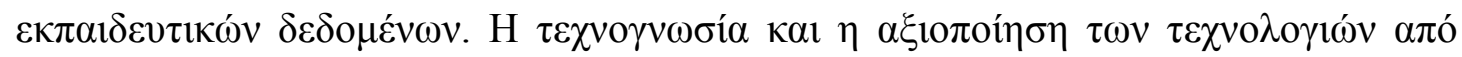

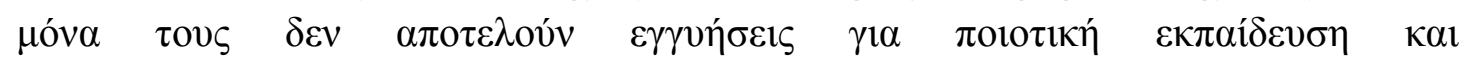

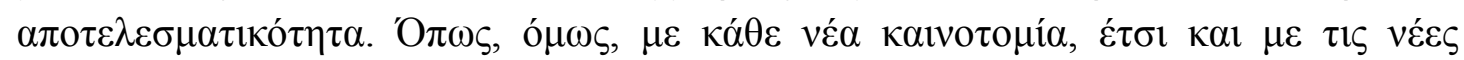

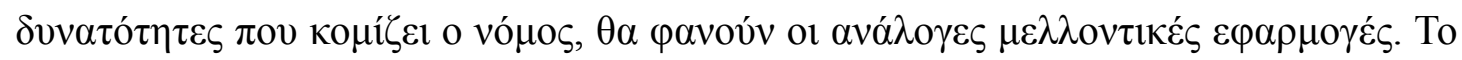

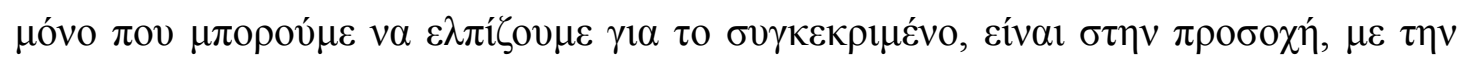

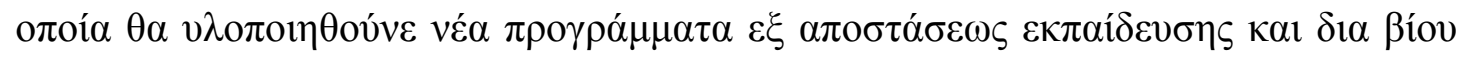
$\mu \alpha ́ \theta \eta \sigma \eta s$.

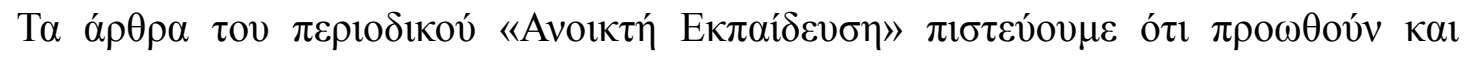

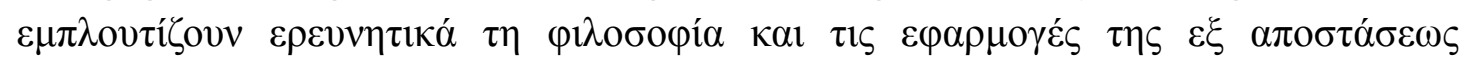

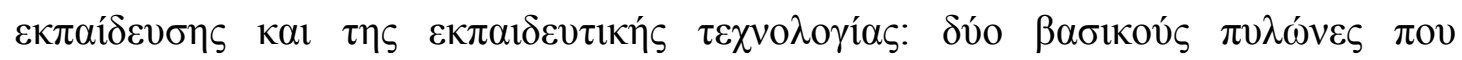

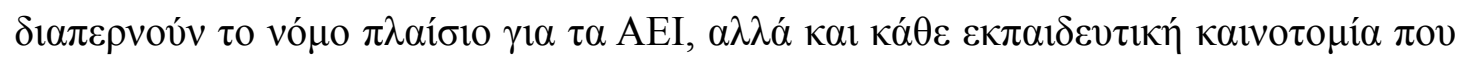

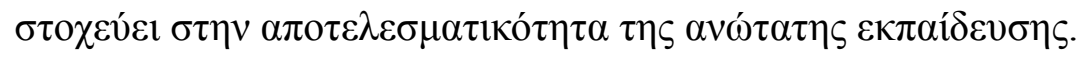




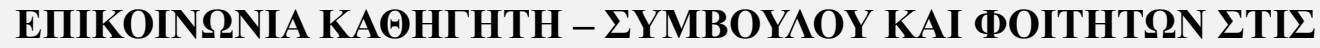 $\Sigma \Pi O Y \Delta E \Sigma$ АПО АПО $\Sigma$ TA $\Sigma H:$

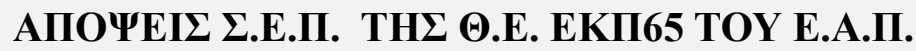

\section{COMMUNICATION BETWEEN TUTORS AND STUDENTS IN D.E.: A case study of the Hellenic Open University}

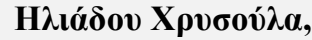

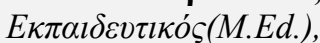
hrysouli@otenet.gr

\section{Пєрí $\eta \psi \eta$}

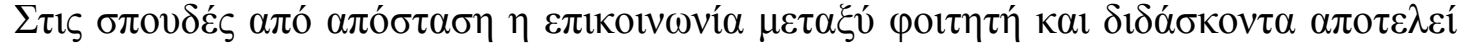

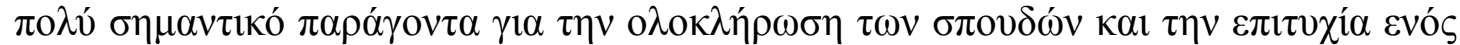

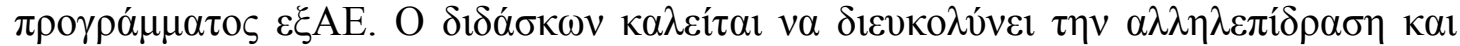

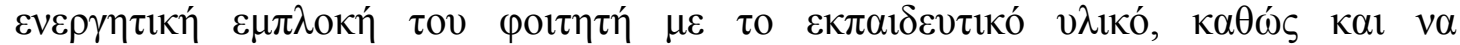

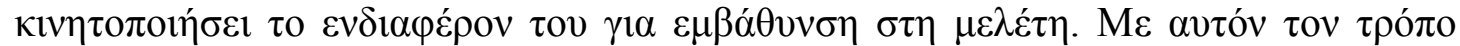

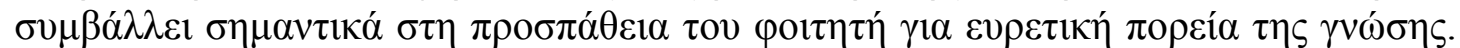

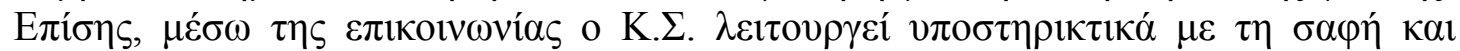

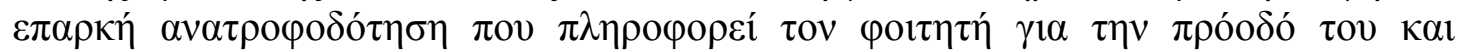

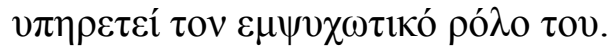

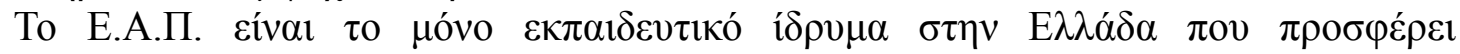

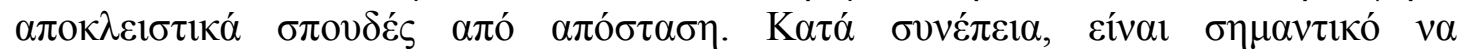

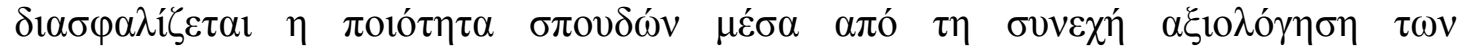

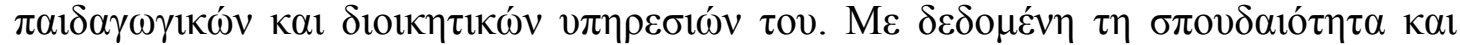

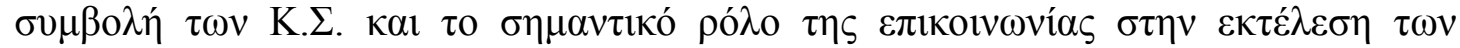

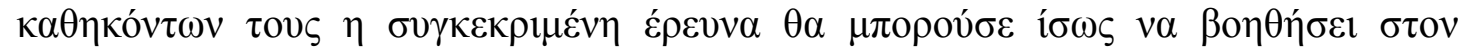

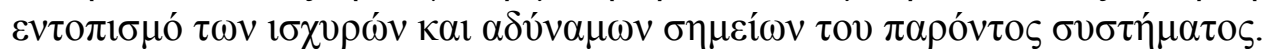

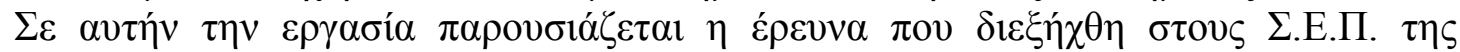

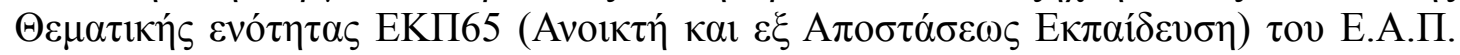

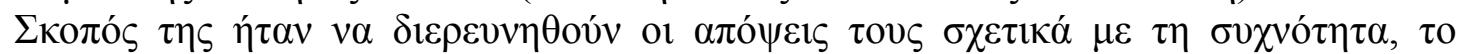

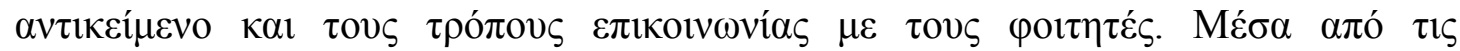

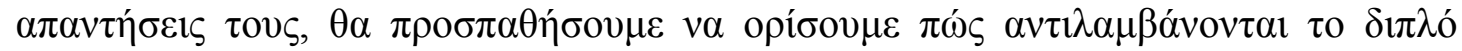

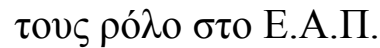

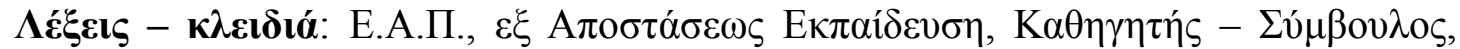

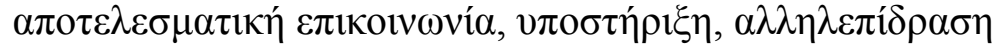

\footnotetext{
Abstract

In Distance Learning programs communication between students and tutors is a key factor contributing to the completion of studies and the success of the programme. By means of this communication the tutor can facilitate the interaction and active involvement of students with the learning material, and motivate their interest in deeper understanding. Thus, they contribute to students' effort towards discovery of knowledge. In addition, effective communication can act as support for the student through clear and adequate feedback, which informs the students of their progress and provides vital encouragement.

In this paper we present the survey which was administered to tutors of the course module EDU65 (Open and Distance Education) of the Hellenic Open University. The objective of the survey was to study their views on the frequency, subject and ways of communication with the students. Through their answers we try to determine their perception of the dual role they play in H.O.U.
} 


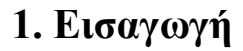

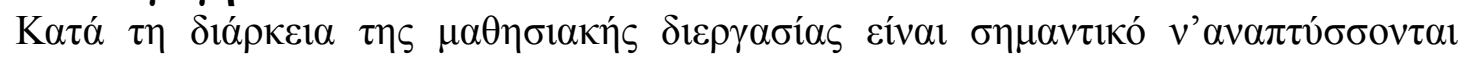

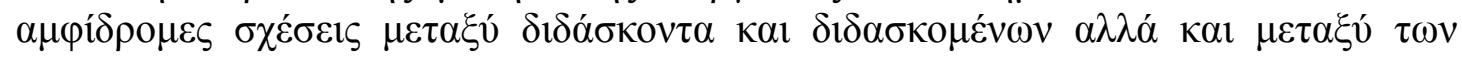

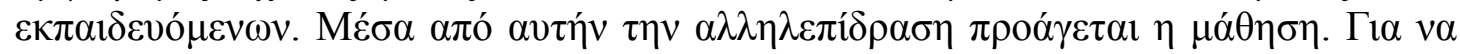

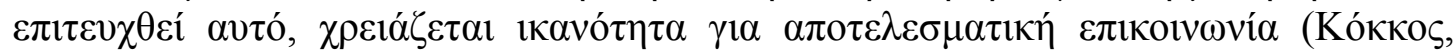

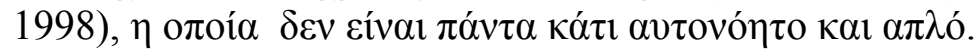

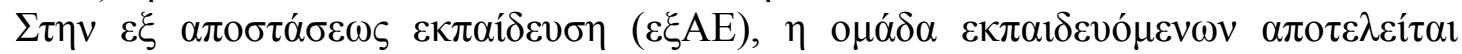

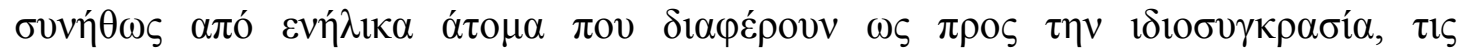

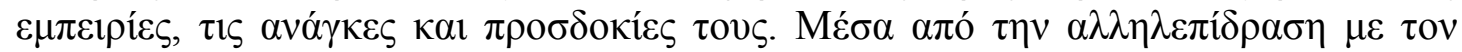

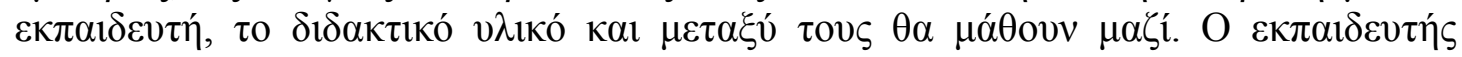

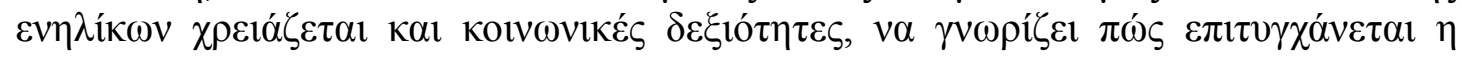

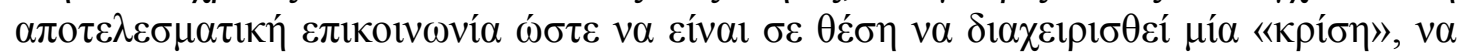

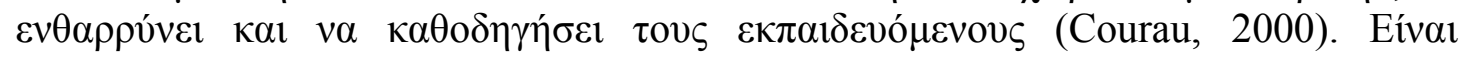

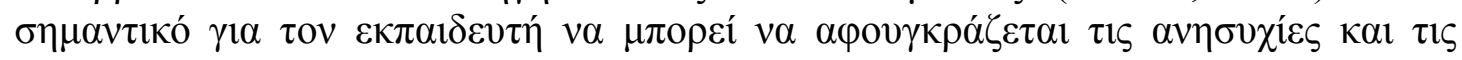

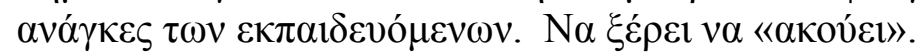

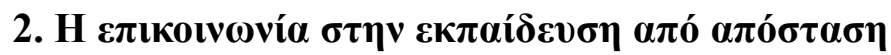

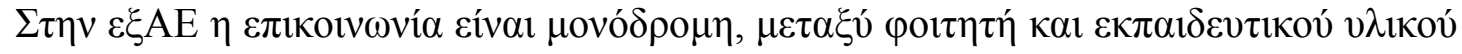

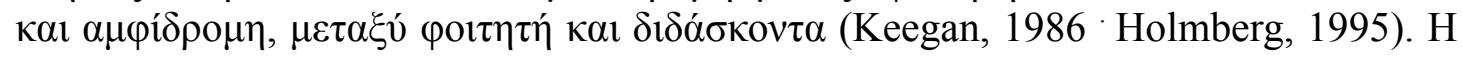

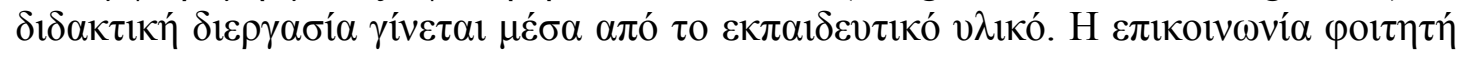

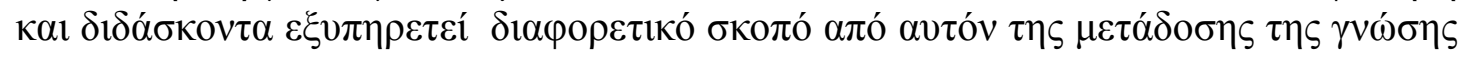

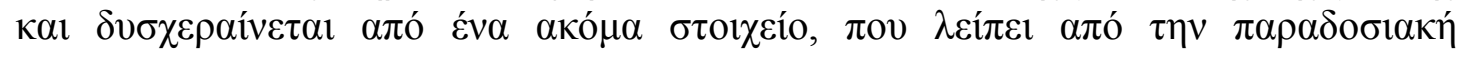

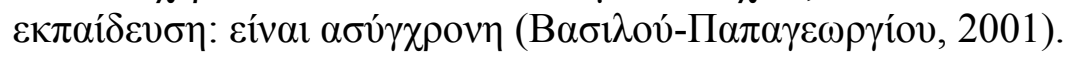

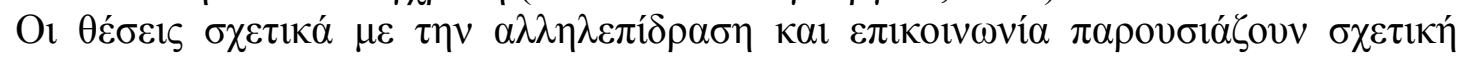

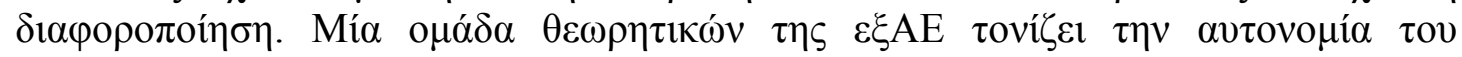

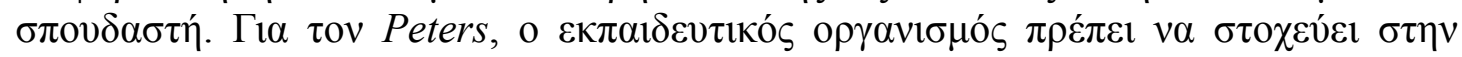

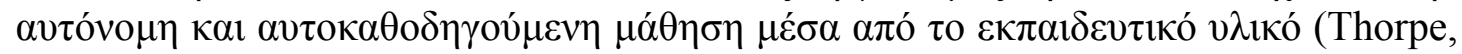

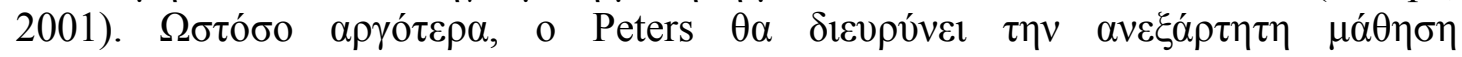

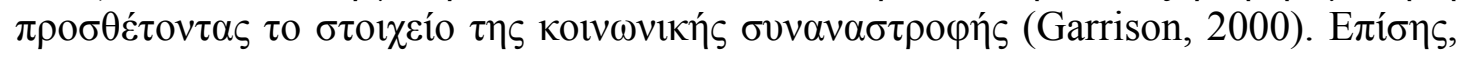

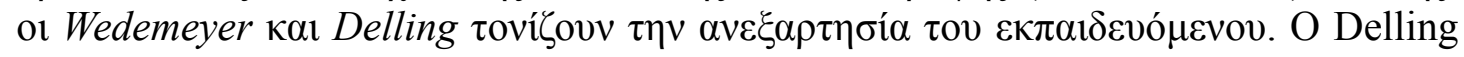

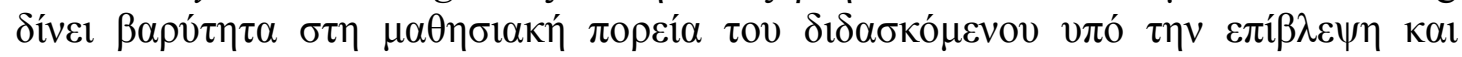

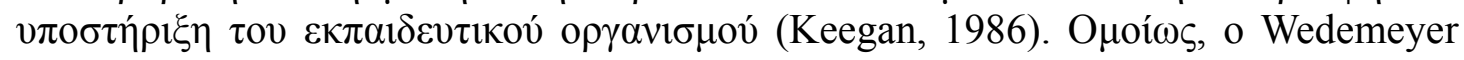

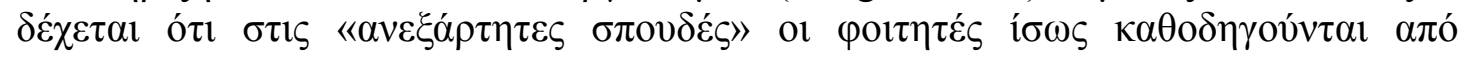

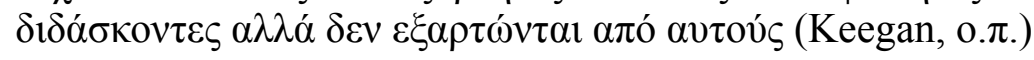

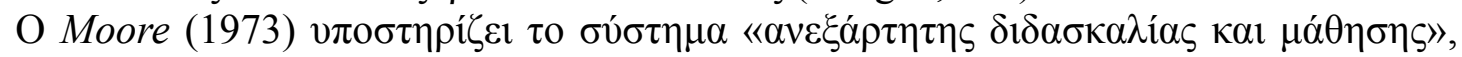

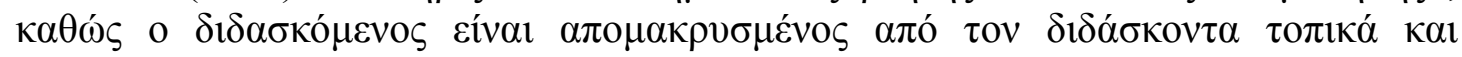

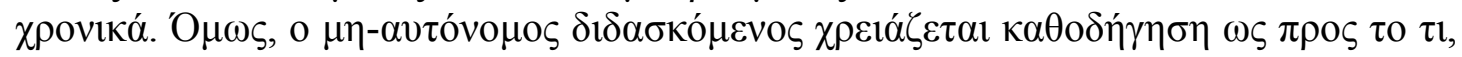

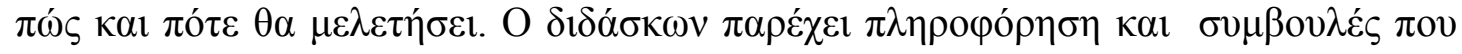

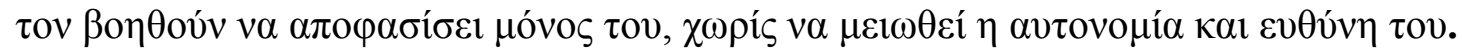

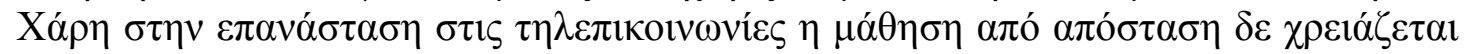

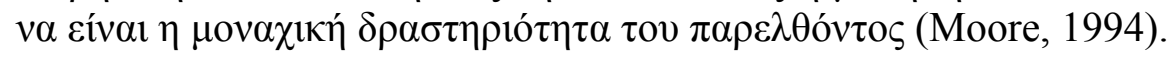

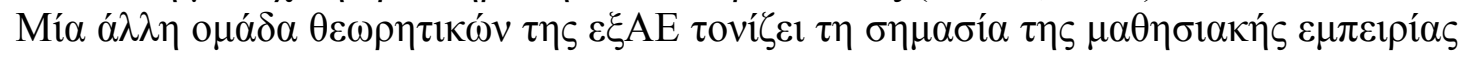

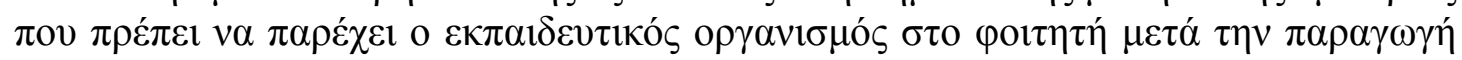

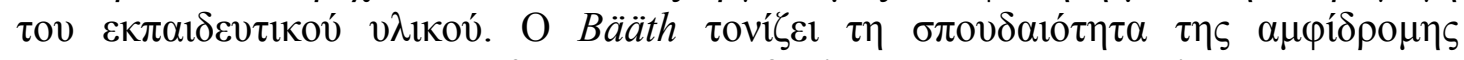

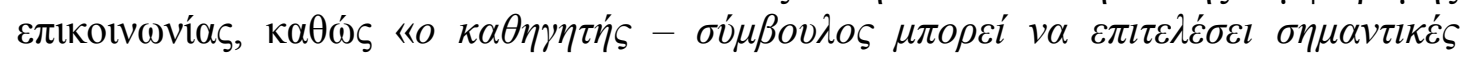

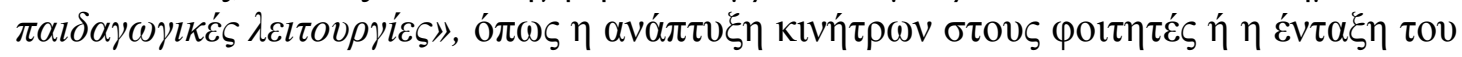

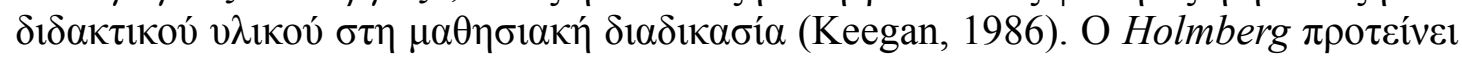

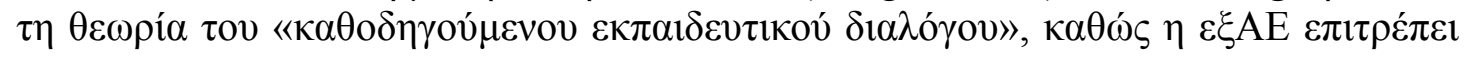




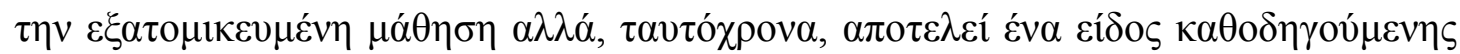

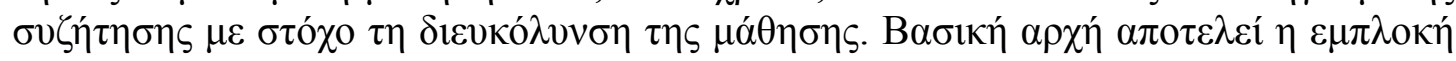

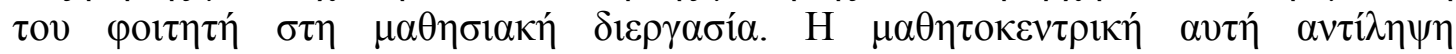

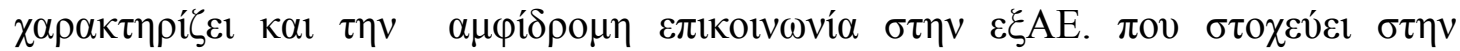

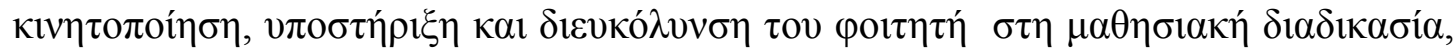

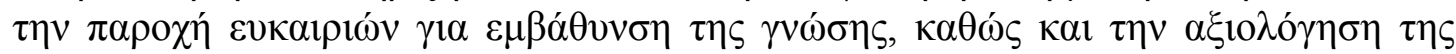

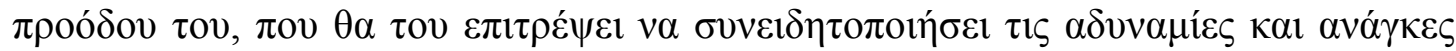

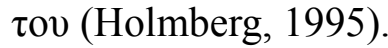

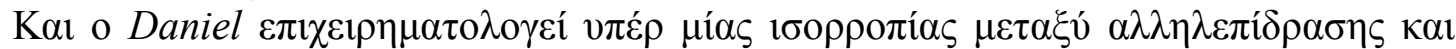

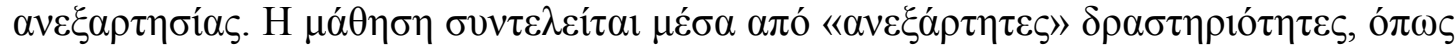

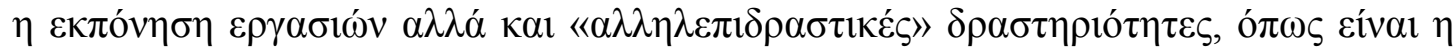

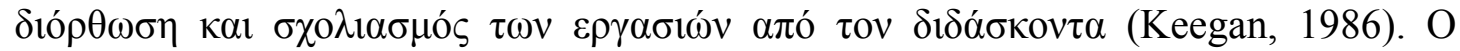

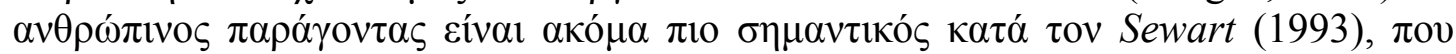

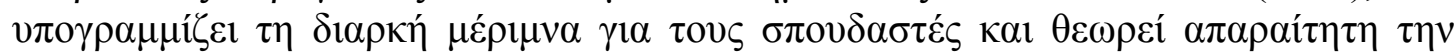

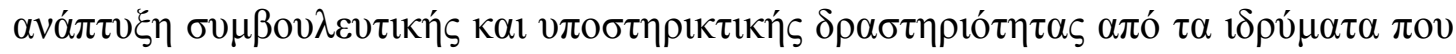

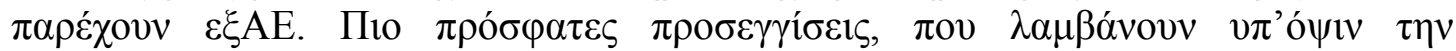

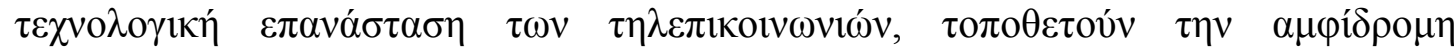

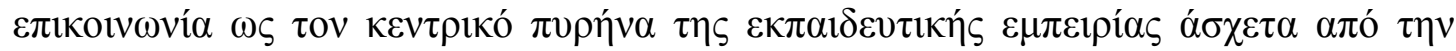

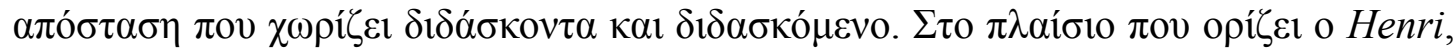

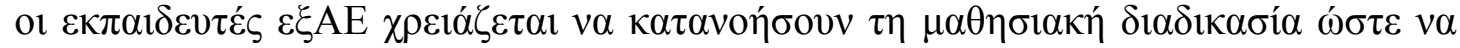

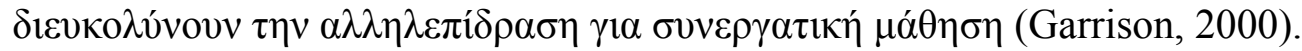

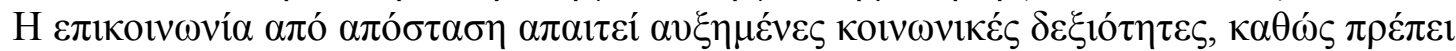

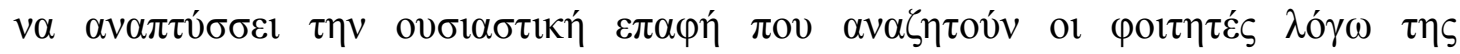

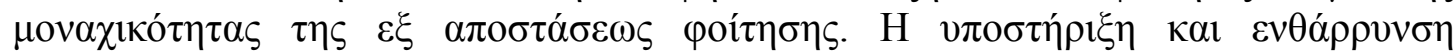

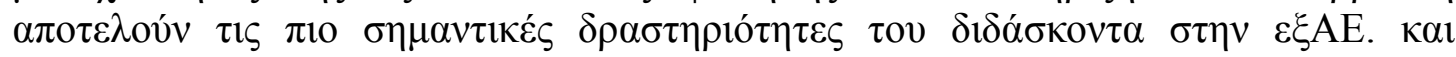

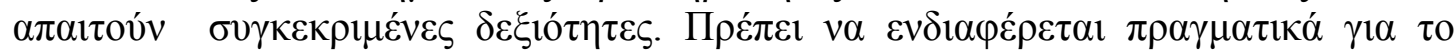

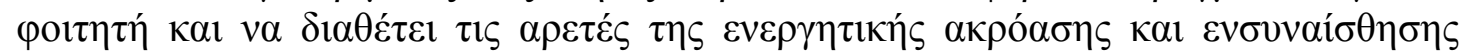

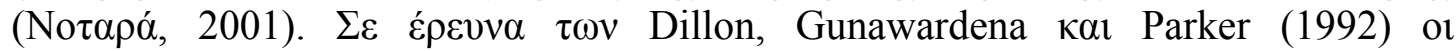

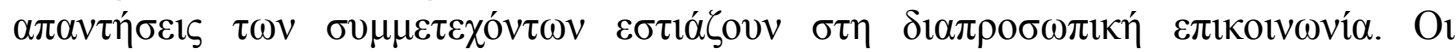

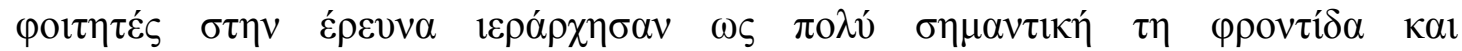

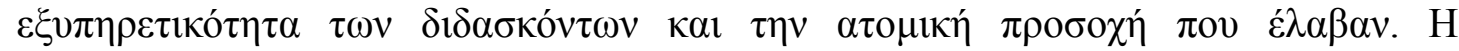

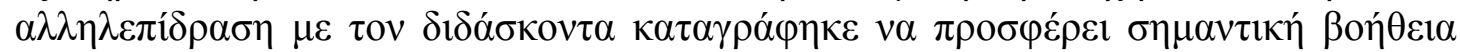

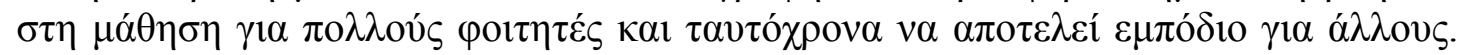

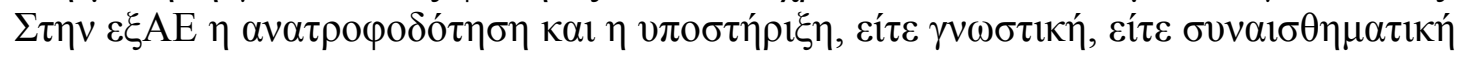

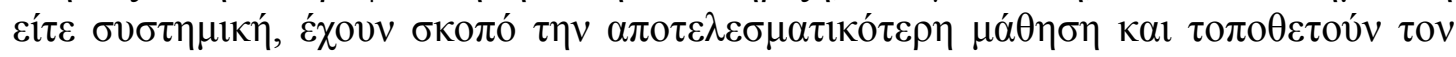


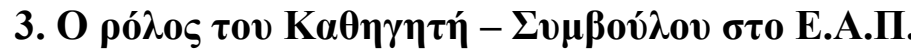

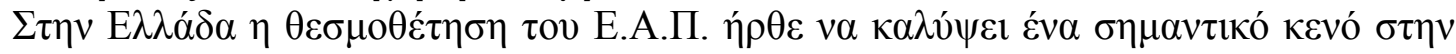

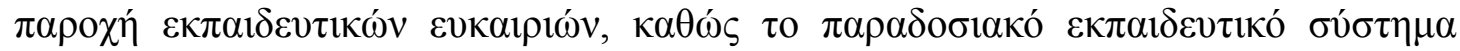

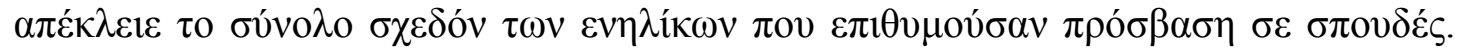

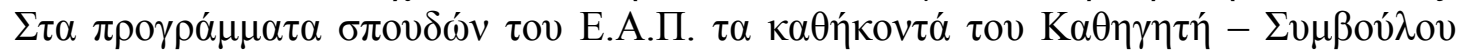

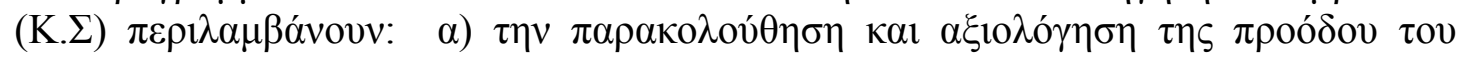

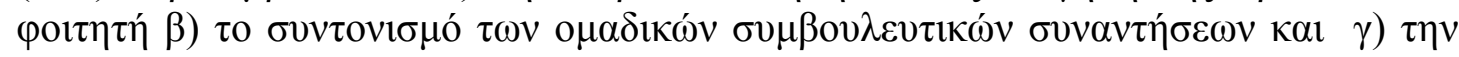

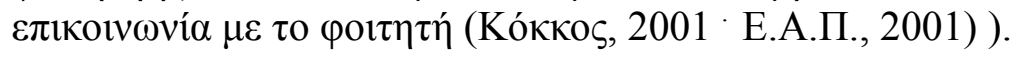

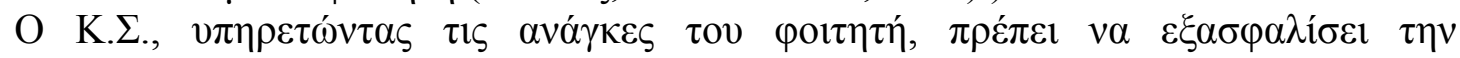

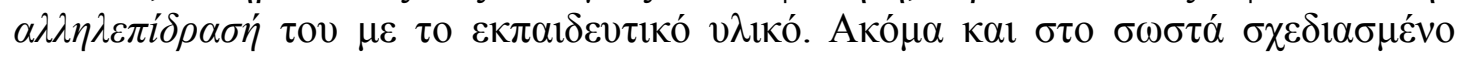

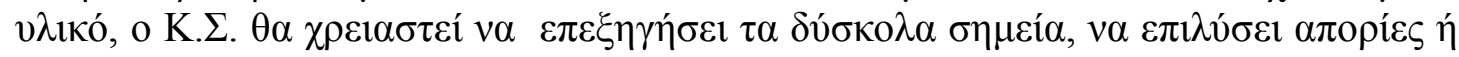

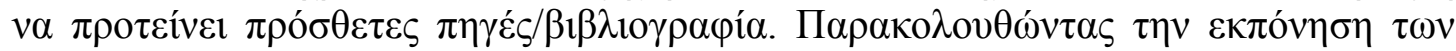

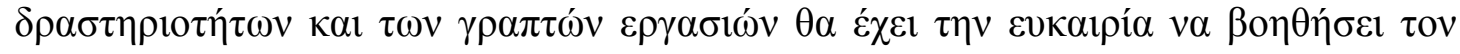




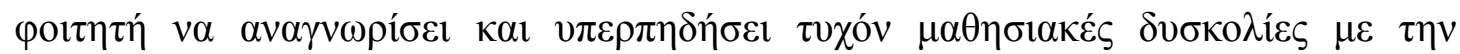

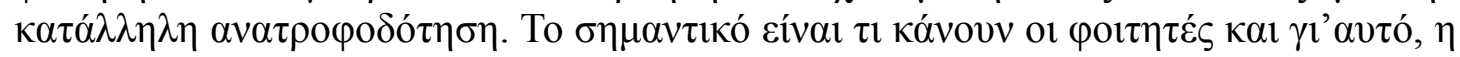

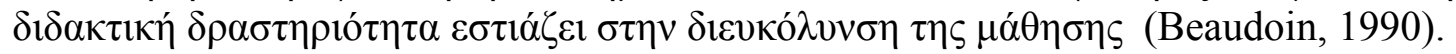

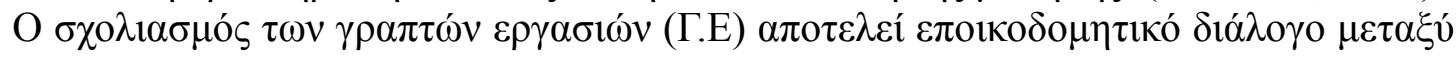

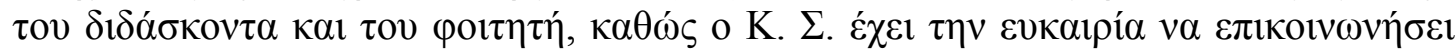

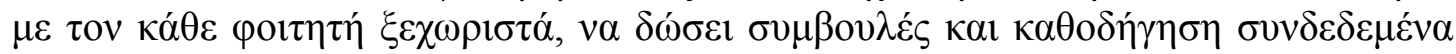

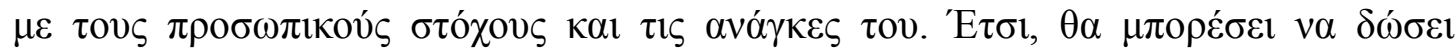

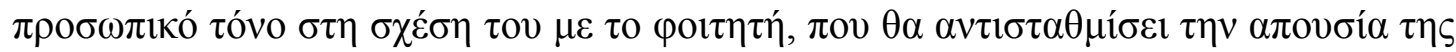

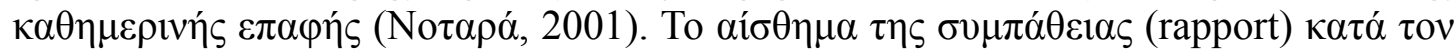

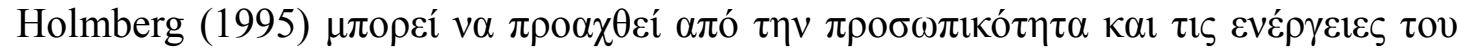

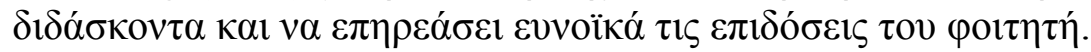

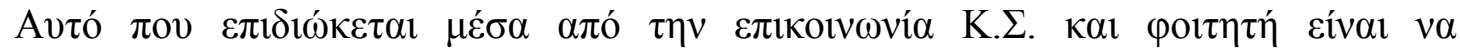

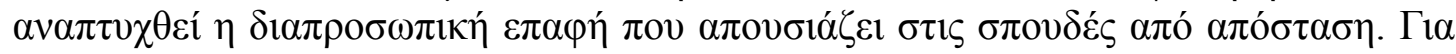

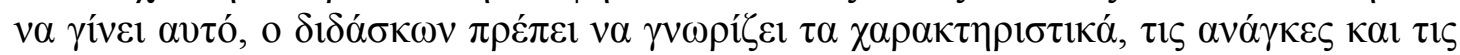

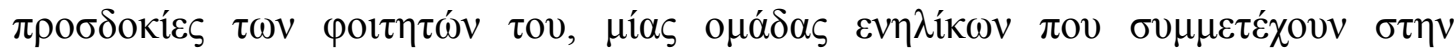
$\varepsilon \kappa \pi \alpha \imath \delta \varepsilon v \tau \imath$ เ

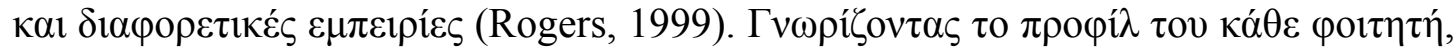

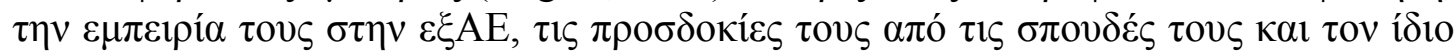

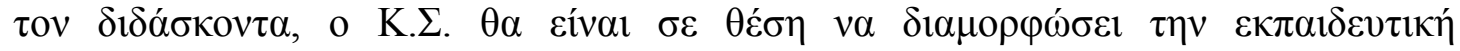

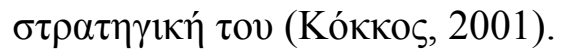

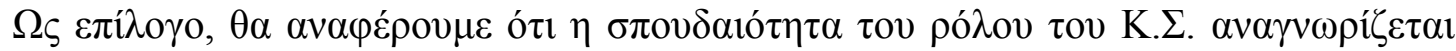

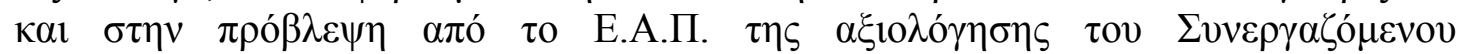

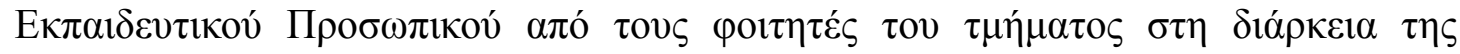

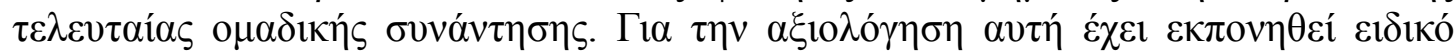

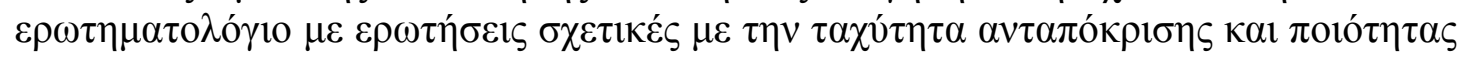

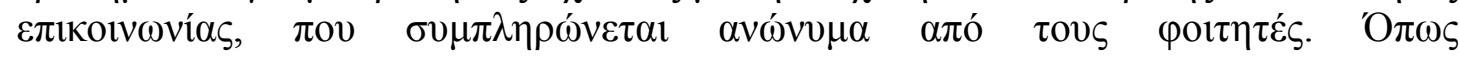

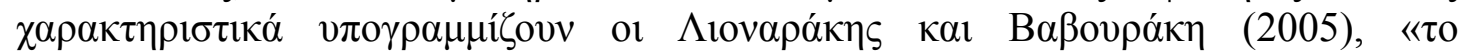

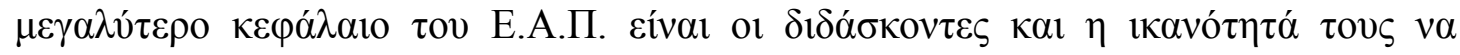

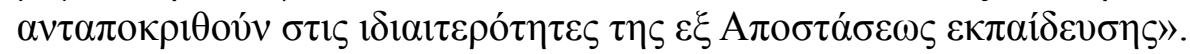

\section{H épevva}

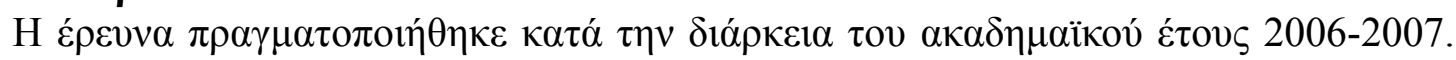

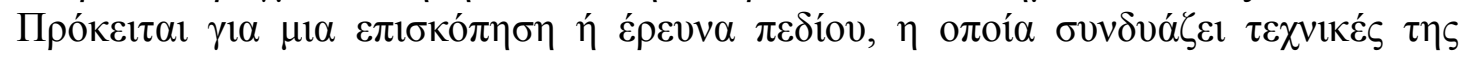

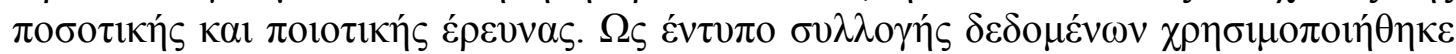

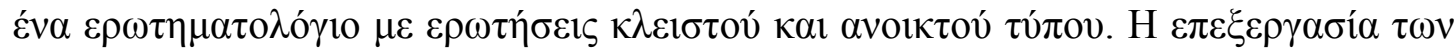

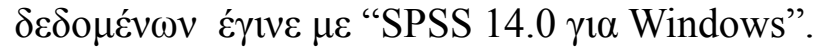

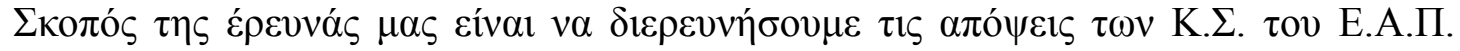

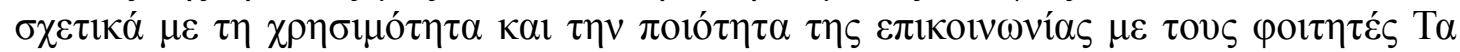

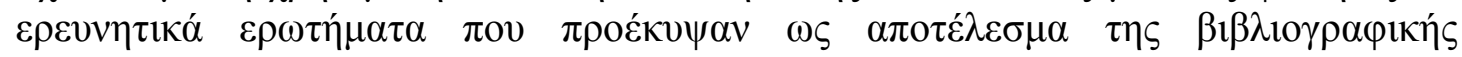

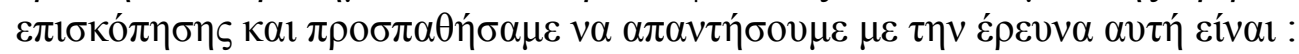

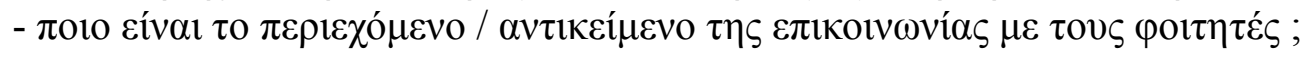

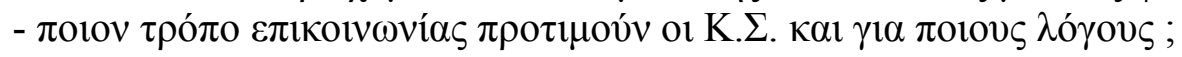

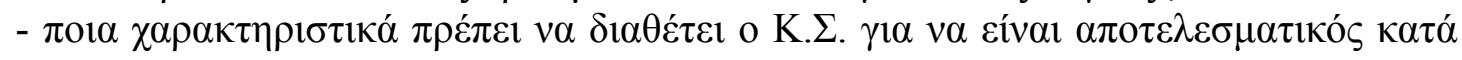

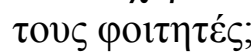

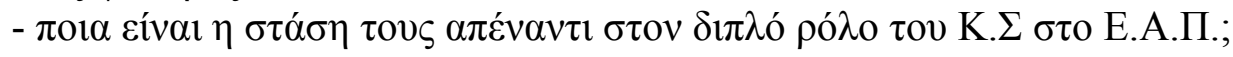

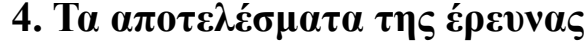

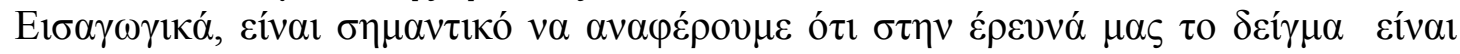

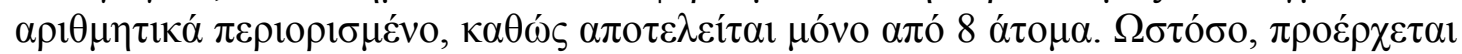

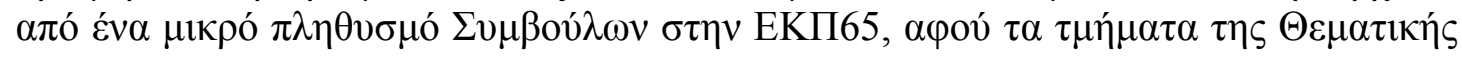




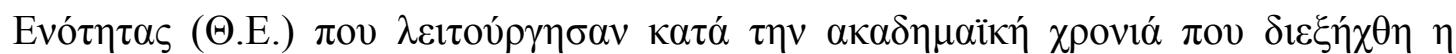

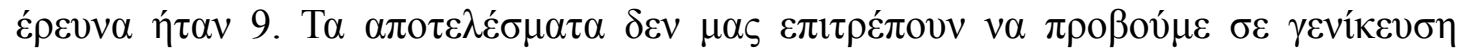

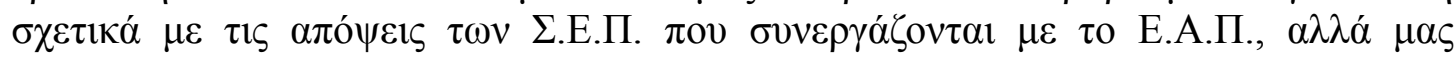

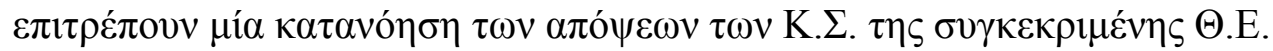

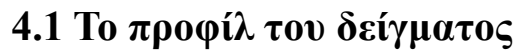

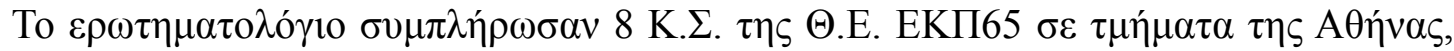

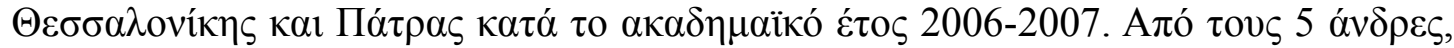

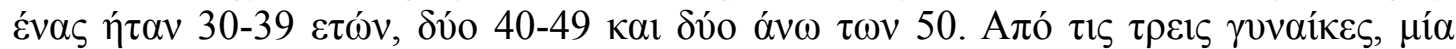

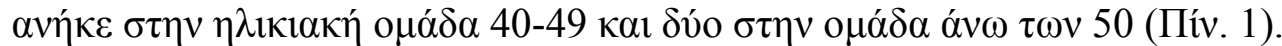

\begin{tabular}{|c|c|c|c|c|c|}
\hline \multirow{2}{*}{\multicolumn{2}{|c|}{ 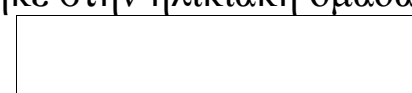 }} & \multicolumn{3}{|c|}{ n入ıкі́a } & \multirow[b]{2}{*}{ 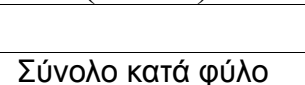 } \\
\hline & & $30-39$ & $40-49$ & $>50$ & \\
\hline \multirow{2}{*}{ 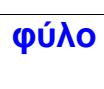 } & ávōpas & 1 & 2 & 2 & 5 \\
\hline & puvaíka & 0 & 1 & 2 & 3 \\
\hline \multicolumn{2}{|c|}{ 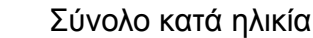 } & 1 & 3 & 4 & 8 \\
\hline
\end{tabular}

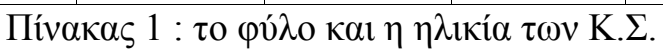

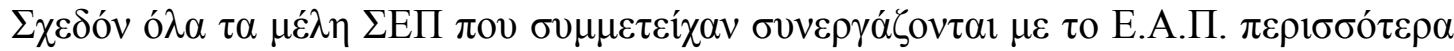

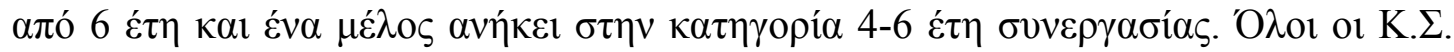

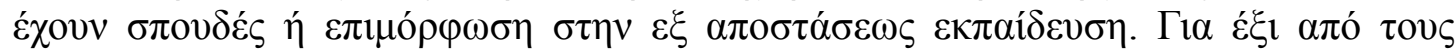

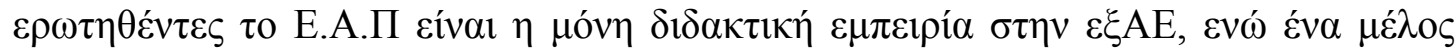

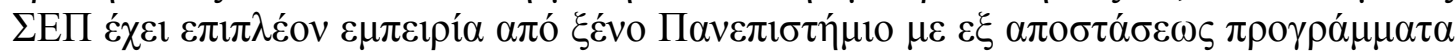

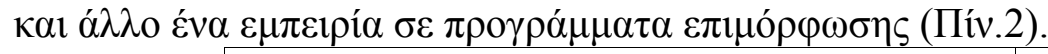

\begin{tabular}{|c|c|}
\hline 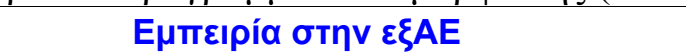 & 乏uxvótпта \\
\hline 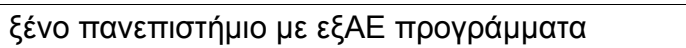 & 1 \\
\hline 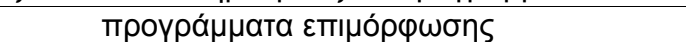 & 1 \\
\hline$\mu$ $\mu$ vo тo EAП & 6 \\
\hline$\Sigma$ ¿́voגo & 8 \\
\hline
\end{tabular}

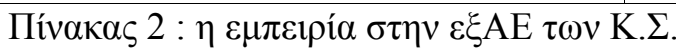

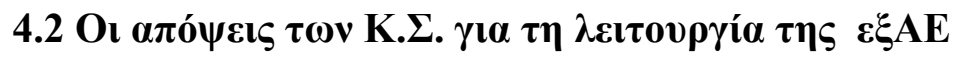

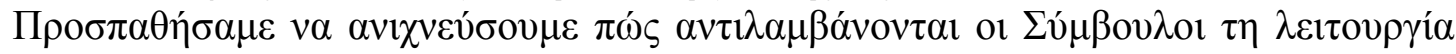

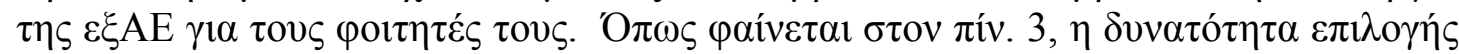

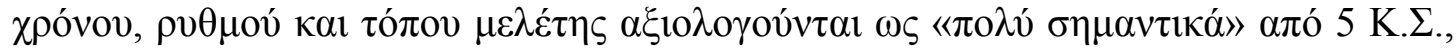

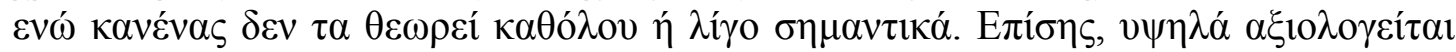

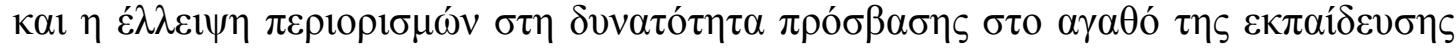

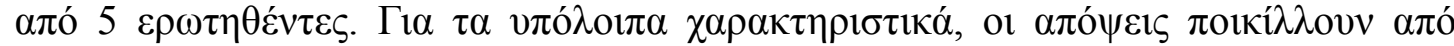

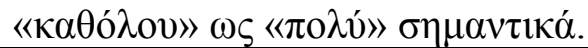

\begin{tabular}{|c|c|c|c|c|c|c|c|}
\hline & 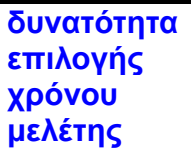 & 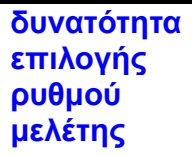 & 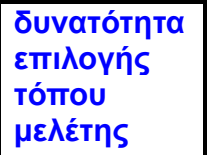 & 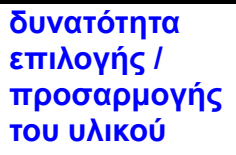 & 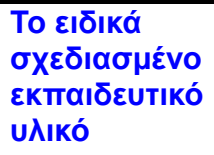 & 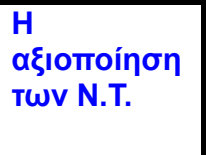 & 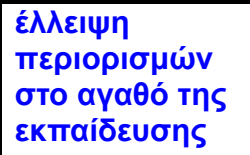 \\
\hline & \multicolumn{7}{|c|}{ 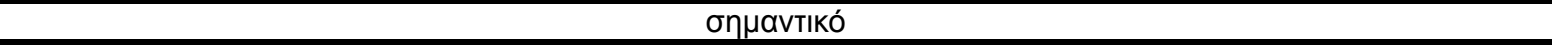 } \\
\hline kaӨóđou & & & & & & & 1 \\
\hline גíyo & & & & 2 & & 3 & 1 \\
\hline$\mu \varepsilon \dot{\tau} \rho \rho \alpha$ & & 1 & 1 & 3 & 2 & 2 & 1 \\
\hline apкहTá & 3 & 2 & 2 & 2 & 3 & 1 & \\
\hline по入и́ & 5 & 5 & 5 & 1 & 3 & 2 & 5 \\
\hline 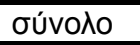 & 8 & 8 & 8 & 8 & 8 & 8 & 8 \\
\hline
\end{tabular}

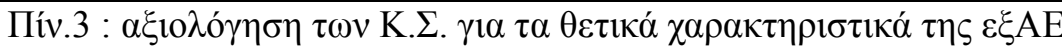

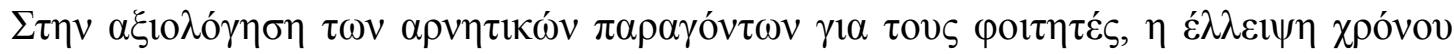

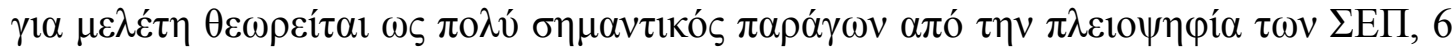

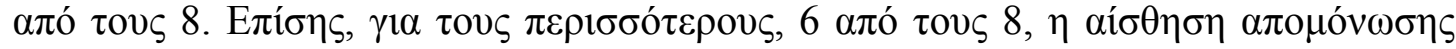

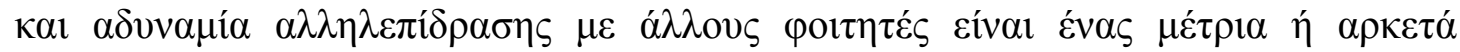




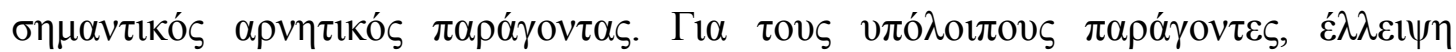

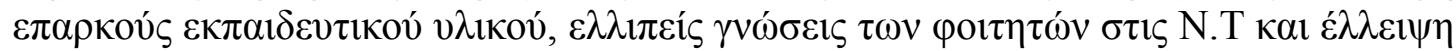

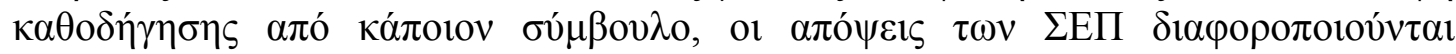

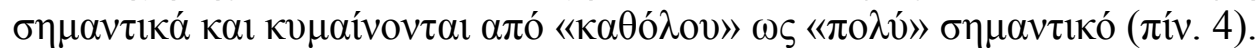

\begin{tabular}{|c|c|c|c|c|c|}
\hline & 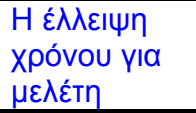 & 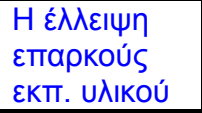 & 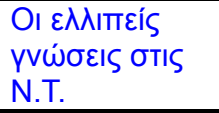 & 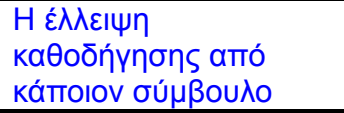 & 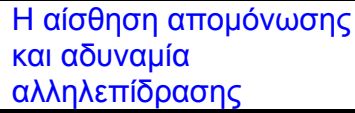 \\
\hline \multicolumn{6}{|c|}{ 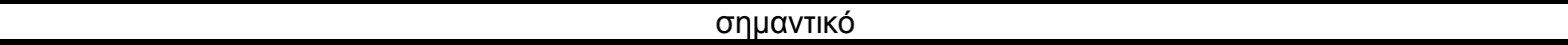 } \\
\hline кaӨó^ou & & 1 & 1 & 2 & \\
\hline Aíyo & & 2 & 2 & 3 & 1 \\
\hline 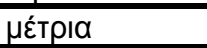 & 1 & 3 & 3 & 1 & 3 \\
\hline 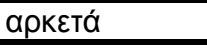 & 1 & 2 & & & 3 \\
\hline по入и́ & 6 & & 2 & 1 & 1 \\
\hline 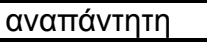 & & & & 1 & \\
\hline oúvoגo & 8 & 8 & 8 & 8 & 8 \\
\hline
\end{tabular}

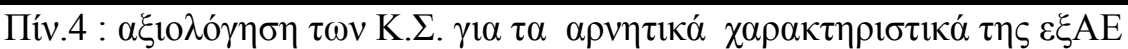

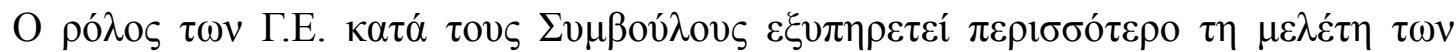

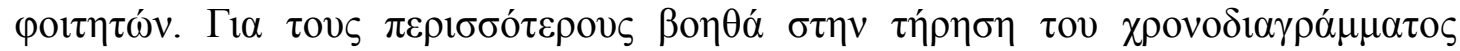

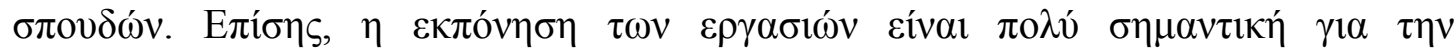

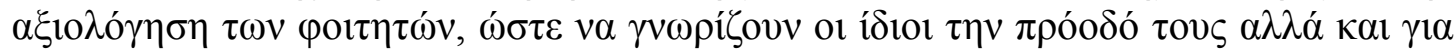

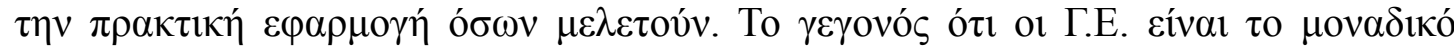

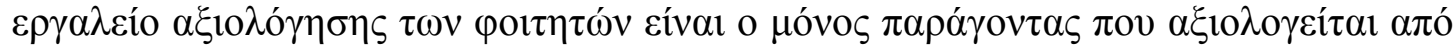

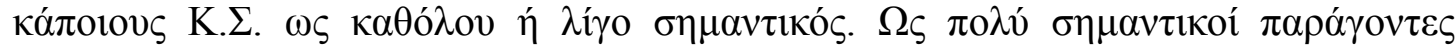

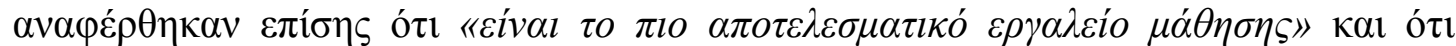

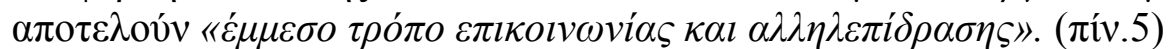

\begin{tabular}{|c|c|c|c|c|c|}
\hline & 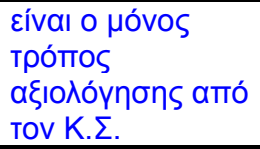 & 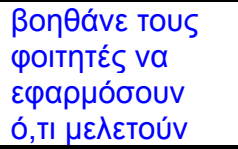 & 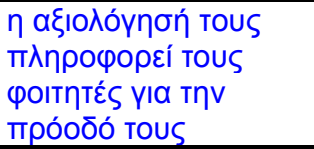 & 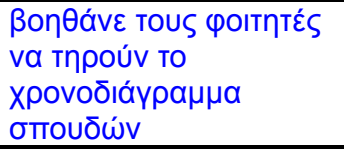 & 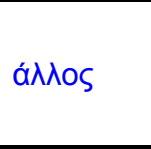 \\
\hline \multicolumn{6}{|c|}{ 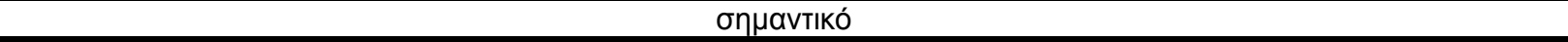 } \\
\hline кaӨóภou & 2 & & & & \\
\hline Aíyo & 1 & & & & \\
\hline 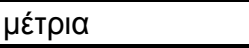 & & 1 & 2 & & \\
\hline аркєтó & 5 & 2 & & 1 & \\
\hline Tாо入ú & & 5 & 6 & 7 & 2 \\
\hline$\sigma u ́ v o \lambda o$ & 8 & 8 & 8 & 8 & \\
\hline
\end{tabular}

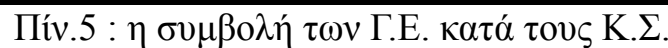

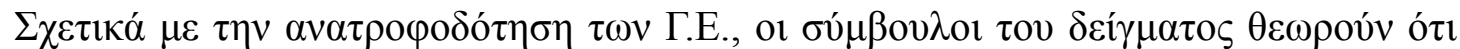

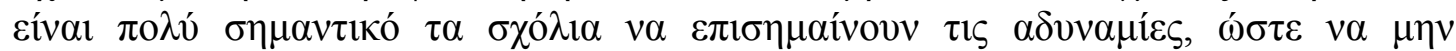

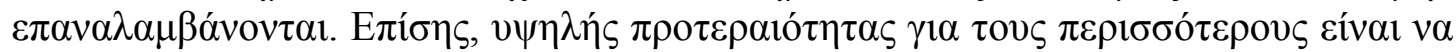

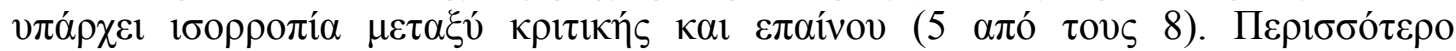

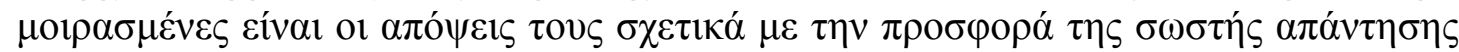

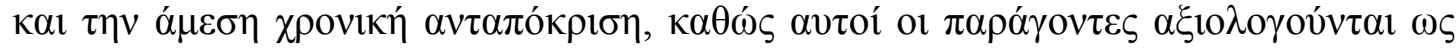

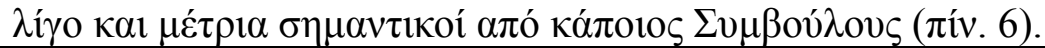

\begin{tabular}{|c|c|c|c|c|}
\hline & 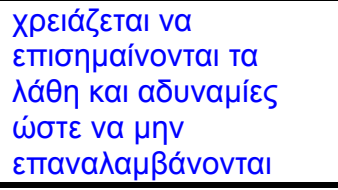 & 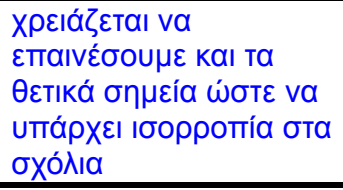 & 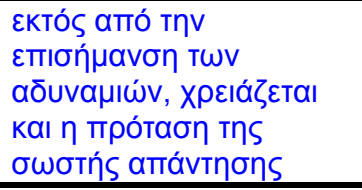 & 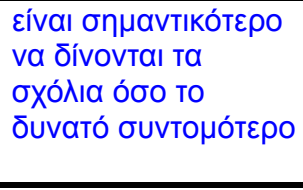 \\
\hline \multicolumn{5}{|c|}{ 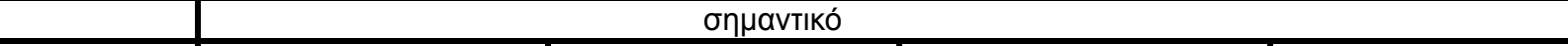 } \\
\hline кaӨó^ou & & & & \\
\hline Aíyo & & & & 1 \\
\hline 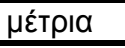 & & 1 & 1 & 4 \\
\hline 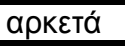 & 1 & 2 & 3 & 1 \\
\hline
\end{tabular}


Open Education - The J oumal for Open and Distance Education and Educational Technology

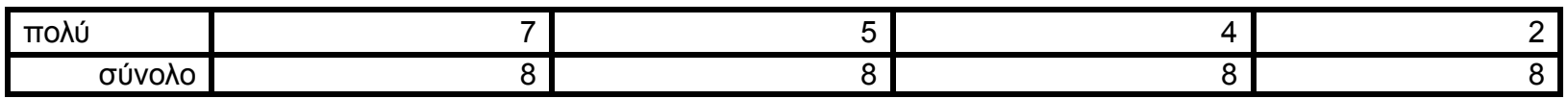

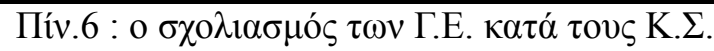

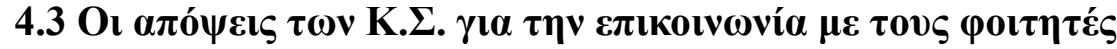

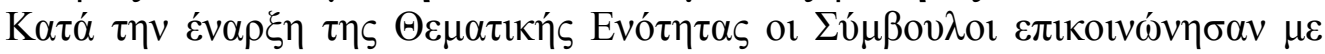

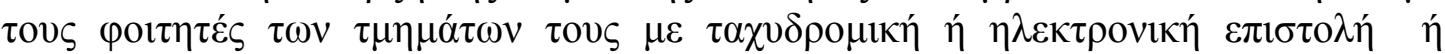

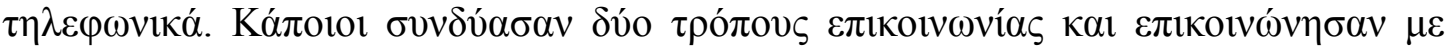

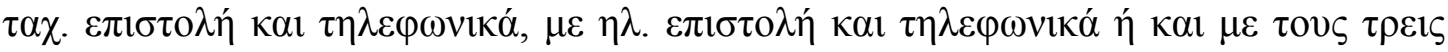

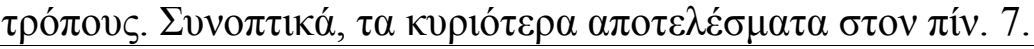

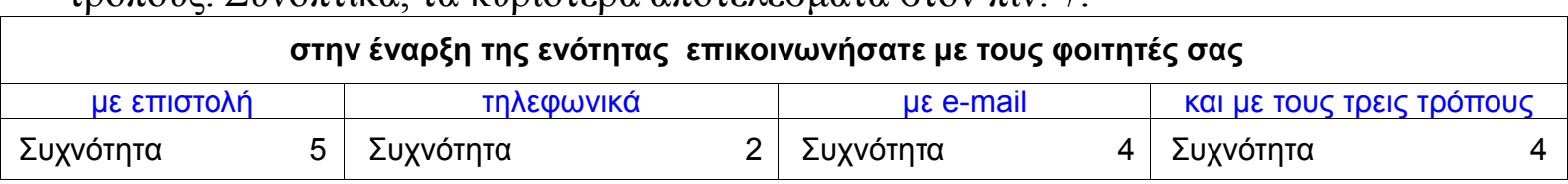

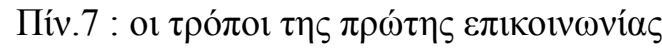

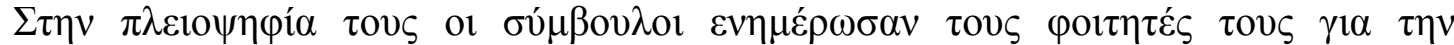

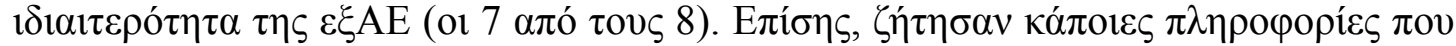

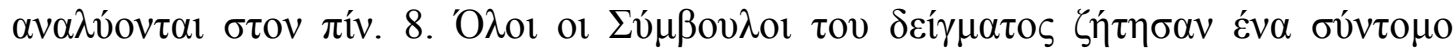

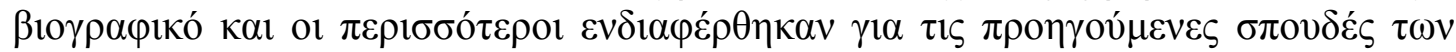

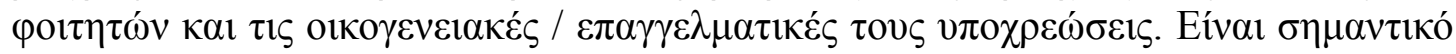

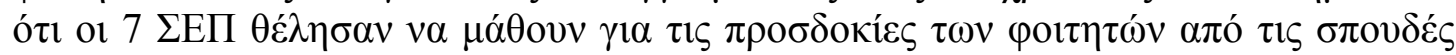

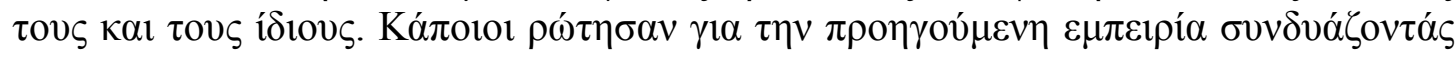

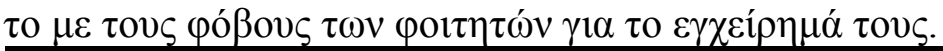

\begin{tabular}{|c|c|}
\hline 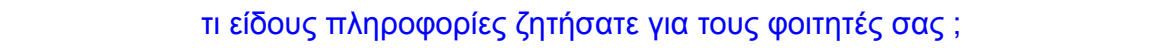 & 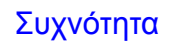 \\
\hline 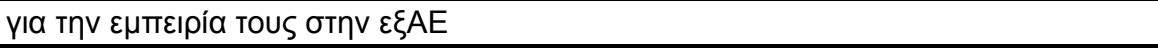 & 4 \\
\hline 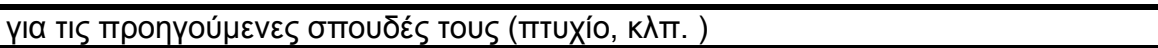 & $\overline{6}$ \\
\hline 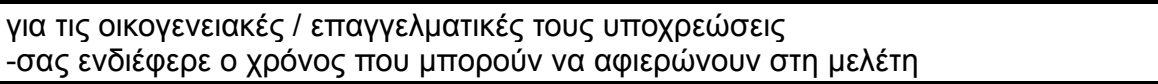 & 6 \\
\hline үıа тоU & 5 \\
\hline 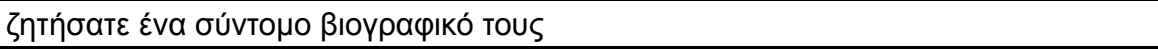 & 8 \\
\hline 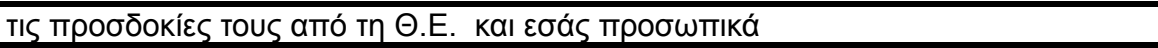 & 7 \\
\hline 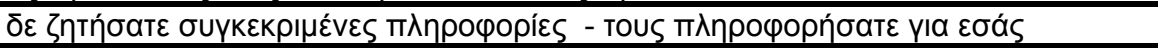 & 1 \\
\hline
\end{tabular}

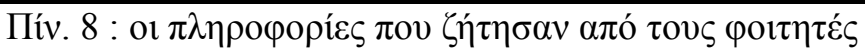

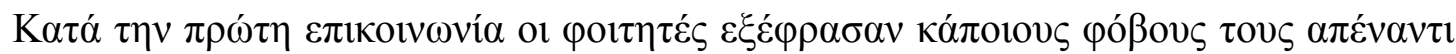

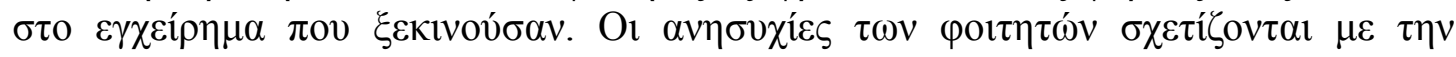

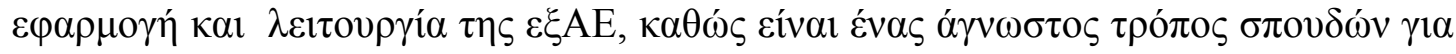

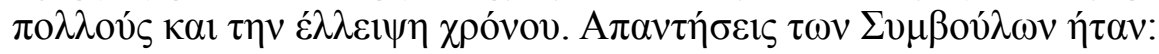

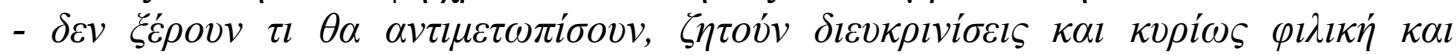

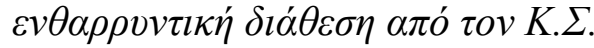

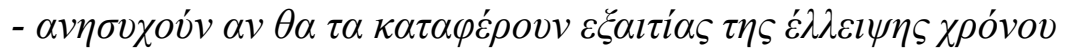

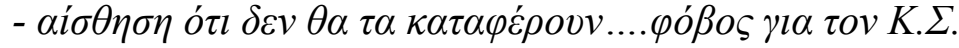

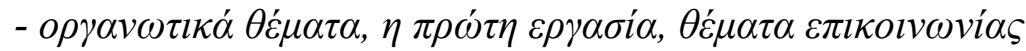

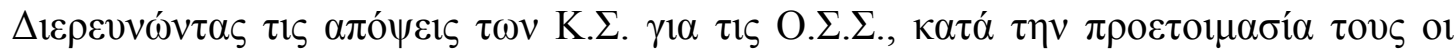

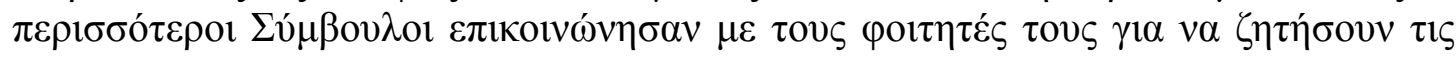

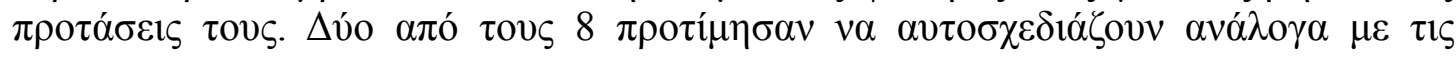

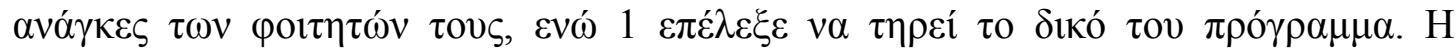

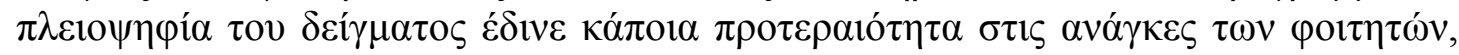

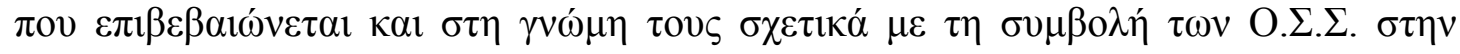

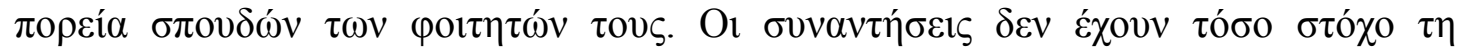

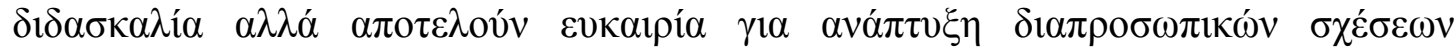


Open Education - The J oumal for Open and Distance Education and Educational Technology

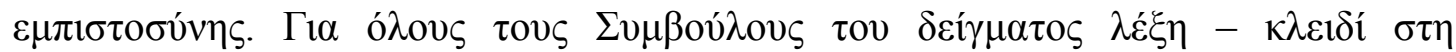

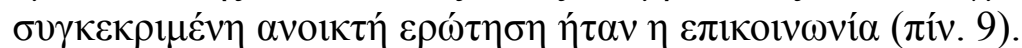

\begin{tabular}{|c|c|}
\hline 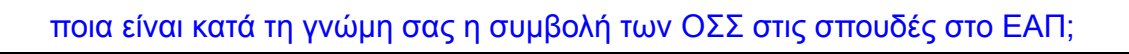 & ¿uxvótпта \\
\hline 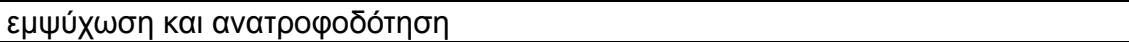 & 1 \\
\hline 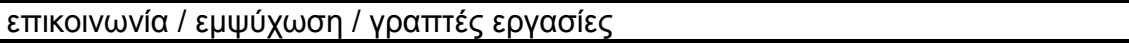 & 2 \\
\hline 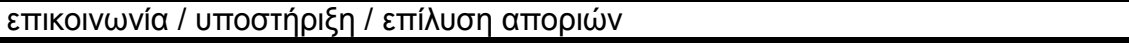 & 2 \\
\hline 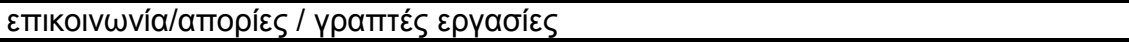 & 1 \\
\hline 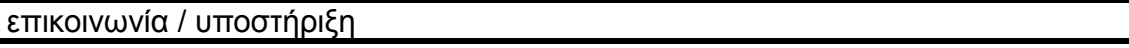 & 1 \\
\hline 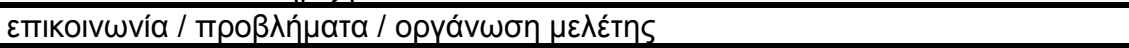 & 1 \\
\hline$\sum$ Évoגo & 8 \\
\hline
\end{tabular}

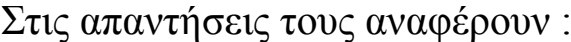

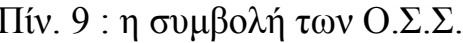

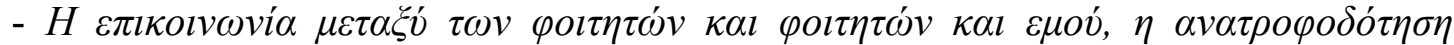

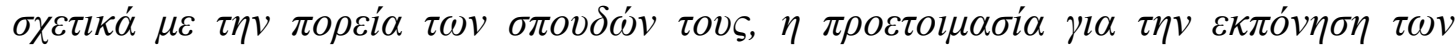
$\varepsilon \rho \gamma \alpha \sigma \iota \omega ́ v, \eta \varepsilon \xi \dot{\alpha} \lambda \varepsilon \imath \psi \eta \varphi o ́ \beta \omega v \kappa \alpha \iota \alpha v \eta \sigma v \chi \imath \omega ́ v$

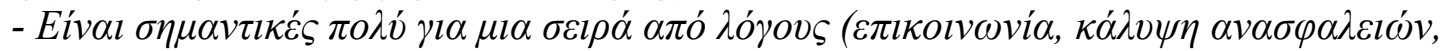

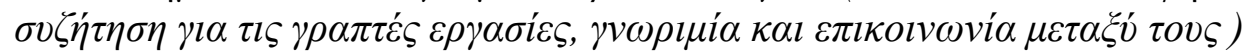

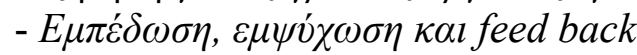

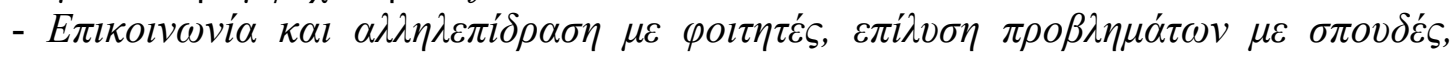

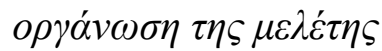

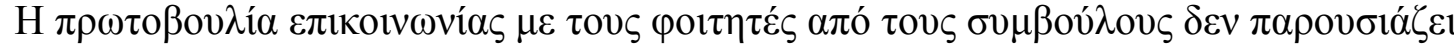

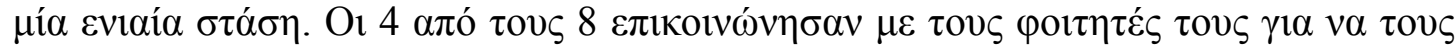

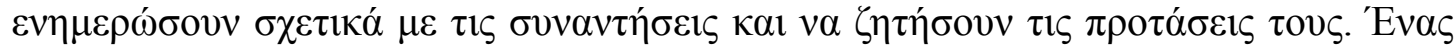

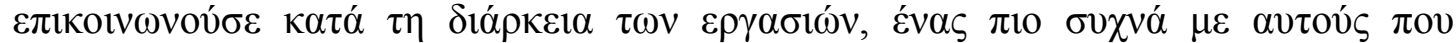

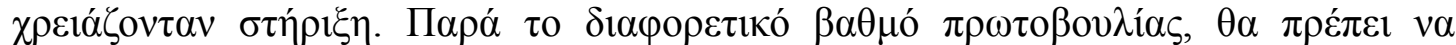

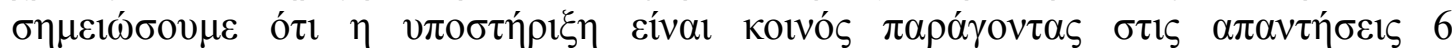

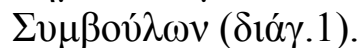

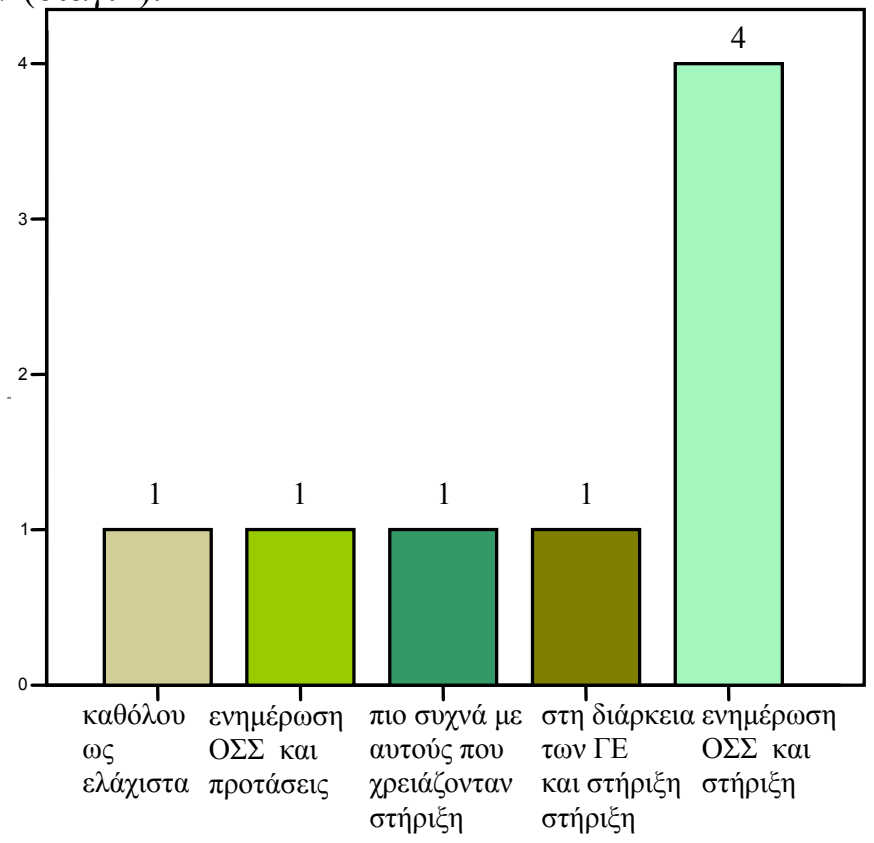

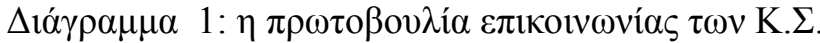

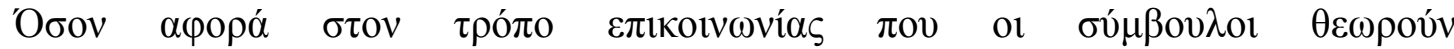

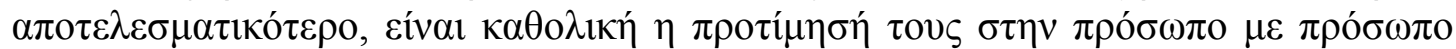

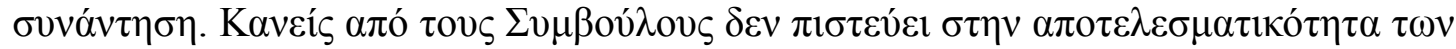

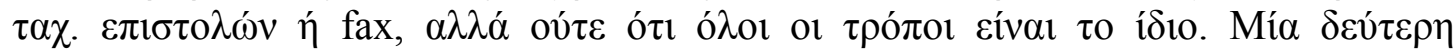

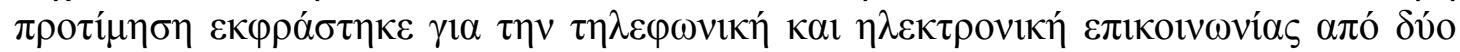




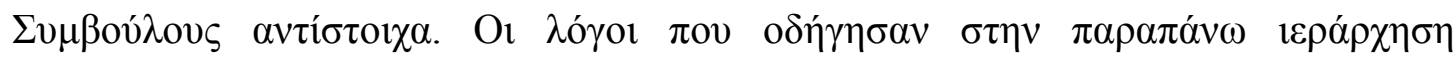

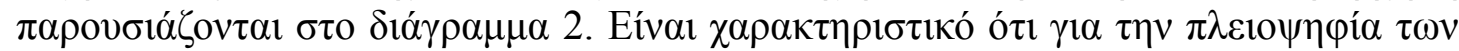

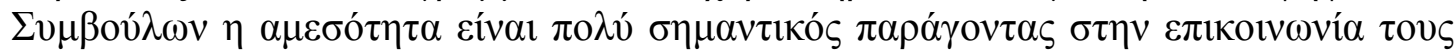

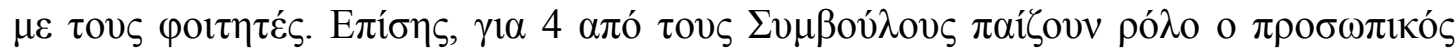

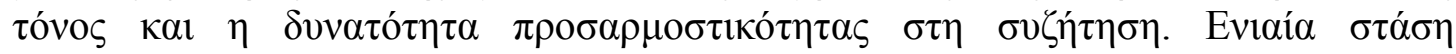

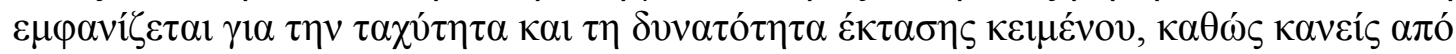

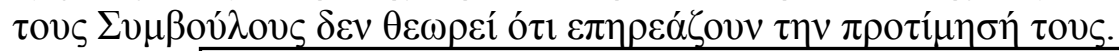

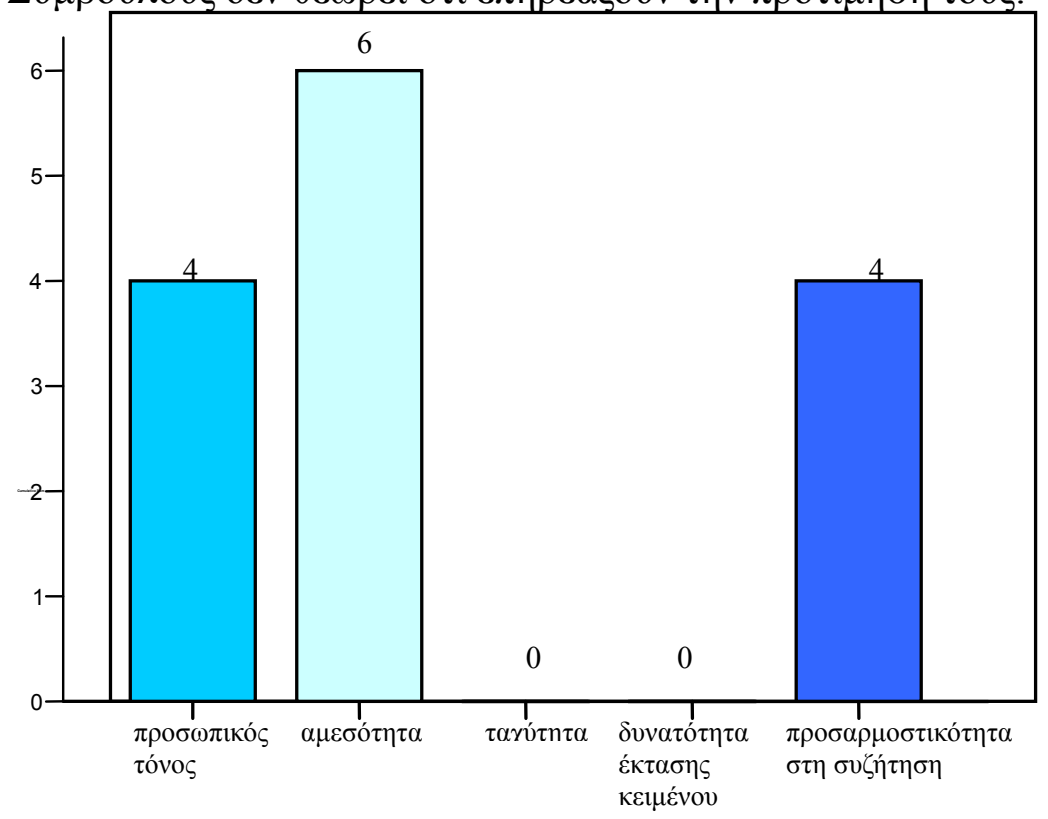

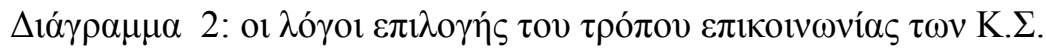

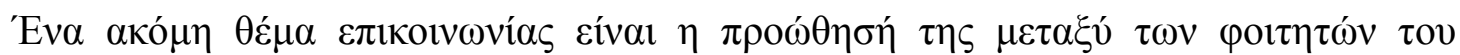

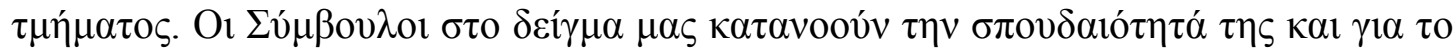

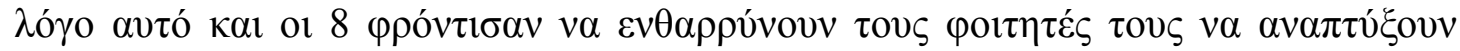

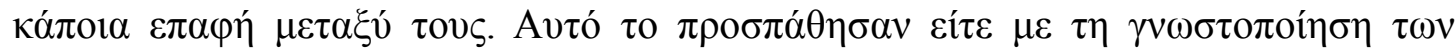

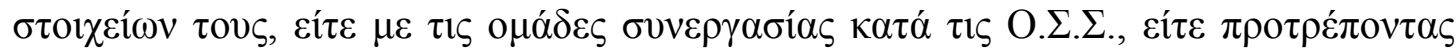

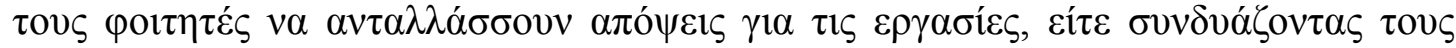

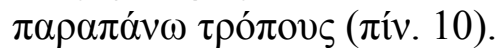

\begin{tabular}{|c|c|}
\hline 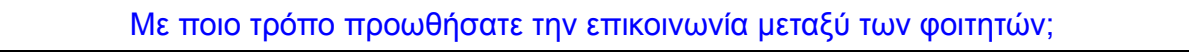 & ¿uхvóтnта \\
\hline 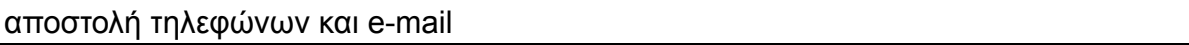 & 2 \\
\hline 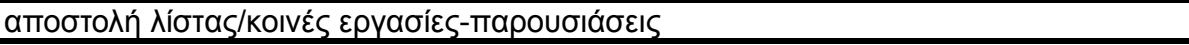 & 1 \\
\hline 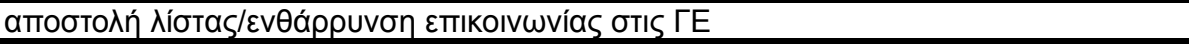 & 1 \\
\hline 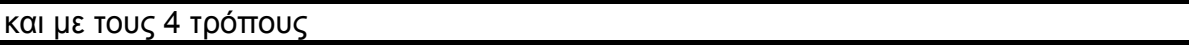 & 3 \\
\hline 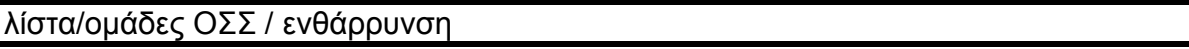 & 1 \\
\hline ¿úvoגo & 8 \\
\hline
\end{tabular}

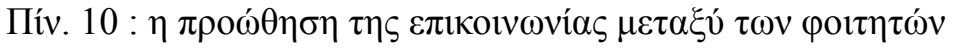

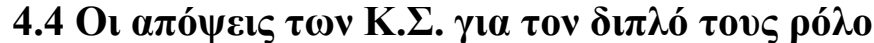

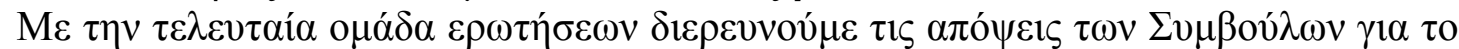

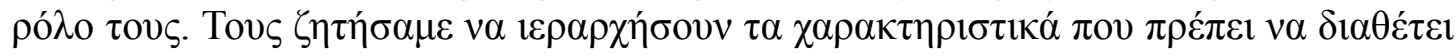

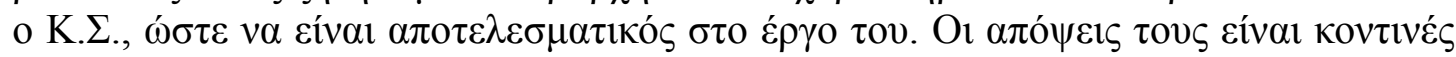

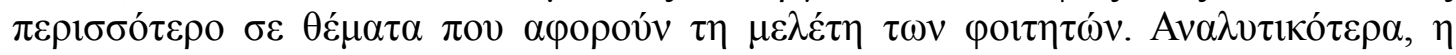

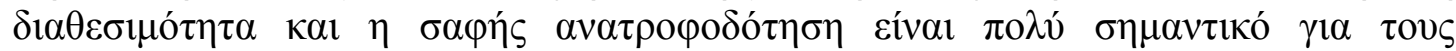

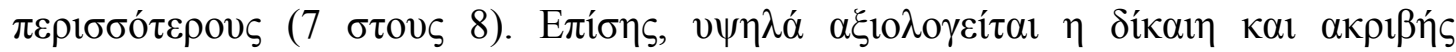
$\alpha \xi$ ı̀

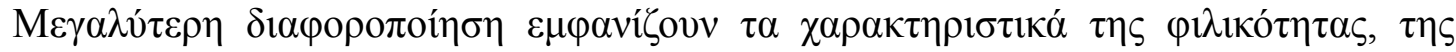


Open Education - The J oumal for Open and Distance Education and Educational Technology

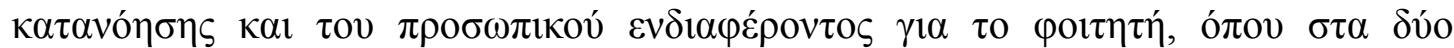

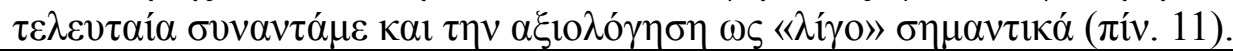

\begin{tabular}{|c|c|c|c|c|c|c|c|}
\hline & 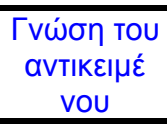 & 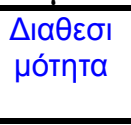 & 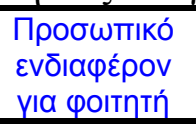 & $\begin{array}{l}\text { ФІ入Іко́ } \\
\text { тпта }\end{array}$ & 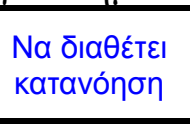 & 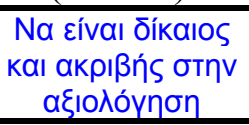 & 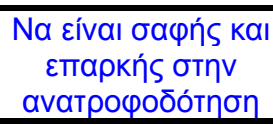 \\
\hline & \multicolumn{7}{|c|}{ 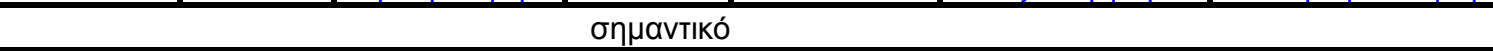 } \\
\hline \multicolumn{8}{|l|}{ kaӨó^ou } \\
\hline Aíyo & & & 1 & & 1 & & \\
\hline 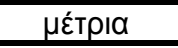 & 1 & & & 1 & & 2 & \\
\hline 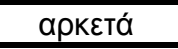 & 2 & 1 & 2 & 3 & 2 & & 1 \\
\hline по入и́ & 5 & 7 & 5 & 4 & 4 & 6 & 7 \\
\hline 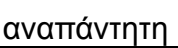 & & & & & 1 & & \\
\hline oúvoגo & 8 & 8 & 8 & 8 & 8 & 8 & 8 \\
\hline
\end{tabular}

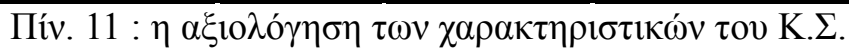

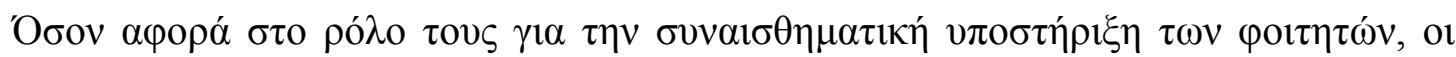

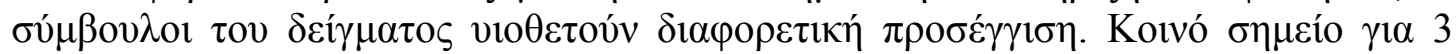

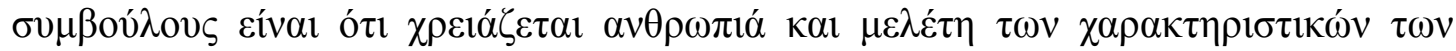

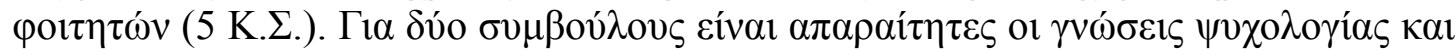

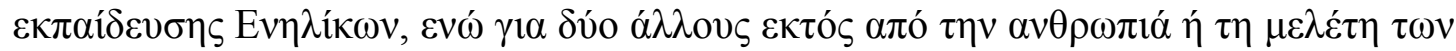

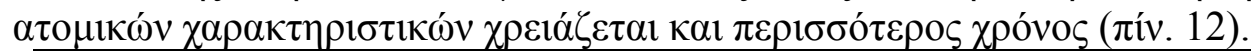

\begin{tabular}{|c|c|}
\hline 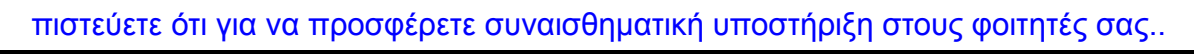 & ¿uxvótnта \\
\hline 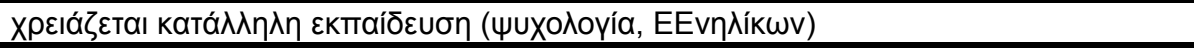 & 2 \\
\hline 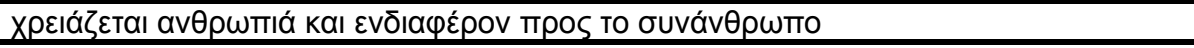 & 1 \\
\hline 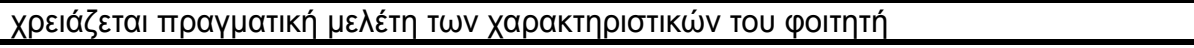 & 2 \\
\hline 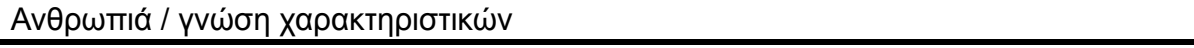 & 1 \\
\hline 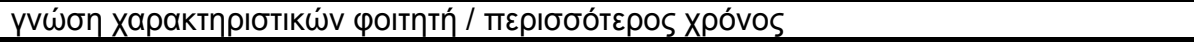 & 1 \\
\hline 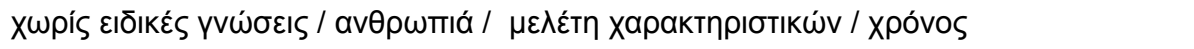 & 1 \\
\hline ¿úvoגo & 8 \\
\hline
\end{tabular}

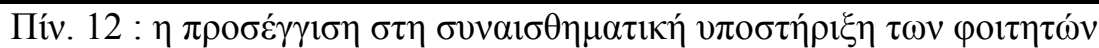

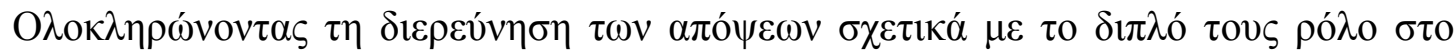
Е.A.П., 4 a

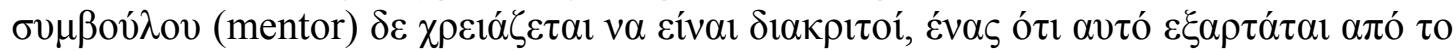

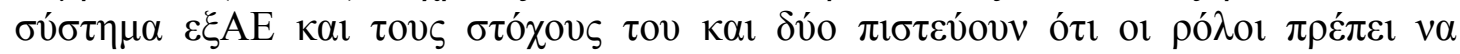

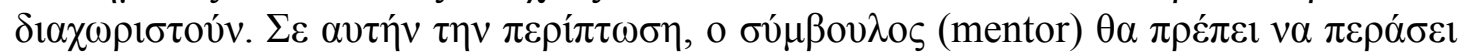

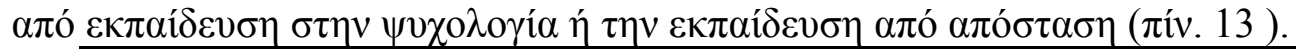

\begin{tabular}{|c|c|}
\hline 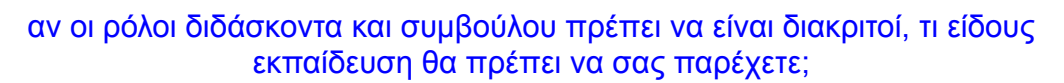 & ¿uxvótпта \\
\hline 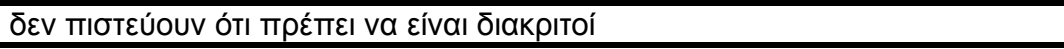 & 4 \\
\hline 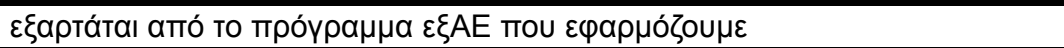 & 1 \\
\hline$\varepsilon \xi A E$ & 1 \\
\hline 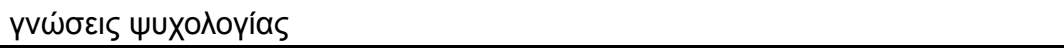 & 1 \\
\hline 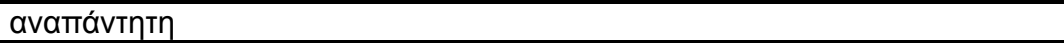 & 1 \\
\hline$\sum$ ¿́voגo & 8 \\
\hline
\end{tabular}

\section{4. $\Sigma v \zeta \eta ́ \tau \eta \sigma \eta \tau \omega v \alpha \pi 0 \tau \varepsilon \lambda \varepsilon \sigma \mu \alpha ́ \tau \omega v$}

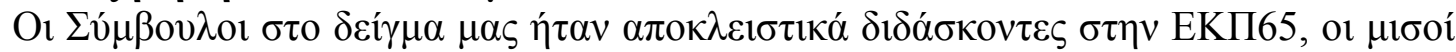

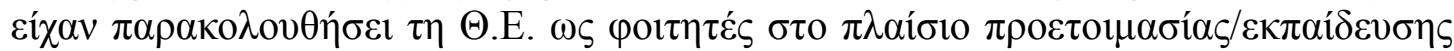

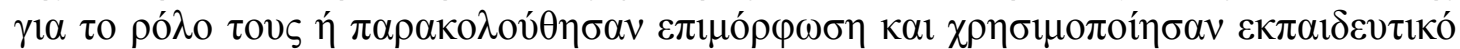

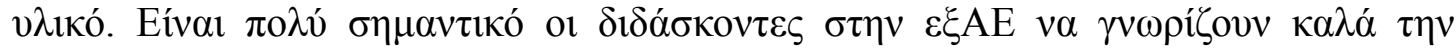

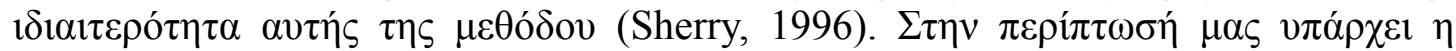

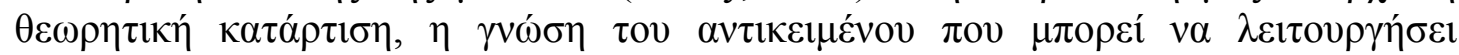




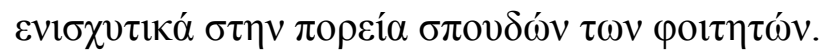

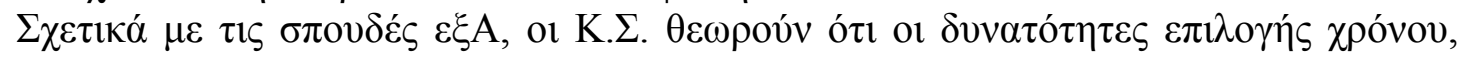

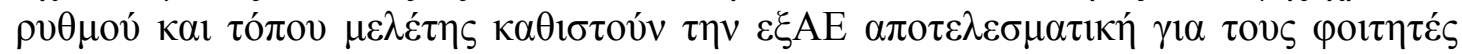

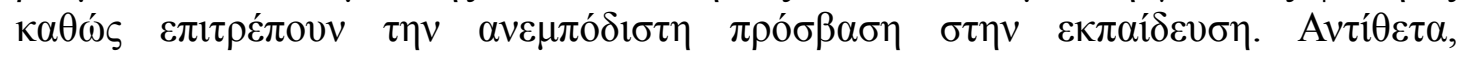

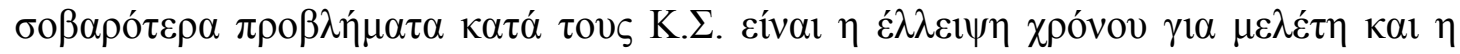

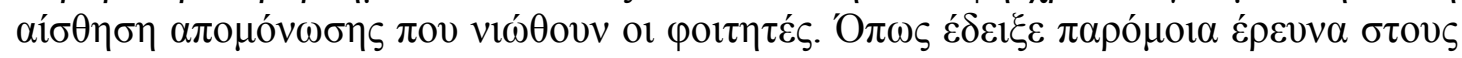

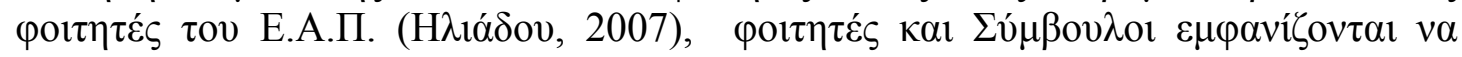

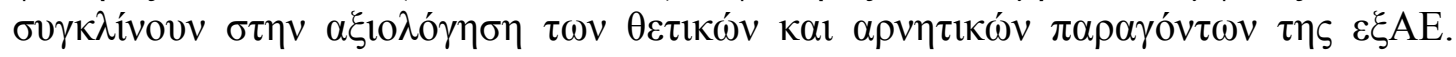

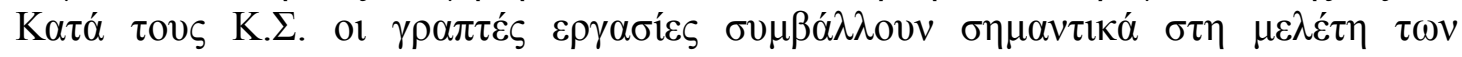

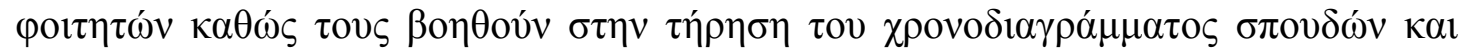

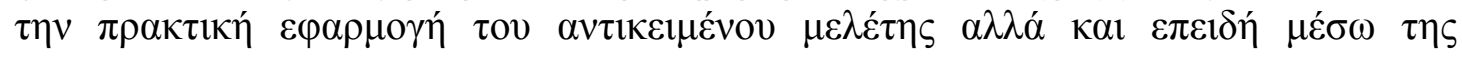

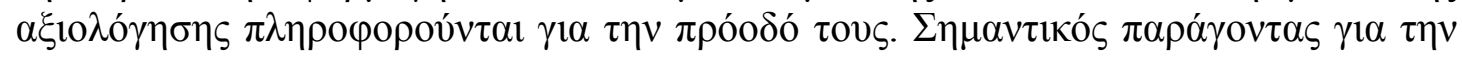

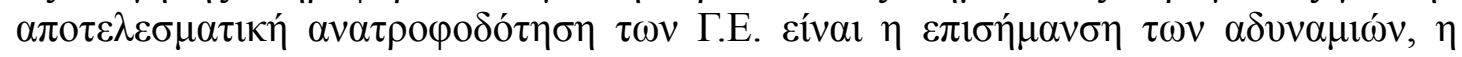

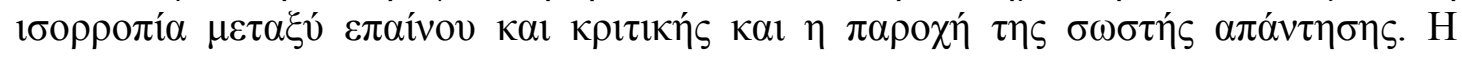

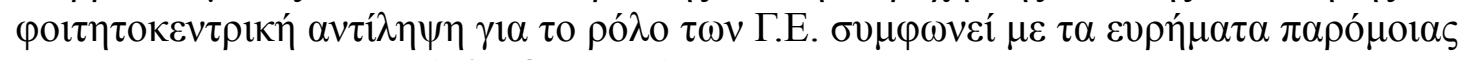

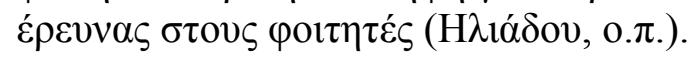

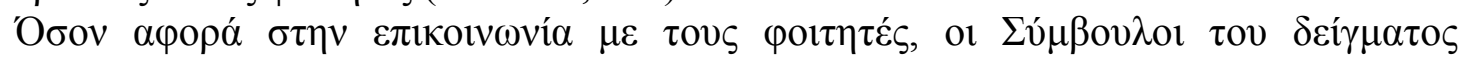

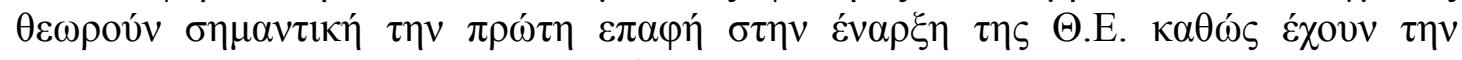

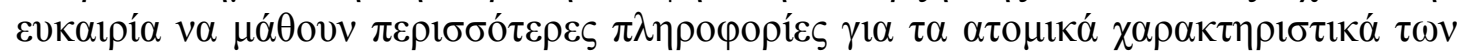

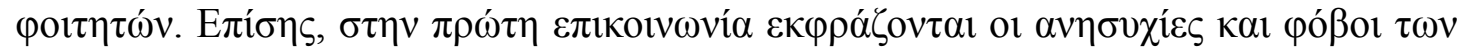

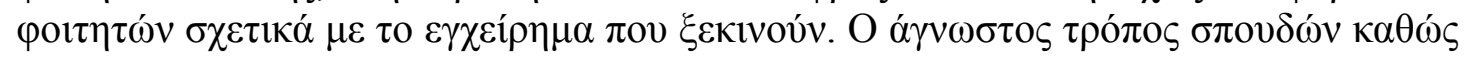

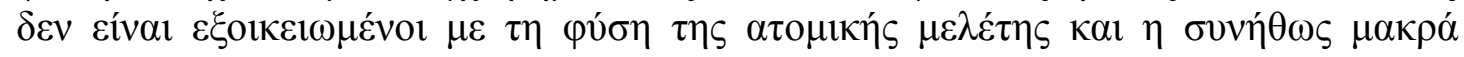

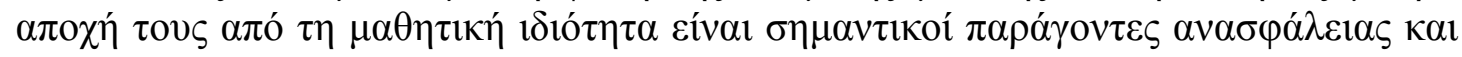

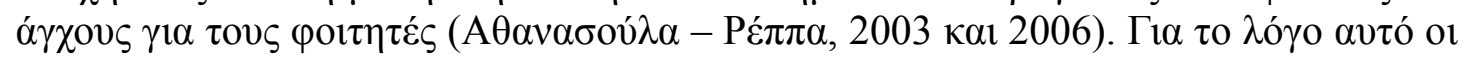

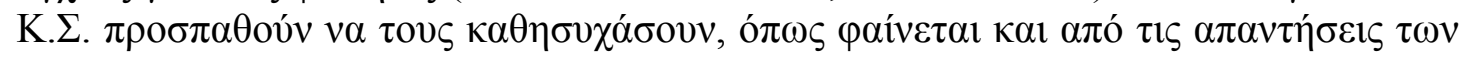

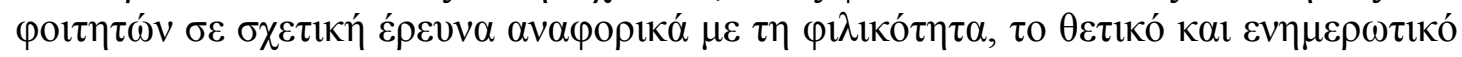

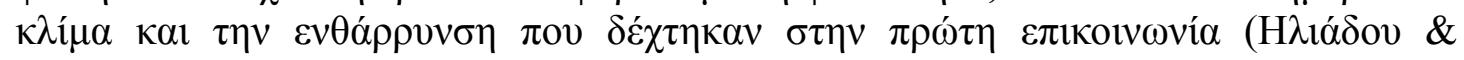

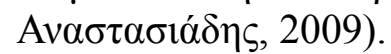

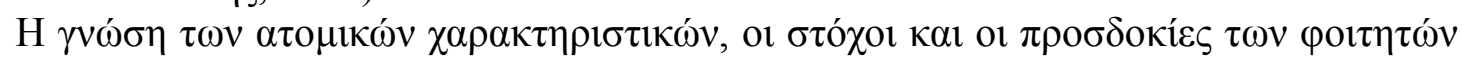

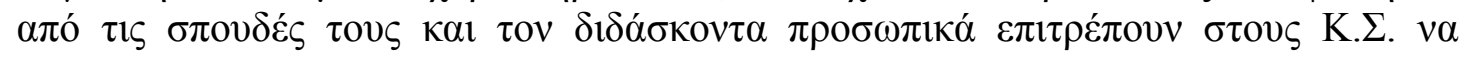

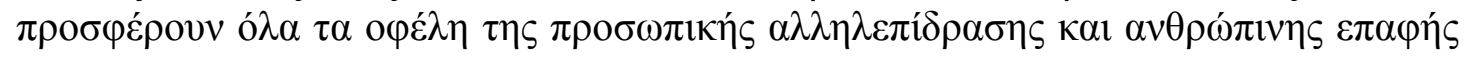

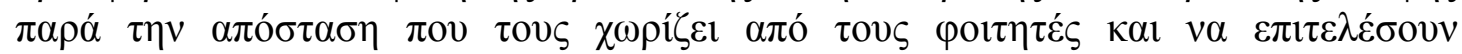

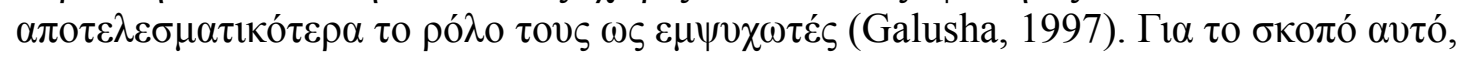

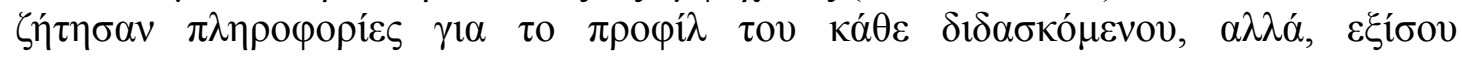

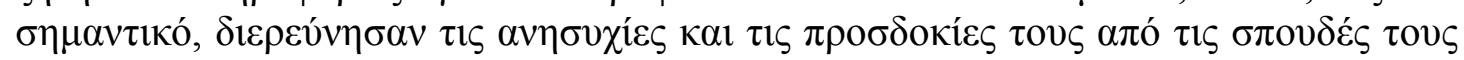

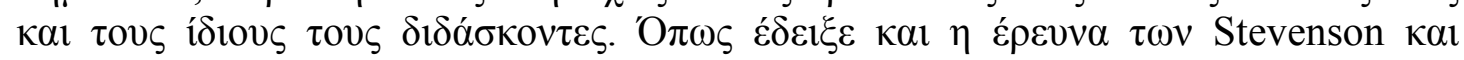

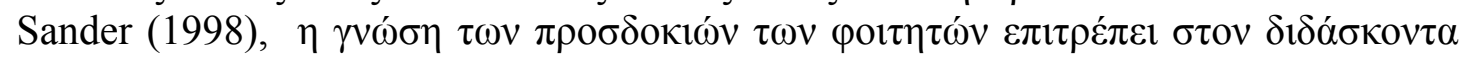

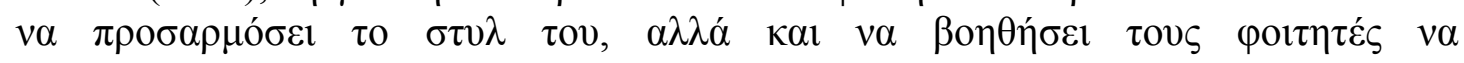

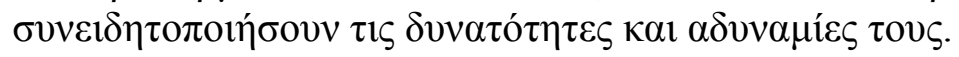

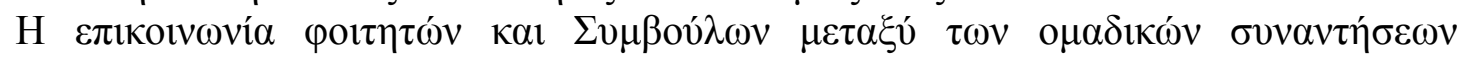

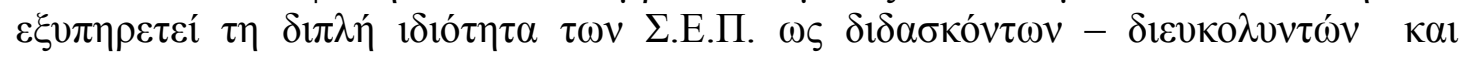

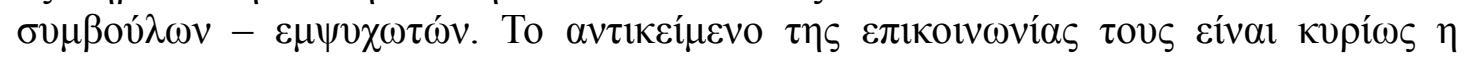
$\varepsilon \pi i ́ \lambda v \sigma \eta$ a

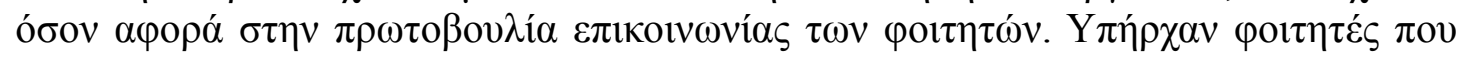

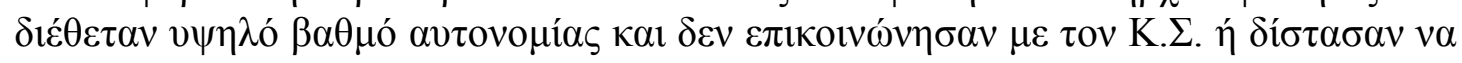

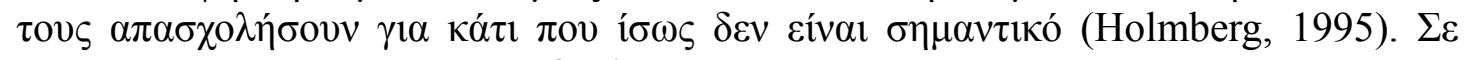

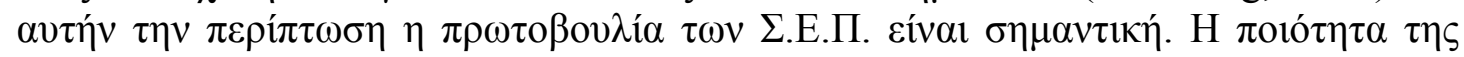

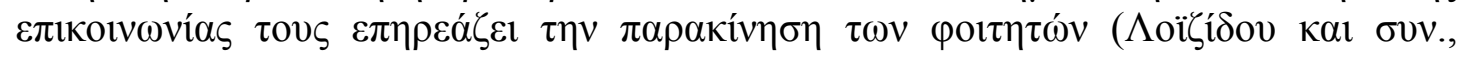

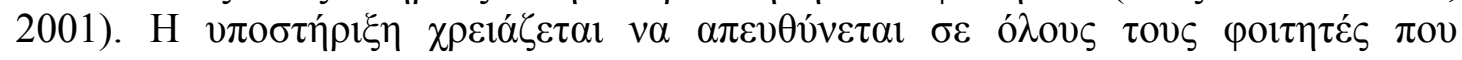

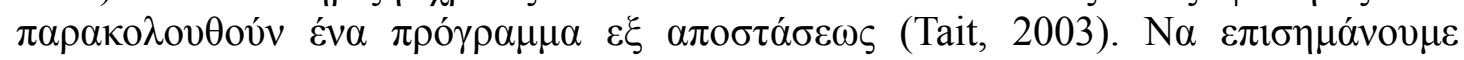




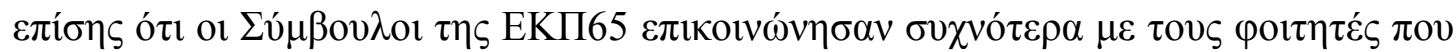

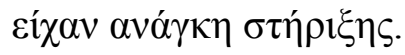

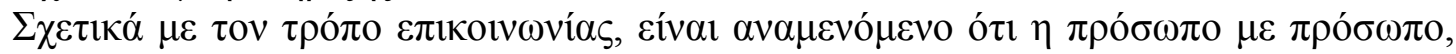

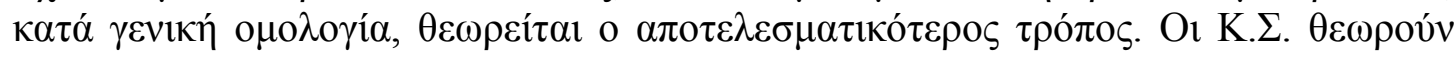

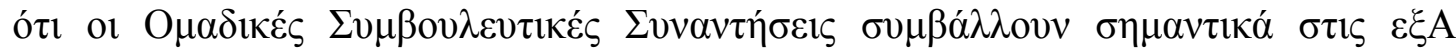

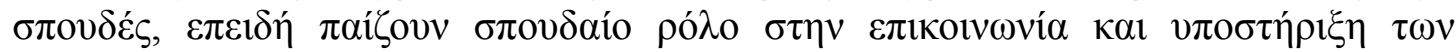

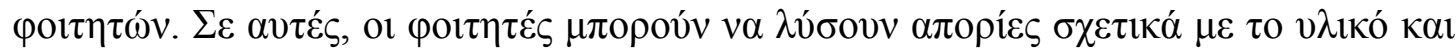

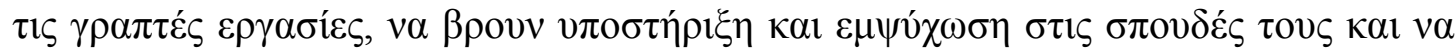

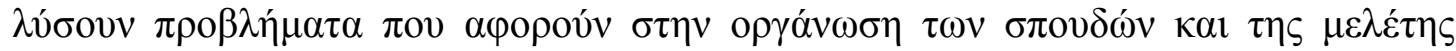

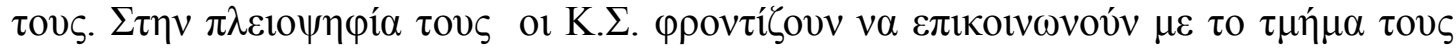

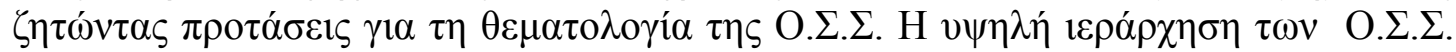

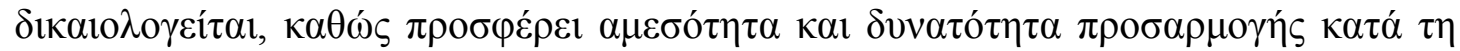

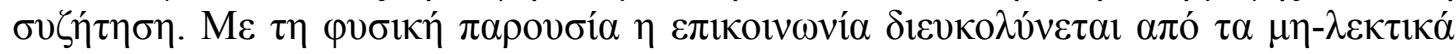

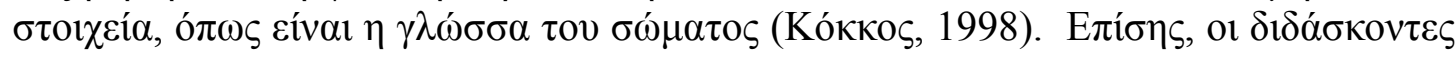

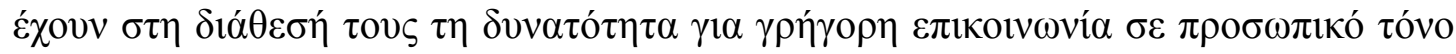

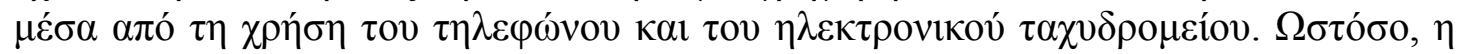

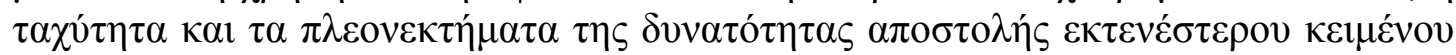

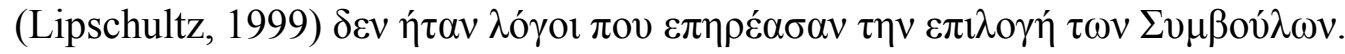

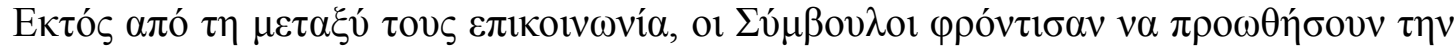

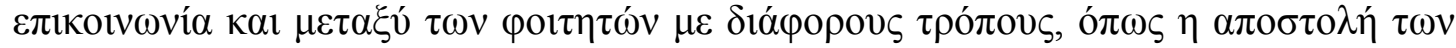

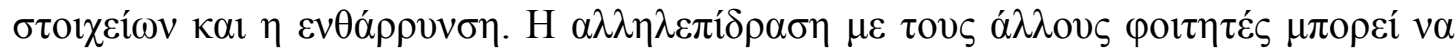

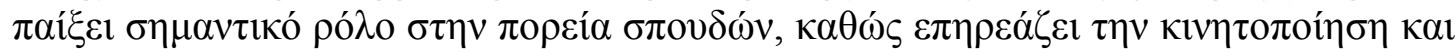

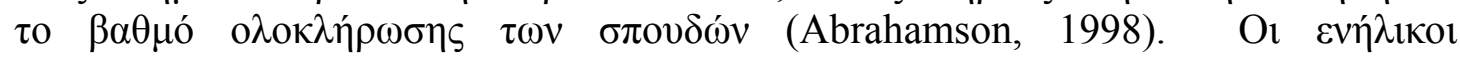

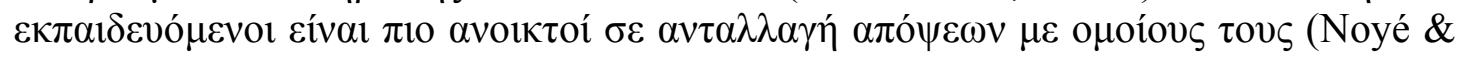

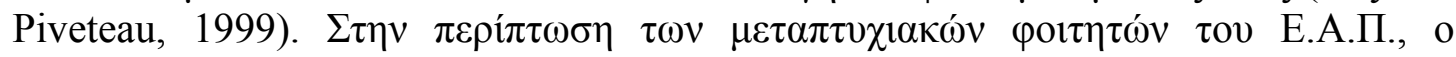

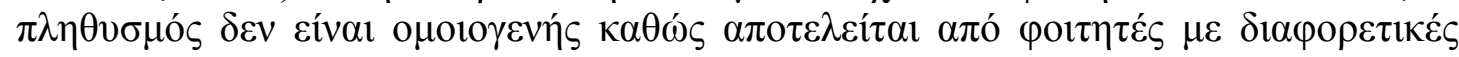

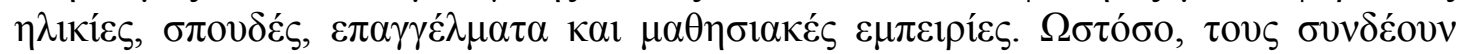

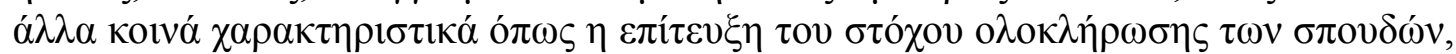

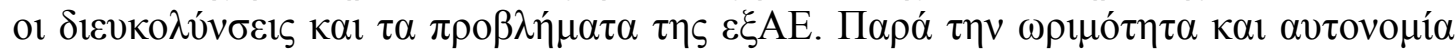

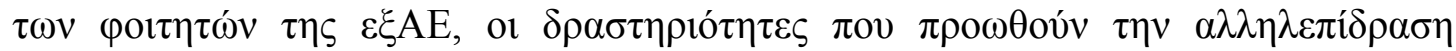

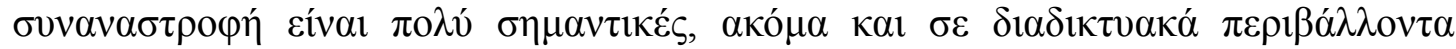

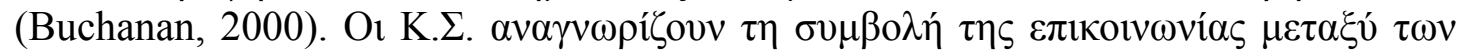

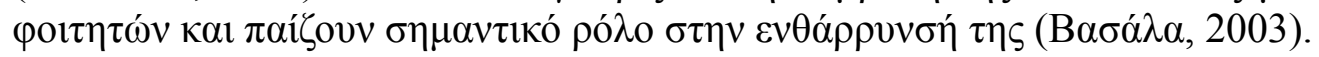

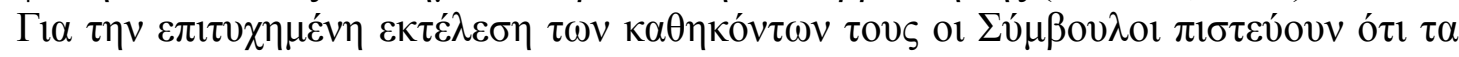

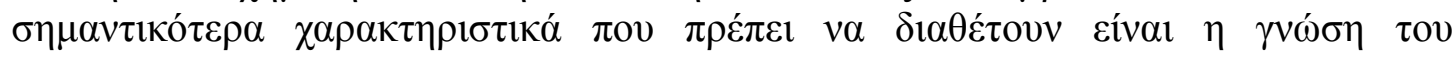

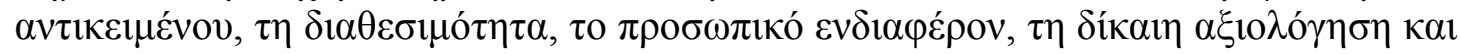

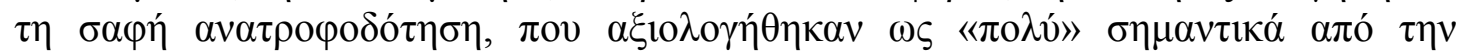

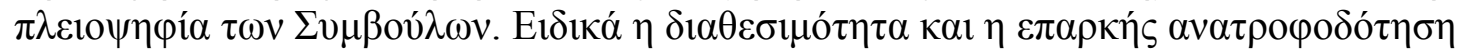

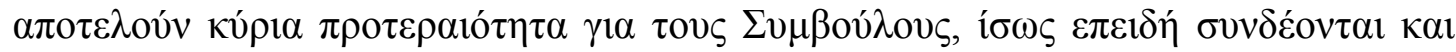

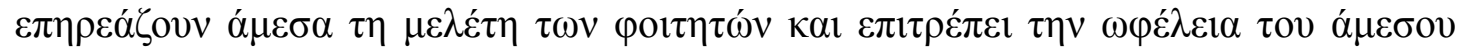

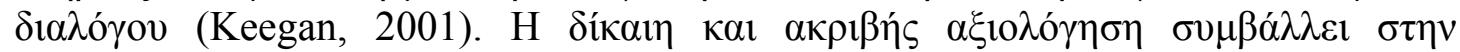

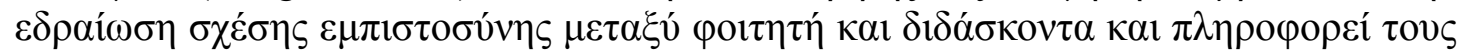

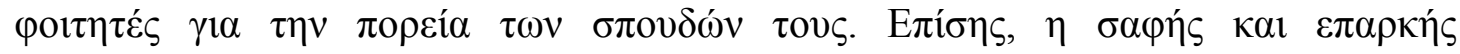

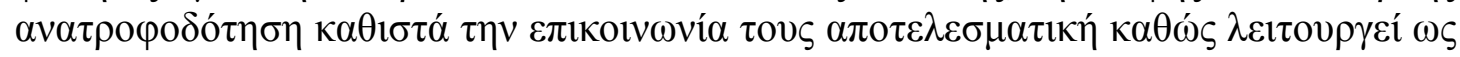

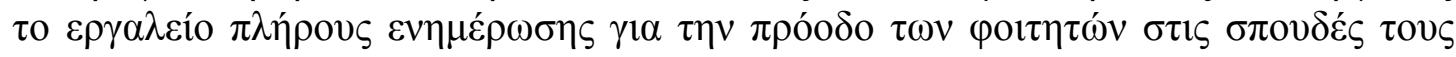

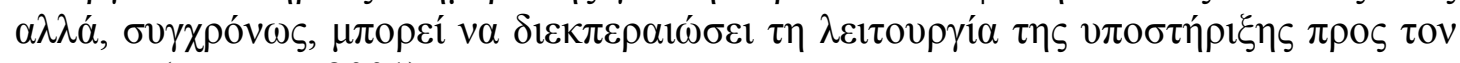

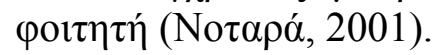

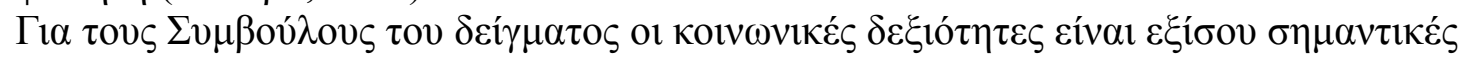

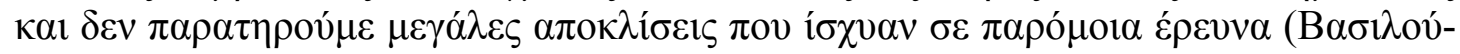

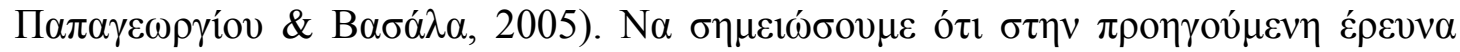




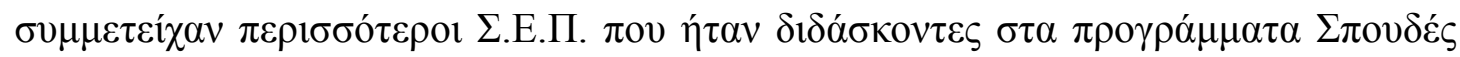

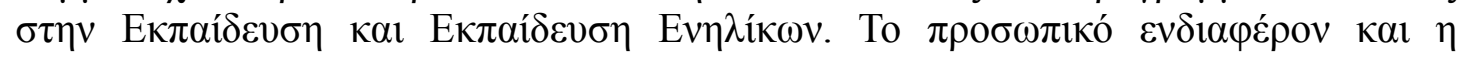

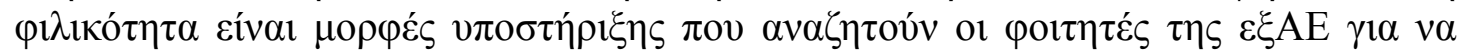

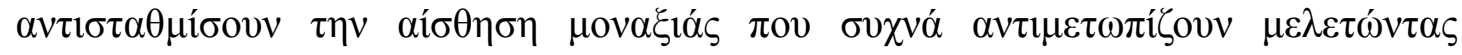

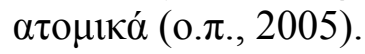

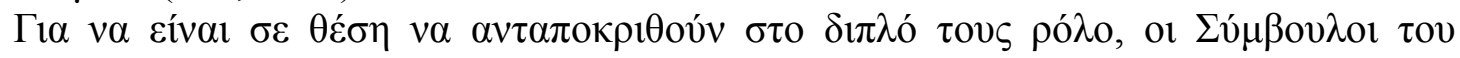

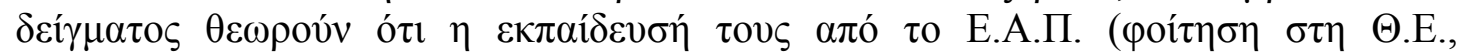

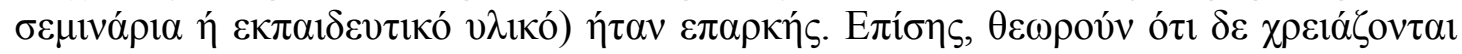

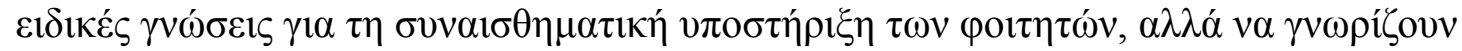

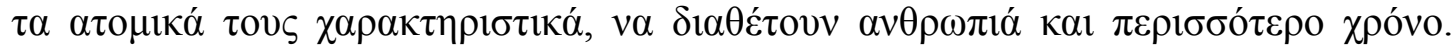

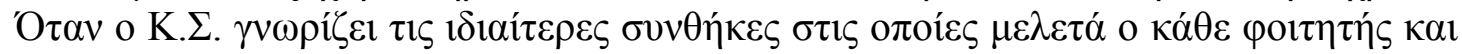

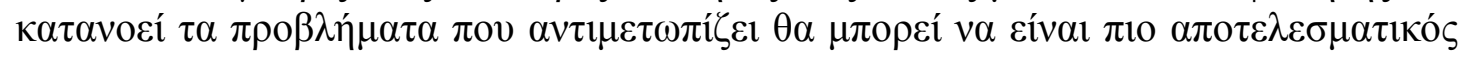

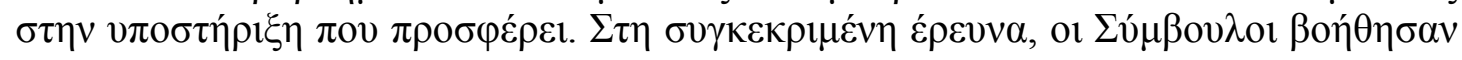

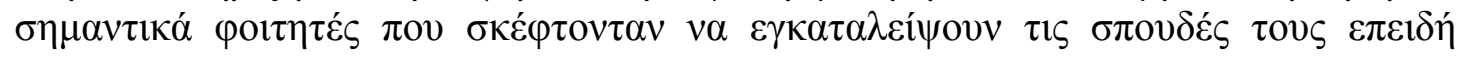

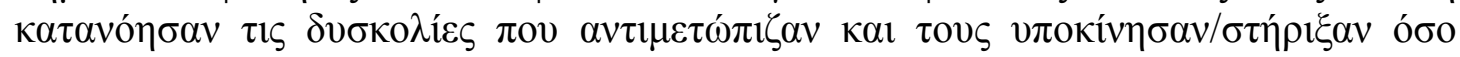

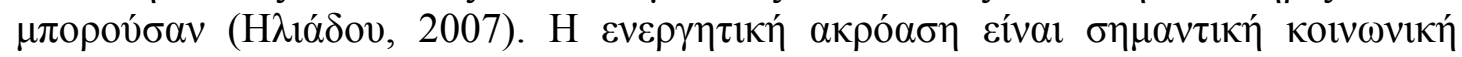

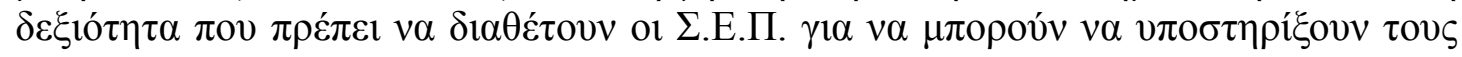

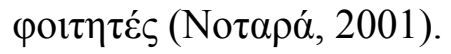

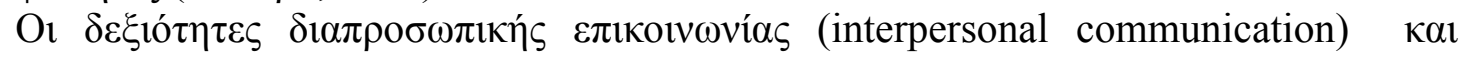

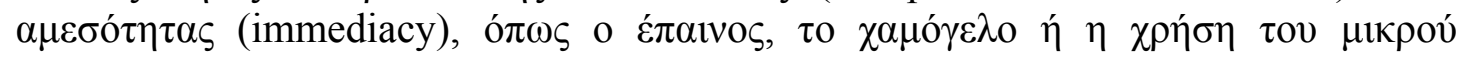

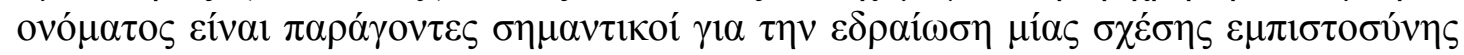

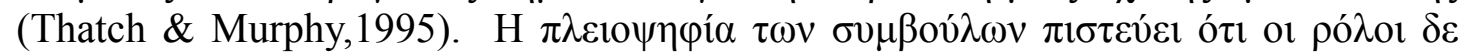

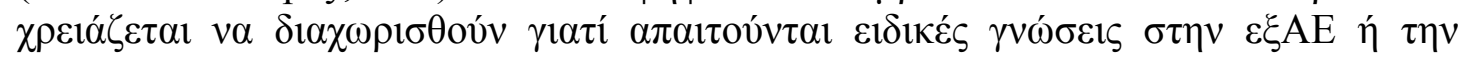

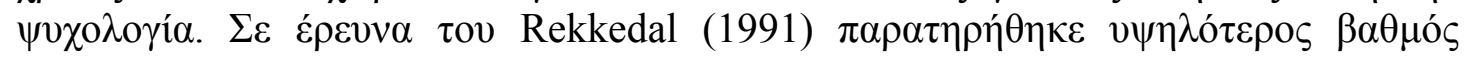

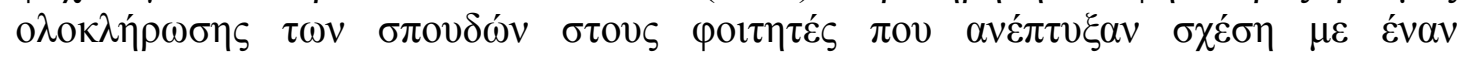

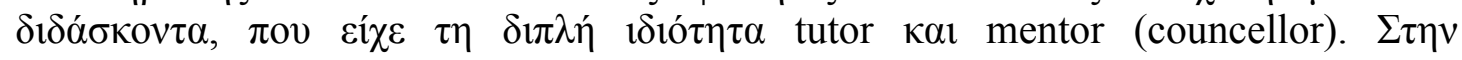

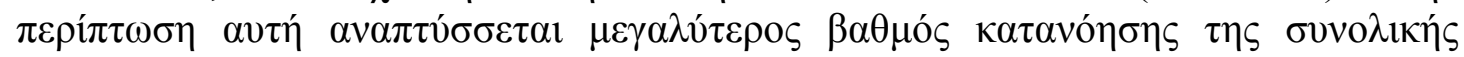

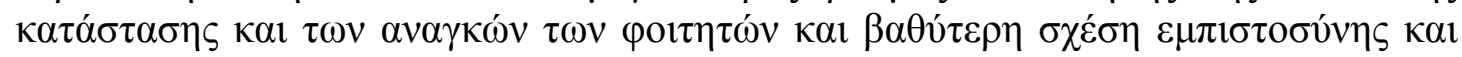

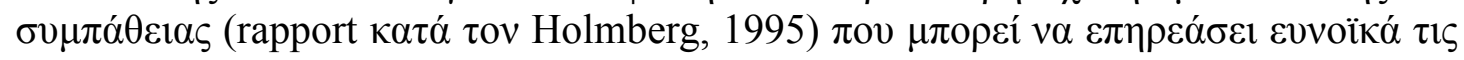

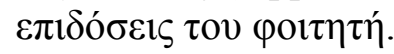

\section{5. $\Sigma v \mu \pi \varepsilon \rho \alpha ́ \sigma \mu \alpha \tau \alpha$}

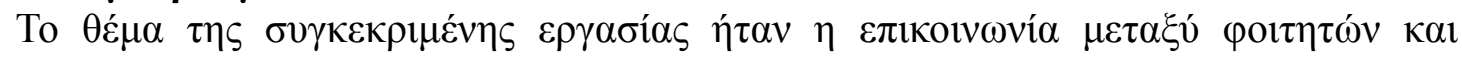

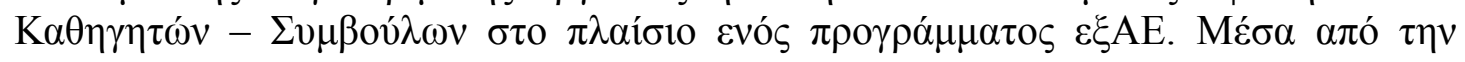

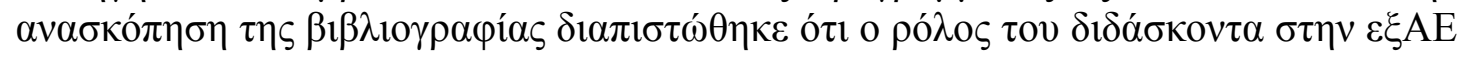

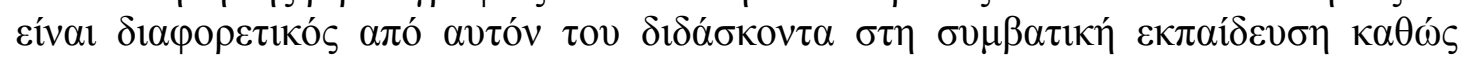

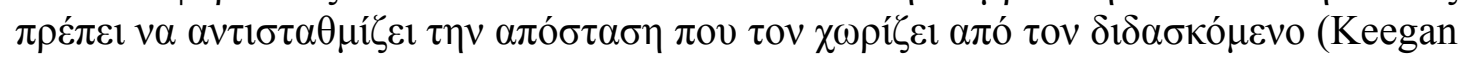

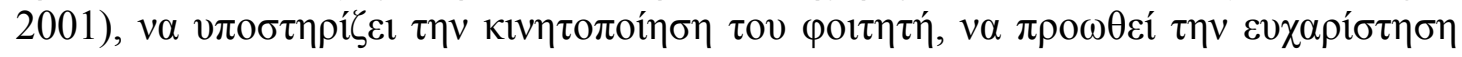

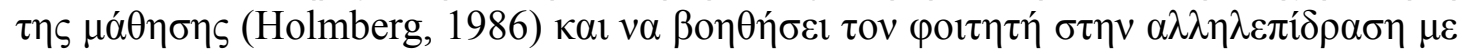

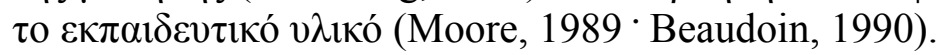

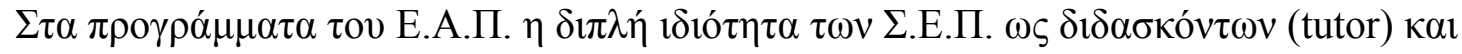

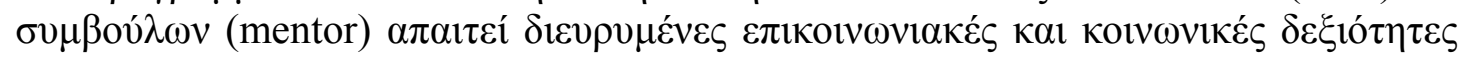

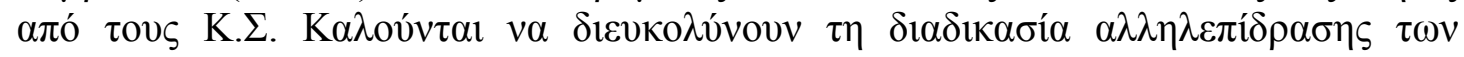

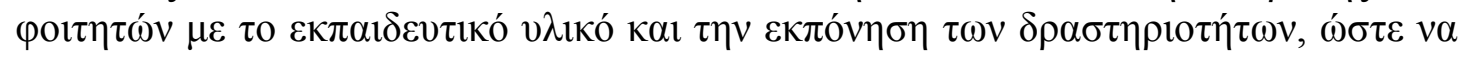

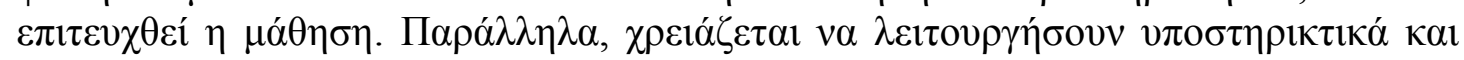

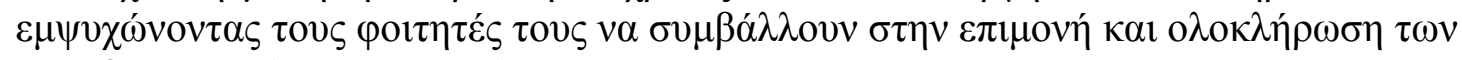
$\sigma \pi \mathrm{ov \delta \omega ́} v \tau 00 \varsigma$ (Е.A.П., 2001).

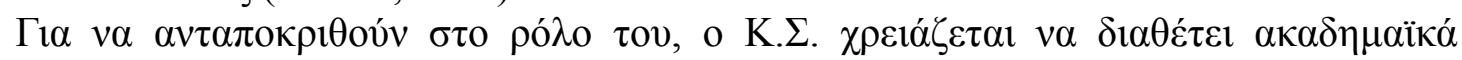

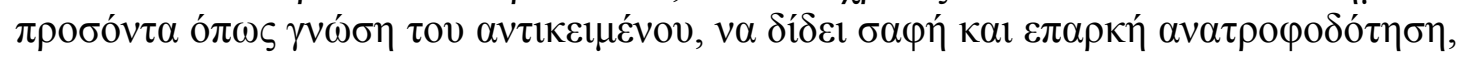

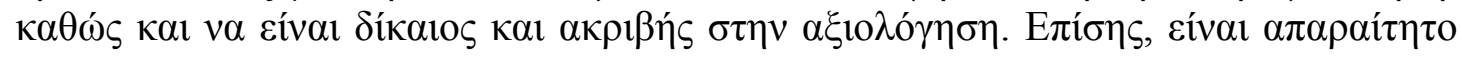




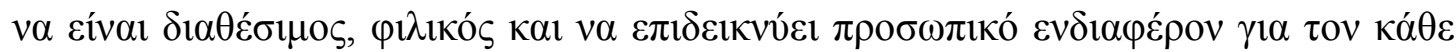

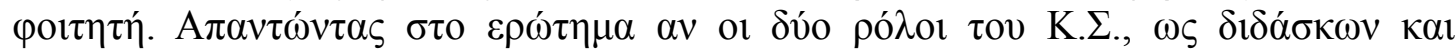

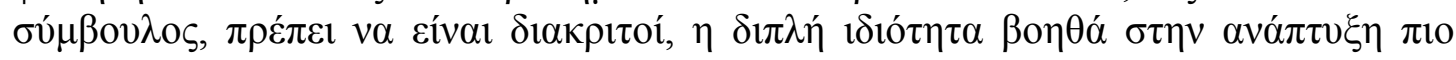

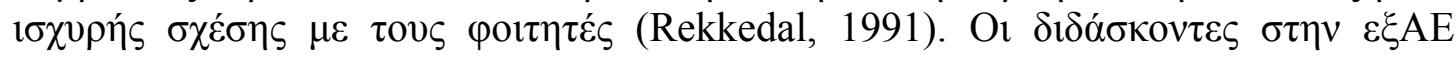

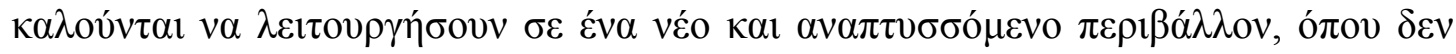

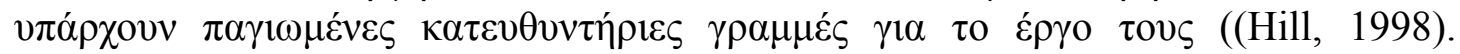

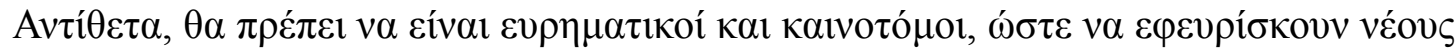

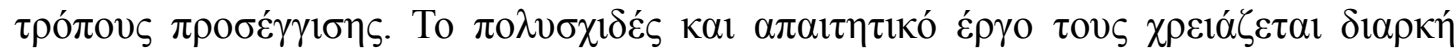

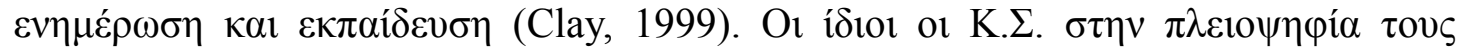

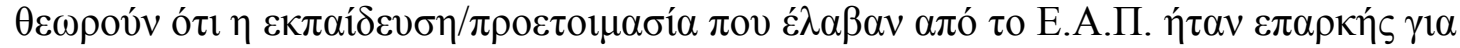

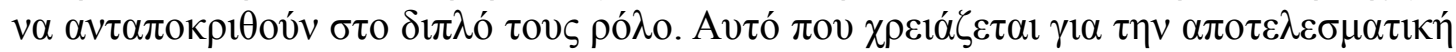

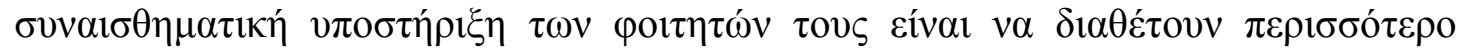

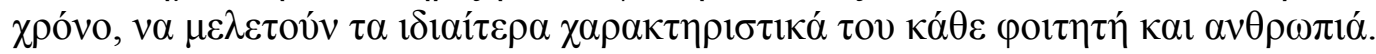

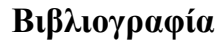

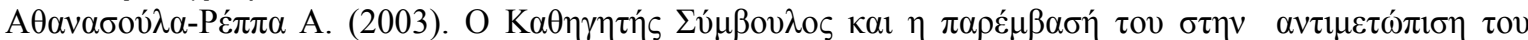

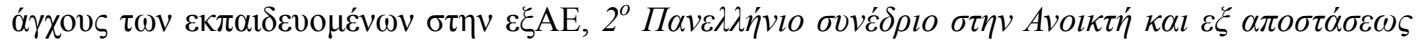

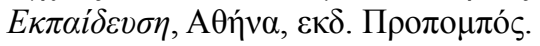

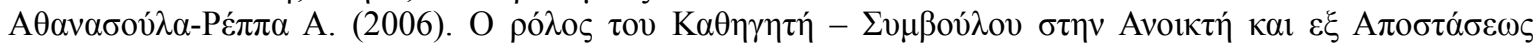

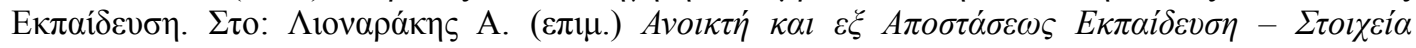

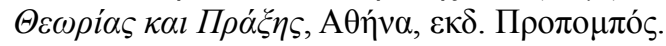

Abrahamson G. (1998). Issues in interactive communication in Distance Education, College Student Journal

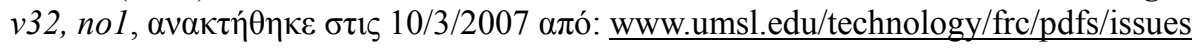

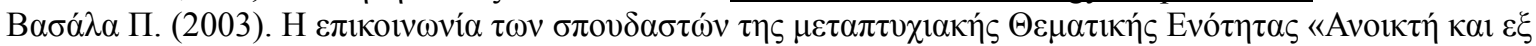

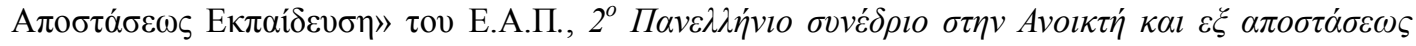

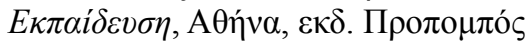

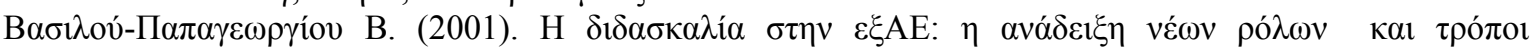

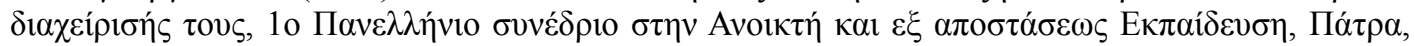

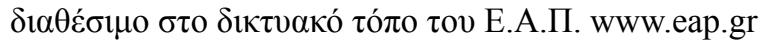

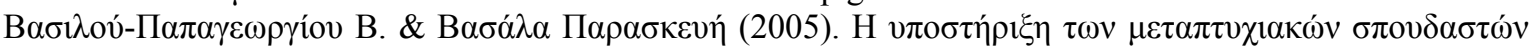

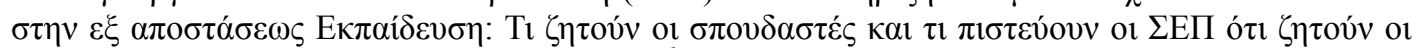

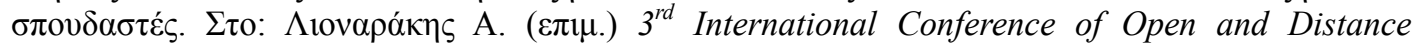

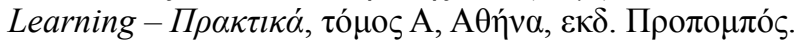

Beaudoin M. (1990). The Instructor's changing role in Distance Education, The American Journal of

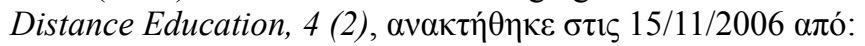

www.uni-oldenburg.de/zef/cde/found/beau90

Clay M. (1999). Development of Training and Support Programs for Distance Education Instructors, $\delta i \alpha \theta \dot{\varepsilon} \sigma \mu$ o $\sigma \tau \mathrm{o}: \mathrm{http}: / / \mathrm{www} . w e s t g a . e d u / \sim$ distance/clay23.html

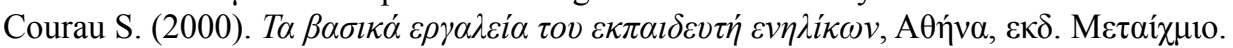

Dillon C.L., Gunawardena C.N., \& Parker R. (1992). Learner support: critical link in distance education,

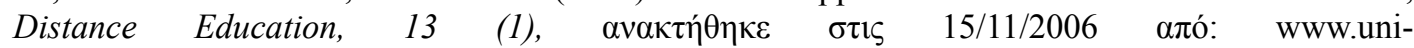
oldenburg.de/zef/cde/support/readings

Galusha J. (1997). Barriers to Learning in Distance Education, Interpersonal Computing and Technology,

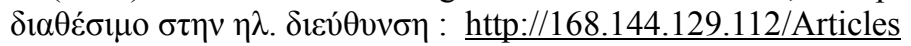

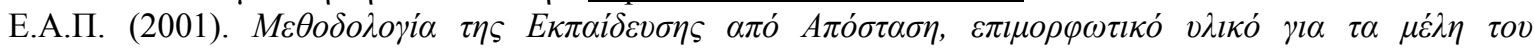

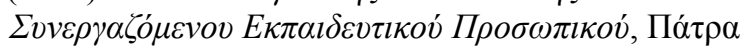

Garrison R. (2000). Theoretical challenges for Distance Education in the $21^{\text {st }}$ century : A shift from structural to transactional issues, International Review of Research in Open and Distance Learning, June

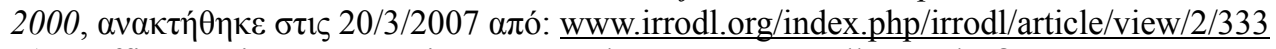

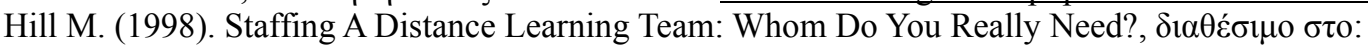

www.westga.edu/ distance/hill11.html

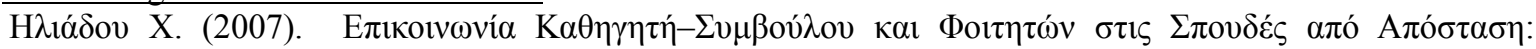

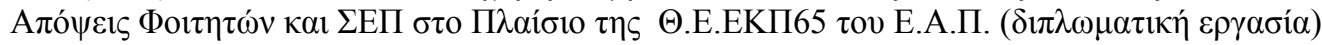

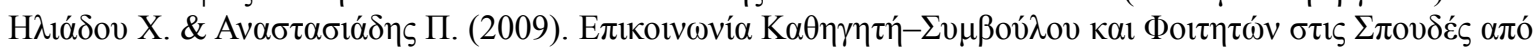

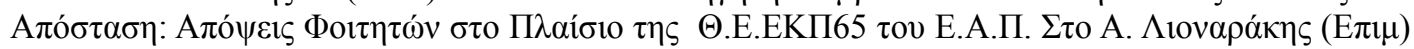

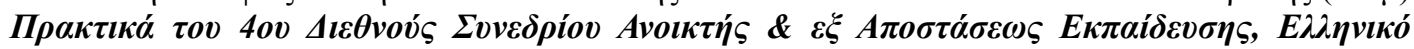

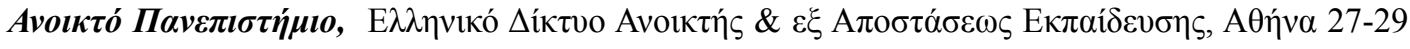


Noєцßpíov 2009.

Holmberg B. (1995). Theory and Practice of Distance Education, London, Routledge.

Keegan D. (1986). Interaction and communication, (Chapter 6, pp.89-107)... $\tau$ o: Keegan, D. (ed.) The

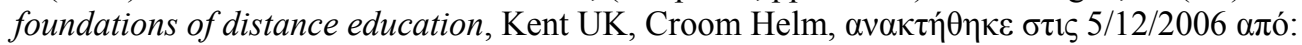
www.uni-oldenburg.de/zef/cde/support/readings

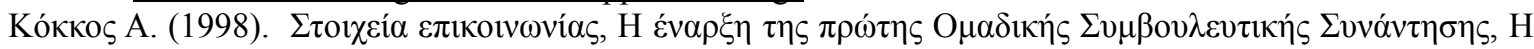

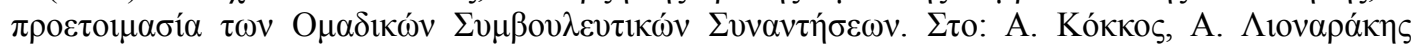

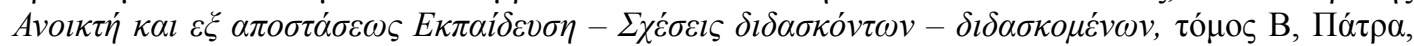

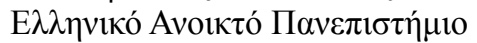

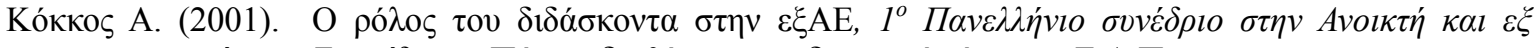

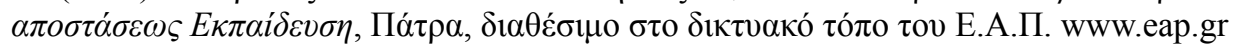

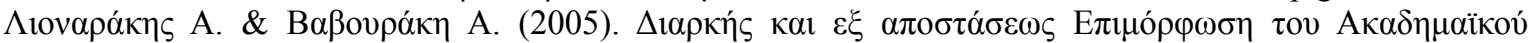

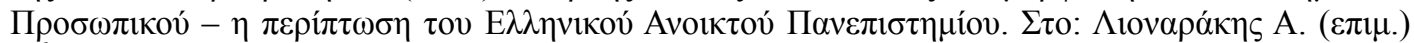

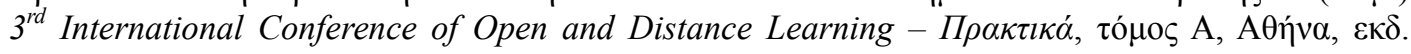
Пролонло́

Lipschultz W. (1999). What can and should we do with e-mail?, The Mentor - An Academic Advising

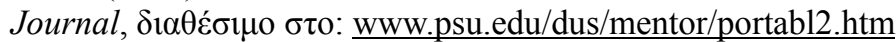

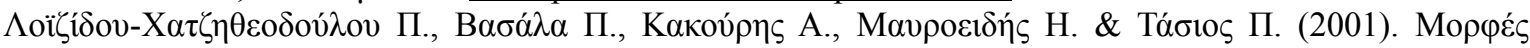

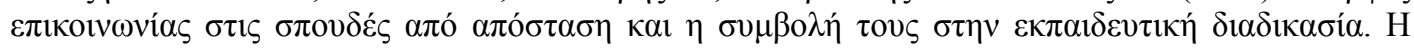

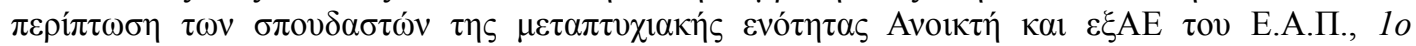

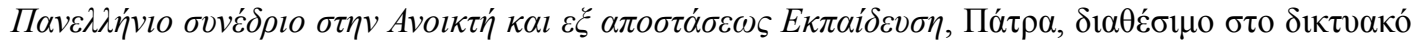

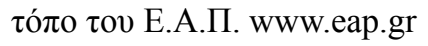

Moore M.G. (1973). Toward a theory of Independent Learning and Teaching, The Journal of Higher Education, vol. 44, pp. 661-680

Moore M.G. (1994). Autonomy and Interdependence, The American Journal of Distance Education, vol. 8,

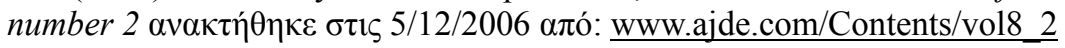

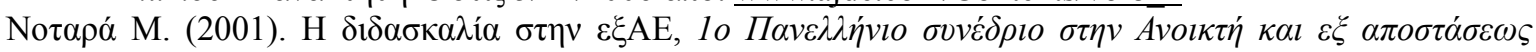

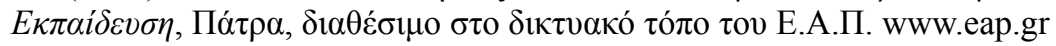

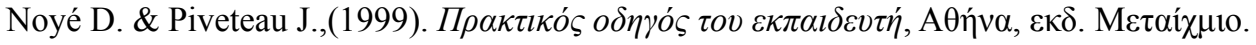

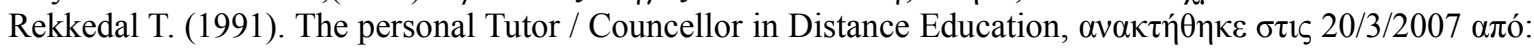
www.nettskolen.com/forskning/25/ziffpers

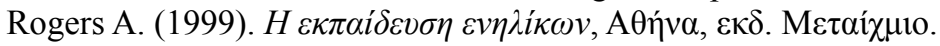

Sewart D. (1993). Student support systems in distance education, Open Learning, 8 (3), 3 - 12

Sherry L. (1996). Issues in Distance Learning, International Journal of Educational Telecommunications, I

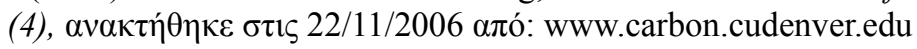

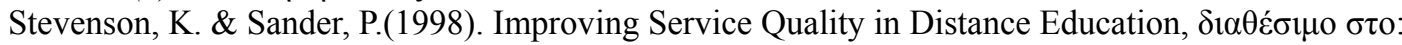

www.eurodl.org/?keyword $=$ distance\%20education\&article $=22$

Tait A. (2003). Reflections on Student Support in Open and Distance Learning, International Review of

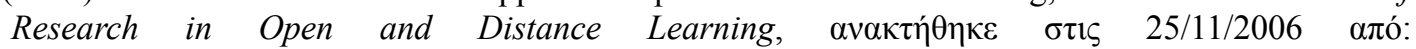
www.irrodl.org/index.php/irrodl/article/view/134/214

Thatch E. \& Murphy K. (1995). Competencies for Distance Education Professionals, Educational Technology Research and Development, vol. 43(1)

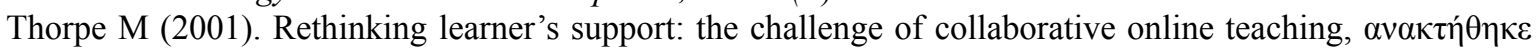

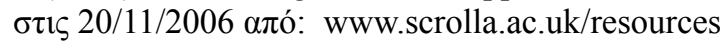




\title{
A Proposed Sketch of a Distance Education Model for the Enrichment of Adult Literacy in Pakistan
}

\author{
Khalid Saleem \\ Assistant Professor University of Education, Lahore, Pakistan \\ khalads@gmail.com \\ Mumtaz Ahmad \\ Ph. D Scholar Allama Iqbal Open University, Islamabad, Pakistan \\ mawatto@gmail.com
}

Abstract

Various efforts have been made to overcome the problem of illiteracy throughout the world, particularly in the developing countries. But, none of these had valuable results. Therefore, in most of the developing countries like Pakistan, governments are concerned about handling the literacy problem effectively. The present paper was conducted in view of the poor literacy condition in Pakistan.it focused upon the analysis of existing literacy situation in Pakistan as well as finding out workable suggestions for overcoming the literacy problem. The study revealed that there was no use of broadcast media or the motivational techniques to attract the illiterate people to the literacy centers. Above all there was no consistency in the literacy programmes due to political factors. The main objectives of the study included to analyze the past literacy programmes in Pakistan and to create a distance education literacy model for Pakistan. The modern way for imparting literacy should be used rather than following the conventional methods. For this purpose a distance education model for enhancing literacy is proposed to be used in Pakistan. This is a theoretical model workable in the low literacy areas with suitable physical provisions.

Key words: adult literacy, distance education, model, enrichment.

\section{Introduction}

Literacy is a basic human need. It has meaning only when it leads to participation in cultural and social activities. It is empowerment that means ability to make decisions and control affairs of one's own life, economically, socially and politically, it is the first step in a life-long earning process of man and women. Life without literacy is life without hope, security and freedom. It is the foundation of all skills and pre-requisite for economic development (Niaz, 1995). Moreover, according to Stromquist (2005, p. 12) in modern societies literacy skills are fundamental to decision-making, personal empowerment, active and passive participation in local and global social community.

The concept of distance education has evolved as an alternative to the formal system of education after an increasing realization that the second one is not perfectly coping with the educational needs and demands of the rapidly growing population. To cope with the increasing demand of education there was need of finding such a way that may help the people in fulfilling their educational thrust which was discovered in the form of distance education. According to Ansari (1992, pp. 25-26); As it was conceived that the formal system of education was not sufficient to meet the increasing demands for education, this led to the search of a via-media which could be alternatively used to expand and fulfill educational needs of all. Instead of 
bringing students to the seats of learning, it was proposed to take schools to the students and hence this concept of distance education came into existence.

In addition to the failure of formal system of education to fulfill educational needs of the people, there are many other causes for the emergence and growth of distance education. Some of the causes according to Perraton (1991, p. 12) are:

First, to cope with the rapidly increasing educational demands of masses. Second, to provide mass education at very low costs. Third, to enable people to acquire education at any stage of their life. Forth, to uplift the professional qualifications of on-the-job people.

The emergence of distance education has provided an alternative that may prove useful in this regard. Hence, according to Wardani (2001, p. 17) 'the department of education in each developing country is challenged to find out ways of eliminating illiteracy and increasing the quality of human resources. One way that needs to be explored is distance education that sounds promising'. It is believed that distance education can reach all illiterate people, even though they reside in very remote areas. Literacy programmes can be developed in the form of printed learning materials, or audio and videocassette, at the national level or at the regional level.

\section{Importance of Literacy}

Literacy is helpful in the development of human relations, the economy and the political-socio structure of nations. Literacy is useful at the individual level in inculcating humanistic etiquettes and manners. The human benefits are related to factors such as the improved self-esteem, empowerment, creativity and critical reflection that can be produced by participation in adult literacy programmes and the practice of literacy. Human benefits are intrinsically valuable and may also be instrumental in realizing other benefits of literacy like improved health, increased political participation and so on (UNESCO, 2006, p. 137).

Bown (1990, p.21) claims that with the acquisition of literacy masses become more confident and courageous. The awareness produced among them because of the newly imparted knowledge help them to demonstrate decisive and confident behaviour. They become more active in their social and private activities. It can also empower learners to take individual as well as collective action in various contexts of their everyday life, such as household, workplace and community (Lind, 1996; Easton, 2005).

Literacy has a wide range of advantages and benefits for economic, social and political development of a country. The positive relationship between economic development and literacy levels and the impact of investment in education on economic growth are well established (Darlene, et al., 2001; Osberg, 2001; Orazem and Gunnarsson, 2003; Sosale, 2000). Likewise, the services of literacy regarding social life are much obvious in four major aspects namely health, gender equality, education, and reproductive behaviour. Above all literacy has implications for the safety of the communities (Maddox, 2005). Along with the traditional components of literacy, citizens may need higher order thinking and problem-solving skills, computer and other related skills, literacy skills necessary for workplace, and literacy skills appropriate for family life.

Literacy has an important relation with the culture. This relation is of two modes. Firstly, literacy influences the culture. It is helpful in bringing the cultural change and preservation of the present cultural values and norms ((Farah, 2005). Moreover, the transformation of culture, in one way or the other, is also dependent of literacy. Secondly, literacy itself is influenced by the prevailing cultural environment 
as planning of a literacy programmes is usually done according to the present culture along with the future needs and requirements. That is why the literacy materials, and the strategies of literacy imparting always correspond with the cultural aspects.

\section{Literacy Efforts in Pakistan}

Since the inception of Pakistan, government has adopted a number of measures for the improvement of literacy rate in the country. But these measures did not prove to be much helpful in improving the literacy rate in the country. According to Ghafoor and Khan (1994, p. 12), since independence there has been a thrust on quantitative expansion of primary education making it accessible to the specific age group (5-9) children by gender and geographic region. But despite hectic efforts the number of illiterates continued to increase at a gradual speed consuming all the endeavours initiated by the government of the Pakistan. For example the number of illiterates increased from 20.25 million in 1951 to 48.8 million in 1998 (UNESCO 2002, p. i).

Literacy and adult education have been recognized as essential elements of human resource development. Therefore, since independence, literacy and adult education have been an integral part of educational policies and national plans. The impact of these policies and plans can be assessed when they are converted into programmes/projects and are actually implemented on the ground (PMLC, 1997, p. $3)$. Following are the various adult literacy drives and schemes launched in Pakistan since independence;

2 Village Agricultural and Industrial Development Programme (1953)

3 Literacy Programme under Basic Democracies (1964-69)

$4 \quad$ Peoples Works Programme, 1973 (Sipah-E-Khidmat)

5 Literacy Programmes through Local Councils in Punjab Province (1974-81)

$6 \quad$ Pakistan Television's Adult Functional Literacy Project 1973.

7 Experimental Pilot Project Integrating Education and Rural Development 1977

8 Rural Education and Development Programme 1982-83

9 Functional Education Project for Rural Areas (1982 to 1985)

$10 \quad 100 \%$ Literate Islamabad Project (1983-84).

11 The National Literacy Plan (1983-93).

12 Television Literacy Centres 1983-84.

13 President Ten Points for Literacy Promotion (1984-85)

14 Iqra Pilot Project 1987-88

15 Nai Roshni Schools Project (1987-89)

16 Training of Unemployed Educated Youth Project (1990-91)

17 Quranic Literacy Project (1991-95).

18 Islamabad Literacy Project 1992-95.

19 Eradication of Illiteracy Project (EIP) 1992-93

20 Ten Year National Literacy Plan (1993-2003)

21 Non-Formal Basic Education Community Schools Project (1995)

22 Crash Literacy Programme (1998)

23 ICT Adult Literacy Project (2001)

24 Adult Literacy Centres (2001-06)

\section{Role of Distance System of Education in Literacy Enhancement}

The process of distance education is currently providing a structured learning environment in which the teacher and student(s) are separated by time and physical 
distance with some form of technology being used for the transmission of all teaching and learning. According to Kaur (1996, p. 1) 'it may be considered a revolutionary development because it marked significant break from the centuries old formal teaching system and led to the development of an innovative multi-media teaching for learning system'.

As far as the relationship of literacy and distance education is concerned, Fleischman (1998, p. 81) states that very little is known about what works and what doesn't when using distance education with Adult Basic Education (ABE). This is probably due to the fact that literacy programmes have historically been the last to access new technology, primarily due to the lack of funding to the literacy field in general (Turner, 1998, p. 68). In the same way distance education can support the literacy in the following ways.

i. Training of literacy personnel.

ii. Enhancement Adult learners' motivation.

iii. Provision of literacy at the doorsteps of illiterates.

iv. Provision of literacy in the cheap mode

v. Public-private partnership for literacy purposes.

vi. Increased possibilities of the use modern technologies

In different countries of the world, distance education has proved itself as effective as the formal system to deliver mass literacy. Drawing upon the well known model of the British Open University, countries such as Pakistan, India and China have combined modern methods of teaching with emerging technologies in order to provide low-cost instruction for basic literacy and job training. Turkey has recently joined those nations involved in large scale distance learning. Only twelve years old, their distance education programme has enrolled almost one million students and is the 6th largest distance education programme in the world. The first programme to provide education out-of-school was the Indian Open School project in 1979, which after ten years became the National Open School (NOS). Pakistan established the Mass Education Programme in 1982 and Bangladesh initiated the Open School of Bangladesh in 1992, both were off-shoots of their national open universities (Edirisingha, 1999, p. 6).

In Indonesia, some aspects of distance education have been implemented for Kejar Paket A, a programme for those who cannot finish formal elementary school. Another experience related to the use of distance and open education is again from India. In 1988, the Salaam Balaak Trust (SBT), a non government organization (NGO), run a centre for street children and working children between the age of three to 18, by applying non-formal and open learning method. In 1990 Vietnam launched literacy and a post-literacy programme (Wagner and Robert 2003, p. 11). In the same way the Gobi Women Project, started in the early 1990s, is a non-formal distance learning programme utilizing print and radio lessons to communicate and renew a number of survival and income-generating skills important to the nomadic women of the Gobi Desert.

Many post-colonial African countries could not expand the conventional schools within their shrinking budgets, even within a generation or more (Perraton, 1982). They chose distance education as an alternative model, which was becoming popular in rich countries at that time. Study Centre model was the particular organizational structure that was established to provide education at a distance in countries in Sub-Saharan Africa. In the initial two examples, learners study at the study centres, under the supervision of facilitators, using the print materials specially prepared for them. Radio and other audio-visual media are also used to some extent 
(Edirisingha, 1999, p. 5). Working on the similar pattern in Kenya a nongovernmental organization named African Medical and Research Foundation (AMREF) started a distance education literacy programme to provide training in the health related issues.

Application of distance education for the promotion of literacy in the modern era is being made with the support of information and communication technologies (ICT). ICT in various forms has been used with some success to support the initial acquisition of literacy skills. For example in the UK, according to Pennells (2005, p. 3 ), ICT has been used to enhance literacy in primary schools under a government initiative. Another literacy programme which adopted the distance education mode was organized by the British Broadcasting Corporation. The first step in this field was taken in Pakistan in 1974 when Allama Iqbal Open University was established. Later on IGNO, Indira Gandhi National Open University, in India also launched some adult literacy programmes.

In the light of the above few examples it is obvious that distance education can be successfully used for literacy purposes. Previously it was considered effective only to enhance the existing skills or the continuing education but, in the present era mixture of styles make it possible to launch literacy campaign through distance education.

\section{Concept and Types of Literacy Models}

A model is not the real world but merely a human construct to help in better understand real world systems. In general, all models have an information input, an information processor, and an output of expected results. As far as the concept of literacy model is concerned, Greane (2005, p. 25) describes in International Reading Association that literacy model is the application of multiple strategies to assist content area teachers/facilitators in developing active and competent readers with in the respective discipline. There are different ways of looking at models for literacy programmes. The most common types of literacy models are;

i. Intensive model: Intensive model is a traditional style classroom delivery with an instructor. Teaching strategies may be customized but the mode of delivery is the classroom experience. This model allows easy measurement of pre- and post-testing.

ii. Immediate model: Immediate Model is the just-in time model. It features a traditional class format, but focuses on a specific, immediate need. The immediate model is not necessarily sequential in nature, and typically has a short life and planning cycle.

iii. Integrated model: In the integrated model work and learning are interconnected. Basic skills programmes analyze what is happening in the workplace and how the basic skills training can support achieving the desired outcomes.

iv. Intervention model: The intervention model is also known as "horizontal" model. The instructor can be at the workplace for one full day.

There is another way of description of literacy models. It deals with the functions of literacy and its components. In different periods of the history, nature and functions of literacy have been changing with the change in individuals' needs and requirements. Moreover, the change in national or local preferences has also forced an amendment in the nature and functions of literacy. Therefore, models of literacy had been undergoing changes with the passage of time.

All the literacy models mentioned above had played an important role at their 
respective places but the need is to upgrade these models so that they can meet the needs and interest of the adult learners in the technologically dominated societies and can enable them to participate in social activities in a productive way.

\section{Statement of the Problem}

The study is designed to propose a sketch of a distance education model for the Improvement of Adult Literacy in Pakistan.

\section{OBJECTIVES OF THE STUDY}

Following were objectives of the study:

i. To assess the need of literacy among Pakistani illiterates.

ii. To specify the role of distance education for the provision of literacy in Pakistan.

iii. To propose a sketch of a distance education model for the improvement of Adult Literacy in Pakistan.

\section{Delimitations of the Study}

In view of the limited time and resources at the disposal of researcher, the study was delimited to: -

i. Propose a sketch of a distance education model for the improvement of Adult Literacy in Pakistan under the administrative control of Allama Iqbql Open University Islamabad.

ii. Adult male illiterates living in the rural areas of the Punjab province of Pakistan.

iii. Literacy instructors and Executive District Officers (E. D. Os) literacy working in Punjab province during 2004-2005.

\section{Procedure of the Study}

This study is descriptive in nature and survey was carried out to collect data for the study,

\section{Population and Sample}

i. Personnel of Department of Distance and Non-formal Education, and Institute of Mass Education of Allama Iqbal Open University Islamabad. Total number of these people was 13 and the whole were taken as sample.

ii. All the 29 Executive District Officers literacy working in different districts of Punjab province were taken as sample.

\section{Development of Instrument}

Separate questionnaires for E. D. Os literacy, personnel from Allama Iqbal Open University and literacy instructors were developed on five point rating scale.

\section{Collection and Analysis of Data.}

i. For data collection questionnaires were personally delivered or mailed to the E. D. Os literacy and personnel from Allama Iqbal Open University. To analyze data collected through the questionnaire of the personnel of AIOU, mean score of their responses was calculated. The following scale value was assigned to each of five responses (Noll, 1965, p.535).

Statements Scale Values




\begin{tabular}{ll}
\hline Strangely Agree (SA) & 5 \\
Agree(A) & 4 \\
Uncertain (UN) & 3 \\
Disagree (D) & 2 \\
Strongly Disagree(SD) & 1 \\
\hline
\end{tabular}

To calculate the mean score following formula was used.

Mean Score $=\underline{5(F \mathrm{SA})+4(F \mathrm{~A})+3(F \mathrm{UNC})+2(F \mathrm{DA})+1(F \mathrm{SDA})}$

Table: 1.1. Analyses of the Data Collected through Questionnaire for the Personnel of AIOU.

\begin{tabular}{|c|c|c|c|c|c|c|}
\hline \multirow{2}{*}{$\begin{array}{l}\text { Item } \\
\text { No. }\end{array}$} & \multirow[t]{2}{*}{ Statement } & \multicolumn{5}{|c|}{ Percentage (\%) of Level } \\
\hline & & SA & A & UN & $\mathrm{D}$ & SD \\
\hline 1 & $\begin{array}{l}\text { Instructors of literacy programme have appropriate } \\
\text { knowledge of adult's psychology. }\end{array}$ & 00 & $\begin{array}{l}18 . \\
1\end{array}$ & $\begin{array}{l}00 . \\
0\end{array}$ & $\begin{array}{l}54 . \\
6\end{array}$ & $\begin{array}{l}27 . \\
3\end{array}$ \\
\hline 2 & $\begin{array}{l}\text { Feedback is provided to learners in existing literacy } \\
\text { programmes. }\end{array}$ & $\begin{array}{l}18 . \\
2\end{array}$ & $\begin{array}{l}00 . \\
0\end{array}$ & $\begin{array}{l}54 . \\
6\end{array}$ & $\begin{array}{l}27 . \\
3\end{array}$ & $\begin{array}{l}27 . \\
3\end{array}$ \\
\hline 3 & $\begin{array}{l}\text { Present literacy materials are interesting for adult } \\
\text { learners }\end{array}$ & 09 & 09 & 00 & $\begin{array}{l}18 . \\
2\end{array}$ & $\begin{array}{l}63 . \\
7\end{array}$ \\
\hline 4 & Evaluation of literacy programmes is made. & 00 & 00 & 00 & $\begin{array}{l}72 . \\
7\end{array}$ & $\begin{array}{l}27 . \\
3\end{array}$ \\
\hline 5 & Continuous feedback is necessary for the adult learners & $\begin{array}{l}72 . \\
7\end{array}$ & $\begin{array}{l}27 . \\
3\end{array}$ & 00 & 00 & 00 \\
\hline 6 & IME can supervise nationwide literacy programme & $\begin{array}{c}36 . \\
4\end{array}$ & $\begin{array}{l}54 . \\
6\end{array}$ & $\begin{array}{l}09 . \\
8\end{array}$ & 00 & 00 \\
\hline 7 & $\begin{array}{l}\text { Telephonic feedback is helpful for solving problems of } \\
\text { illiterates }\end{array}$ & $\begin{array}{c}18 . \\
2\end{array}$ & $\begin{array}{l}45 . \\
4\end{array}$ & $\begin{array}{l}18 . \\
2\end{array}$ & $\begin{array}{l}09 . \\
1\end{array}$ & $\begin{array}{ll}09 . \\
1\end{array}$ \\
\hline 8 & $\begin{array}{l}\text { IME and NGOs can work collaboratively to run a } \\
\text { literacy programme }\end{array}$ & $\begin{array}{c}18 . \\
2\end{array}$ & 00 & 00 & $\begin{array}{l}54 . \\
6\end{array}$ & $\begin{array}{l}27 . \\
3\end{array}$ \\
\hline 9 & $\begin{array}{l}\text { Early experiences of adult learners are utilized to make } \\
\text { them literate }\end{array}$ & $\begin{array}{c}54 . \\
6\end{array}$ & $\begin{array}{l}36 . \\
4\end{array}$ & 00 & 00 & 00 \\
\hline 10 & $\begin{array}{l}\text { It is possible to launch literacy programme through } \\
\text { distance education mode }\end{array}$ & $\begin{array}{c}54 . \\
6\end{array}$ & $\begin{array}{l}45 . \\
4\end{array}$ & 00 & 00 & 00 \\
\hline 11 & $\begin{array}{l}\text { Television assisted teaching proves helpful to teach } \\
\text { writing skills }\end{array}$ & $\begin{array}{c}54 . \\
64\end{array}$ & $\begin{array}{l}27 . \\
3\end{array}$ & $\begin{array}{c}18 . \\
2\end{array}$ & 00 & 00 \\
\hline 12 & $\begin{array}{l}\text { The skill of reading can be taught through television } \\
\text { programmes }\end{array}$ & $\begin{array}{c}54 . \\
6\end{array}$ & $\begin{array}{l}27 . \\
3\end{array}$ & 00 & $\begin{array}{l}18 . \\
2\end{array}$ & 00 \\
\hline 13 & Adult learners are cooperative to their fellow learners & $\begin{array}{c}54 . \\
6\end{array}$ & $\begin{array}{l}27 . \\
3\end{array}$ & 00 & $\begin{array}{ll}18 . \\
2\end{array}$ & 00 \\
\hline 14 & $\begin{array}{l}\text { Literacy programmes through distance education reduce } \\
\text { administrative problems }\end{array}$ & $\begin{array}{c}54 . \\
6\end{array}$ & $\begin{array}{l}27 . \\
3\end{array}$ & $\begin{array}{c}18 . \\
2\end{array}$ & 00 & 00 \\
\hline 15 & Distance education literacy programme are economical & $\begin{array}{l}36 . \\
4\end{array}$ & $\begin{array}{l}36 . \\
4\end{array}$ & $\begin{array}{c}18 . \\
2\end{array}$ & $\begin{array}{ll}09 . \\
1\end{array}$ & 00 \\
\hline 16 & $\begin{array}{l}\text { Literacy programmes through distance education can } \\
\text { prove successful in Pakistani situation }\end{array}$ & $\begin{array}{c}36 . \\
4\end{array}$ & $\begin{array}{l}54 . \\
6\end{array}$ & $\begin{array}{c}09 . \\
1\end{array}$ & 00 & 00 \\
\hline 17 & $\begin{array}{l}\text { Casual meetings between adult learners and instructors } \\
\text { are necessary to solve literacy problems }\end{array}$ & $\begin{array}{c}36 . \\
4\end{array}$ & $\begin{array}{l}45 . \\
4\end{array}$ & 00 & $\begin{array}{l}18 . \\
2\end{array}$ & 00 \\
\hline
\end{tabular}




\begin{tabular}{|c|c|c|c|c|c|c|}
\hline 18 & $\begin{array}{l}\text { Mass media motivates adult illiterates to join literacy } \\
\text { programme }\end{array}$ & $\begin{array}{c}36 . \\
4\end{array}$ & $\begin{array}{l}36 . \\
4\end{array}$ & $\begin{array}{c}18 . \\
2\end{array}$ & $\begin{array}{l}09 . \\
1\end{array}$ & 00 \\
\hline 19 & $\begin{array}{l}\text { Radio helps in creating awareness about literacy } \\
\text { programmes }\end{array}$ & $\begin{array}{c}27 . \\
3\end{array}$ & $\begin{array}{l}45 . \\
4\end{array}$ & $\begin{array}{c}27 . \\
3\end{array}$ & 00 & 00 \\
\hline 20 & Computer can be applied to teach literacy skills & $\begin{array}{c}36 . \\
4\end{array}$ & $\begin{array}{l}27 . \\
3\end{array}$ & $\begin{array}{l}18 . \\
2\end{array}$ & $\begin{array}{l}18 . \\
2\end{array}$ & 00 \\
\hline
\end{tabular}

Data shown in Table No. 1.1 reflects that AIOU personnel agreed with the Statements No.5 (72.7\%), 6 (54.6\%), 9 (54.6\%), 10 (54.6\%), 11 (54.6\%), $12(54.6 \%), 13$ $(54.6 \%), 14(54.6 \%) 15(54.6 \%)$ and16 (54.6\%). In the same way, these personnel showed their disagreement towards the Statements No. 1(54.6\%), 3(63.7\%), 4, $(72.7 \%)$, and $8(54.6 \%)$. However, majority of the personnel remained uncertain to the Statement No. 2(54.6\%).

It is reflected from the analysis of data for the questionnaire of AIOU personnel that despite the importance of feedback and availability of instructors at telephone the adult learners are not properly guided. Moreover AIOU personnel agreed that the distance education mode for the provision of literacy is best alternative for the developing countries as it reduces the administrative problems and saves funds. Similarly, it was agreed by the AIOU personnel that through the use of broadcast media, especially radio and television, literacy skills like reading and writing can be imparted. Therefore, through the use of broadcast media and casual meetings between adult learners and the literacy instructors literacy programmes can be easily launched in Pakistan.

Table: 1.2. Analyses Of The Data Collected Through The Questionnaire For E. D. Os

Literacy.

\begin{tabular}{|c|c|c|c|c|c|c|}
\hline \multirow{2}{*}{$\begin{array}{l}\text { Item } \\
\text { No. }\end{array}$} & \multirow[t]{2}{*}{ Statement } & \multicolumn{5}{|c|}{ Percentage $(\%)$ of Level } \\
\hline & & SA & A & UN & D & SD \\
\hline 1 & $\begin{array}{l}\text { Community surveys are conducted for need assessment } \\
\text { before launching a literacy programme }\end{array}$ & 66.7 & 20.8 & 00 & 8.3 & 4.2 \\
\hline 2 & $\begin{array}{l}\text { The literacy centres are approachable for the adult } \\
\text { illiterates }\end{array}$ & 58.3 & 25.0 & 8.3 & 4.2 & 4.2 \\
\hline 3 & $\begin{array}{l}\text { The instructors' attitude toward the adult illiterates is } \\
\text { polite }\end{array}$ & 58.3 & 16.7 & 4.2 & 8.3 & 12.5 \\
\hline 4 & $\begin{array}{l}\text { Instructor is able to build up teamwork environment } \\
\text { during the teaching and learning process }\end{array}$ & 8.3 & 8.3 & 00 & 54.2 & 29.2 \\
\hline 5 & $\begin{array}{l}\text { Instructors are capable of creating pleasant teaching } \\
\text { learning environment at literacy centres }\end{array}$ & 20.8 & 20.8 & 00 & 41.7 & 16.7 \\
\hline 6 & The progress of learners is regularly evaluated & 20.8 & 12.5 & 00 & 58.3 & 8.3 \\
\hline 7 & Tap recorder is used during teaching & 8.3 & 8.3 & 00 & 54.2 & 29.2 \\
\hline
\end{tabular}


Open Education - The J oumal for Open and Distance Education and Educational Technology

\begin{tabular}{|l|l|l|l|l|l|l|}
\hline 8 & $\begin{array}{l}\text { VCP/VCR is provided at literacy centre to teach adult } \\
\text { learners }\end{array}$ & 00 & 00 & 00 & 29.2 & 70.8 \\
\hline 9 & Supervisors facilitate the instructors at literacy centre & 00 & 00 & 8.3 & 62.5 & 29.2 \\
\hline 10 & Literacy programmes are launched on regular basis. & 00 & 00 & 00 & 16.7 & 83.3 \\
\hline 11 & $\begin{array}{l}\text { Instructors highlight the importance of literacy in the } \\
\text { society }\end{array}$ & 20.8 & 8.3 & 00 & 45.8 & 25.0 \\
\hline 12 & Instructors are punctual & 41.7 & 37.5 & 00 & 16.6 & 4.2 \\
\hline 13 & $\begin{array}{l}\text { The instructors need training in how to deal with the } \\
\text { illiterate persons }\end{array}$ & 33.3 & 45.8 & 00 & 20.8 & 00 \\
\hline 14 & $\begin{array}{l}\text { The administration provides learning material to the adult } \\
\text { learners free of cost }\end{array}$ & 33.3 & 50.0 & 00 & 16.7 & 00 \\
\hline 15 & Local community avoids from literacy personnel & 16.7 & 16.7 & 00 & 50.0 & 16.7 \\
\hline 16 & Instructors are appointed from local community & 41.7 & 33.3 & 00 & 25.0 & 00 \\
\hline 17 & $\begin{array}{l}\text { Instructor motivates the people to join literacy } \\
\text { programmes }\end{array}$ & 54.2 & 25.0 & 00 & 12.5 & 8.3 \\
\hline 18 & $\begin{array}{l}\text { Broadcast media provide supplementary support to the } \\
\text { literacy programme }\end{array}$ & 4.2 & 4.2 & 00 & 62.5 & 29.2 \\
\hline 20 & Audio-visual material is available at literacy centres & 8.3 & 8.3 & 00 & 16.7 & 66.7 \\
\hline 22 & The material developed for literacy is easy to understand & 37.5 & 50.0 & 00 & 12.5 & 00 \\
\hline 19 & The supervising staff of literacy programmes is sufficient & 87.5 & 12.5 & 00 & 00 & 00 \\
\hline
\end{tabular}

Analysis of data in Table No. 1.2 shows that E. D. Os literacy agreed with the Statements No.1 (66.7\%), 2 (58.3\%), 3 (58.3\%), $17(54.2 \%)$ and 22(87.5\%). Similarly, these officers showed their disagreement towards the Statements No. $4(54.2 \%), 6(58.3 \%), 7,(54.2 \%), 8(70.8 \%), 9(62.5 \%), 10(83.3 \%), 18(62.5 \%)$ and $21(66.7 \%)$. The analysis of the responses of E. D. Os literacy showed that the literacy programmes in Pakistan are poorly funded with inadequate facilities for the adult learners as well as the literacy instructors. There is no support material available at the literacy centres. The aspect of evaluation and feedback was almost missing in these programmes although it was agreed by the E. D. Os literacy that there is sufficient staff available for this purpose. Despite the local appointment of the instructors they are unable to motivate the people to join literacy programmes.

\section{Conclusions}

i. There exists need for literacy among Pakistani illiterates and due importance is given to literacy by them. 
ii. Moreover, local people cooperate with the literacy personnel and community welcomes the literacy programmes.

iii. Community surveys are not conducted for need assessment before launching a literacy programme in Pakistan and no proper arrangements are made for the mobilization of community.

iv. New coming governments do not continued the on-going literacy programme of the previous governments

v. Despite insufficient funding from the government for literacy programmes free of cost learning material and financial assistance is provided to the adult learners

vi. Literacy materials for the literacy programmes in Pakistan are not locally prepared.

vii. Literacy instructors are appointed on merit from the local community. Therefore, they highlight the importance of literacy in the society. Occasionally volunteers are deputed to teach adult illiterates

viii. literacy instructors are not trained to teach adult illiterates and because of the absence of training they;

a. face difficulties in handling adult learners

b. do not motivate adult illiterates to join literacy class

c. never relate the literacy work to the everyday life of adult learners

d. are not familiar with the needs of adult learners

e. do not show polite attitude towards the adult illiterates

f. are not punctual

ix. There are no proper facilities at literacy centre as there is lack of furniture and unavailability of A. V. Aids, like tap recorder, VCP/VCR teaching machines and Flip chart / Flash cards. In the same way literacy centres are not established at an easy access of learners.

$\mathrm{x}$. There is no use of broadcast media to supports literacy programmes.

xi. Literacy programmes through distance education can prove successful in Pakistani situation as;

a. basic infrastructure to launch such programme is available

b. literacy programmes through distance education reduce administrative problems

c. literacy programmes through distance education are economical, and

d. casual meetings between adult learners and instructors can solve their literacy problems

xii. Any distance education literacy programme can be made successful by the effectiveness of media support, telephonic feedback, usefulness of print material, face to face interaction and through the guidance and counselling of adult learners instructors and supervisors

xiii. IME and IET can mutually cooperate for the need analysis, planning, development of print materials and provision of media support to the adult learners moreover; Regional Directorates can help IME during the phases of training and Evaluation

\section{Recommendations}

i. Literacy should be seen as a continuous process that requires sustained learning and application. There are no magic lines to cross from illiteracy into literacy. All policies and programmes should be defined to encourage sustained participation and celebrate progressive achievement rather than focusing on one-off provision with a single end point. 
ii. Governments have to lead responsibility in meeting the right to adult literacy and in providing leadership, policy frameworks, an enabling environment and resources. They should:

a. ensure cooperation across all relevant ministries and linkages to all relevant development programmes,

b. work in systematic collaboration with experienced civil society organizations,

c. ensure linkages between all the relevant agencies, especially at the local level, and ensure relevance to the issues in learners' lives by promoting the decentralization of budgets and of decision-making over curriculum, methods and materials.

iii. The Ministry of Education along with the EFA Wing should co-operate the agencies and institutions who desire to contribute in the literacy enhancement efforts.

iv. Such NGOs should be encouraged who wish to work under the umbrella of an established institution and supports the literacy efforts at the grass root level.

v. Governments should take responsibility to stimulate the market for production and distribution of a wide variety of materials suitable for new readers, for example working with publishers / newspaper producers. They should balance this with funding for local production of materials, especially by learners, facilitators and trainers.

vi. For effective literacy instruction trained literacy instructors are needed who are expert in adult psychology and the teaching of adults.

vii. It is needed to make the literacy programme consistent and regular. Even the change of governments should not affect the literacy programmes.

viii. There is need to increase funding for the literacy programmes.

ix. There is need to involve the local community in the literacy programme to make it acceptable by all sections of society.

Proposed Distance Education Model for Enhancement of Literacy in Pakistan 


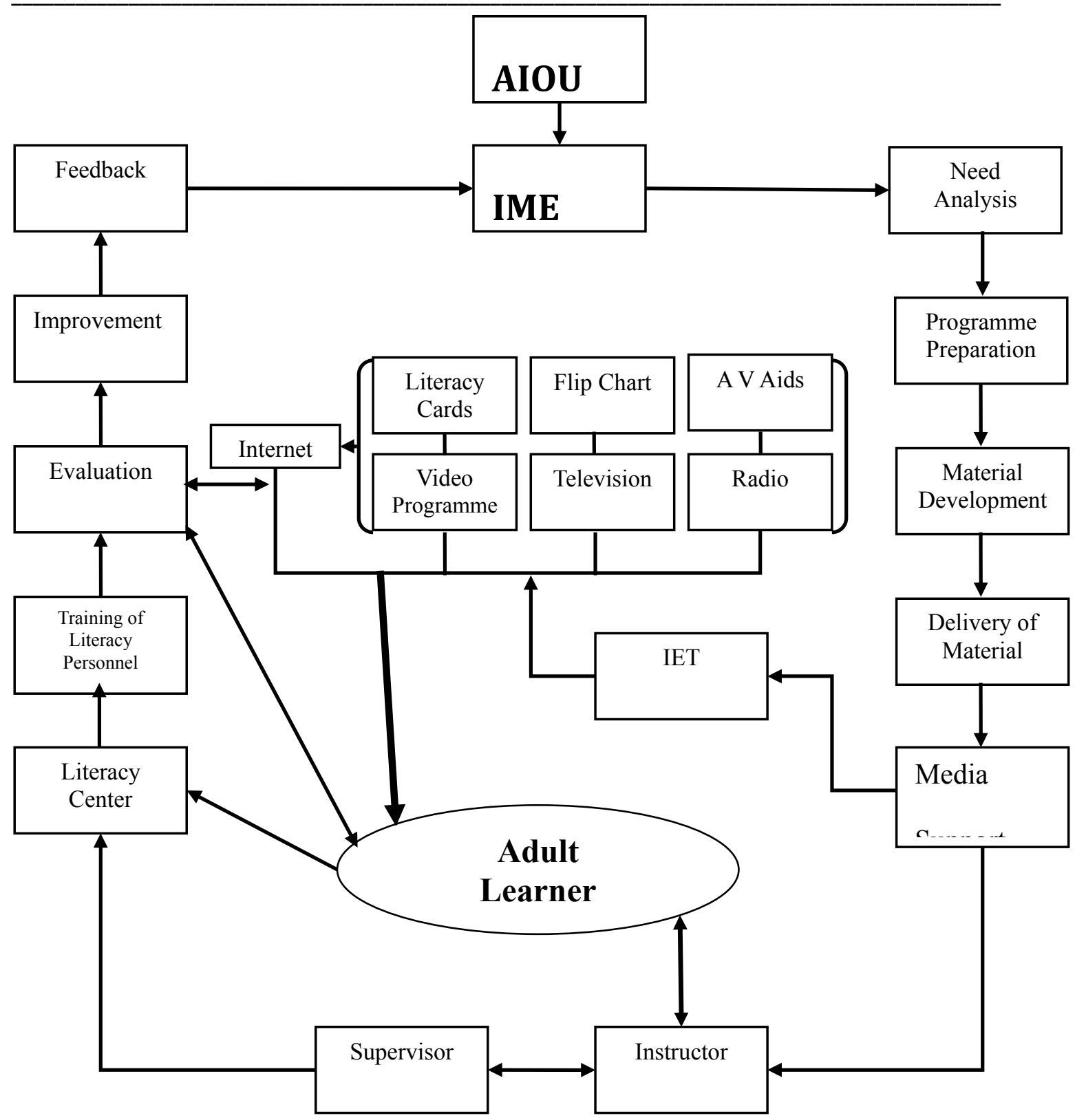

The description of the model is given on the next page. 
The distance education model for enhancement of literacy runs as

Allama Iqbal Open University is the mother institute for distance education literacy model and the Institute of Mass Education is the fundamental element of the model.

Need analysis is made by the IME.

On the bases of need analysis literacy programme is planned and prepared.

- Material for the literacy programme is then prepared both for the adult learners and the literacy personnel in the form of literacy primers and manuals.

The developed material is then delivered to the concerned persons.

- Media support is provided after delivering the material. For the media support Institute of Educational technology is responsible to develop- the Radio, Television and Video programmes along different types of non-broadcast media including A. V. Aids like Flip Charts and Literacy Cards. All the developed materials are available through Internet to the adult learners as well as the instructors and other officials.

This media support is provided both to the adult learners and the literacy instructors.

Adult learners have casual visits to the literacy center for face to face meeting with the literacy instructor.

- Literacy instructors have two way contact with both the adult learners and the supervisor who on the other hand is also responsible for supervising the literacy center.

- Training is provided to the personnel involved in the process of literacy impartation

- Evaluation of the adult learners and media support is conducted.

- On the bases of evaluation improvement is made and finally feedback is provided to the IME. 


\section{References}

Ansari, M. M. (1992). Economics of distance education, New Delhi: Concept Publishing Company.

Bown, L. (1990). Preparing the Future: women, literacy and development. London: ActionAid Development Report No. 4., ActionAid/ODA.

Darlene B. et al. (2001). Education and enlightenment: Literacy Awareness Kit, Ottawa: Department of Human Resources Development, Canada.

Easton, P. B. (2005). Literacy and empowerment: Raising Key Issues. Paper presented at the Sixth Meeting of the Working Group on Education for All, UNESCO, 1921 July 2005.

Edirisingha, P. (1999). Reaching the unreached through distance education: Costs, Outcomes and Sustainability, Beijing: Regional Seminar on the Use of Simple and Modern Media for Rural Education, Sponsored by the UNESCO Regional Office, 12-16 Dec 1999.

Farah, I. (2005). The cultural benefits of literacy, Background paper for EFA Global Monitoring Report 2006, through the University of East Anglia, Norwich.

Fleischman, J. (1998). Distance learning and adult basic education, In C. Hopey (Ed.) Technology, Basic Skills, and Adult Education: Getting Ready to Move Forward, The Ohio State University: The Center on Education and Training for Employment, College of Education.

Ghafoor, A. \& A. S. Khan (1994). Literacy efforts in Pakistan, from Rhetoric to Action, Islamabad, National Education and Training Commission, Ministry of Education.

Greaney, V. (2005). Reading achievement levels and reading habits of Indonesian Pupils, Unpublished paper, Washington, DC: World Bank.

Kaur, A. S. (1996). Managing distance education, New Delhi: Deep and Deep Publications

Lind, A. (1996). Free to speak up - overall evaluation of the national literacy programme in Namibia. Edited by Directorate of Adult Basic Education, Ministry of Basic Education and Culture. Windhoek:, Gamsberg MacMillan.

Maddox, B. (2001). Literacy and the market: The economic uses of literacy among the peasantry in North-West Bangladesh, in B. Street (ed.), "Literacy and Development: Ethnographic Perspectives", London: Routledge.

Niaz, I. (1995). How to establish the literacy resource centre for girls and women and effectively manage IT, Dhaka, Bangladesh: Presented at LRC Training Workshop 21-25 August 1995.

Orazem, P. F. \& Gunnarsson, V. (2003). Child labour, school attendance and academic performance: A Review, Working Paper, International Programme on the Elimination of Child Labour. Geneva, International Labour Office.

Osberg, L. (2001). Schooling, literacy and individual earnings, Seventh International Adult Literacy Survey Monograph, Statistics Canada.

Pennells J. (2005). Literacy, Distance Learning and ICT, Cambridge: IEC.

Perraton, H (1982). Alternative routs to formal education; distance teaching for school equivalency, London, Johns Hopkins University Press

Perraton, H. (1991). Administrative structure for distance education, London: Common Wealth of Learning.

PMLC. (1997). Adult literacy in Pakistan: Quranic Literacy Project, Islamabad:rime Minister's Literacy Commission, Ministry of Education Government of 
Pakistan.

Sosale, S. (2000). Trends in private sector development in world bank education Projects, Washington, D.C: Policy Research Working Paper 2452. Human Development Network, Education Team, World Bank.

Stromquist, N. (2005). Gender and literacy development, In: D. A. Wagner, R. L. Venezky and B. V. Street (eds.), "Literacy: An International Handbook. Boulder", Colo: Westview Press.

Turner, T. C. (1998). Technology in adult education programs, In C. Hopey (Ed.) Technology, Basic Skills, and Adult Education: Getting Ready to Move Forward, The Center on Education and Training for Employment, College of Education, The Ohio State University.

UNESCO (2002). Literacy trends and statistics in Pakistan, Islamabad, UNESCO Office.

UNESCO. (2006). Education for All Global Monitoring Report 2006: Paris, UNESCO.

Wagner, D. A. and Robert K. (2003). New technologies for literacy and adult education: A Global Perspective, International Literacy Institute, National Center for Adult Literacy, University of Pennsylvania.

Wardani, I. G. A. K. (2001). Literacy campaign through distance education, the First Seameo Education Congress Bangkok, 26-29 March 2001. 


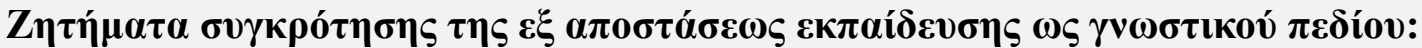

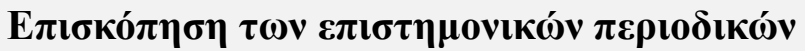 \\ Issues in the disciplinary constitution of open and distance learning: A review of the field's academic journals
}

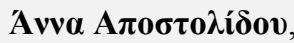

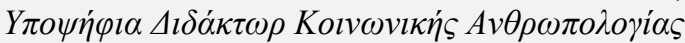
University College London, annapostolidou@gmail.com

\begin{abstract}
This paper focuses on a selection of international printed and electronic ODL-related journals that cover the domains of distance learning, e-learning and educational technology in order to point out certain problematic areas in the disciplinary constitution of Open and Distance Learning today. Attempting an application of discourse analysis and content analysis on the journals' scope, structure and content, the paper presents a critical discussion of four main issues: a) The formation of hegemonic and peripheral educational cultures, which reflect different educational needs and agendas; $b$ ) the issue of access to education, especially concentrating on social exclusion and the developing world; c) the concept of educational technology and its ambiguous uses, with reference to learning needs and pedagogical practices; and d) the epistemological and scientific identity of Open and Distance Learning and the necessity for careful theoretical and methodological reflection on its founding principles. Acknowledging that the future character of education is being shaped by contemporary strategic decisions, the present inspection seeks to demonstrate the analytic value of the study of ODL-related academic journals and its contribution to the field's reflective awareness.
\end{abstract}

Keywords: Journal, educational technology, educational culture, access, epistemological identity.

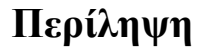

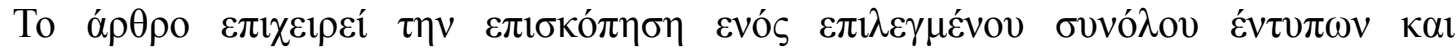

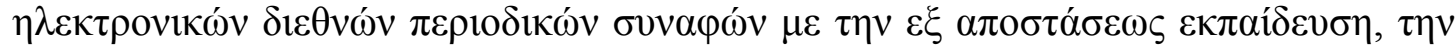

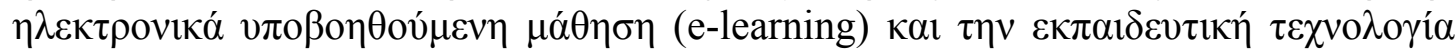

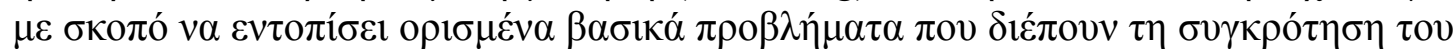

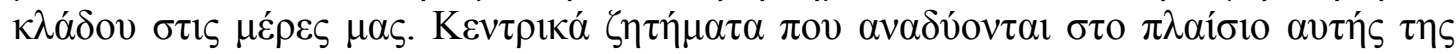

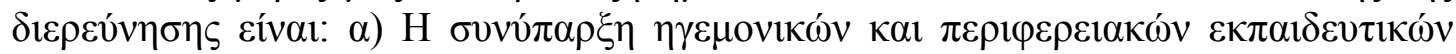

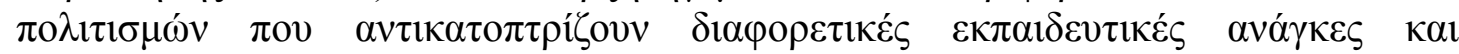

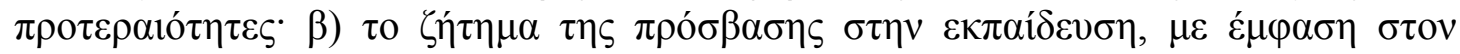

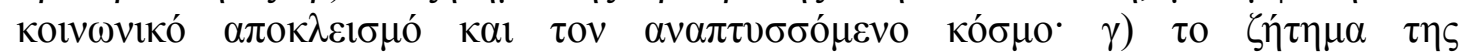

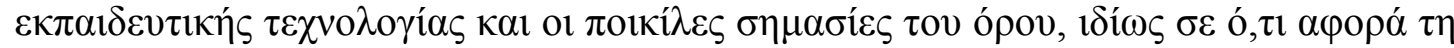

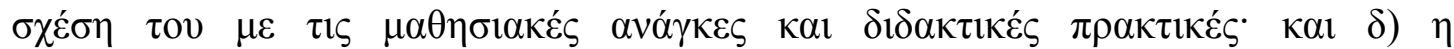

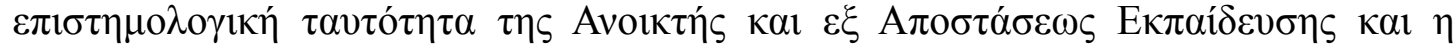

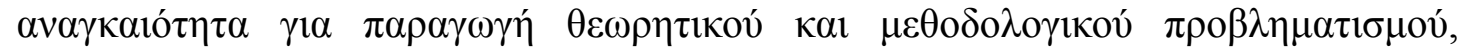

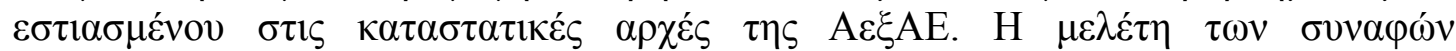

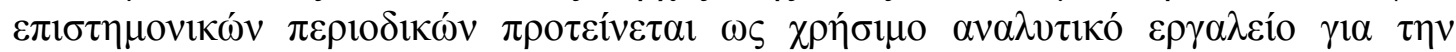

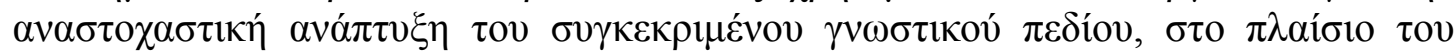

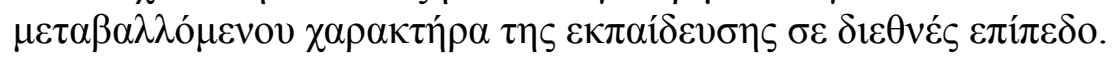

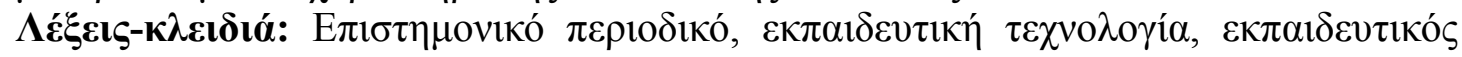




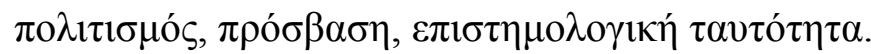

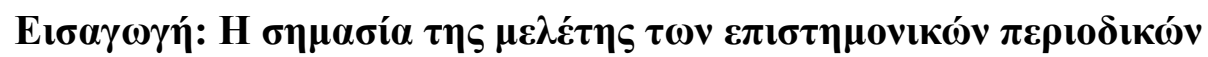

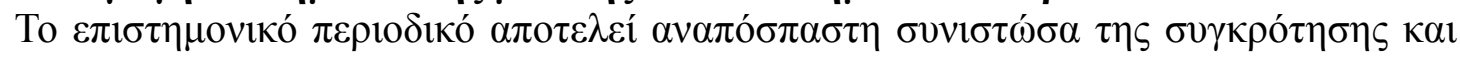

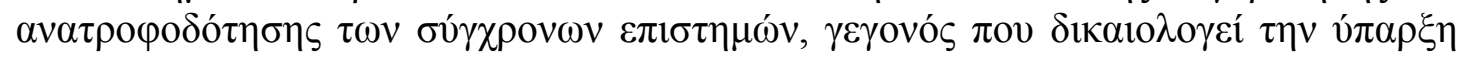

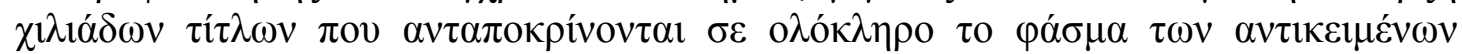

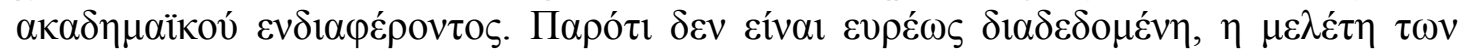

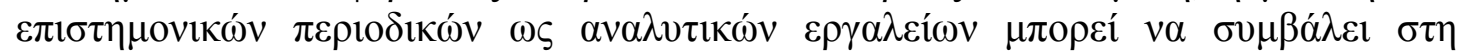

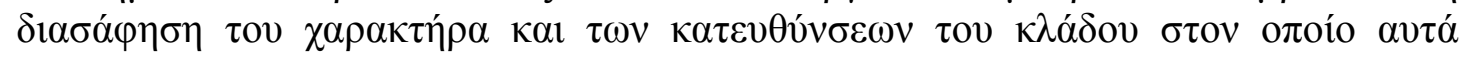

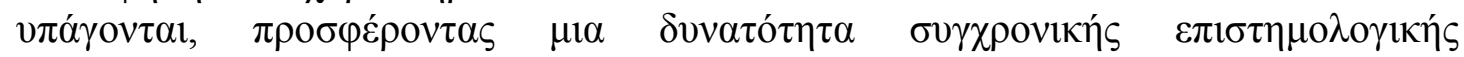

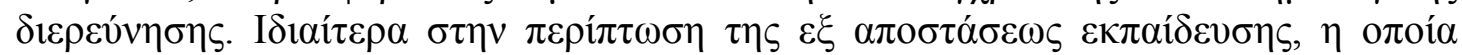

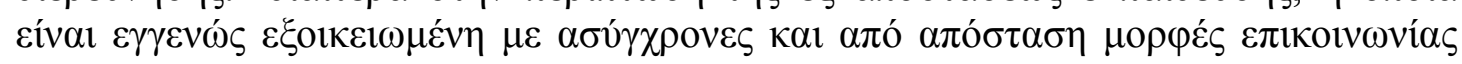

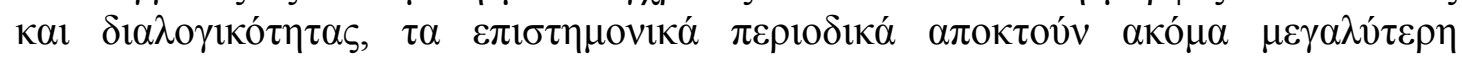

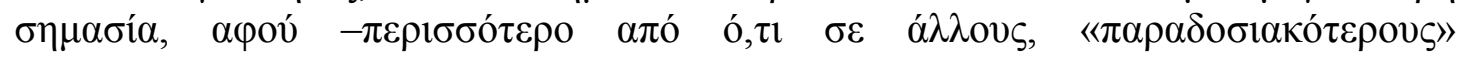

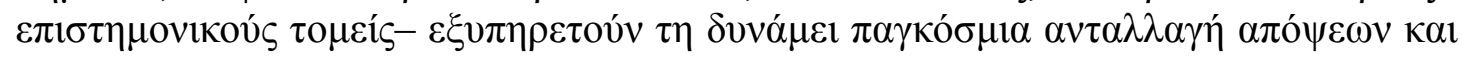

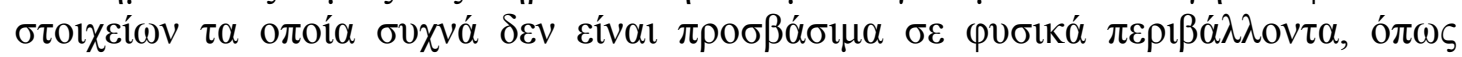

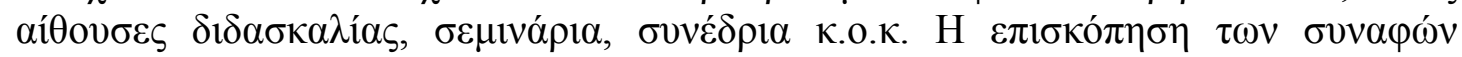

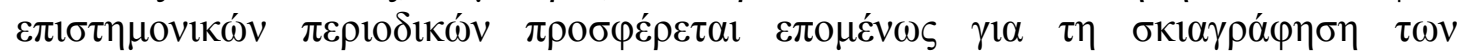

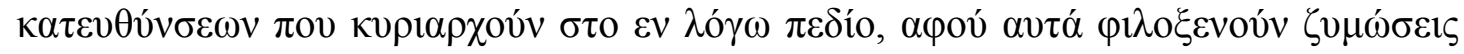

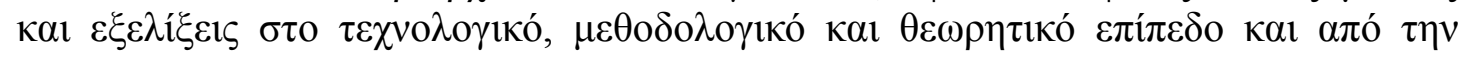

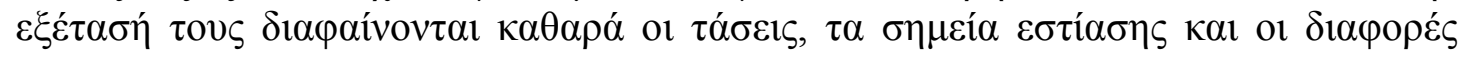

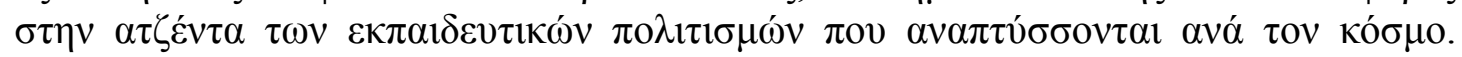

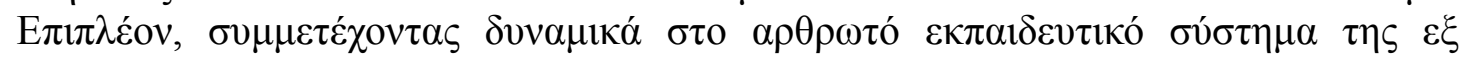

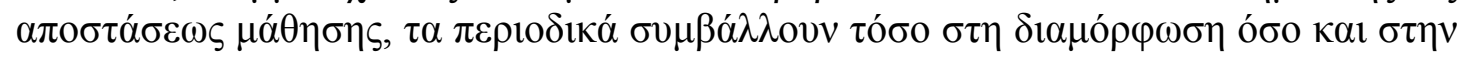

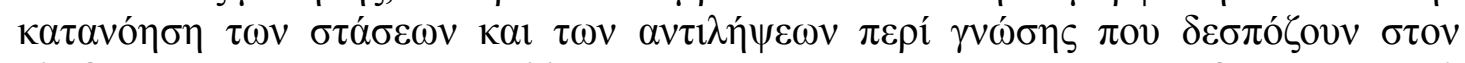

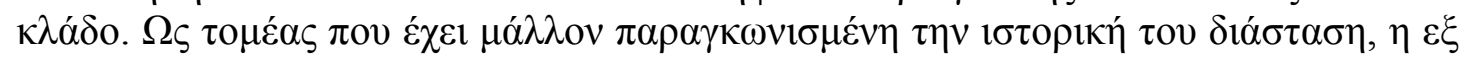

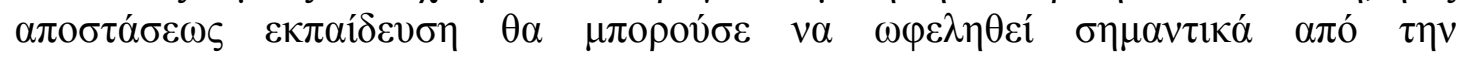

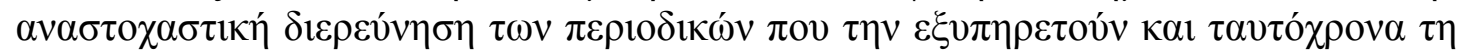

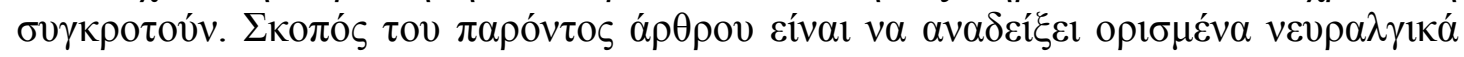

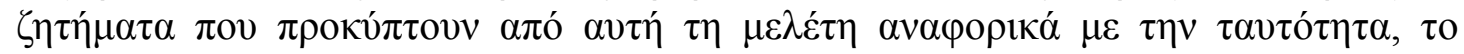

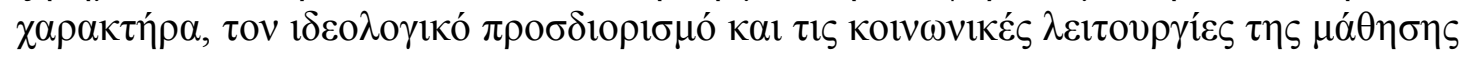

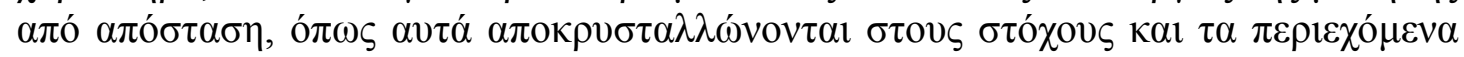
$\tau \omega \nu \pi \varepsilon \rho 10 \delta ı \kappa \omega v ~ \sigma \varepsilon \delta 1 \varepsilon \theta v \eta \dot{~ \kappa \lambda i ́ \mu \alpha \kappa \alpha . ~}$

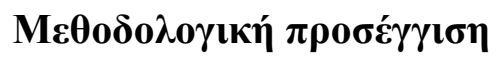

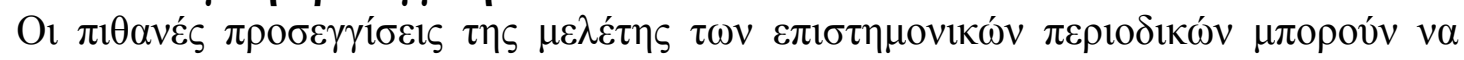

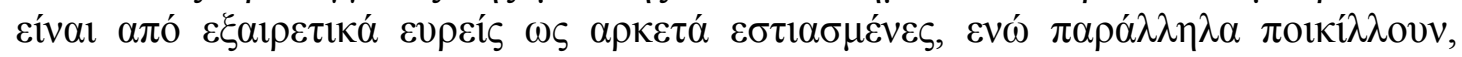

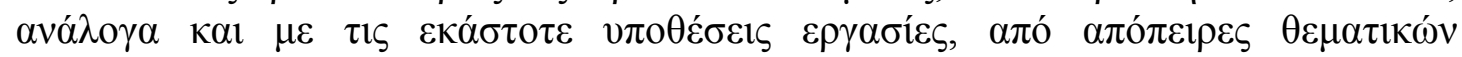

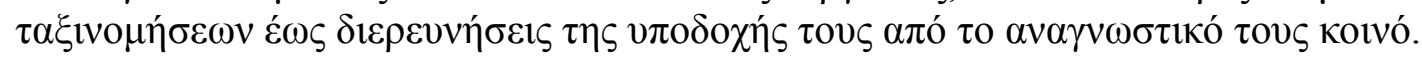

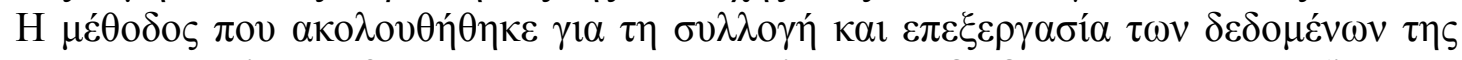

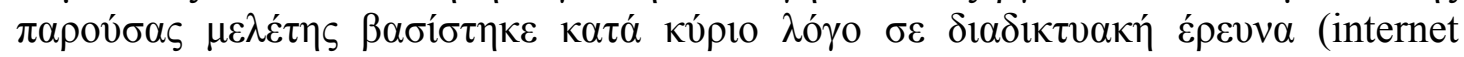

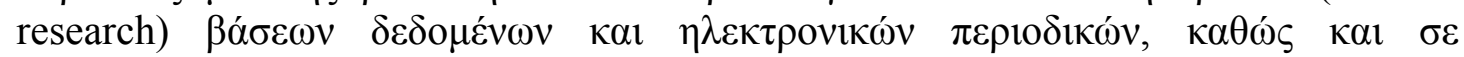

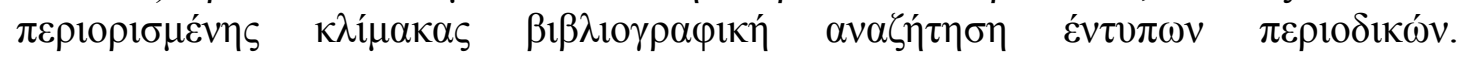

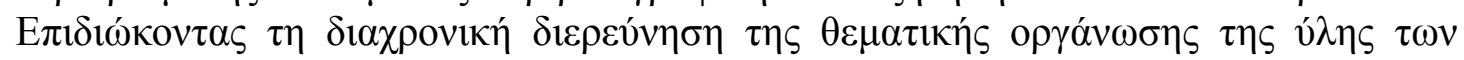

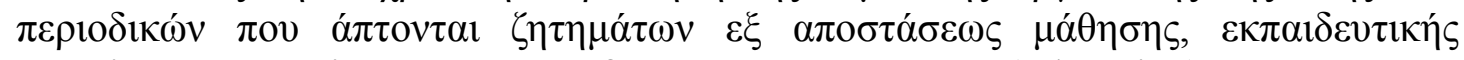

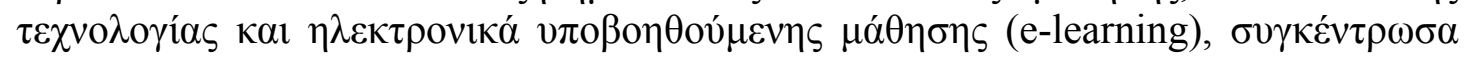

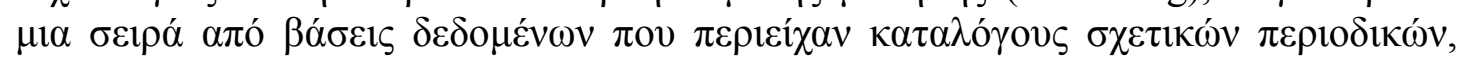

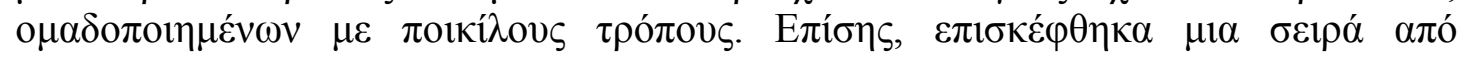

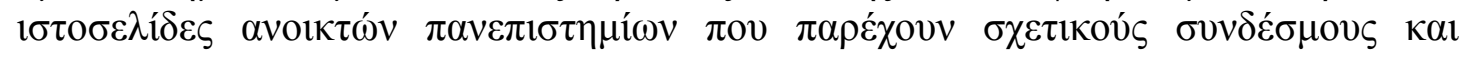




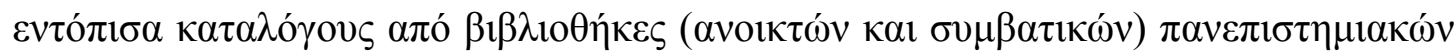

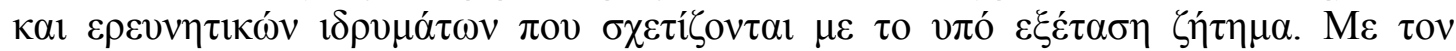

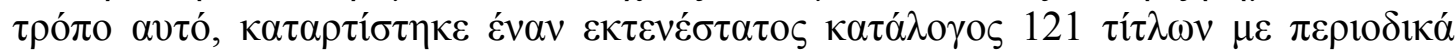

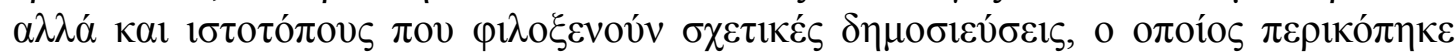

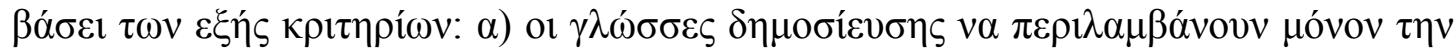

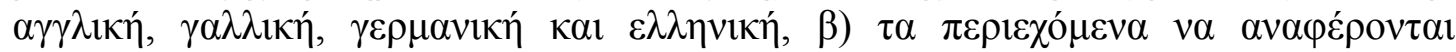

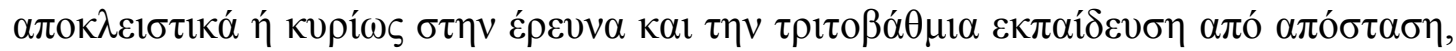

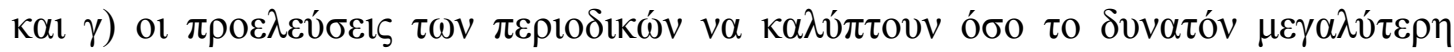

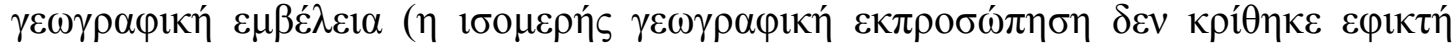

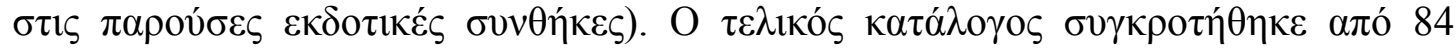

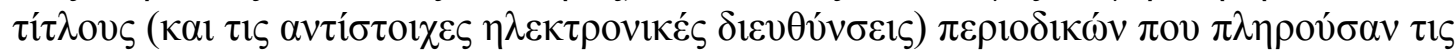

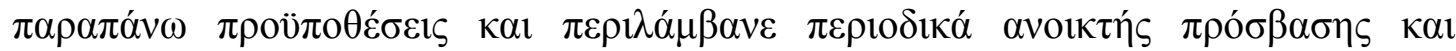

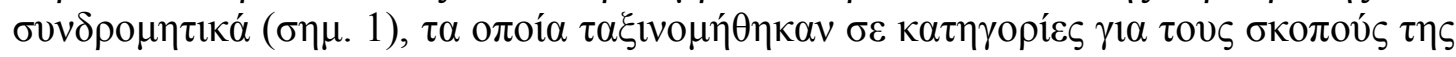

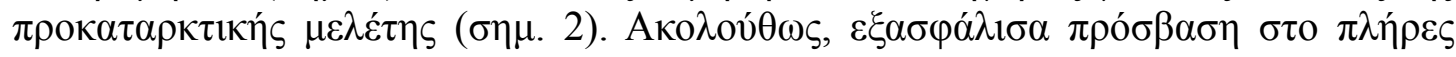

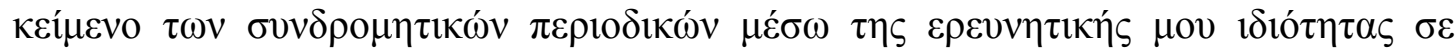

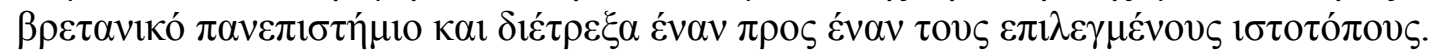

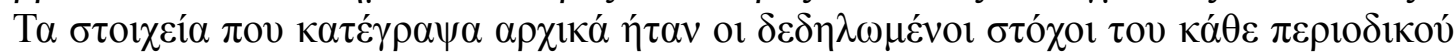

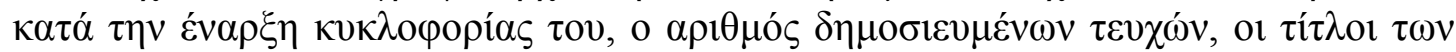

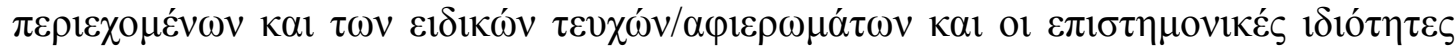

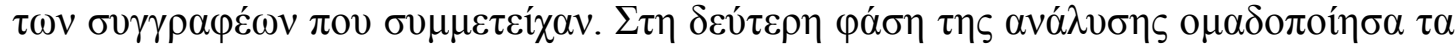

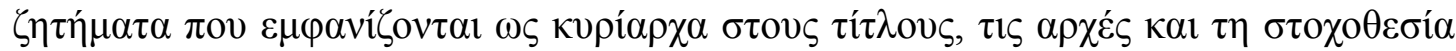

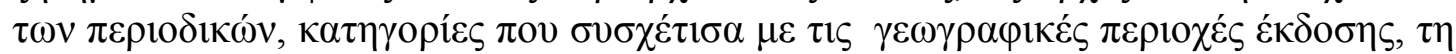

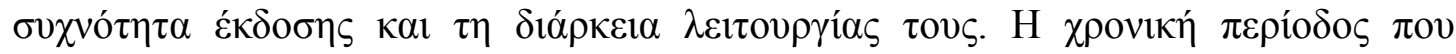

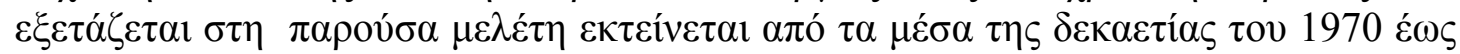

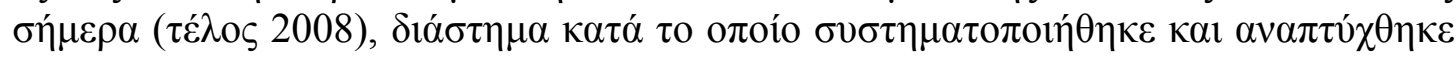

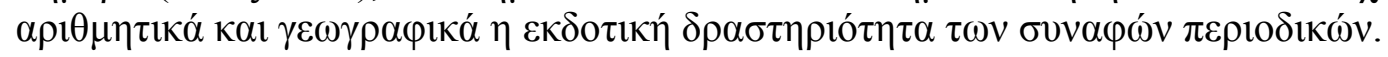

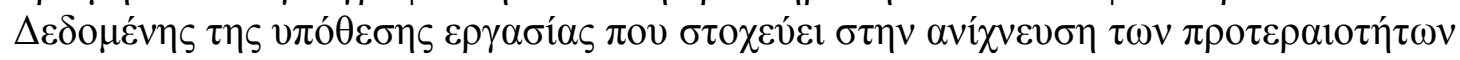

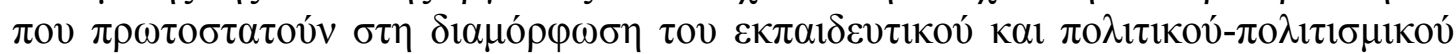

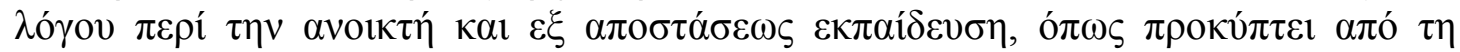

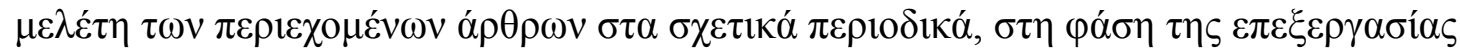

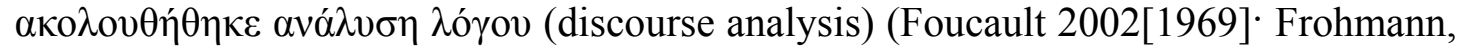

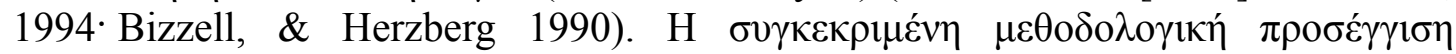

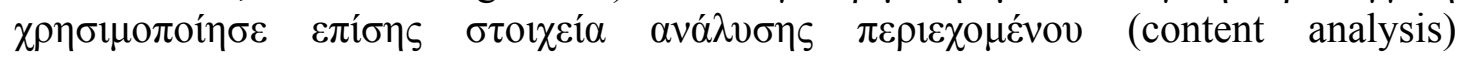

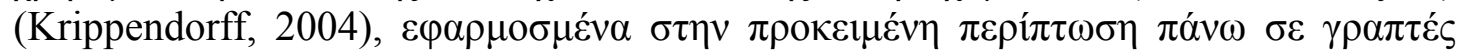

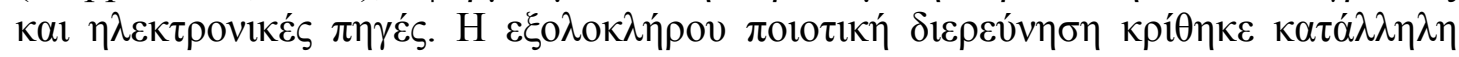

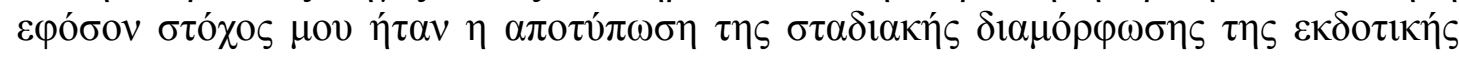

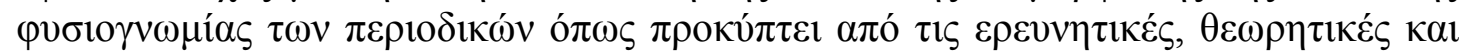

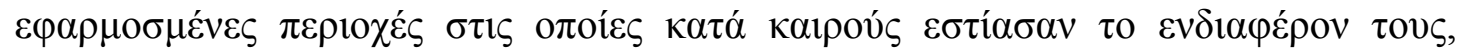

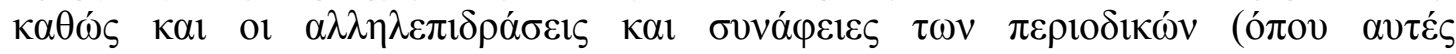

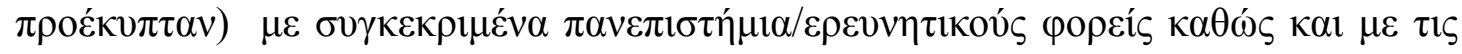

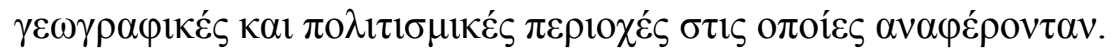

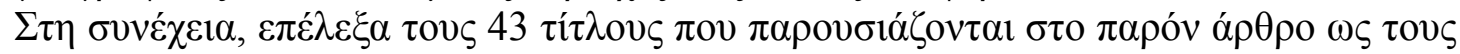

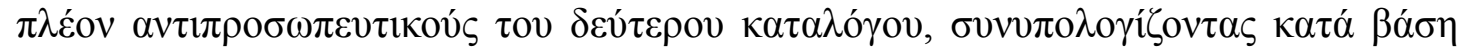

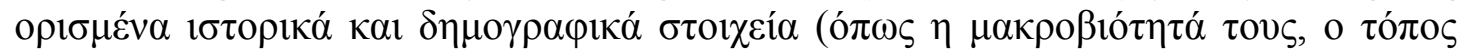

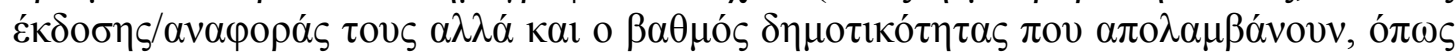

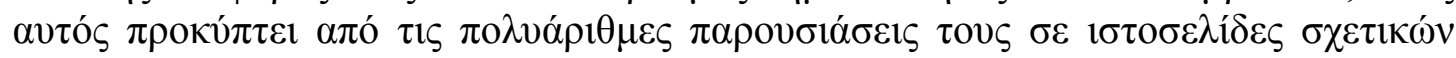

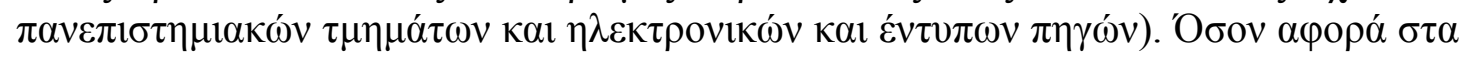

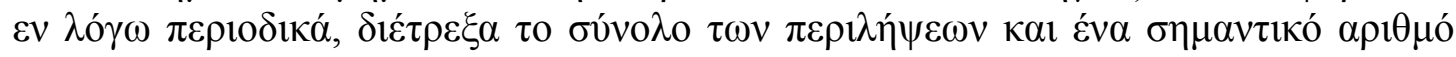

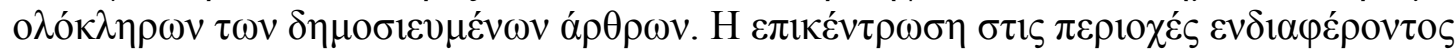

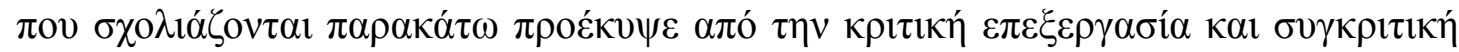




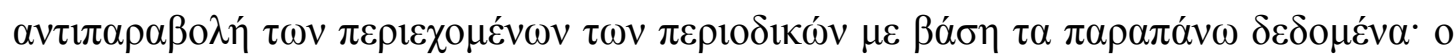

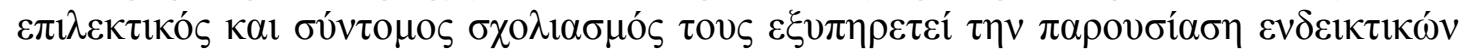

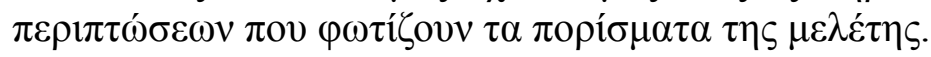

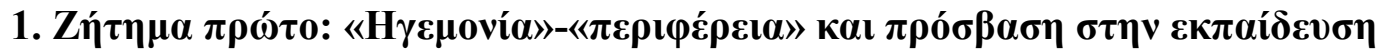

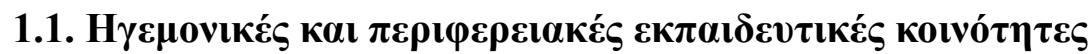

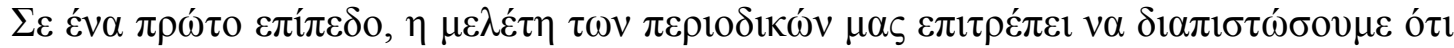

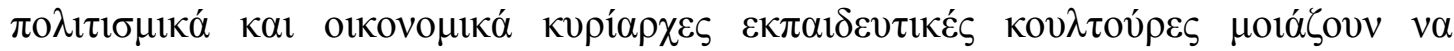

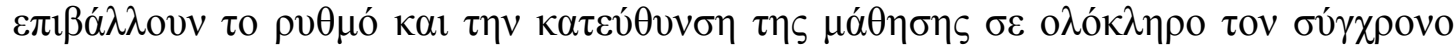

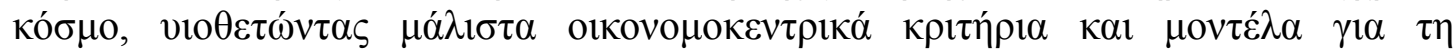

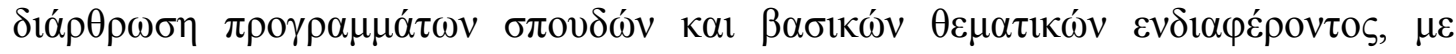

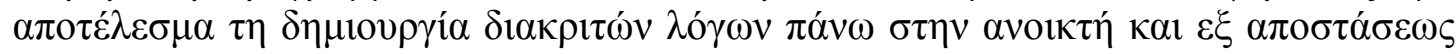

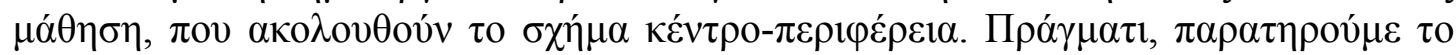

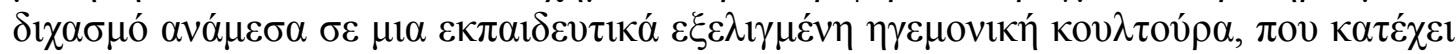

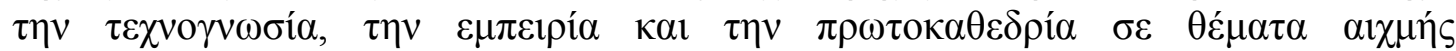

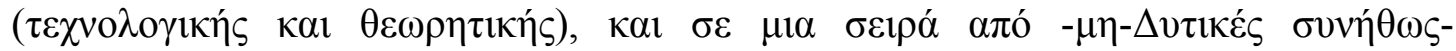

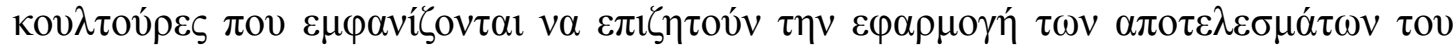

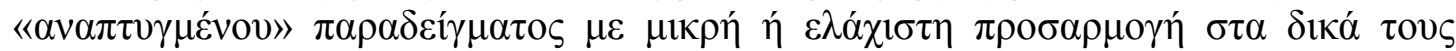

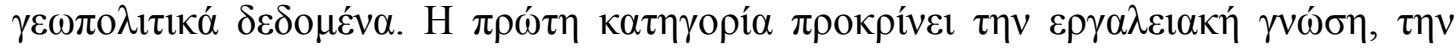

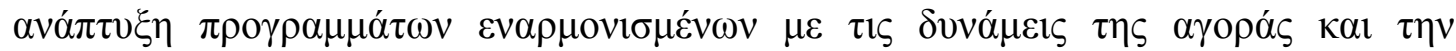

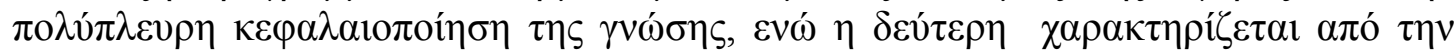

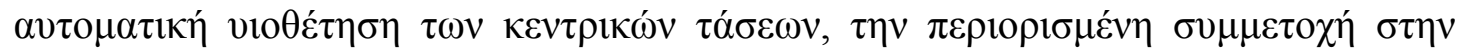

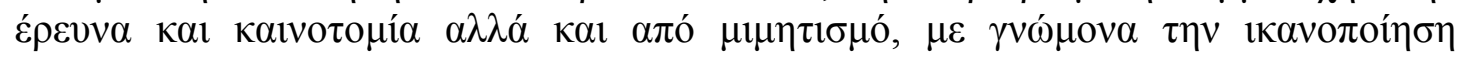

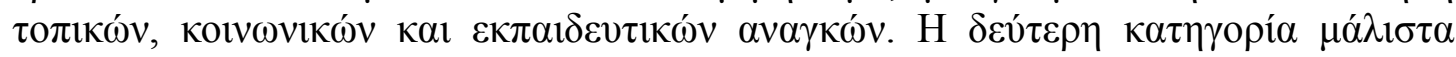

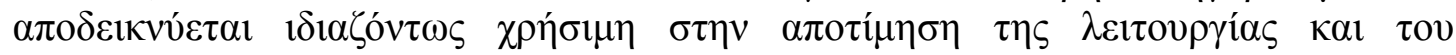

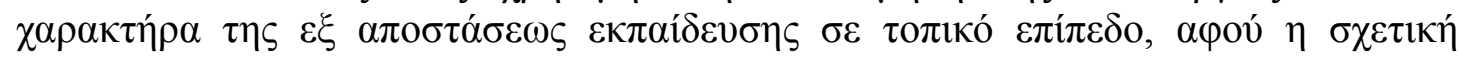

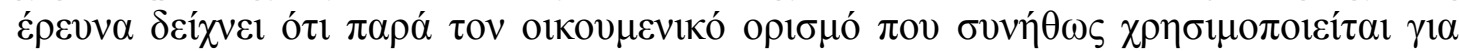

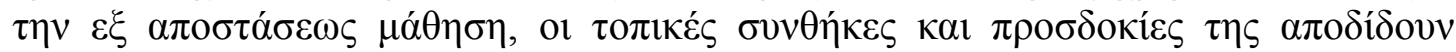

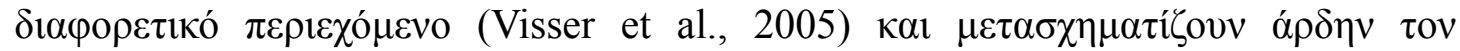

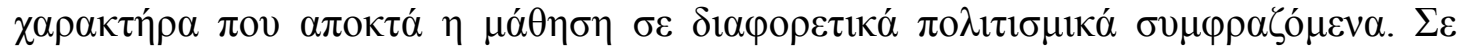

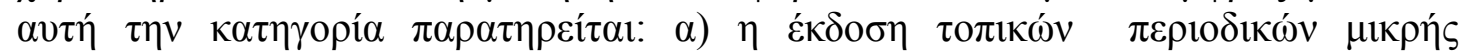

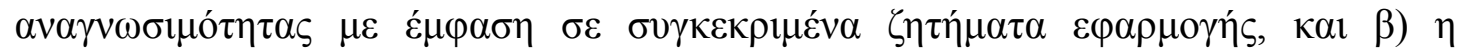
$\delta \eta \mu 10 v \rho \gamma i ́ \alpha$ a

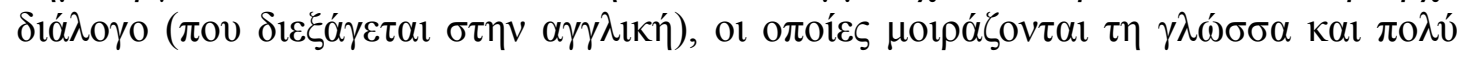

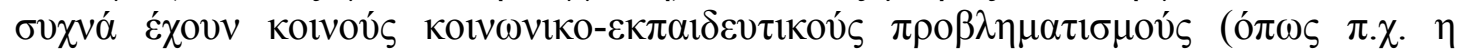

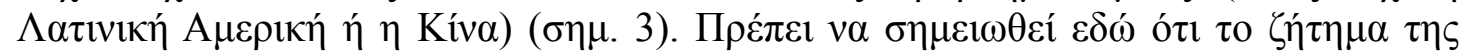

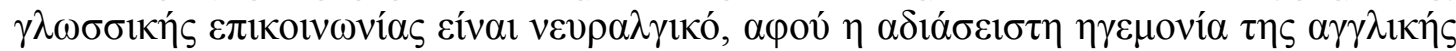

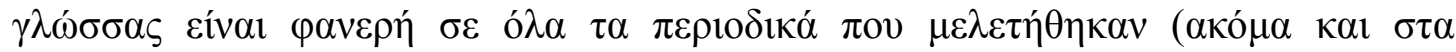

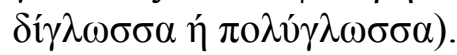

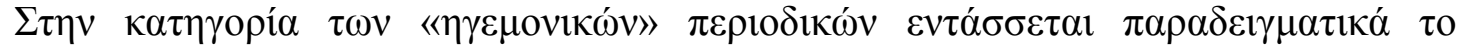

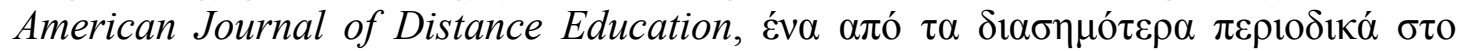

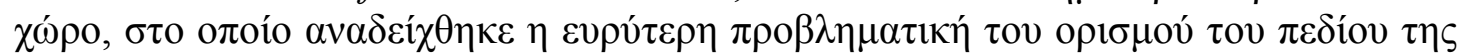

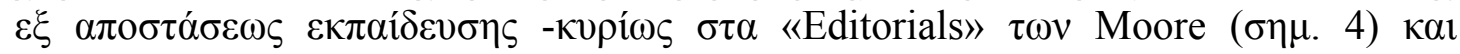

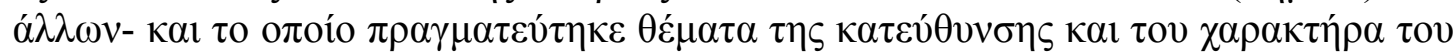

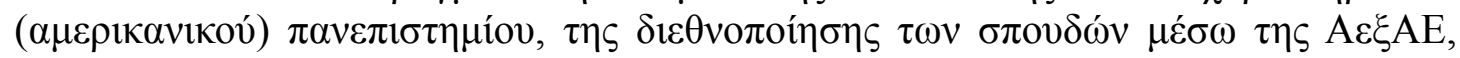

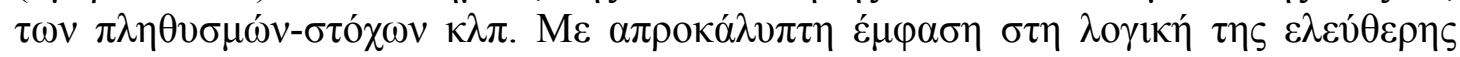

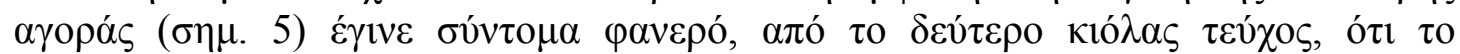

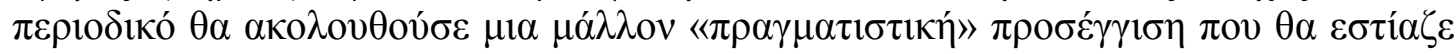

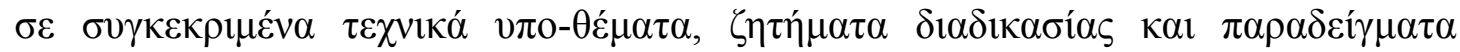




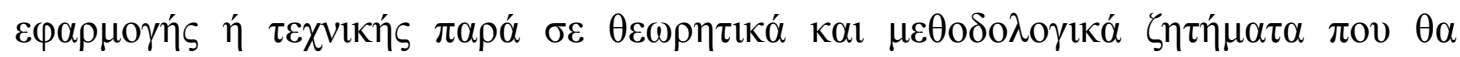

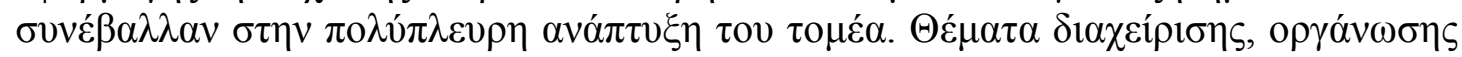

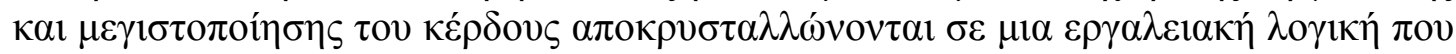

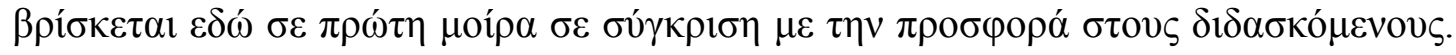

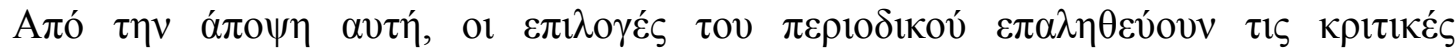

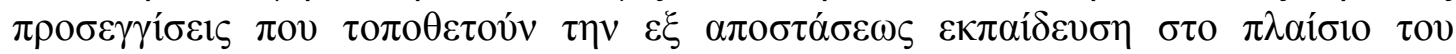

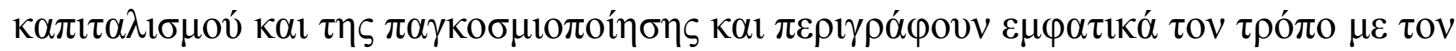

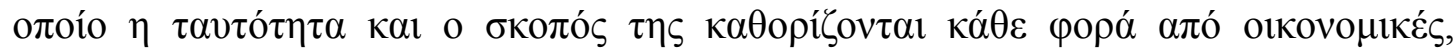

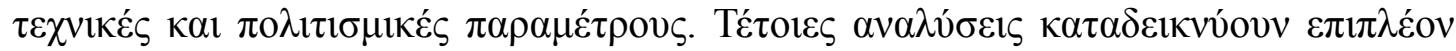

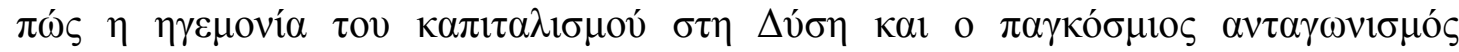

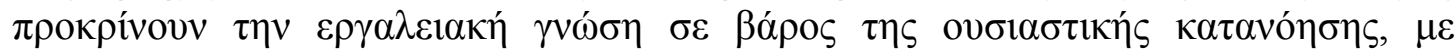

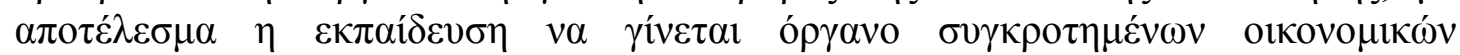

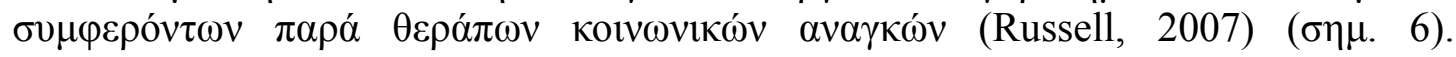

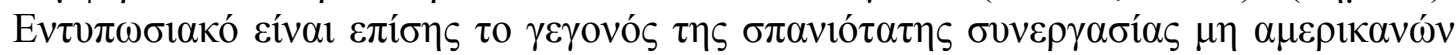

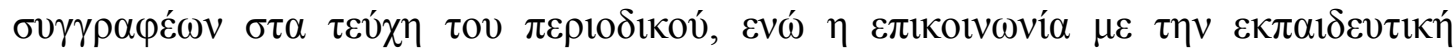

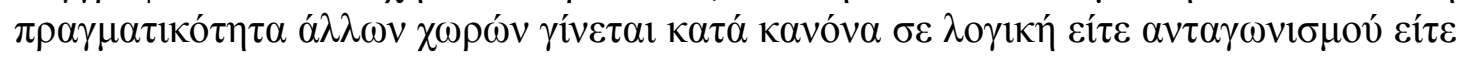

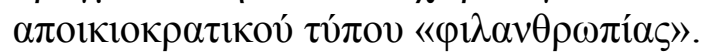

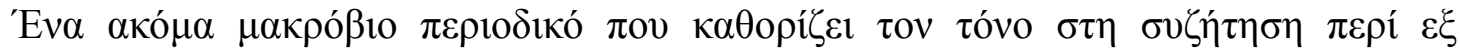

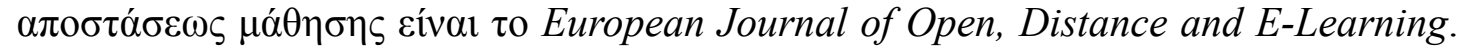

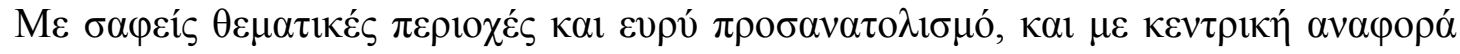

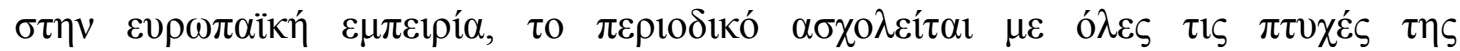

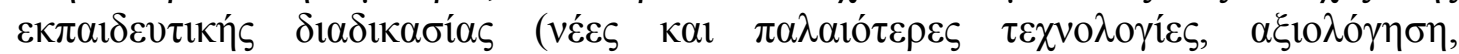

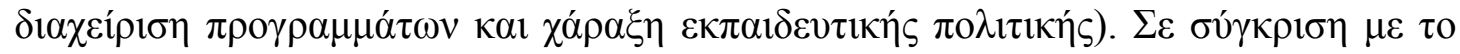

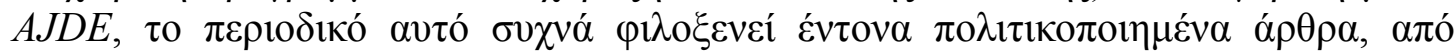

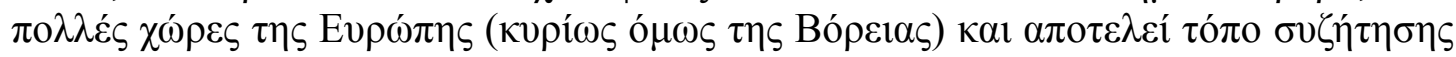

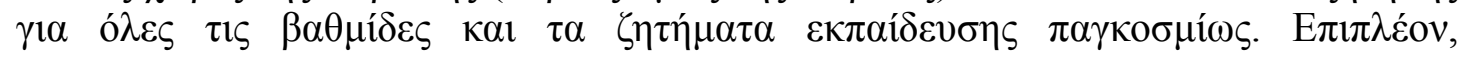

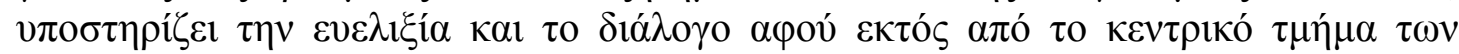

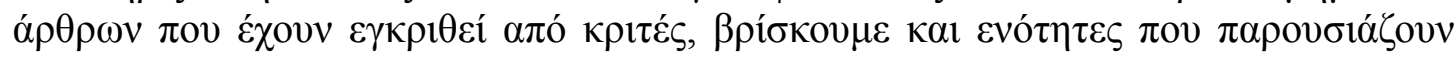

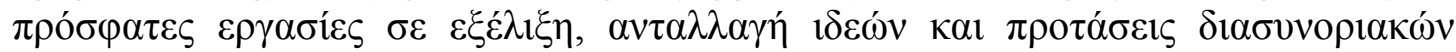

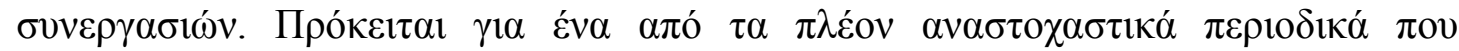

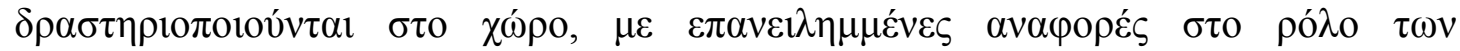

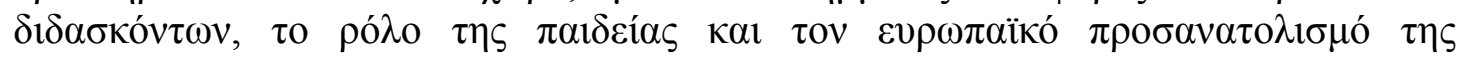

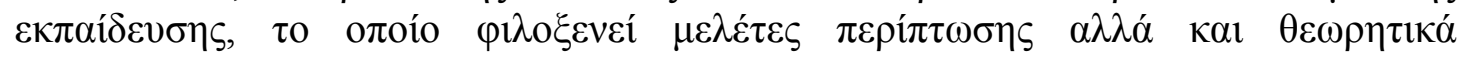

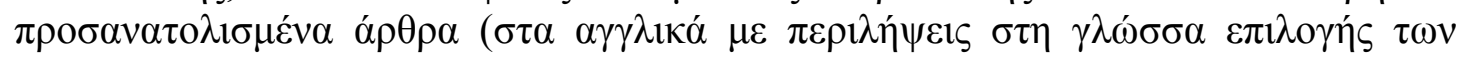

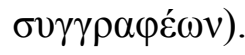

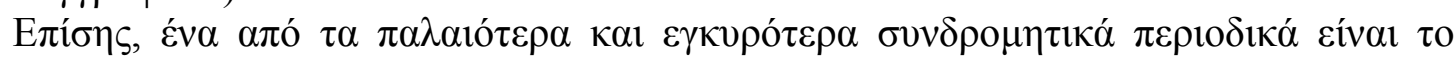

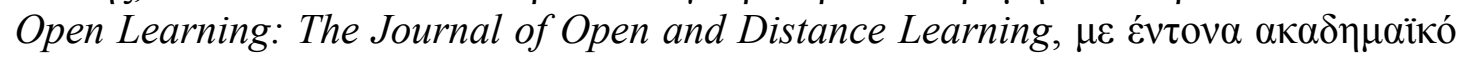

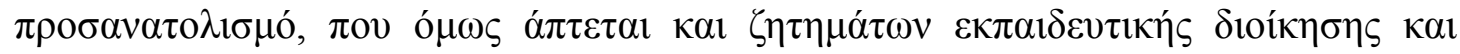

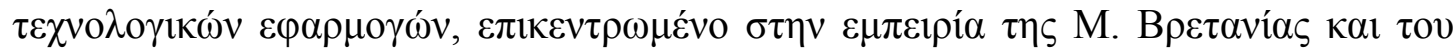

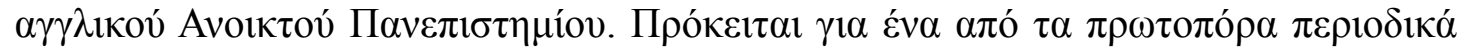

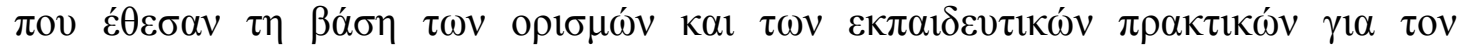

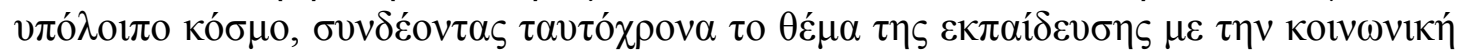

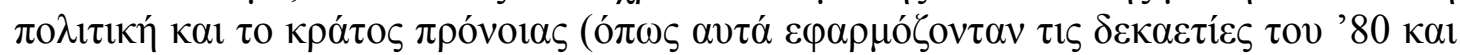

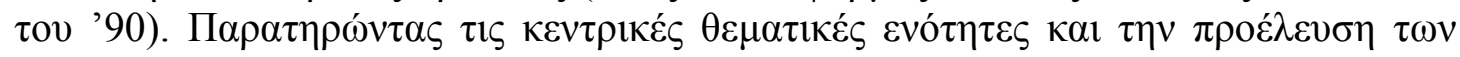

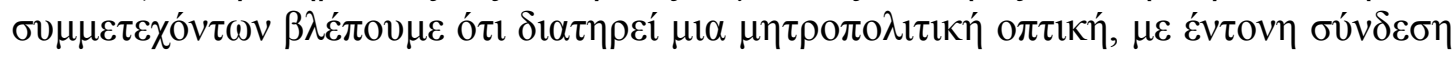

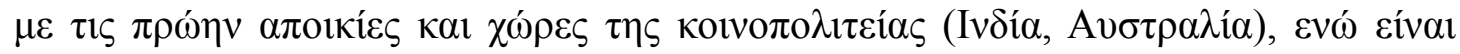

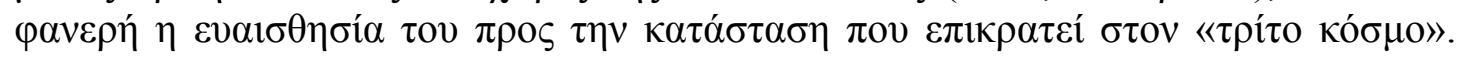

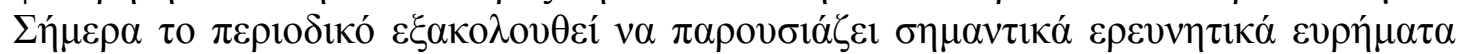

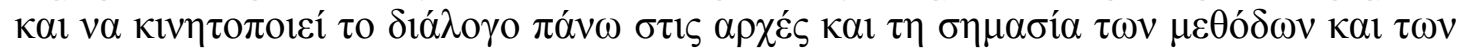

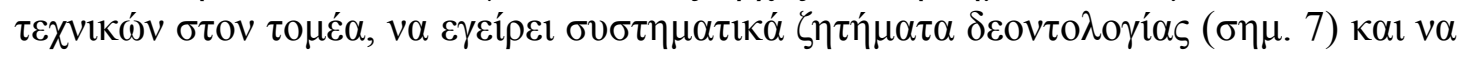




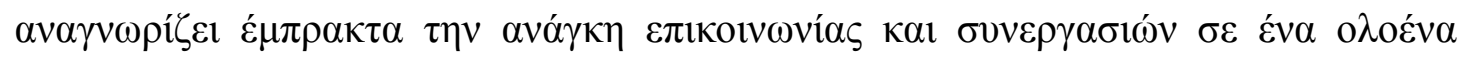

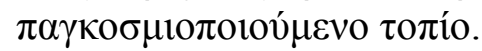

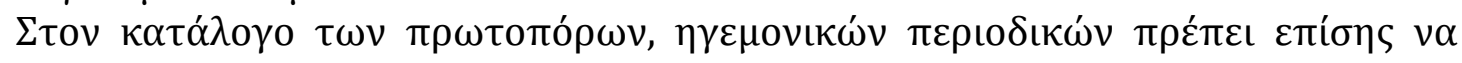

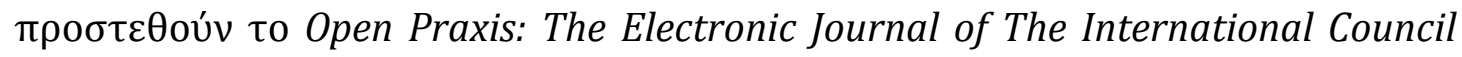

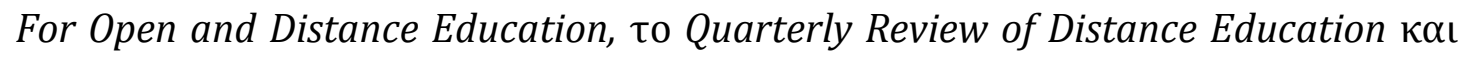

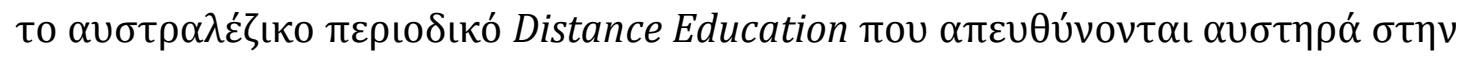

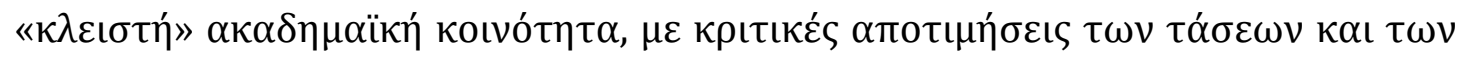

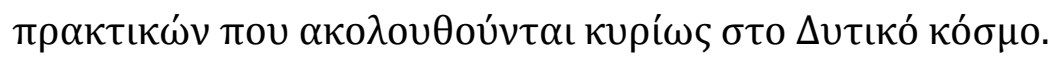

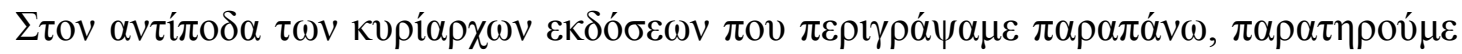

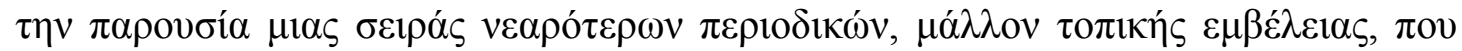

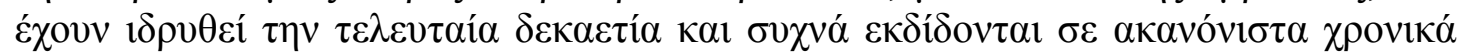

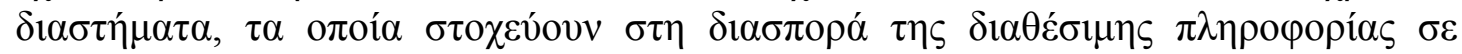

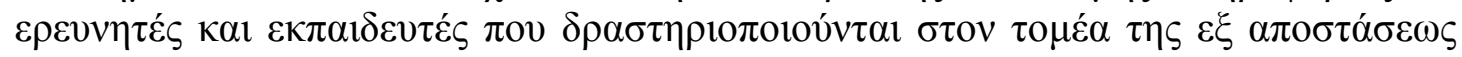

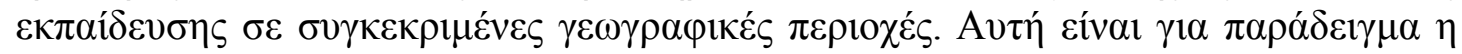

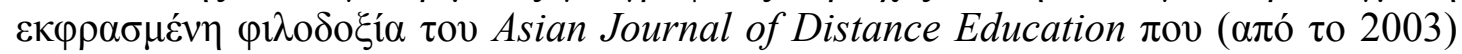

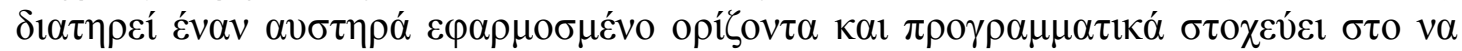

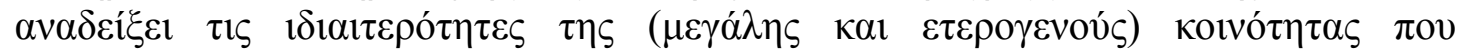

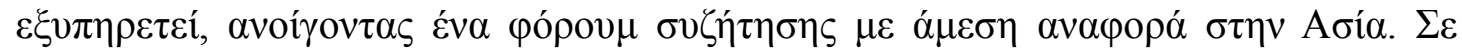

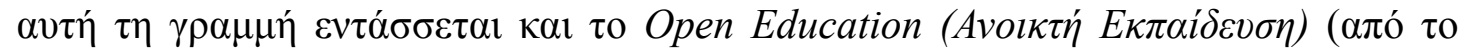

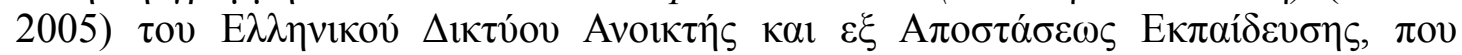

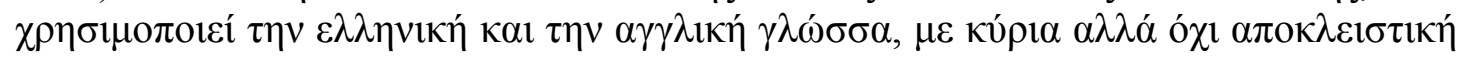

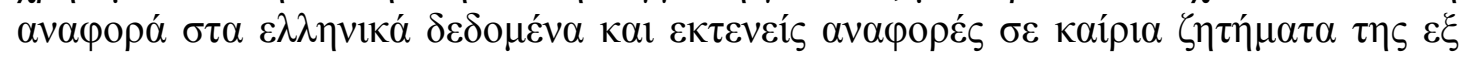

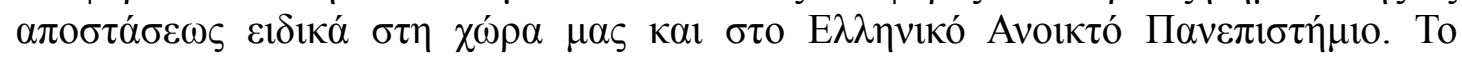

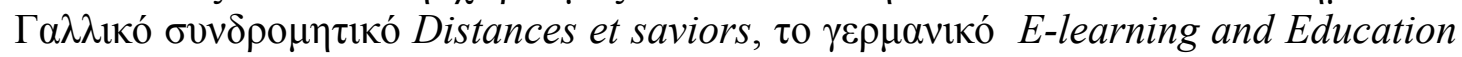

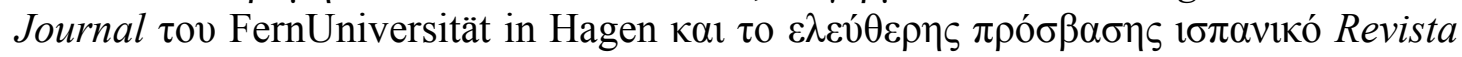

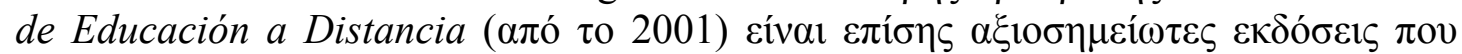

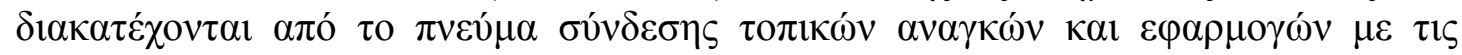

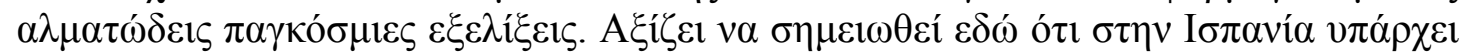

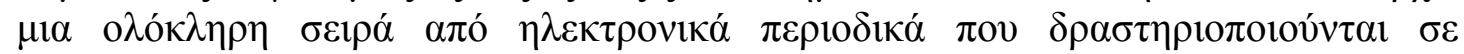

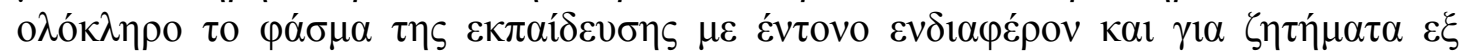

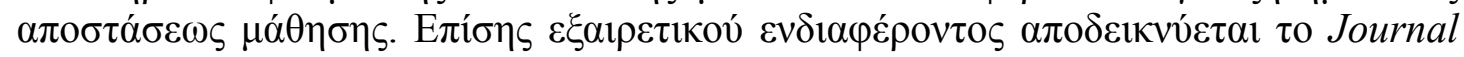

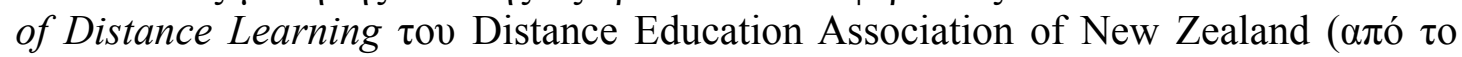

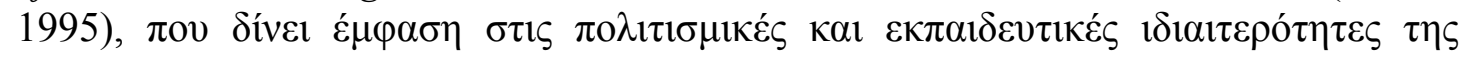

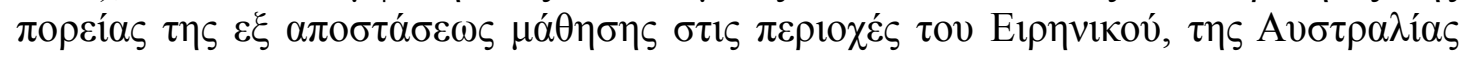

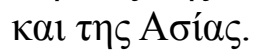

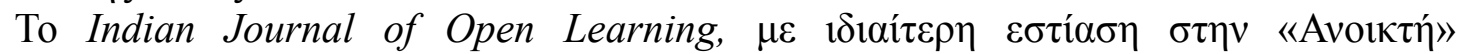

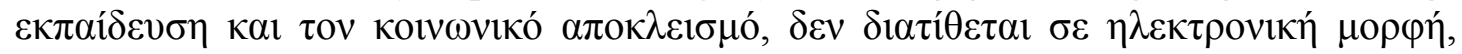

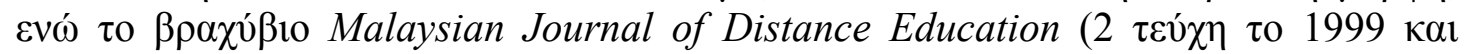

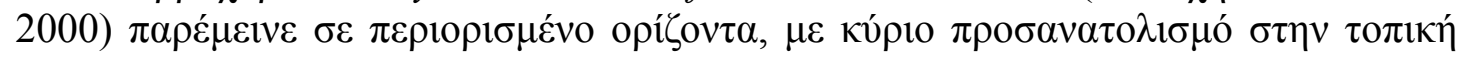

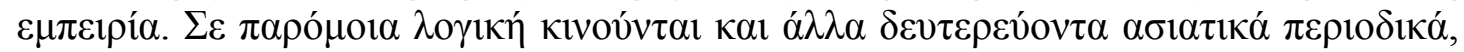
ó $\pi \omega \varsigma \tau \alpha$ Indian Journal of Distance Education (Panjab University, Ivoía), Kakatiya Journal of Distance Education (Kakatiya University, Ivoí $\alpha$ ), Media and Technology

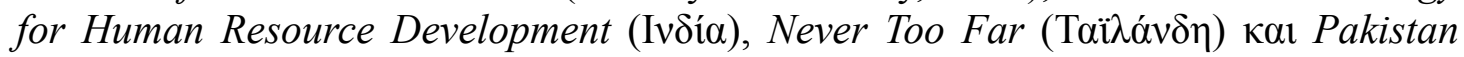

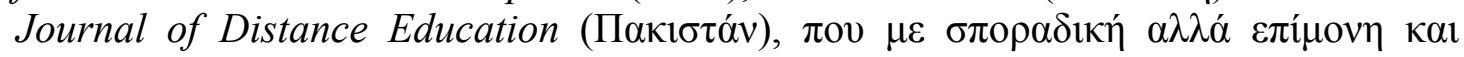

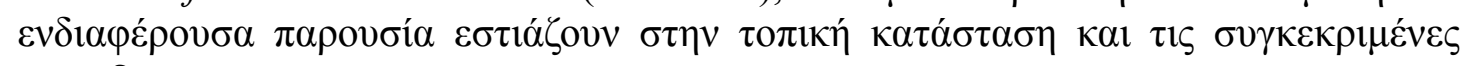
$\varepsilon \kappa \pi \alpha 1 \delta \varepsilon v \tau 1 \kappa \varepsilon ́ \zeta \alpha \nu \alpha ́ \gamma \kappa \varepsilon \varsigma$.

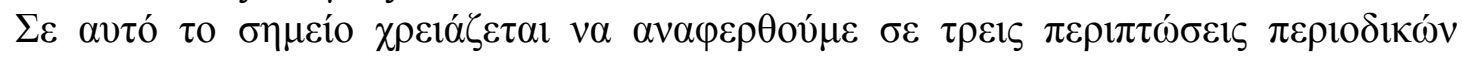

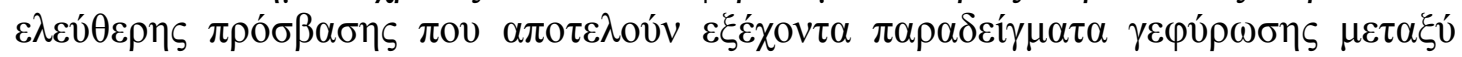

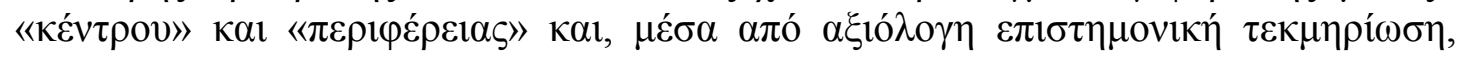




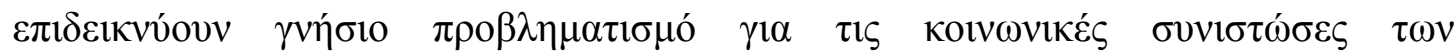

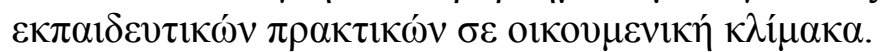

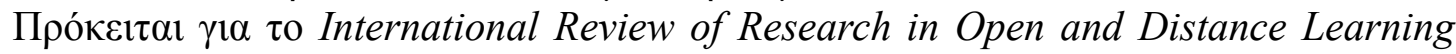

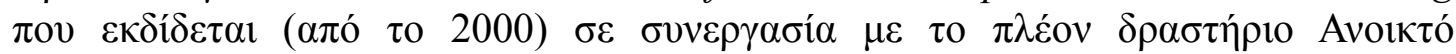

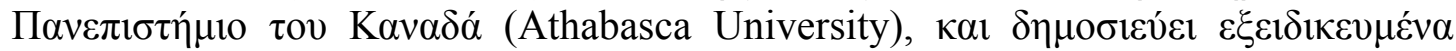

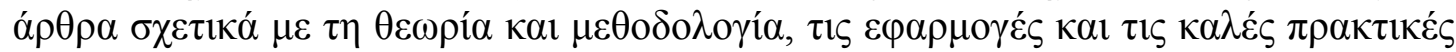

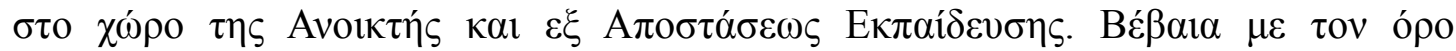

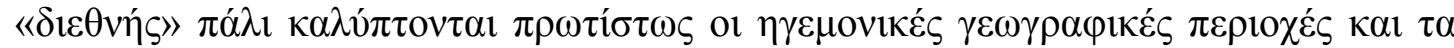

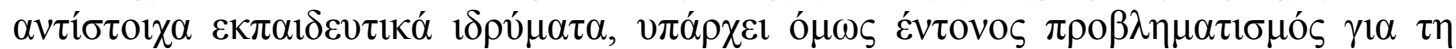

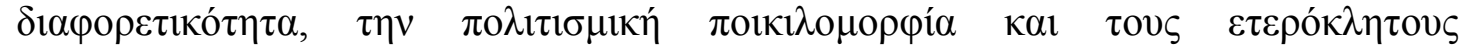

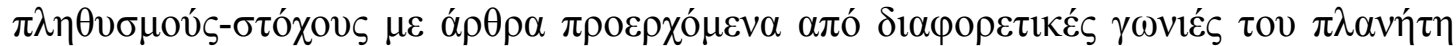

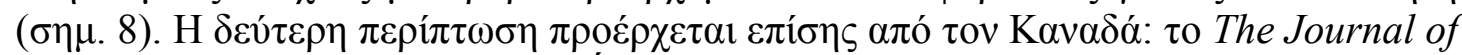

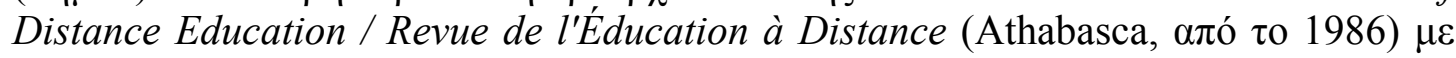

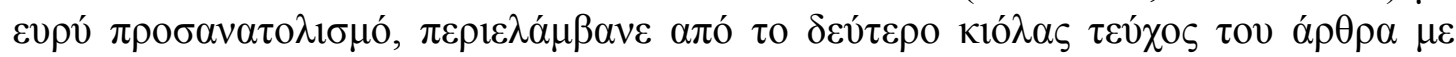

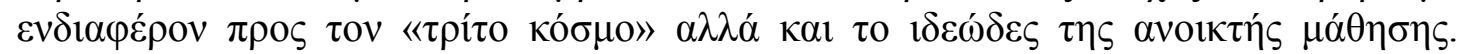

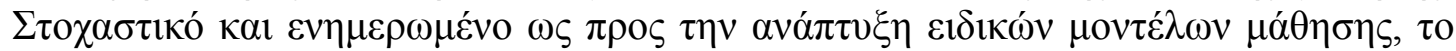

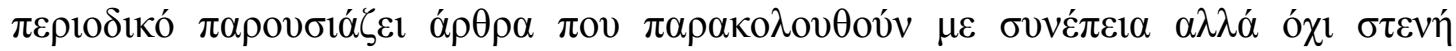

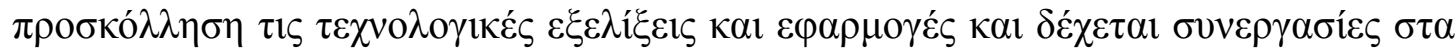

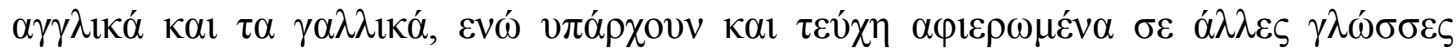

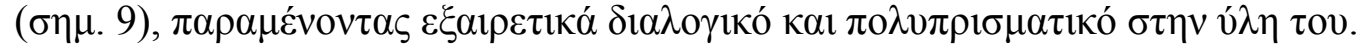

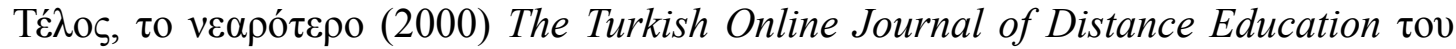

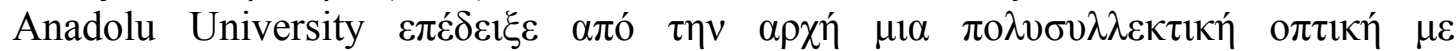

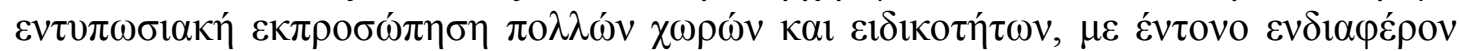

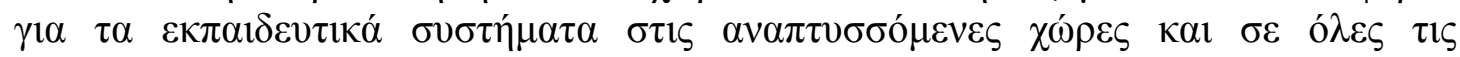
$\varepsilon \kappa \pi \alpha \iota \delta \varepsilon v \tau \iota \kappa \varepsilon ́ \varsigma \beta \beta \theta \mu i ́ \delta \varepsilon \varsigma$.

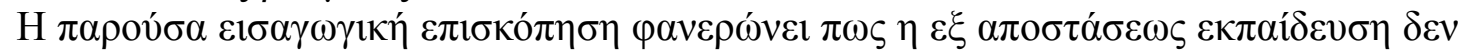

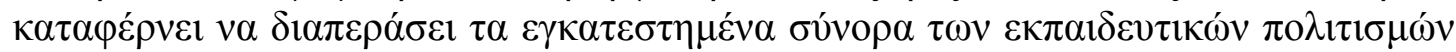

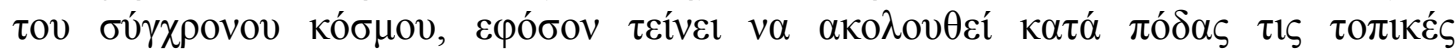

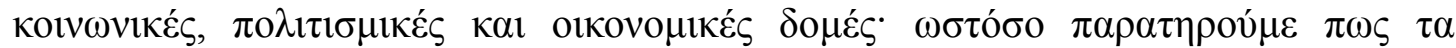

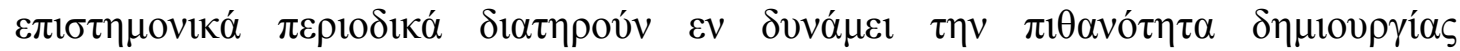

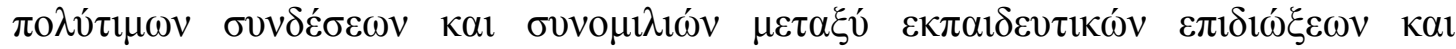

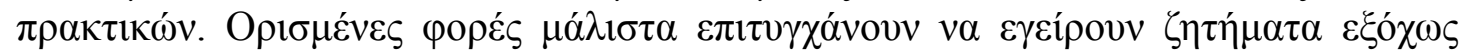

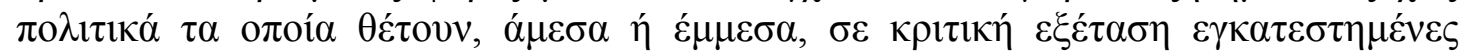

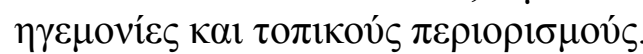

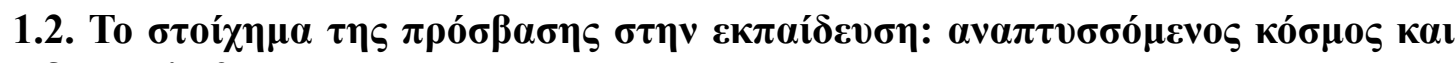

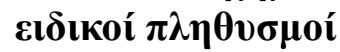

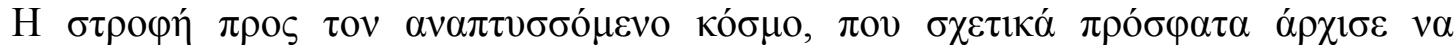

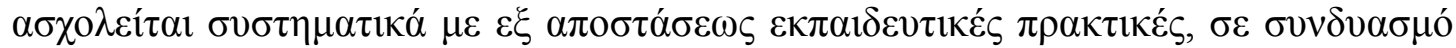

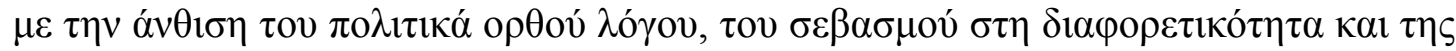

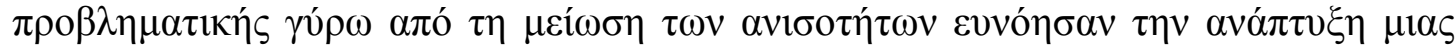

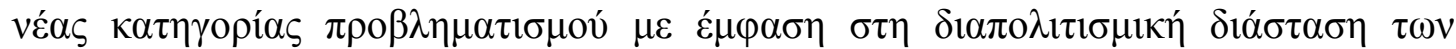

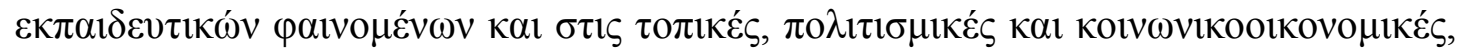

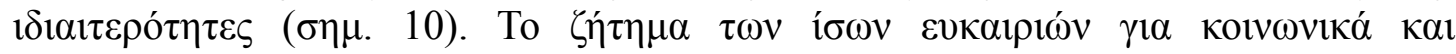

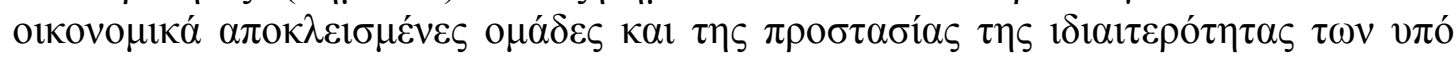

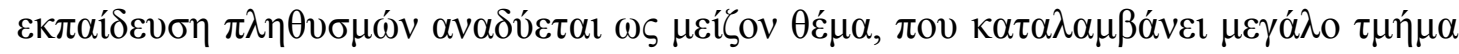

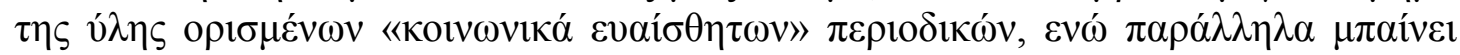

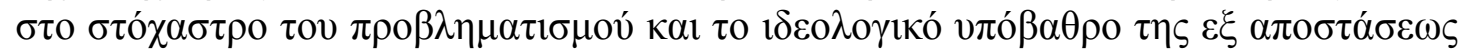

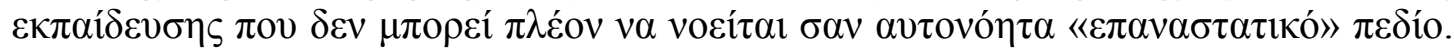

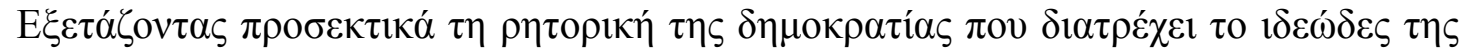




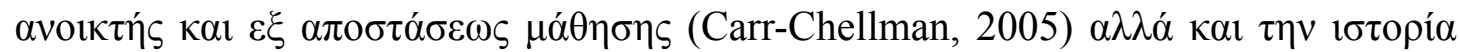

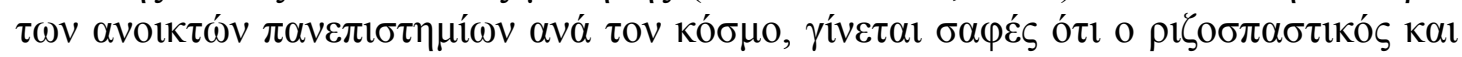

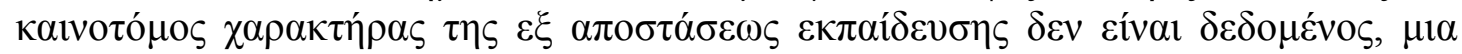

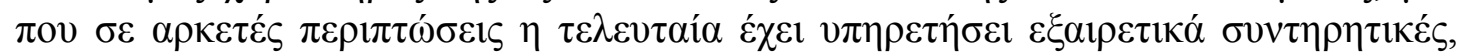

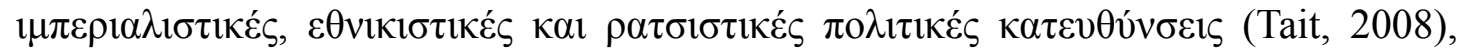

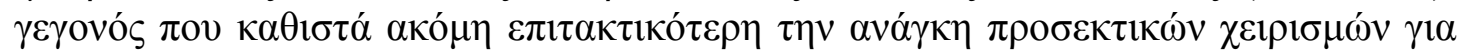

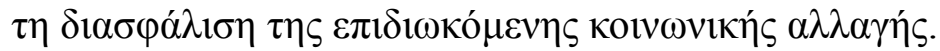

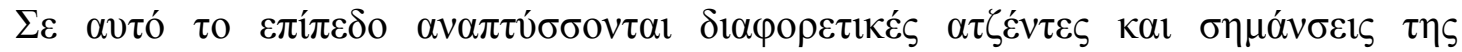

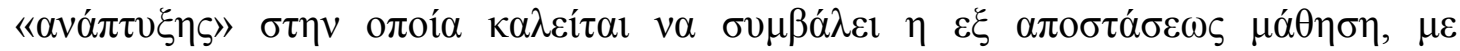

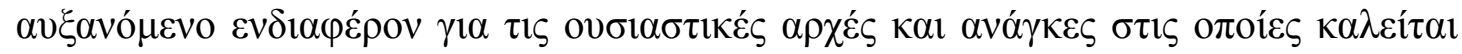

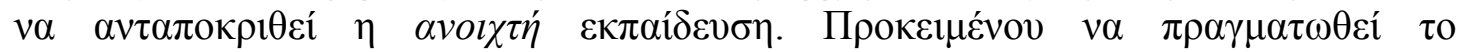

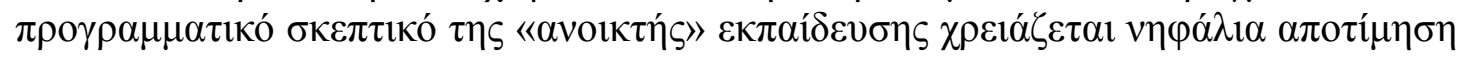

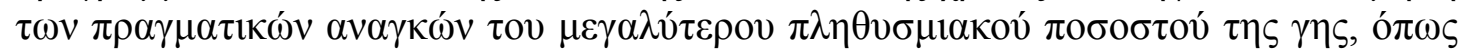

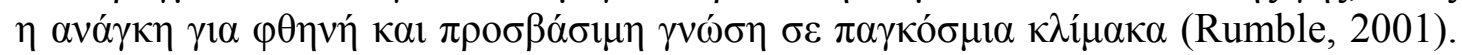

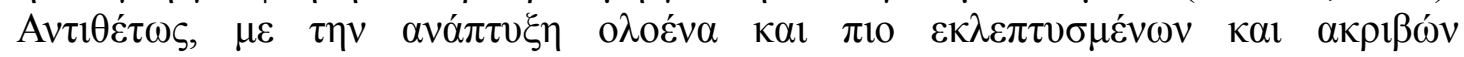

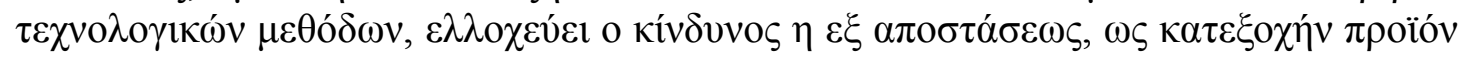

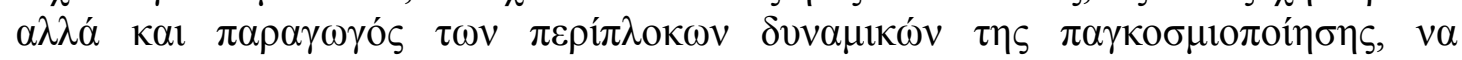

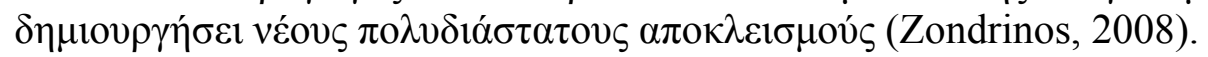

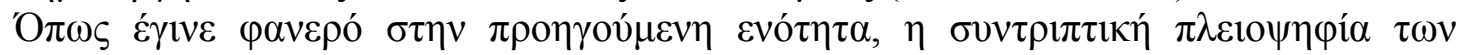

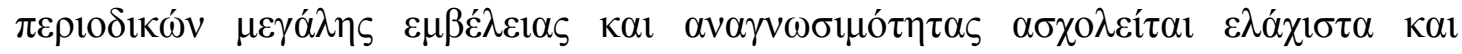

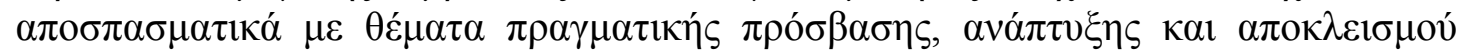

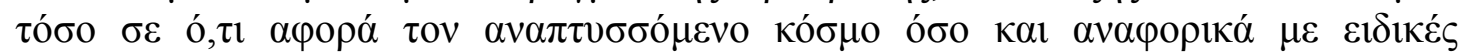

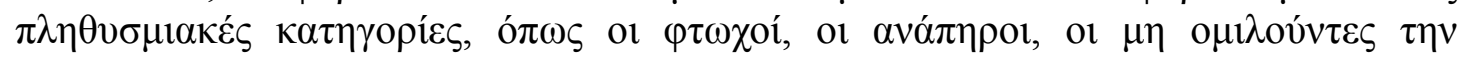

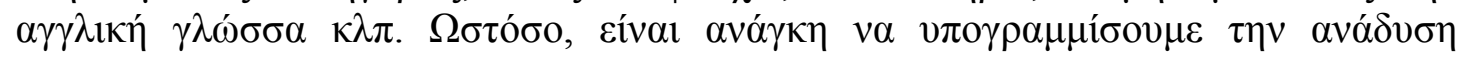

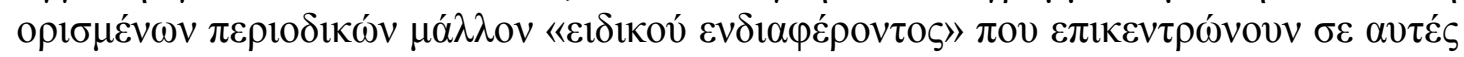

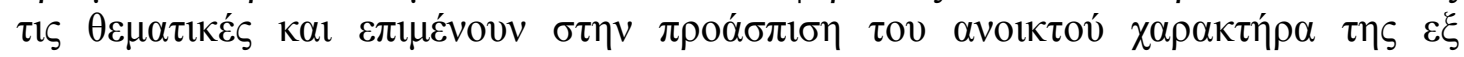

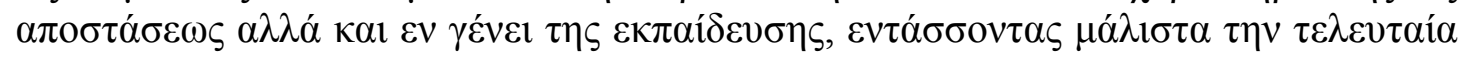

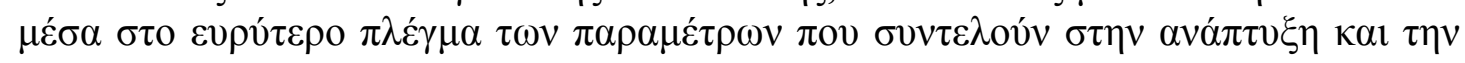

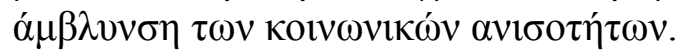

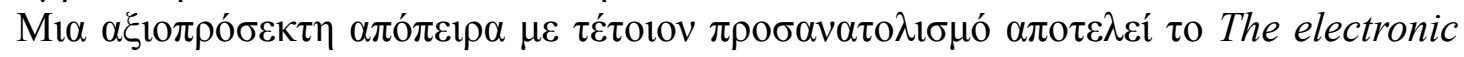

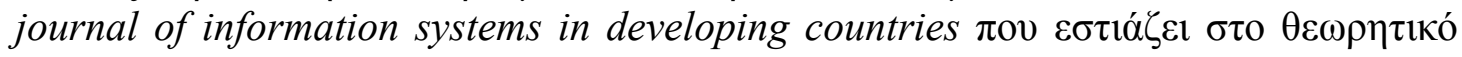

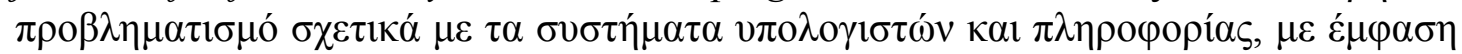

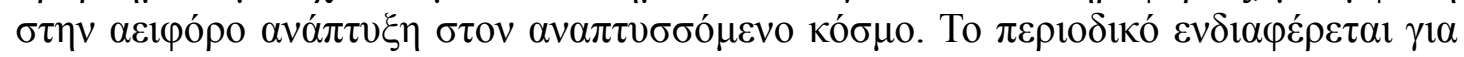

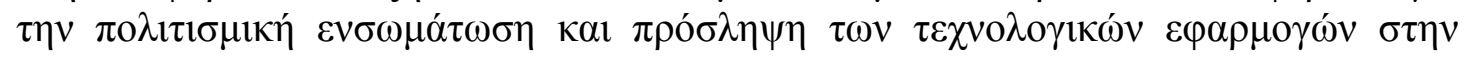

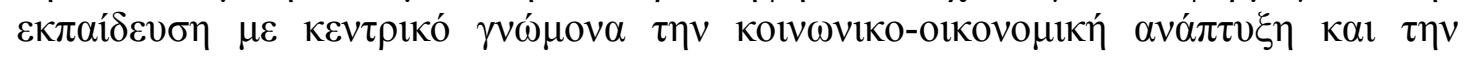
$\alpha \dot{\alpha} \beta \lambda v v \sigma \eta \tau \omega v \alpha v i \sigma o \tau \eta \dot{\tau} \omega v$.

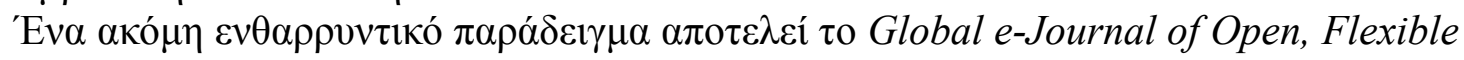

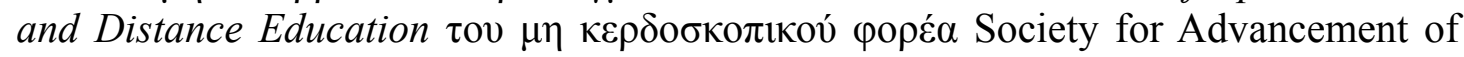

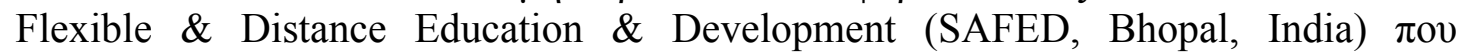

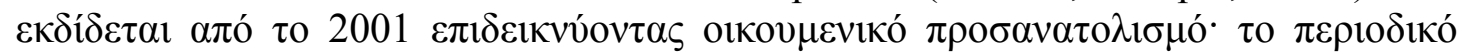

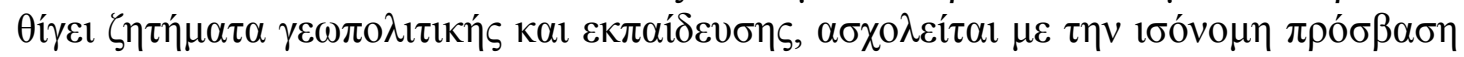

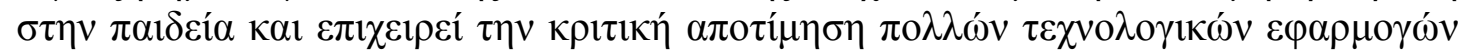

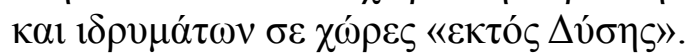

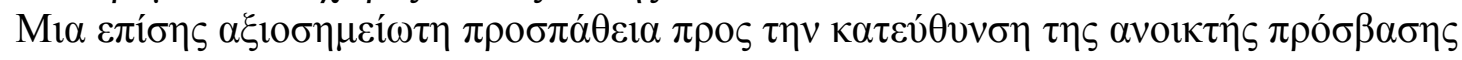

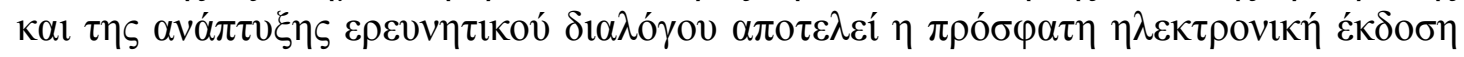

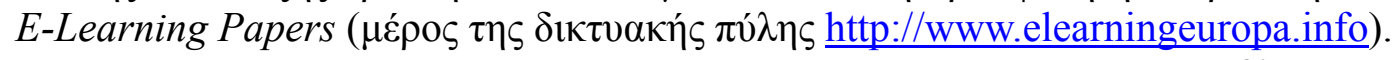

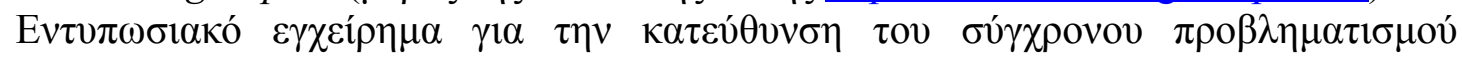

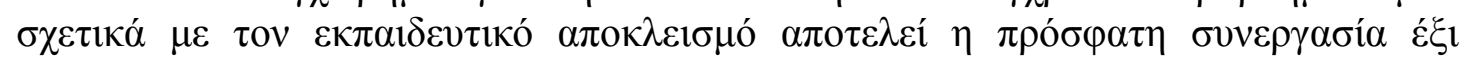

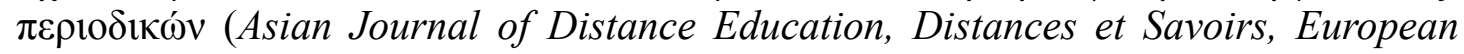
Journal of Open Distance and E-learning, Journal of Asynchronous Learning 
Networks, International Review of Research in Open and Distance Learning, Open

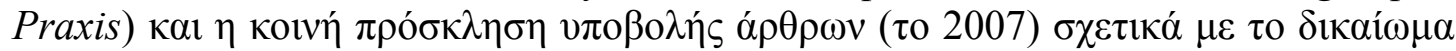

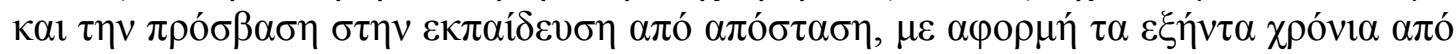

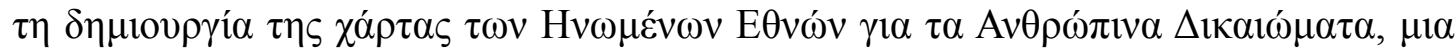

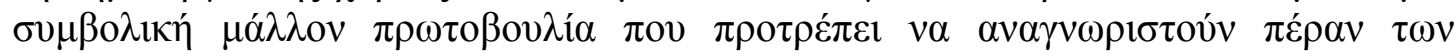

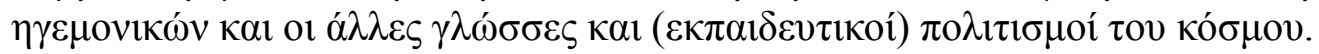

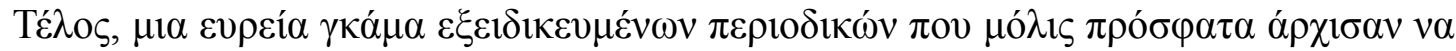

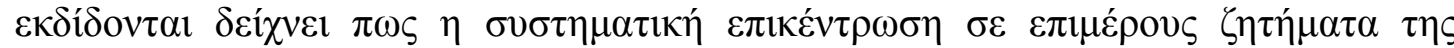

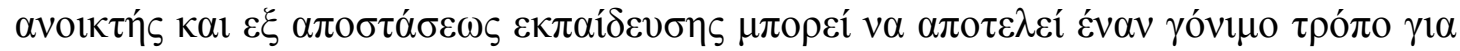

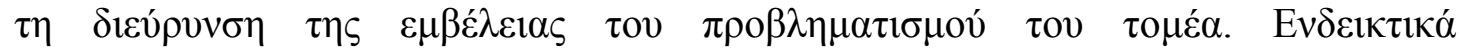

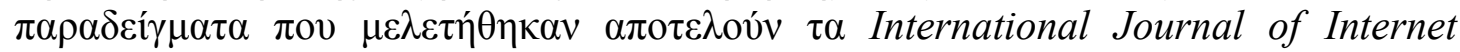

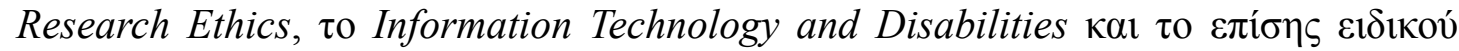

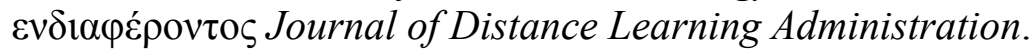

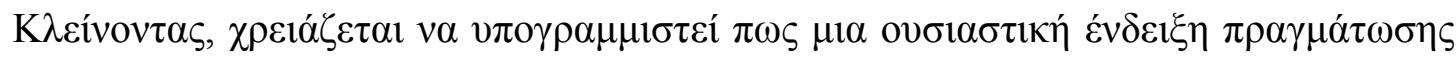

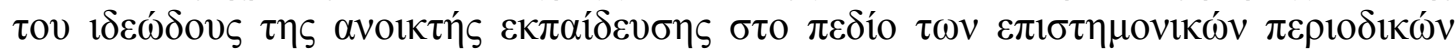

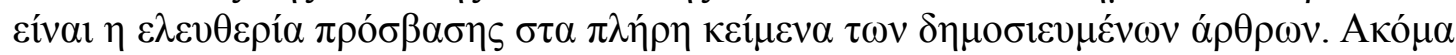

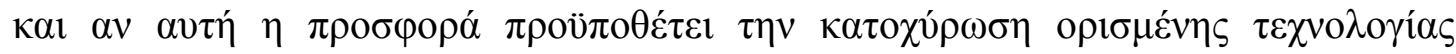

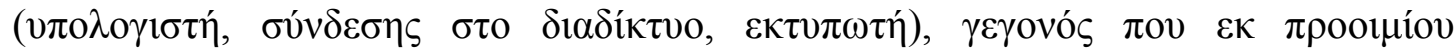

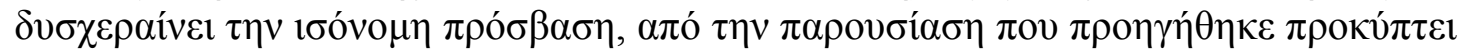

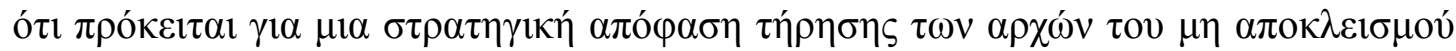

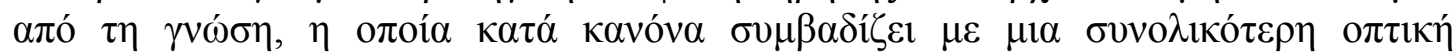

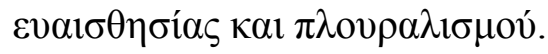

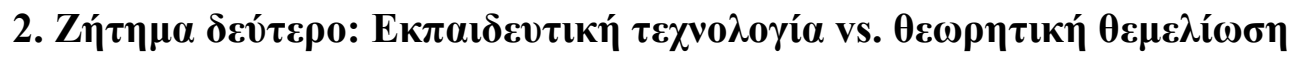

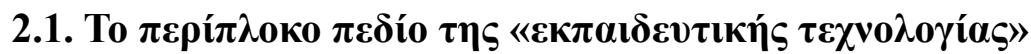

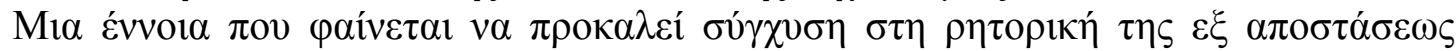

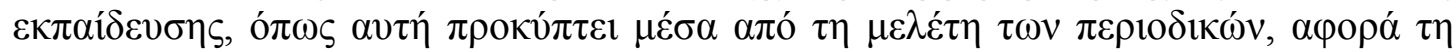

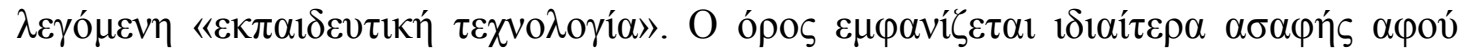

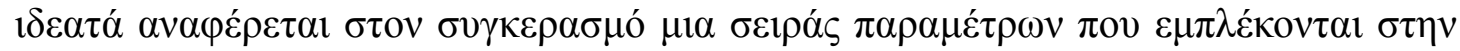

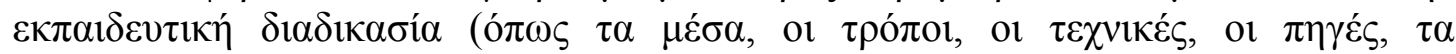

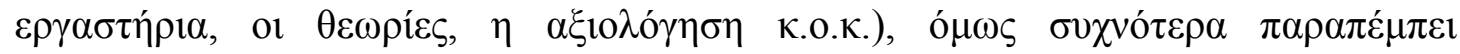

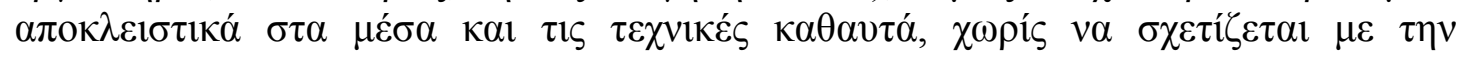

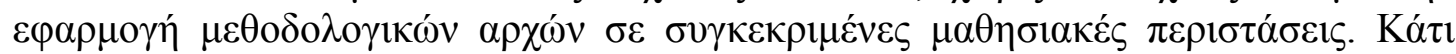

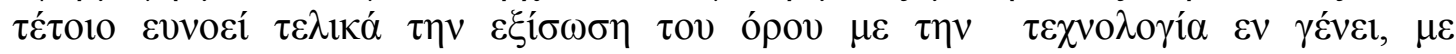

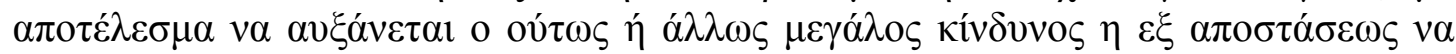

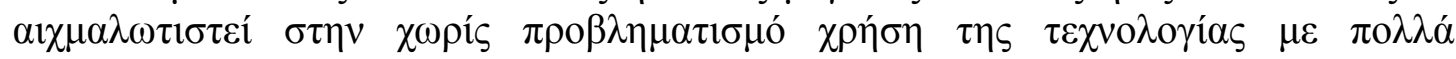

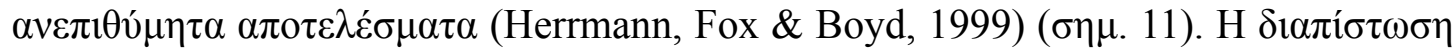

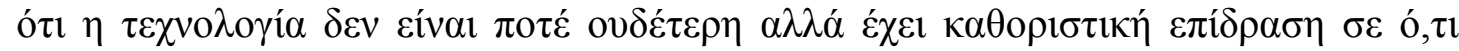

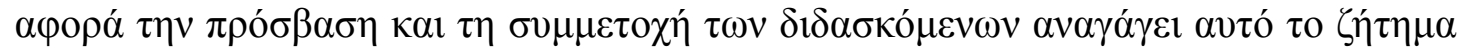

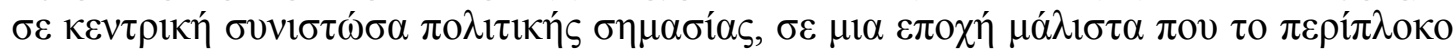

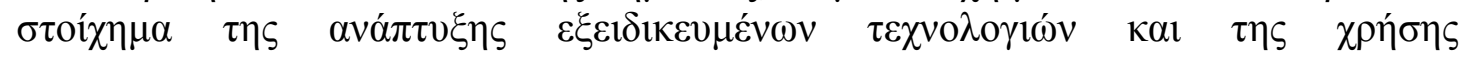

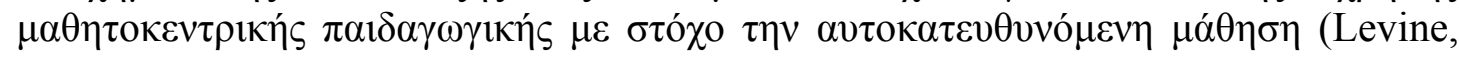

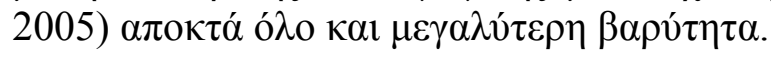

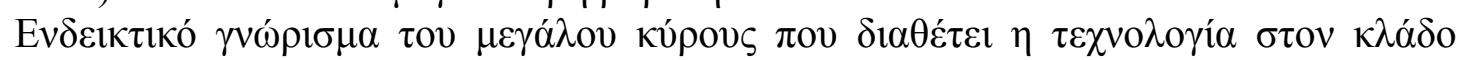

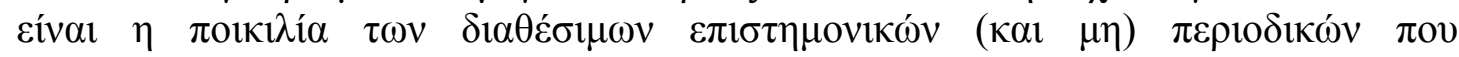

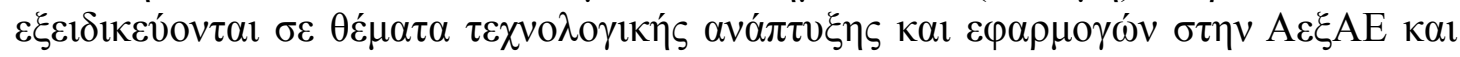

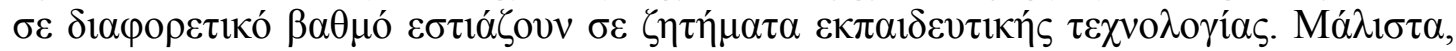

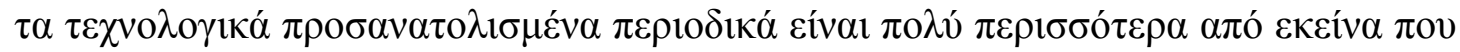




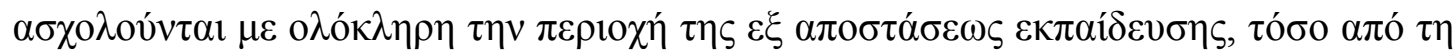

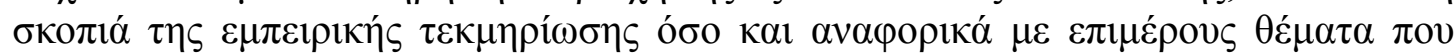

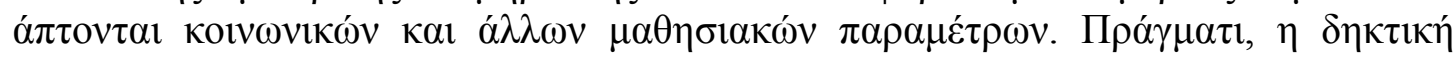

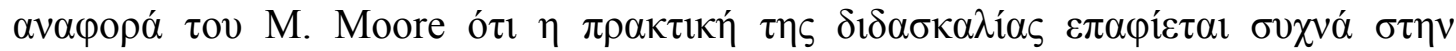

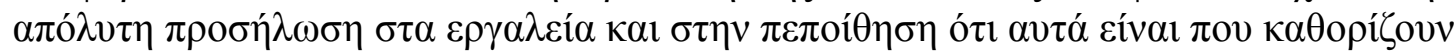

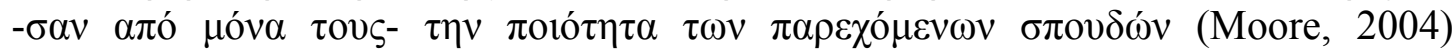

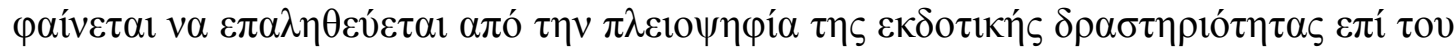
$\alpha \nu \tau \iota \kappa \varepsilon \mu \mu \varepsilon ́ v o v$.

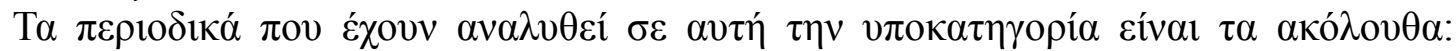
Journal of Asynchronous Learning Networks, Educational Technology Review, Contemporary Issues in Technology and Teacher Education, Educational Technology and Society, Journal of Research on Technology in Education (JRTE), Elearn

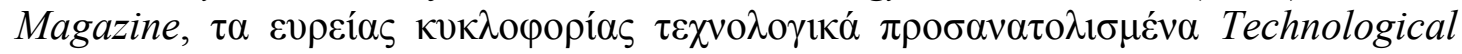
Horizons in Education (T.H.E.) Journal, Learning Technology, Technology Periodical

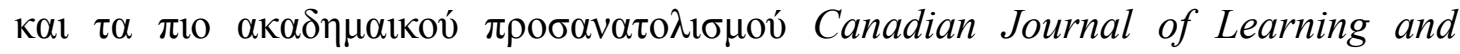
technology / La Revue Canadienne de l'Apprentissage et de la technologie $\kappa \alpha$

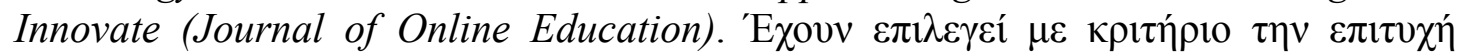

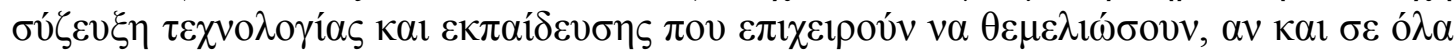

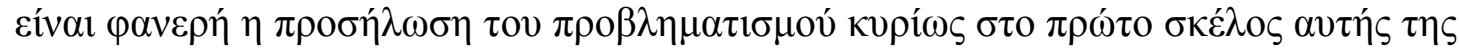

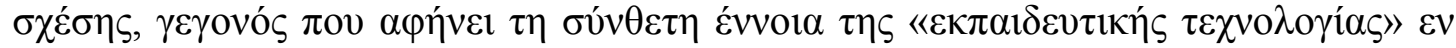

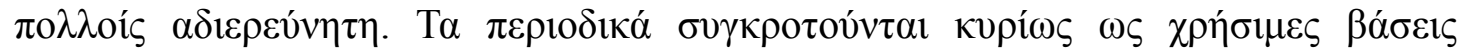

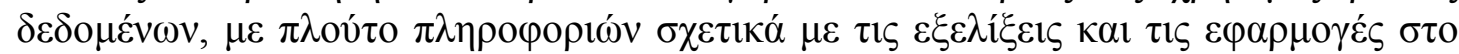

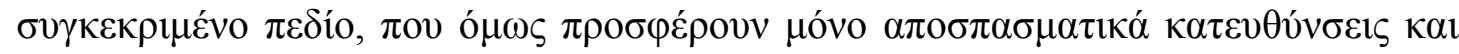

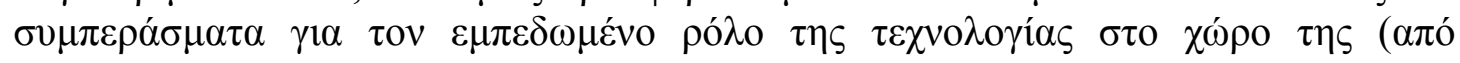
$\alpha \pi$ ó $\tau \alpha \sigma \eta) \varepsilon \kappa \pi \alpha i ́ \delta \varepsilon v \sigma \eta \varsigma$.

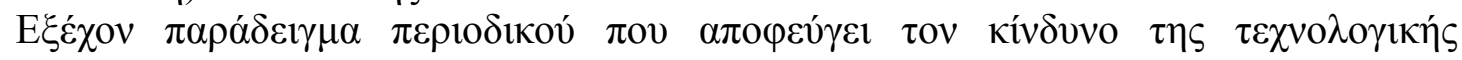

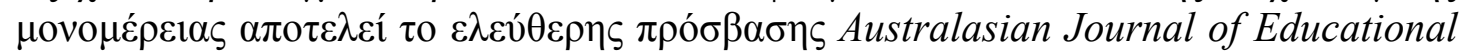

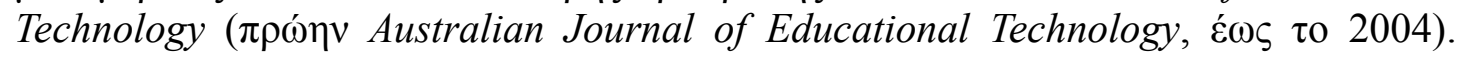

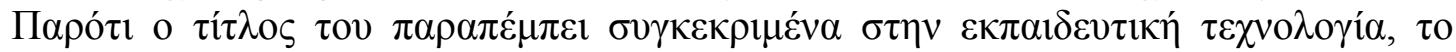

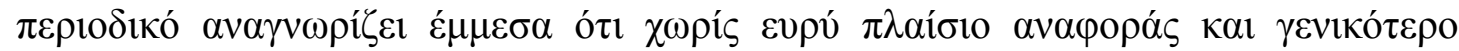

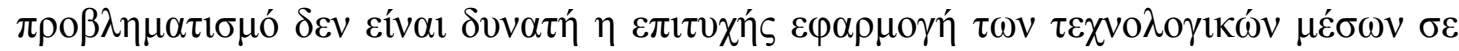

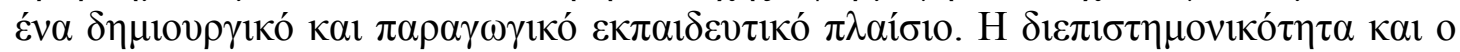

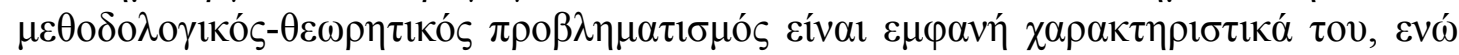

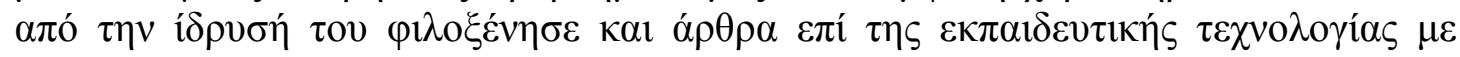

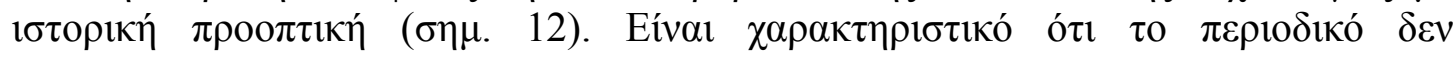

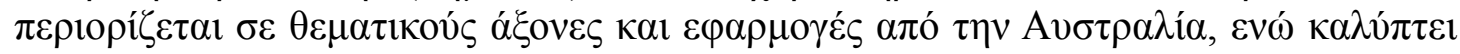

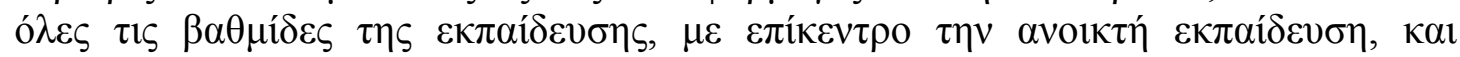

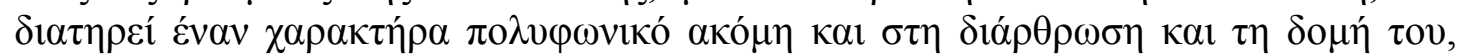

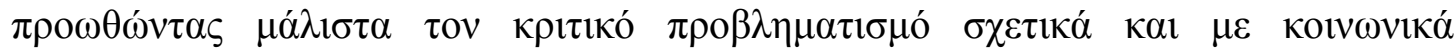

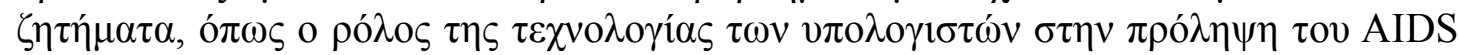

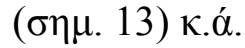

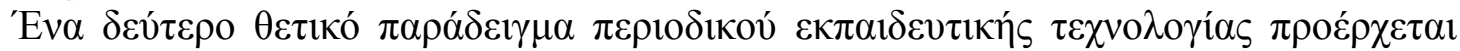

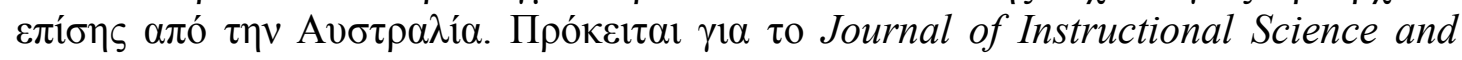

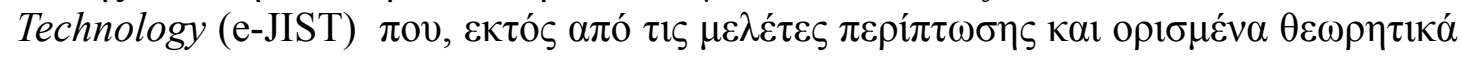

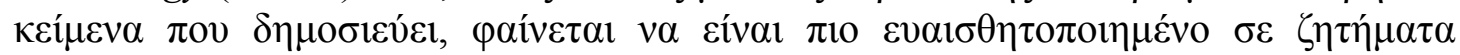

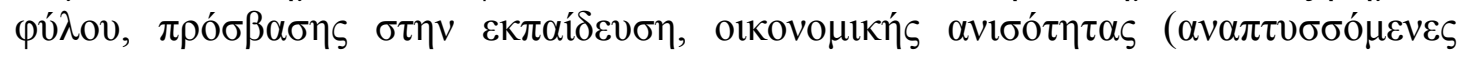

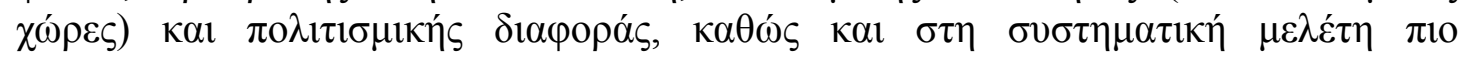

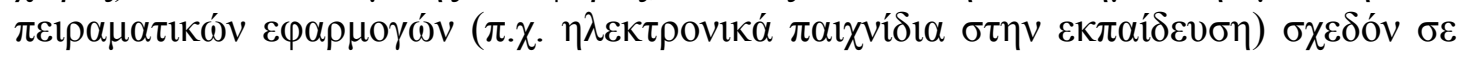

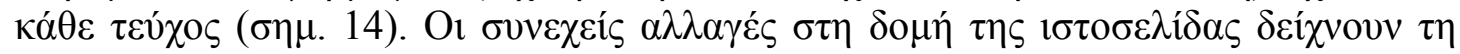

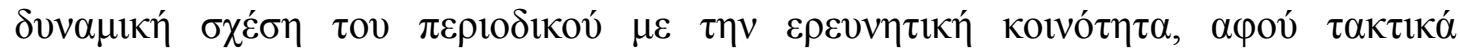




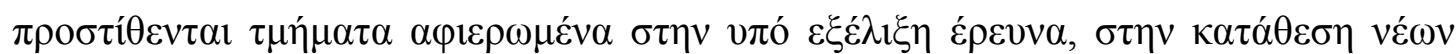

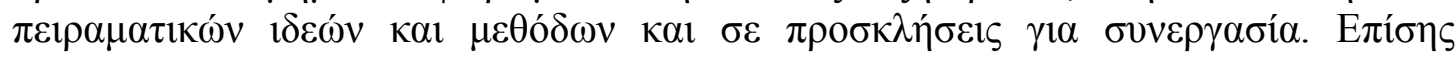

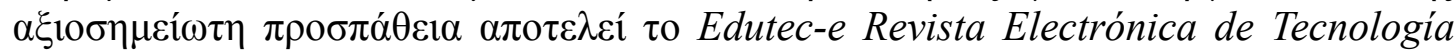

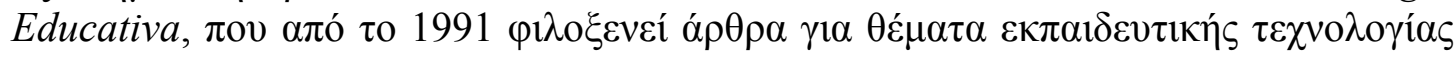

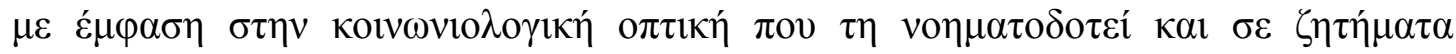

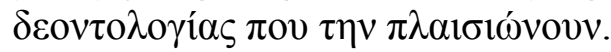

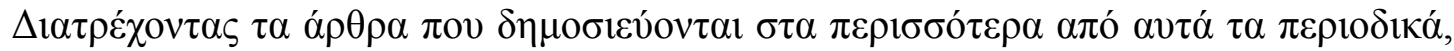

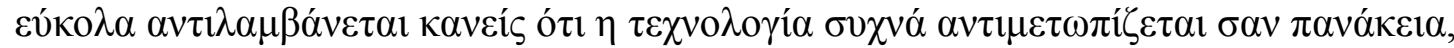

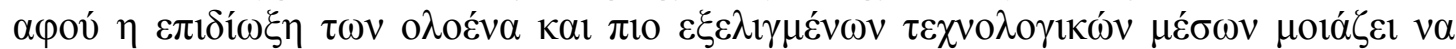

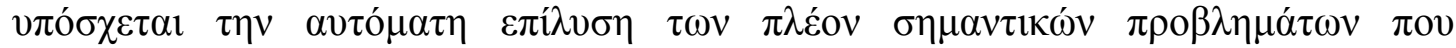

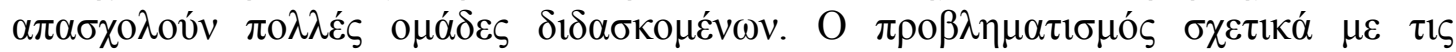

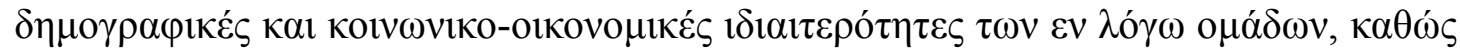

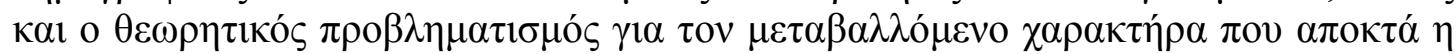

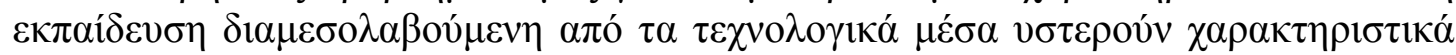

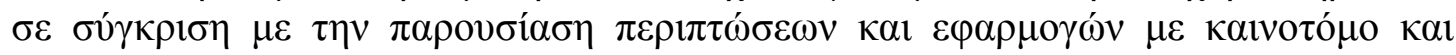

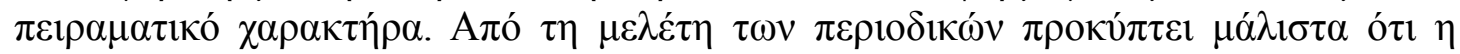

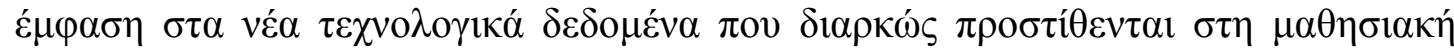

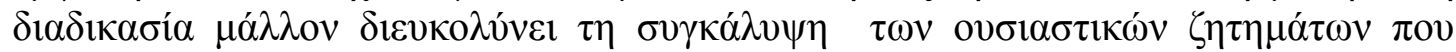

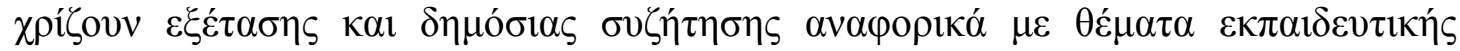

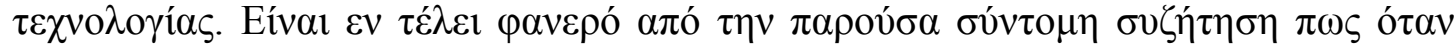

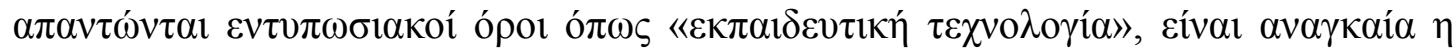

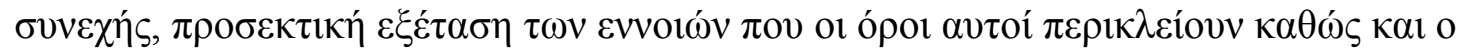

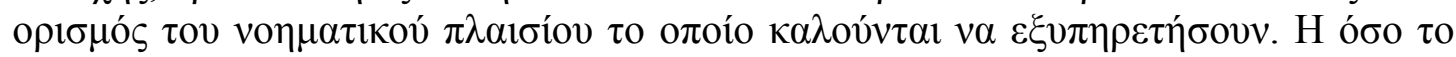

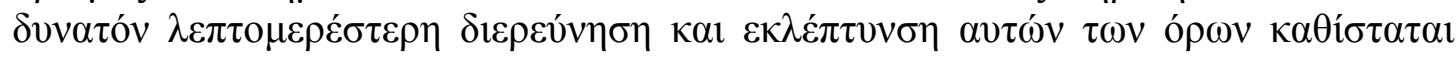

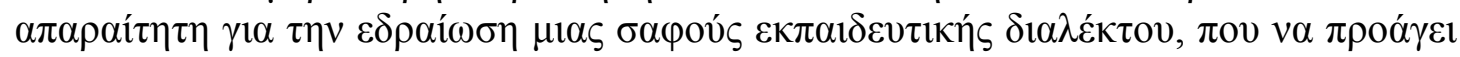

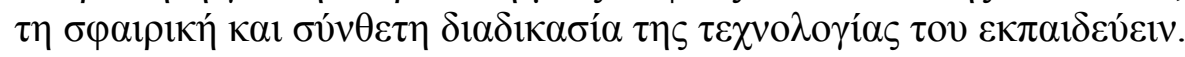

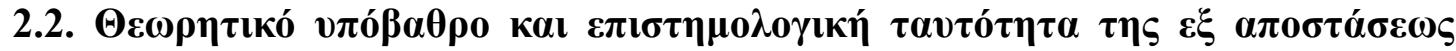 $\varepsilon \kappa \pi \alpha i ́ \delta \varepsilon v \sigma \eta \varsigma$}

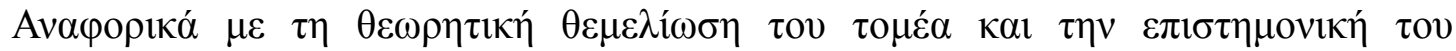

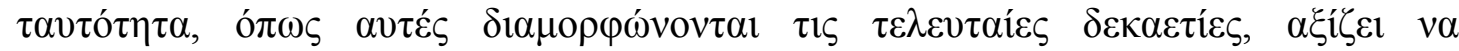

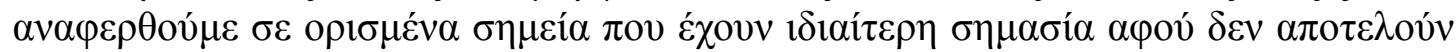

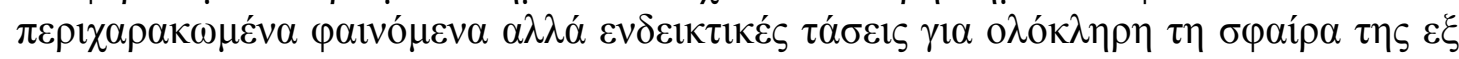

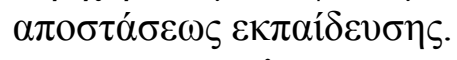

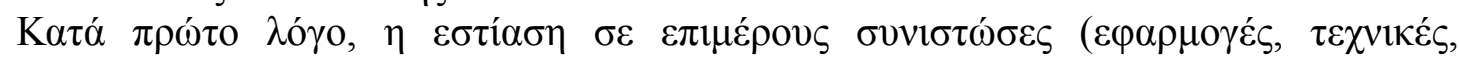

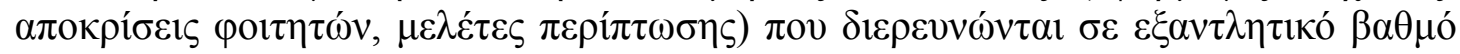

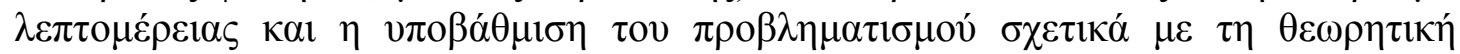

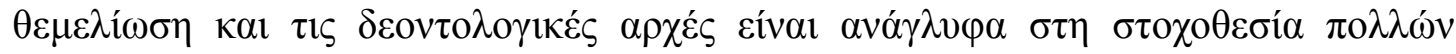

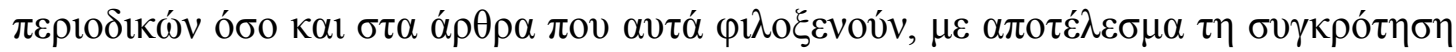

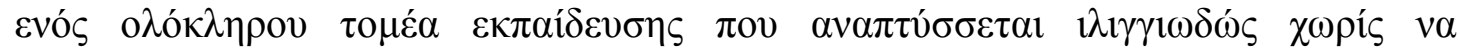

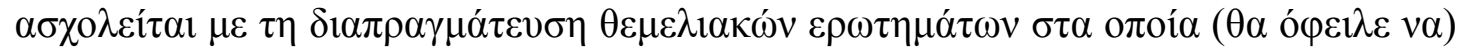
$\beta \alpha \sigma i \zeta \varepsilon \tau \alpha 1$.

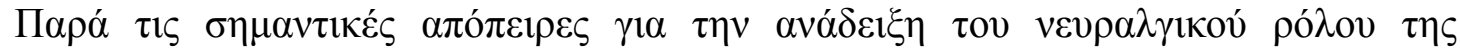

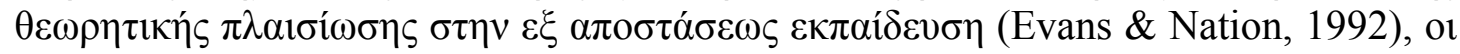

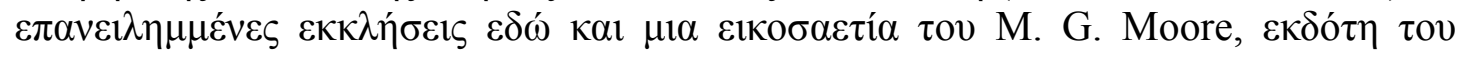

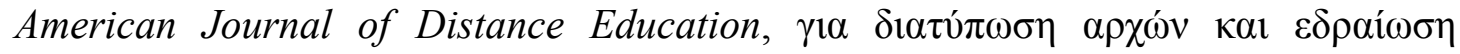

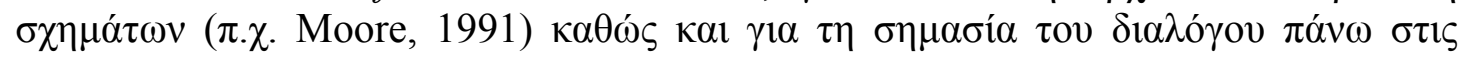

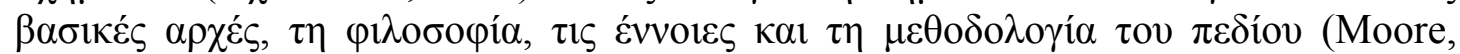

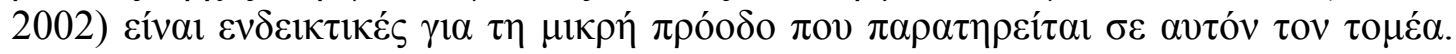

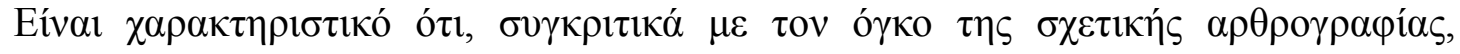




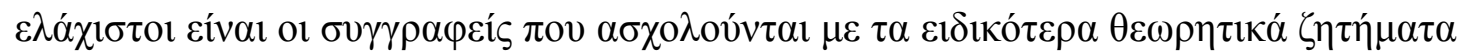

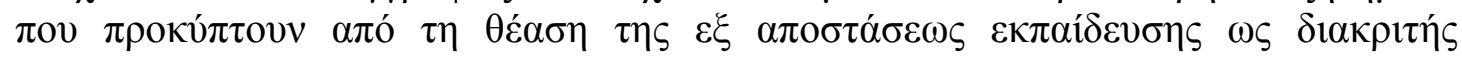

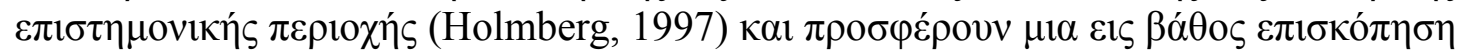

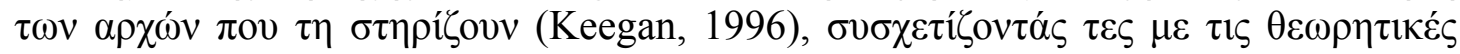

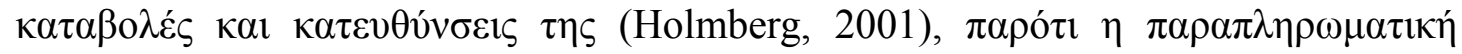

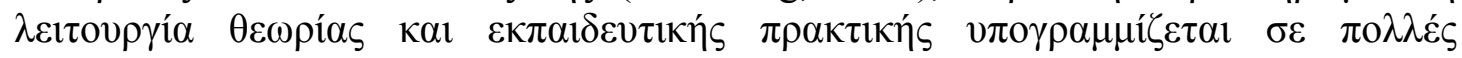

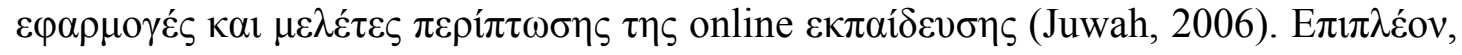

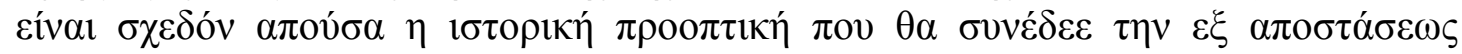

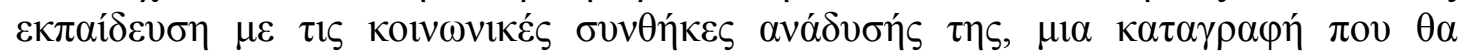

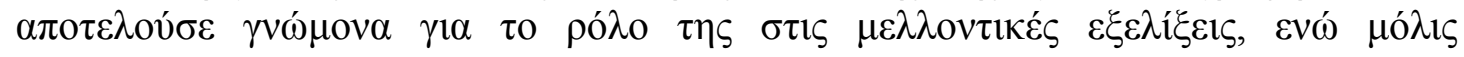

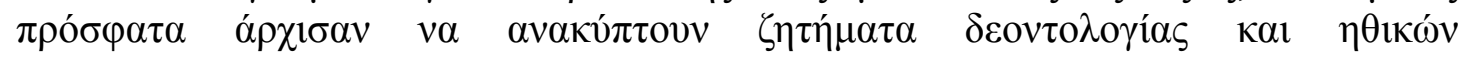

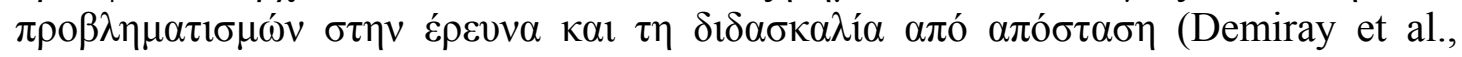

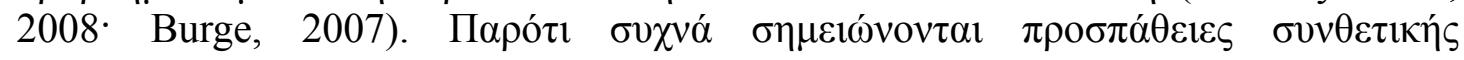

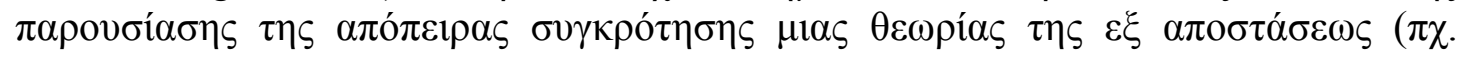

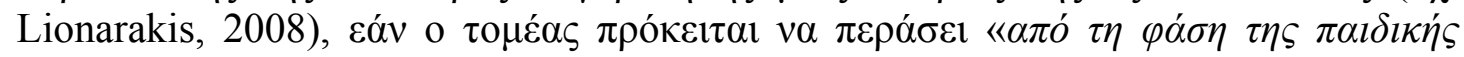

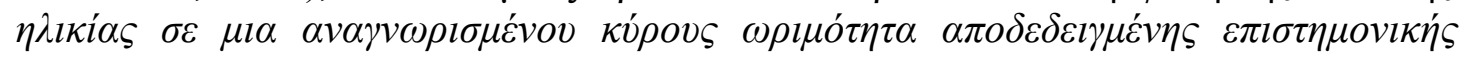

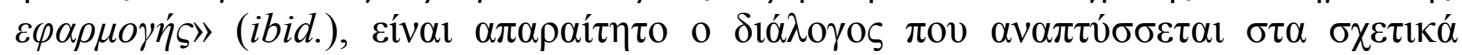

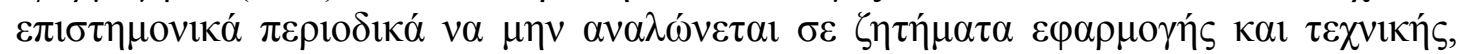

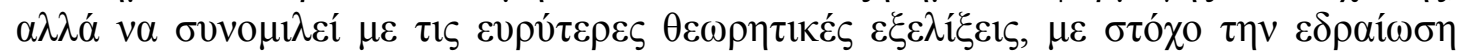

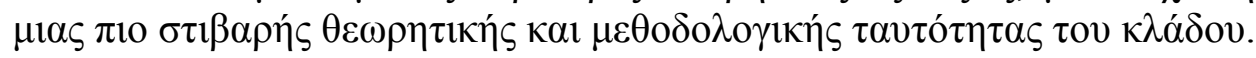

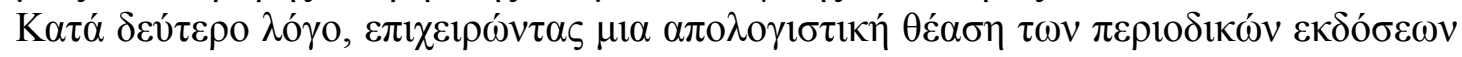

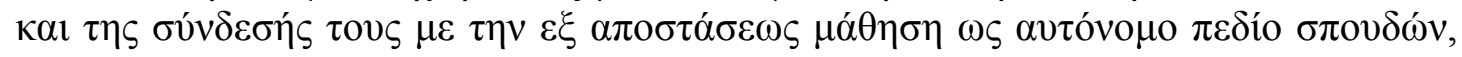

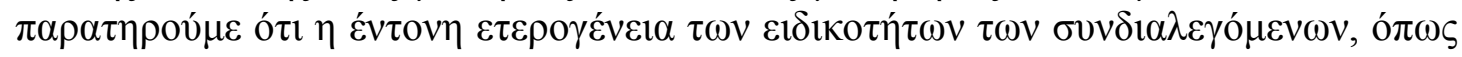

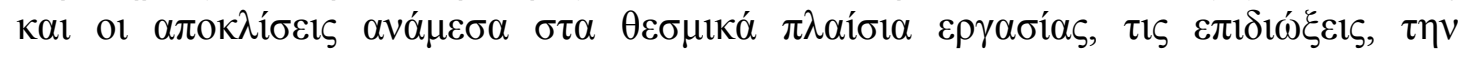

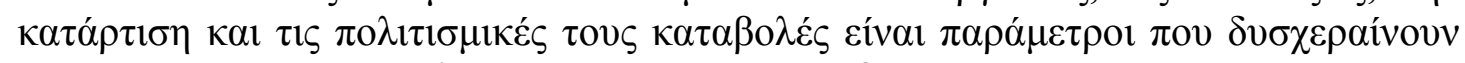

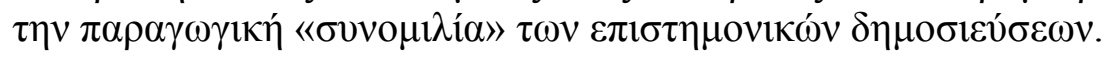

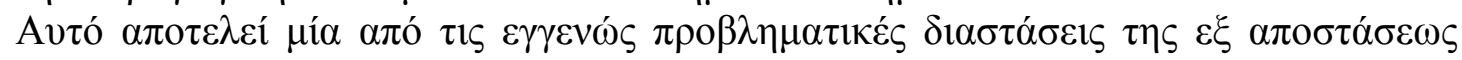

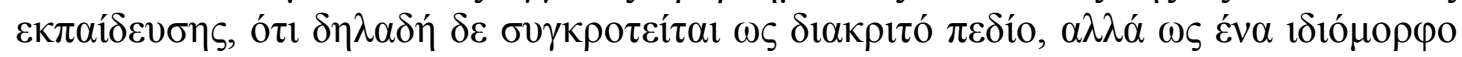

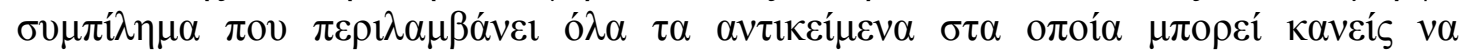

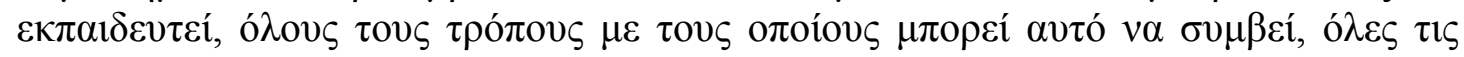

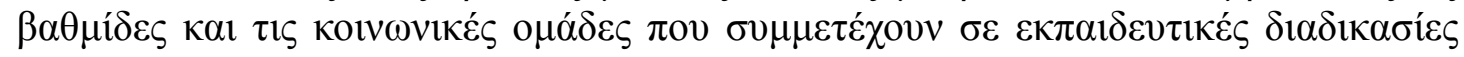

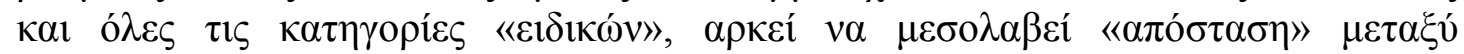

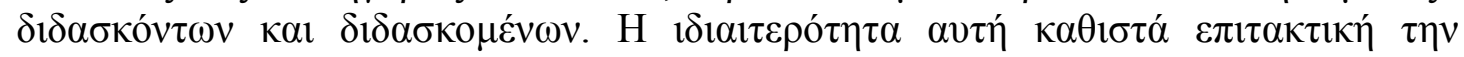

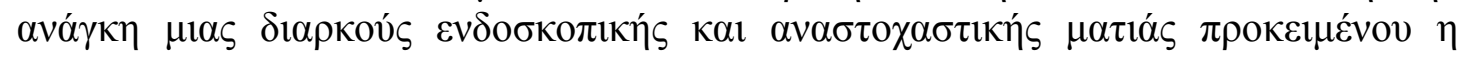

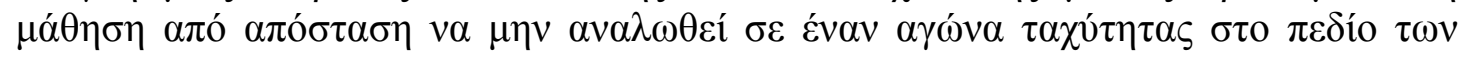

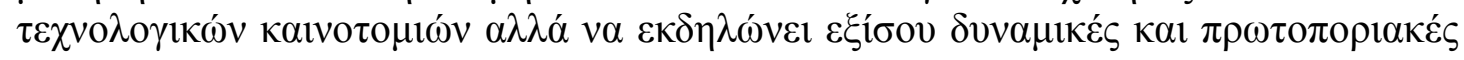

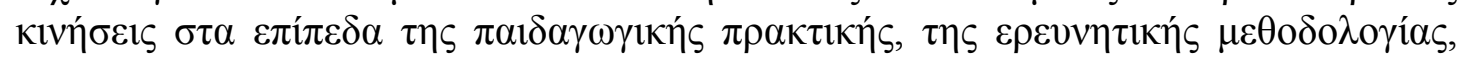

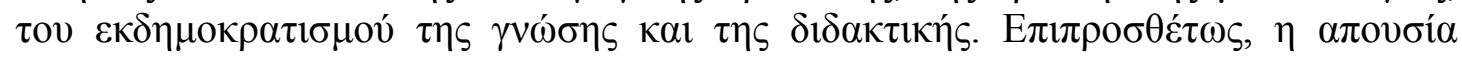

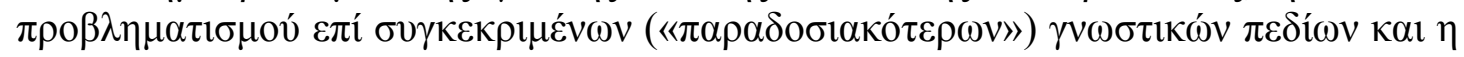

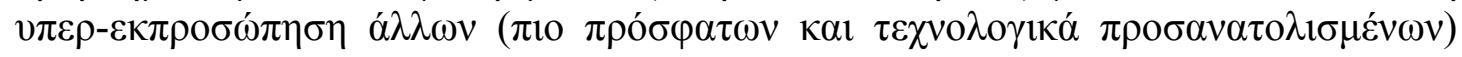

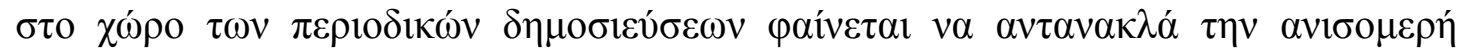

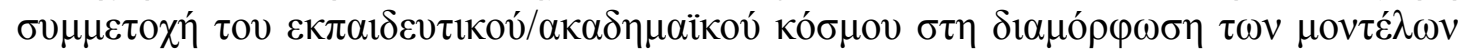

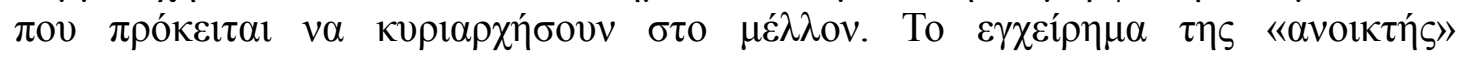

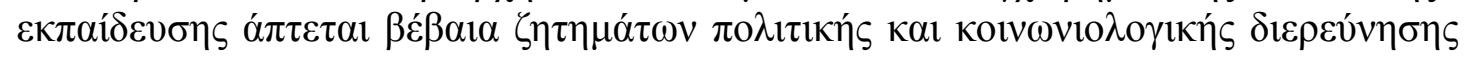

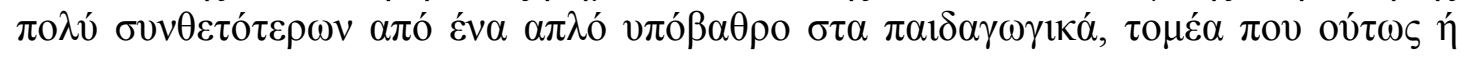

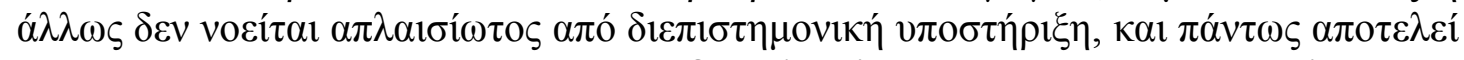

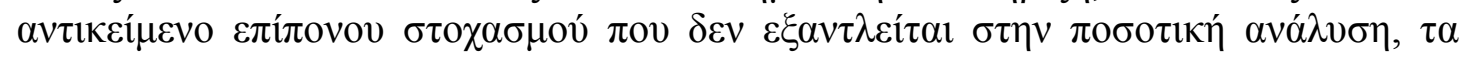

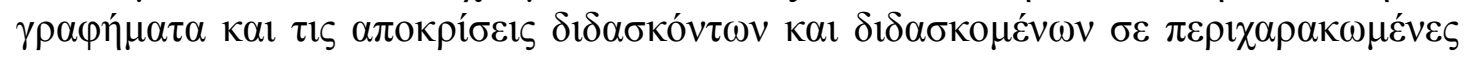

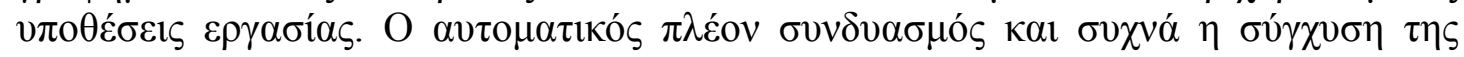

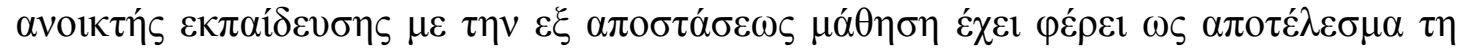




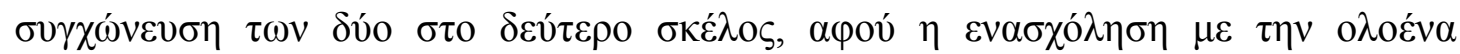

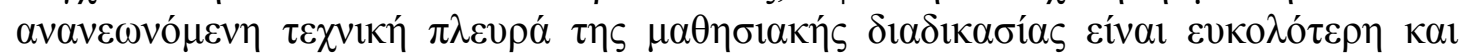

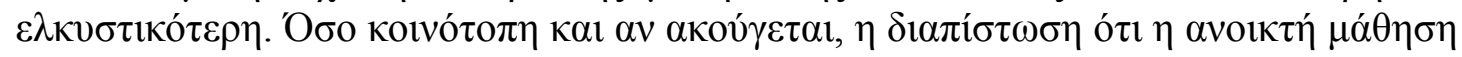

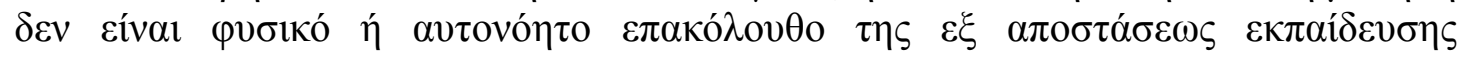

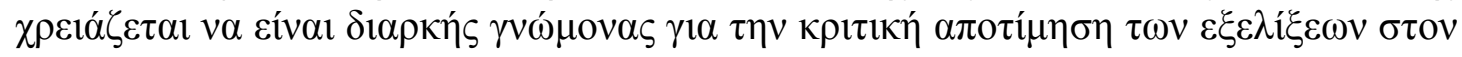
$\tau о \mu \varepsilon ́ \alpha$.

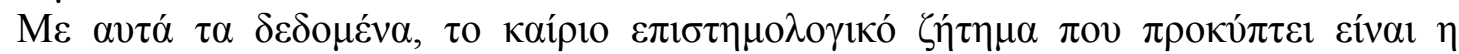

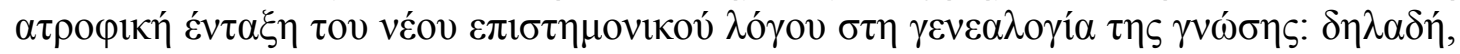

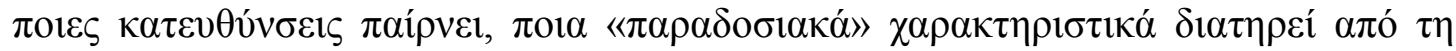

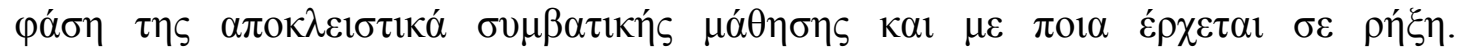

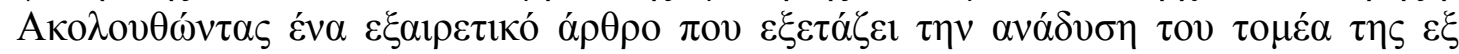

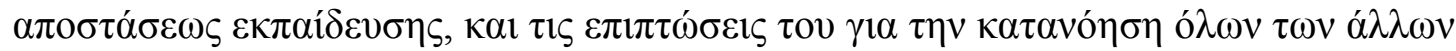

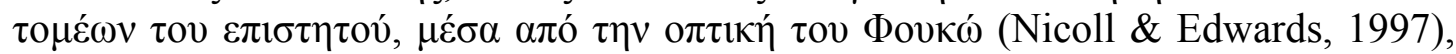

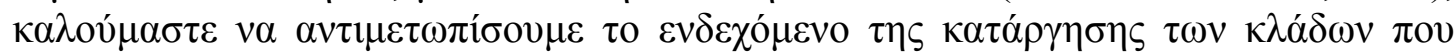

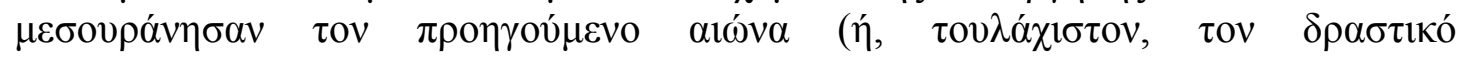

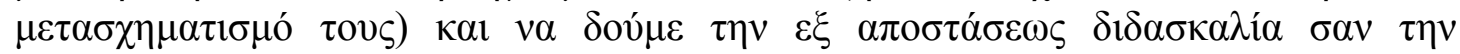

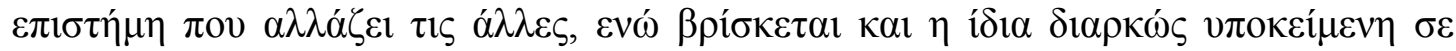

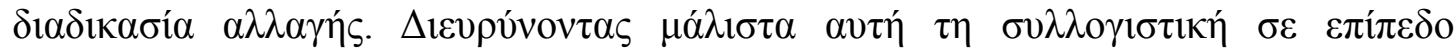

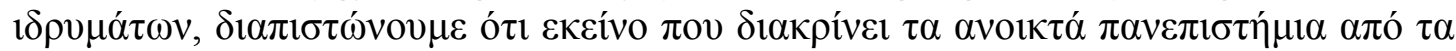

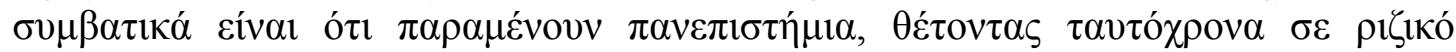

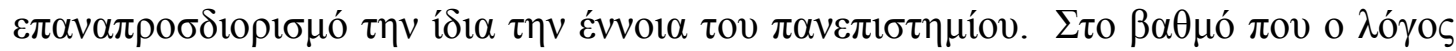

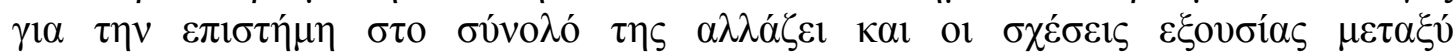

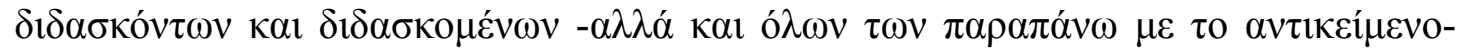

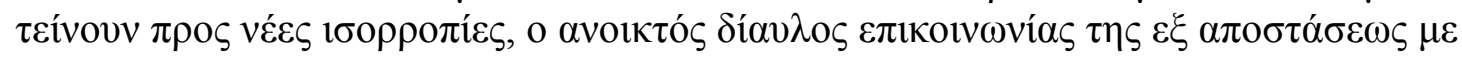

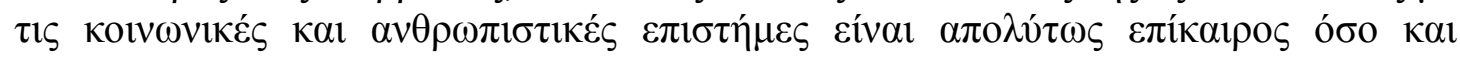

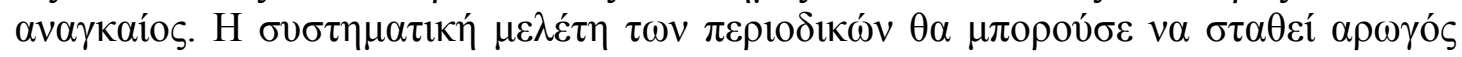

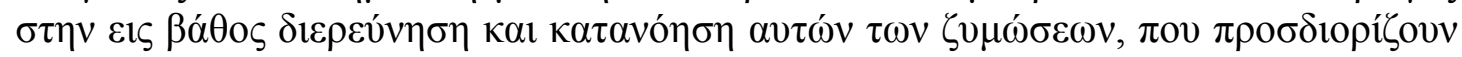

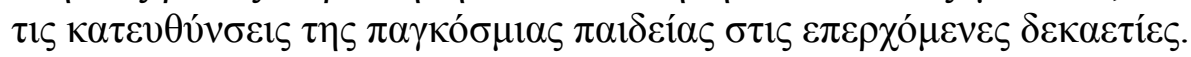

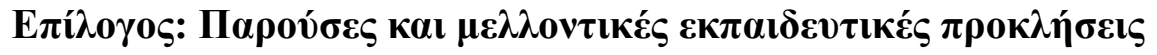

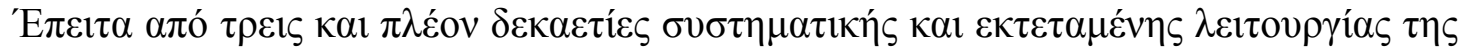

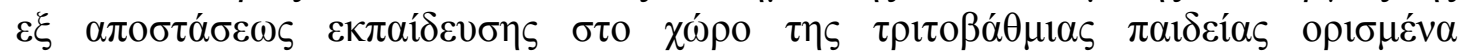

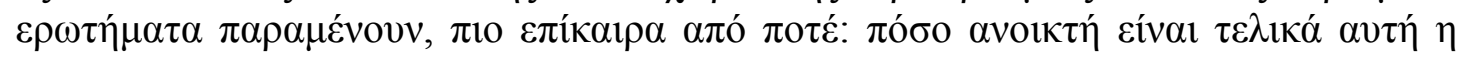

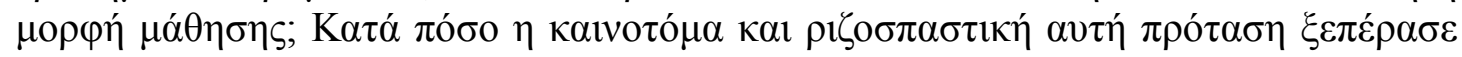

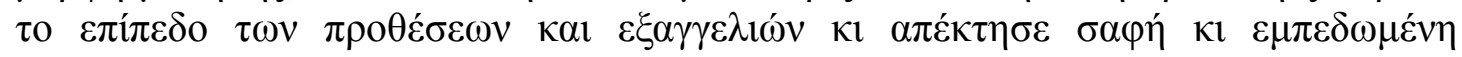

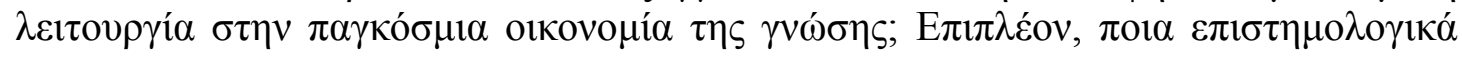

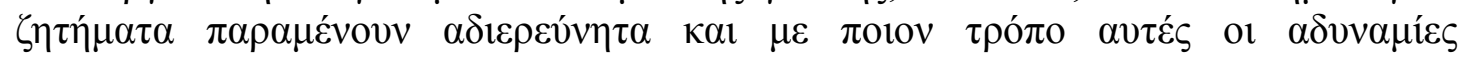

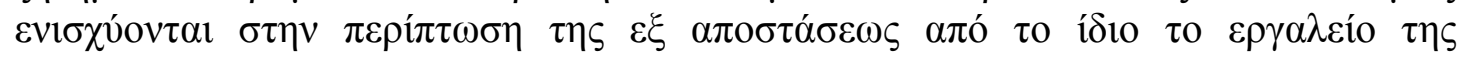

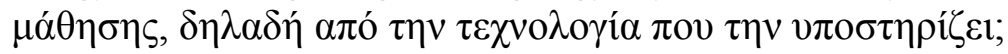

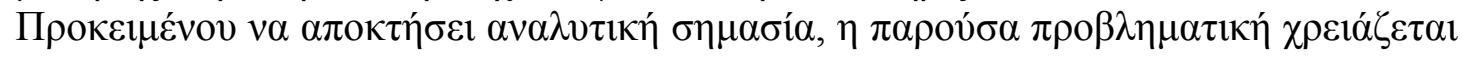

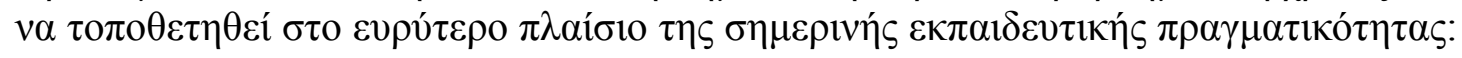

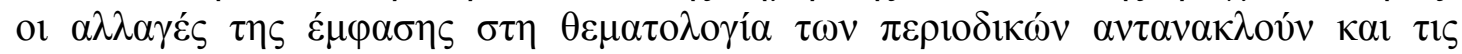

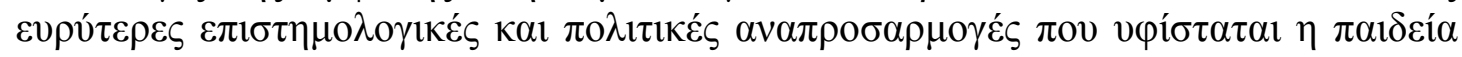

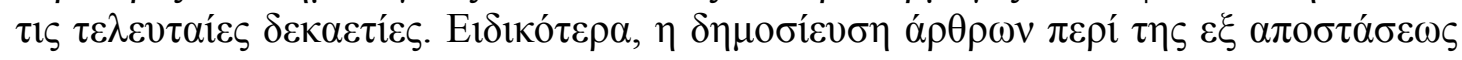

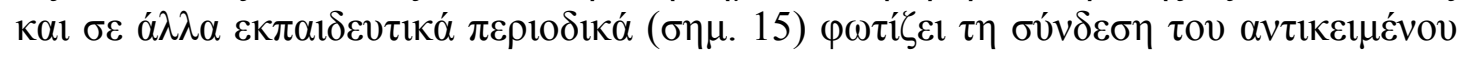

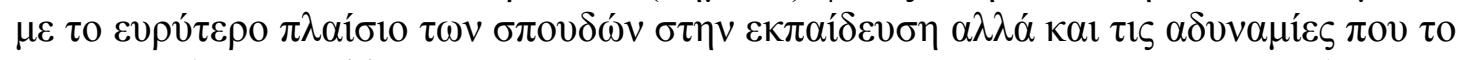

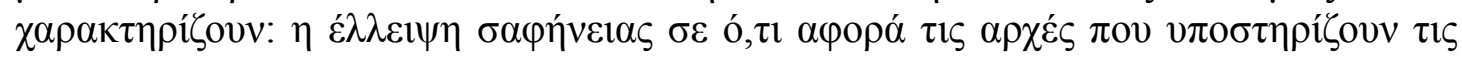

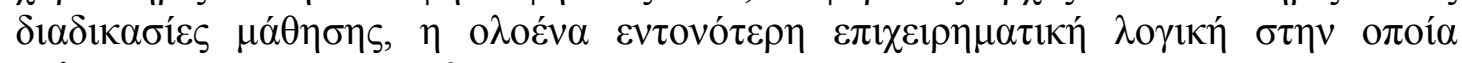

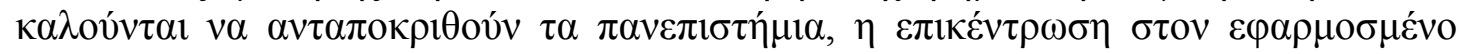

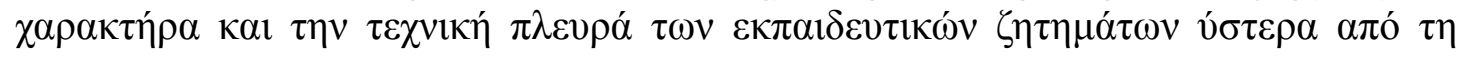




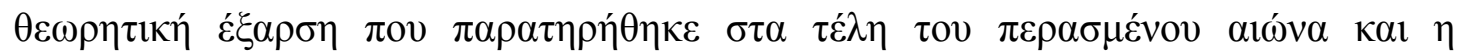

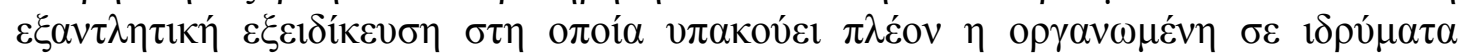

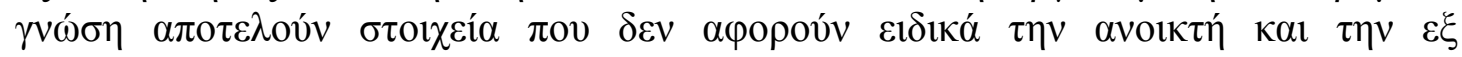

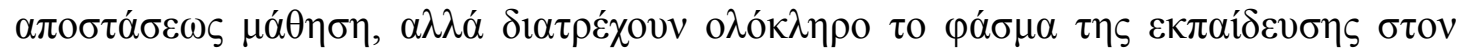

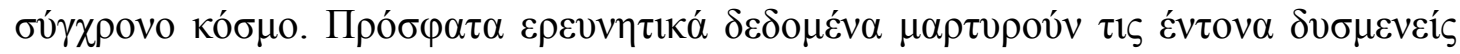

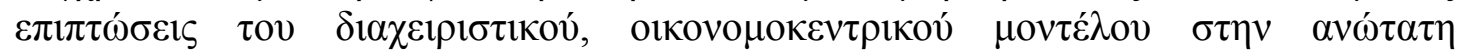

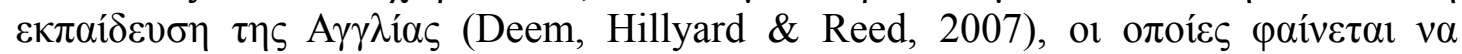

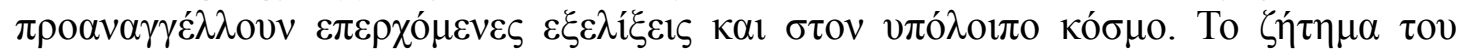

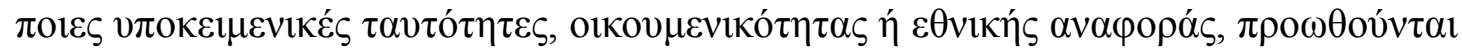

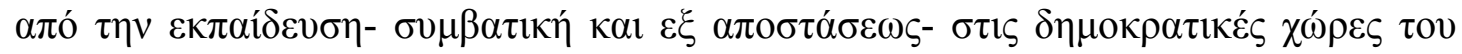

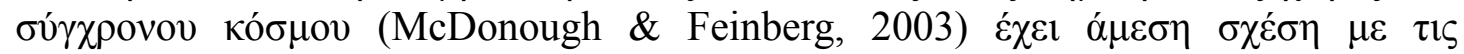

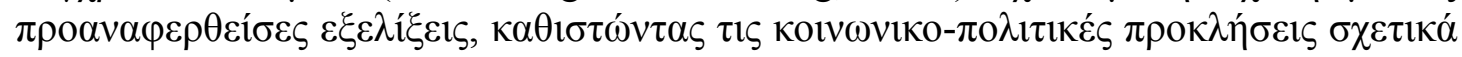

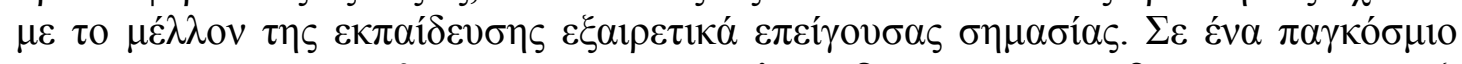

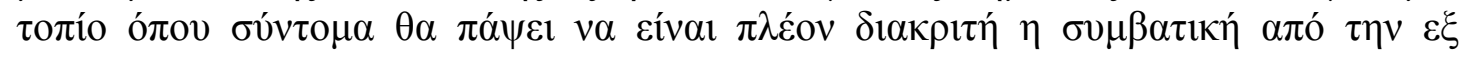

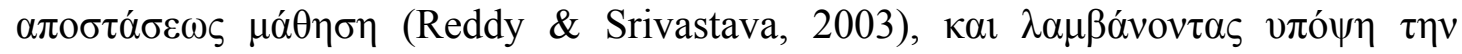

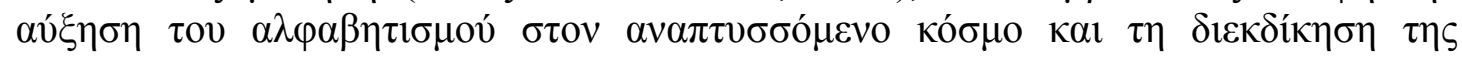

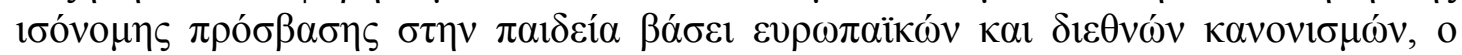

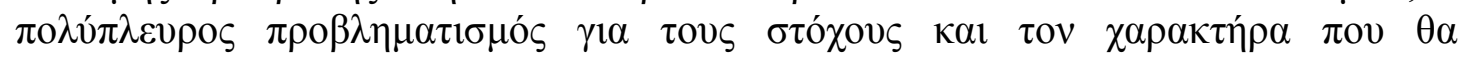

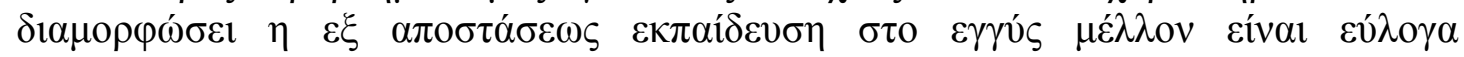

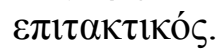

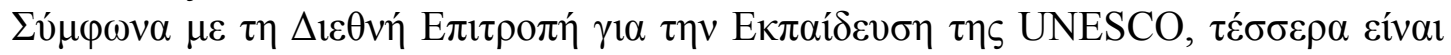

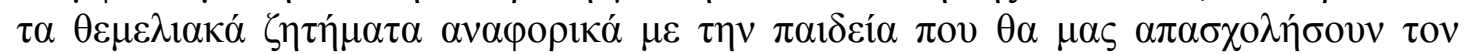

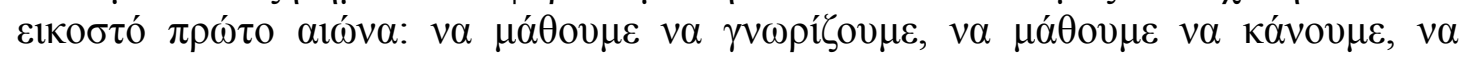

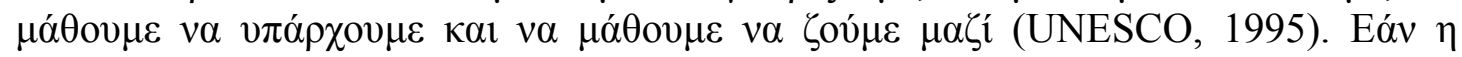

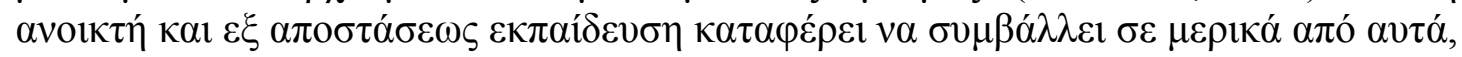

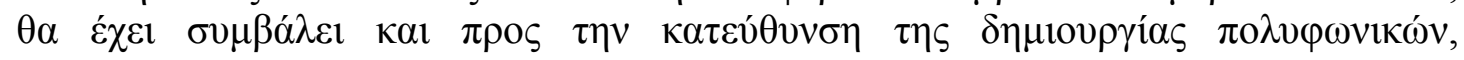

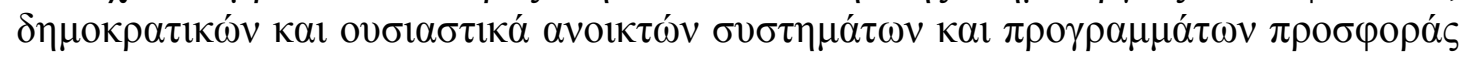

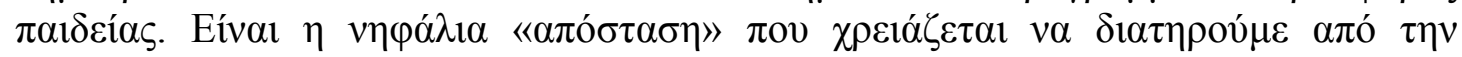

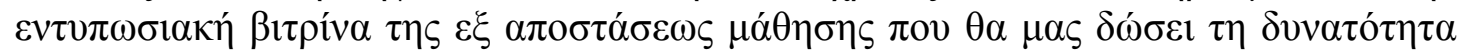

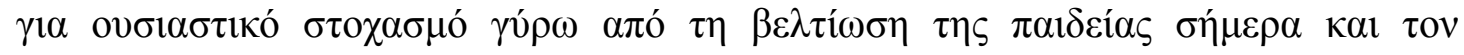

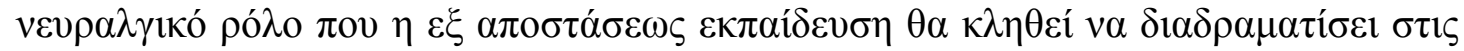

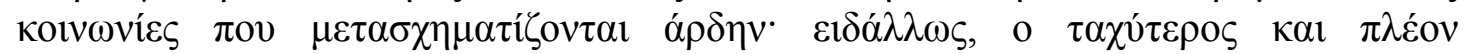

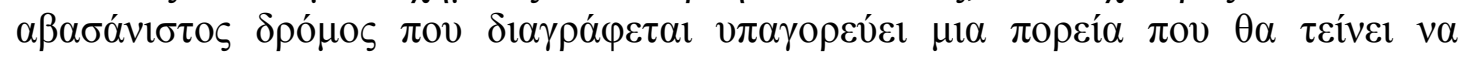

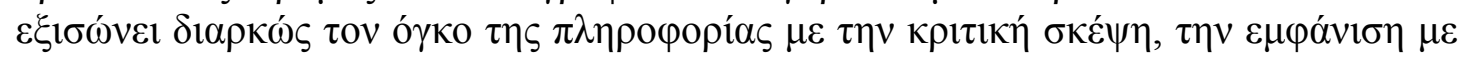

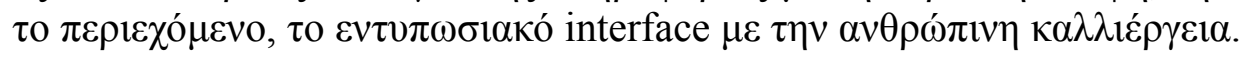

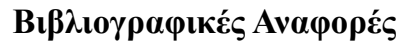

Bizzell, P., \& Herzberg, B. (1990). The Rhetorical Tradition: Readings from Classical Times to the Present. Boston: Bedford Books of St. Martin's Press.

Burge, E. J. (2007). Considering ethical issues. Open Learning: The Journal of Open and Distance Learning, 22(2), 107-115.

Carr-Chellman, A. A. (Ed.). (2005). Global Perspectives in E-Learning: Rhetoric and Reality. Thousand Oaks, CA: Sage.

Demiray, U. \& Ramesh, C. S. (Eds.). (2008). Ethical Practices and Implications in Distance Learning. Hershey: Information Science Reference.

Dikshit, H. P., Garg S., Panda S., \& Vijayshri (Eds.). (2002). Access and Equity: Challenges for Open and Distance Learning. New Delhi: Kogan Page.

Evans, T. \& Nation, D. (1992). Theorising Open and Distance Education. Open Learning: The Journal of Open and Distance Learning, 7(2), 3-13.

Foucault, M. (2002 [1969]). The Archeology of Knowledge. London \& New York: Routledge.

Frohmann, B. (1994). Discourse Analysis as a Research Method in Library and Information Science. Library and Information Science Research, 16, 119-138. 
Gaskell, A. (2006). Editorial: Intercultural and interpersonal understanding, Open Learning: The Journal of Open and Distance Learning, 21(3), 187-189.

Guri-Rosenblit, S. (2001). The Tower of Babel Syndrome in the Discourse of Information Technologies in Higher Education. Global E-Journal of Open, Flexible and Distance Education, 1(1).

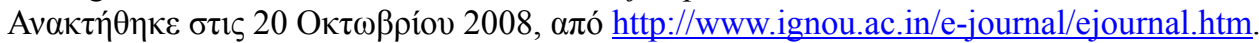

Herrmann, A., Fox. R., \& Boyd A. (1999). Benign Educational Technology? Open Learning: The Journal of Open and Distance Learning, 14(1), 3-8.

Hine, C. (Ed.). (2005). Virtual Methods: Issues in Social Research on the Internet. Oxford: Berg.

Holmberg, B. (1997). Distance Education Theory again. Open Learning: The Journal of Open and Distance Learning, 12(1), 31-39.

Holmberg, B. (2001). Distance Education in Essence. Oldenburg, Germany: Biblioteksund informationssystem der Universität Oldenburg.

Jones, S. (Ed.). (1999). Doing Internet Research: Critical Issues and Methods for Examining the Net. London: Sage.

Juwah, C. (Ed.). (2006). Interactions in Online Education: Implications for Theory and Practice. Oxon \& New York: Routledge.

Keegan, D. (1996). Foundations of Distance Education. London: Routledge.

Kember, D. (2007). Reconsidering Open and Distance Learning in the Developing World. Abingdon: Routledge.

Krippendorff, K. (2004). Content Analysis: An Introduction to Its Methodology. Thousand Oaks, CA: Sage Publications.

Levine, J. S. (Ed.). (2005). Making Distance Education Work: Understanding Learning and Learners at a Distance. Okemos, Michigan: LearnerAssociates.net.

Lionarakis, A. (2008). The theory of distance education and its complexity. European Journal of Open,

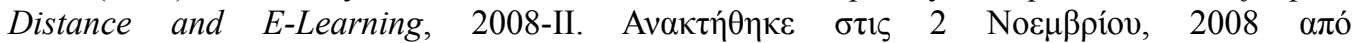
http://www.eurodl.org/materials/contrib/2008/Lionarakis.htm.

Lundin, R. (1998). Being Unreal: Epistemology, Ontology, and Phenomenology in a Virtual Educational

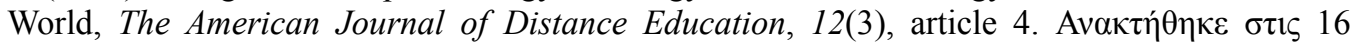

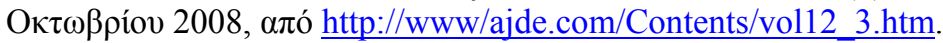

Monolescu, D., Schifter C., \& Greenwood L. (2004). The Distance Education Evolution: Issues and Case Studies. Hershey \& London: Information Science Publishing.

Moore, M. G. (1991). Distance Education Theory. The American Journal of Distance Education, 5(3).

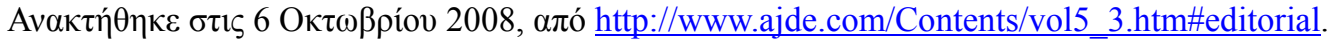

Moore, M. G. (2002). Editorial: Learning the Necessary Principles. The American Journal of Distance

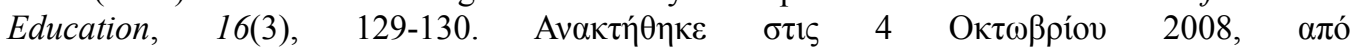
http://www.ajde.com/Documents/AJDE1603_1.pdf.

Moore, M. G. (2004). Editorial: Constructivists: Don't Blame the Tools! The American Journal of

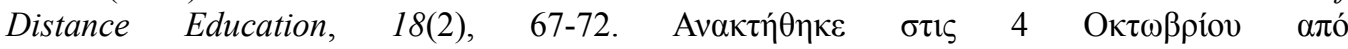
http://www.ajde.com/Documents/pp67-72.pdf.

Nicoll, K., \& Edwards R. (1997). Open Learning and the demise of discipline. Open Learning: The Journal of Open and Distance Learning, 12(3), 14-24.

Oxford, R. \& Florini, B. (1990). Editorial: What Distance Education Can Learn from Other Disciplines.

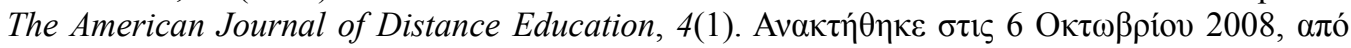
http://www.ajde.com/Contents/vol4_1.htm\#editorial.

Perraton, H. (2007). Open and distance learning in the developing world. Routledge Studies in Distance Education. Abingdon: Routledge.

Reddy, V., \& Srivastave M. (2003). ICT and the Future of Distance Education. Turkish Online Journal of

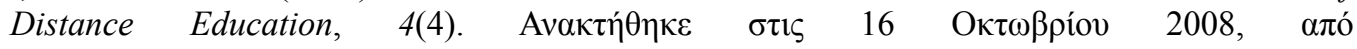
http://tojde.anadolu.edu.tr/tojde12/index.htm.

Rumble, G. (2001). Just How Relevant is E-education to Global Educational Needs? Open Learning: The Journal of Open and Distance Learning, 16(3), 223-232.

Russell, G. (2007), Globalization, Distance Education and Hegemonic Futures, Turkish Online Journal of

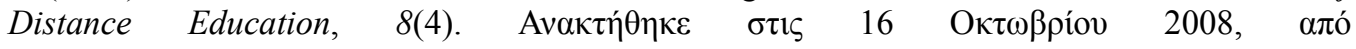
http://tojde.anadolu.edu.tr/tojde28/index.htm.

Sharma, R. C., \& Mishra S. (Eds.). (2007). Cases on Global E-Learning Practices: Successes and Pitfalls. Information Science Publishing.

Stewart, D., Keegan D., \& B. Holmberg (Eds.). (1988). Distance education: International perspectives. New York: Routledge.

Tait, A. (2008). What are open universities for? Open Learning: The Journal of Open and Distance Learning, 23(2), 85-93. 
Open Education - The J oumal for Open and Distance Education and Educational Technology

UNESCO (1995). International Commission on Education for the Twenty-First Century. Paris: UNESCO.

Visser Y. L, Visser L., Simonsin M., \& Amirault R. (2005). Trends and Issues in Distance Education: International Perspectives. Greenwich, CT: Information Age Publishing.

Zondrinos, D. (2008). Online, distance education and globalization: Its impact on educational access,

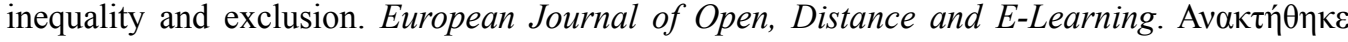

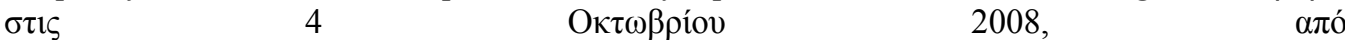
http://www.eurodl.org/materials/special/2008/Dimitris_Zondiros.htm.

\section{$\Sigma \eta \mu \varepsilon เ \omega ́ \sigma \varepsilon ı \varsigma$}

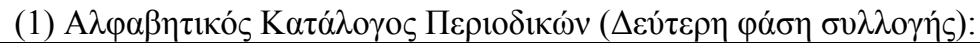

\begin{tabular}{|c|}
\hline 1. Active Learning \\
\hline 2. American Journal of Distance Education \\
\hline 3. Annual Conference on Distance Teaching and Learning \\
\hline 4. Asian Journal of Distance Education \\
\hline 5. $\quad$ Association for Learning Technology Journal \\
\hline 6. Australasian Journal of Educational Technology \\
\hline 7. Campus Technology \\
\hline 8. Canadian Journal of Learning and Technology \\
\hline 9. Computers and Education \\
\hline 10. Contemporary Issues in Technology \& Teacher Education \\
\hline 11. DEOSNEWS (Best Practices for Faculty Who Teach Online) \\
\hline 12. DESIEN - Distance Education Systemwide Interactive Electronic Newsletter \\
\hline 13. Distance education report \\
\hline 14. Distance Education \\
\hline 15. Distance Educator \\
\hline 16. Distances et saviors: Une revue scientifique sur l'enseignement à distance \\
\hline 17. Distances \\
\hline 18. E-Content Magazine \\
\hline 19. Education and Information Technologies \\
\hline 20. Education.au Reports \\
\hline 21. Educational Technology \& Society \\
\hline 22. Educational Technology Research \& Development \\
\hline 23. EDUCAUSE Quarterly \\
\hline 24. Edutec-e Revista Electrónica de Tecnología Educativa \\
\hline 25. E-learning Age Magazine \\
\hline 26. E-learning Age Research Reports \\
\hline 27. European Journal of Open, Distance and E-Learning \\
\hline 28. Global Journal of Open, Flexible \& Distance Education \\
\hline 29. Indian Journal of Distance Education \\
\hline 30. Indian Journal of Open Learning \\
\hline 31. Information Technology and Disabilities \\
\hline 32. Innovate \\
\hline 33. Interactive Educational Multimedia \\
\hline 34. International journal of distance education technologies \\
\hline 35. International Journal of Educational Technology \\
\hline 36. International Journal of Emerging Technologies in Learning \\
\hline 37. International Journal of Instructional Technology and Distance Learning \\
\hline 38. International Journal of Internet Research Ethics \\
\hline 39. International Journal on E-Learning \\
\hline 40. International Review of Research in Open and Distance Learning \\
\hline 41. Journal of Asynchronous Learning Networks \\
\hline 42. Journal of Computer Assisted Learning \\
\hline 43. Journal of Computer Mediated Communication \\
\hline 44. Journal of Distance Education/Revue de l'enseignement à distance (CADE) \\
\hline 45. Journal of Distance Learning Administration \\
\hline 46. Journal of Educational Multimedia and Hypermedia \\
\hline
\end{tabular}


Open Education - The J oumal for Open and Distance Education and Educational Technology

\begin{tabular}{|c|}
\hline 47. Journal of e-Learning and Knowledge Society \\
\hline 48. Journal of Instructional Science and Technology \\
\hline 49. Journal of Interactive Learning Research \\
\hline 50. Journal of Interactive Media in Education \\
\hline 51. Journal of Library \& Information Services in Distance Learning \\
\hline 52. Journal of Research on Technology in Education \\
\hline 53. Journal of Technology and Teacher Education \\
\hline 54. Journal of University Teaching and Learning Practice \\
\hline 55. Kakatiya Journal of Distance Education \\
\hline 56. Learning Technology \\
\hline 57. Malaysian Journal of Distance Education \\
\hline 58. Media and Technology for Human Resource Development \\
\hline 59. Never Too Far \\
\hline 60. Online Chronicle of Distance Education \& Communication \\
\hline 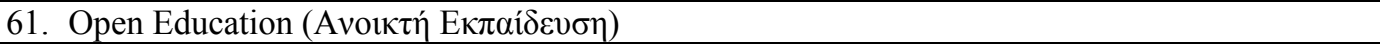 \\
\hline 62. Open Learning \\
\hline 63. Open Praxis \\
\hline 64. Pakistan Journal of Distance Education \\
\hline 65. SAMTEC - South African Educational Technology and e-Learning for Development (portal) \\
\hline 66. Sloan-C View: Perspectives in Quality Online Education \\
\hline 67. $\mathrm{T}+\mathrm{D}$ Magazine \\
\hline 68. Technological Horizons in Education (T.H.E.) Journal \\
\hline 69. Technology and Learning On-line \\
\hline 70. The Electronic Journal on Information Systems in Developing Countries \\
\hline 71. The Information Society \\
\hline 72. The International Journal of Education and Development \\
\hline 73. The Internet and Higher Education \\
\hline 74. The Journal of Distance Learning \\
\hline 75. The Journal of Library Services for Distance Education \\
\hline 76. The Knowledge Tree e-journal of Flexible Learning in Vocational Education and Training \\
\hline 77. The Quarterly Review of Distance Education \\
\hline 78. The Technology Source \\
\hline 79. The Turkish Online Journal of Distance Education \\
\hline 80. The Virtual University Gazette \\
\hline 81. Theory and Practice of Online Learning (Athabasca University) \\
\hline 82. Transformative Dialogues: Teaching and Learning Journal \\
\hline 83. Virtual University Journal \\
\hline 84. World Association for Online Education - WAOE e-Bulletin \\
\hline
\end{tabular}

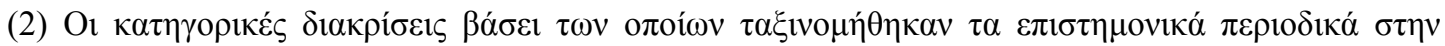

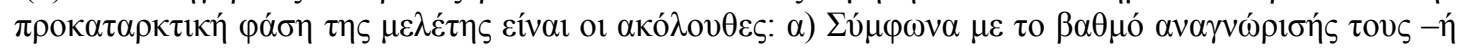

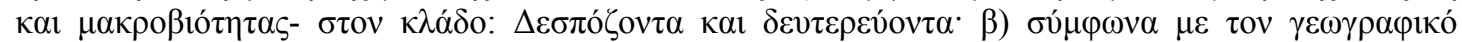

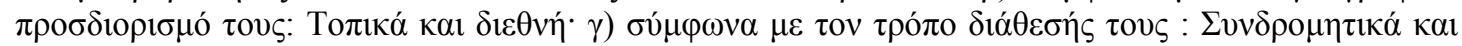

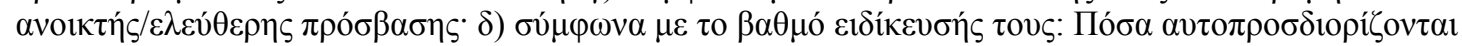

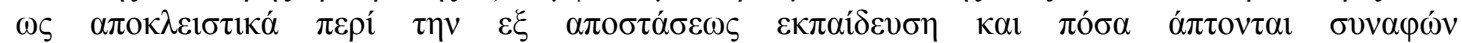

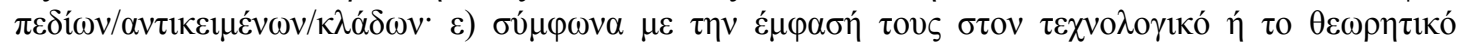

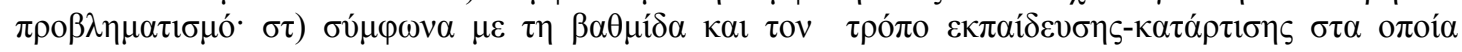

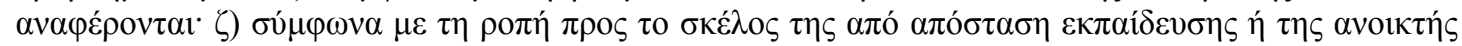

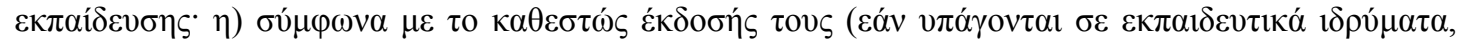

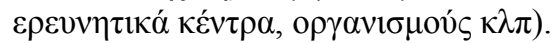

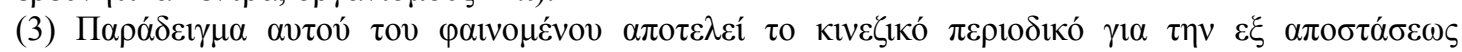

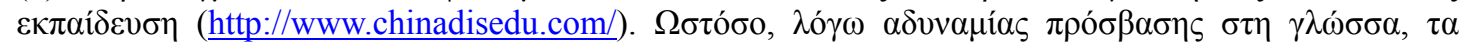

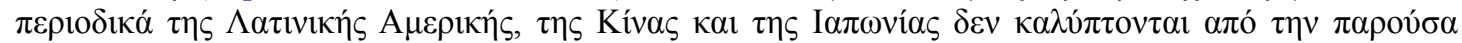

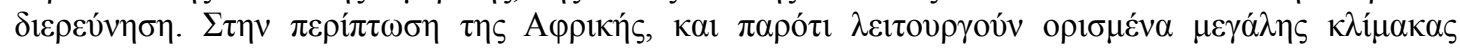

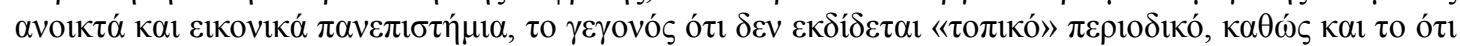

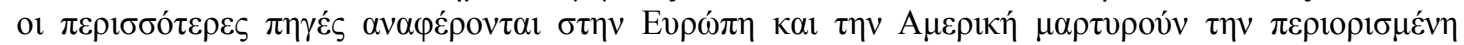

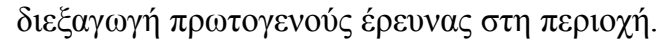

(4) American Journal of Distance Education 4(1), 1990. 
(5) American Journal of Distance Education 2(2), 1988· кal 4(2), 1990.

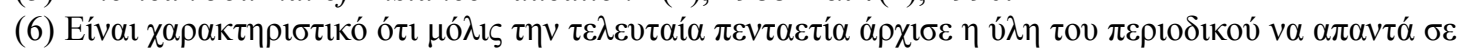

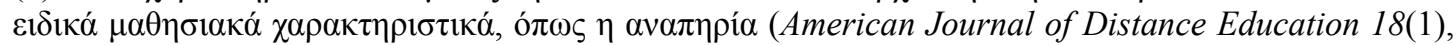

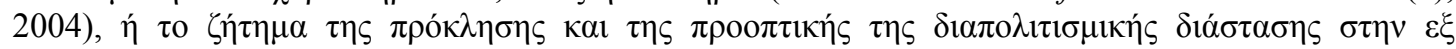

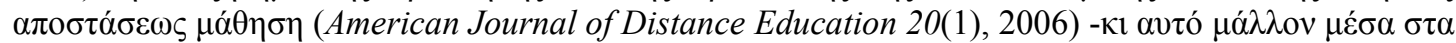

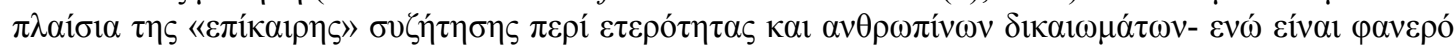

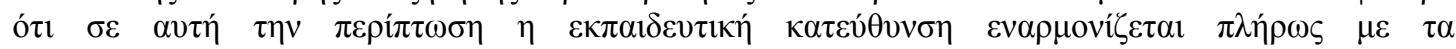

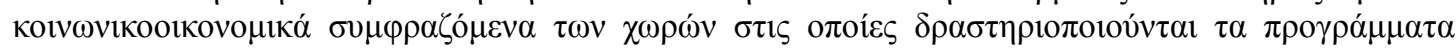

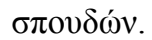

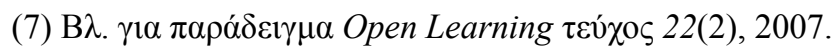

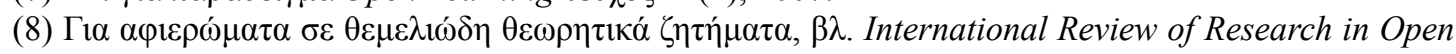
and Distance Learning 7(2), 2006 8(2), 2007· 9(1), 2008. Гı

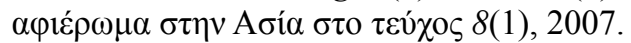

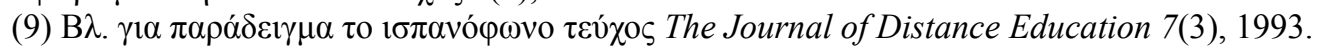

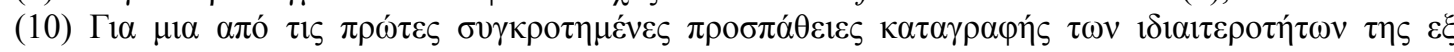

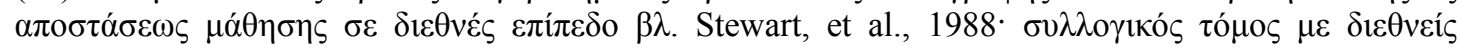

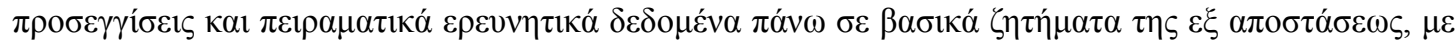

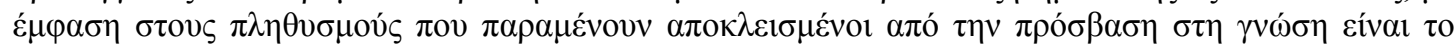

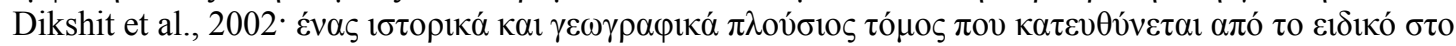

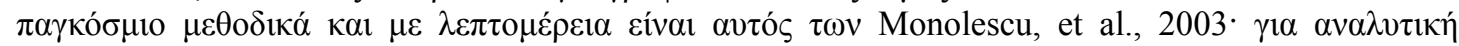

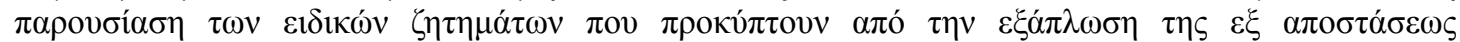

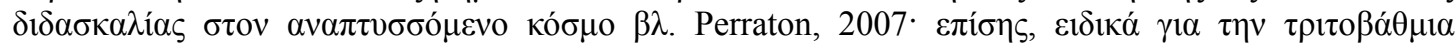

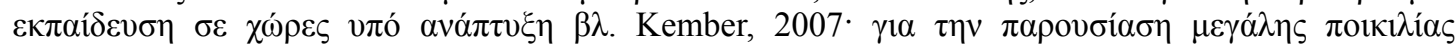

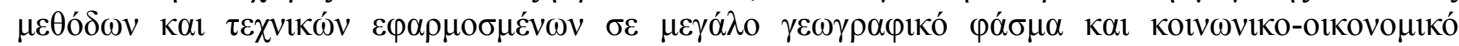

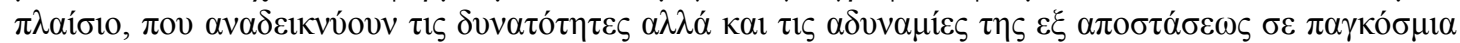

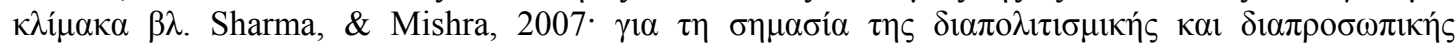

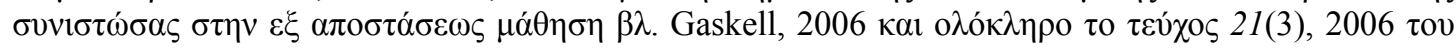
Open Learning: The Journal of Open and Distance Learning.

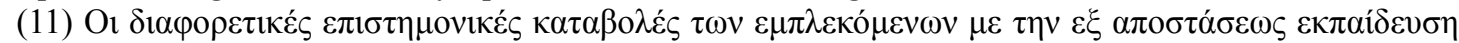

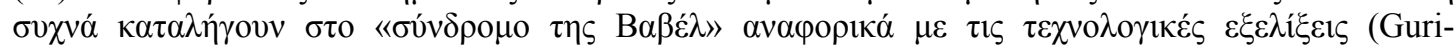

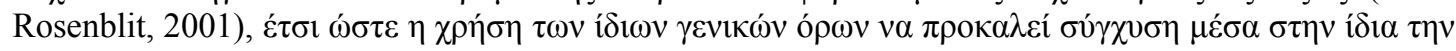

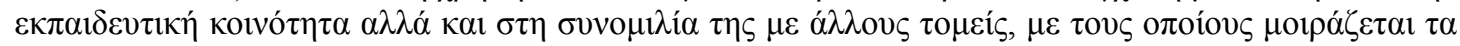

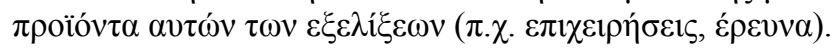

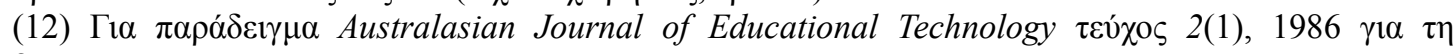

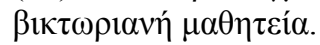

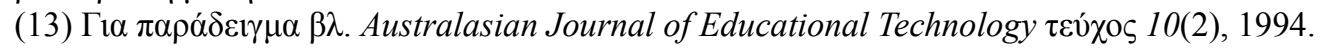

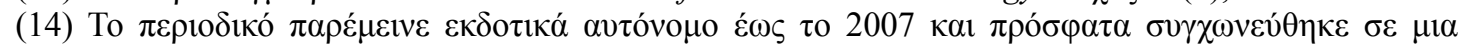

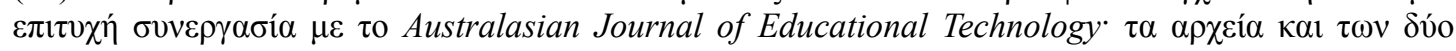

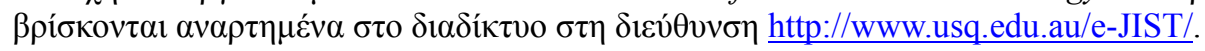

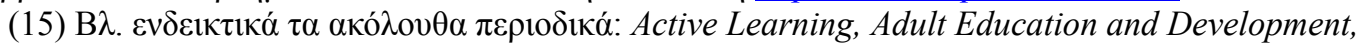
American Educational Research Journal, American Educational Research Journal, Anthropology and Education Quarterly, Citizenship and Social Justice, Complicity: An International Journal of

Complexity in Education, Current Issues in Education, Education and Culture, Education,

Educational Research Review, International Journal of Research and Method in Education. 


\section{What kind of Haptic devices and applications are needed in education? Requirements, Specifications and hands-on experience derived from an IST project}

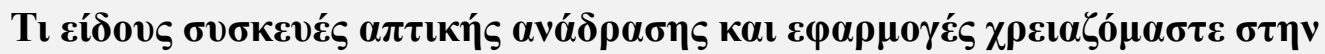

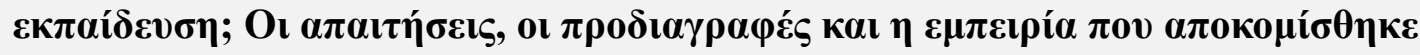
$\alpha \pi$ óva IST $\pi \rho o ́ \gamma \rho \alpha \mu \mu \alpha$

S. P. Christodoulou

HPCLab, Computer Engineering \& Informatics Department, University of Patras, 26500 Rion, Patras, Greece spc@hpclab.ceid.upatras.gr

D. M. Garyfallidou

The Science Laboratory, School of Education, University of Patras, 26500 Rion, Patras, Greece

D.M.Garyfallidou@upatras.gr

G. S. Ioannidis

The Science Laboratory, School of Education University of Patras, 26500 Rion, Patras, Greece gsioanni@upatras.gr

T. S. Papatheodorou

HPCLab, Computer Engineering \& Informatics Department University of Patras, 26500 Rion, Patras, Greece tsp@hpclab.ceid.upatras.gr

E. A. Stathi

HPCLab, Computer Engineering \& Informatics Department, University of Patras, 26500 Rion, Patras, Greece stathie@hpclab.ceid.upatras.gr

\section{Пері́ $\eta \psi \eta$}

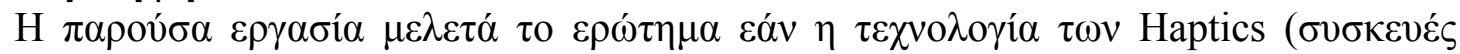

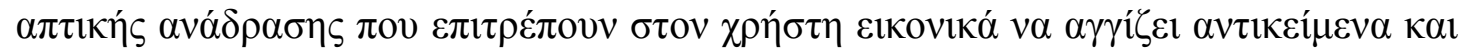

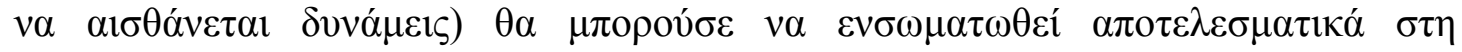

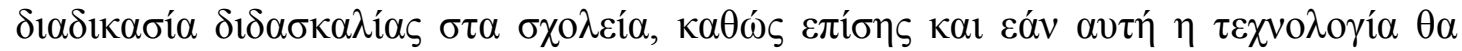
$\mu \pi$

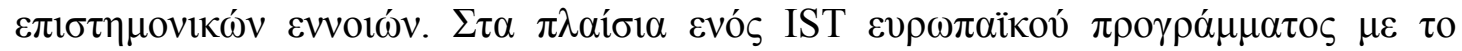

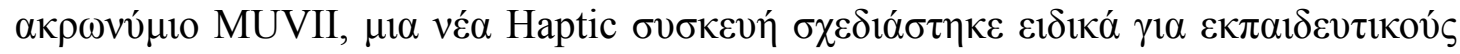

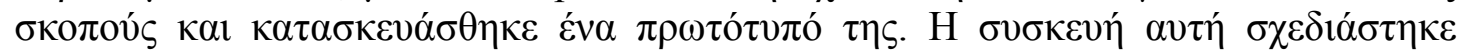

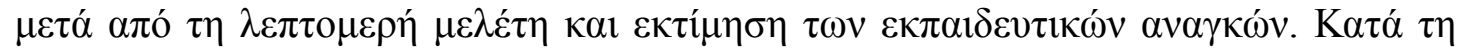

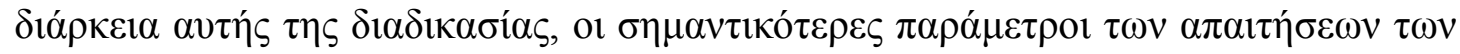

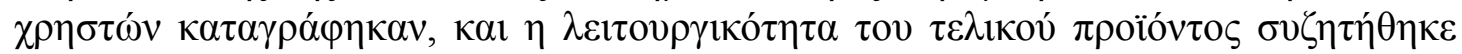

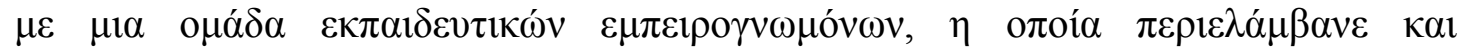

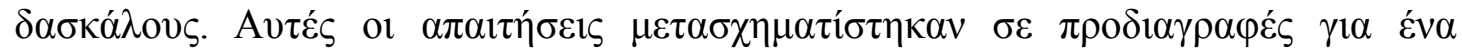

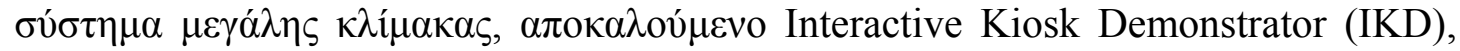

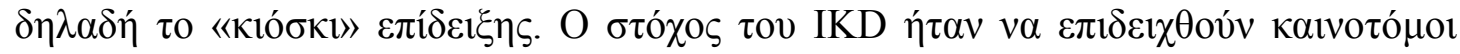

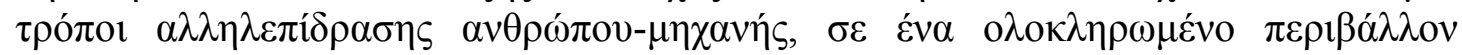

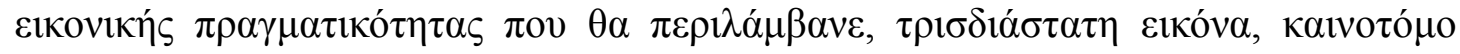

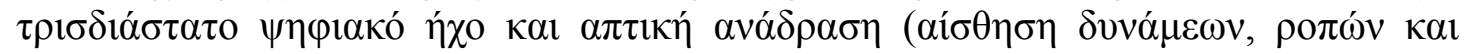

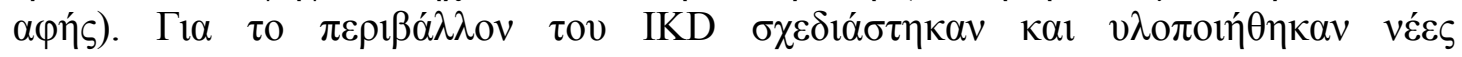

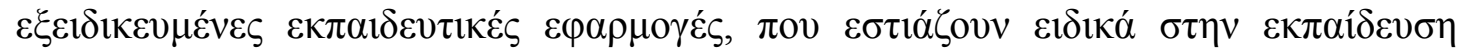

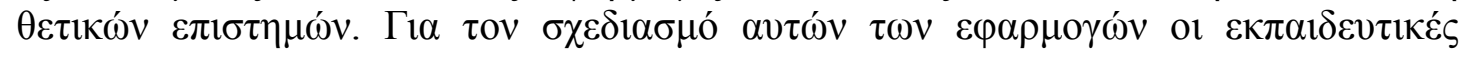




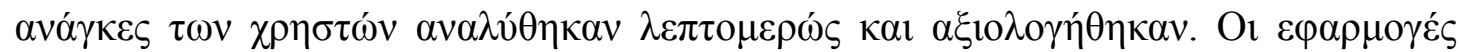

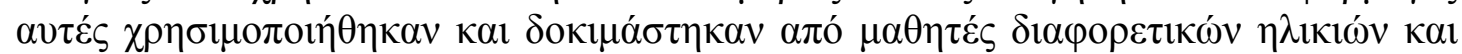

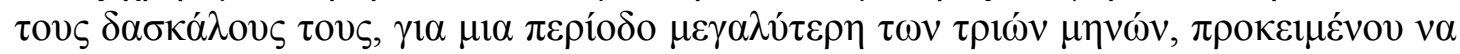

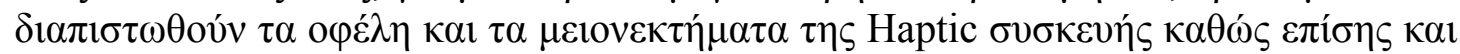

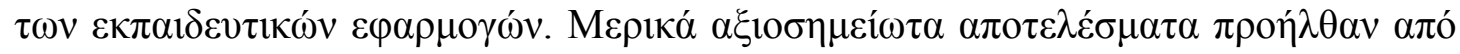

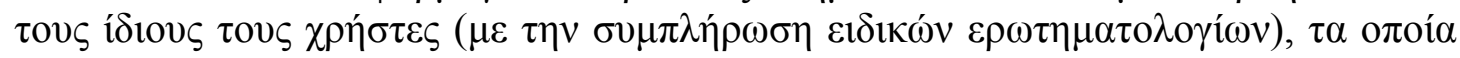

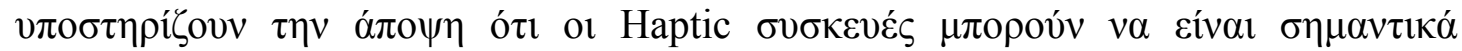

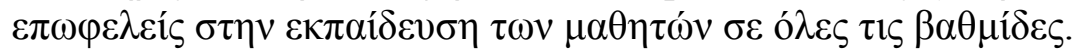

\section{bstract}

In this paper the question whether Haptics technology (virtually touching objects and feeling forces), could be effectively implemented in the teaching procedure is studied, as well as if this technology can be used in improving the understanding of certain scientific concepts. Under the framework of an IST European program called MUVII, a new haptic device was designed especially for educational purposes and a prototype was implemented. This device was designed after thorough consideration of the educational needs. During this process, the most important parameters of the end user requirements were drawn up, and the functionality of the end product was discussed with a team of educational experts (the team also included teachers). These requirements were converted into specifications for a large scale system, called the Interactive Kiosk Demonstrator (IKD). IKD's objective was to demonstrate new interactive paradigms forming a novel integration of the following modalities: 3Dvision, 3D-audio and haptic (force, torque, and tactile) feedback. For the application software design (focusing especially on the context of science education) the educational needs of the end users were thoroughly analyzed and evaluated and, subsequently, some applications were designed and developed. These applications have been used and tested by students of different ages, and their teachers, for a period of over three months in order to divulge the benefits and the drawbacks of the haptic device as well as the applications. Some remarkable results were derived from the users' feedback, which support the view that haptic interfaces can be significantly beneficial to the students' education.

Keywords. Haptic Devices, Science Educational Applications, Specifications, User requirements, Virtual Reality.

\section{Introduction to Haptics, 3D Graphics and 3D Sound}

Initially, it is useful to define some terms of Haptics, 3D Graphics and $3 D$ Sound, since most haptic devices use this kind of technology.

The term haptics was popularized in the United States by the philosopher Gilles Deleuze and comes from the Greek verb "haptesthai", which means to touch and to handle. The technology refers to the ability of touching and handling virtual objects. In a more general sense, it is an interface that enables users to understand the weight, the shape, the volume of an object and the forces acting on it. By using special input/output devices (joysticks, data gloves, or other devices), users can receive feedback from computer applications in the form of sensations felt on the fingers, the hand, or other parts of the body. The term "tactile feedback" refers only to the sensory input on the fingertips of the user. 
With $3 D$ (three-dimensional) graphics we refer to a space where objects (polygons) are made up by a series of dots which are referred to as corners. The coordinates of these corners are specified by three values: $x, y$ and $z$. The representation of the $3 \mathrm{D}$ space on each of user's eyes is always a $2 \mathrm{D}$ image obtained through the rendering process. The impression of $3 \mathrm{D}$ is created in the human mind after some (quite elaborate) computation, albeit without conscious effort from the part of the user. Many parameters are used as input to this computation, like the different $2 \mathrm{D}$ image in each eye, different colouring (hue) of objects as distance increases, common assumptions as to the size and speed of various objects etc.

As far as $3 D$ sound is concerned, true $3 \mathrm{D}$ sound has genuine depth and width to it. Just like 3D graphics, 3D sound can also be recreated by just two speakers and some very advanced mathematics! The use of 3D graphics and 3D sound combined with haptic feedback create a multi-sensory immersion for the users.

\section{MUVII project description}

MUVII stands for the Multi User Virtual Interactive Interface and is the acronym of the IST Project IST-2000-28463. The key objective of MUVII project (WWW) was to develop on the one hand two new Man-Machine-Interface Devices featuring haptic feedback, called Haptic-3D-Interface (H3DI), and on the other hand a prototype of an innovative integrated platform using the device: the Interactive Kiosk Demonstrator (IKD). University of Patras (HPCLab - High Performance Information Systems Laboratory) was responsible for the design and integration of the IKD platform as well as the development of the 3D haptic applications. In cooperation with The Science Laboratory of the School of Education of University of Patras, provided the specifications of the device and carried out the testing of the whole platform with pupils and teachers. The other partners of the project were: Laval Mayenne Technopole (France), CEA - Commissariat a l'Energie Atomique (France), SINTEF The Foundation for Scientific and Industrial Research at the Norwegian Institute of Technology (Norway), De Pinxi (Belgium), Institut für Kommunikationsakustik Ruhr University of Bochum (Germany), ONDIM (France), CompuTouch (Norway), Centre PIC (Russia).

Nowadays, educational content and applications are developed everywhere. The use of web-based applications to institutions and universities of higher education has been extensively used, especially on distance education universities, like Hellenic Open University (Papadakis et. al., 2005). However, when talking about teaching in primary and secondary schools the opportunity of having a natural "look and feel" environment for teaching purposes is very promising indeed.

The purpose of MUVII IKD was to demonstrate new interactive paradigms in a novel integration of the following modalities as these interfaced interactively with the user: $3 D$-vision, 3D-audio and haptic (force and tactile) feedback. The process followed in order to design, implement and test the IKD was:

1. User requirements and constraints for the IKD device and applications were gathered and analyzed. 
2. Technically feasible specifications of the IKD device, applications and platform were defined in detail.

3. Design and development of the IKD device

4. Design of the modular architecture of IKD supporting platform.

5. Design and development of IKD Applications.

6. Integration of the hardware and software modules.

7. Educational Testing of the IKD, for more than three months, with an adequate sample of more than 300 pupils, and some teachers.

In the present paper the first two steps of this process are described, and some conclusions regarding the issues raised in them are drawn, which can be used as a useful guide for those interested in developing haptic interfaces and applications for educational purposes.

\section{User Requirements Gathering}

In order to collect the most important user requirements, several discussions with potential users (mostly with teachers and to a lesser extent with students) were held. It is interesting to note that although students normally have a better knowledge of computers than their teachers, they are also "dreamers" and ask for features that are not feasible yet.

During these discussions the concepts behind the current haptic technologies (force and tactile feedback) as well as our ideas were explained. In order to judge the pupils' reaction towards virtual reality environments involving haptic feedback interfaces, some trials were performed using both children (of various ages) as well as adults, and a setup involving commercial haptic interface devices (like Phantom by SensAble Technologies and I-Feel-Mouse by Logitech) was utilised. The results of those trials were most encouraging, especially considering that these devices have a "feel" a lot less natural than the one expected from the H3DI of the MUVII IKD.

Moreover, regarding the tactile feedback, it was demonstrated that the tactile motors of CompuTouch (one of the partners in MUVII project) gave the sensory input expected when they were integrated in a common mouse. The users got feedback on their index while interacting with a windows application and the capabilities and limitations of such tactile motors were studied in detail.

The teachers were fascinated with the idea of using haptics in their classes since this technology gives the opportunity to observe, test and simulate phenomena that could not be performed in a class or in a school laboratory (Ioannidis and Garyfallidou, 2001). This is due to several reasons such as safety problems (e.g. it is too risky to use explosives or certain chemicals in an ordinary lab) or too difficult and perhaps impossible experiments ("switch off" friction for instance, driving any type of vehicle and feel the forces of a collision, construct a certain machine etc.).

One of the primary interests during this phase was to specify the best shape and functionalities of the IKD device. The main requirements for the IKD device was the movement independence, the feeling of force feedback independently on each finger, the precision of the movement so that the haptic device could function "transparently" 
as an extension of the user's hand. Apart from that, users expressed their interest in the use of two devices, one for each hand. Moreover, users wanted a large workspace (i.e. actual space where the device would be active).

As far as the potential applications were concerned, the users described the most educationally preferable software as one that used a scenario that could not be easily performed in a class, but with a high educational value nevertheless, while being exciting enough to attract the student's attention. Many different ideas for educational scenarios were put forward and were exhaustively discussed. Finally two applications were selected and implemented for the IKD, as described in a following section.

\section{User Requirements Analysis}

In the ensuing analysis, it was revealed that as far as the device was concerned, users wanted to use advanced wearable (not ground-based) haptic interfaces instead of joysticks. The characteristics most users required involved grasping, manipulating and throwing objects in the virtual space, while feeling forces and tactile feedback on as many fingers as possible (but at least on the two essential ones: thumb and index). The users required to be able to investigate and explore various 3D objects and feel their material, surface, size, shape, etc. Another very important characteristic, for the educational use of the IKD was deemed to be its realism, (something that is normally ignored by game developers). Special emphasis was given in support of accurate hand and finger movement.

Considering the projected educational use of the device, users wanted an "easy-touse" device that did not require in-depth knowledge of computers, robotics, or physics. Another important factor was the weight of the device - the need to be as light as possible so that young children can handle it - and the freedom of movements. The users wanted the device to be a "natural" continuation of their hand, which they can freely move and operate in the application's environment.

Regarding $3 D$ sound features, users found very interesting the idea of hearing the various sound cues of the application and being able to easily perceive their direction, distance and volume, while at the same time they can communicate with the other users by using open air headphones. Furthermore, Haptic related sounds (i.e. sounds produced directly from the interaction of the user's virtual hand with the objects) should also be supported by the sound subsystem of the IKD.

One of the most challenging requirements was to support two users interacting simultaneously in the context of the same application. The users should be able to jointly manage the common viewpoint, but each user should be able to independently move inside the 3D world of the application. The two users should be able to act simultaneously either on the same or on different objects.

The analysis of the user requirements led to the specifications of the IKD haptic device, the IKD platform and the IKD applications, as described in the next sections.

\section{IKD Haptic Device Specifications}


The MUVII IKD Haptic 3D Interface (H3DI) is the human-interface part of the IKD platform and plays an extremely important role in the functionality of the whole system. The IKD H3DI should respect some specific constraints, regarding the various ages of the final users (children as well as adults), and some requirements that are determined by the IKD applications. The analysis of these requirements and constraints led to the following specifications:

Size of H3DI: How small should the body of the H3DI be? The main concern is the use of the device by young children (primary school). As the size of their hand is smaller, we cannot expect young children to be able to handle with ease (or at all) a device created with a fully-grown male in mind. Therefore, the best possible solution is to create a device that is adjustable in size, so it can be used by adults and children as well. This could be achieved by having detachable pads attached to the main body.

Weight: It should be as light as possible, approximately 100 - 150 grams. The balance of this weight is of considerable importance, as it should not be very top - heavy, or in anyway out of balance. A top-heavy H3DI would feel unnatural to use and, therefore, the user would find it hard to "immerse" in the use of it.

Priorities to be kept in the design: The balance and fit are probably more important parameters than size and weight. Any inevitable compromises made should keep this in mind.

Type of movements: The users should have the capacity to move their hand (with the device worn) freely in space without strict movement restrictions. Moreover, the users should be able to use at least two of their fingers (thumb and index) independently. This way, it was observed that they could easily manipulate virtual objects (albeit in a rather unnatural fashion). Taking into consideration that in this trial a new haptic interface was evolved, this is acceptable. It also turned out that the user could easily ajust him/herself to this situation, e.g. the use of only two fingers to manipulate objects). Furthermore, the precision of the movement is an important factor, because it allows the user to perceive the haptic device as an extension of his hand.

Force feedback: Users should be able to manipulate objects using their two fingers and feel forces acting on them, independently on each finger.

Tactile feedback: Users should be able to get tactile feedback (feel the surface contours and textures) on their two fingertips. For the MUVII IKD H3DI this tactile feedback is provided by two tactile motors integrated on the H3DI. These motors (see Figure 1) were developed by CompuTouch, a MUVII partner. The integration on the H3DI allows good force feedback on finger, while keeping fingertips free for tactile feedback. 
Open Education - The J oumal for Open and Distance Education and Educational Technology Volume 7, Number 1, 2011 Section one. (C) Open Education ISSN: 1791-9312
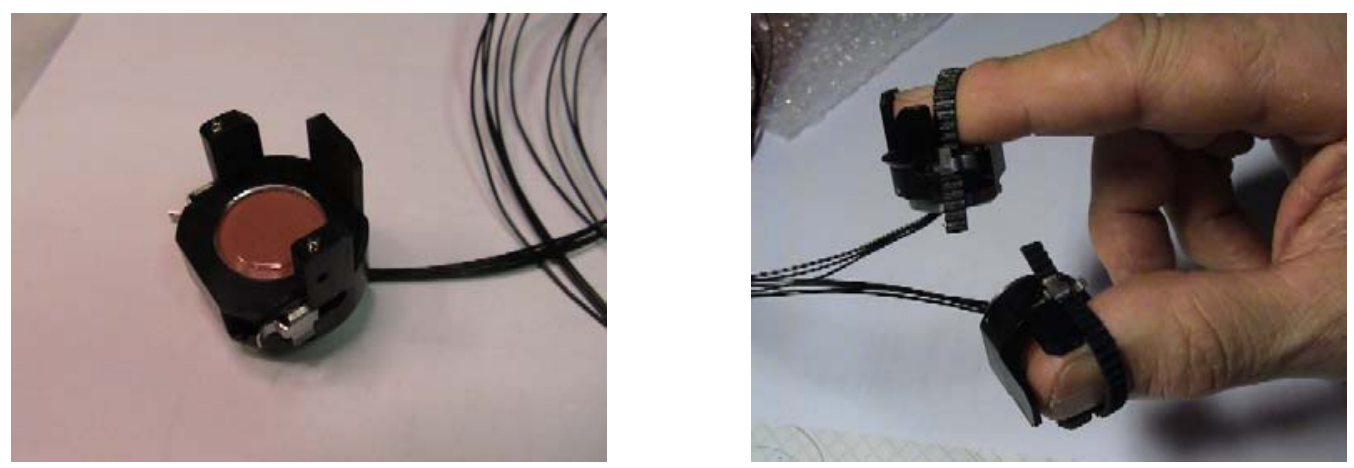

Figure 1: Tactile Motors (Courtesy of CompuTouch $(W W W)$ ) 
Type of actions: By exploiting the force-feedback of the H3DI, users should be able to select, pick-up, hold, move, orient, release/place, pull/push and throw objects, while they feel forces acting on their fingers (weight, torque, collisions, etc.). By exploiting the tactile feedback users should be able to investigate and explore various $3 \mathrm{D}$ objects and feel their surface material, shape, etc.

Workspace: This is the actual physical space where the device would be operational (its position can be tracked). Minimum requirements are $600 \mathrm{~mm}$ (width), $600 \mathrm{~mm}$ (length), and $400 \mathrm{~mm}$ (height). Obviously, the greater the final workspace is, the better for the users.

Robustness: A certain amount of robustness is required especially as we envisage children using it. Ideally, it should be able to withstand a drop from $80-100 \mathrm{~cm}$ height onto a hard floor without breaking or needing adjustment or realignment.

\section{IKD Platform Specifications}

Three modalities were involved in MUVII interactive environment: visual, haptic, and aural (i.e. sound). To achieve the best virtual reality immersion the best solutions for each one of these three modalities involved were selected and (most importantly) integrated together. Indeed, MUVII IKD demonstrated new interaction paradigms and a novel integration of these three modalities: 3D-vision, 3D-audio, and haptic (force, torque, and tactile) feedback.

Innovations of IKD included:

- The multimodality of haptics, 3D-audio and 3D-graphics, to provide an integrated, natural "look and feel" immersion environment for edutainment purposes.

- Design and implementation of a special haptic feedback device (see Figure 2) that support tactile \& 3DOF force feedback, especially designed for educational virtual environments.

- An extensible and modular architecture of the platform that can support the integration of two such haptic feedback devices, thus providing multi-user ability (either teacher-pupil or pupil-pupil) to enhance the teaching procedure and the collaboration among pupils.

- Support for motion capture / tracking for hand and head of two users.

- Sophisticated 3D-sound: use of open headphones, head-tracking and real-time reproduction of individual 3D sound for each user.

- Innovative haptic interaction metaphors, like the combination of visual with haptic or audio with haptic stimuli, aiming at the creation of a multi-sense educational environment. These metaphors give emphasis on events that cause haptic and tactile feedback to the users, mainly through "real-world" and "non-real-world" audio cues, sound effects and visual effects.

- Rapid application development support through the integration and customization of a commercial tool (Virtools), the MUVII IKD library of predefined generic objects \& behaviours and the MUVII IKD audio authoring toolkit.

- Innovative Educational Demo Applications: These applications introduce several innovative features and their primary purpose is to demonstrate the capabilities of the H3DI and to build on its functionality, aiming at the rapid adaptation of users in the characteristics (and in the way of use) of the device. 

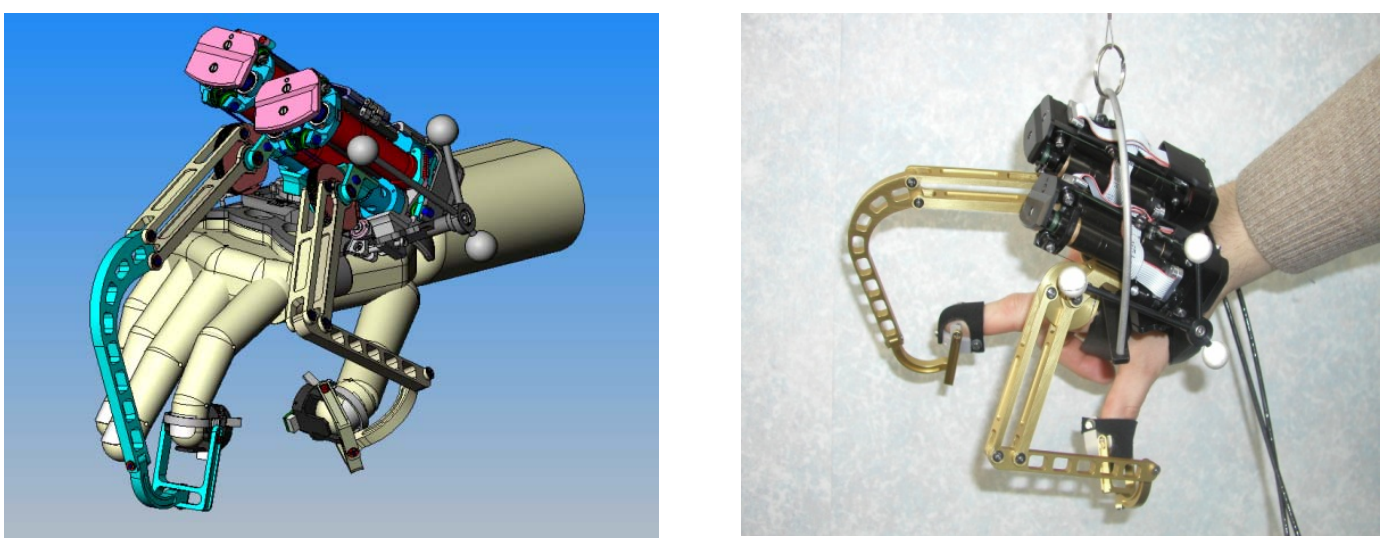

Figure 2: IKD H3DI concept and realization respectively (Courtesy of CEA (WWW))

Figure 3 shows the hardware architecture of the IKD, showing PC hosts in charge of each module and network communication connecting these hosts.

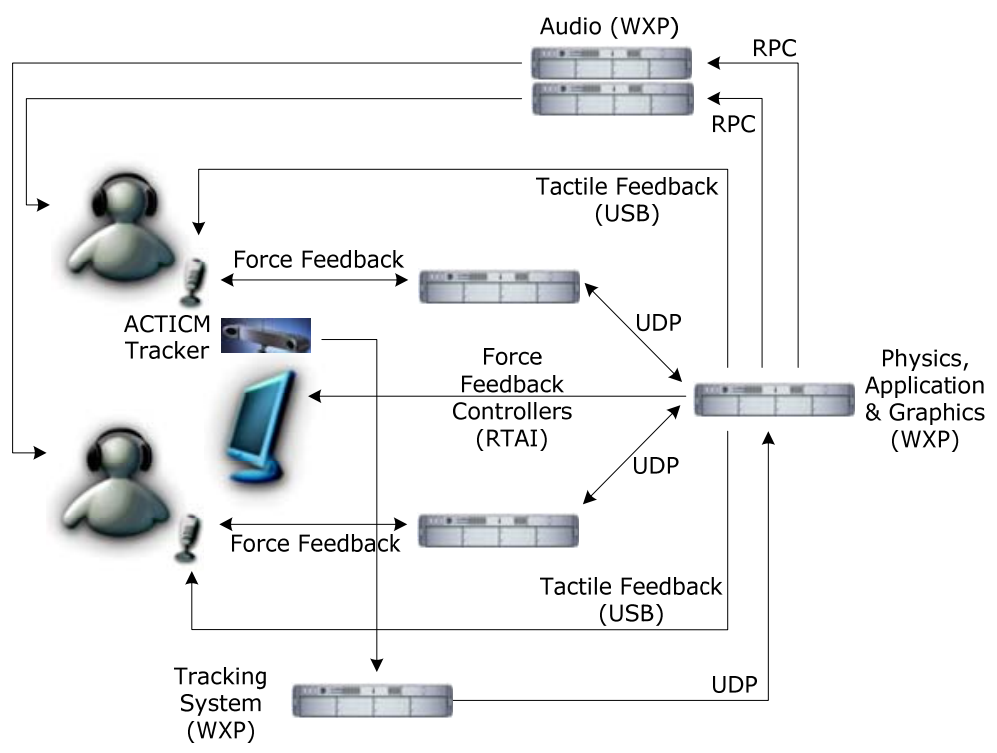

Figure 3: IKD hardware architecture

The really hard and technically challenging issue for IKD was to keep the latency time between visual, haptic, tactile and audio feedback as small as possible in order to achieve a great degree of realism. Very short time latency was also required for the hand tracker that was used for the accurate positioning of the real human hand and the real-time rendering of the virtual hand inside the 3D world of the application. The problem is getting harder, if you consider that these subsystems of the IKD platform are implemented by different pieces of software which are being executed in different computers and in some cases under different operating systems all connected to form a single system.

\section{Haptics and Education}


In a conventional class environment, when instructing a pupil on the way to achieve a manual task, the teacher will occasionally resort to demonstration through a direct mechanical contact (especially so for younger pupils). Unlike visual demonstration or verbal instruction, mechanical or haptic demonstration is designed to communicate directly to the pupil's hand. Common examples of this kind of teaching occur in sports and music instruction.

Traditionally most of our daily activities were accomplished by the use of the human hand. Computers may have brought significant changes to our daily life but the interface is still carried out through a keyboard and a mouse which are data input devices, that do not offer to the user any information related to the "object" he/she manipulates, and this is equally so even if we speak about virtual reality. Computer never sends data to the keyboard or the mouse because these could not create haptic reaction to the user's hand. Haptic devices come to eliminate this limitation.

"Unlike traditional interfaces that provide visual and auditory information, haptic interfaces generate mechanical signals that stimulate human kinesthetic and touch channels. Haptic interfaces also provide humans with the means to act on their environment." (Hayward et al, 2004).

But could haptics extend the teaching possibilities, esp. for science concepts?

Some research groups like NanoScale Science Education Research Group (WWW), investigate the impact of haptics on students' learning of science concepts, and their results so far are very encouraging. According to their research, when a student uses haptic devices receives two kinds of stimulants distinguished in kinesthetic and tactile perception. Both stimulants are dynamic, which means that they change as the user is operating the objects, no matter if they are physical or virtual. This active touch involves intentional actions that an individual chooses to do, whereas passive touch can occur without any initiating action. For educators, involving students in consciously choosing to investigate the properties of an object is a powerful motivator and increases attention to learning.

Moreover, it is experimentally proved by Sathian et al (1997) and Itan (2005) that where vision is the dominating sense in form perception (macro-geometry) of bodies, touch excels in defining their texture (micro-geometry).

Other researchers (Okamura et al, 2002) showed that a purely audio-visual environment, even if it is highly interactive, can present difficulties for "haptic learners". By addressing the sense of touch, haptic interfaces are promising tools for helping students with haptic cognitive styles obtain an understanding of mathematical and physical phenomena. 


\section{IKD Educational Applications}

Haptic technologies offer an enormous variety of applications starting from "virtual" school laboratories and going as far as learning auto driving, surgery, or even space ship manoeuvres. In such a virtual reality environment that supports haptics, the applications could provide the users with the capability to:

- investigate and explore various 3D objects. (Tactile feedback, which involves Material, Surface of objects, Size, Shape, Weight etc.)

- feel force and torque feedback (caused by weight, torque etc)

- $\quad$ select, pick-up, hold, move, orient and release/place objects

- hear sounds that emulate sounds produced by surfaces rubbing against each other or by surface-collision events etc.

The educational applications that were developed in the framework of MUVII project were built to exploit the advanced features of MUVII IKD platform and device and to investigate their educational potential. Indeed, the didactical opportunities offered by the system (as it gradually developed) were painstakingly analyzed and evaluated. Subsequently, two educational applications were selected for implementation in the IKD: (A) Newtonian Physics, Trajectories and the Solar System, and (B) Virtual Model Assembly - Gears (each one with learning mode, recapitulation mode and edutainment mode).

From the educational point of view, both educational applications were based on the constructivist theory (for an overview of the theory see Ioannidis et al, 2005, section 3.5). The basis of the constructivist theory of learning and instruction is formed by the writings of Piaget (Piaget, 1972 and Piaget et al 1995) and Vygotsky (1978), along with the work of Ausubel (1968) and Bruner (1990). According to this theory students are responsible for their own learning, and meaningful learning demands that pupils construct their own knowledge.

Computer aided learning and virtual reality environments allow students to learn by following his/her own pace, or even according to their interest. Using the MUVII IKD educational applications in their "active manipulation" mode, students can manipulate objects after consciously deciding to do so. Thus, users interact with the objects they choose in the way they choose, and feel the feedback from their actions. This stimulates their interest and increases their attention. Our study showed that the knowledge remaining in the student's mind after such a learning activity is higher than what is left after teaching the same subject using traditional methods of teaching, where the student passively listens to the teacher teach or watch a science video.

Within the application of Newtonian Physics and the solar system, the user interactively (and virtually) navigates through the solar system, while collecting information about anything that interests him/her. The user experiences the effect of the forces when accelerating objects (e.g. by trying to throw them off their course) as well as the strength of the gravitational forces applied to objects at different distances from the sun or from a certain planet. Obviously, for the purpose of such an interaction the user is endowed with "super-powers". With the use of haptics the pupils are able to experience, feel and gradually learn the way the laws of simple mechanics in the way these are applied at the scale of our solar system. Figure 4 shows a screenshot of the application. 
Regarding the Virtual Model Assembly - Gears, the user is offered a lesson of the history of cogs, gears and their applications through the ages. They can also try to assemble some selected applications by combining gears. The users experience the effect of forces like those caused by weight, friction, motion, rotation etc. This application can also be used to enhance students' understanding of phenomena like the transmission of motion from one part of a machine to another. Figures 5 and 6 show two screenshots of this application, the second one using the watermill paradigm.

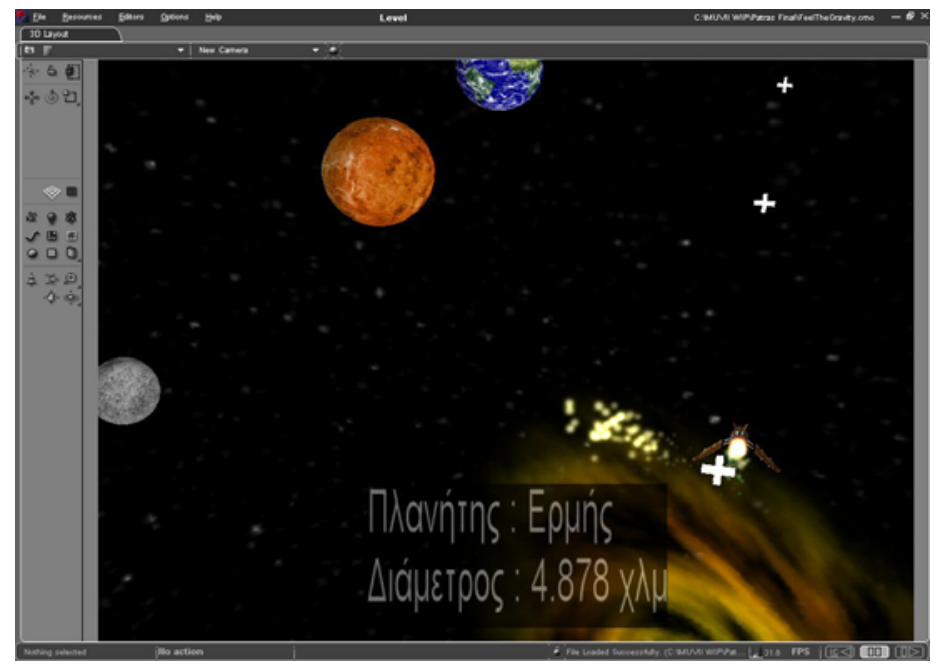

Figure 4: Feeling the gravity

(Planet: Hermes Diameter: $4.878 \mathrm{~km}$ )

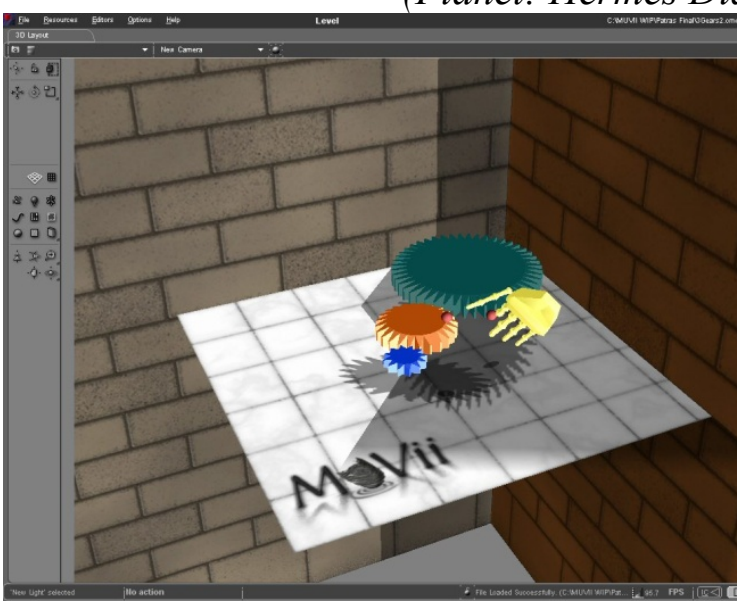

Figure 5: Feeling moving gears

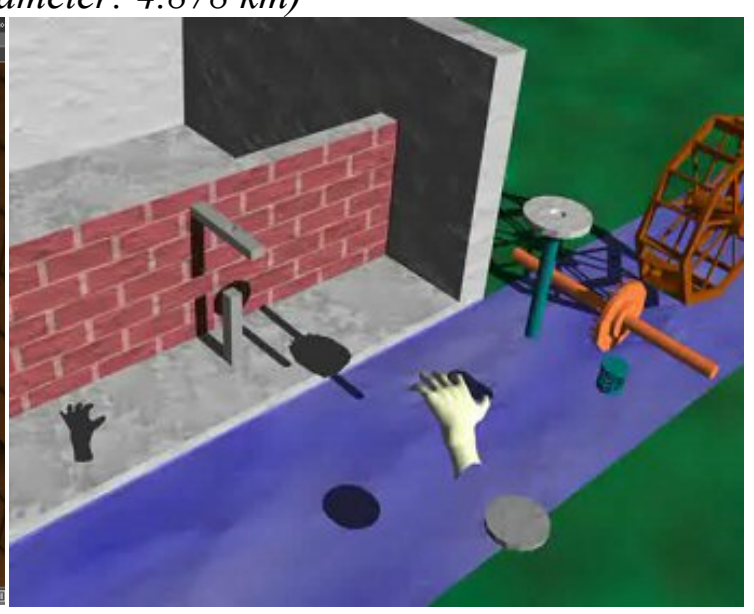

Figure 6: Assembling a windmill

\section{IKD Experimental Validation}

The first public experimental validation of IKD H3DI, software infrastructure and applications, took place in May 2004 in Laval Virtual (see Figure 7 and 8) exhibition event, one of the biggest annual exhibitions in Europe for Virtual Reality.

Despite the fact that the device was aimed at children of ages above 10 years old, it 
occurred that even younger children (e.g. 7-10 years old) could use it effectively. During the tests in Laval the users were very enthusiastic and attracted by the device and the applications. Almost no-one gave up trying to finish the application, despite the difficulties everyone faces when coming in contact with a new technology and a virtual space he/she has to interact with. The testers were invited to write their opinion and impression about the IKD, some of which are presented here:

Kevin, 12:

I find it very good fun, and I think that this system could be used to control/test objects in space or on planets.

Christian, 36 :

Very good sensation of the movements in space and of the grapping. Very realistic force feedback. Thank you

Nicolas, 24:

Feelings almost real, impressive sensation of resistance. I think that many useful applications can be developed.

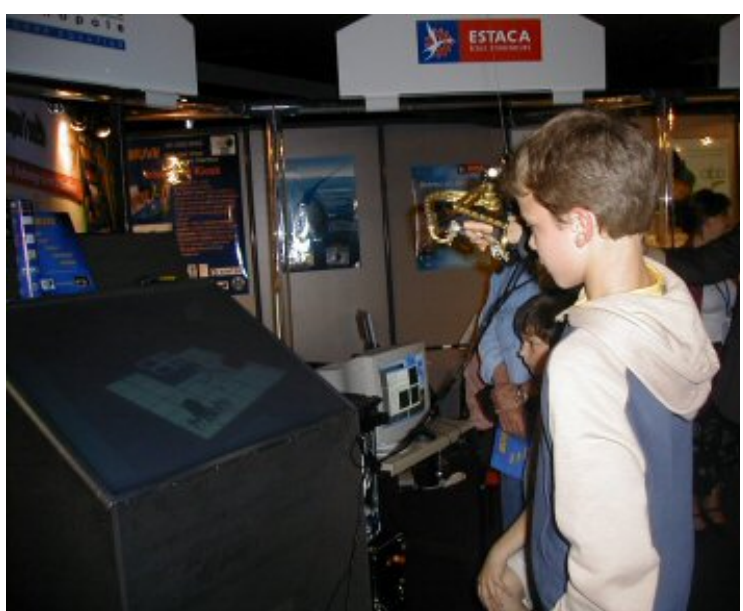

Figure 7: Laval Virtual demonstration, a young user

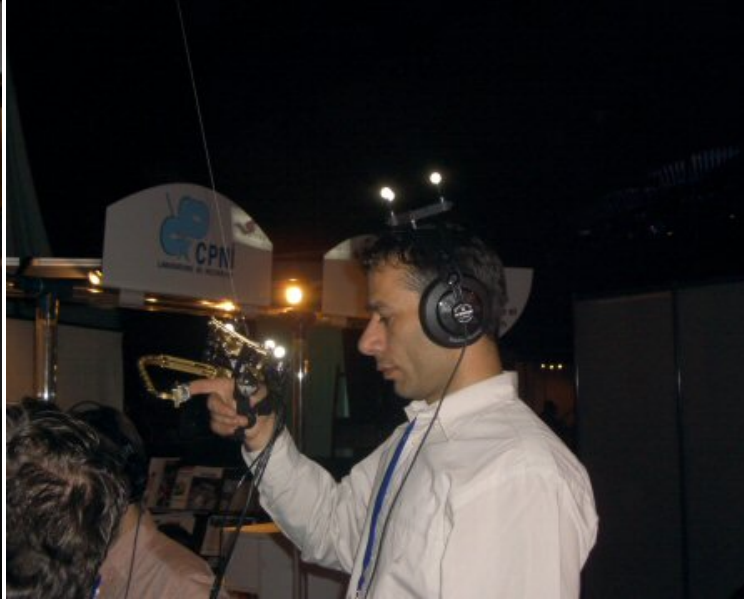

Figure 8: Laval Virtual demonstration, an adult user

After this initial demonstration of IKD, the educational trial of the IKD system took place, for more than three months, with an adequate sample of more than 300 pupils, and some teachers.

IKD H3DI, software infrastructure and applications have been tested, against functional and performance issues, with schoolchildren that have been randomly selected from various schools of Patras and Achaia prefecture. It should be mentioned here that mostly due to the equipment size, and the lack of suitable space in schools, the students that participated in the testing procedure had to visit the device which was set at laboratory at the University of Patras (HPCLab). Members of the Science Laboratory (School of Education) were responsible for the educational trial.

In this educational trial 163 students participated:

64 of which were primary school pupils,

74 were lower secondary school students, and 
25 were upper secondary (i.e. lyceum) school students.

For the educational trail all the international accepted practices concerning research in education were followed. The "exercises" for each group of students were chosen in accordance to their age. The educational results of this teaching approach, as well as the feedback derived from the users were presented in (Christodoulou et. al., 2005). We summarise the most important ones below.

- A large proportion of children found teaching with the use of a haptic device interesting. Students responded quite well to the use of this haptic prototype, although this could be made to look "friendlier".

- Students were also pleased and seemed to be even amused by their experience. They would like to repeat the experience they have had and they would also like to see the haptic device extend its abilities; in addition they wanted to see haptics and the whole IKD device in particular being used in other (more varied) applications.

- No student found the experiment boring or too difficult (which is a big compliment!).

- Assessing the learning achieved using the application on the Newton's law on gravity, we observe most students choosing the correct answer most of the time. By comparing these answers with these of the pre-test (a questionnaire filled by students before the trial), an overall increase in understanding is obvious. This really is "understanding by feeling"!

- The great majority of students were interested in the haptic application (as well as the device) one way or another. Younger children by being naturally "enthusiastic" found the curriculum interesting in higher percentages than the older ones.

- The majority of the students were willing to participate in future lessons using devices with haptic interfaces, while a sizable proportion of them would also wished to have had such a device available for their personal use at home.

- It was also observed that generally the girls seemed to consider (e.g. to think about) their hand-movements before they made them, and as a result, their handling of the haptic device was steadier. The boys seemed to be more impulsive (- anxious even) and made quick movements (almost jerky, sometimes). The above are general observations made by the researchers for the bulk of the students, while it should be stressed that individual handling skill differed amongst students.

Overall, the encouraging result from this investigation is that we can easily use IT and haptics to teach science (and perhaps other subjects) even with primary school pupils.

\section{Conclusions}

The multi-sensory environment of a haptic device can greatly improve the existing teaching methods, by offering tools of enhanced quality suitable for deeper understanding of the entities taught. Students seem to have adjusted well to the new system and have enjoyed using it. Currently there are no other applications with capabilities similar to the ones specified in MUVII project, incorporating characteristics like multi-user collaboration among students and teachers, efficient 
3D-sound sub-system, targeting children of various ages, efficient haptic interaction metaphors etc.

Some significant requirements and specifications for haptic devices are outlined in this paper, mainly for applications that will be used in education. Those who seek to develop haptic interfaces, as well as application software for education, will find the specifications presented extremely useful. In addition to that, this paper will help potential users (teachers, students) become more acquainted with the use of haptic interfaces in education, understand the power (and limitations) of haptically-enhanced virtual reality educational systems, and provide some useful feedback on the prospective implementation in their everyday school practice.

\section{References}

Ausubel, D. (1968). Educational psychology: A cognitive view. New York: Holt, Rinehart \& Winston. Bruner, J. (1990). Acts of meaning. Cambridge, MA: Harvard University Press

Christodoulou S. P., Garyfallidou D. M., Gavala M. N., Ioannidis G. S., Papatheodorou T. S., and Stathi E. A. (2005), "Haptic devices in Virtual Reality used for Education: Designing and educational testing of an innovative system", Proceedings of International Conference ICL2005 (Interactive Computer Aided Learning) September 28 - 30, 2005 - Villach, Austria

Hayward V., Astley O.R., Cruz-Hernandez M., Grant D. and Robles-de-la-Torre G. (2004) Haptic interfaces and devices. Sensor Review 24(1), Emerald Group Publishing Ltd., ISSN 0260-2288, pp. 16-29

Ioannidis G. S. and Garyfallidou D. M. (2001), Education using information and communication technology (ICT), and ICT education: categories methods and trends, In: Auer M. and Auer U. (Eds.) Proc. ICL2001 workshop: "Interactive Computer aided Learning, Experiences and visions", Villach, Austria, Kassel University Press, (2001) ISBN 3-933146-67-4

Ioannidis G.S., Garyfallidou D.M., and Spiliotopoulou-Papantoniou V. (2005) Streaming Media in Education, and their impact in teaching and learning Educational best practices and some first observations of their implementation. Published by Education Highway, ISBN 3 - 9500247 - 4 3, (First Edition, July 2005), also available at http://estream.schule.at/

Itan, Y. (2005) Human motion perception mechanisms: A functional magnetic resonance imaging experiment applying Computational, Psychophysical and Physical methods, available at: http://www.homepages.ucl.ac.uk/ ucbpyit/Essay3PDF.pdf (visited in February 2007).

Okamura A.M., Richard C. and Cutkosky M.R. (2002) Feeling is believing: Using a force-feedback joystick to teach dynamic systems, ASEE Journal of Engineering Education, 92, 3 (Jul 2002), pp. 345-349

Papadakis S., Xenos M., Mitsou E., "Experiences and technical issues from the delivery of computerbased learning materials in the Hellenic Open University", Open Education: the Journal for Open and Distance Education and Educational Technology, Vol. 1, No. 2, pp. 12-28, 2005.

Piaget, J. (1972). The psychology of the child. New York: Basic Books.

Piaget, J., Gruber, H. (Ed.), \& Voneche, J. J. (Ed.) (1995). The essential Piaget Publisher: Jason Aronson; 100th Anniversary Ed

Sathian, K., Zangaladze, A., Hoffman, J. M. and Grafton, S.T. (1997) Feeling with the mind's eye, NeuroReport, 8, (1997), pp. 3877-3881

Vygotsky, L.S. (1978). Mind in society: The development of higher psychological processes. Harvard University Press.

CEA - Commisariat a l'Energie Atomique (WWW), http://www.cea.fr

CompuTouch AS (WWW), http://www.computouch.no/

MUVII Web Site (WWW), IST Project: Multi User Virtual Interactive Interface, http://muvii.hpclab.ceid.upatras.gr, visited in March 2007

Nano-Scale Science Education research group (WWW), College of education, NC State University, http://ced.ncsu.edu/nanoscale/haptics.htm, visited in March 2007 
Open Education - The J oumal for Open and Distance Education and Educational Technology

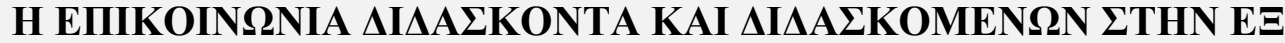

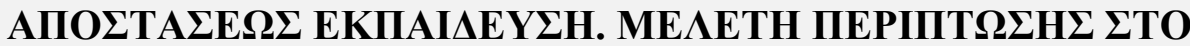 ПРОГРАММА ЕКПАI
}

\section{COMMUNICATION BETWEEN TUTORS AND STUDENTS IN DISTANCE EDUCATION. THE CASE OF THE TRAINING PROGRAMME FOR INSTRUCTORS OF THE CENTER FOR CONTINUING EDUCATION AND ADULT'S DISTANCE EDUCATION}

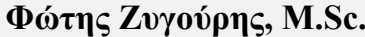

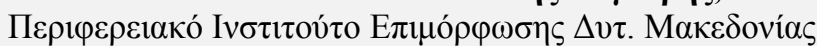 \\ e-mail: zygourisfotis@yahoo.gr

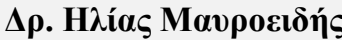

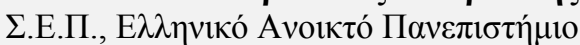 \\ e-mail: imavr@tee.gr
}

\section{Пері́ $\eta \psi \eta$}

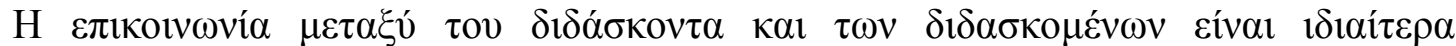

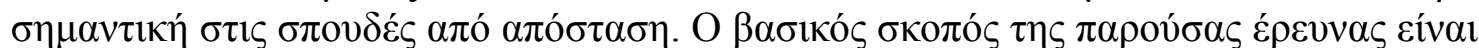

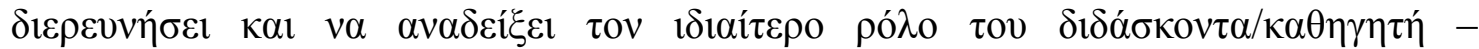

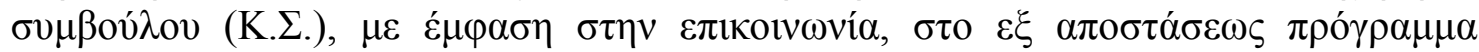

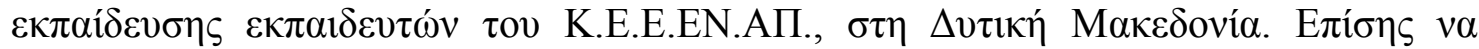

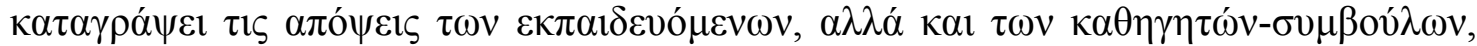

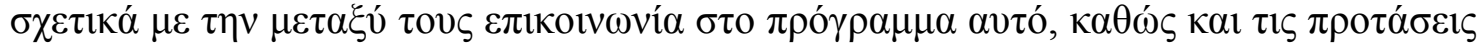

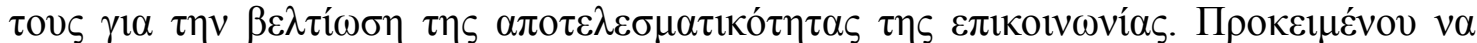

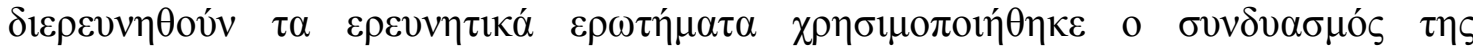

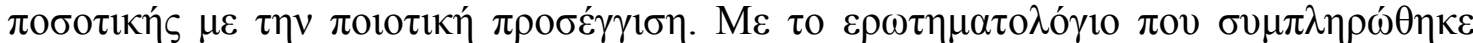

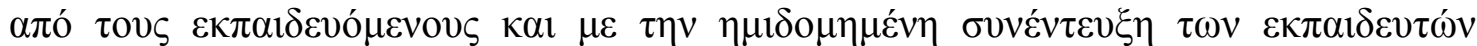

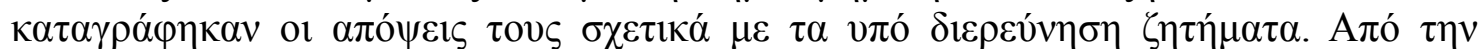

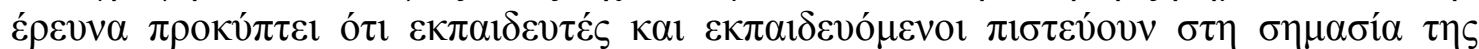

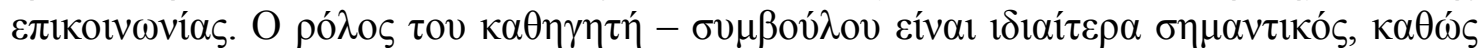

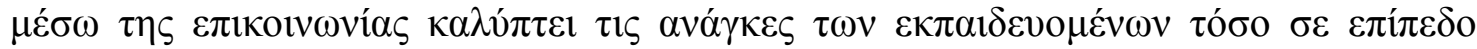

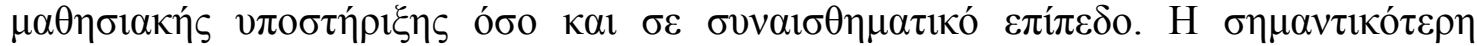

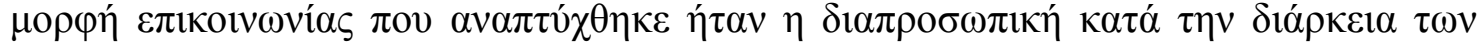

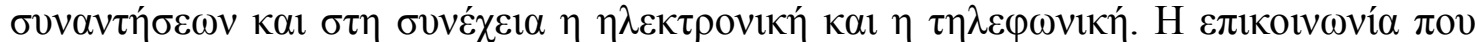

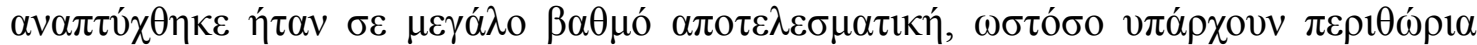

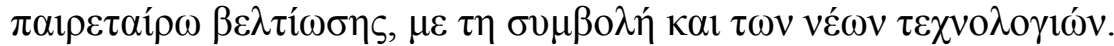

\begin{abstract}
In distance learning, communication is very important for bridging the distance between the tutor and the students. The role of the tutor is particularly important in this respect, especially for providing the students with the necessary support, encouragement, and feedback so that they can actively participate in the learning process. The main objective of the present work is to explore the role of the tutor in the training programme for instructors of the Center for Continuing Education and Adult's Distance Education (C.C.E.A.D.E.) in the region of Western Macedonia, Greece, focusing on communication. Furthermore, to examine the opinions of students and tutors on several aspects of their communication during the programme, including their proposals for improving the effectiveness of communication. A combination of
\end{abstract}


quantitative and qualitative approaches was used: a questionnaire was answered by the students and a semi- structured interview was addressed to the tutors. The results show that both students and tutors believe in the importance of communication. The role of tutors is particularly important in addressing, through communication, the needs of the students with respect to both instructional and emotional support. The most important form of communication developed was interpersonal communication during the meetings, followed by communication through e-mail and telephone respectively. Communication was perceived to be effective to a great extent. However, the results show that there is a need for further improvement. Appropriate use of new technologies can be very useful in this respect.

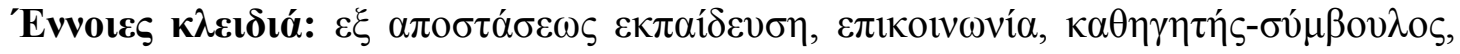

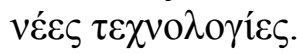

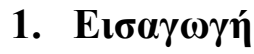

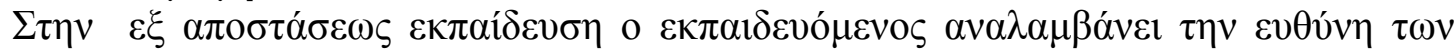

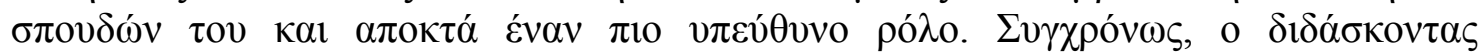

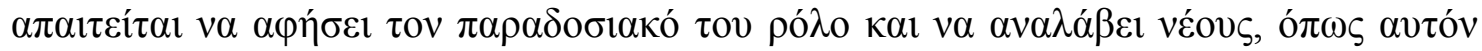

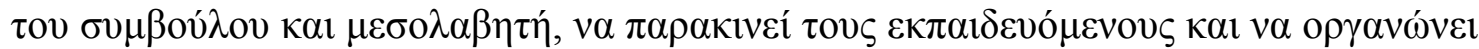

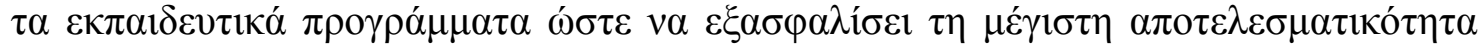

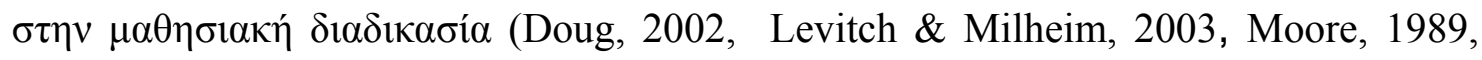

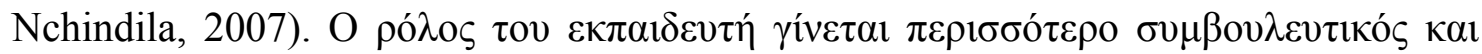

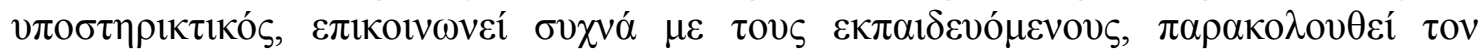

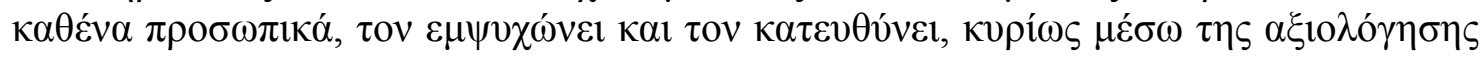

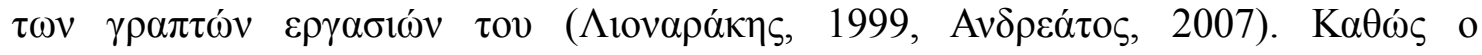

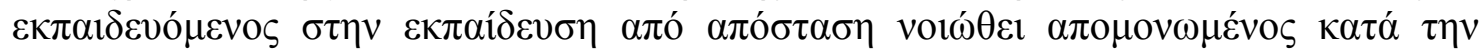

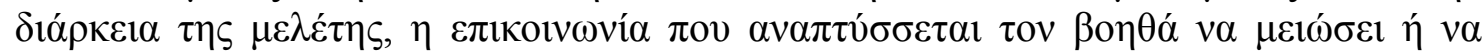

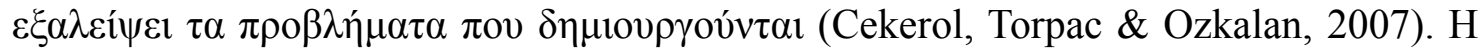

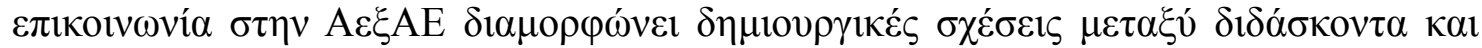

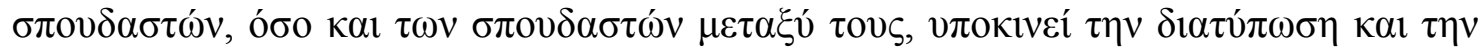

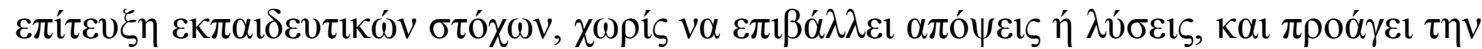

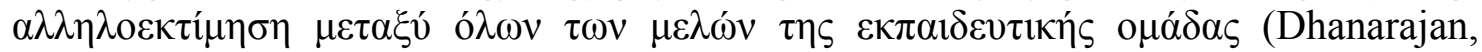
1996).

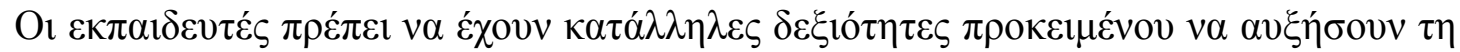

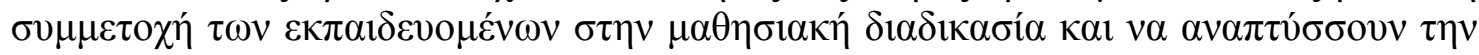

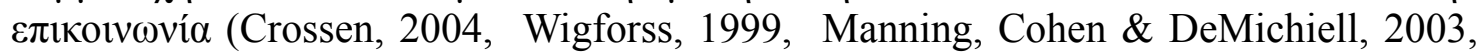

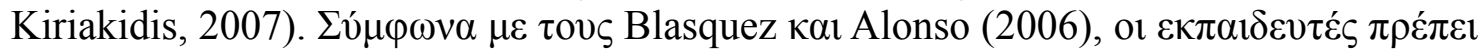

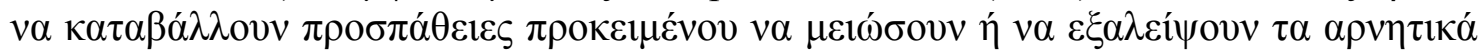

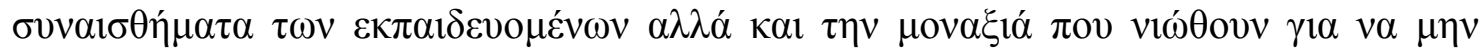

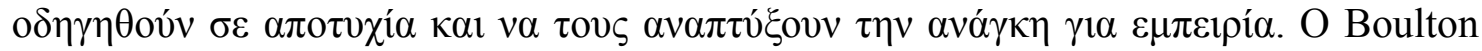

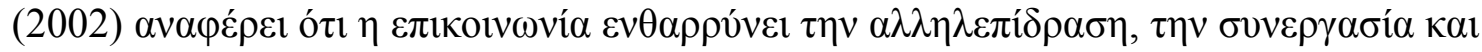

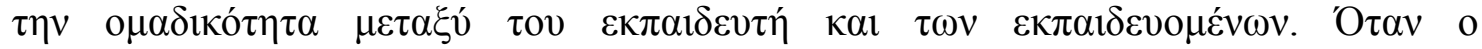

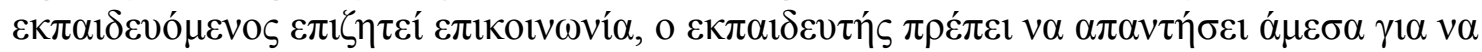

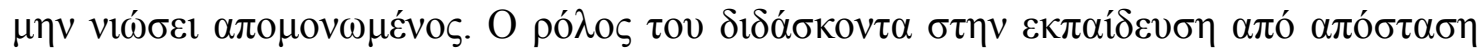

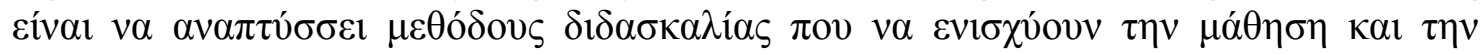

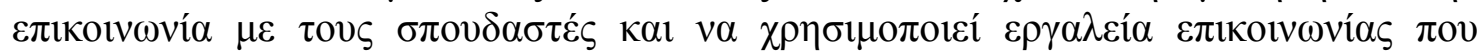

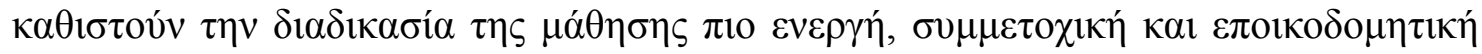

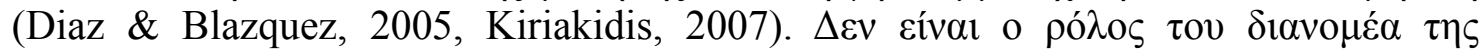

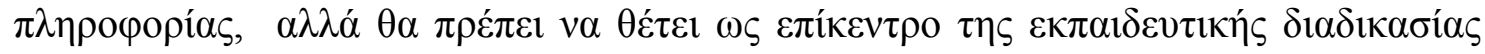




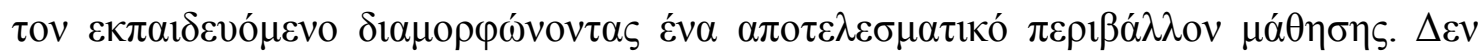

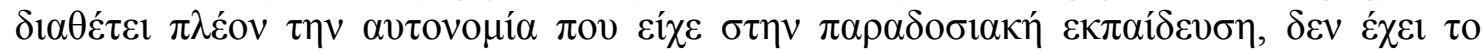

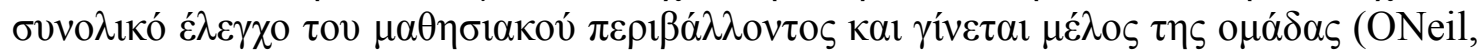

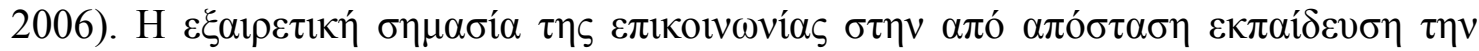

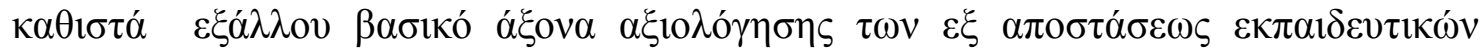
$\pi \rho о \gamma \rho \alpha \mu \mu \alpha \tau \omega v$ (Trentin \& Scimeca, 1999).

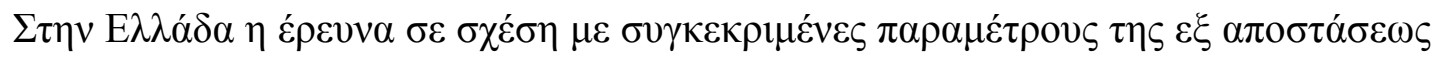

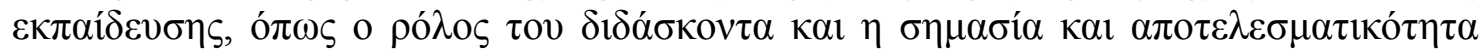

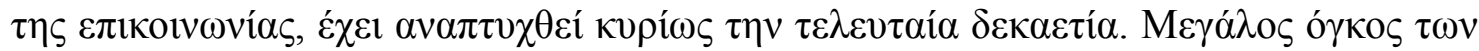

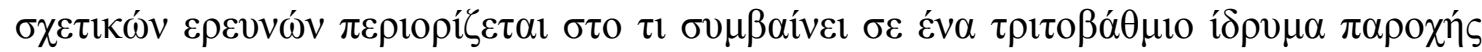

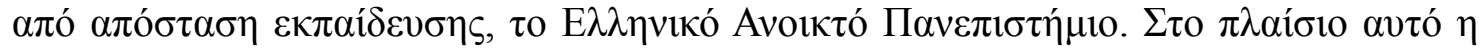

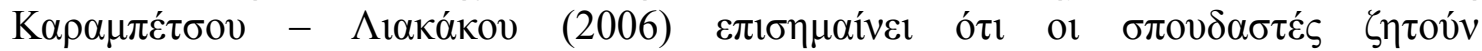

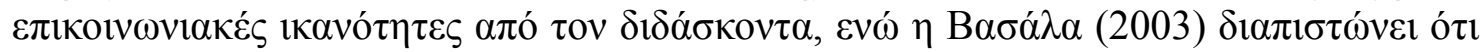

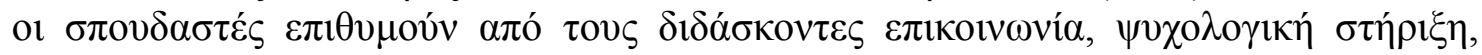

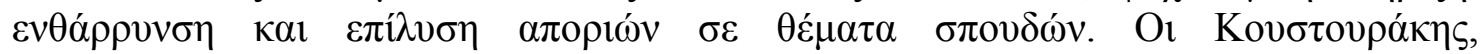

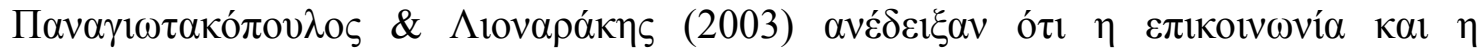

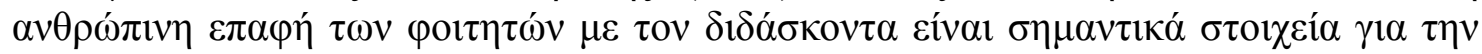

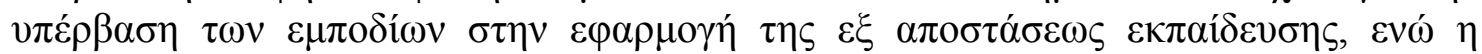

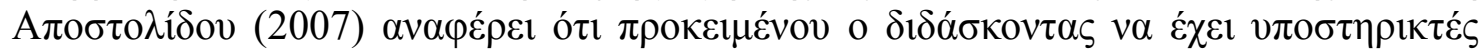

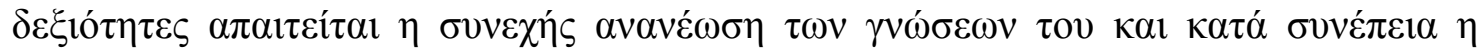

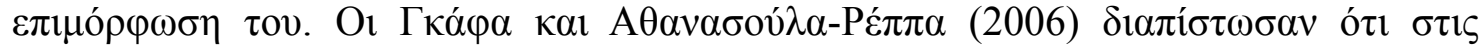

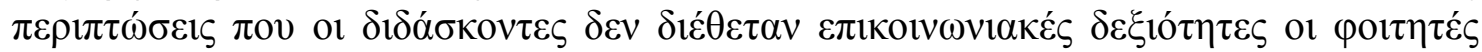

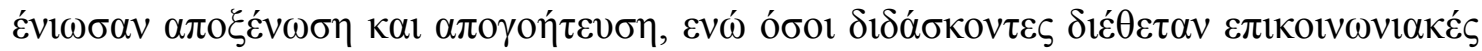

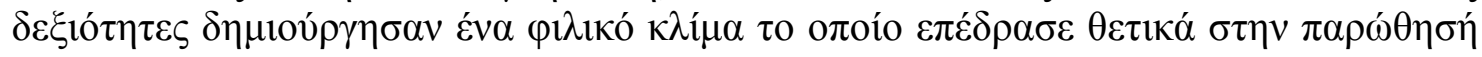
tovs.

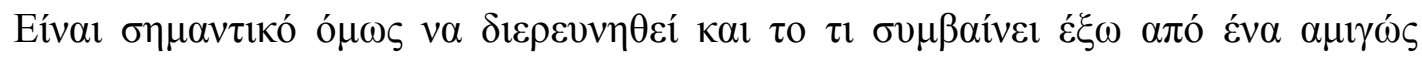

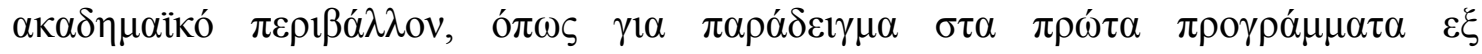

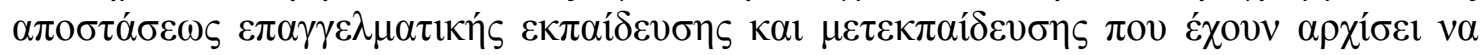

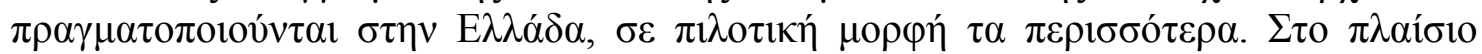

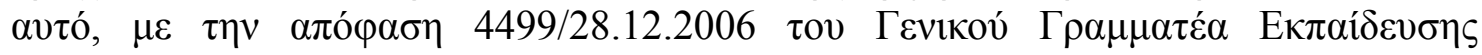

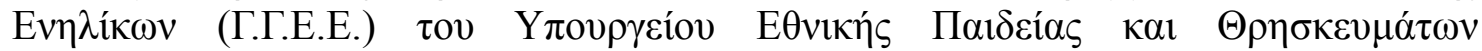

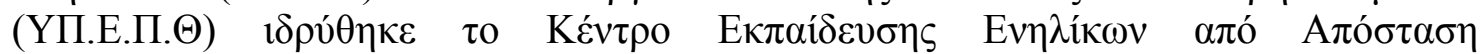

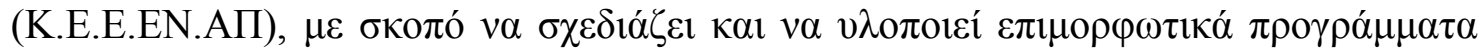

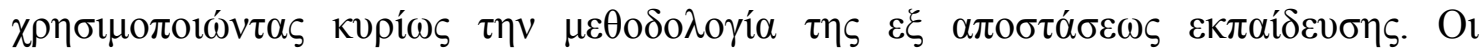

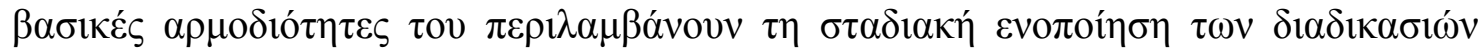

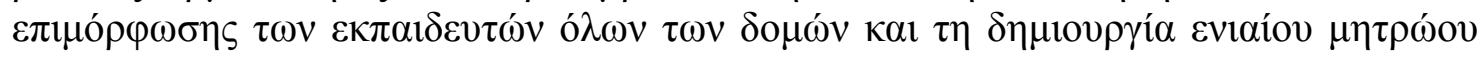

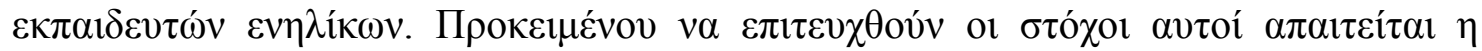

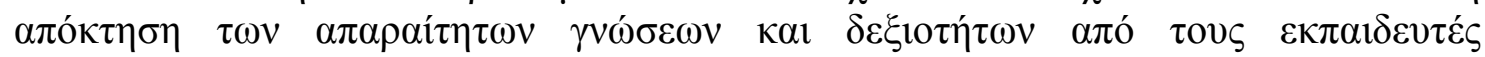

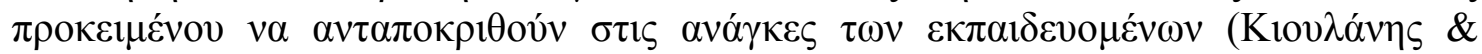

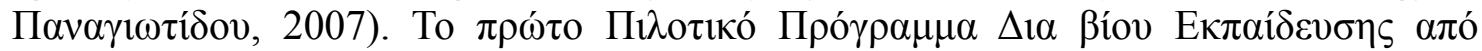

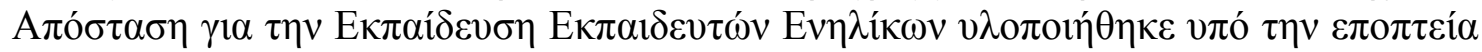

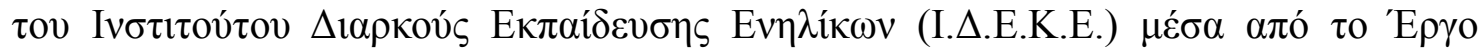

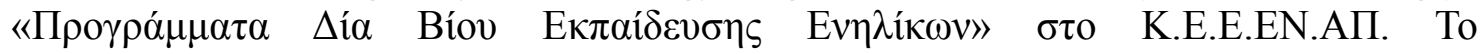

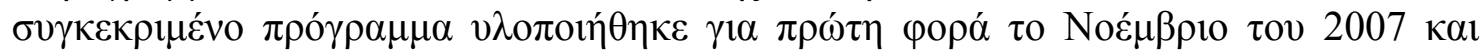

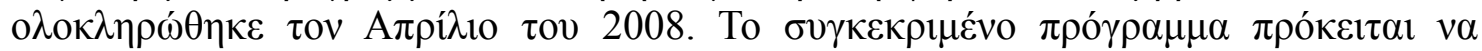

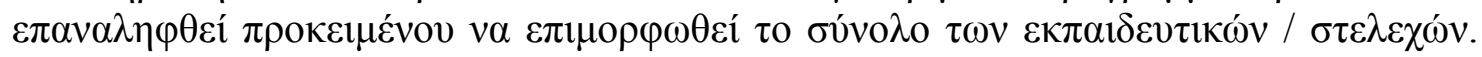

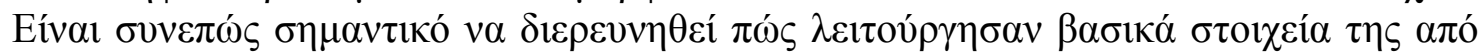

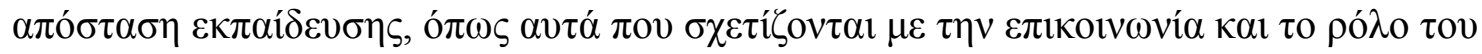

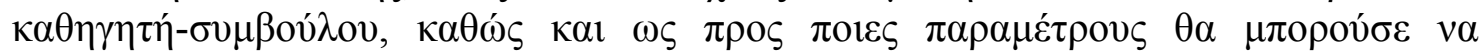




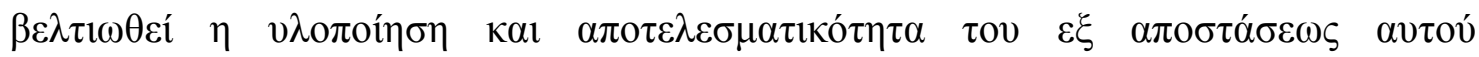

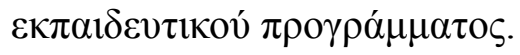

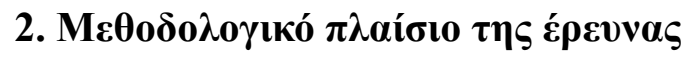

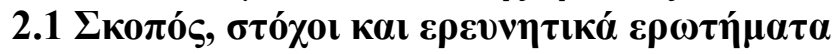

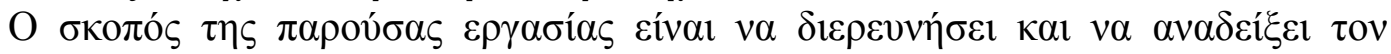

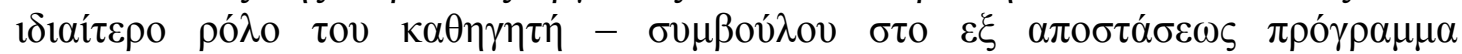

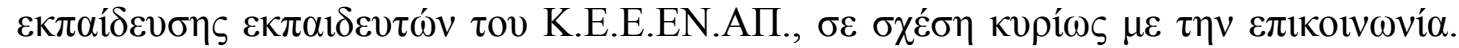

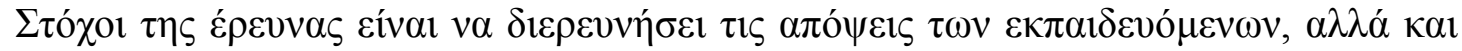

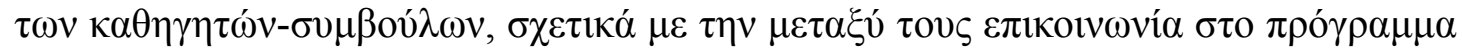

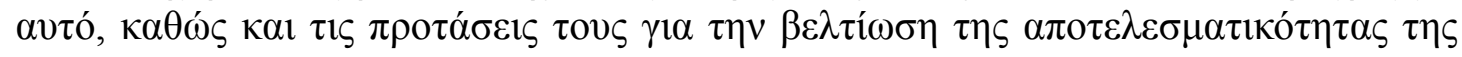

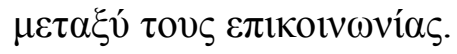

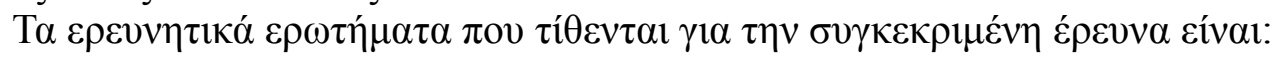

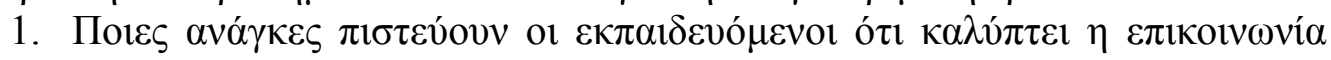

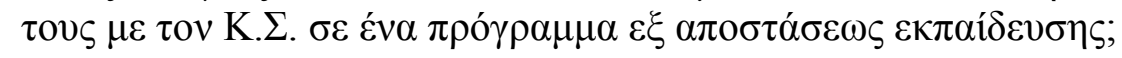

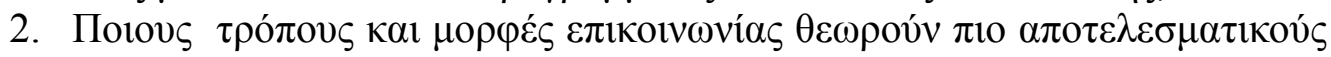

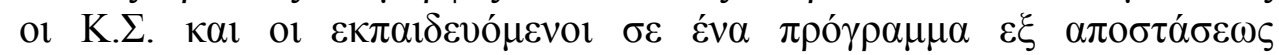
$\varepsilon \kappa \pi \alpha i \delta \varepsilon \varepsilon \sigma \eta \zeta ;$

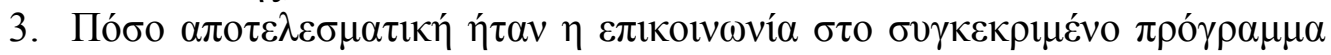

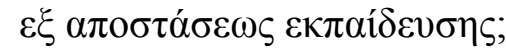

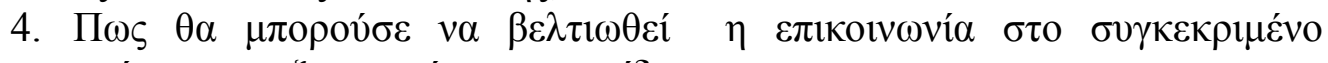

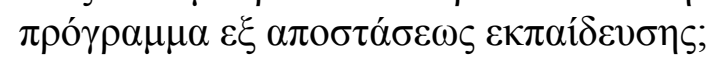

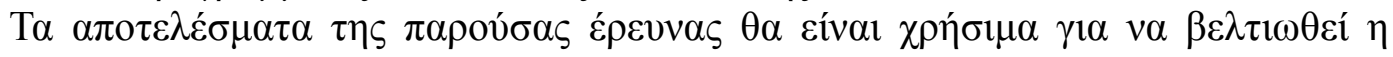

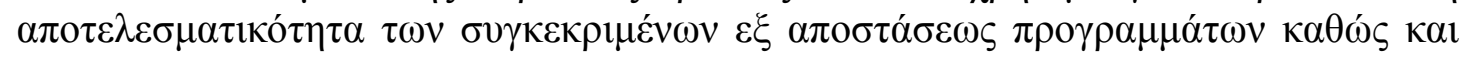

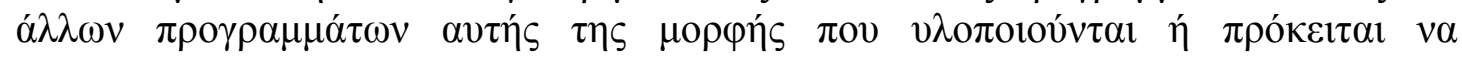

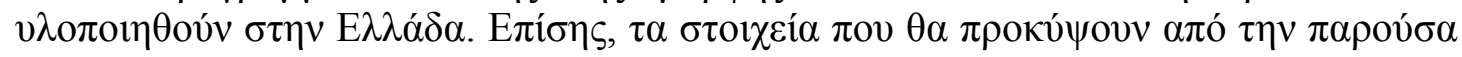

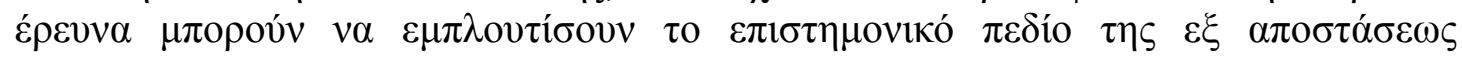

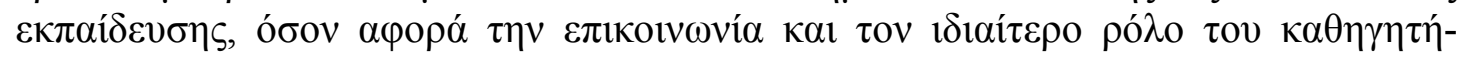

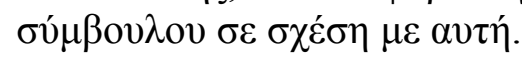

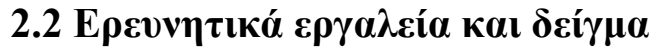

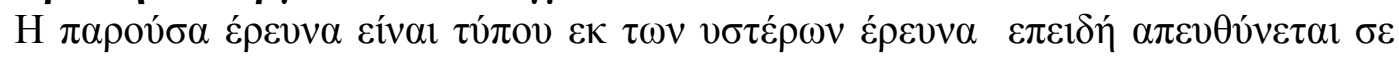

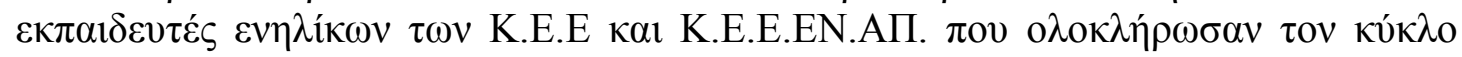

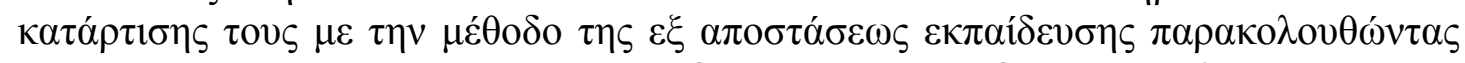

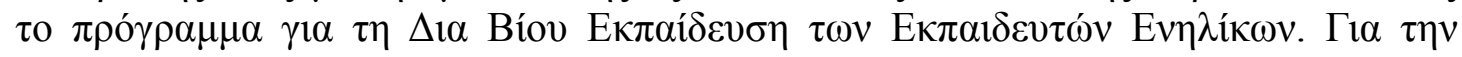

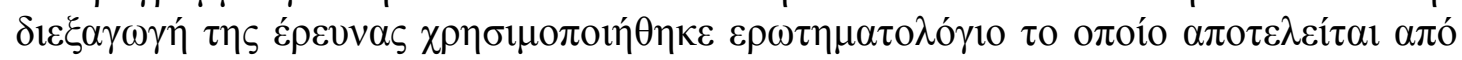

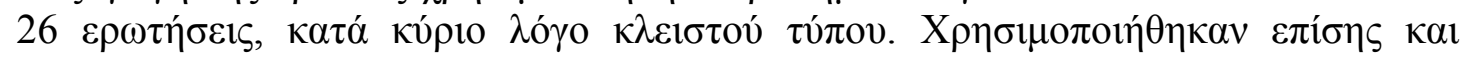

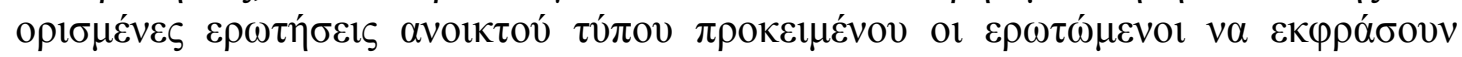

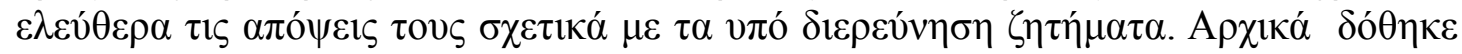

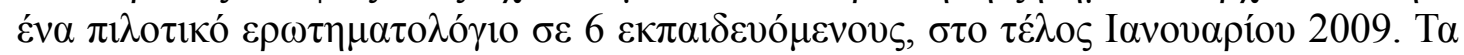

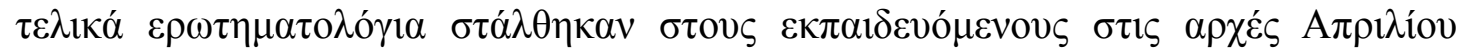

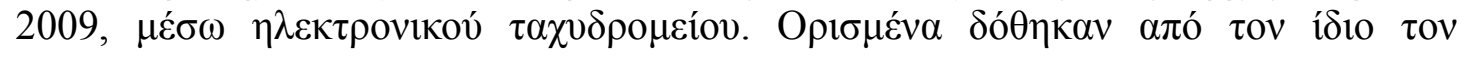

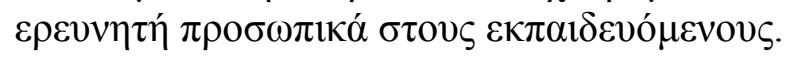

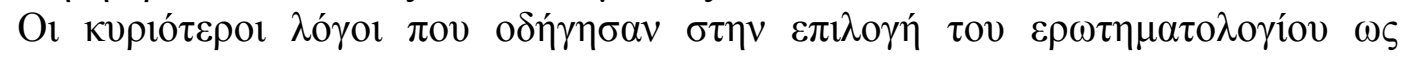

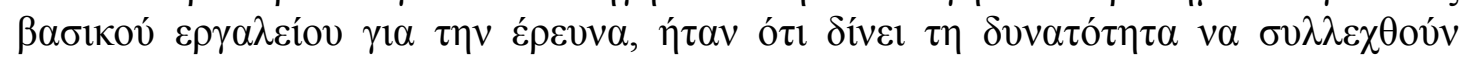

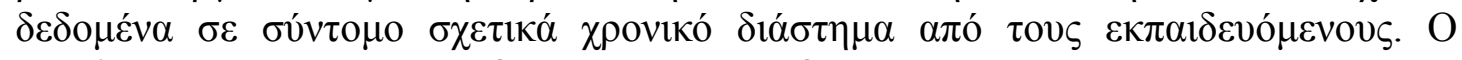

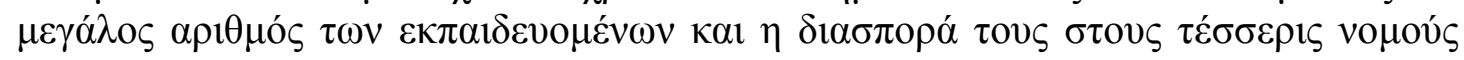

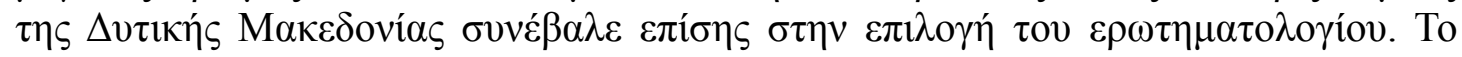

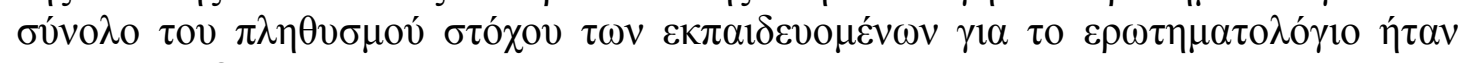

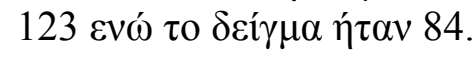




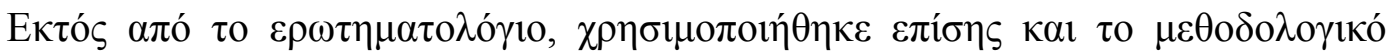

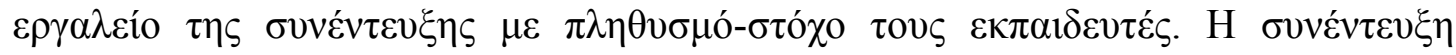

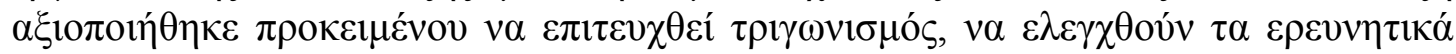

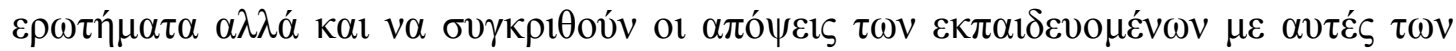

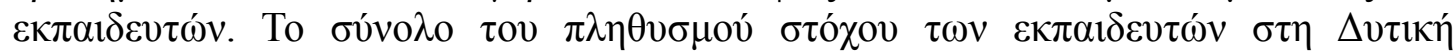

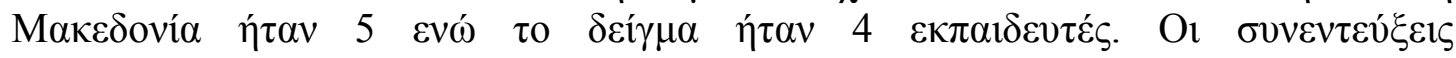

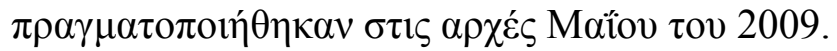

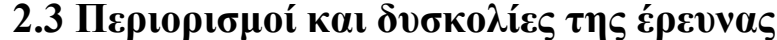

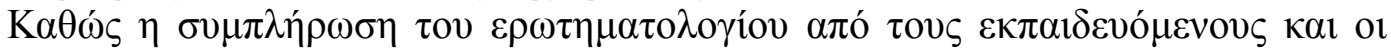

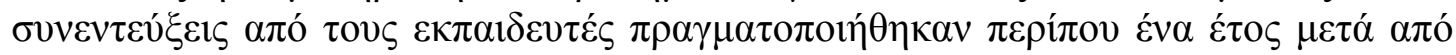

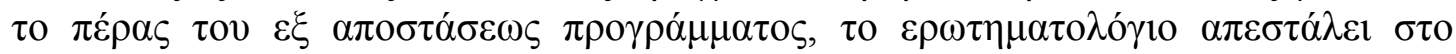

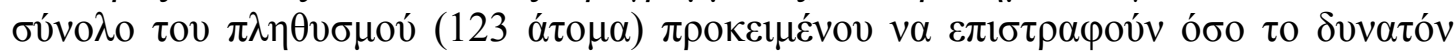

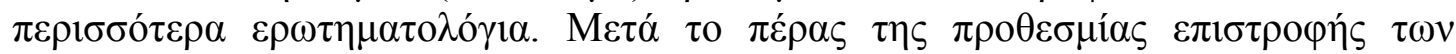

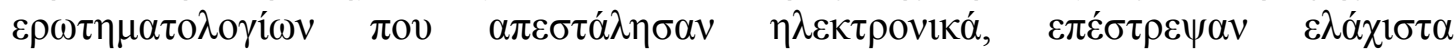

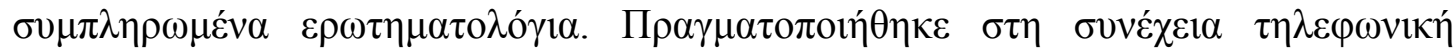

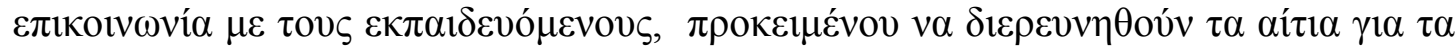

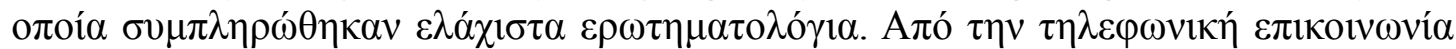
$\varepsilon v \tau о \pi i ́ \sigma \tau \eta \kappa \varepsilon$ ó

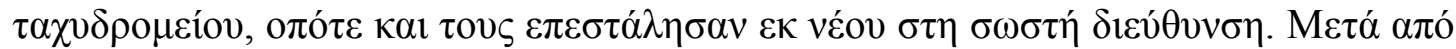

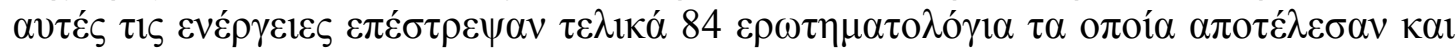

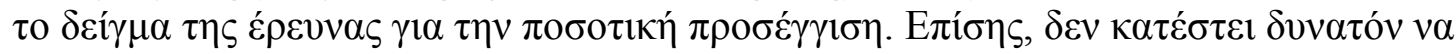

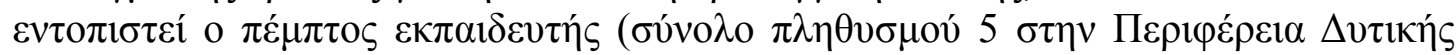

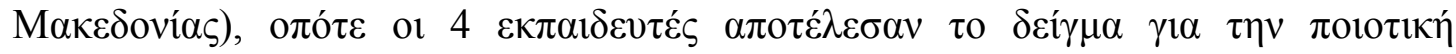
$\pi \rho \circ \sigma \varepsilon ́ \gamma \gamma i \sigma \eta$.

\section{3. $А \pi 0 \tau \varepsilon \lambda \varepsilon ́ \sigma \mu \alpha \tau \alpha$}

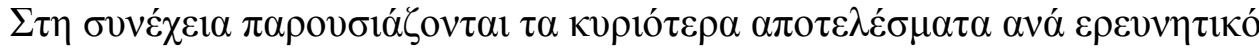
$\varepsilon \rho \omega ́ \tau \eta \mu \alpha$.

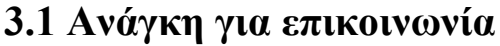

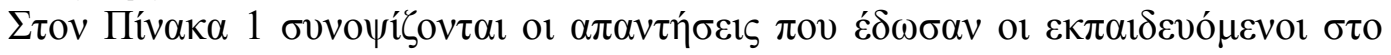

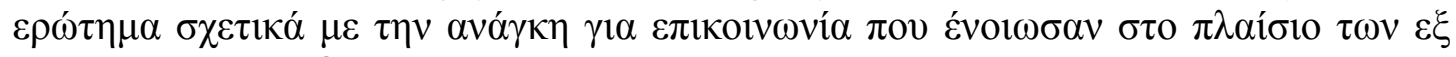

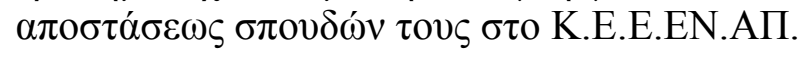

\begin{tabular}{|c|c|c|c|c|c|}
\hline 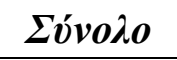 & 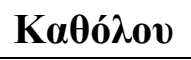 & Míyo & 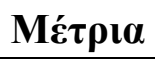 & 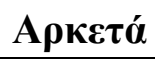 & 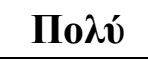 \\
\hline 84 & 2 & 10 & 20 & 41 & 11 \\
\hline $100,00 \%$ & $2,38 \%$ & $11,90 \%$ & $23,81 \%$ & $48,81 \%$ & $13,10 \%$ \\
\hline
\end{tabular}

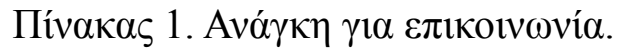

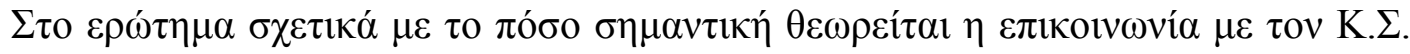

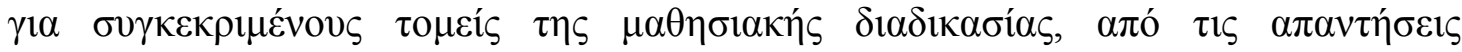

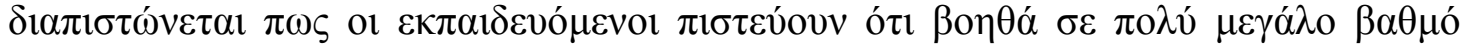

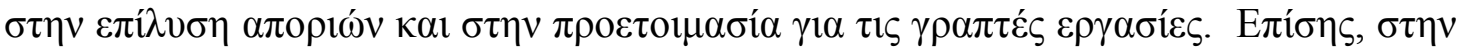

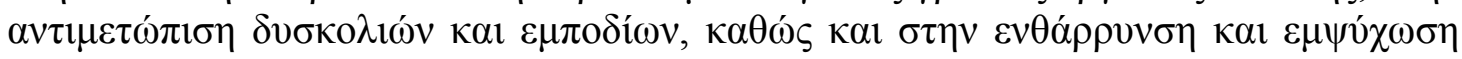

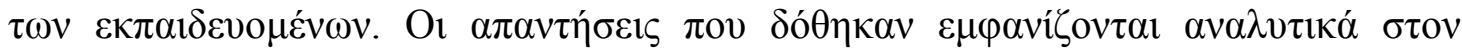

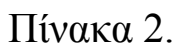




\begin{tabular}{|c|c|c|c|c|c|}
\hline 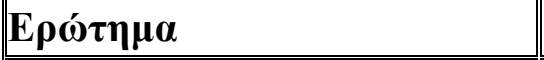 & 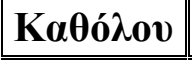 & Míyo & 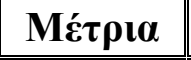 & 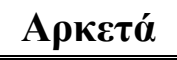 & 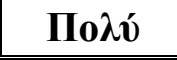 \\
\hline 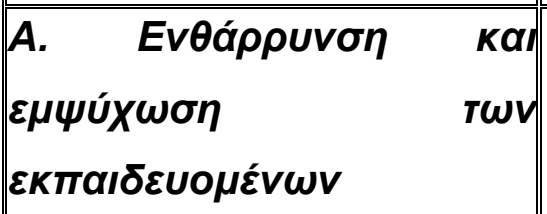 & $\begin{array}{c}1 \\
(1,19 \%)\end{array}$ & $\begin{array}{c}3 \\
(3,57 \%)\end{array}$ & $\begin{array}{c}5 \\
(5,95 \%)\end{array}$ & $\begin{array}{c}39 \\
(46,43 \%)\end{array}$ & $\begin{array}{c}36 \\
(42,86 \%)\end{array}$ \\
\hline 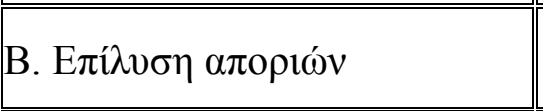 & ---- & ---- & $\begin{array}{c}5 \\
(5,95 \%) \\
\end{array}$ & $\begin{array}{c}28 \\
(33,33 \%) \\
\end{array}$ & $\begin{array}{c}51 \\
(60,71 \%) \\
\end{array}$ \\
\hline 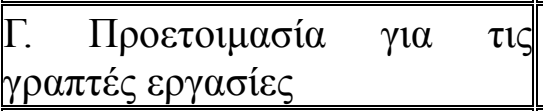 & ---- & $\begin{array}{c}4 \\
(4,76 \%)\end{array}$ & $\begin{array}{c}9 \\
(10,71 \%) \\
\end{array}$ & $\begin{array}{c}27 \\
(32,14 \%) \\
\end{array}$ & $\begin{array}{c}44 \\
(52,38 \%) \\
\end{array}$ \\
\hline 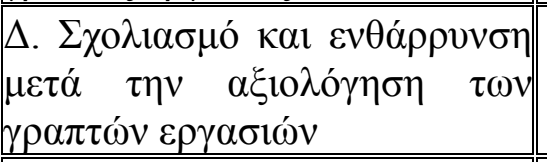 & $\begin{array}{c}1 \\
(1,19 \%) \\
\end{array}$ & $\begin{array}{c}3 \\
(3,57 \%)\end{array}$ & $\begin{array}{c}10 \\
(11,90 \%)\end{array}$ & $\begin{array}{c}36 \\
(42,86 \%)\end{array}$ & $\begin{array}{c}34 \\
(40,48 \%)\end{array}$ \\
\hline 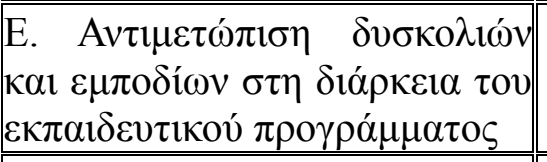 & $\begin{array}{c}1 \\
(1,19 \%) \\
\end{array}$ & $(1,19 \%)$ & $\begin{array}{c}8 \\
(9,52 \%)\end{array}$ & $\begin{array}{c}37 \\
(44,05 \%)\end{array}$ & $\begin{array}{c}37 \\
(44,05 \%)\end{array}$ \\
\hline$\Sigma \mathrm{T} . \mathrm{A}^{\mathrm{A}} \lambda \lambda_{\mathrm{o}} \ldots$ & ---- & ---- & ---- & ---- & ---- \\
\hline
\end{tabular}

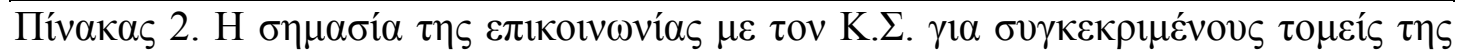

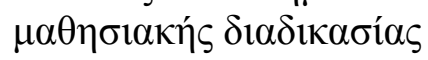

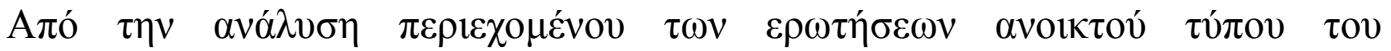

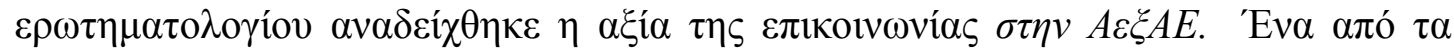

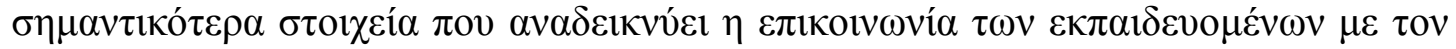

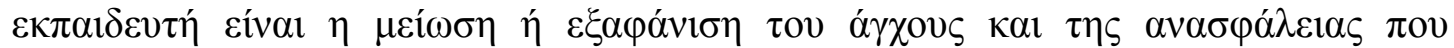

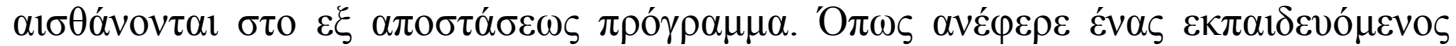

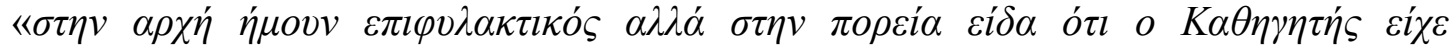

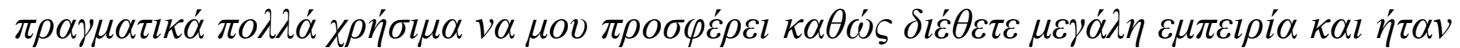

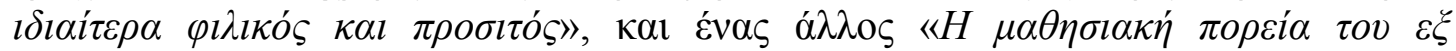

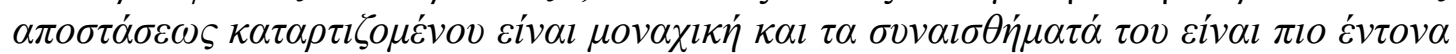

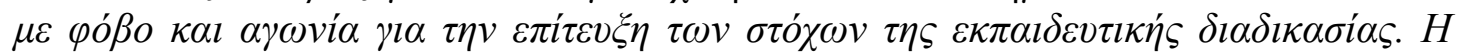

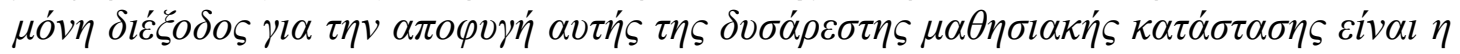

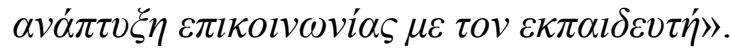

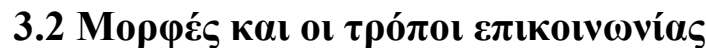

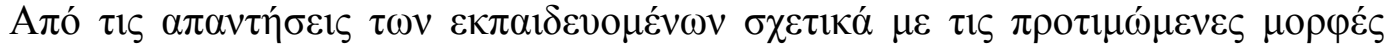

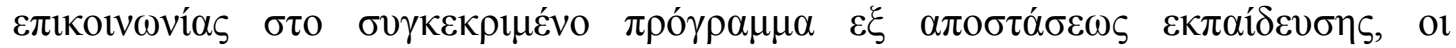

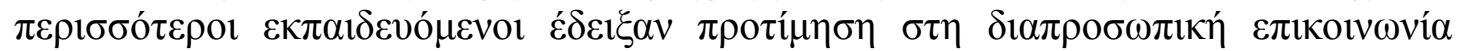

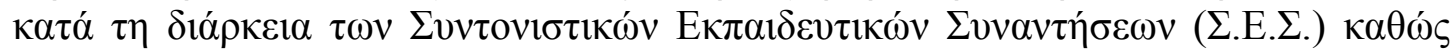

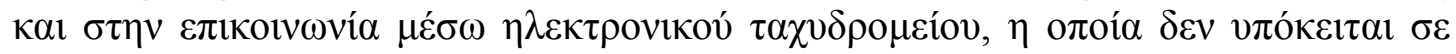

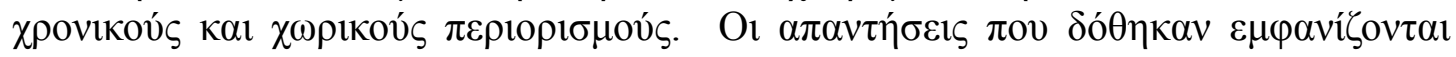

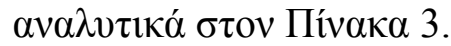

\begin{tabular}{|c|c|c|c|c|c|}
\hline 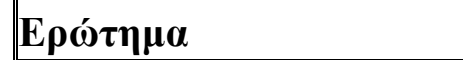 & 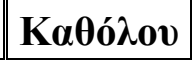 & Líyo & 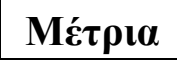 & Аркєта́ & Подv́ \\
\hline 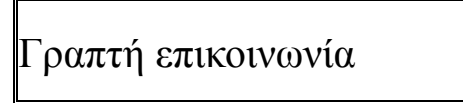 & $\begin{array}{c}30 \\
(36,14 \%)\end{array}$ & $\begin{array}{c}14 \\
(16,87 \%)\end{array}$ & $\begin{array}{c}20 \\
(24,10 \%)\end{array}$ & $\begin{array}{c}13 \\
(15,66 \%)\end{array}$ & $\begin{array}{c}6 \\
(7,23 \%)\end{array}$ \\
\hline 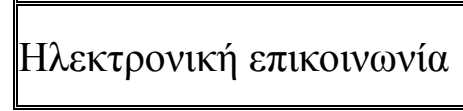 & $\begin{array}{c}2 \\
(2,38 \%)\end{array}$ & $\begin{array}{c}2 \\
(2,38 \%)\end{array}$ & $\begin{array}{c}8 \\
(9,52 \%)\end{array}$ & $\begin{array}{c}29 \\
(34,52 \%)\end{array}$ & $\begin{array}{c}43 \\
(51,19 \%)\end{array}$ \\
\hline
\end{tabular}


Open Education - The J oumal for Open and Distance Education and Educational Technology

\begin{tabular}{|c|c|c|c|c|c|}
\hline 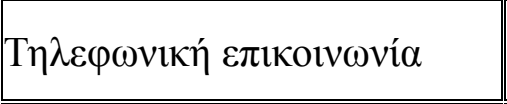 & $\begin{array}{c}19 \\
(22,89 \%) \\
\end{array}$ & $\begin{array}{c}15 \\
(18,07 \%)\end{array}$ & $\begin{array}{c}8 \\
(9,64 \%) \\
\end{array}$ & $\begin{array}{c}25 \\
(30,12 \%) \\
\end{array}$ & $\begin{array}{c}16 \\
(19,28 \%)\end{array}$ \\
\hline$\Phi \alpha \xi$ & $\begin{array}{c}65 \\
(78,31 \%)\end{array}$ & $\begin{array}{c}9 \\
(10,84 \%)\end{array}$ & $\begin{array}{c}7 \\
(8,43 \%) \\
\end{array}$ & ---- & $\begin{array}{c}2 \\
(2,41 \%)\end{array}$ \\
\hline 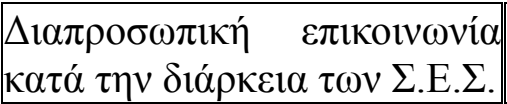 & $\begin{array}{c}1 \\
(1,22 \%)\end{array}$ & $\begin{array}{c}4 \\
(4,88 \%)\end{array}$ & $\begin{array}{c}9 \\
(10,98 \%)\end{array}$ & $\begin{array}{c}20 \\
(24,39 \%)\end{array}$ & $\begin{array}{c}48 \\
(58,54 \%)\end{array}$ \\
\hline$A \lambda \lambda 0 \ldots$ & ---- & ---- & ---- & ---- & ---- \\
\hline
\end{tabular}

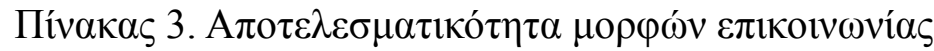

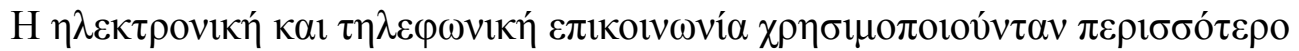

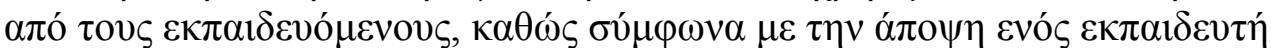

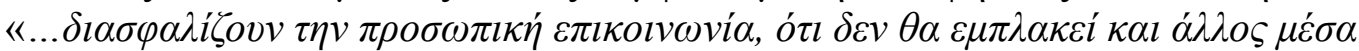

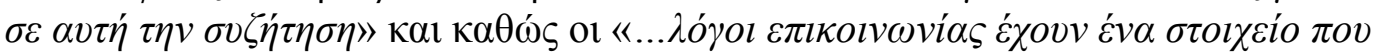

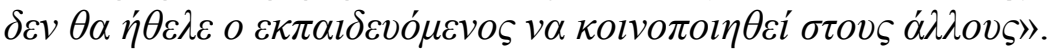

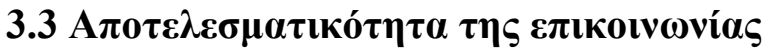

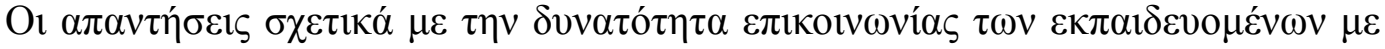

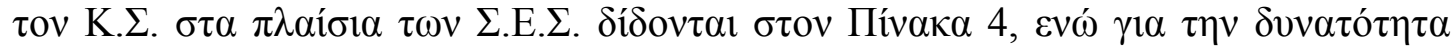

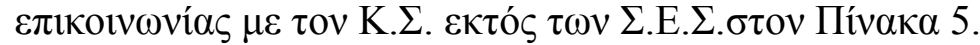

\begin{tabular}{|c|c|c|c|c|c|}
\hline 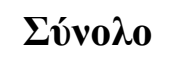 & 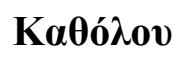 & 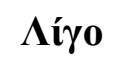 & 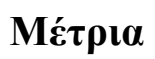 & 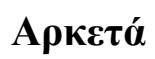 & 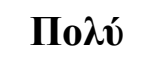 \\
\hline 83 & ---- & 6 & 11 & 38 & 28 \\
\hline $100,00 \%$ & ---- & $7,23 \%$ & $13,25 \%$ & $45,78 \%$ & $33,73 \%$ \\
\hline
\end{tabular}

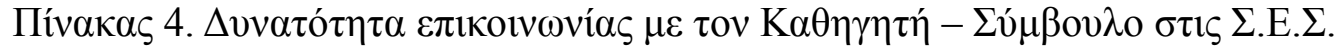

\begin{tabular}{|c|c|c|c|c|c|}
\hline 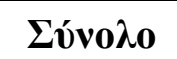 & 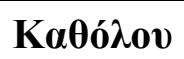 & 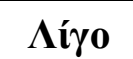 & 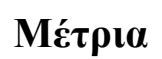 & 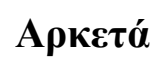 & 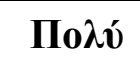 \\
\hline 84 & 1 & 8 & 16 & 36 & 23 \\
\hline $100,00 \%$ & $1,19 \%$ & $9,52 \%$ & $19,05 \%$ & $42,86 \%$ & $27,38 \%$ \\
\hline
\end{tabular}

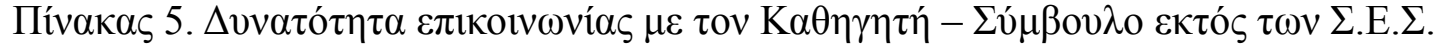

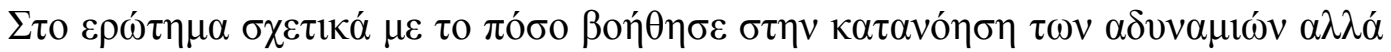

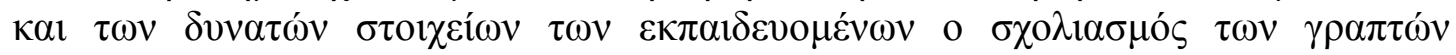

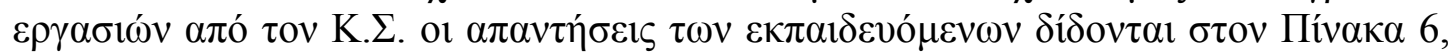

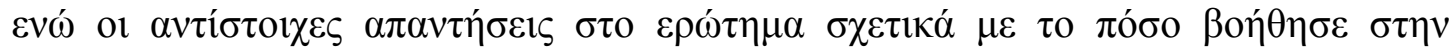

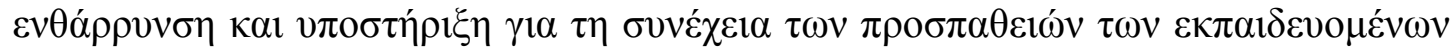

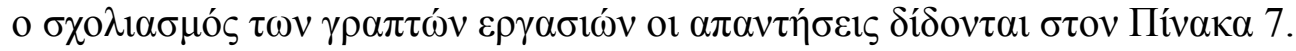

\begin{tabular}{|c|c|c|c|c|c|}
\hline 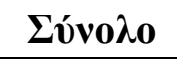 & 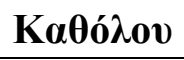 & Aífo & 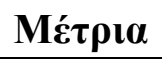 & 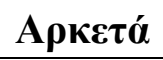 & Подv́ \\
\hline 83 & 3 & 5 & 9 & 41 & 25 \\
\hline $100,00 \%$ & $3,61 \%$ & $6,02 \%$ & $10,84 \%$ & $49,40 \%$ & $30,12 \%$ \\
\hline
\end{tabular}

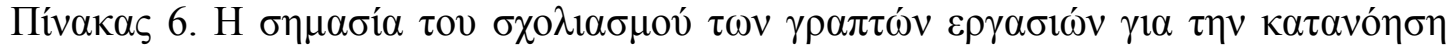


Open Education - The J oumal for Open and Distance Education and Educational Technology Volume 7, Number 1, 2011 Section one. (c) Open Education

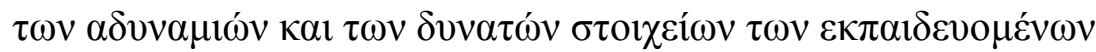

\begin{tabular}{|c|c|c|c|c|c|}
\hline 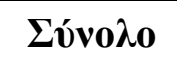 & 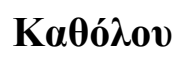 & Mífo & M⿻́㇒́ & 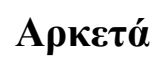 & Подv́ \\
\hline 83 & 3 & 7 & 10 & 34 & 29 \\
\hline $100,00 \%$ & $3,61 \%$ & $8,43 \%$ & $12,05 \%$ & $40,96 \%$ & $34,94 \%$ \\
\hline
\end{tabular}

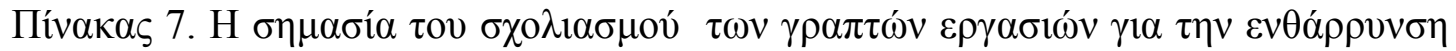

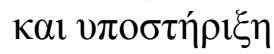

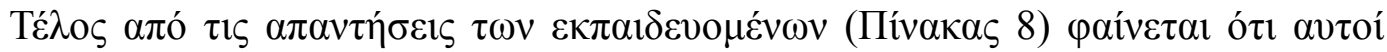

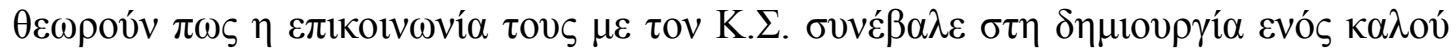

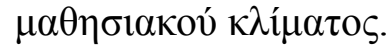

\begin{tabular}{|c|c|c|c|c|c|}
\hline 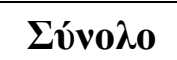 & 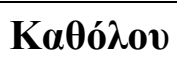 & Míyo & Mécpı & 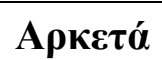 & Пoגv́ \\
\hline 83 & 2 & 4 & 12 & 28 & 37 \\
\hline $100,00 \%$ & $2,41 \%$ & $4,82 \%$ & $14,46 \%$ & $33,73 \%$ & $44,58 \%$ \\
\hline
\end{tabular}

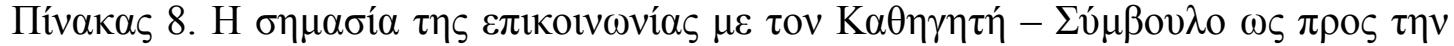

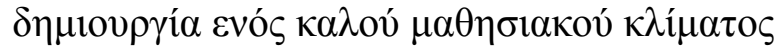

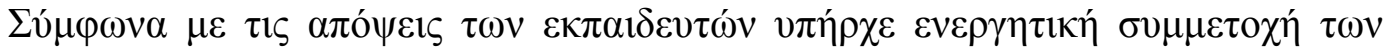

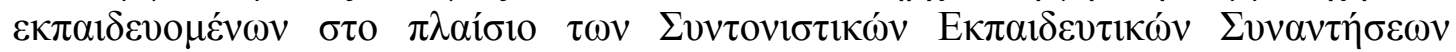

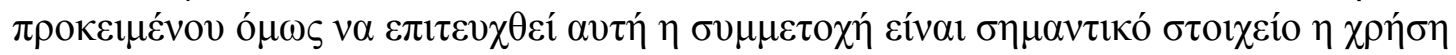

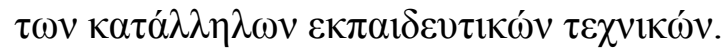

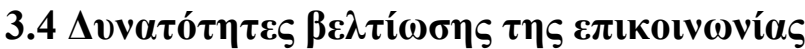

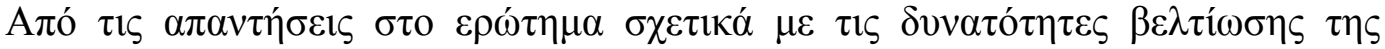

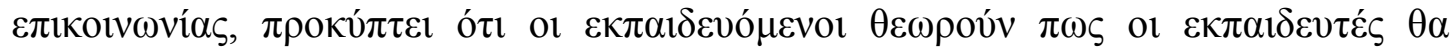

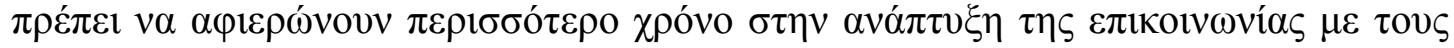

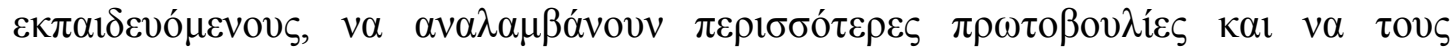

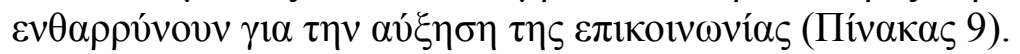

\begin{tabular}{|c|c|c|c|c|c|}
\hline \multicolumn{6}{|c|}{ 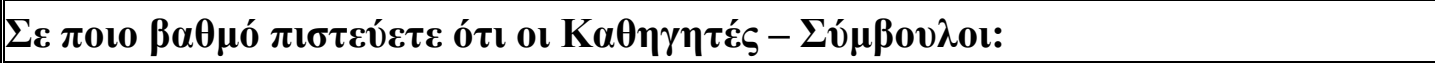 } \\
\hline 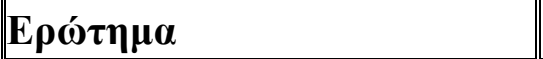 & 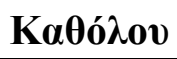 & 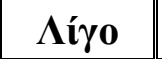 & 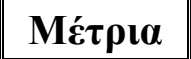 & 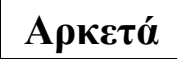 & Подv́ \\
\hline 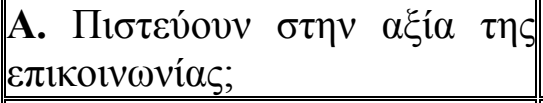 & $\begin{array}{c}1 \\
(1,20 \%) \\
\end{array}$ & $\begin{array}{c}7 \\
(8,43 \%) \\
\end{array}$ & $\begin{array}{c}11 \\
(13,25 \%) \\
\end{array}$ & $\begin{array}{c}29 \\
(34,94 \%) \\
\end{array}$ & $\begin{array}{c}35 \\
(42,17 \%) \\
\end{array}$ \\
\hline 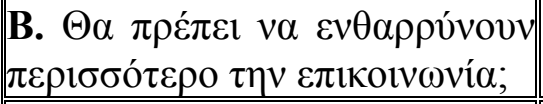 & $\begin{array}{c}1 \\
(1,20 \%) \\
\end{array}$ & $\begin{array}{c}3 \\
(3,61 \%) \\
\end{array}$ & $\begin{array}{c}14 \\
(16,87 \%) \\
\end{array}$ & $\begin{array}{c}25 \\
(30,12 \%) \\
\end{array}$ & $\begin{array}{c}40 \\
(48,19 \%) \\
\end{array}$ \\
\hline 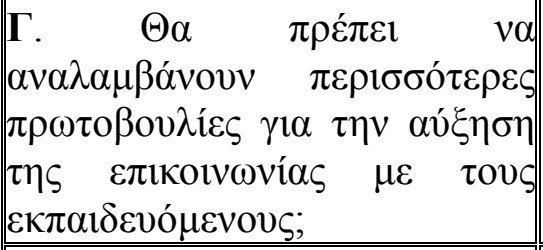 & $(1,20 \%)$ & $\begin{array}{c}3 \\
(3,61 \%)\end{array}$ & $\begin{array}{c}5 \\
(6,02 \%)\end{array}$ & $\begin{array}{c}29 \\
(34,94 \%)\end{array}$ & $\begin{array}{c}45 \\
(54,22 \%)\end{array}$ \\
\hline 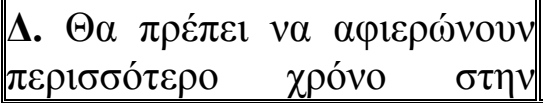 & $\begin{array}{c}1 \\
(1,20 \%) \\
\end{array}$ & $\begin{array}{c}4 \\
(4,82 \%)\end{array}$ & $\begin{array}{c}8 \\
(9,64 \%) \\
\end{array}$ & $\begin{array}{c}23 \\
(27,71 \%) \\
\end{array}$ & $\begin{array}{c}47 \\
(56,63 \%) \\
\end{array}$ \\
\hline
\end{tabular}




\begin{tabular}{|c|c|c|c|c|c|}
\hline 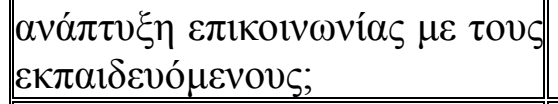 & & & & & \\
\hline 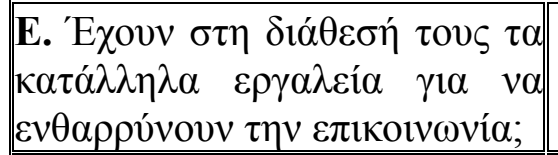 & $\begin{array}{c}1 \\
(1,20 \%)\end{array}$ & $\begin{array}{c}10 \\
(12,05 \% \\
)\end{array}$ & $\begin{array}{c}20 \\
(24,10 \%)\end{array}$ & $\begin{array}{c}26 \\
(31,33 \%)\end{array}$ & $\begin{array}{c}26 \\
(31,33 \%)\end{array}$ \\
\hline 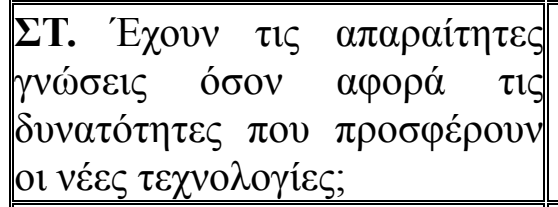 & ---- & $\begin{array}{c}3 \\
(3,61 \%)\end{array}$ & $\begin{array}{c}19 \\
(22,89 \%)\end{array}$ & $\begin{array}{c}27 \\
(32,53 \%)\end{array}$ & $\begin{array}{c}34 \\
(40,96 \%)\end{array}$ \\
\hline Z. $A \lambda \lambda \lambda_{0} \ldots .$. & & & & & \\
\hline
\end{tabular}

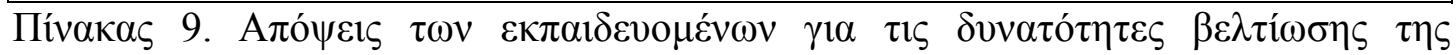

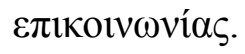

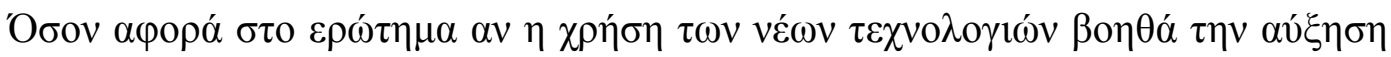
$\tau \eta \varsigma \varepsilon \pi$ «

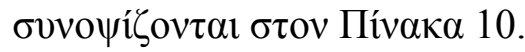

\begin{tabular}{|c|c|c|c|c|c|}
\hline 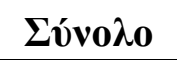 & 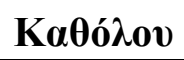 & Aífo & 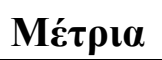 & 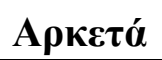 & Пoגv́ \\
\hline 80 & --- & 1 & " ---- & 16 & 63 \\
\hline $100,00 \%$ & ---- & $1,25 \%$ & ---- & $20,00 \%$ & $78,75 \%$ \\
\hline
\end{tabular}

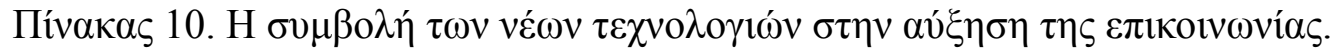

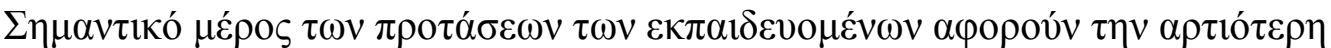

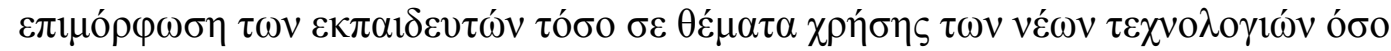

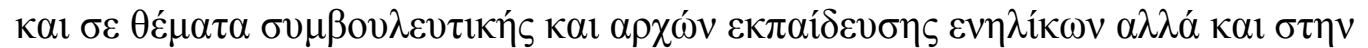

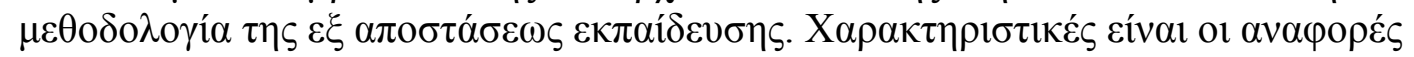

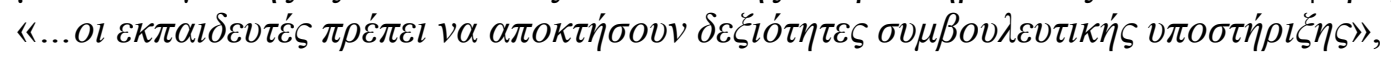

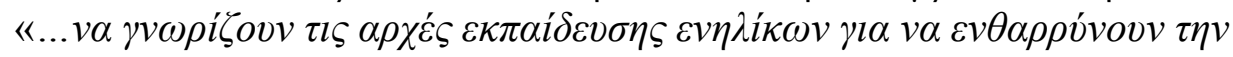

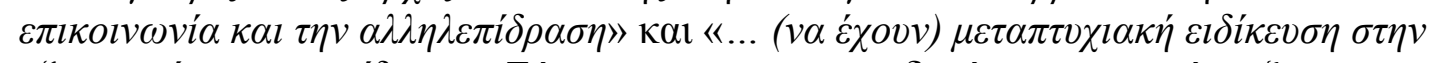

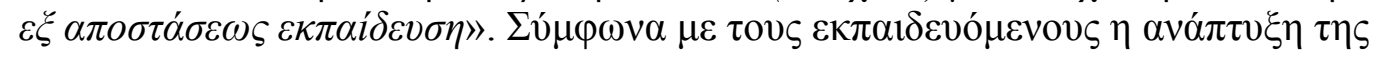

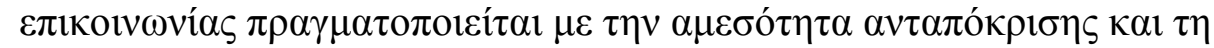

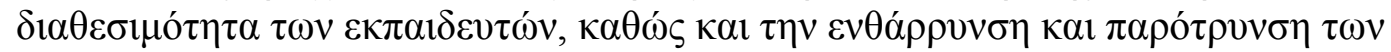

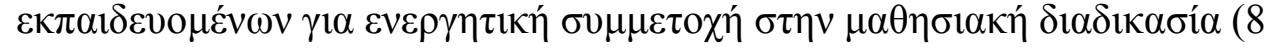

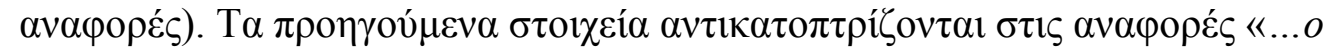

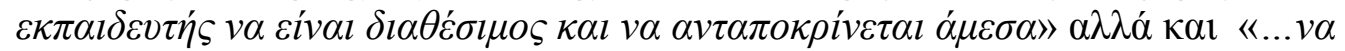

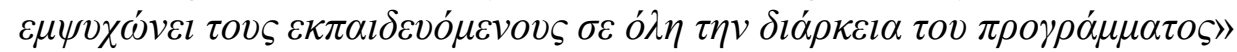

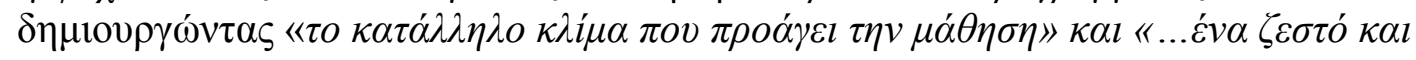

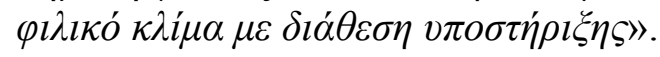

\section{4. $\Sigma v \zeta \eta ́ \eta \eta \eta \eta ~ \alpha \pi 0 \tau \varepsilon \lambda \varepsilon \sigma \mu \alpha ́ \tau \omega v$}

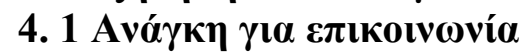

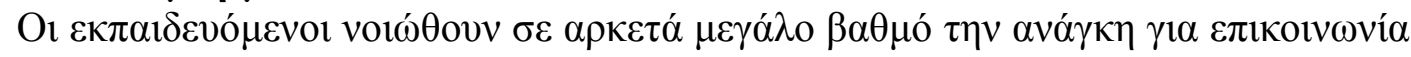

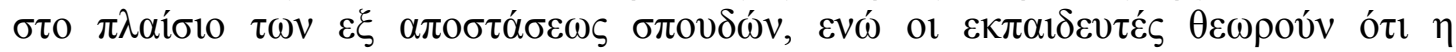

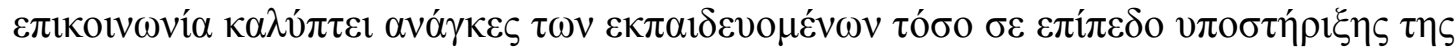

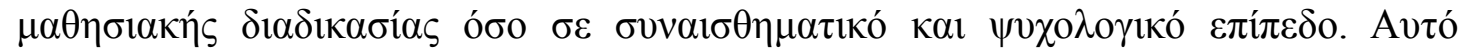

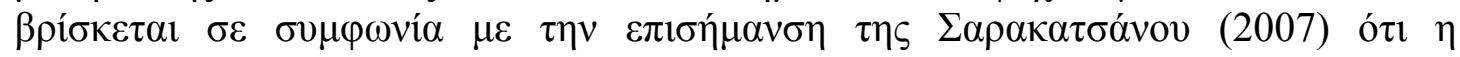




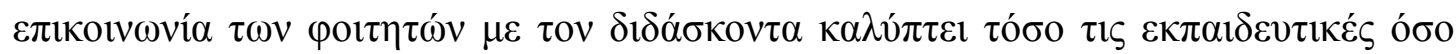

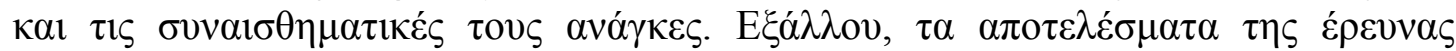

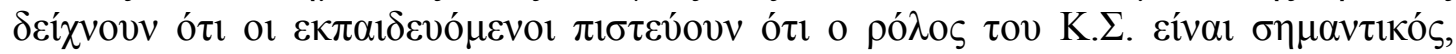

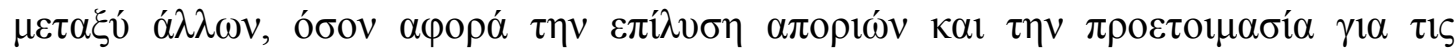

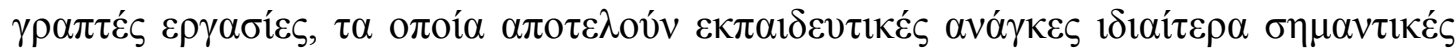

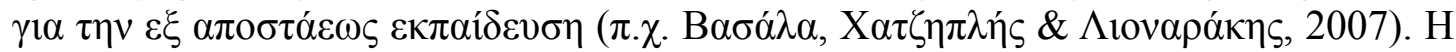

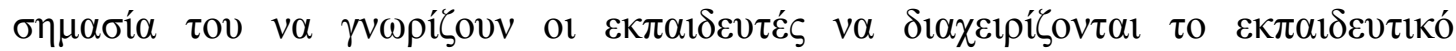

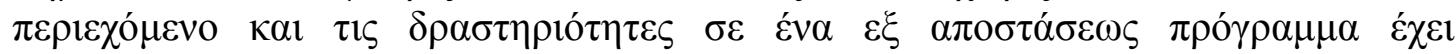

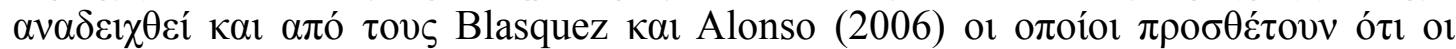

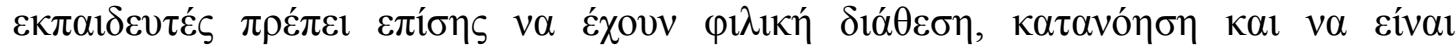

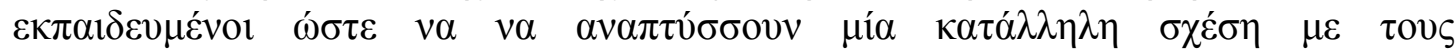
$\delta 1 \delta \alpha \sigma \kappa o ́ \mu \varepsilon v o v \varsigma$.

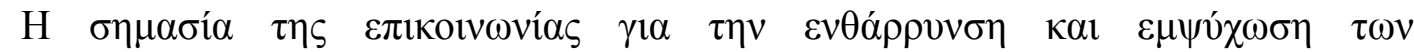

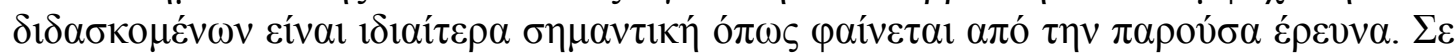

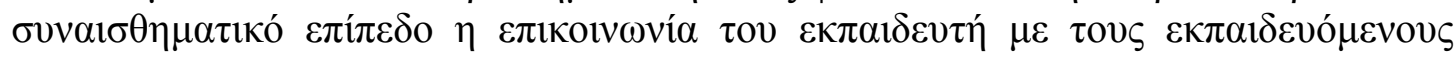

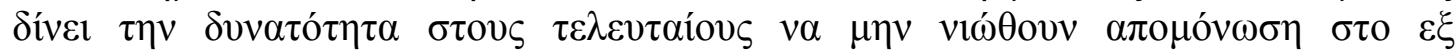

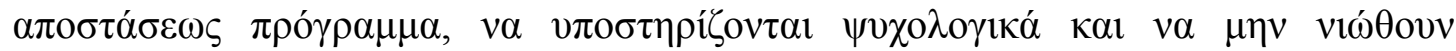

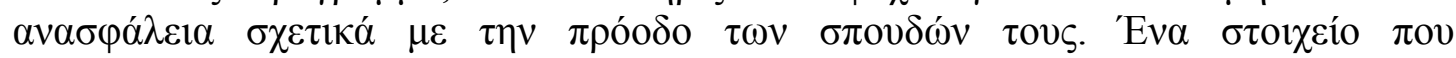

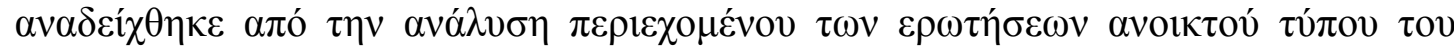

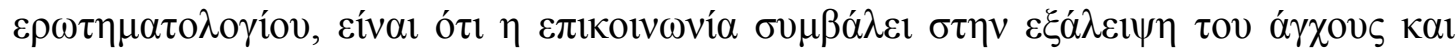

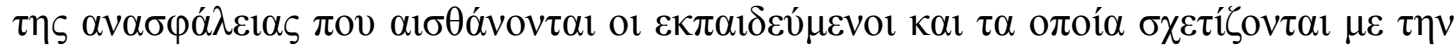

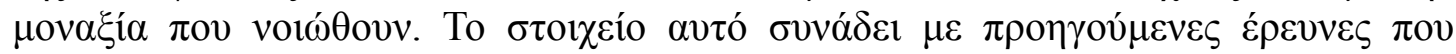

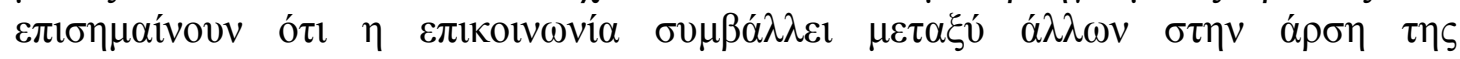

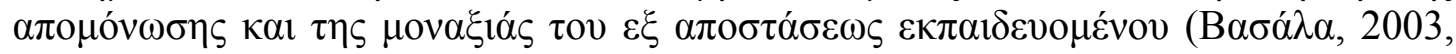

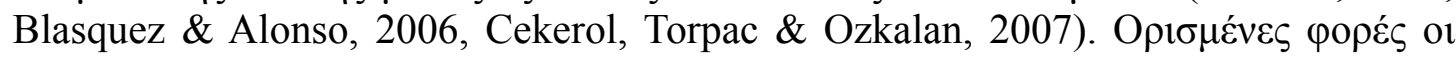

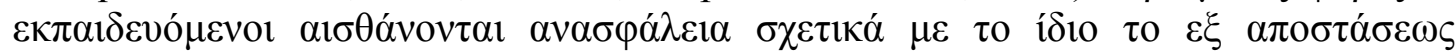

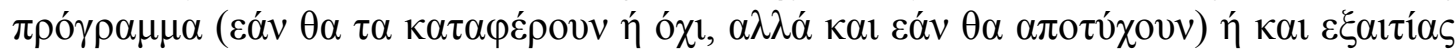

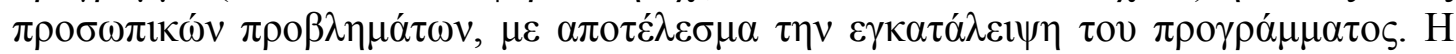

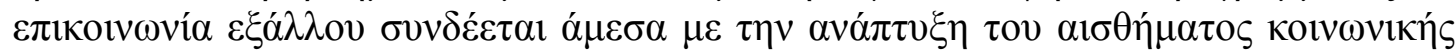

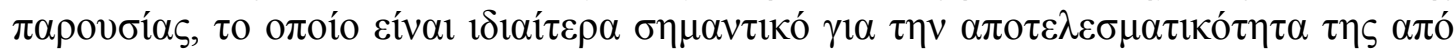

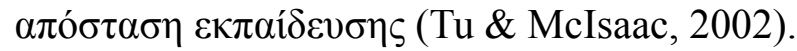

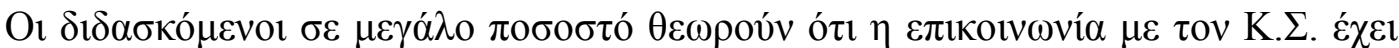

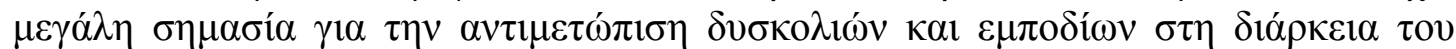

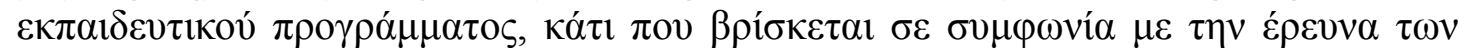

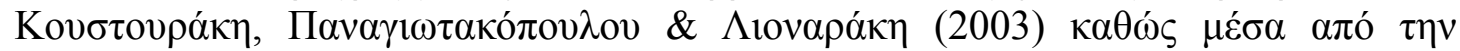

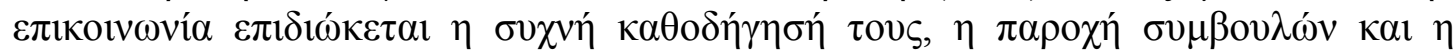

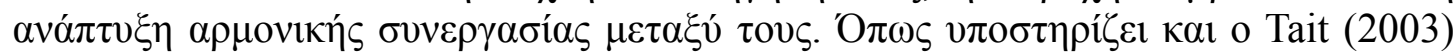

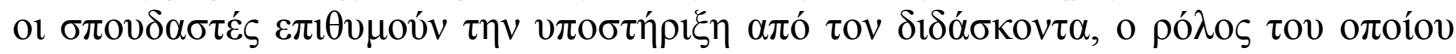

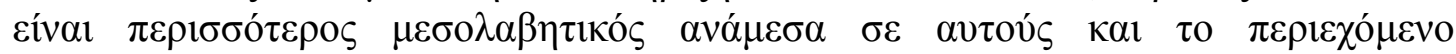

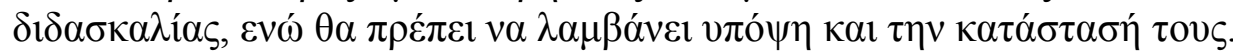

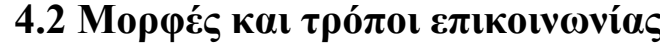

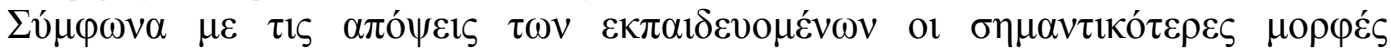

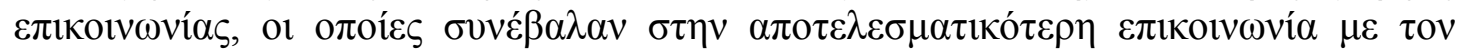

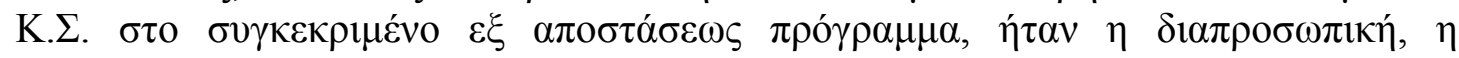

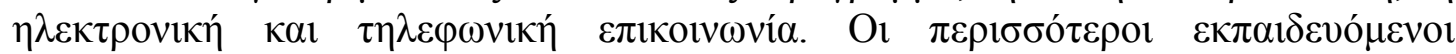

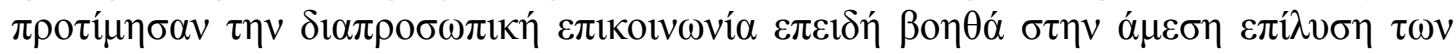

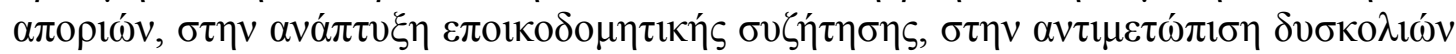

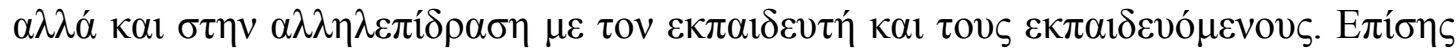




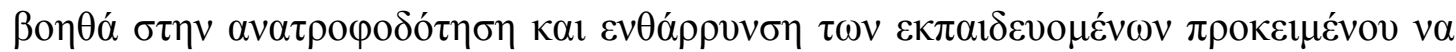

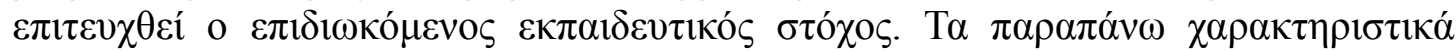

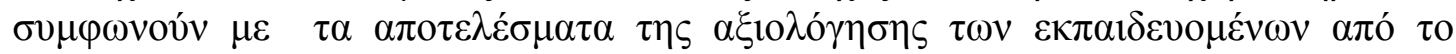

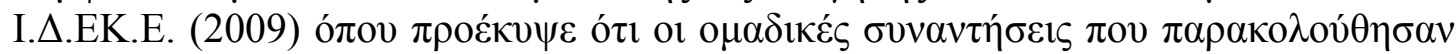

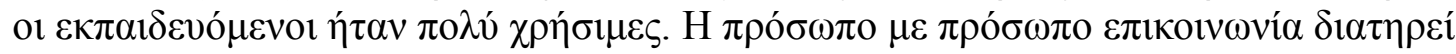

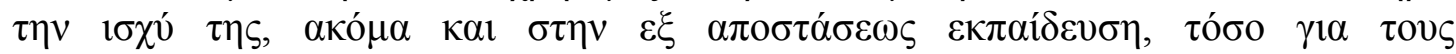

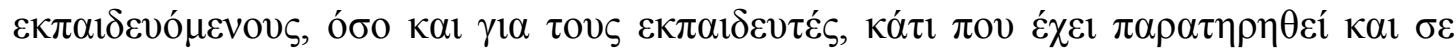

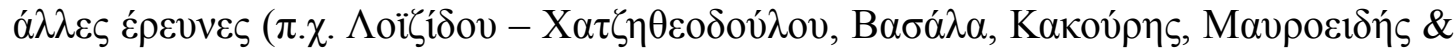

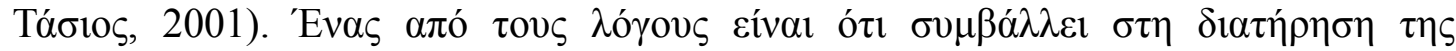

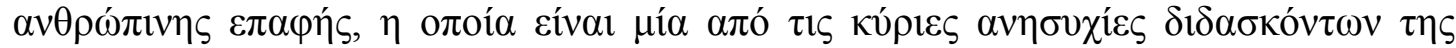

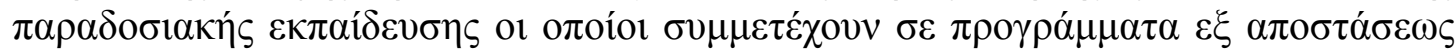

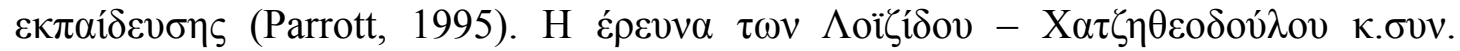

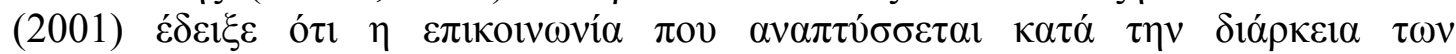

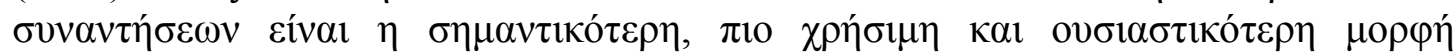

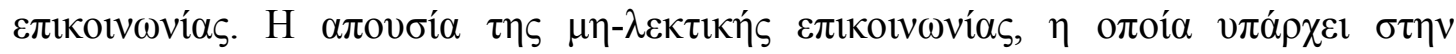

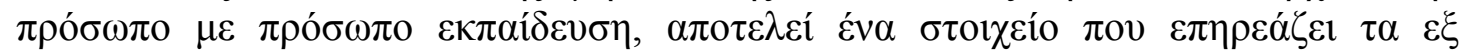

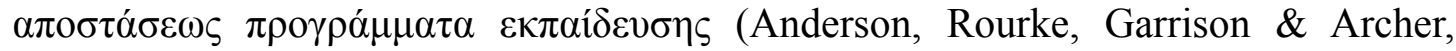

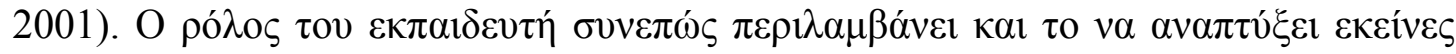

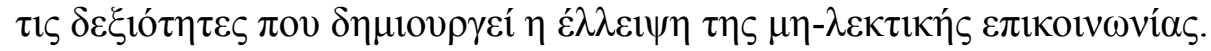

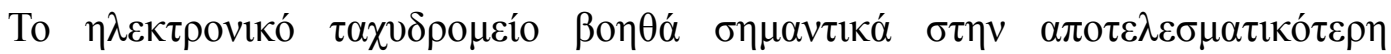

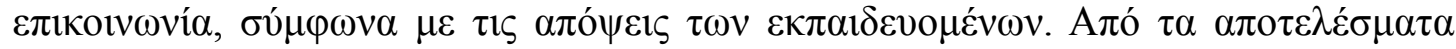

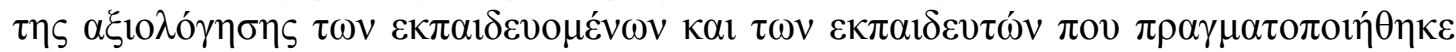

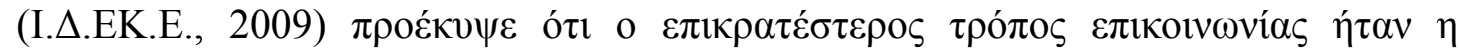

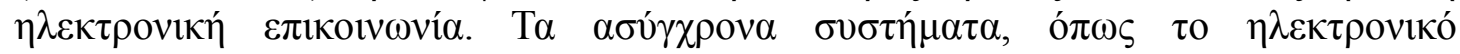

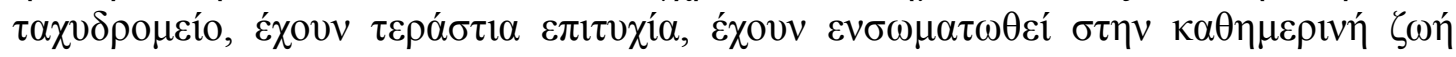

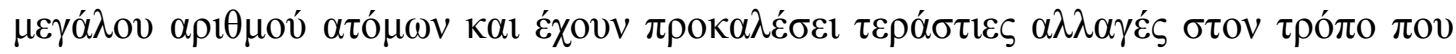

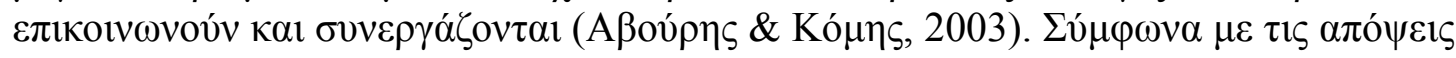

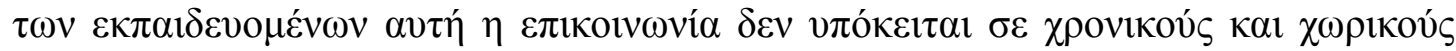

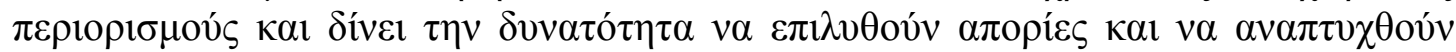

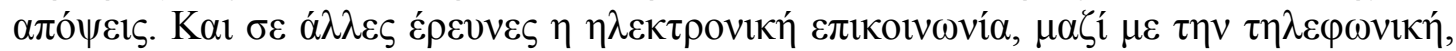

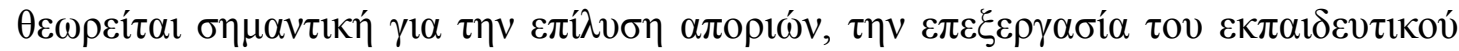

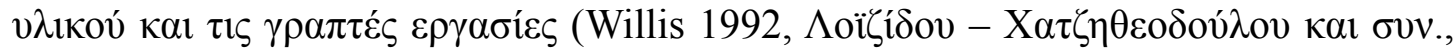

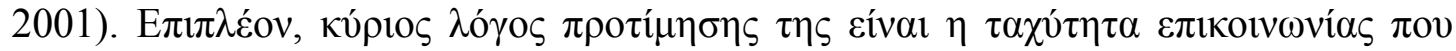

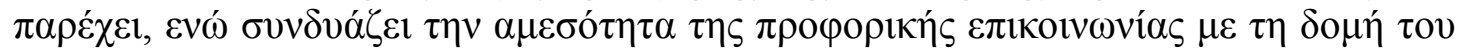

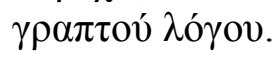

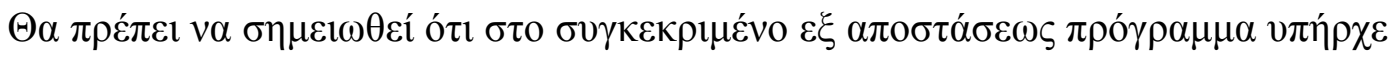

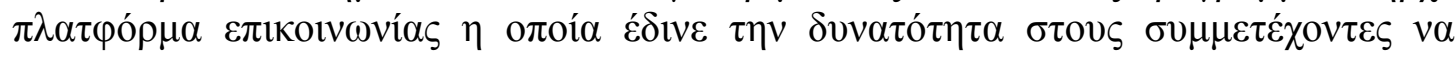

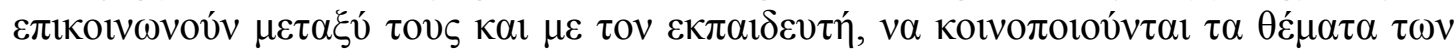

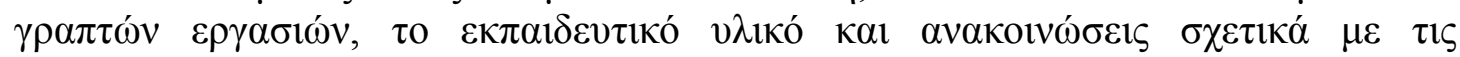

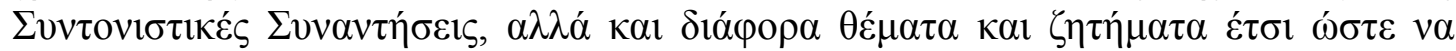

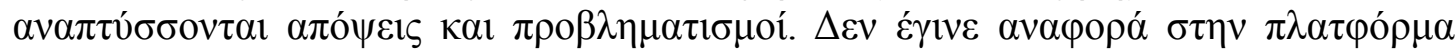

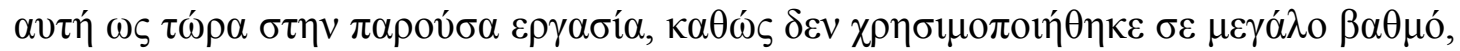

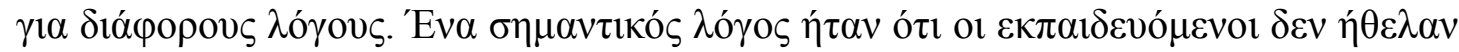

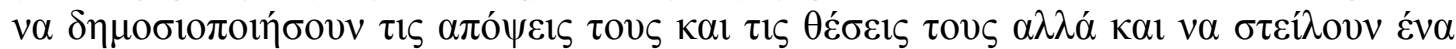

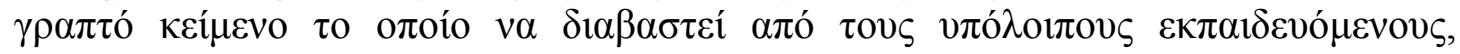

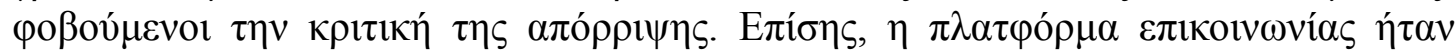

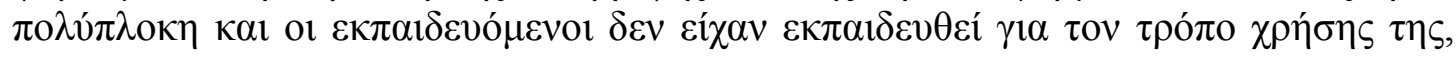

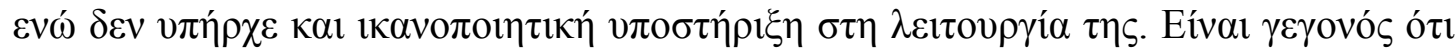

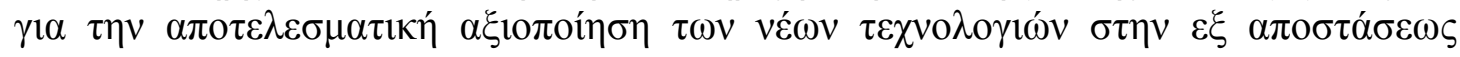




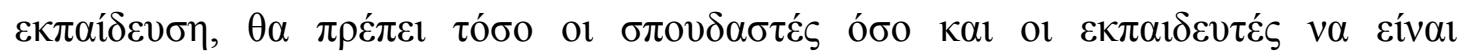

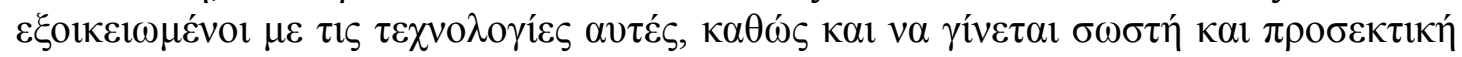

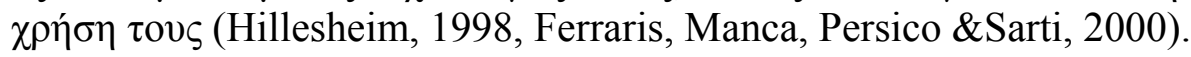

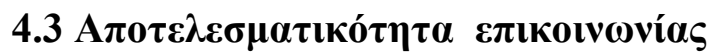

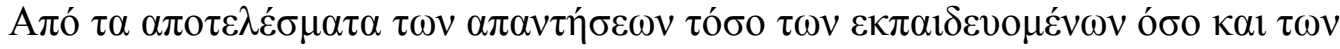

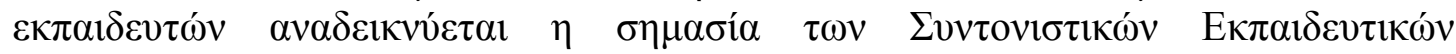

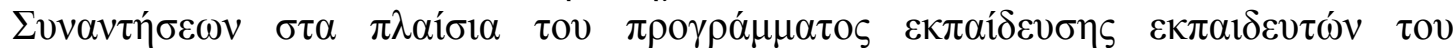

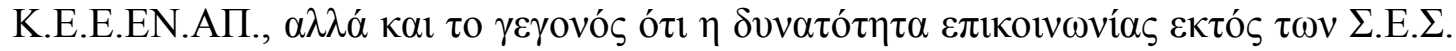

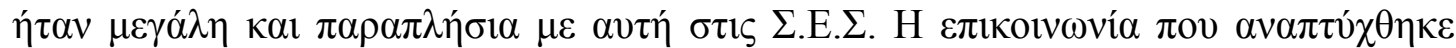

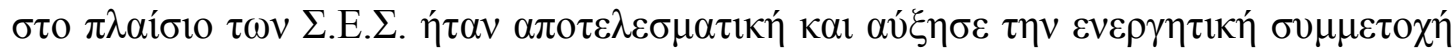

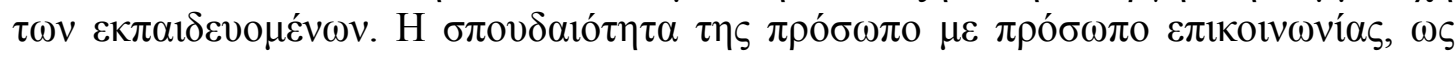

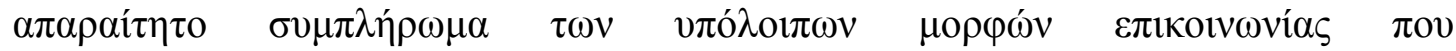

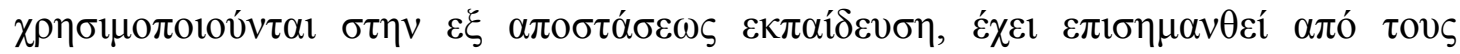

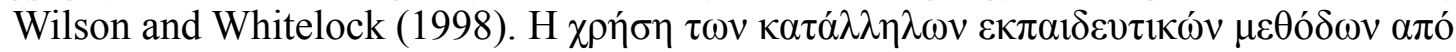

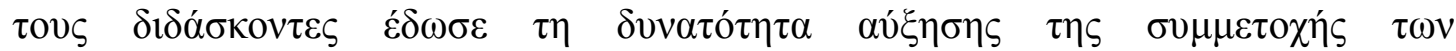

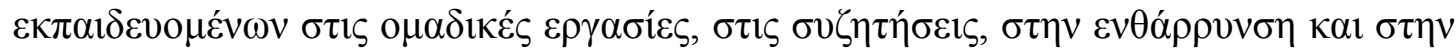

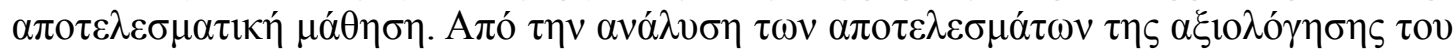

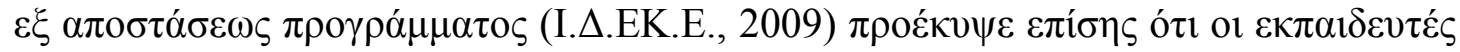

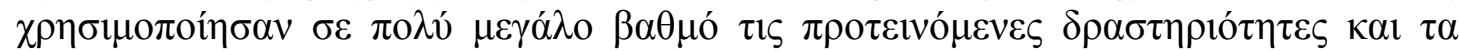

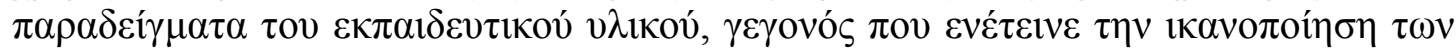

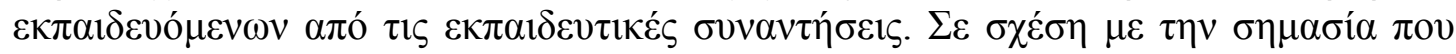

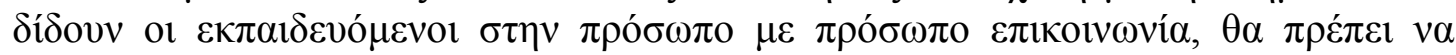

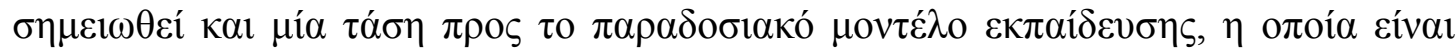

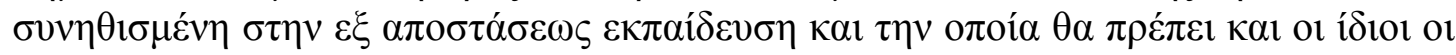

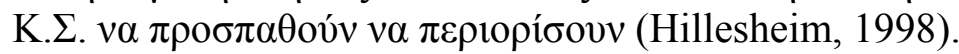

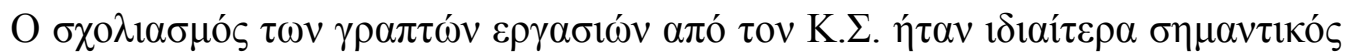

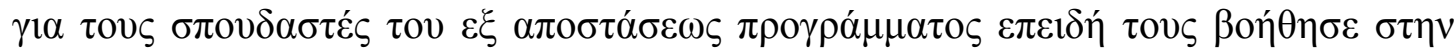

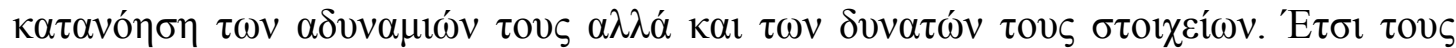

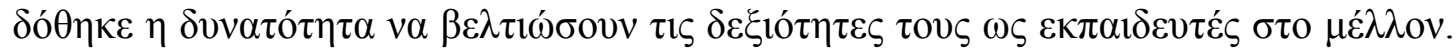

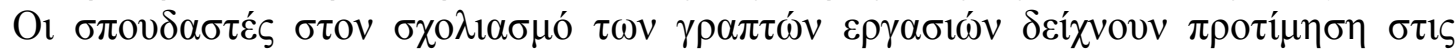

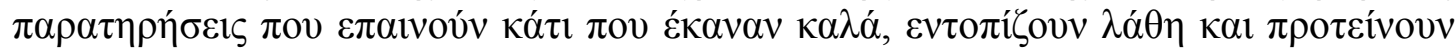

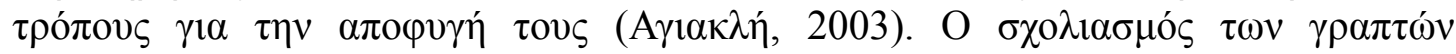

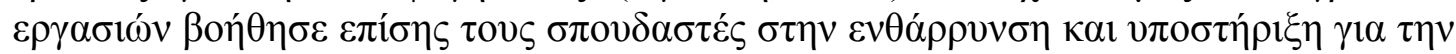

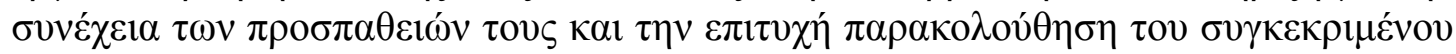

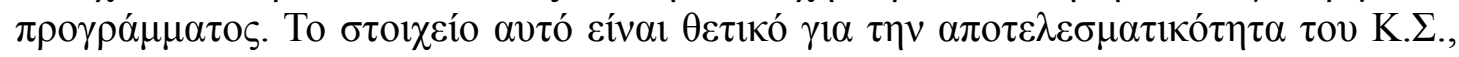

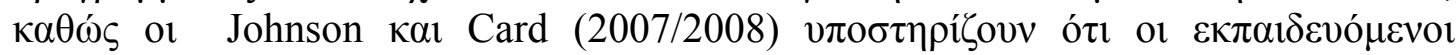

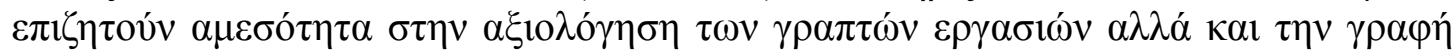

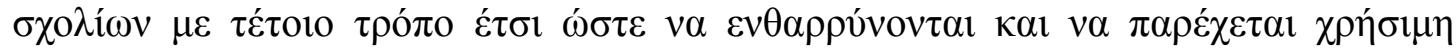

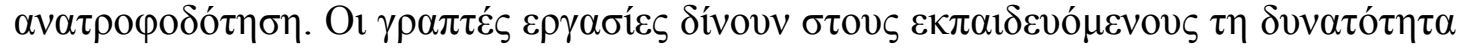

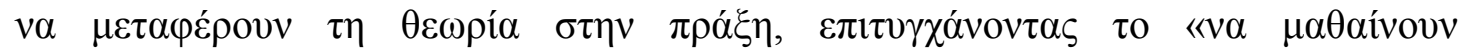

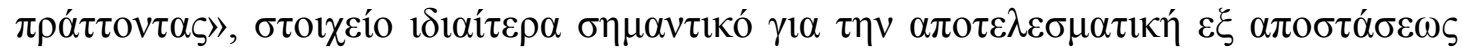

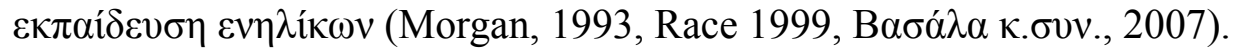

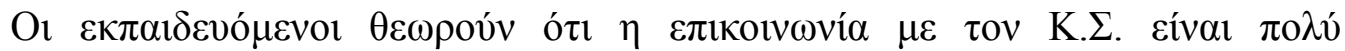

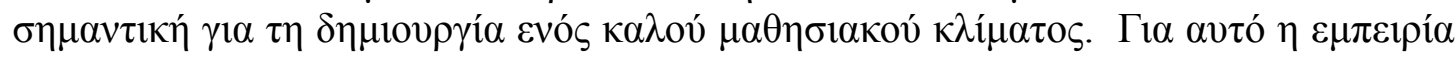

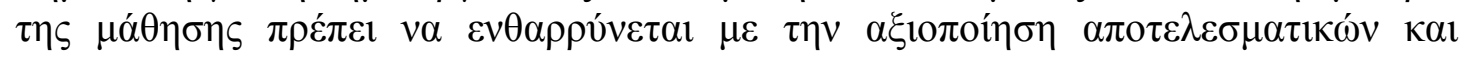

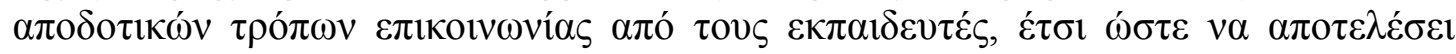

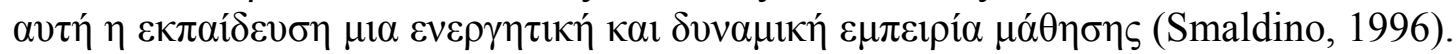

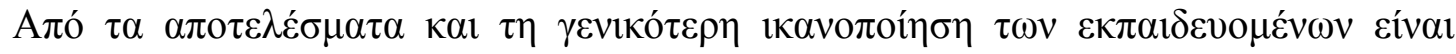




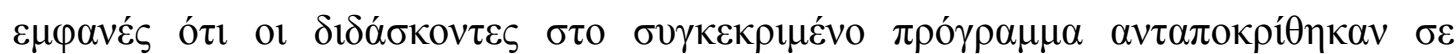

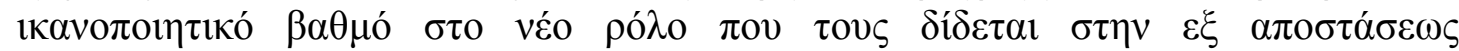

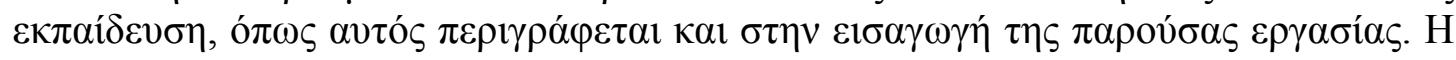

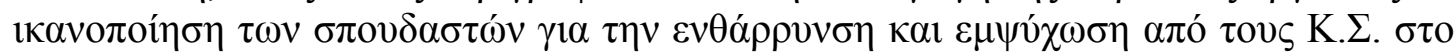

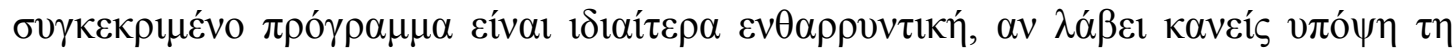

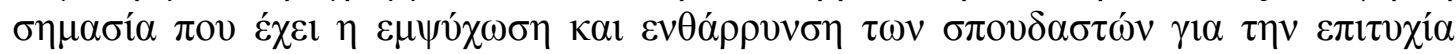

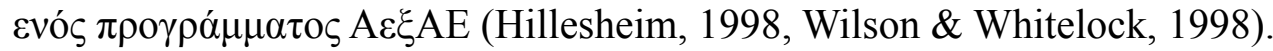

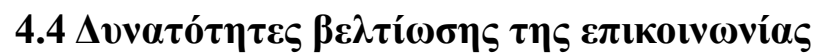

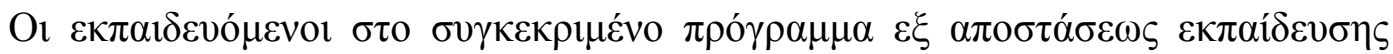

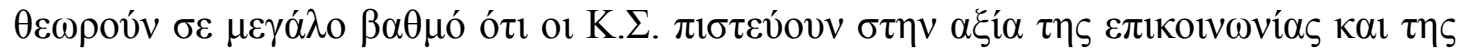

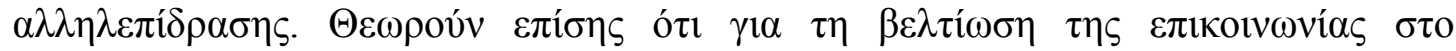

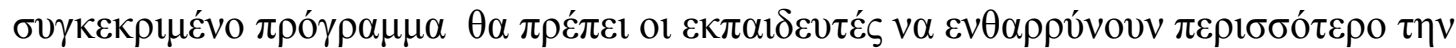

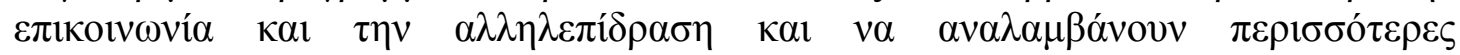

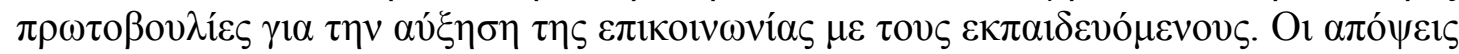

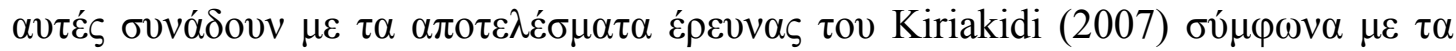

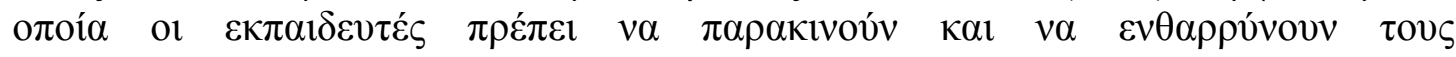

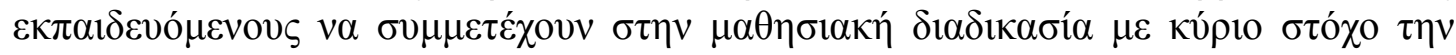

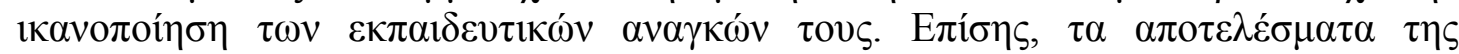

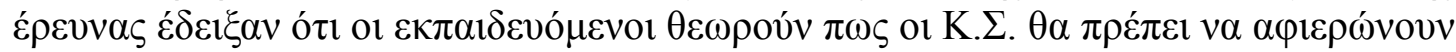

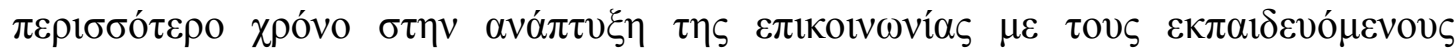

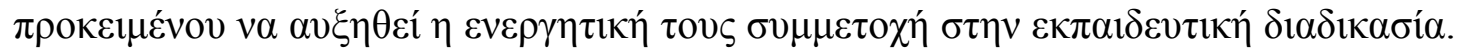

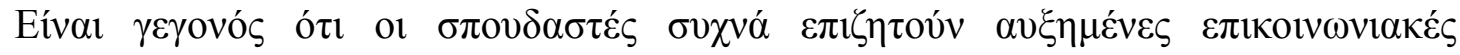

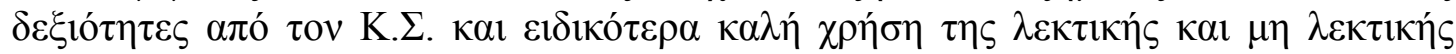

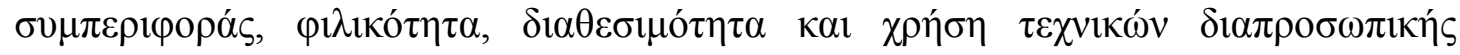

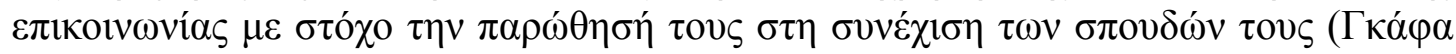

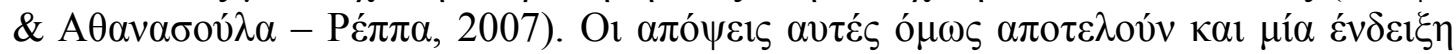

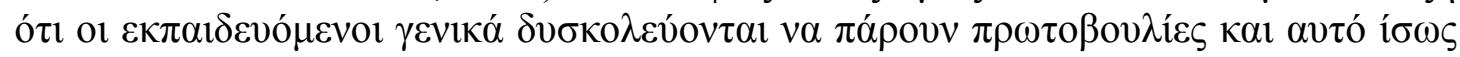

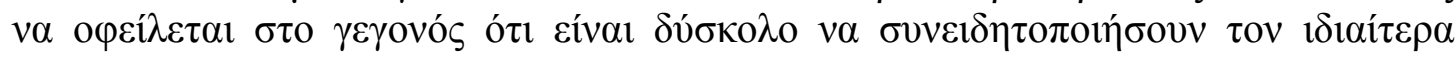

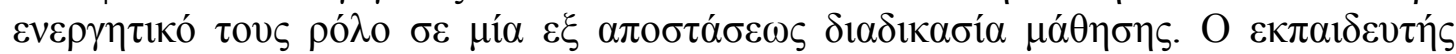

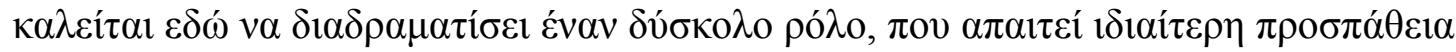

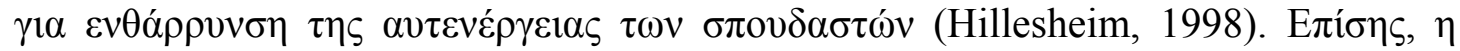

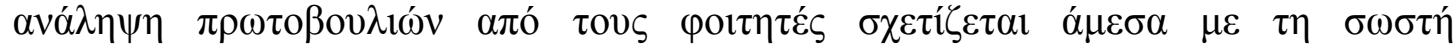

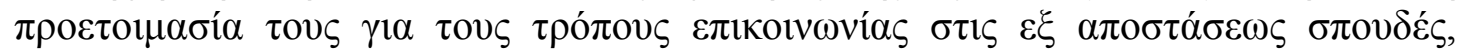

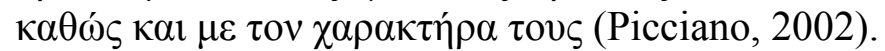

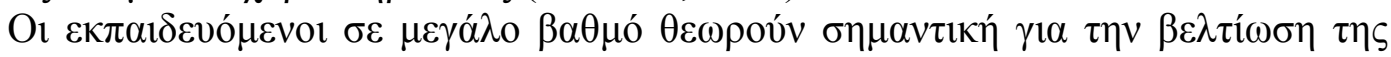

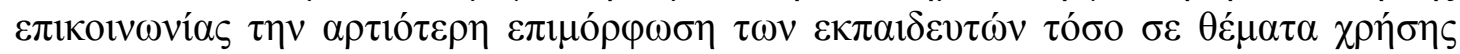

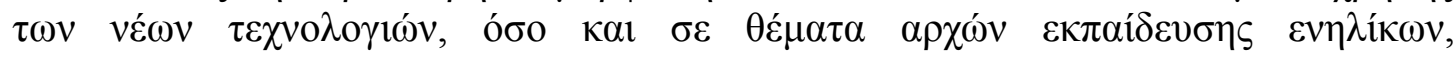

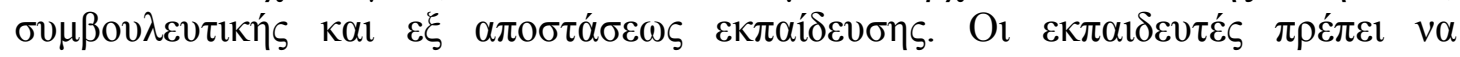

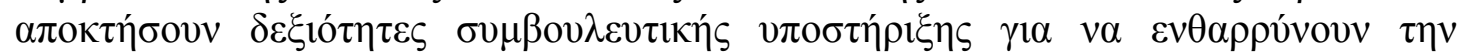

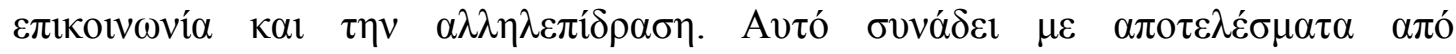

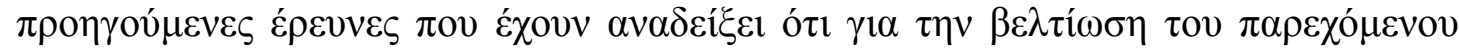

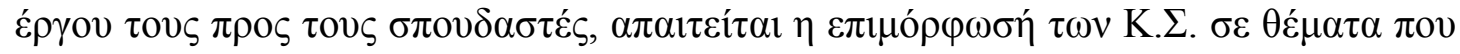

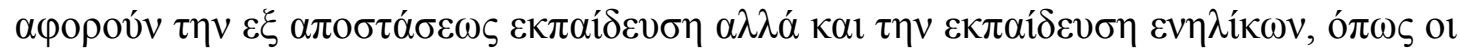

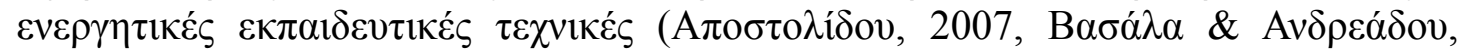

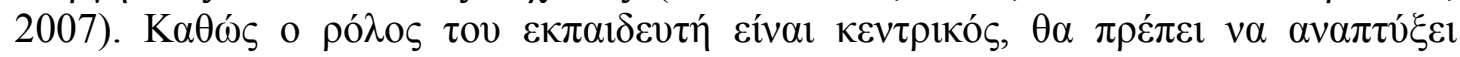

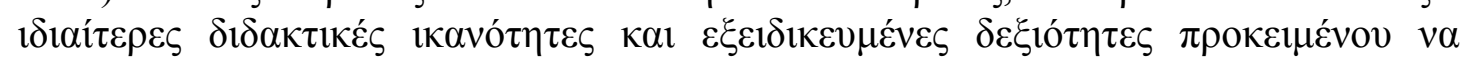

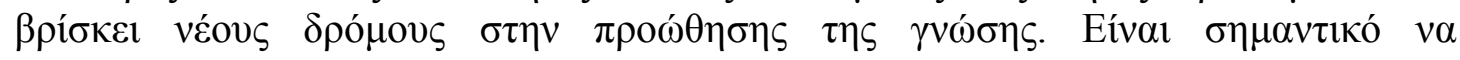

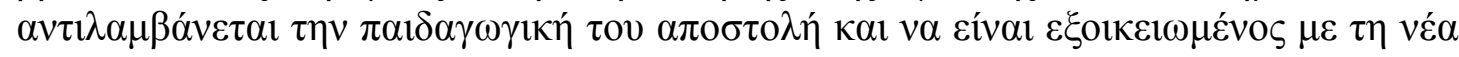




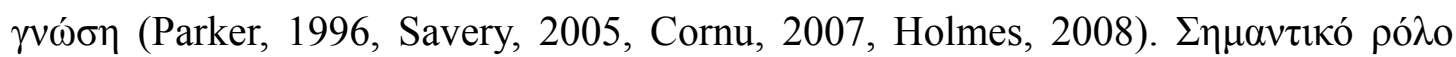

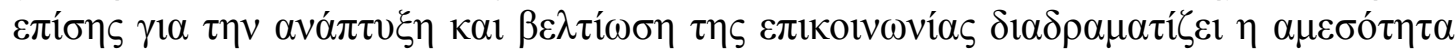

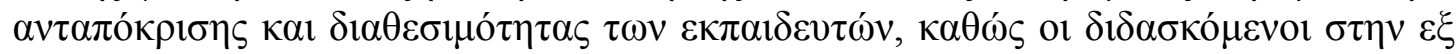

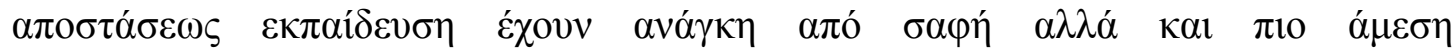

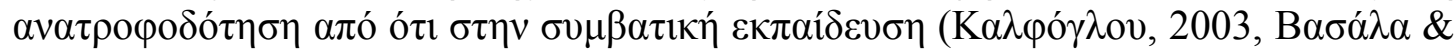

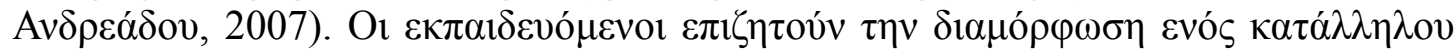

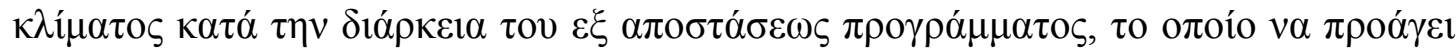

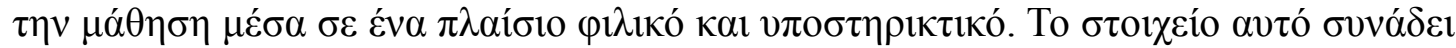

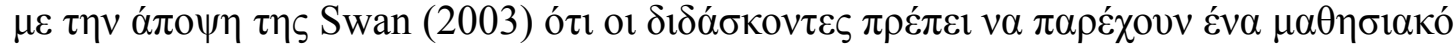

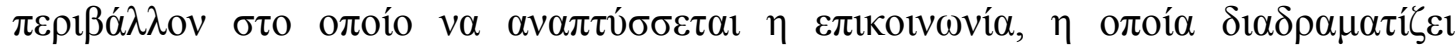
$\alpha \pi \circ \varphi \alpha \sigma \imath \sigma \tau 1 \kappa o ́ ~ \rho o ́ \lambda o ~ \sigma \tau \eta \nu ~ \varepsilon \kappa \pi \alpha i ́ \delta \varepsilon v \sigma \eta ~ \alpha \pi o ́ ~ \alpha \pi o ́ \sigma \tau \alpha \sigma \eta$.

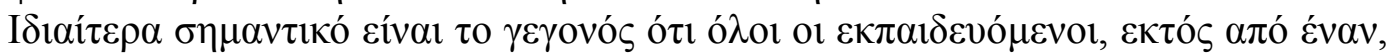

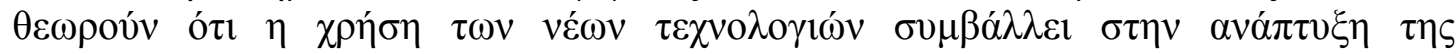

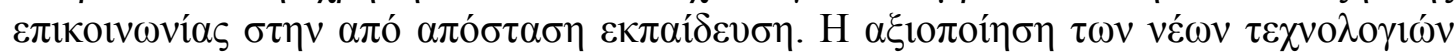

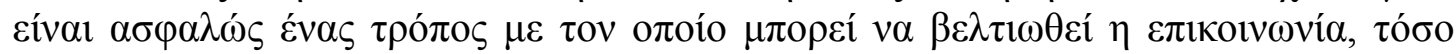

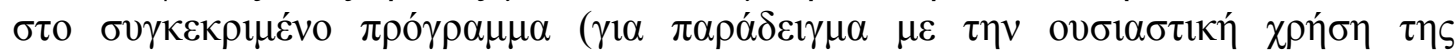

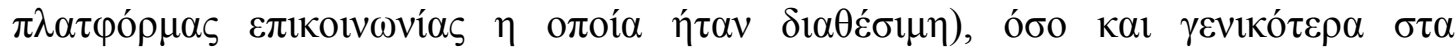

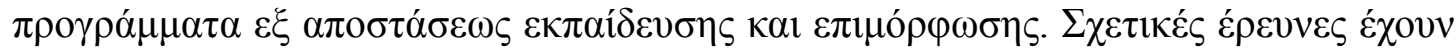

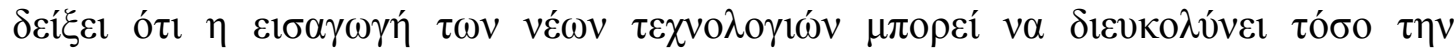

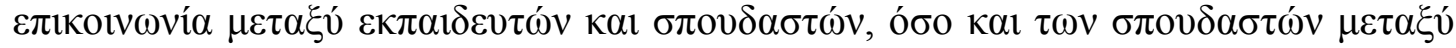

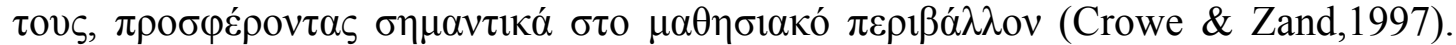

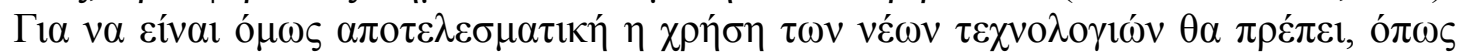

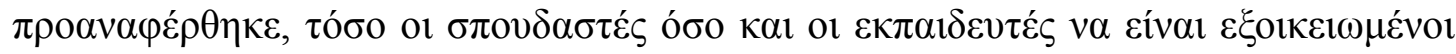

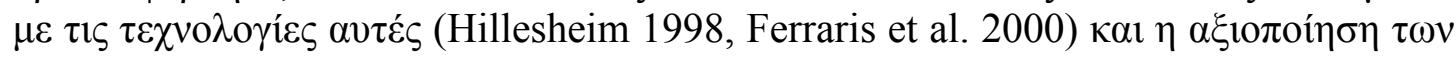

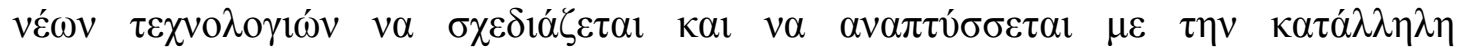

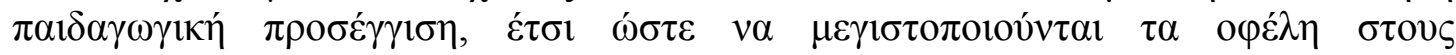

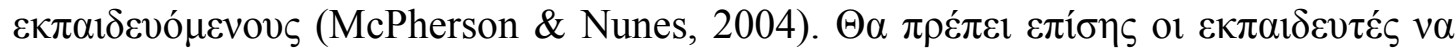

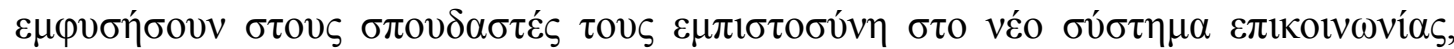

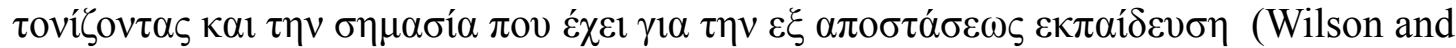

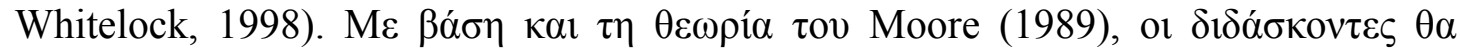

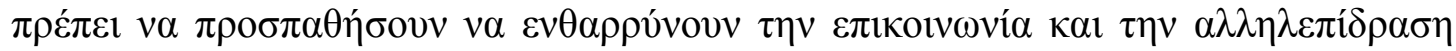

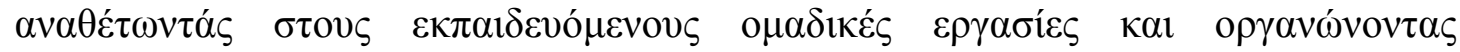

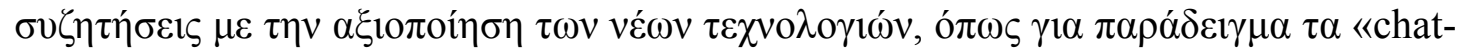
rooms» (Kelsey \& D'souza, 2004).

\section{5. $\Sigma v \mu \pi \varepsilon \rho \alpha ́ \sigma \mu \alpha \tau \alpha$}

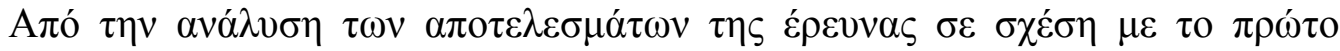

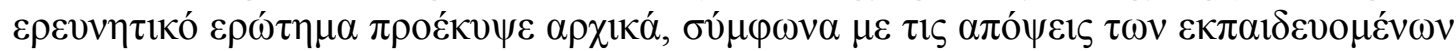

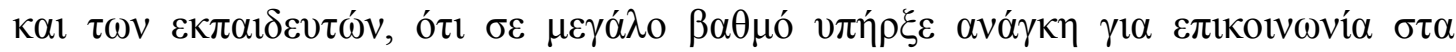

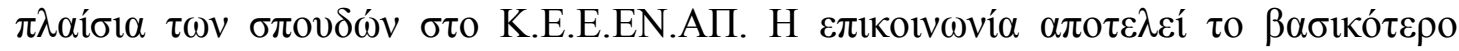

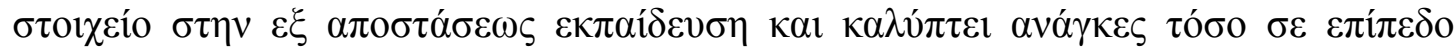

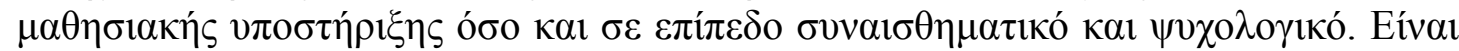

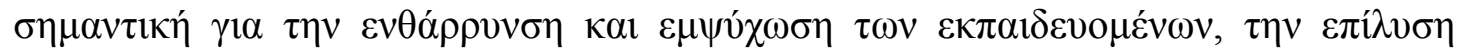

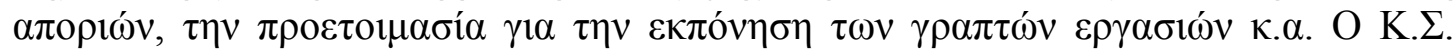

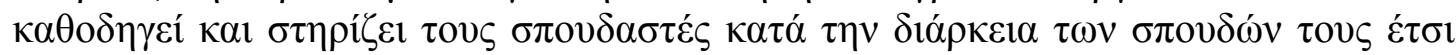

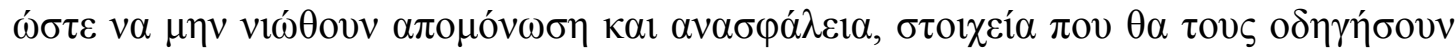

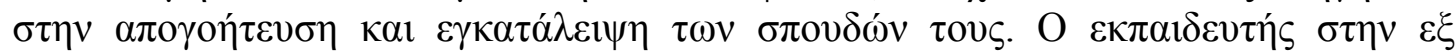

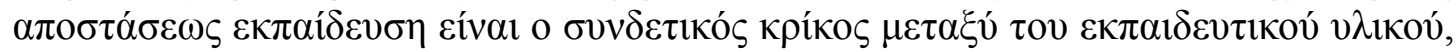

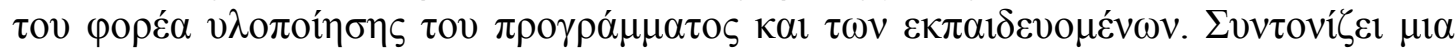

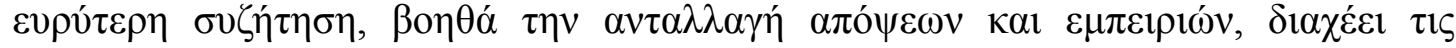




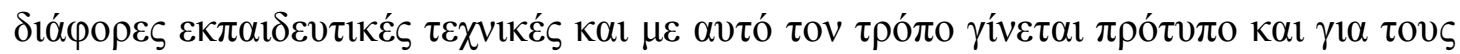

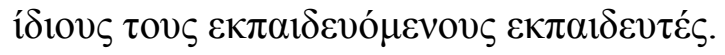

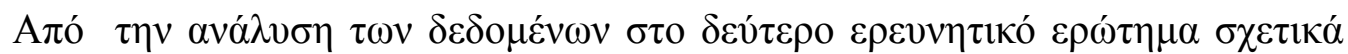

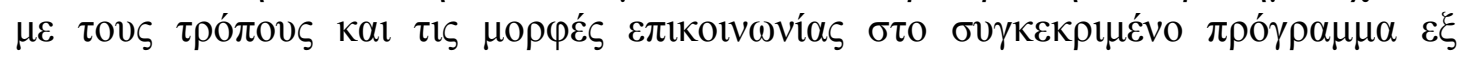

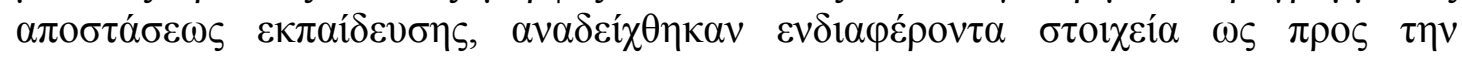

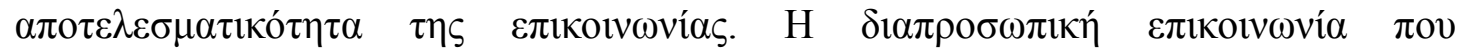

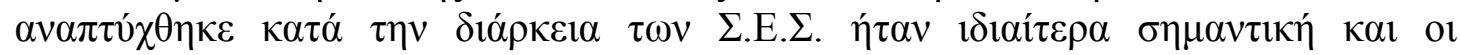

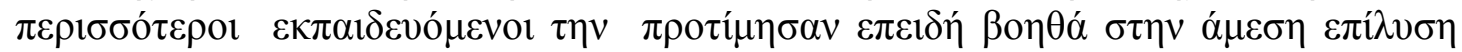
$\tau \omega \nu$ a

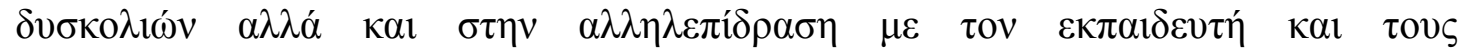

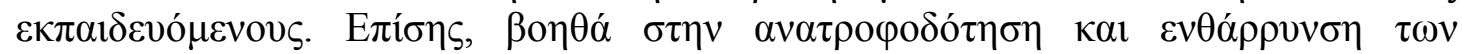

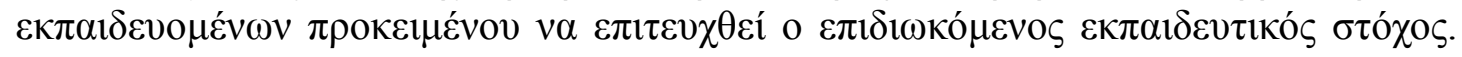

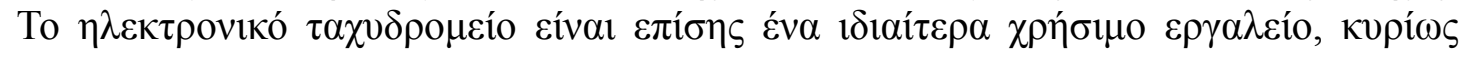

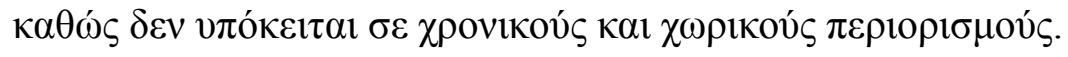

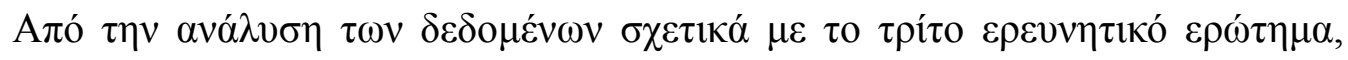

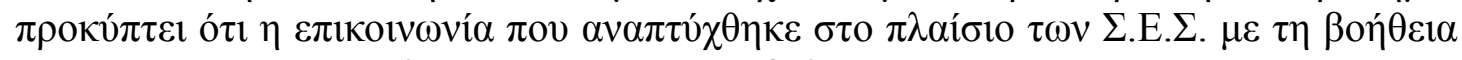

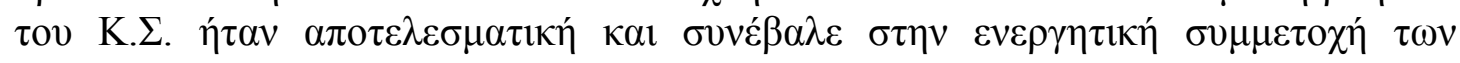

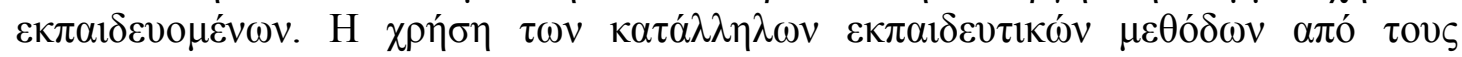

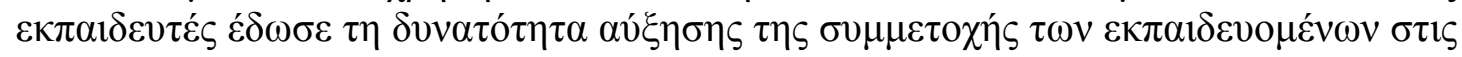

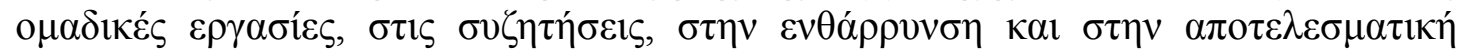

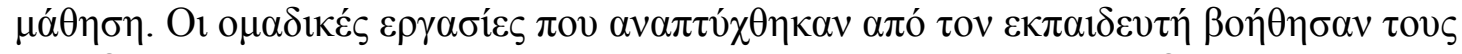

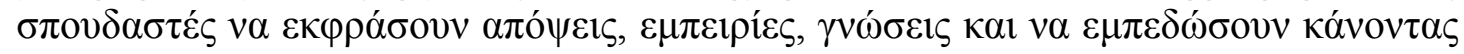

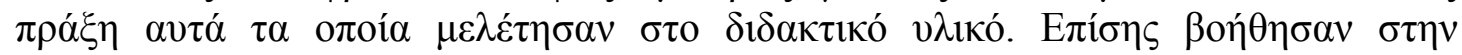

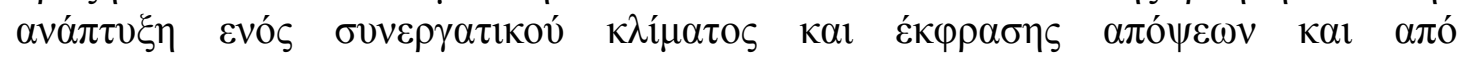

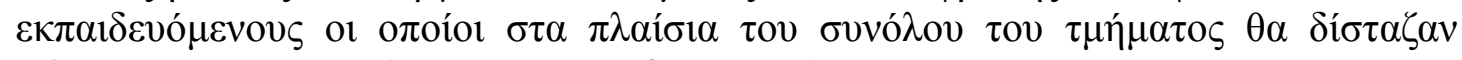

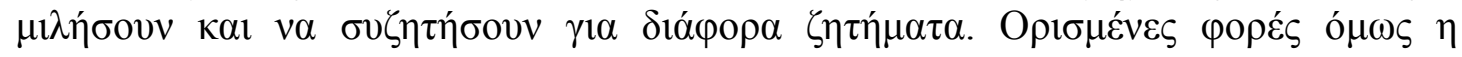

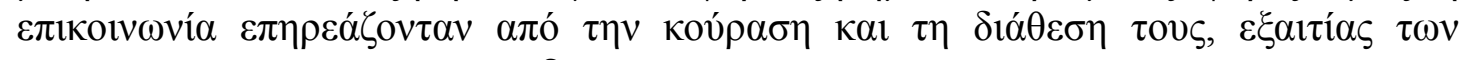

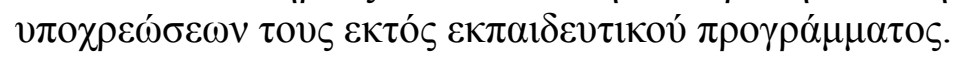

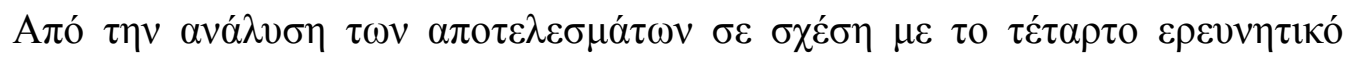

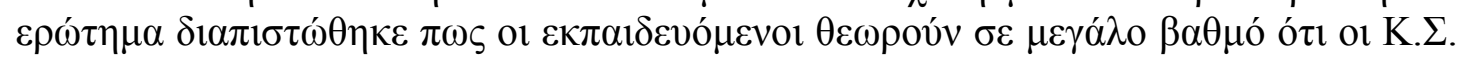

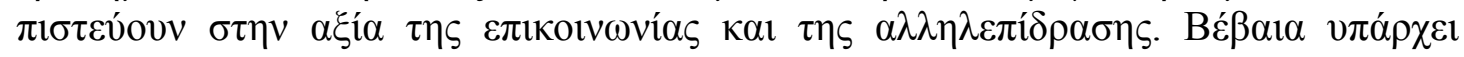

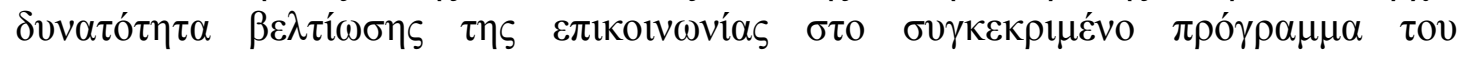

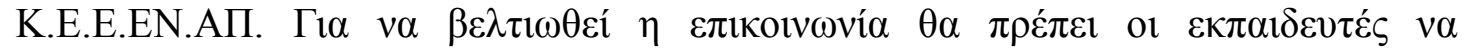

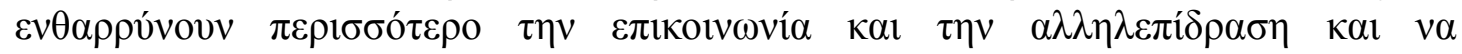

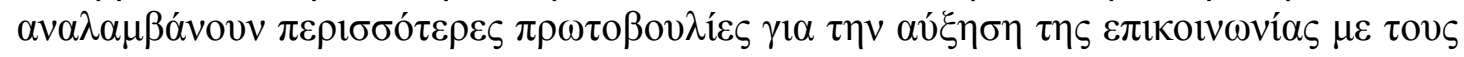

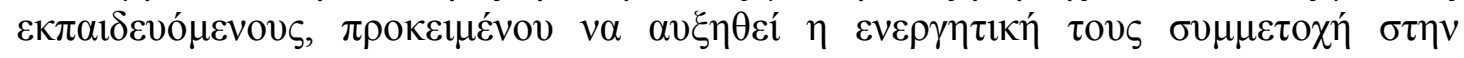

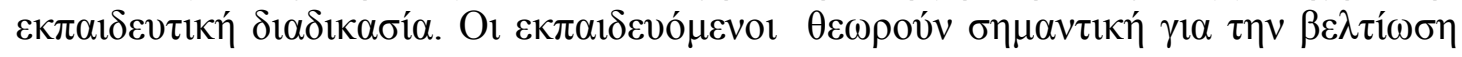

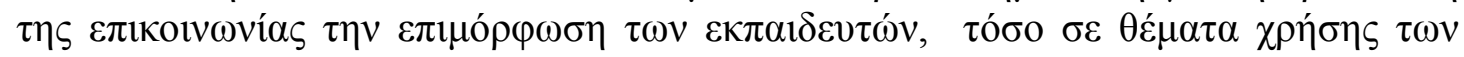

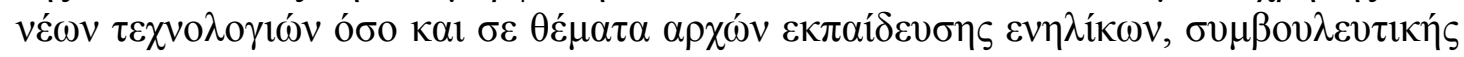

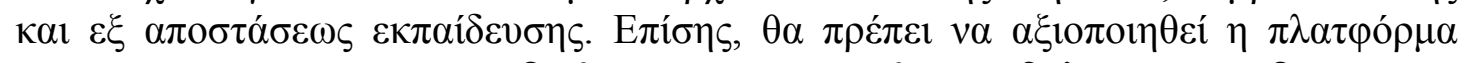

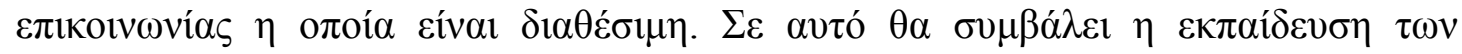

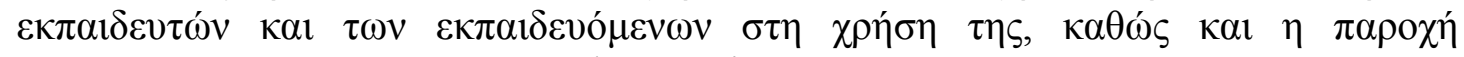

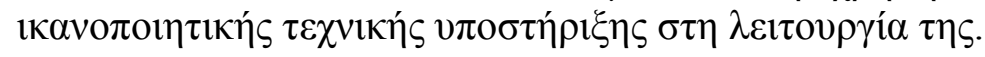

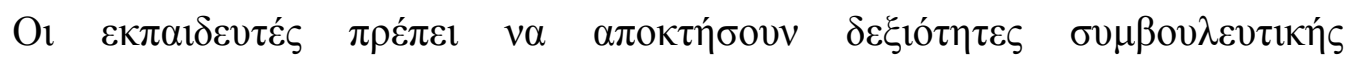

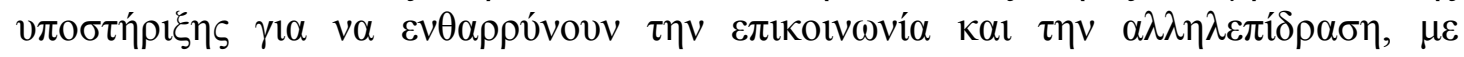

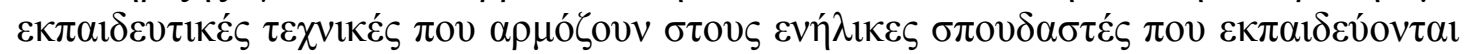

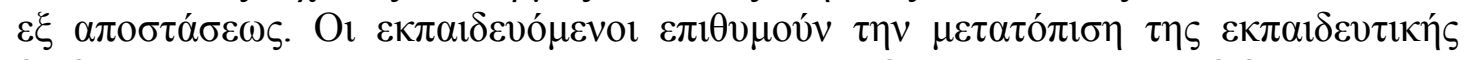

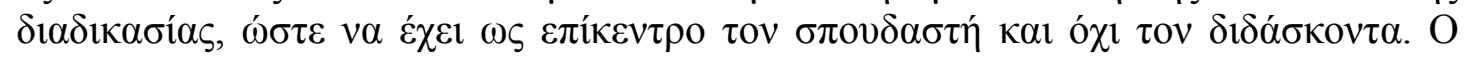




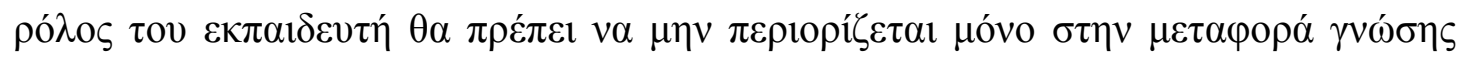

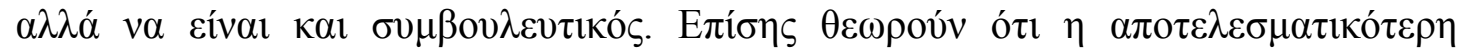

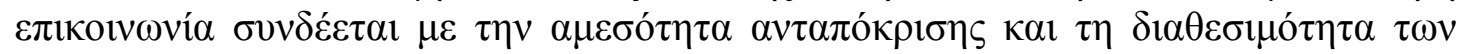

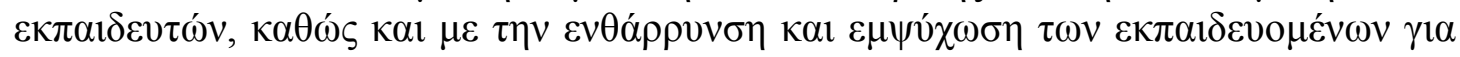

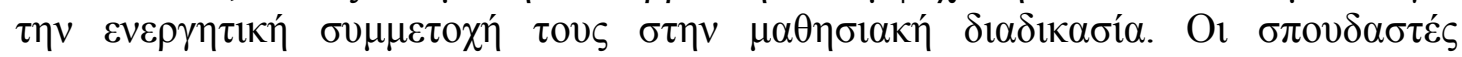

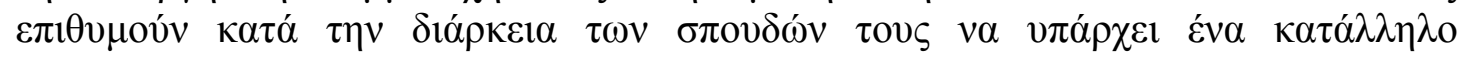

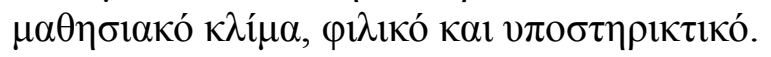

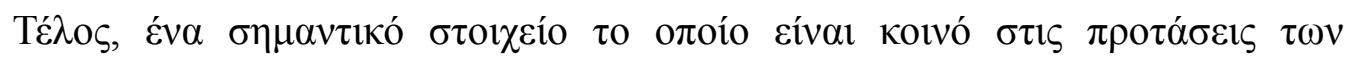

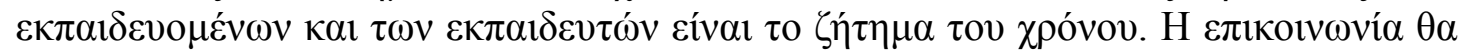

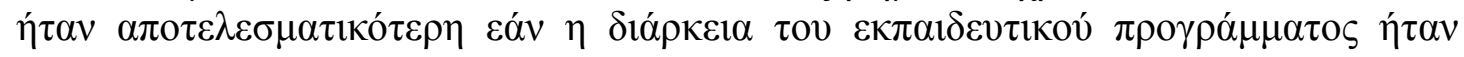

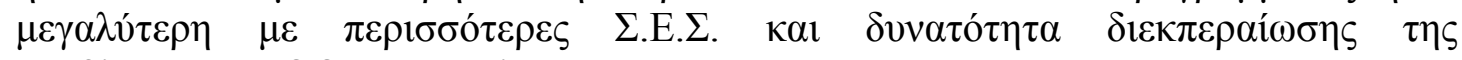

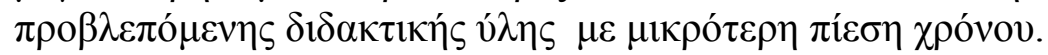

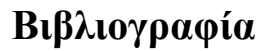

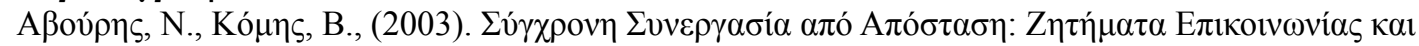

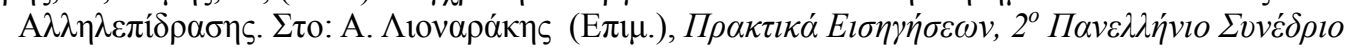

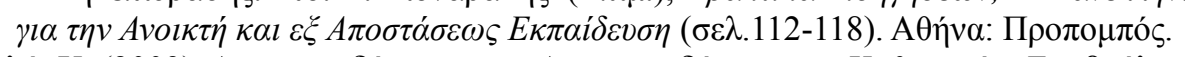

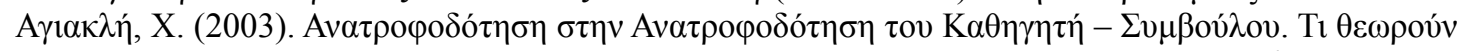

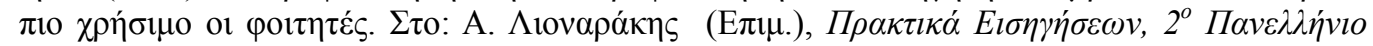

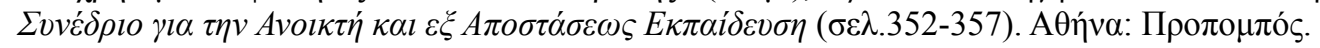

Anderson,T., Rourke,L., Garrison,R., \& Archer,W. (2001). Assessing Teaching Presence in a Computer Context. Journal of Asynchronous Learning Networks, 5(2). Retrieved 10/11/2008 from http://www.sloan-c.org/publications/jaln/v5n2/pdf/v5n2 anderson.pdf

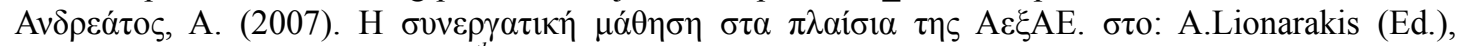

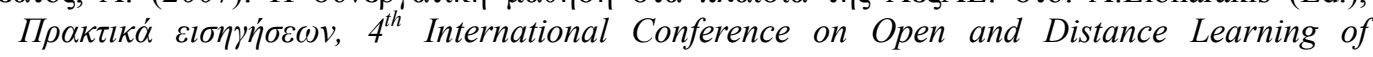

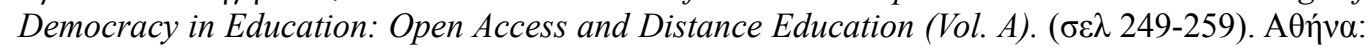
Пролонто́s.

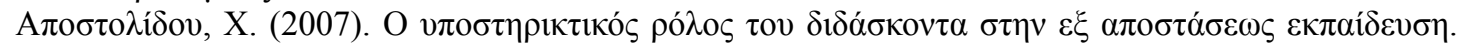

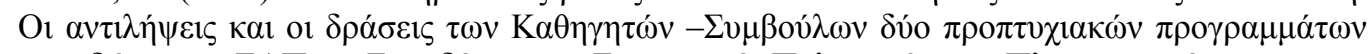

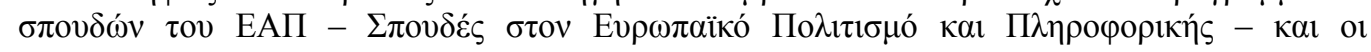

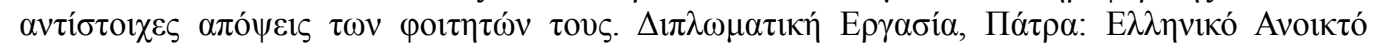

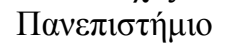

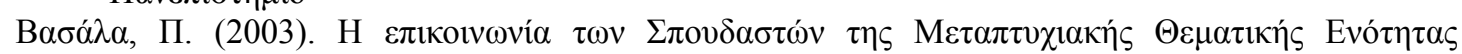

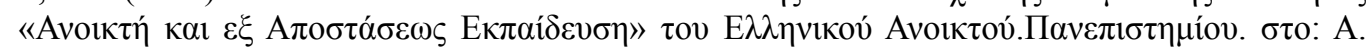

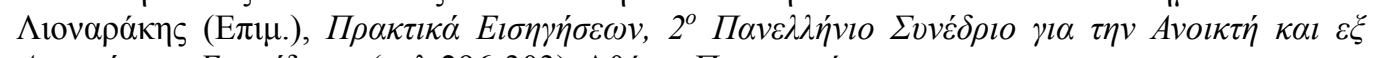

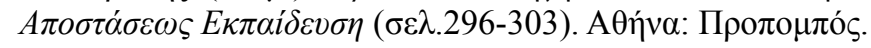

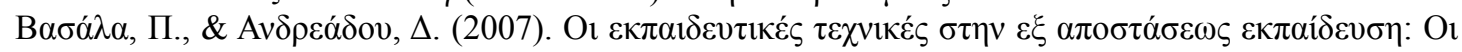

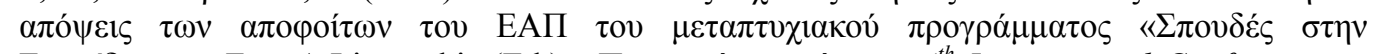

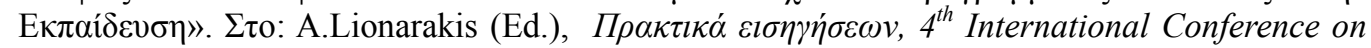
Open and Distance Learning of Democracy in Education:Open Access and Distance Education

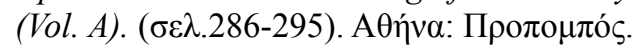

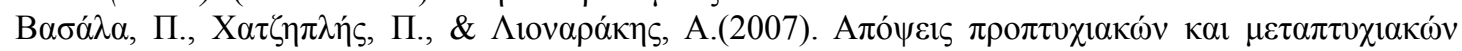

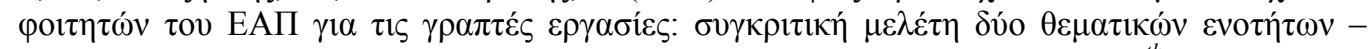

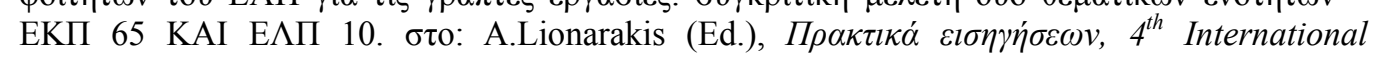
Conference on Open and Distance Learning of Democracy in Education: Open Access and

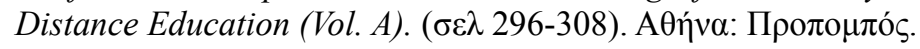

Blazquez, F.E., \& Alonso, L.D. (2006). A training Proposal for e-Learning Teachers. European Journal of Open, Distance and E-Learning Retrieved 13/11/2008 from http://www.eurodl.org/materials/contrib/2006/Blazquez_and_Alonso.htm

Boulton,J. (2002). Web-Based Distance Education: Pedagogy, Epistemology and Instructional Design. Retrieved 27/11/2008 from http://www.usask.ca/education/coursework/802papers/boulton/

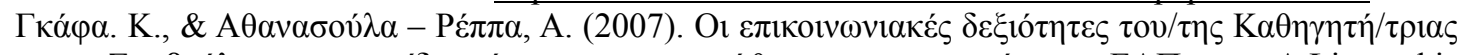

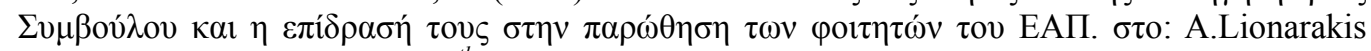

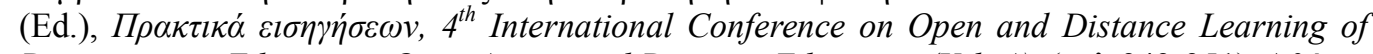

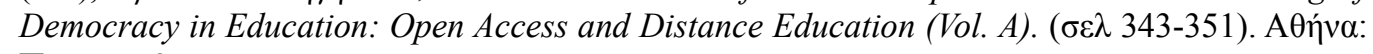
Пролонто́s. 
Cekerol, K., Torpak, E., \& Ozkanal, B. (2007). The Planning and Application of a Communication Model for Quality and Effective Learning in Distance Education. Proceedings, EADTU's $20^{\text {th }}$ Anniversary Conference "International courses and services online: Virtual Erasmus and a new generation of Open Educational Resources for a European and global outreach". 8-9 November, Lisbon, Portugal. Retrieved 29/10/2008 from http://www.eadtu.nl/conference-2007/files/SAA6.pdf

Cornu,B. (2007). New Media and Open and Distance Learning: New Challenges for Education in a Knowledge Society. Informatics in Education, 6(1), 43-52. Retrieved 10/11/2008 from http://www.mii.lt/informatics in education/pdf/INFE095.pdf

Crossen, T. (2004). Distance Education as Communication System. Retrieved 13/11/2008 from http://www.pde.state.pa.us/able/lib/able/fieldnotes04/fn04adistancesys.pdf

Crowe, D., and Zand, H. (1997). Novices entering mathematics-I. The impact of new technology, Computers and Education, 28 (1), 43-54.

Dhanarajan, G. (1996). Distance Education: Status and Issues. In: Proceedings of the Regional Seminar on Capacity Building in Distance Education for Training of Primary School Teachers. Bangkok.

Diaz, L. \& Blazquez,E. (2005). The Role of Virtual Teacher: How to Use Properly CommunicationTools. Proceedings, International Conference on Multimedia and ICT in Education, Lisbon, Portugal. Retrieved 22/11/2008 from http://www.formatex.org/micte2005/149.pdf

Doug, V. (2002). Distance Learning: Promises, Problems, and Possibilities. Online Journal of Distance Learning Administration, 5(3). Retrieved 10/11/2008 from http://www.westga.edu/ distance/ojdla/fall53/valentine53.html

Ferraris, M., Manca, S., Persico, D. and Sarti, L. (2000). Managing the change from face-to face to distance training for SMEs, Computers and Education, 34, 77-91.

Hillesheim, G. (1998). Distance Learning: Barriers and Strategies for Students and Faculty, The Internet and Higher Education, 1(1), 31-44.

Holmes,D. (2008). Social Presence Created in Distance Education. University of West Georgia. Retrieved 09/11/2008 from http://debbieholmes.files.wordpress.com/2008/05/literature-reviewproject.pdf

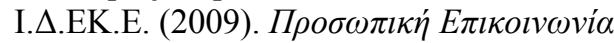

Johnson, E. J., \& Card, K. (2007/2008). The Effects of Instructor and Students Immediacy Behaviors in Writing Improvement and Course Satisfaction in a Web-based Undergraduate Course. Mountainrise, 4(2). Retrieved 10/11/2008 from http://mountainrise.wcu.edu/archive/vol4no2/html/johnson-card.pdf

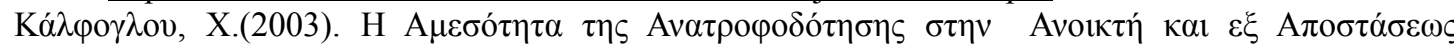

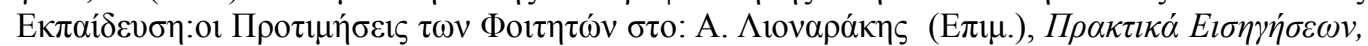

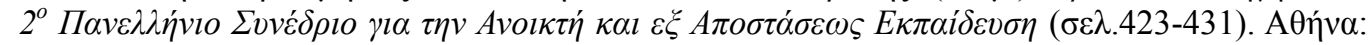
Пролонло́s.

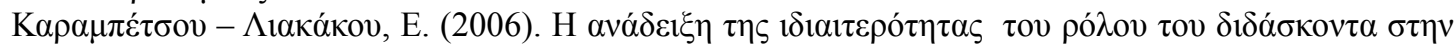

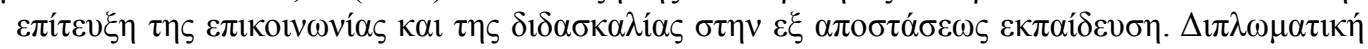

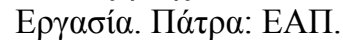

Kelsey, K. and D'souza, A. (2004). Student Motivation for Learning at a Distance: Does Interaction Matter?, Online Journal of Distance Learning Administration, 7(2). State University of West Georgia, Distance Education Center.

Kiriakidis, P. (2007). Online Learner Satisfaction: Learner -Instructor Discourse. Proceedings, $12^{\text {th }}$ annual TCC Worldwide Online Conference: Voyaging into a new era!. Retrieved 11/10/2008 from http://etec.hawaii.edu/proceedings/2007/kiriakidis.pdf

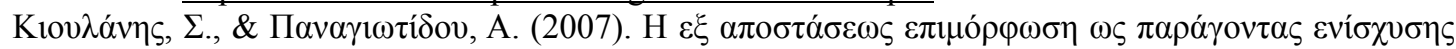

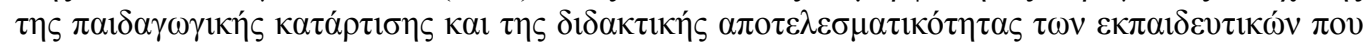

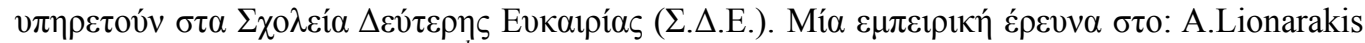

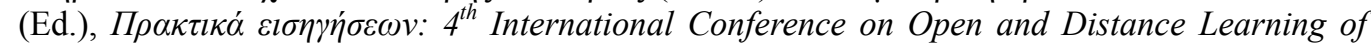

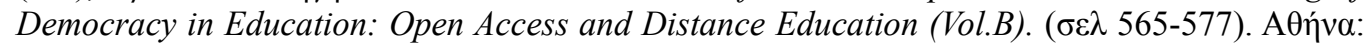
Пролонло́s.

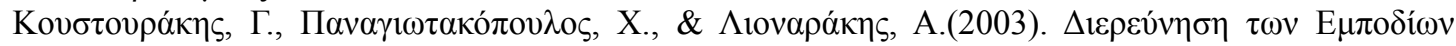

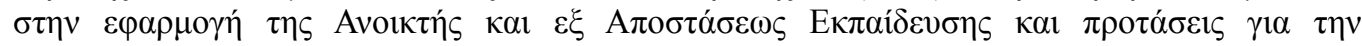

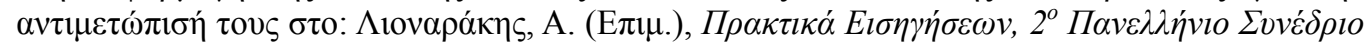

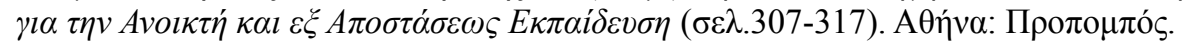

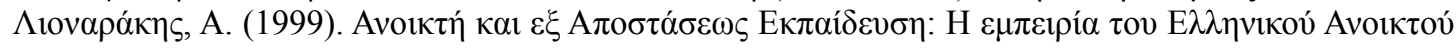

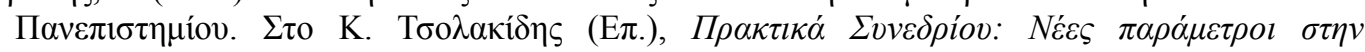

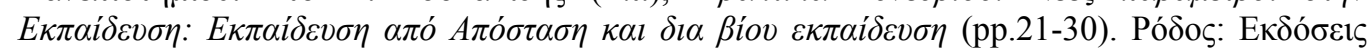

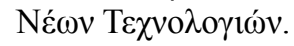




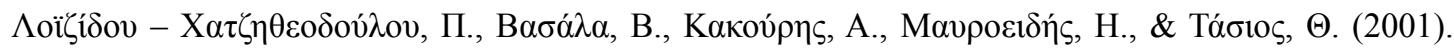

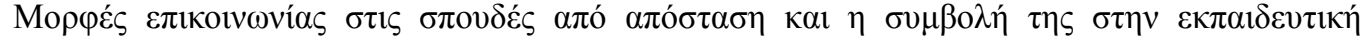

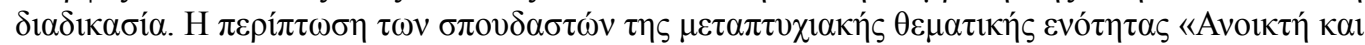

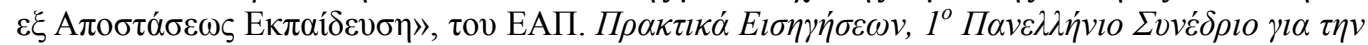

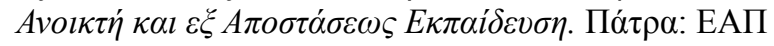

Levitch, S., \& Milheim, W. (2003) Transitioning Instructor Skills to the Virtual Classroom. Retrieved 30/10/2008 from http://www.acslearning.com/EdTecnArticle.pdf

Manning, R.D., Cohen, M.S., \& DeMichiell., R.L. (2003). Distance Learning: Step by Step. Journal of Information Technology \& Education, 2. Retrieved 26/10/2008 from http://jite.org/documents/Vol2/v2p115-130-96.pdf

McPherson,M.,\& Nunes,M.B. (2004). The Role of Tutors as an Integral Part of Online Learning Support. European Journal of Open, Distance and E-Learning. Retrieved 20/10/2008 from http://www.eurodl.org/materials/contrib/2004/Maggie_MsP.html

Moore, G.M. (1989). Editorial: Three types of interaction. The American Journal of Distance Education, 3(2). 1-6. Retrieved 10/11/2008 from http://www.ajde.com/Contents/vol3_2.htm\#editorial

Morgan, A. (1993). Improving your student's learning. Reflections on the experience of study. London, Kogan Page.

Nchindila, B. (2007). The Role of the Tutors in the Teaching of Online English for Business Programmes in South Africa: A Case Study. Journal of Online Learning and Teaching, 3(4). Retrieved 14/11/2008 from http://jolt.merlot.org/vol3no4/nchindila.htm

ONeil,D.G. (2006). How Distance Education has Changed Teaching and the Role of the Instructor. Retrieved 12/11/2008 from http://www.g-casa.com/download/ONeil_Distance Education.pdf

Parker,A. (1996). A Distance Education How-To Manual. Recommendations from the Field. Retrieved 12/10/2008 from http://ad.informatik.uni-freiburg.de/bibliothek/proceedings/webnet96/Html/340.htm

Parrott, S. (1995). Future Learning: Distance Education in Community Colleges. ERIC Digest No. ED385311.

Picciano, A.G. (2002). Beyond Students Perceptions: Issues of interaction, presence and performance in an online course. Journal of Asynchronous Learning Networks, 6(1). Retrieved 14/10/2007 from http://aln.org/alnweb/journal/vol6 issue1/picciano.htm

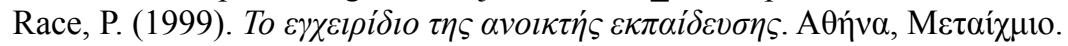

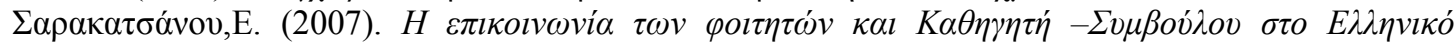

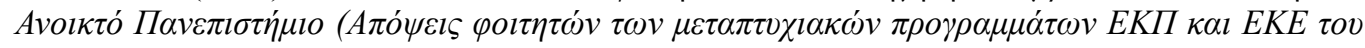

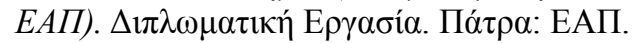

Savery,J. (2005). BE VOCAL: Characteristics of Succesfull Online Instructors. Journal of Interactive Online Learning, 4(2). Retrieved 15/11/2008 from http://www.ncolr.org/jiol/issues/PDF/4.2.6.pdf

Smaldino,S. (1996). Effective Techniques for Distance Education Instruction. Proceedings of the Conference Frontiers in Education 1996, Salt Lake City, Utah. Retrieved 23/10/2008 from http://fie-conference.org/fie96/papers/225.pdf

Swan, M. (2003). Distance Delivery: Impact on Learning. AITC Consortium. Retrieved 17/11/2008 from http://agclassroom.org/consortium/pdf/ag literacy/2003/distance delivery.pdf

Tait, A. (2003). Reflections on Student Support in Open and Distance Learning. The International Review of Research in Open and Distance Learning, 4(1). Retrieved 15/11/2008 from http://www.irrodl.org/index.php/irrodl/article/viewArticle/134/214

Trentin, G. and Scimeca, S. (1999). The roles of tutors and experts in designing online education courses, Distance Education, 20(1), 144 - 161.

$\mathrm{Tu}, \mathrm{C.H}, \&$ McIsaac, M. (2002). The relationship of social presence and interaction in online classes. The American Journal of Distance Education, 16(3), 131-150.

Wigforss, E.(1999). The Role of Communication in Learning Technologies.Retrieved 21/10/2008 from http://www.ascilite.org.au/conferences/brisbane99/papers/wigforss.pdf

Willis, B. (1992). Strategies for teaching at a distance. ERIC Digest No. ED 351008.

Wilson, T. and Whitelock, D. (1998). What are the perceived benefits of participating in a computermediated communication environment for distance learning computer science students?, Computers and Education, 30(3/4), 259-269. 


\title{
DIGITAL RADIO BROADCAST: NEW TECHNOLOGICAL RESOURCES TO PRODUCE EDUCATIONAL PROGRAMS ONLINE ${ }^{1}$
}

\author{
Teixeira, Marcelo Mendonça \\ Universidade do Minho, Portugal \\ Doctoral Student in Educational Technology \\ marcelo.uminho.pt@gmail.com
}

Silva, Bento Duarte Universidade do Minho, Portugal Associate Teacher Doctor bento@iep.uminho.pt

\begin{abstract}
The radio as a vehicle of mass communication has undergone many changes over the years through the development of informatics and cybernetics. The process of digitization suffered by conventional broadcasters and the availability of its content on the Internet, produced the latest step in the recent history of media - the Web Radio. In turn, the education has been used in the new technological resources to produce educational programs multidisciplinary in several areas of knowledge and in different parts of the world. Therefore, the present educational-communicative paradigm requires a new way of thought about the pedagogic models and new intervention strategies in society, which are able to respond to contemporary educational and intervenient processes. In this sense, the radio has been a great ally for Education for over one hundred and fifty years, taking the information and knowledge to the most inhospitable places. Nowadays, with a web format, the radio makes available, in virtual space, an ample group of technological interfaces with an alternative and complementary environment of teaching-learning. Appears, therefore, learning through the Internet radio, providing the virtual space and a set of interfaces for technology that teachers can disseminate their scientific work, suggest readings, stimulate debate on issues related to discipline (forums discussion), information notes, reviews, interviews with local experts and invited, promotion of academic events (congresses, seminars, lectures, conferences or meetings), lessons in podcast, beyond the possibility of synchronous communication with the network, through email, blog, facebook, forum, messenger, chat, myspace, twitter and others. In this communication, we will make a reflection about the web radio, showing its present panorama as an educational-communicative media and highlighting the case study of the Rádio Universitária do Minho (RUM).
\end{abstract}

Key-Words: Radio Web, E-Learning, Collaboration, Multimedia Sharing, Interactivity.

\footnotetext{
${ }^{1}$ Text produced under the individual program Doctor Foundation for Science and Technology of Portugal (FCT), integrated in the Center for Research in Education (CIED) - University of Minho. Project: Information Society, Innovation and educational processes.
} 


\section{Introduction}

The virtual environments can be considered as one of the factors driving the transformation of mass media, associating new forms in network communication and making the information more accessible to any person that has access to the worldwide network of computers, promoting the production and dissemination of information in science and technology for teaching and research in all areas of knowledge and different sectors of society. According to Castells (2009), it is fundamental to think of communication as a necessary element of sociability, understanding the act of communicating from its symbolic nature and inherently cultural. Lévy (2010) adds to that, asserting that the Collective Intelligence is a determining factor in competitiveness, creativity and human development in a knowledge based economy, or in an information economy in the Network Society.

Communications technologies allow for the annihilation of distance and for globalization; the potential for rapid, synchronous and asynchronous communication also changes the relationship to time (Castells, 2000a). This is because communication technologies, such as the Internet, allow for decentralization of operations and focusing of control, increasing the effectiveness of networks relative to hierarchical structures (Castells, 2000b).

However, it was the ability to connect these technologies together, giving rise to such networks, which expanded and integrated the individual and groups into a wider setting and new standards of globalization. As regards education, it is considered that this new configuration allows communication to expand the territory of the local school to deterritorialized areas of knowledge, enabling viewing school as a true learning community (Silva, 2002).

Commenting on the link between information and learning, Pinto (2002, p.185) concludes that "information is present, more and more complex and frequent, in the environment surrounding the learner. His/her perceptions build in a structured manner (education) an informational model that he/she uses to survive in this very environment". When it comes to mass media that develop sociocultural activities of informal and non-formal education, they almost always include formal programs when oriented directly to the school's curriculum. That is the case of school and college web-broadcasted radio stations, which have an informative nature, but are very much biased towards formativeness, establishing mixed-type educommunicative configurations.

The educommunicative paradigm online requires a new way of thought about the pedagogic models and new intervention strategies in society, which are able to respond to contemporary education. The radio as a vehicle of mass communication has undergone many changes over the years through the development of informatics and cybernetics. The process of digitization suffered by conventional broadcasters and the availability of its content on the Internet produced the latest step in the recent history of media: The web radio. In turn, the education has been used in the new technological resources to produce educational programs that are multidisciplinary in several areas of knowledge and in different parts of the world, with a proposal of rupturing from the traditional educational model based on a linear transmission of knowledge. 
Hence, the main activity yet to be developed by educators is to advise educational institutions on the use of new technologies as a didactic support, promoting and spreading their educational applications inside and outside classrooms. That is the case of web radio representing a valuable space for the popularization of information, education and the socialization of culture, and that can be accessed at anytime and anywhere in the world.

In this sense, we will make a reflection on the web radio presenting its present panorama as an educational-communicative media and highlighting the case study of the Rádio Universitária do Minho (RUM).

\section{Methodology Adopted in the Investigation}

Given the characteristics of the study, the research methodology used was of qualitative, descriptive and interpretative, using the technique of content analysis (analysis of programs online), and random samples by the application of a questionnaire survey with 203 students from different courses at the University of Minho on their perceptions about the web radio (the research was conducted on the Campus of University of Minho).

As techniques and instruments for data collection: Information survey on the web (analysis of university web radios in Portugal); document analysis (through the technique of content analysis - program grids); the observation of portuguese university radio programs, and the analysis of the formative tendency of Rádio Universitária do Minho (RUM). The data collection was processed in the second quarter of 2009, and the treatment of data's was made at this time by using at statistic program SPSS 17.0.

We also used the classification of radiophonic categories from Filho (2003), which could be categorized in: Journalistic (note, news, bulletin, reporting, interview, comment, editorial, chronicle, journalistic documentary, round-tables or debates, police program, sportive and techno-scientific programs); Educative-Cultural (autobiography, educative-cultural documentary, thematic program); Entertainment (musical program, fictional program and artistic event); Publicity (spot, jingle, testimonial, promotion piece); Advertising (public action radio-phonic piece, electoral and religious programs); Service (notes of public utility and service); Special (children's program, varieties program).

\section{Results}

\subsection{The concept of Web Radio}

The web radio can be defined as the radiophonic emission on the Internet in real time, usually in audio formats (MP3 or MP4, OGG Vorbis, WebPlayer, Real Audio, Windows Media Audio and HE-ACC). Different from traditional radio, your transmission could be followed by images, videos, texts, pictures and links. This advance allows the listener to do much more than just listen, making communication much more dynamic. Currently, it is possible to conduct online education, offering didactic material in PDF files or Word documents, video, podcast, and have access to 
up-to-date information through the feed RSS, clear up doubts with the instructor / educator through messenger, e-mail, chat, twitter, forums, as well as the interactivity in real time, through audio-conference or video-conference. It is about the combination of various elements: Ubiquity; flexibility; low cost; emission in real time; synchronous and asynchronous communication; multi-directed connectivity; multimedia sharing; streaming; collaboration and the interactivity integrated with elearning.

Often, the online reproduction of hertz signal through codification in the personal computer, through streaming, reproduces the emission on the Internet. The data is sent from personal computer packages for audio, video, text, images for Internet, which are stored on the platform online and made available to the public, which has access to a range of interactive resources. To Lee (2005) and Geller (2007), the web radio is nothing more than digital radio with support of the Internet, which allows the presence of audio, video, text, and promoting the emergence of new genres and new forms of interaction.

Thus, the main differences between traditional radio and Internet radio are the ways of accessing radio: By the computer; the flexibility of synchronous and asynchronous programming; geographical coverage (from local to global); the quality of emissions (without interference or noise), and active participation of the public. The user not only listens, but reads, writes and assists the programs of radio, having at their disposal a set of integrated interfaces. Finally, the interactive multimedia together with the audio in virtual environment is the essence of what has been called "web radio", which has the potential advantage of network, enriching its programming with multimedia content and additional resources, allowing a constant interaction transmitter-receptor (Perona Páez \& Veloso, 2007).

In some countries, the web radio is being used as an educational interface in virtual learning environments responsible for the divulging of various cultural activities on schools or universities, with programs dedicated to music, theater, cinema, education, science, technology, politic, poetry, literature, economy, news and transmission of popular festivals.

\subsection{University Web Radios in Portugal}

The first university radio in the world appeared on $19^{\text {th }}$ November 1923 in Argentina it was called the Radio Universidad. Since then, university radios followed up the evolutionary course of the common radio over the decades, representing the interests of the academic community. Such as web educational and training radio projects that emerged in the late 90's, the online broadcasters, brought up in the university departments, and the university radios began to supply a range of services and applications that did not exist till then. Unlike conventional radio, Internet radio immediately sparked the interest of the broadcasting companies, once it did not impose limits on the listener's geographical range, nor on the exclusive audio format programming.

It can be said that communication is an element which acts on individuals, as it is through it that people interact with each other, exchanging information. Therefore, understanding communication results in the perception of human relations, in a 
process involving individualities, stories, feelings, values and ways of perceiving the world, bringing on changes in the way society individuals feel, think and act.

In Portugal exists a reasonable number of academic broadcasters which are primarily characterized by a great range of varied programming, including numerous fields in which it is possible to find all kinds of genres and issues. In addition, university radios are broadcasters that foster radio-creation becoming themselves real alternatives to the general radio dominant content, as they offer a vast collection of "information, education, culture and entertainment". This radio stations are characterized by presenting a programmatic heterogeneous grid with respect to content, thus emerging as another option to the ordinary programming of the major conventional broadcasters. In fact, among the generalist portuguese radios, the Information and the Entertaining and Cultural Magazines hold $60 \%$ of the total offers, while sport only represents little more than $5 \%$. The remaining percentage is reserved for Music and participation spaces and, to a lesser extent, for Education and other minority genres $(35 \%)$, with only a sporadic presence on university radio stations. This programmatic uniformity becomes even more apparent if we bear in mind that the different networks choose to transmit the same contents at the same time schedule, affecting a vast majority of broadcasters, including those that have been managed in recent years. Along with a worldwide trend and according to researches carried out within this area, online radios should not be restricted to "generalist" functions, but should also be a combining tool with a complementary interface aimed at people's education, whether it is instructive or informative.

According to Teixeira (2009), the Rádio Universitária de Coimbra (www.ruc.pt), from Universidade de Coimbra; the Rádio Universitária do Marão (www.universidade.fm), from the Trás-os-Montes and Alto Douro Region; the Rádio Universitária do Algarve (www.rua.pt), from the Universidade do Algarve; the Rádio Universitária Beira do Interior (www.rubi.ubi.pt), from the Universidade Beira do Interior; the Radio Zero (www.radiozero.pt), from the Instituto Superior Técnico and the Rádio Universitária do Minho (www.rum.pt), from the Universidade do Minho, are the Portuguese University Web Radios. In global terms, it is possible to assert that they share similar objectives, but have different and heterogeneous structures and program typologies (Cordeiro, 2005).

These radios have not delayed in employing the technological resources of web radio, fact that can be sustained by the quantitative growth of online platforms, through which people can have access to the ordinary programming in real time by means of a great variety of devices connected to the Internet. We will proceed with the presentation of the results in accordance with the objectives of this research, now analysing the web radios in the Portuguese academic universes. For that purpose, we made a selection of some experiments using Perona Páez and Veloso's selection criteria of "Radio Educational Modalities" (2007).

\subsubsection{Structure of Operation}

The Rádio Universitária de Coimbra (RUC) exists since 1986 and was one of the first university radios in Portugal with web emission. Nowadays, it develops its activities based on the following categories: the formative, the informative, the academic and the cultural-educative. The formative category is established through periodic courses 
of formation and recycling for speakers, editors/speakers and technicians, besides the realization of didactic programs in collaboration with public and private institutions. The informative category is a space focused on the debate of questions related to the Universidade de Coimbra and to up-to-date news. About the academic life at the Universidade de Coimbra, the RUC dedicates an ample space in its program grid, transmitting the main occurrences of the learning institution. Finally, the cultural category is responsible for the divulging of various cultural activities going on in Coimbra, at the North Region or in the rest of the country, with programs dedicated to music, theater, cinema, poetry, literature and the transmission of concerts, shows and popular festivals.

The Rádio Universidade do Marão (FM Universidade) gave its first steps in the virtual world in 2000 (Cordeiro 2005), and since then the objective of the broadcasting station was to become the first university radio totally digital in Portugal. However, for technical reasons, this evolution to the web was only firmed in posterior years (Leão, 2007). Presently, the Rádio Universidade do Marão is focused on the academic public of the Trás-os-Montes and Alto Douro Region, with a purely informative focus.

In 2003, the Rádio Universitária do Algarve (RUA) arouse in a partnership between the Academic Association and the Universidade do Algarve. In the web, it has as an objective to divulge and promote the academic activities and cultural and musical events of the South Region in Portugal. Different from "RUC" and "RUA", it is grounded in three pillars to develop its activities: the Academy, the Culture and Alternative Music. The objective of the programs directed to the Academy is to show to the national Portuguese community the contributions that the Universidade do Algarve offers to the region and to the country, related to teaching and research. The Culture is centered on the promotion and divulging of cultural events that take place in the Algarve Region (popular festivals, concerts, shows, cinema, literature, theater). The Alternative Music programs promote the work of artists and bands unknown to the public in general and to the communication means.

Still in 2003 the Rádio Universitária Beira do Interior (RUBI) was created as a laboratory of the discipline radio-phonic Journalism at the Universidade Beira do Interior, only with an internal character. In 2004, the RUBI starts to emit its radiophonic program on the web, and started to be called "RUBIweb". But only in 2006 that the RUBI decided to bet on the diversity of categories in its program grid, aggregating multimedia interfaces to its web radio platform, such as the podcast and e-mail.

According to the Filho's (2003), radio-phonic classification of categories, Rádio Universitária Beira do Interior is exclusively generalist and informative, functioning as the "voice" of the Departments from the Universidade Beira do Interior, as well as of the local community, Covilhã.

The Radio Zero is part of the Association of the Instituto Superior Técnico (IST), with its headquarters in Lisbon (before 2006 it was called RIIST - Internal Radio of IST). Being originally a radio of university character, its objectives are centered in offering radio-phonic formation to the students interested in working in radio, as well as inform the "IST" academic community about the main events going on at the 
university, in a cultural, scientific and educative ambit (http://www.radiozero.pt/projecto/). It has more than 40 programs on its online program grid of journalistic, cultural-educative and entertainment character (in its majority).

Taking advantage of the Internet interactive potentials, university radio stations seem to demonstrate a certain sensitivity in ensuring the rights of access and participation, something that has been increasingly neglected in other communicative fields.

\subsubsection{The RUM}

The Rádio Universitária do Minho (RUM) exists since 1989, and since 2006 it started to transmit via web, with a clearly heterogeneous program offer, on which spaces of purely formative-instructive character are mixed with others that explore different categories and formats, closer to some ongoing experiences in Europe. According to Leão (2007), the RUM launched two crucial interfaces in the context of its strategy to conquer and gain the loyalty of new public: the website and the online emission. The consolidation of the online emission, particularly, revealed as an alternative to the "conventional receptors", emphasizing culture, debates on education, science, economy, politics, news, local informs, chronicles, interviews, and specialized reports.

In its relationship to the Universidade do Minho, the RUM makes available the virtual space and a group of technological interfaces for the lecturers to divulge their scientific works, suggest readings, stimulate the debate on themes related to their disciplines (discussion forums), to inform grades, tests, interviews, divulge local, national and international academic events (congresses, seminars, talks, colloquiums, meetings...), store lectures in podcast (in a way that the student can have access to the discipline contents in any part of the world), besides the possibilities of synchronous communication with the broadcasting station, through e-mail, blog, messenger, twitter, chat, facebook or myspace.

Besides, it is on the program grid that the RUM is most different from other Portuguese university radios, for its thematic diversity and of the programs dedicated to the educational-cultural and journalistic categories:

Caixa de Ferramentas (Tool Box) and the Diferença em $1^{\circ}$ Plano (Difference in $1^{\text {st }}$ Level) - debates and interviews and the promotion of specialized support services to attend the peculiarities of people with special needs;

Ciência para Todos (Science for All) and the Universidade Sem-Muros; (No boundaries University) at the Democracia Viva (Living Democracy) - promotion and divulging of the university's cultural and scientific activities;

Campus Verbal (serves as a radio-phonic laboratory, where the students from the Institute of Literature and Science of the Universidade do Minho, of French and German areas, produce radio programs based on what they have learned in the lecture rooms, and store the contents at the university's website in a podcast format;

Olhar no Feminino (View on the Feminine) - discusses themes related to the female world; 
Magazine da Educação (Education Magazine) and the Livros com RUM (Books with $R U M)$ - information and reflection on the Portuguese and international literary situation, with interview from critics, authors and specialists in literature;

Praça Município / Café com Blogs (Municipal Square / Coffee with Blogs) - debate about the Portuguese political scenario;

Eco RUM (Ecology RUM) - program focused on the protection and conservation of the environment;

Rumo Económico (Economic Route) - interviews and reports on the national and international economic panorama;

Cultura Impressa (Printed Culture) - the main topics of printed media are debated on the program;

Cultura Crónica (Chronics Culture) - program focused on stage arts, cinema, literature and shows;

Escola de Rádio (Radio School), where courses on the radio-phonic universe are developed (the courses are given by communication experts from the Rádio Universitária do Minho and lecturers of the Universidade do Minho), with more than 60 specialized programs divided by categories and for all public.

This is the Rádio Universitária do Minho, functioning as a social communication vehicle of local communities and as a valuable space for the divulgation, socialization and popularization of science and technology, produced by different departments at. According with the researcher Paula Cordeiro (2005), the contribution and influence of university radios in the development of the future professionals' formation, allied to the importance in the context of radio-phonic communication in general are incontestable, and, in a context where the main concern is the profit-making of the station, university radios appear as elements that offer alternatives of program and formation.

Based on a cooperative work, with interactivity among the participants and sharing of ideas, the interface "web radio" is presented as a mean through which the students feel an important and active part of the educational-communicative process. "This takes us to a proposal of rupture from the traditional educational model based on a linear transmission of discipline content, in which emission and reception are separate" (Fernandes \& Silva, 2004:379).

Therefore, the alternative nature of the analysed broadcasting companies is marked by the exploitation of contents that can hardly be found in other grids, such as programs dealing with the problem of social exclusion, those which make science and knowledge popular, as well as those that emphasize less commercial music or that bring together cinema, literature and art. On the other hand, it presents a wide range of educational programs, ranging from formal and informal classes to professional and technical training courses and languages, thus providing an alternative and complementary teaching and learning background (Perona Páez \& Veloso, 2007). 
For students, it represents an extension of knowledge and an interactive room outside the traditional classrooms, approaching Lévy's concept (1997) on "Cyberculture", or even, on the virtualization process society faces nowadays, in which the virtual is not opposed to reality, but it complements it (Lévy, 2010). Classrooms today are no longer the only standard teaching, still less the exclusive learning area. Geographical boundaries are diluted; the world today is interconnected by the simultaneity of the new information and communication technologies. Thus, it is necessary to understand the contemporary changes at a social, political and economic level and the way they affect human relations in all society spheres.

Fig. 1. - Cultura RUM Website

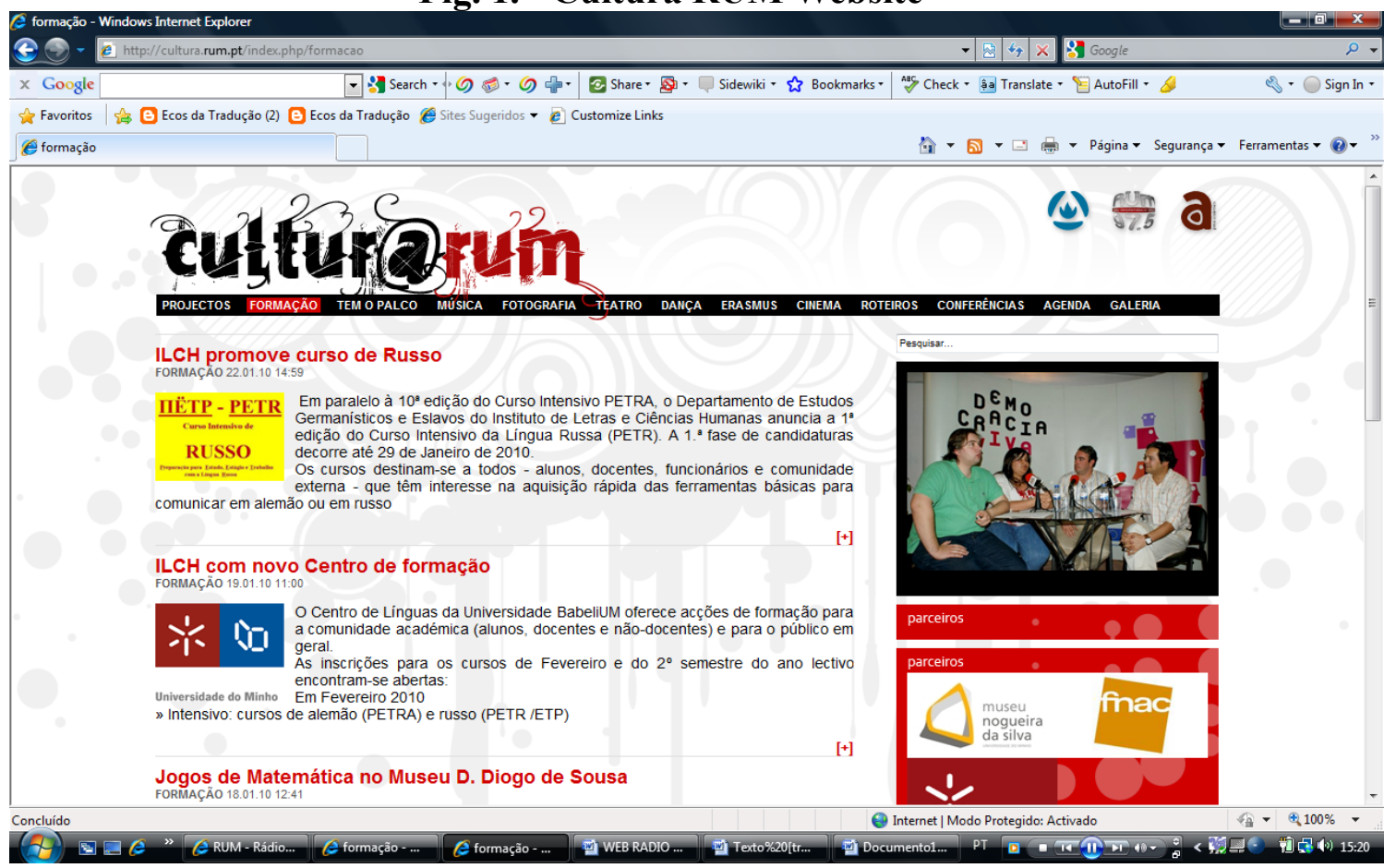

Font: (http://cultura.rum.pt/index.php/formacao).

As said by Díez (2009), the medias are instruments of public service, proving, at the same time, to be very useful in stimulating debate and in making easier for the students to understand the contents transmitted in the classroom. It is also reasonable to argue that teachers' new space and time perceptions, as well as those of the teaching and learning process as a whole, are of a crucial importance in establishing a school under new paradigms, achievable through the application of electronic age concepts, such as E-learning, Open School, University Without Walls $(U W W)$ and Social Web, i.e. the socialization phenomenon already taking place at a global scale in an environment of sharing experiences, information and online learning (Correia \& Tomé, 2007). In the contemporary context, exploring web radio educational potentials seems to be a new challenge for educommunicators.

According Perona Páez (2009), the university radios are not restricted to generalist or informative functions anymore, but figure as a complementary or alternative mean for people's formation.

\section{Conclusions}


The universities and schools through a web radio can provide educational programs in podcast with different themes for different courses or areas of knowledge, which will be available online and can be accessed at anytime and anywhere in the world. Through this technological resource, there is no possibility of losing the program if the person is busy, the programs are available online and can be accessed when necessary or possible.

Such tendency is present in the objectives of the Rádio Universitária do Minho, through its social, cultural, educative and formative activities, both on conventional and web formats. However, the understanding of the web radio as an educationalcommunicative media has been followed by some difficulties related to the international academic community, in face of the still restricted investigation about the potentialities of online radio. Besides that, due to the similarity of its basic characteristics, it is common for the student public to confound podcast with web radio.

Another question to be considered is the need to create its own identity as a means of mass communication on the web, once it comes from a traditional media format. Even though it is still in need of a solid methodological-theoretical basis, the use of the web radio as a formative interface has been expanding significantly in the world.

In general, we believe that the radio web use as an educational interface has being significantly expanded throughout the world, though there is still a lack of a solid theoretical and methodological basis. In this sense, it becomes evident the need to more fully investigate the teaching and learning process through the web radio in educational institutions. In the Portuguese case, more specifically, it is crucial to deepen studies on the academic radio educational and training potentials and their contributions to the country. This is this study main suggestion for future research.

Despite the abovementioned obstacles, any course or discipline in school or at university, made available in a virtual learning environment, could use the radiophonic program, or produce, in a local radio station, contents to be shared by the students. It is only question of believing in the potential of the radio on the Internet and its potentialities.

\section{References}

Castells, M. (2009). Communication power. New York: Oxford University Press.

Castells, M. (2000a). Materials for an exploratory theory of the network society. British Journal of Sociology Vol. No. 51 Issue No. 1 (January/March 2000) pp. 5-24.

Castells, M. (2000b). The Rise of the Network Society, Second Edition. U.S.: Blackwell Publishing.

Cordeiro, P. (2005). Experiências de rádio produzidas para e por jovens: o panorama português das rádios universitárias. Proceedings of the I Encuentro Iberoamericano de Radios Universitarias, 13-14 March 2005, Granada, pp.1-9.

Correia, C., Tomé, I. (2007). O que é e-learning. Lisboa: Plátano Editora.

Díez, D. (2009). Las rádios universitárias en España. Transformación al mundo digital. Telos - Cuadernos de Comunicación e Innovación, Julho-Setembro de $2009, \mathrm{n}^{\circ} 80$.

Fernandes, S., Silva, M. (2004). Criar e desenvolver uma rádio online na escola: 
Interatividade e cooperação no ambiente de aprendizagem, Revista da FAEEBA - Educação e Contemporaneidade, XIII (22), pp. 375-384.

Filho, A. (2003). Gêneros radiofónicos - Os formatos e os programas em áudio. São Paulo: Paulinas.

Geller, V. (2007). Creating powerful radio: getting, keeping and growing audiences. Burlington: Focal Press.

Lee, E. (2005). How internet radio change the world. New York: iUniverse.

Lévy, P. (2010). From social computing to reflexive collective intelligence: The IEML research program. Information Sciences, 180, 71-94.

Lévy, P. (1997). Cyberculture. Paris: Odile Jacob.

Perona Páez, J. (2009). Edu-webs radiofónicas: experiencias españolas de educación en medios. Comunicar, 13, Vol.XVII, Revista Científica de Educomunicación, 107-114.

Perona Páez, J., \& Veloso, M. (2007). Modalidades educativas de la radio en la era digital. Icono 14 - Revista de Comunicación Y Nuevas Tecnologías. Retrieved on April 2008 from http://www.icono14.net/revista/num9/articulos/08.pdf.

Silva, B. (2002). A Glocalização da Educação: Da escrita às comunidades de aprendizagem. In: O particular e o global no virar do milénio, Cruzar saberes em educação. Proceedings of the V Congresso da Sociedade Portuguesa de Ciências da Educação. Porto: Sociedade Portuguesa de Ciências da Educação, pp. 779-788.

Pinto, M. (2002). Práticas educativas numa sociedade global. Porto: Asa.

Teixeira, M. (2009). Análise da rádio web como uma interface dinamizadora da prática educativa: Estudo de Caso da RUM. Master Dissertation. Braga: University of Minho. 


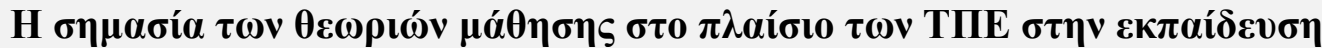 Learning theories importance in the framework of Information and Communication Technologies in education
}

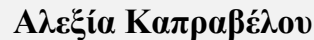

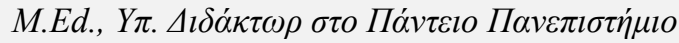
akaprave@gmail.com, akaprave@,sch.gr

\begin{abstract}
In this article psychological and pedagogical learning theories implicated in teaching with the use of computers are presented, critically examined, and compared. Behaviorism suggests hierarchically organized educational software, aim-centered, that points on content revision, unopened exercises, right answers, correction of mistakes, and that assesses students-computer users by injunctions, rewards-penalties, so that they would improve their knowledge. Cognitive contructivism's implications are the student-centered approach, de-inculpation of students' mistakes, modelization/simulation/microworlds, problem-solving, use and production of open inter-thematic software, utilization of previous learning, gradual spiral-learning, epagogic, experimental, self-directed, self-exploring learning. Sociocultural theory's implications are the group-collaborative environment, knowledge connection to praxis, holistic learning, interactive and authentic environments of apprenticeship and practice. Critical-emancipatory theorists' view across new technologies is objurgatory, as technologies are used by the dominant class for its own benefits and for increase of exploitation and subordination of non-privileged. Concerning that education is not socially neutral, the present paper is exploring questions, such as what are the pros and cons of every theory, how theories look alike or differ, what the profits are and who win from the new technologies today, how they are used for/against people. In conclusion, I shall try to prove that the future of new technologies that serve each human being belongs to socio-cultural and critical-emancipatory orientations.
\end{abstract}

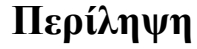

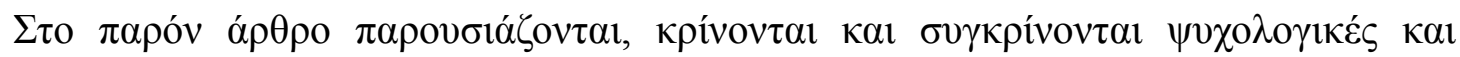

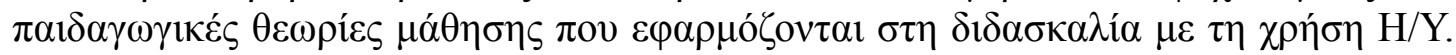

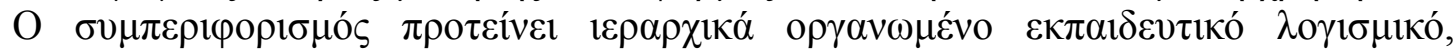

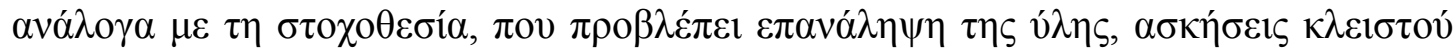

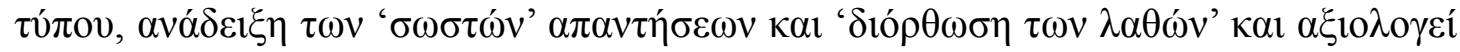

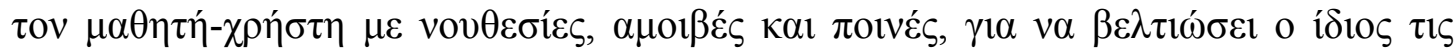

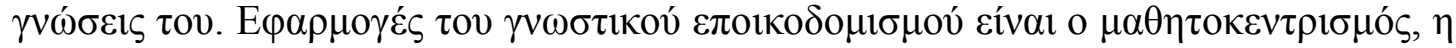

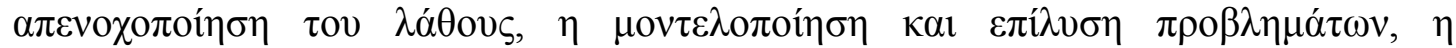

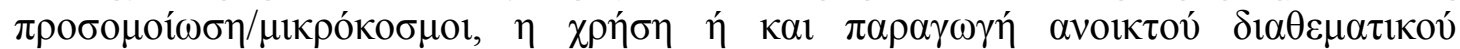

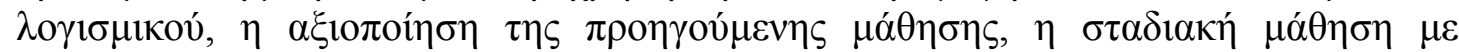

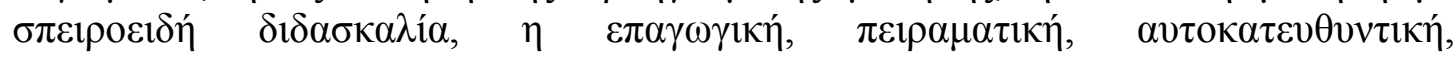

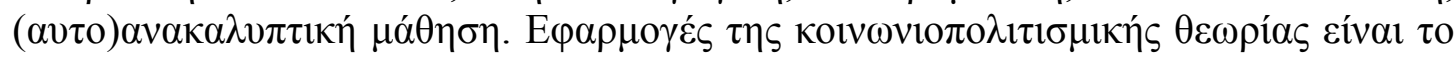

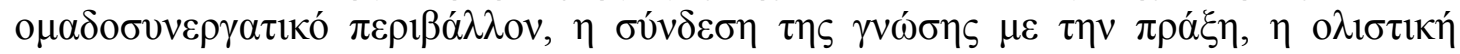

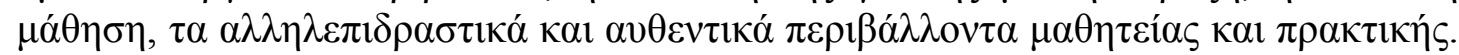

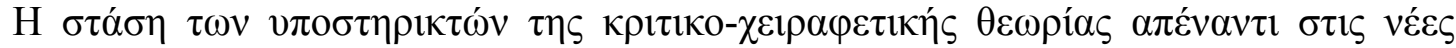

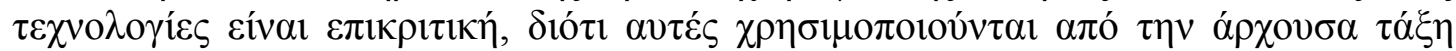

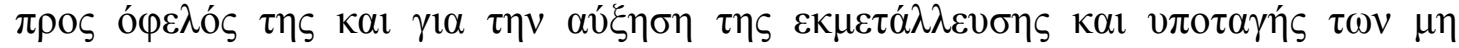




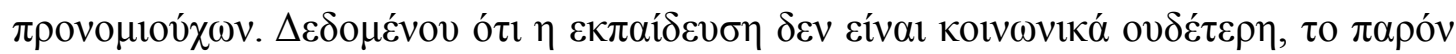

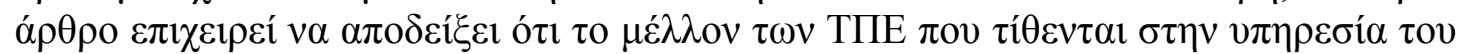

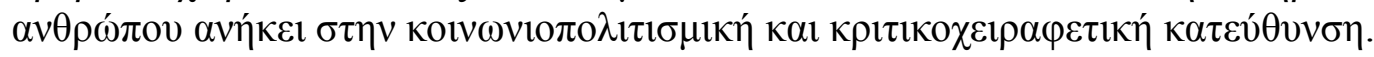

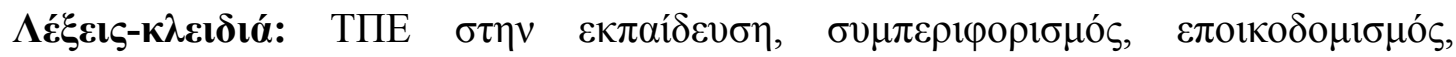

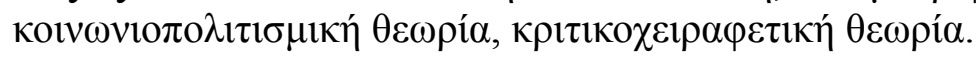

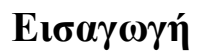

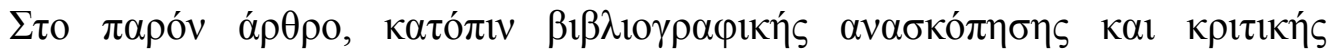

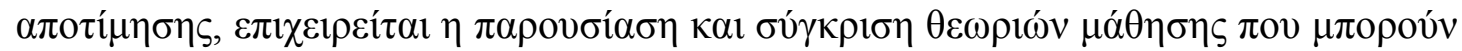

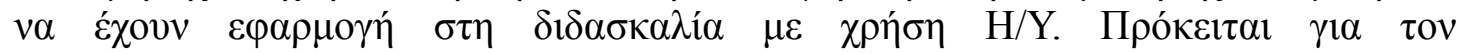

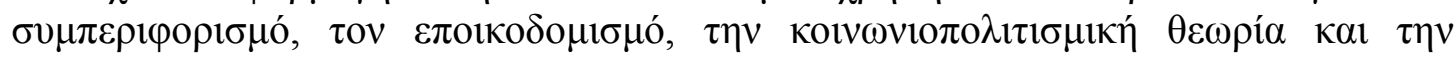

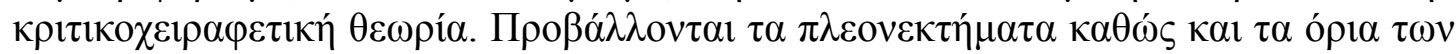

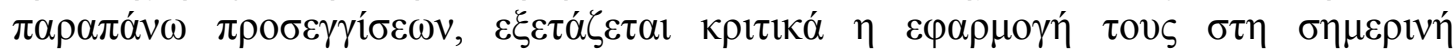

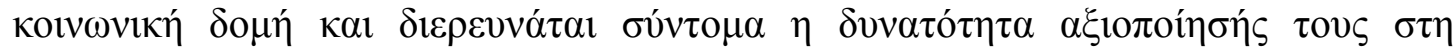

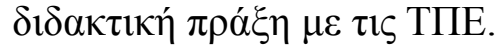

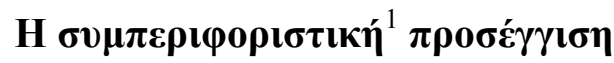

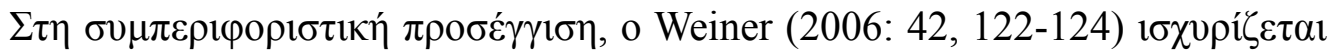

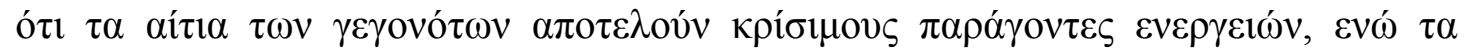

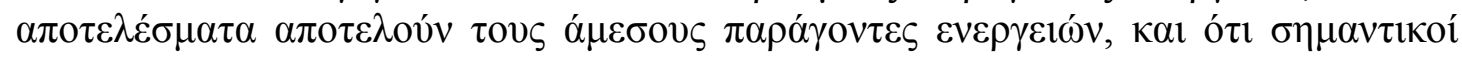

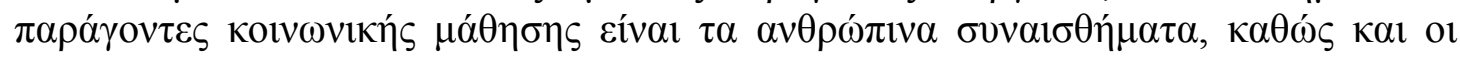

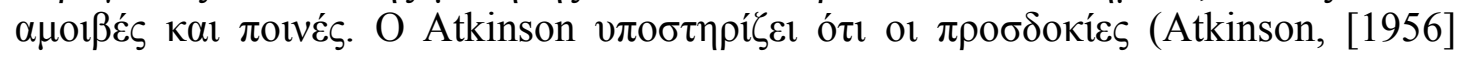

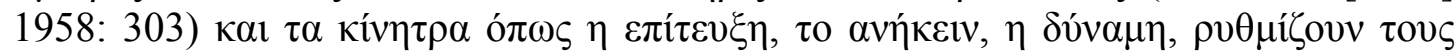

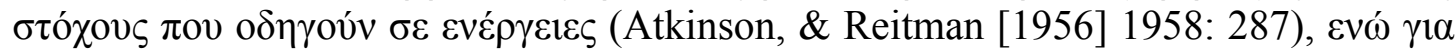

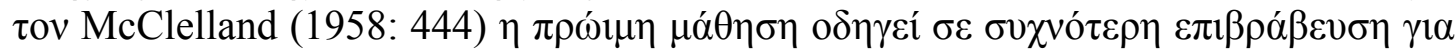

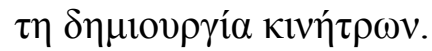

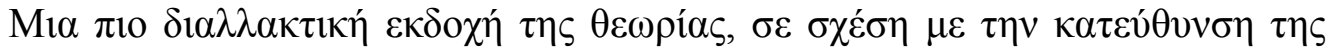

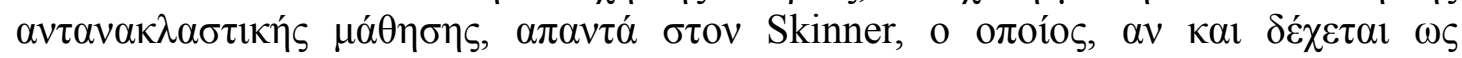

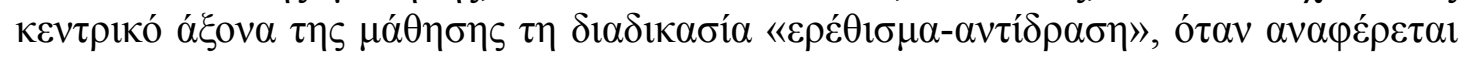

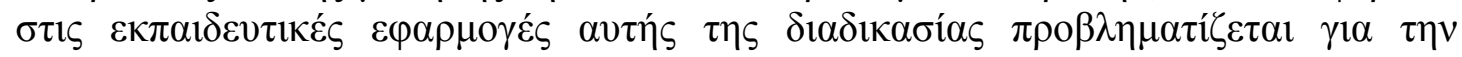

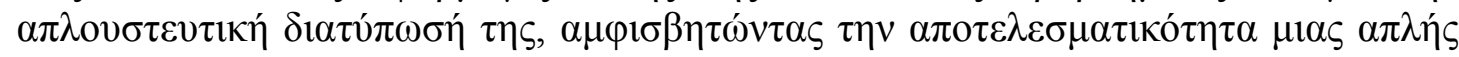

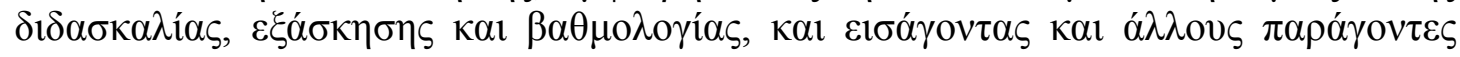

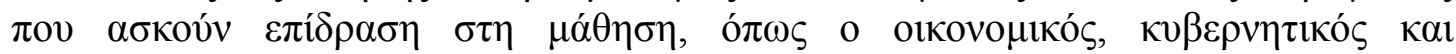

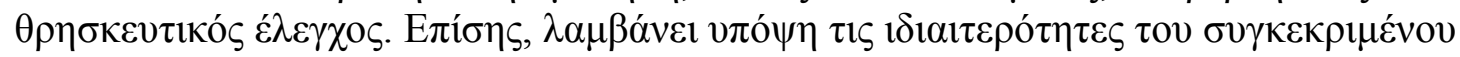

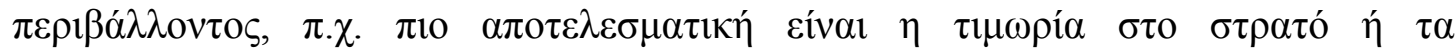

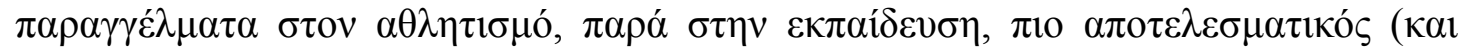

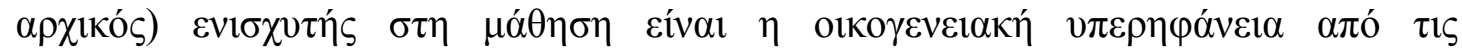

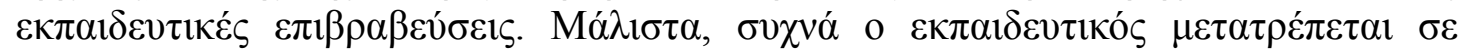

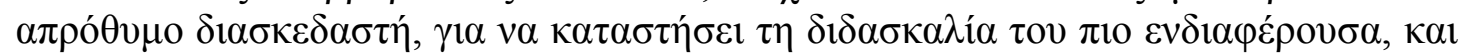

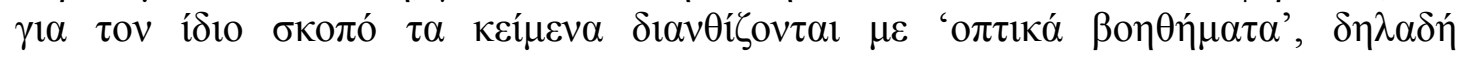

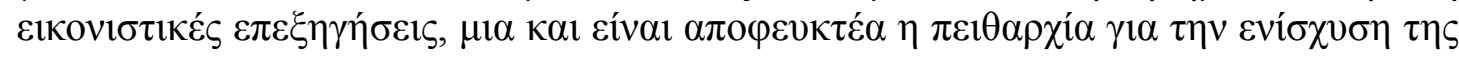

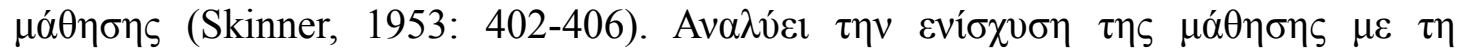

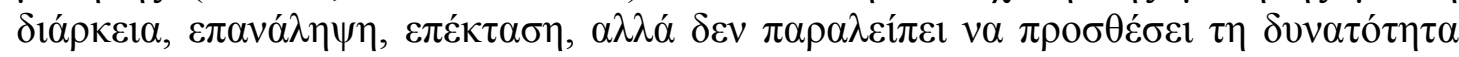

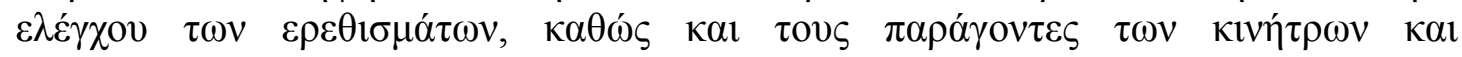

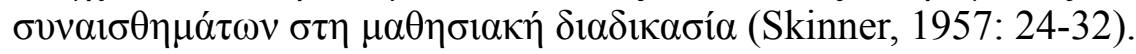

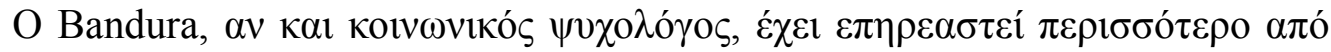




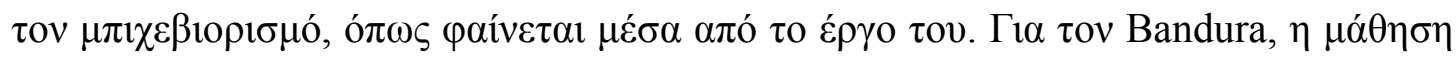

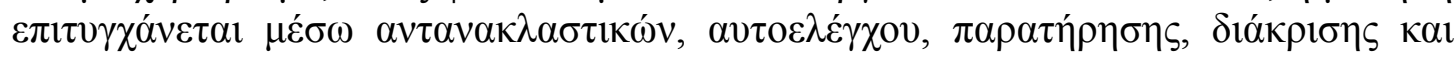

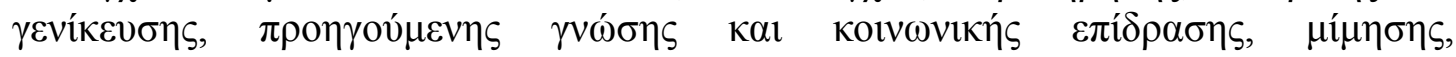

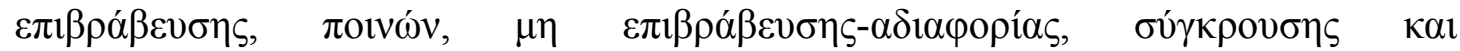

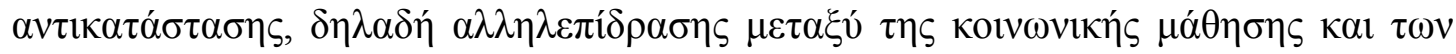

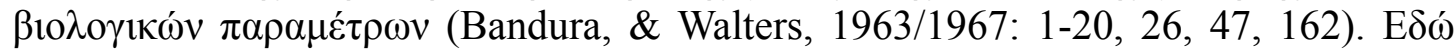

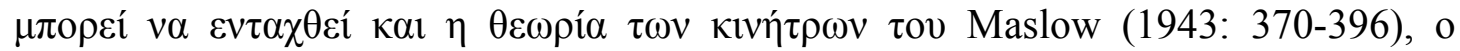

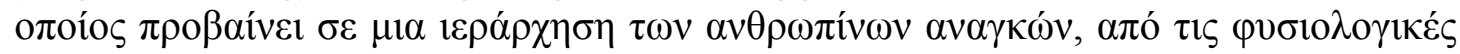

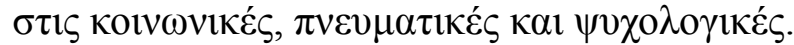

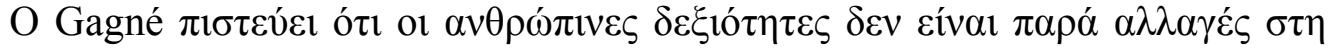

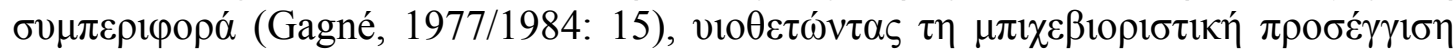

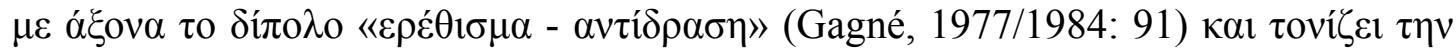

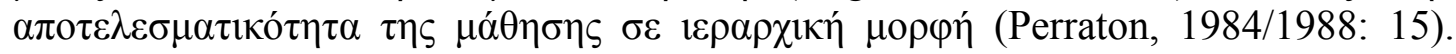

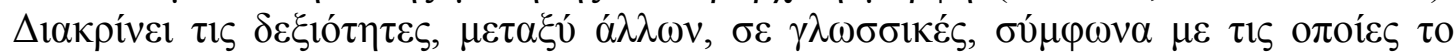

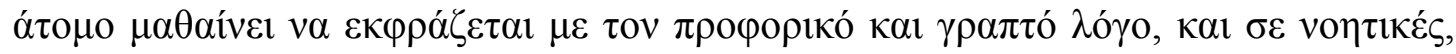

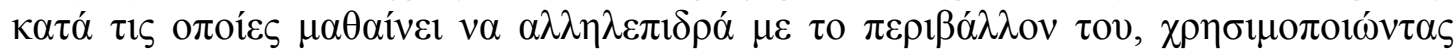

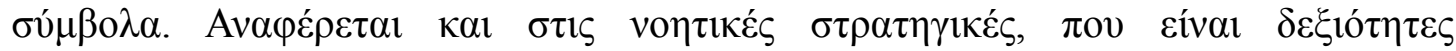

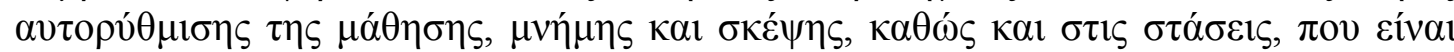

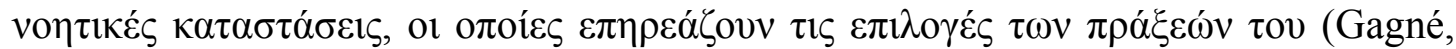

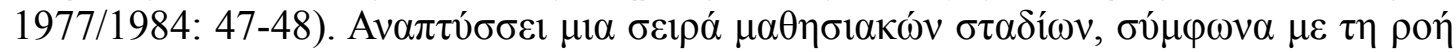

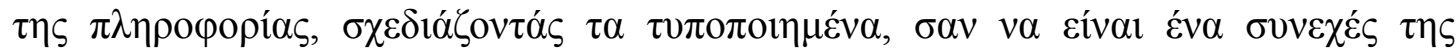

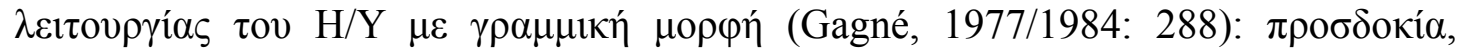

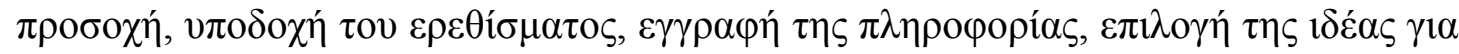

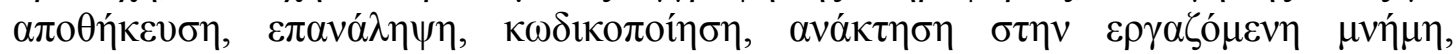

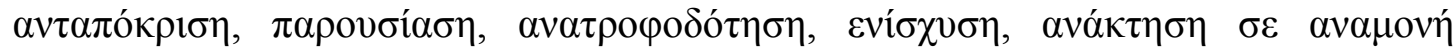

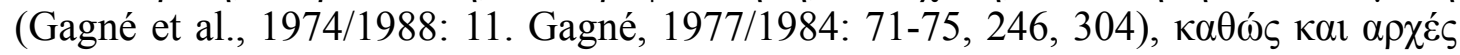

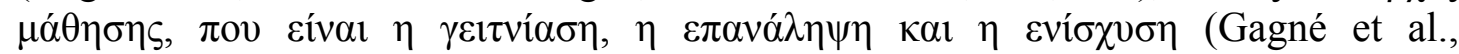

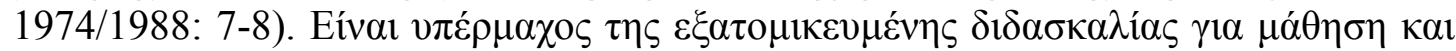

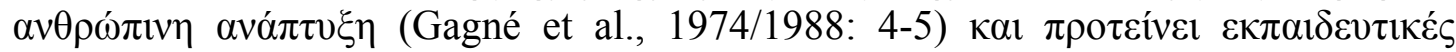

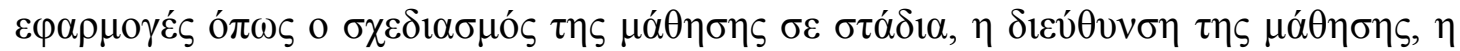

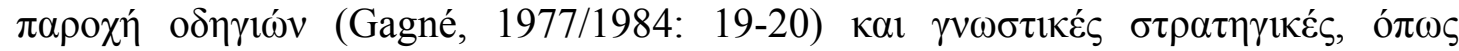

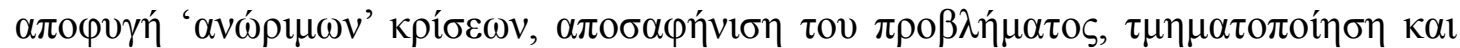

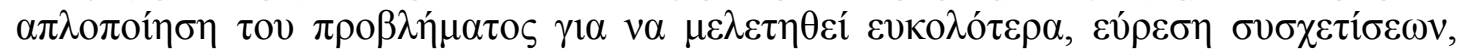

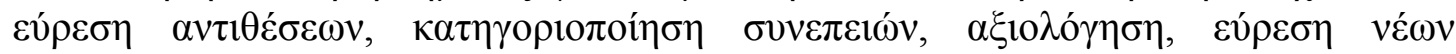

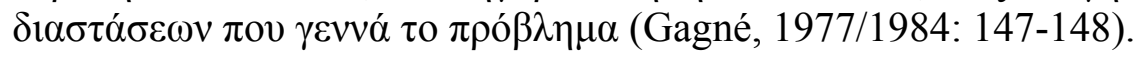

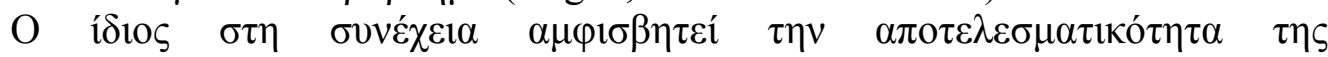

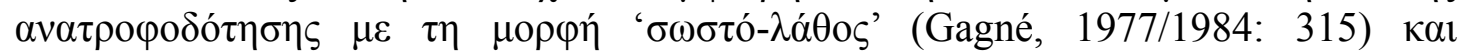

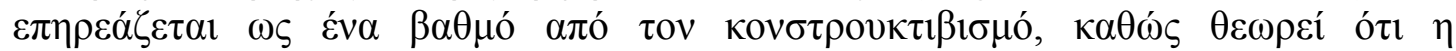

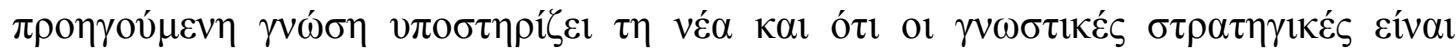

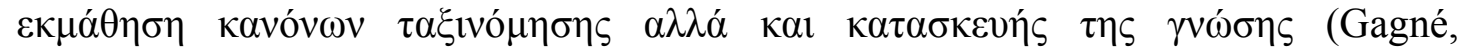

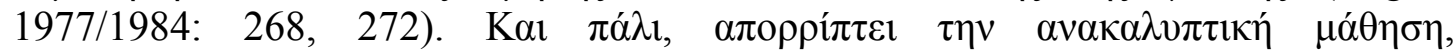

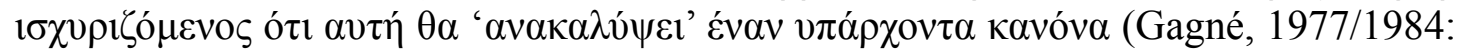

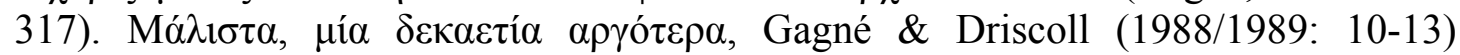

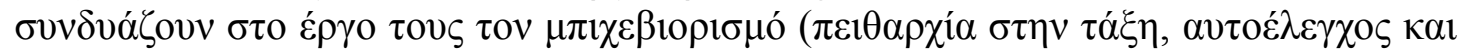

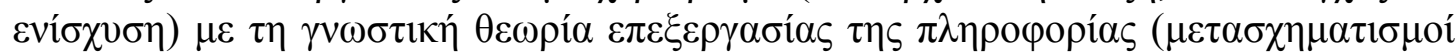

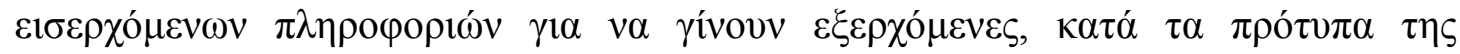

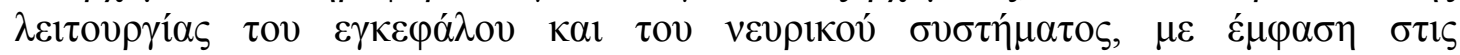

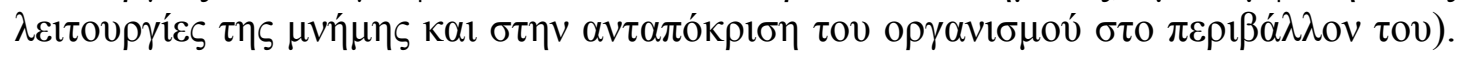

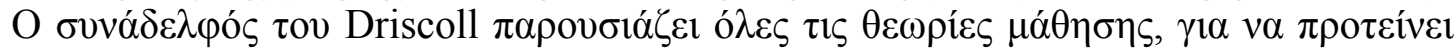

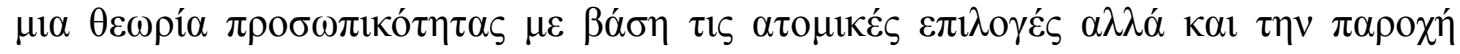




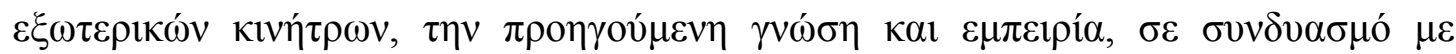

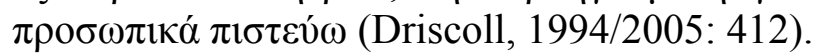

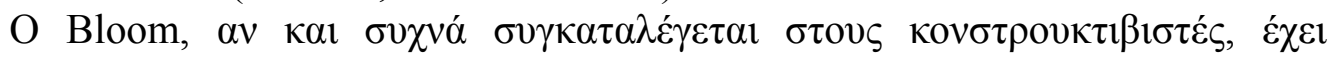

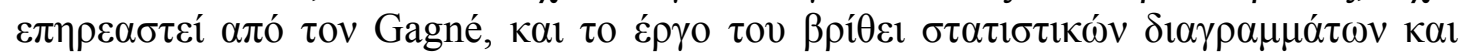

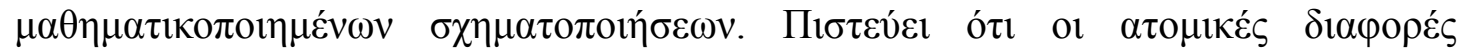

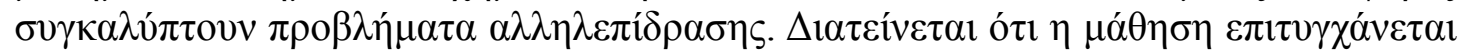

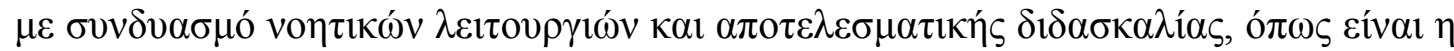

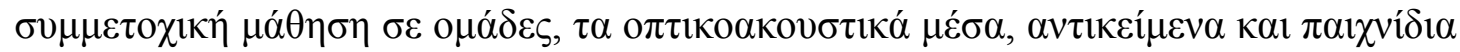

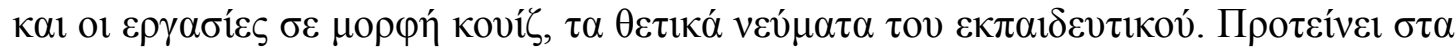

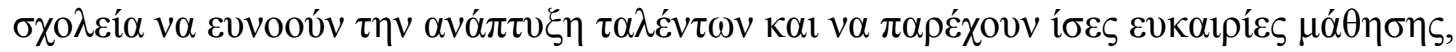

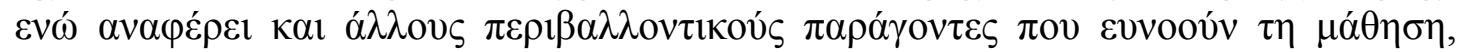

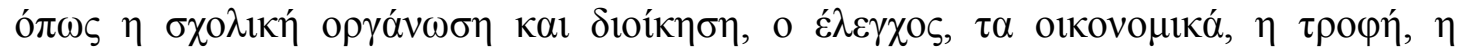

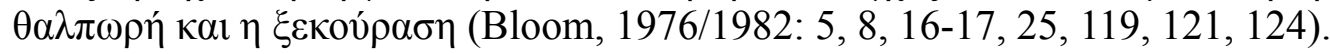

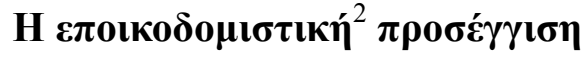

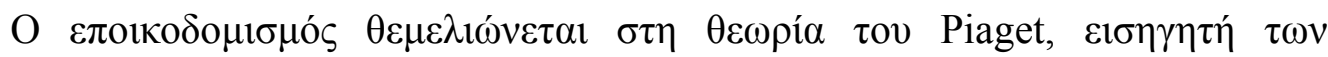

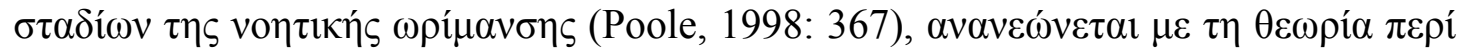

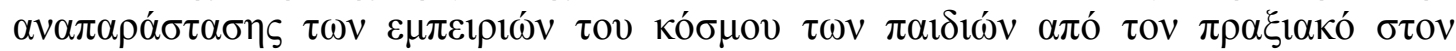

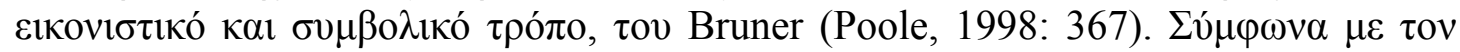

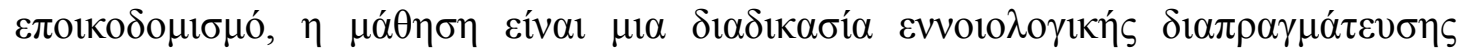

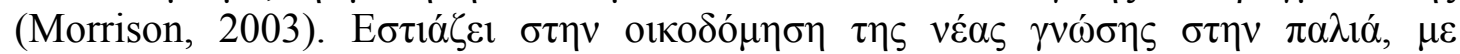

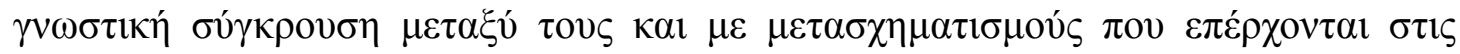

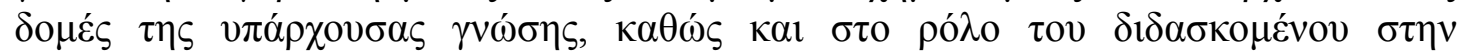

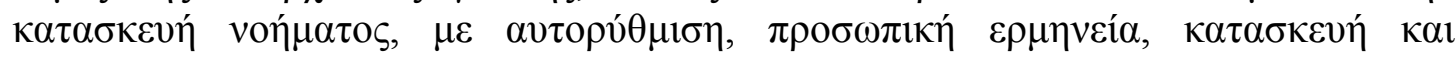

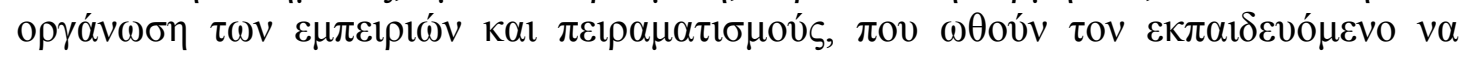

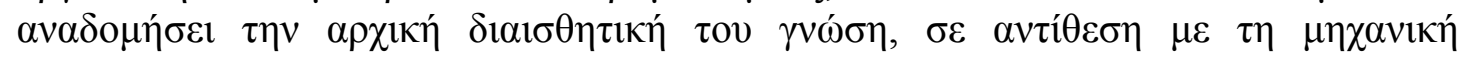

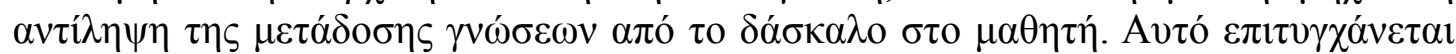

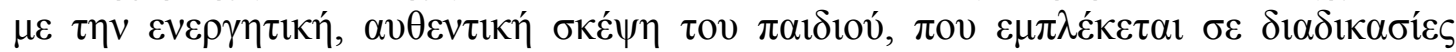

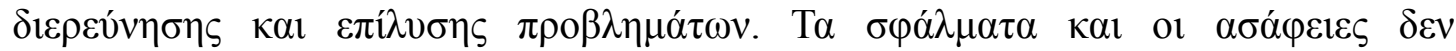

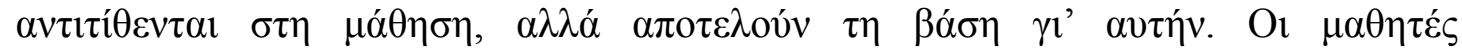

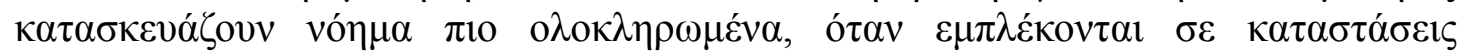

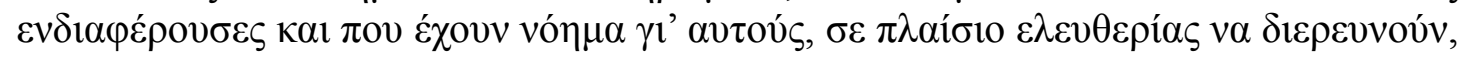

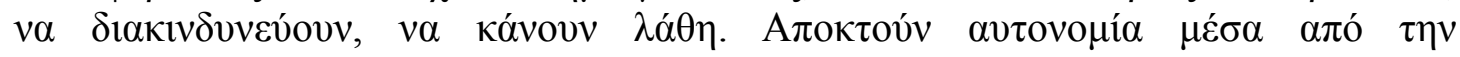

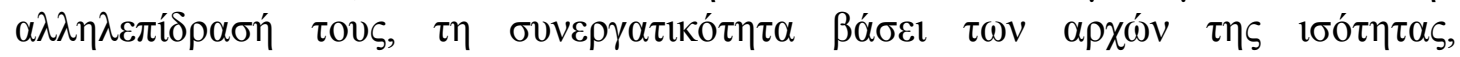

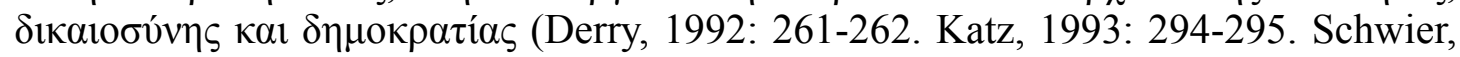
1995: 119. Kahn, \& Friedman, 1998: 164-165. Poole, 1998: 15. Kanuka, \& Anderson, 1999. Koivusaari, 1999: 312. Cantero, 2000: 6. Papert, 2000: 229. Wilson, \& Lowry, 2000: 79).

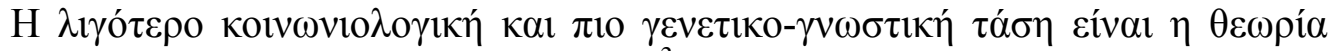

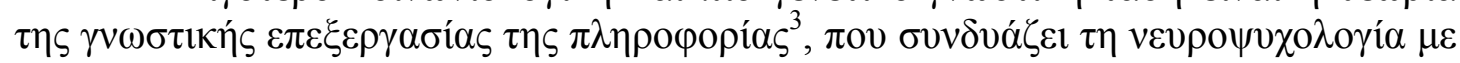

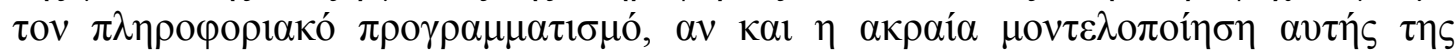

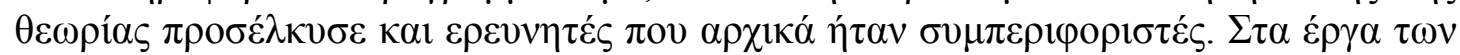

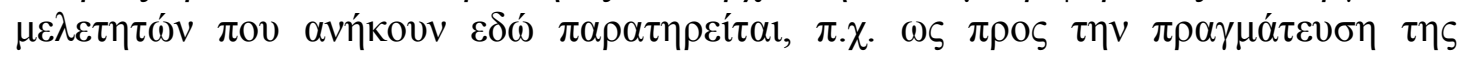

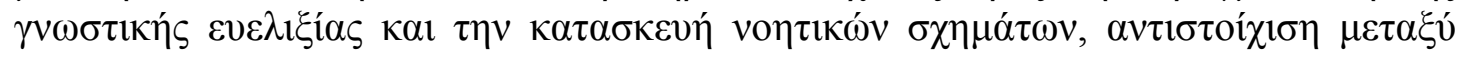

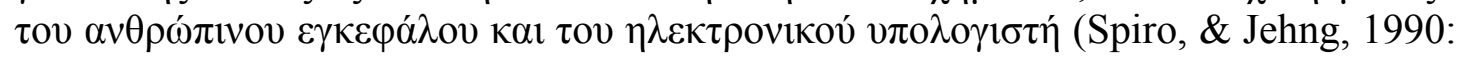

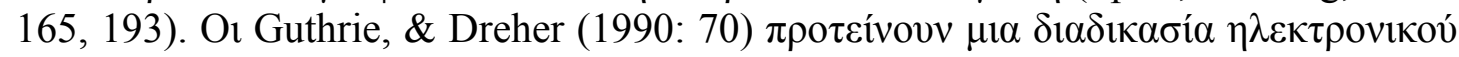

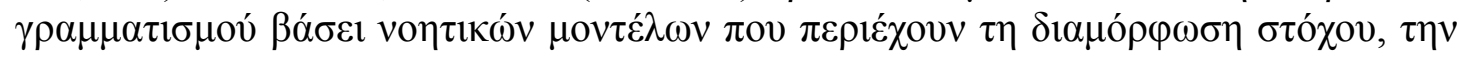

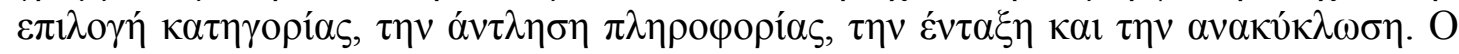

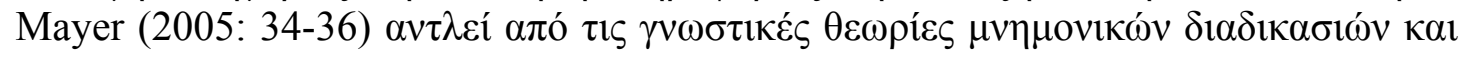




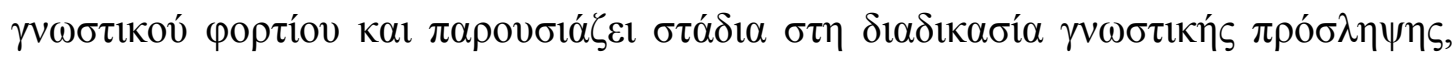
ó

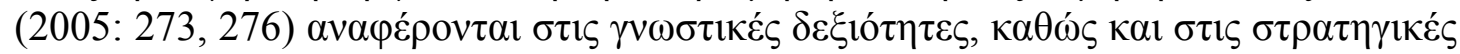

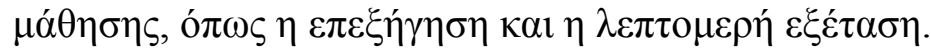

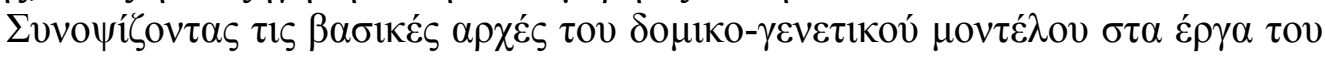

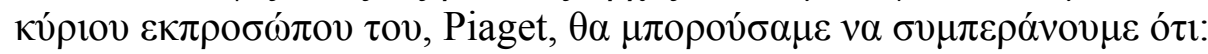

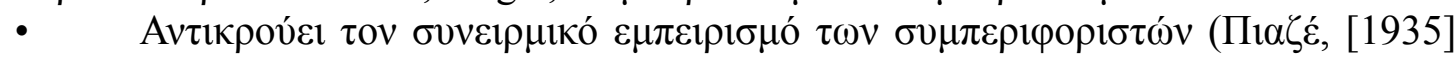

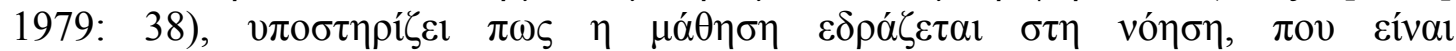

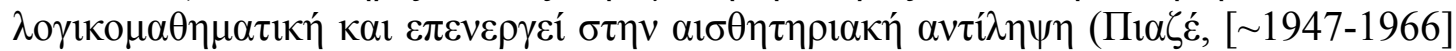

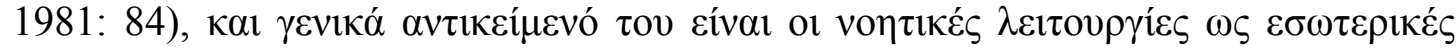

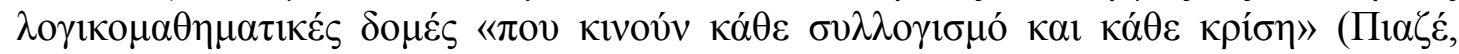
[1935] 1979: 39).

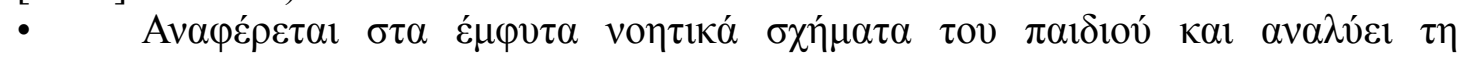

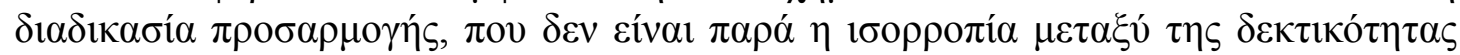

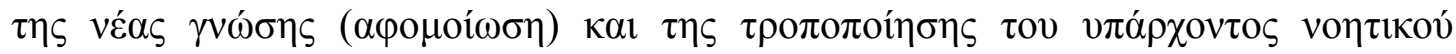

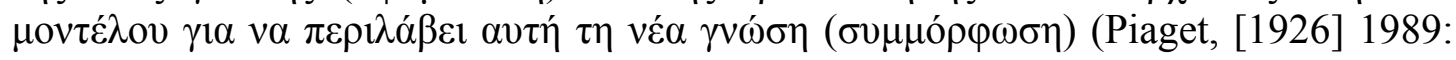
8, 32. Piaget, 1951: 283).

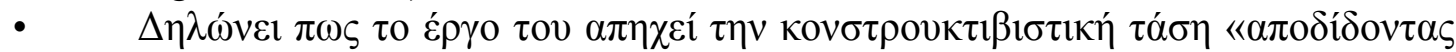

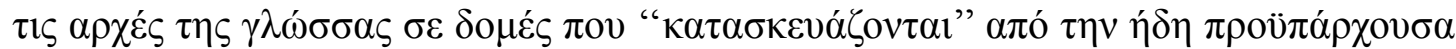

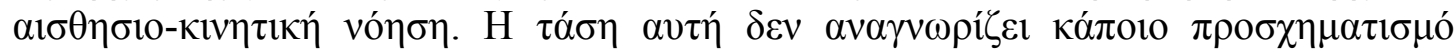

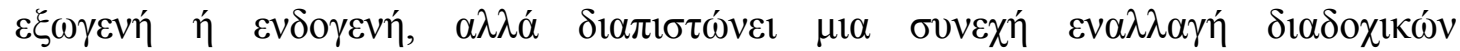

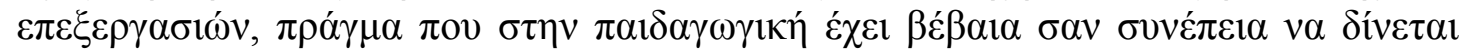

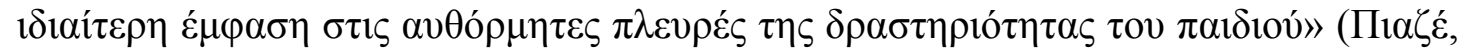
[1971] 1979: 18).

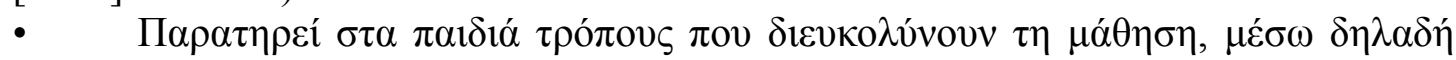

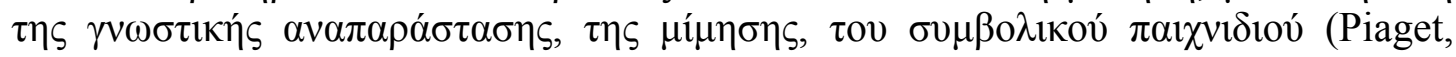
1951: 273).

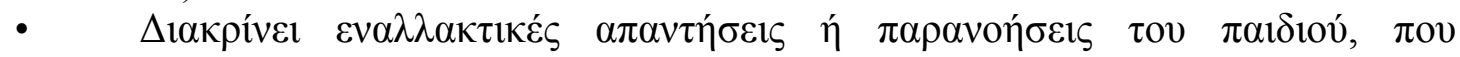

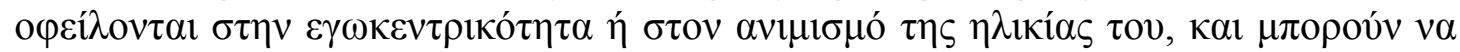

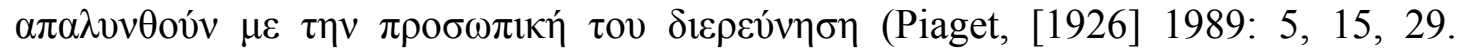
Piaget, [1923] 1989).

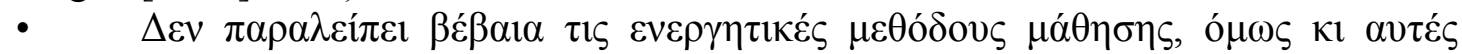

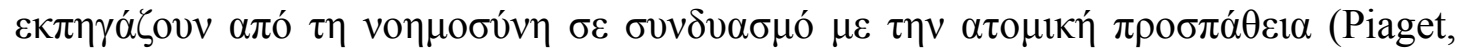
[1930] 2000: 57-58).

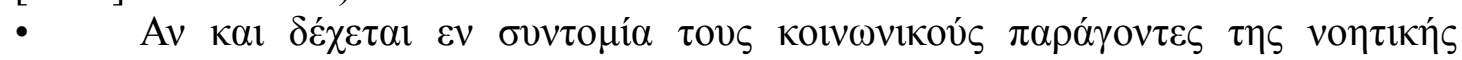

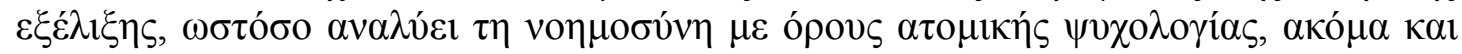

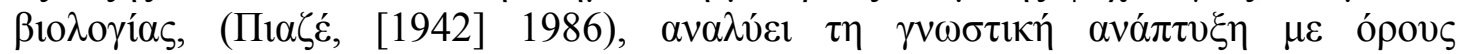

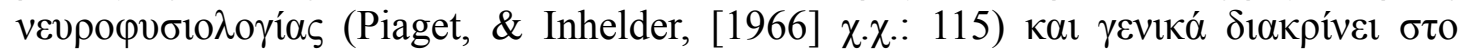

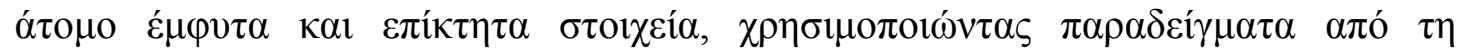

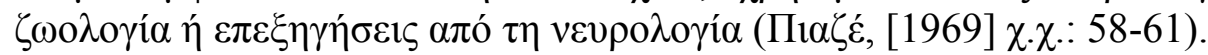

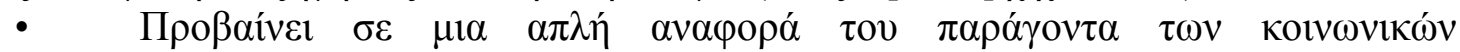

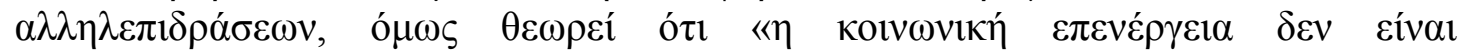

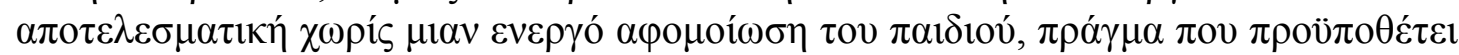

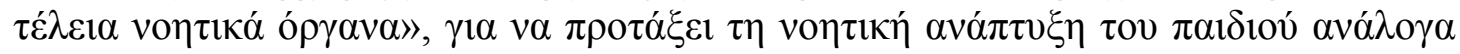

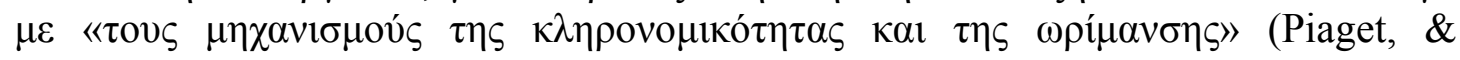

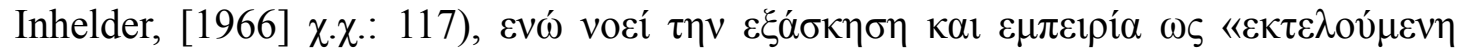

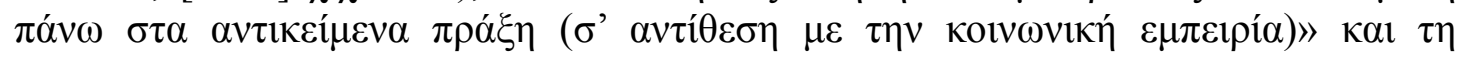

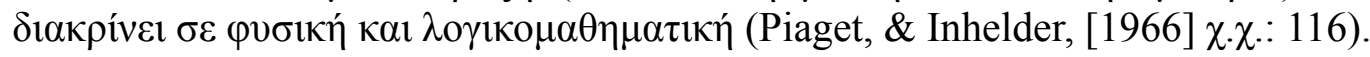

O Lev Semyonovich Vygotsky $\kappa \alpha$ o Alexander Romanovich Luria, $\pi$ ov

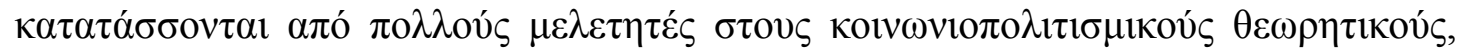




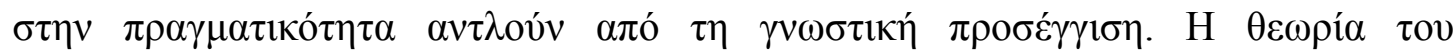

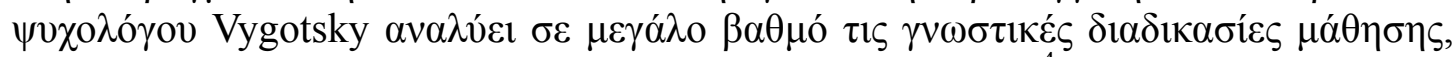

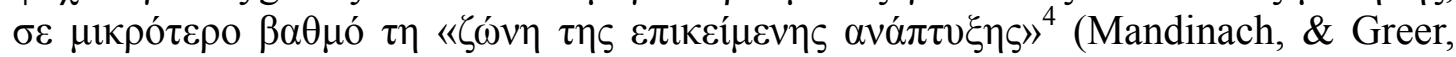
1992: 252. Katz, 1993: 294-295. Crook, 1996: 88, 101. Dillenbourg, 1996: 170. Davis et al., 1997: 25. Poole, 1998: 15. Mercer, \& Wegerif, 1999: 79. Seufert, 2003: 229)

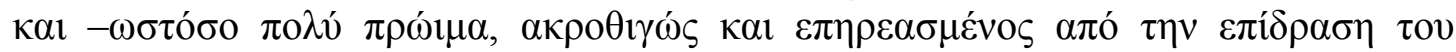

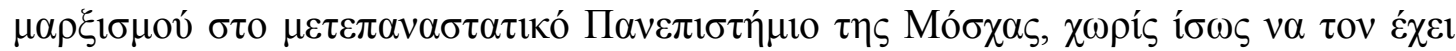

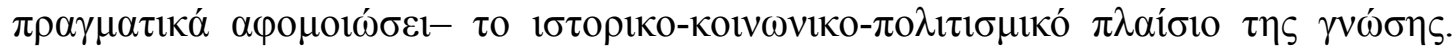

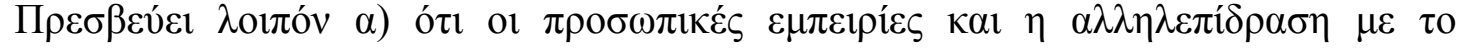

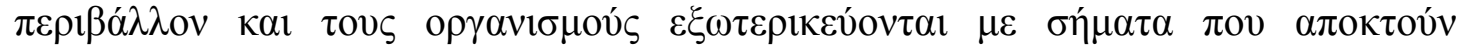

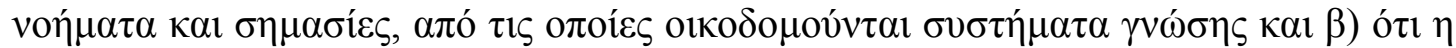

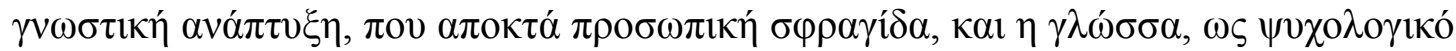

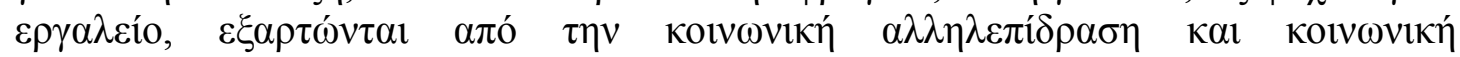

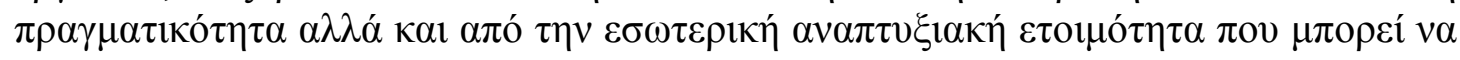

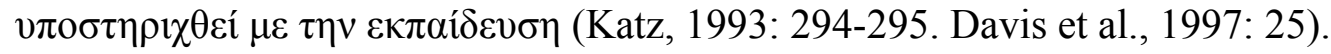

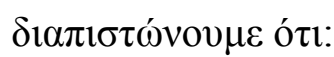

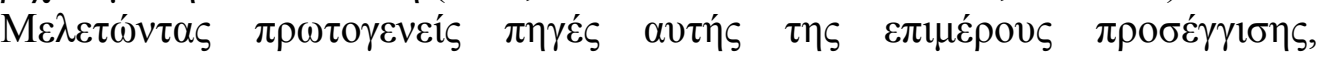

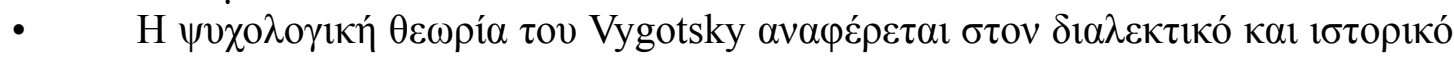

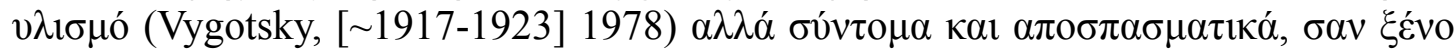

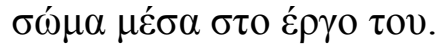

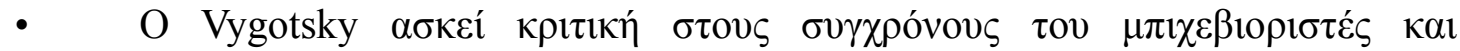

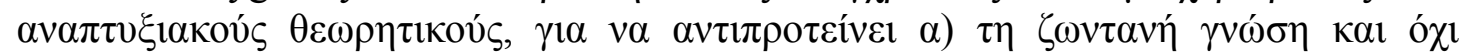

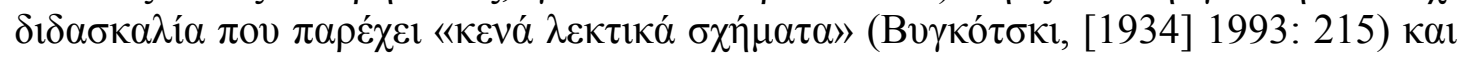

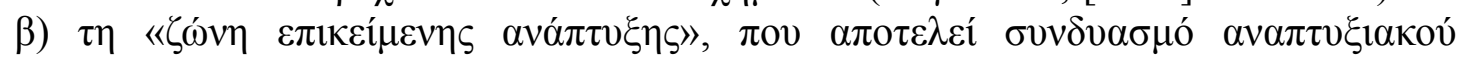

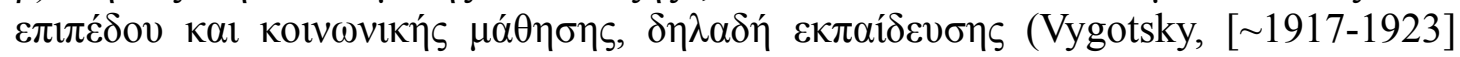

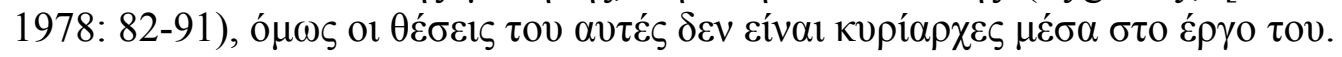

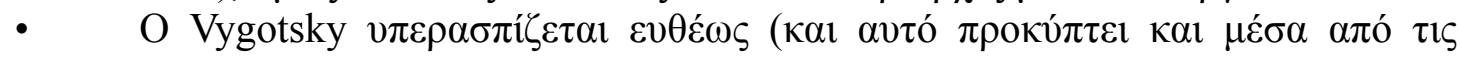

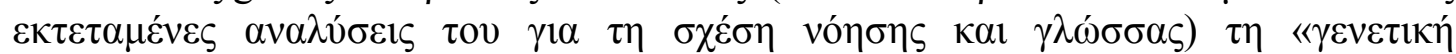

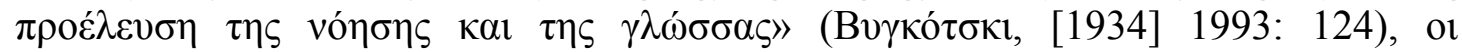

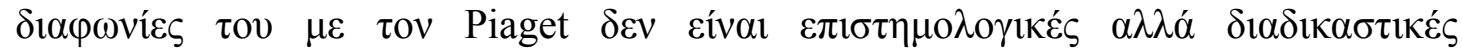

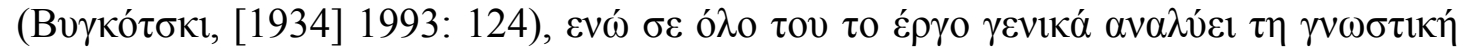
$\varepsilon \xi \dot{\xi} \lambda \lambda_{1} \xi \eta \tau$

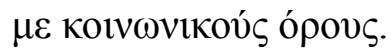

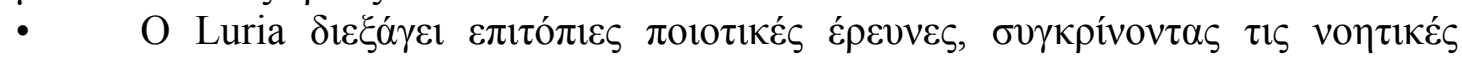

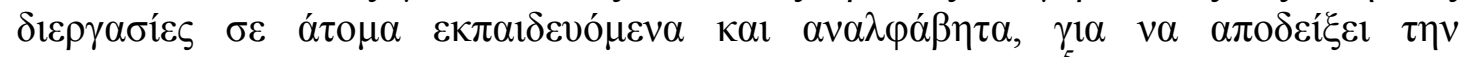

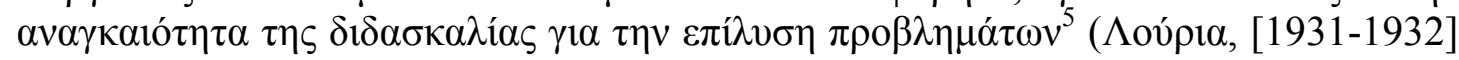
1992).

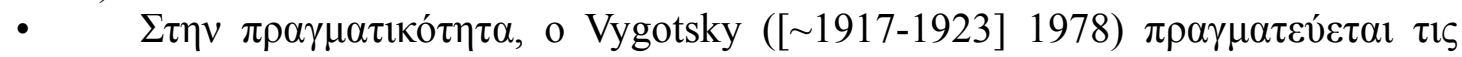

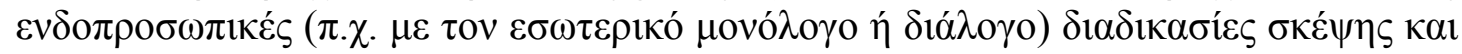

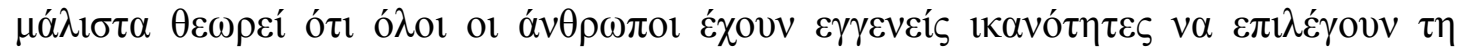

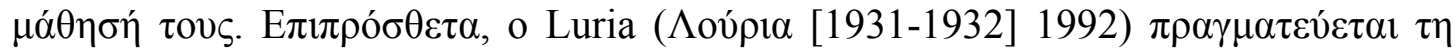

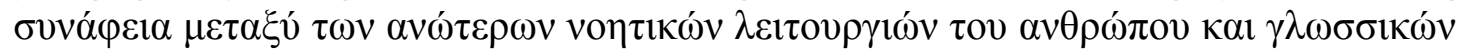

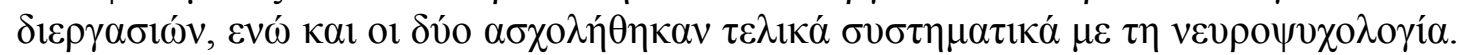

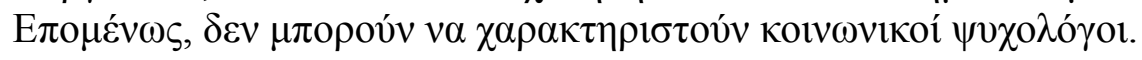

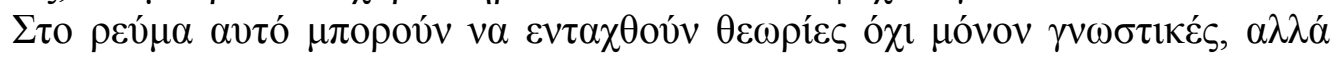

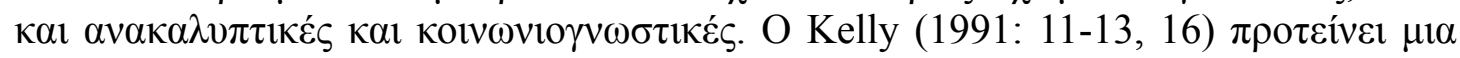

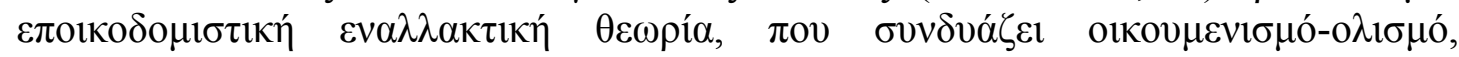

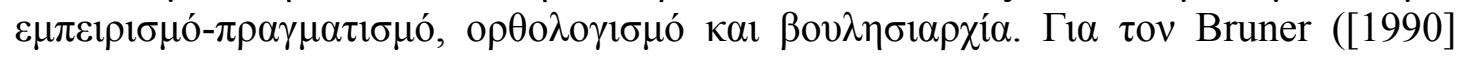

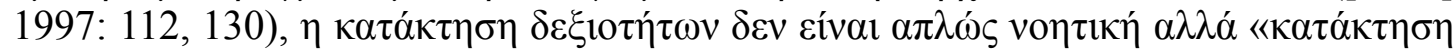

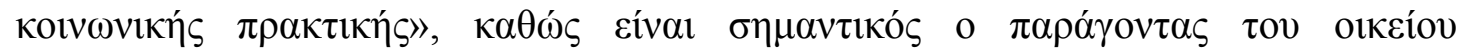




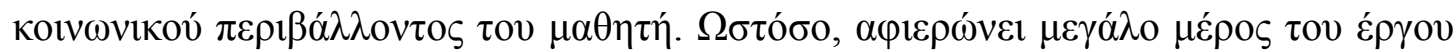

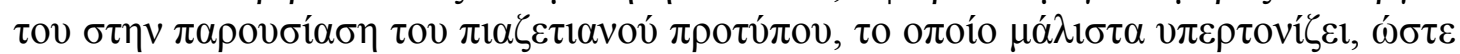
$\eta \theta \varepsilon \omega \rho i ́ \alpha$

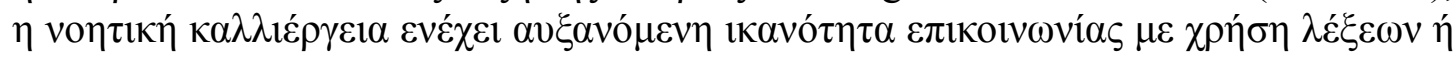

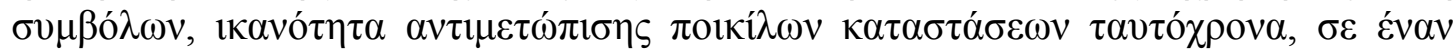

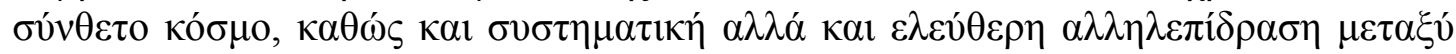

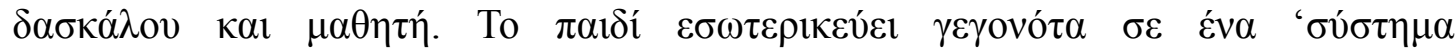

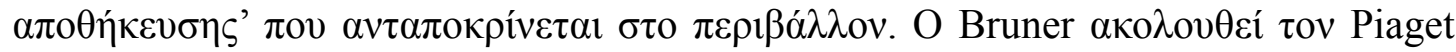

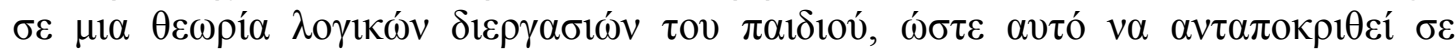

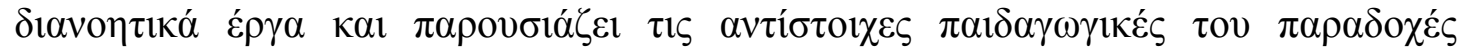
(Bruner, 1960/1977: 33, 37, 48, 52, 60, 73, 84):

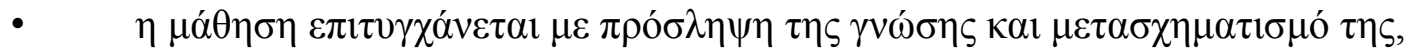

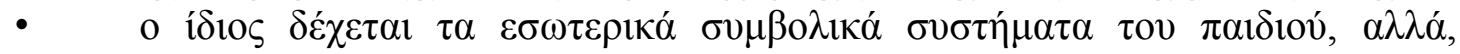

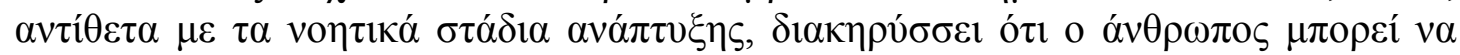

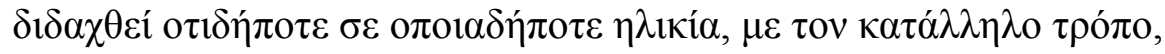

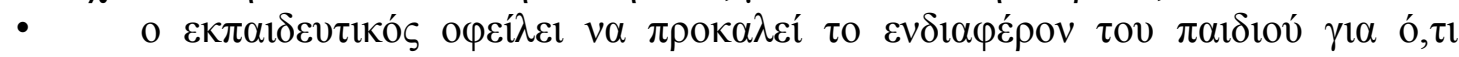

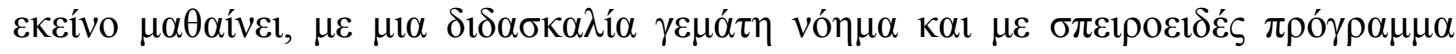

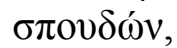

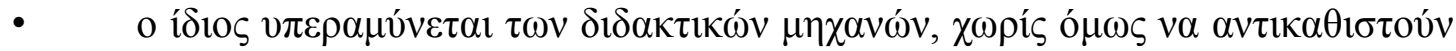

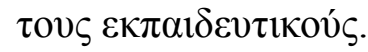

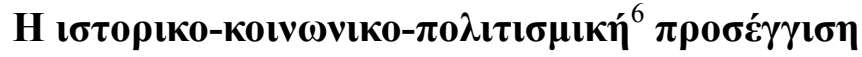

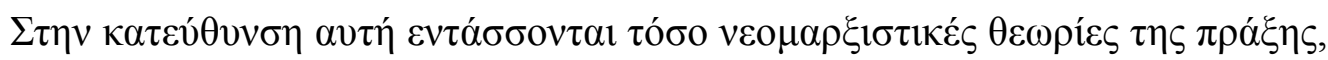

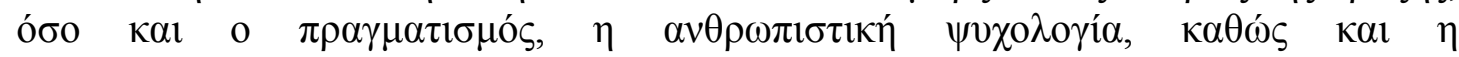

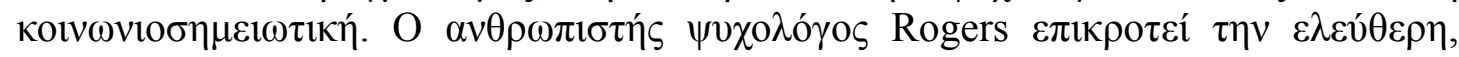

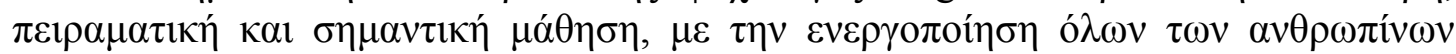

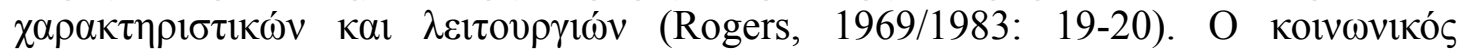

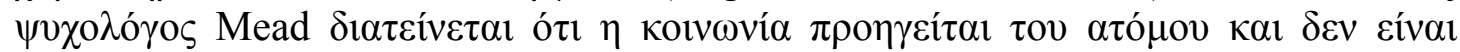

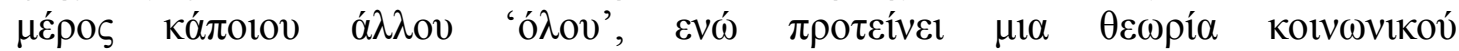

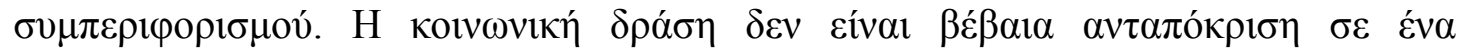

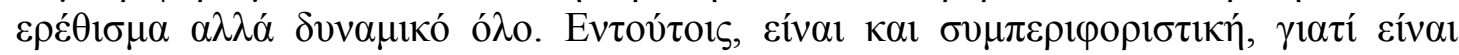
$\pi \alpha \rho \alpha \tau \eta р \dot{\sigma ı \mu \eta ~(M e a d, ~ 1934 / 1965: ~ 115-187) . ~ O 1 ~ D o i s e ~ \& ~ M u g n y ~([1981] ~ 1987: ~ 43-47, ~}$

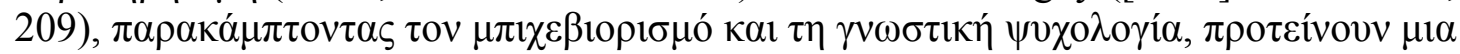

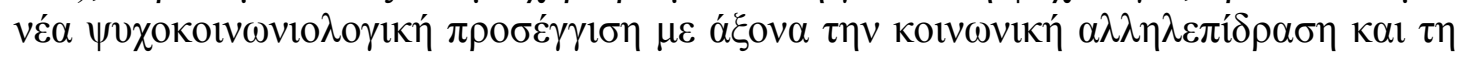

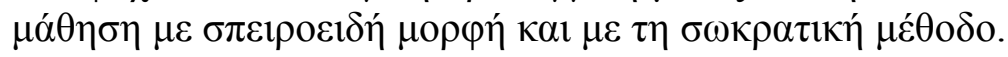

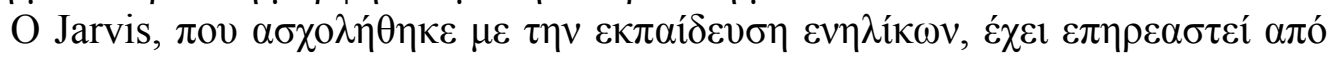

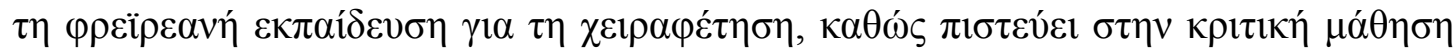

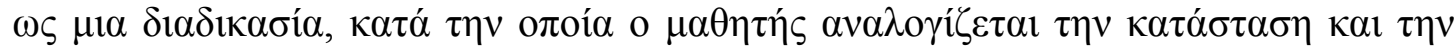

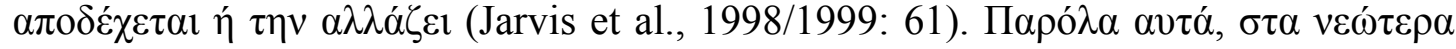

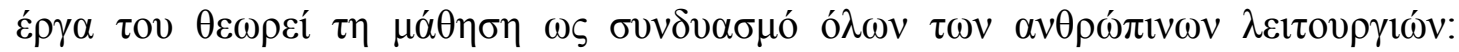

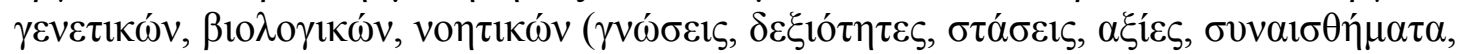

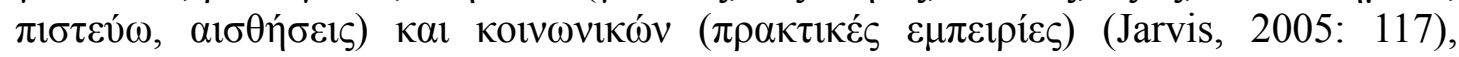

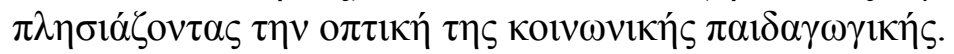

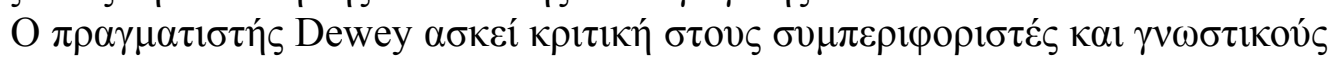

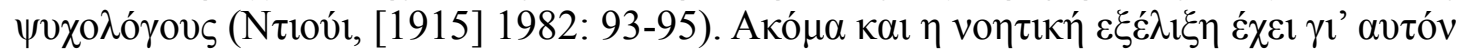

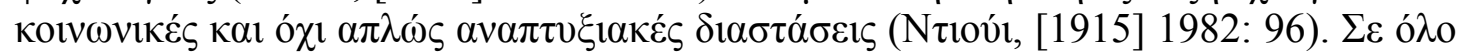

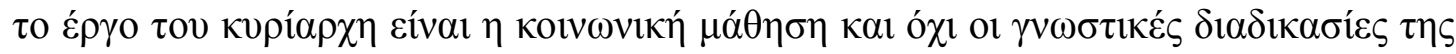

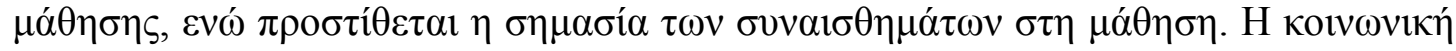

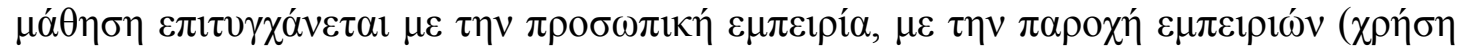


$\pi \alpha \rho \alpha \delta \varepsilon 1 \gamma \mu \alpha \dot{\tau} \omega v, \pi \rho \alpha \kappa \tau \imath \kappa \eta ́ ~ \varepsilon \kappa \pi \alpha i ́ \delta \varepsilon v \sigma \eta, \pi \alpha \imath \chi v i ́ \delta \imath)$ a

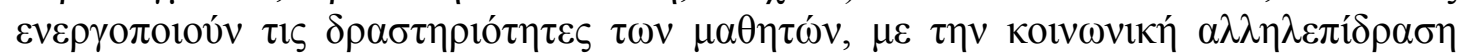

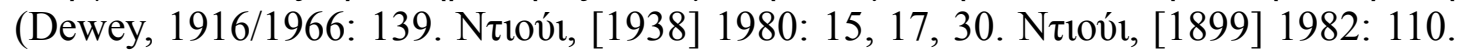

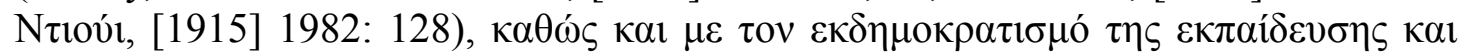

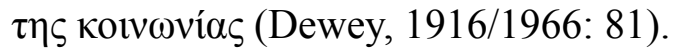

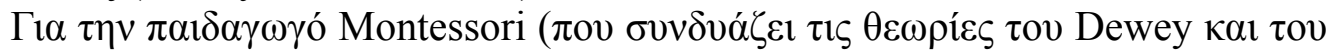

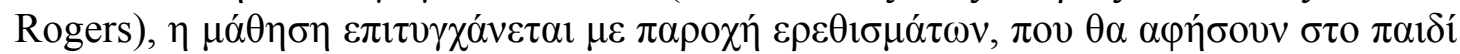

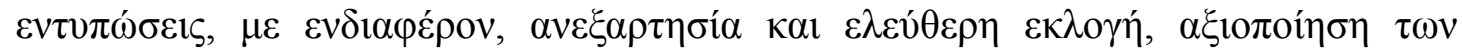

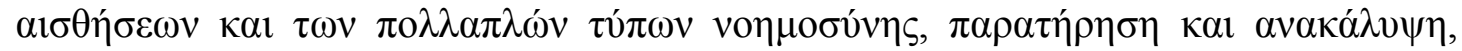

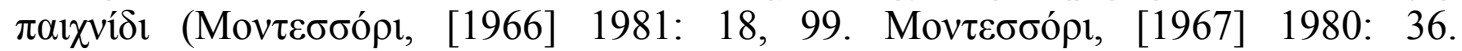

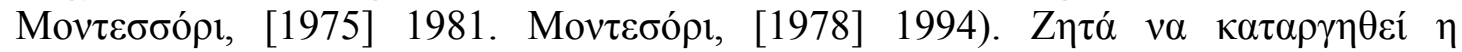

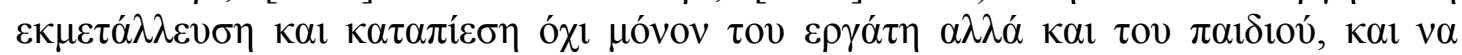

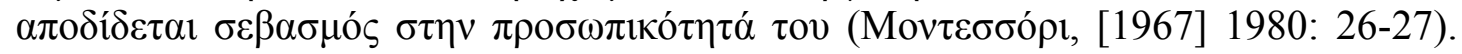

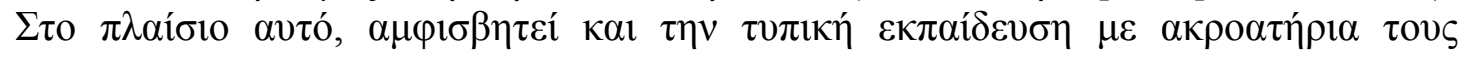

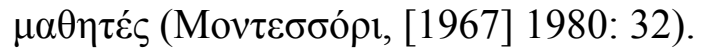

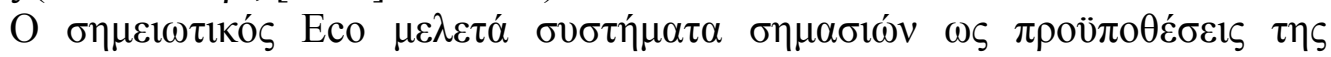

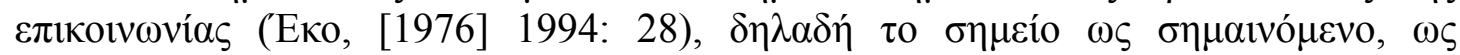

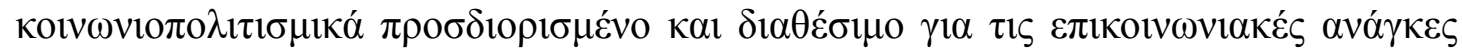

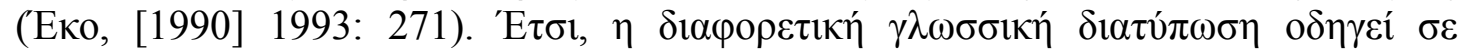

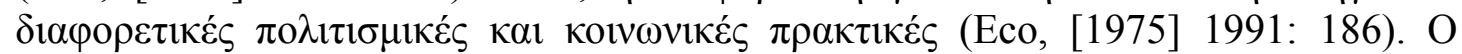

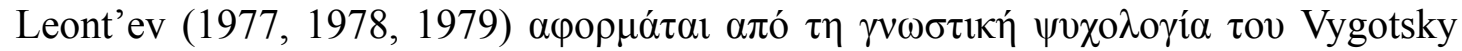

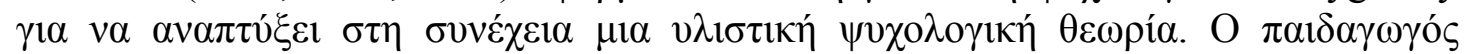

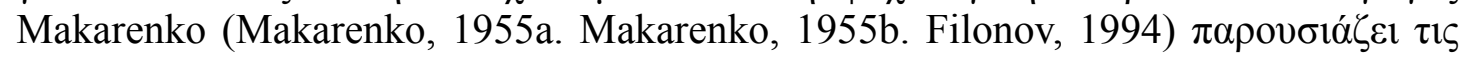

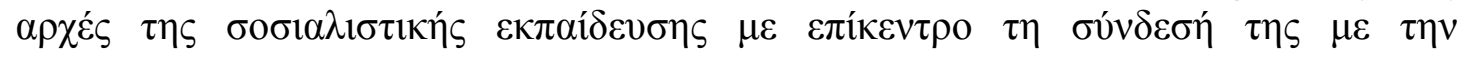

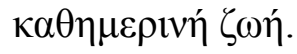

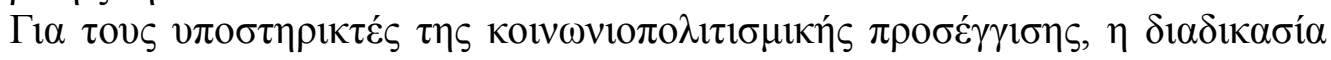

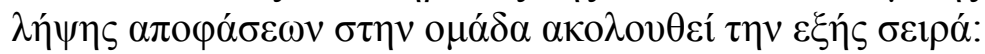

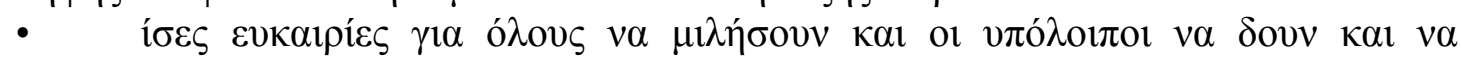

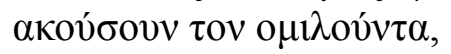

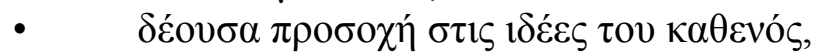

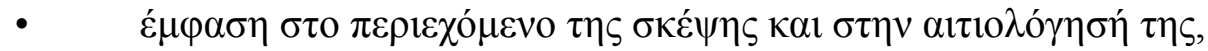

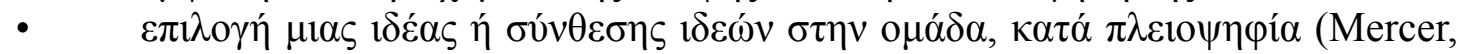

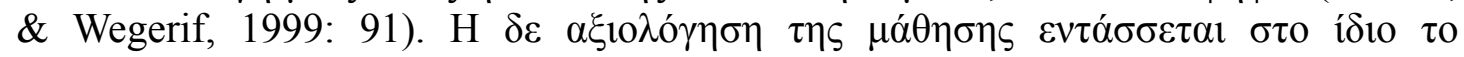

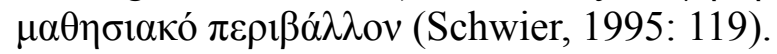

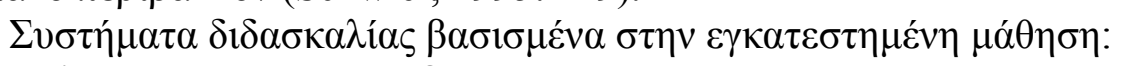

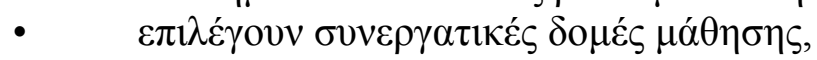

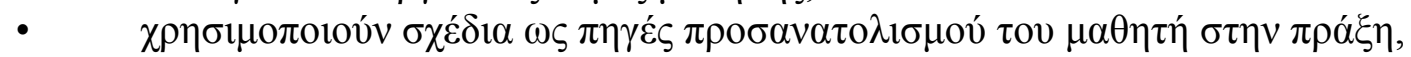

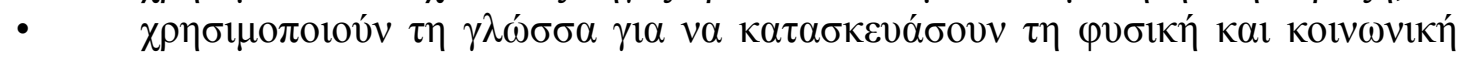
$\pi \rho \alpha \gamma \mu \alpha \tau \iota \kappa o ́ \tau \eta \tau \alpha$,

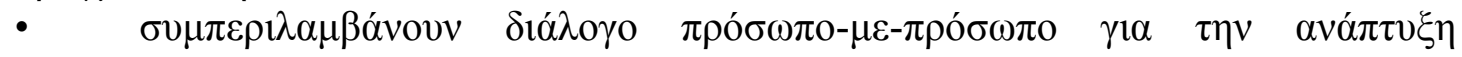
$\delta \varepsilon \xi 10 \tau \eta \dot{\tau} \omega v$

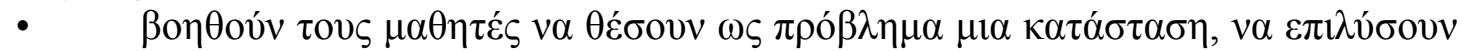

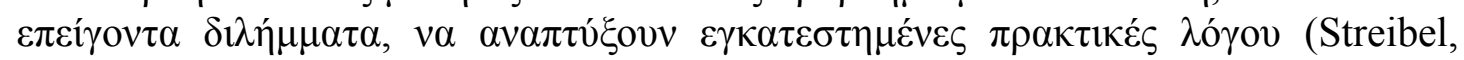
1995: 154).

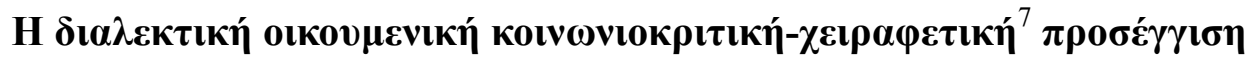

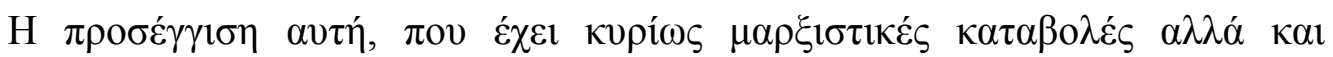

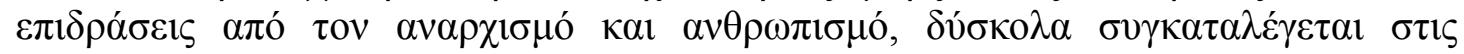

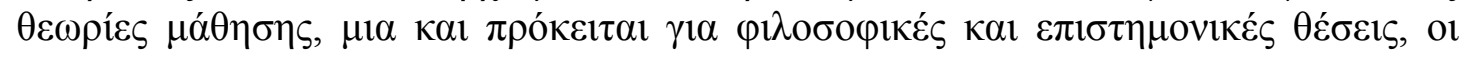




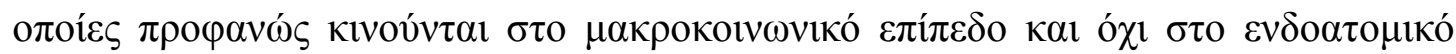

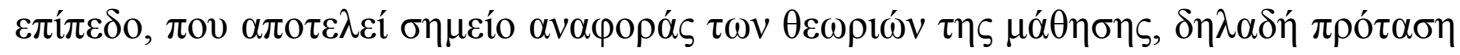

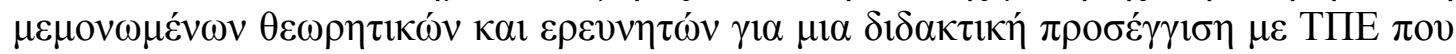

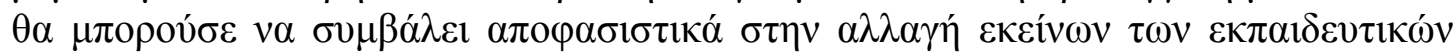

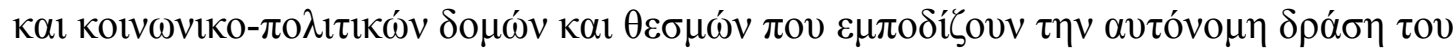

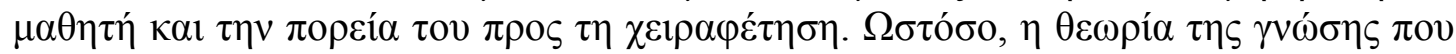

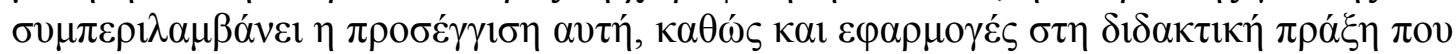

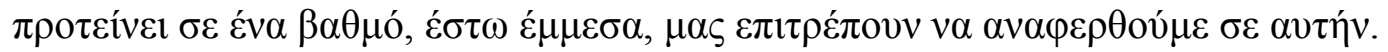

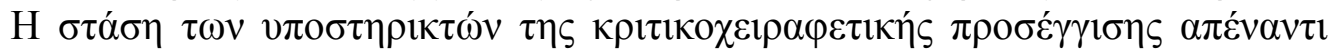

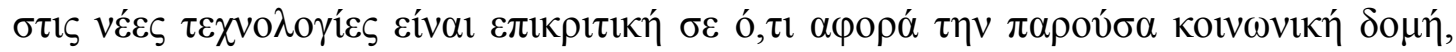

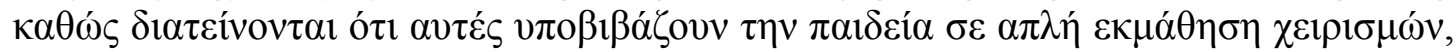

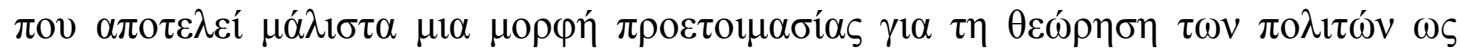

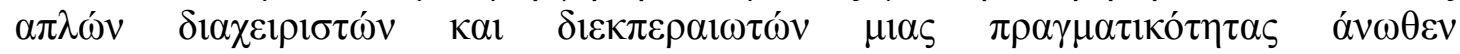

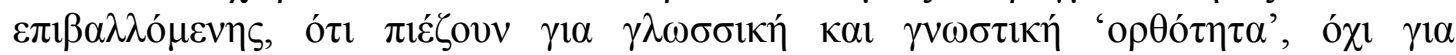

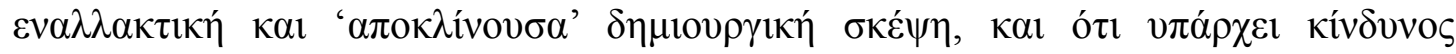

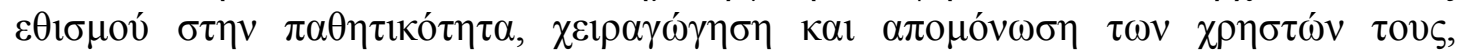

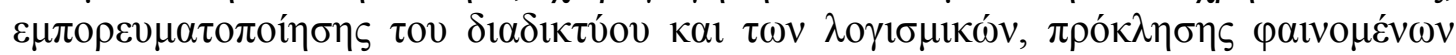

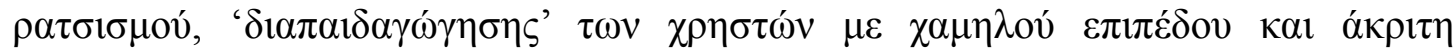

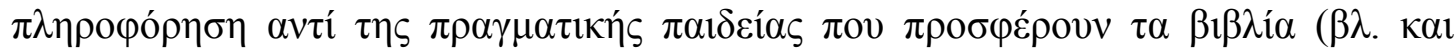
Aldrich et al., 1998: 321-322, 324. Poole, 1998: 375-376. Kern, \& Warschauer, 2000:

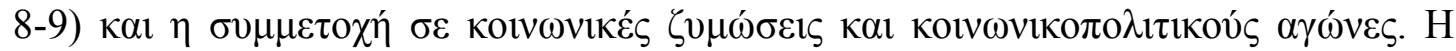

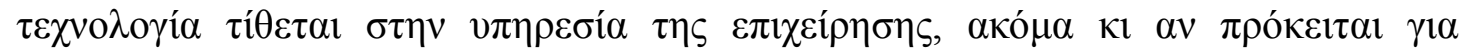

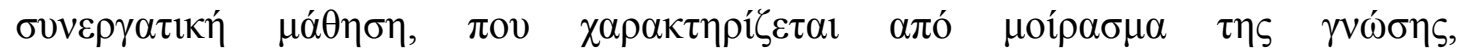

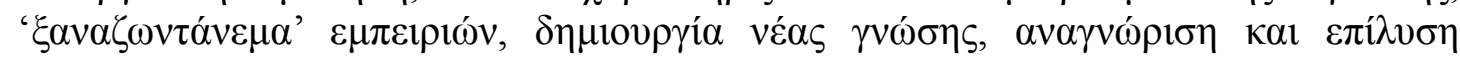

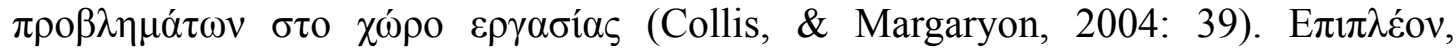

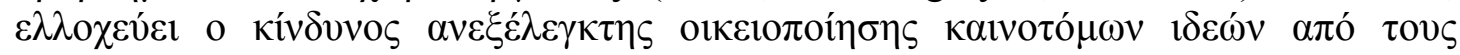

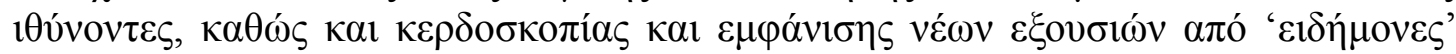

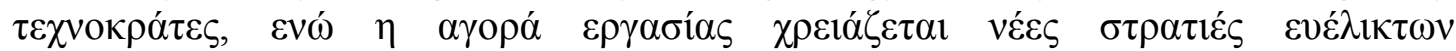

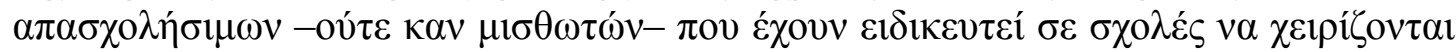

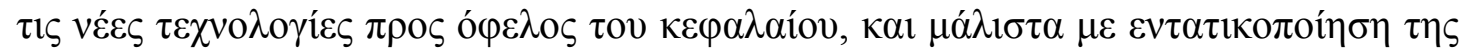

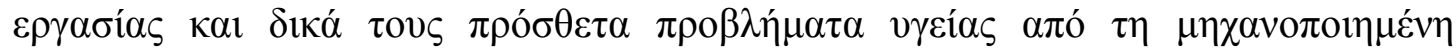
$\varepsilon \rho \gamma \alpha \sigma i ́ \alpha$.

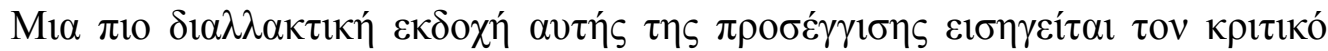

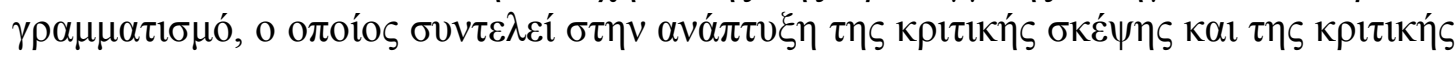

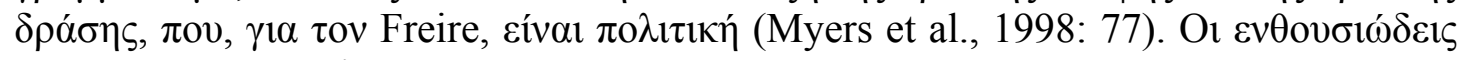

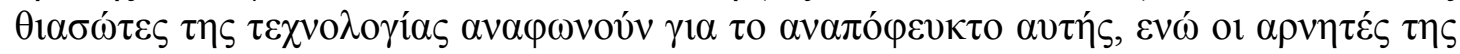

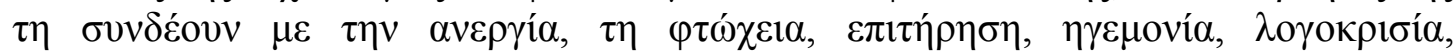

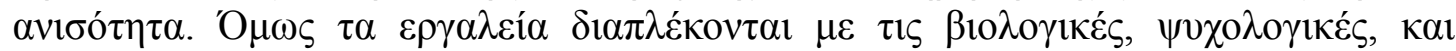

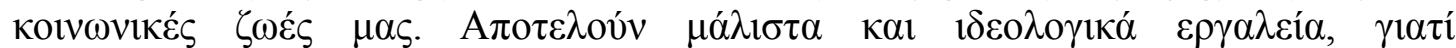

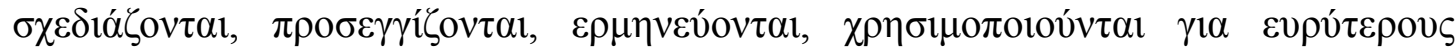

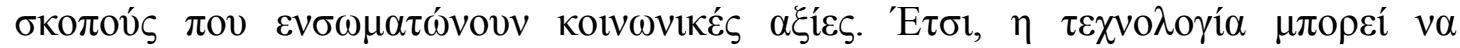

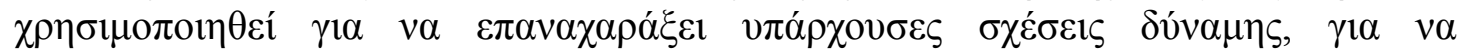

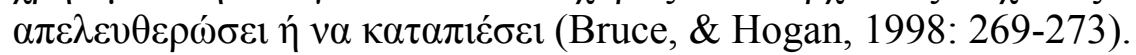

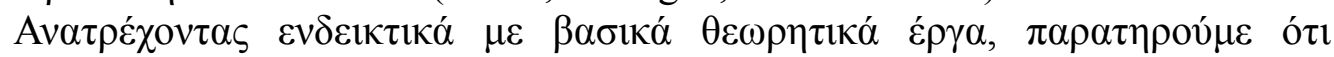

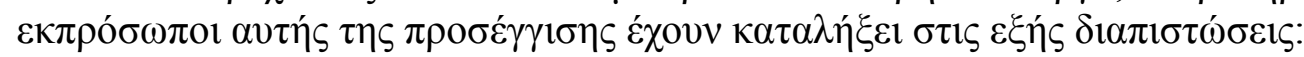

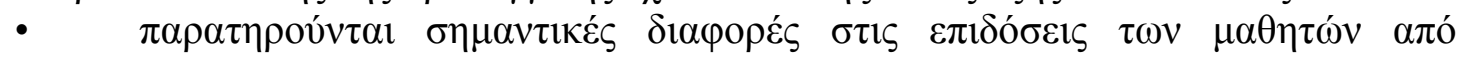

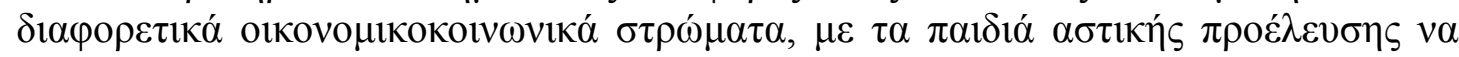

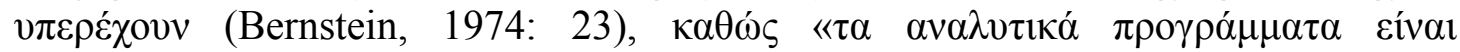

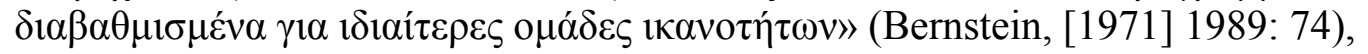




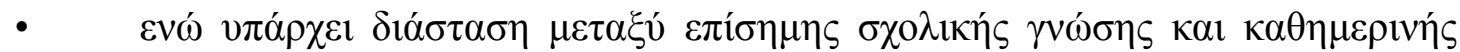

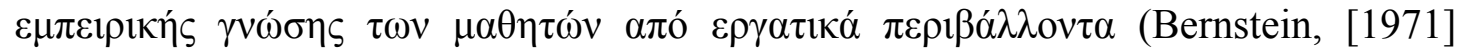

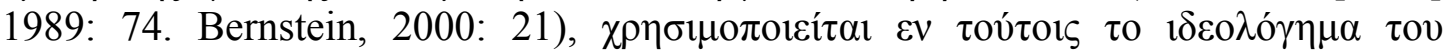

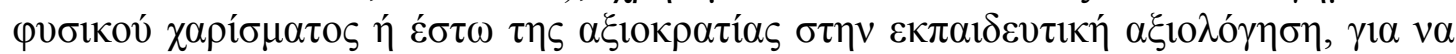

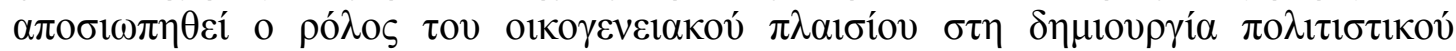

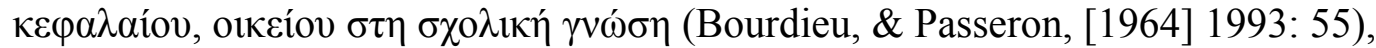

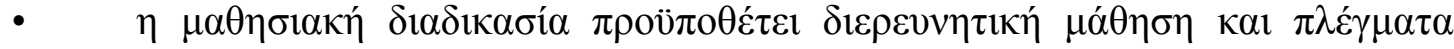

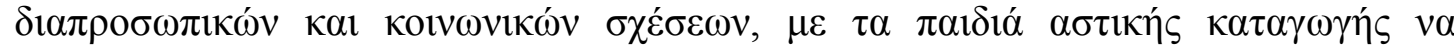

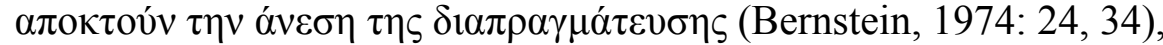

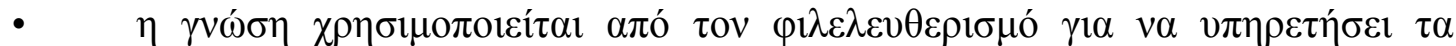

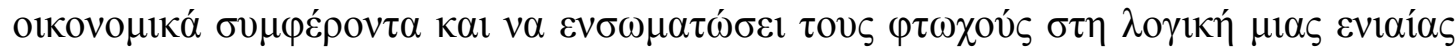

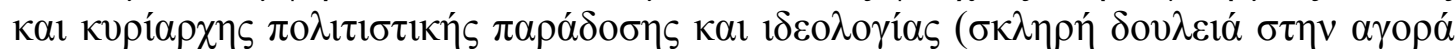

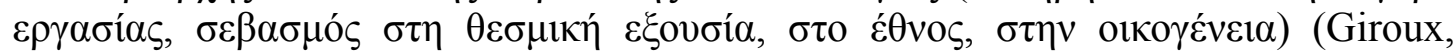
1987/2001: 2-3),

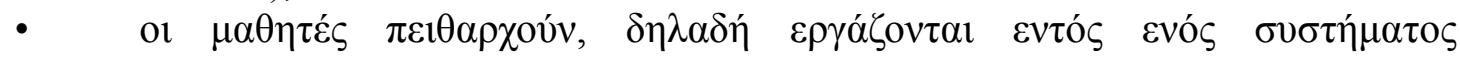

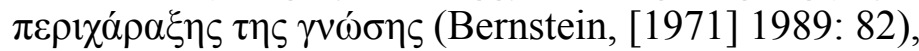

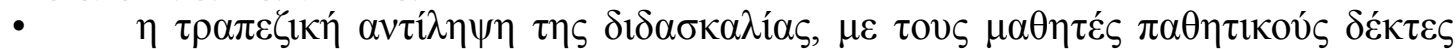

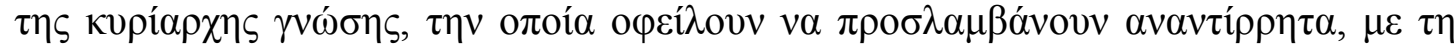

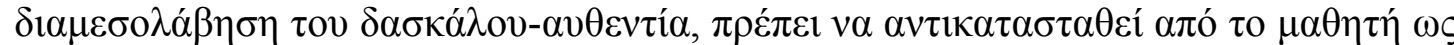

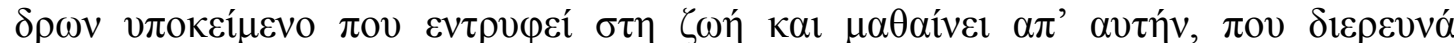

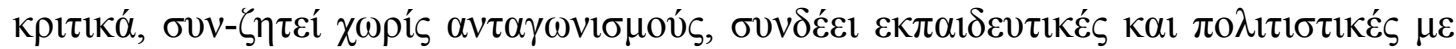

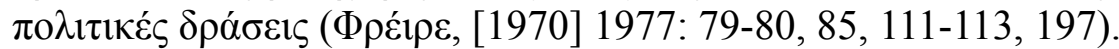

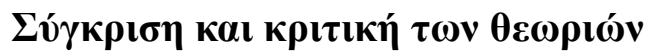

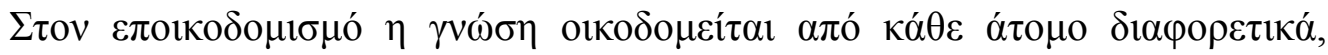

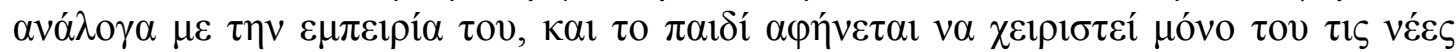

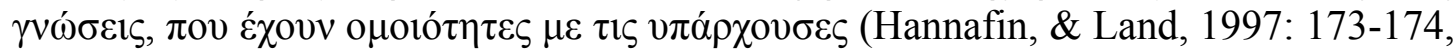

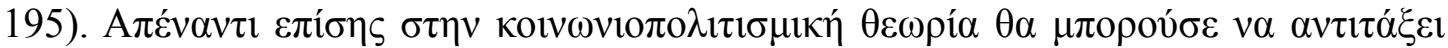

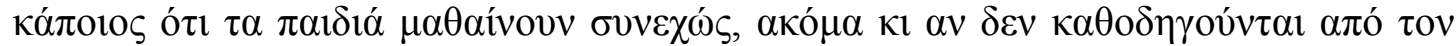

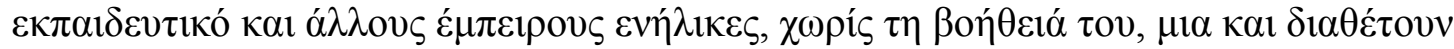

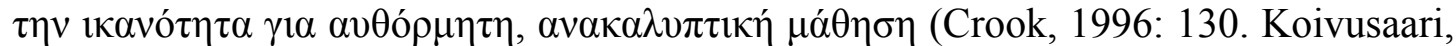

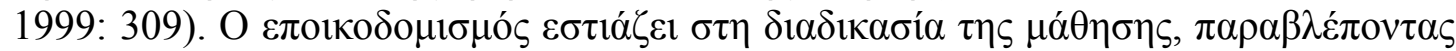

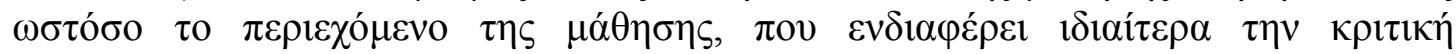

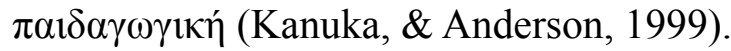

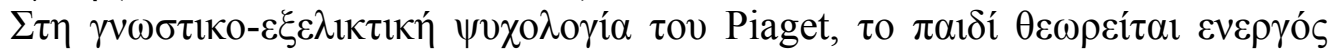

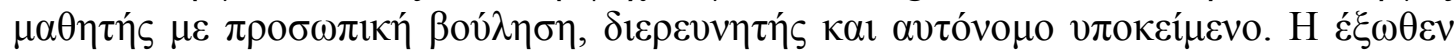

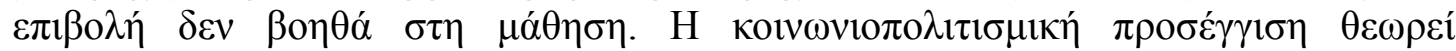

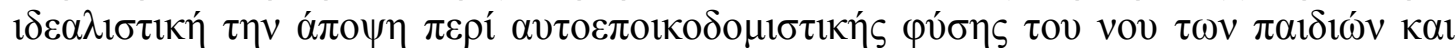

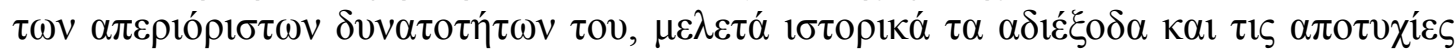

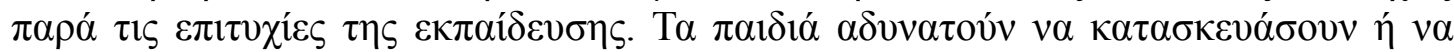

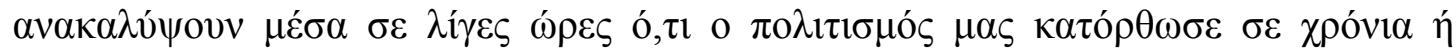

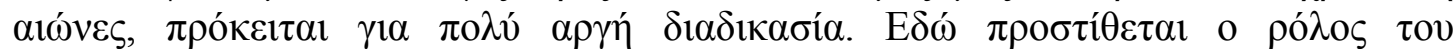

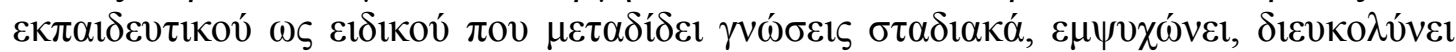

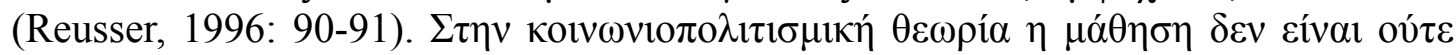

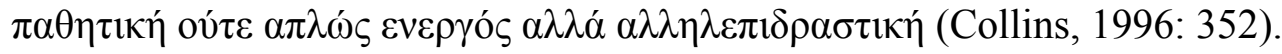

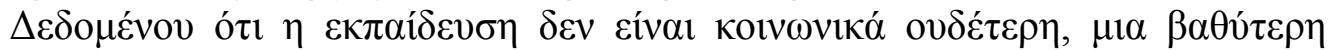

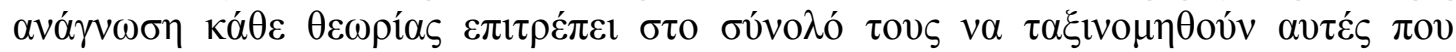

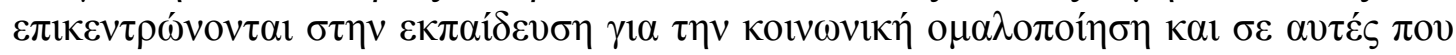

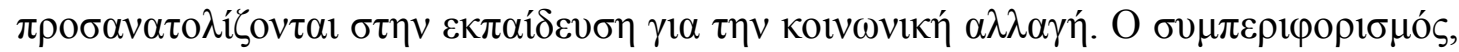




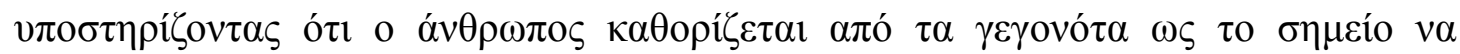

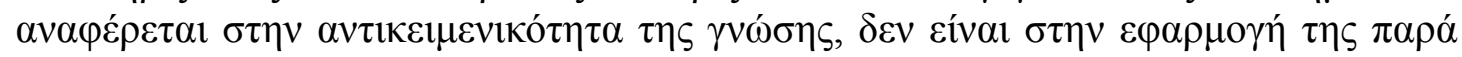

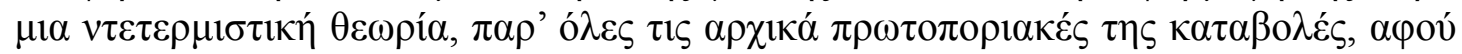

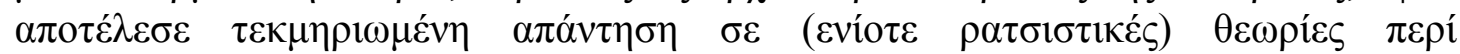

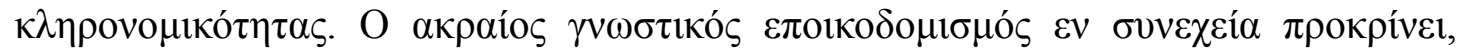

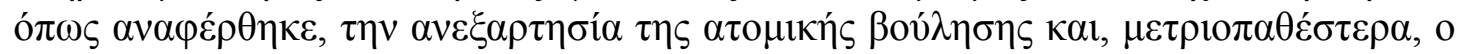

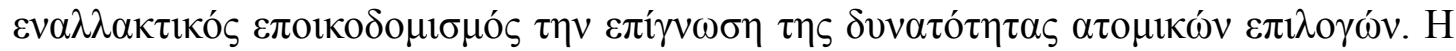

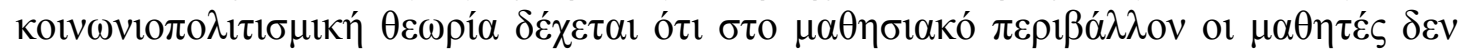

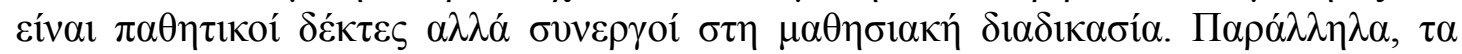

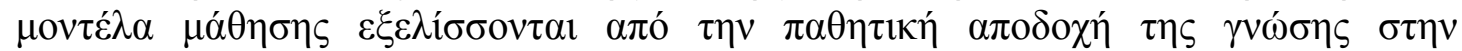

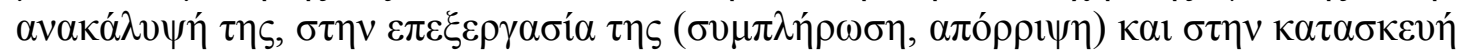
$\tau \eta \varsigma$ (Shuell, 1992: 21-22).

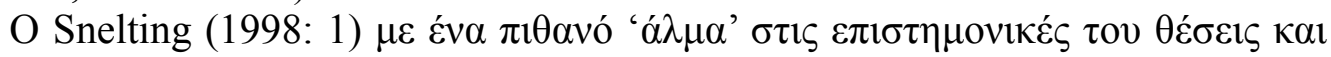

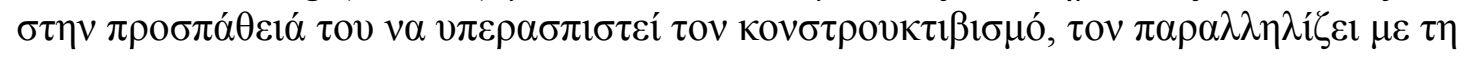

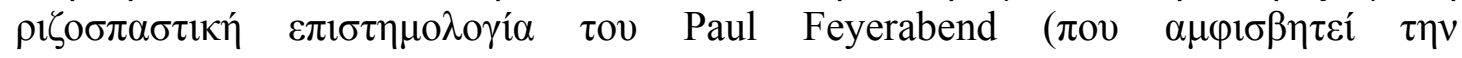

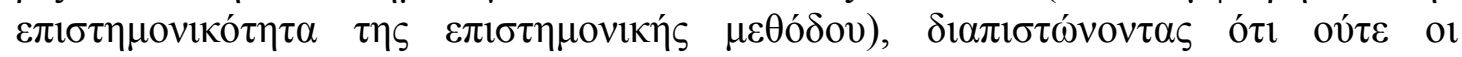

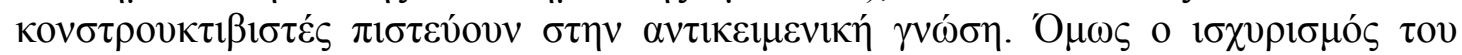

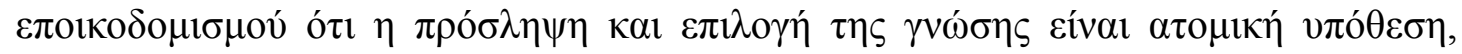

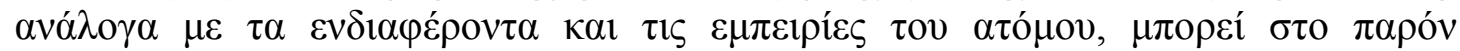

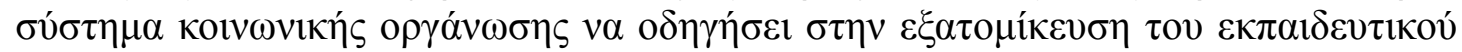

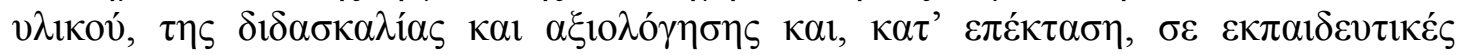

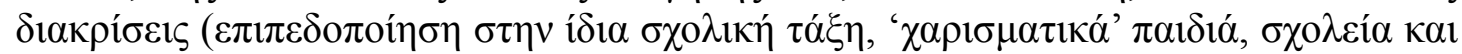

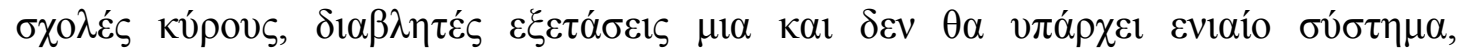

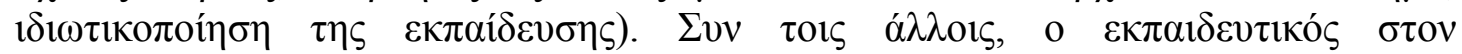

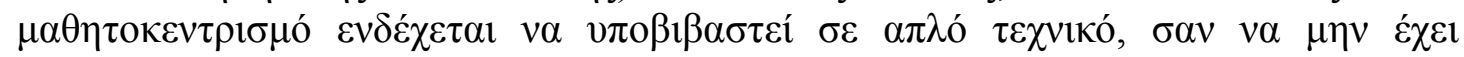

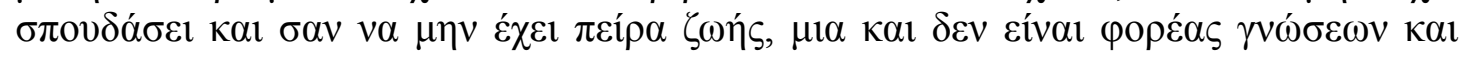

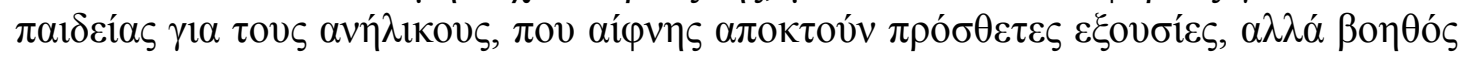

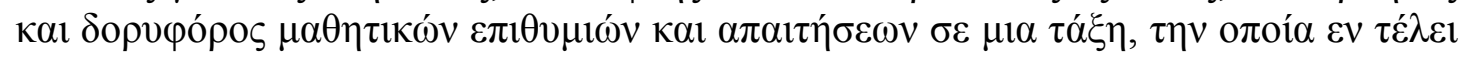

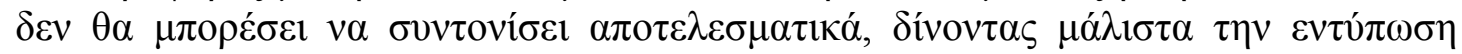

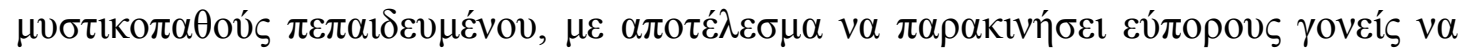

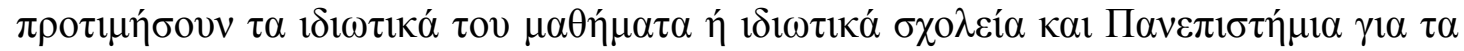

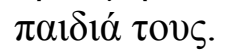

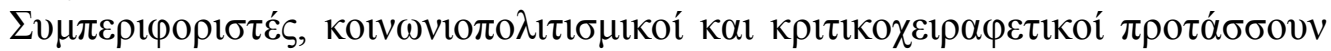

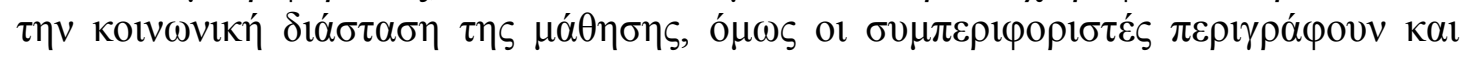

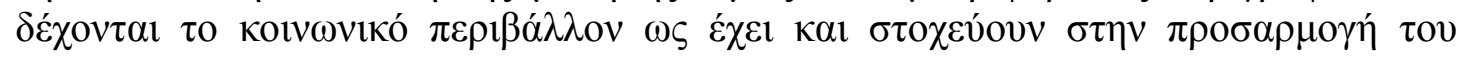

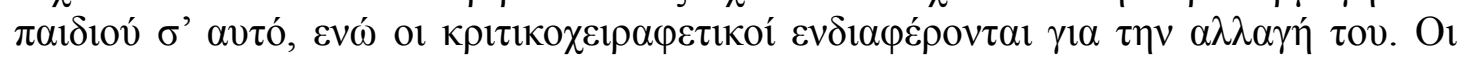

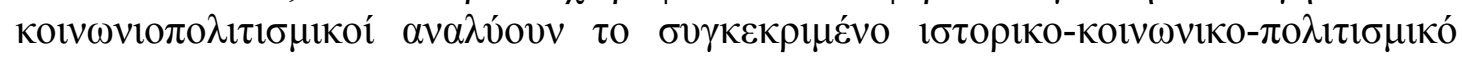

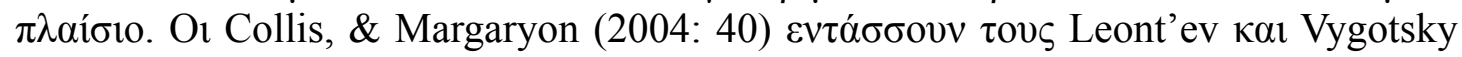

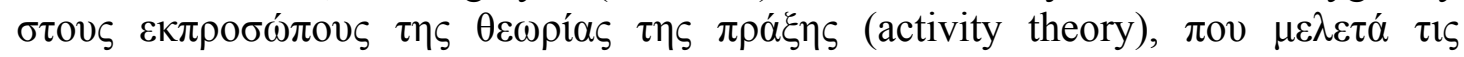

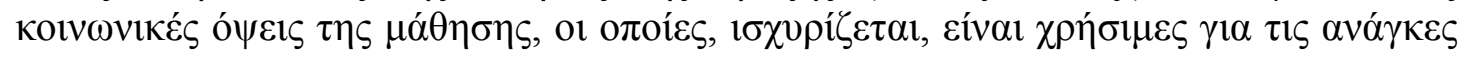

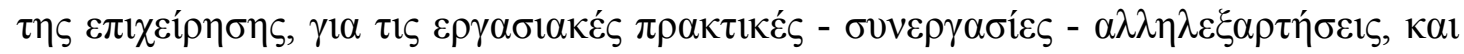

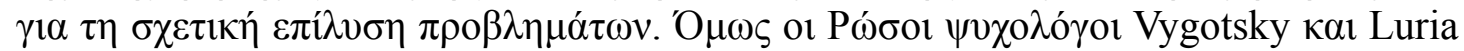

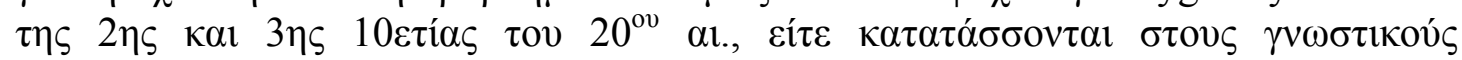

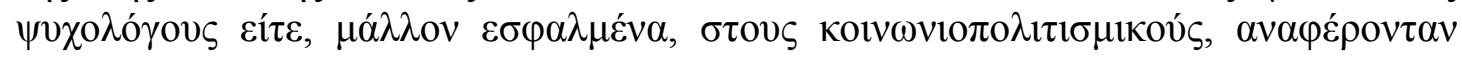

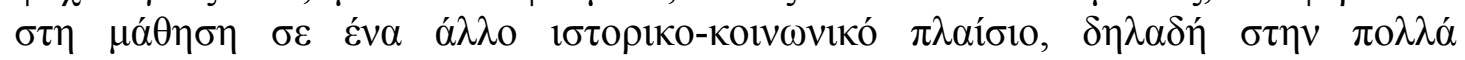

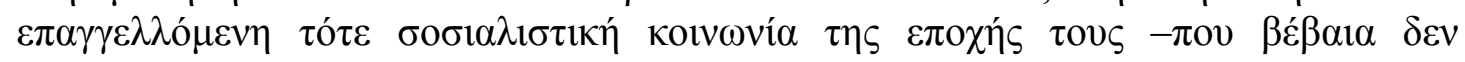

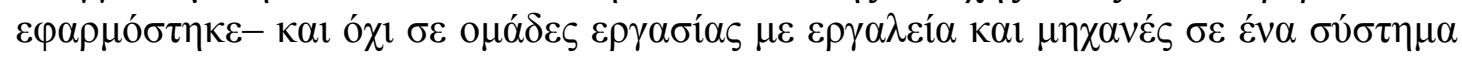

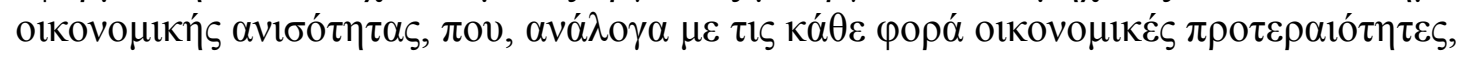

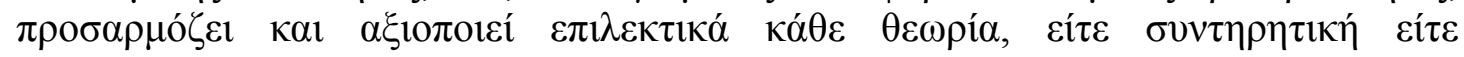
 


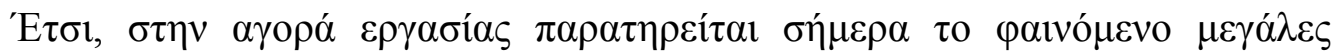

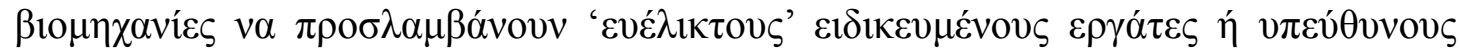

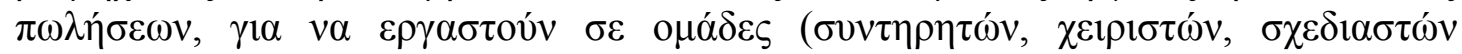

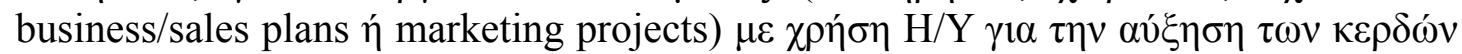

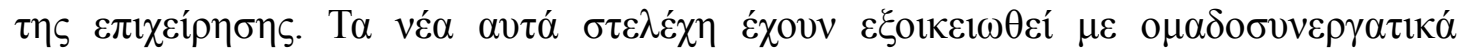

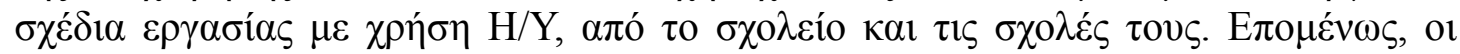

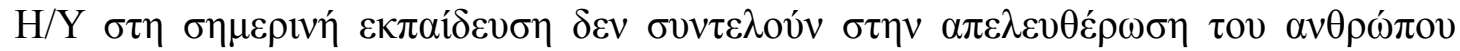

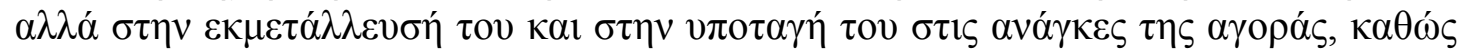

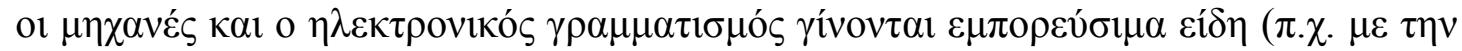

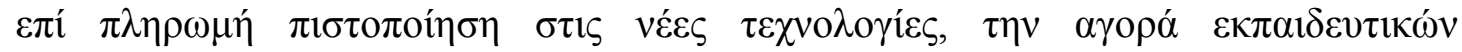

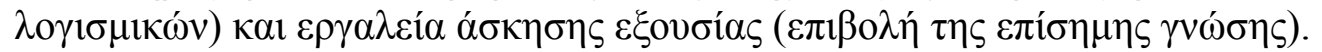

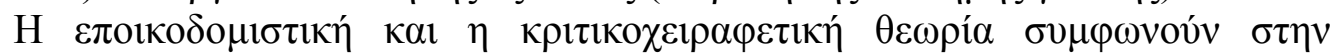

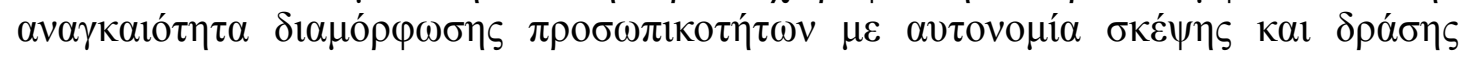

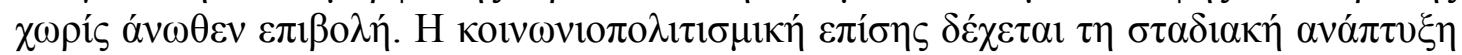

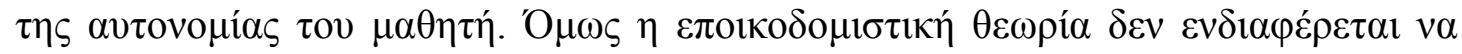

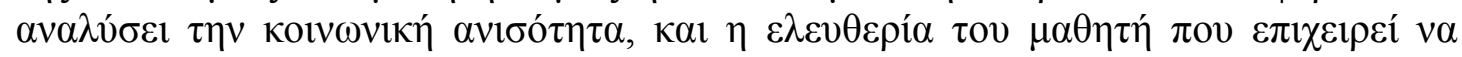

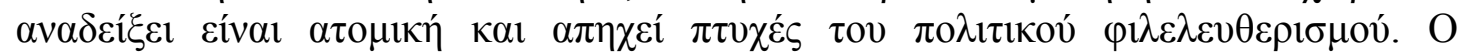

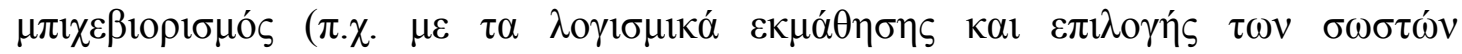

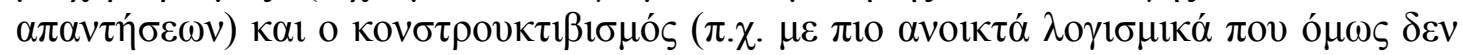

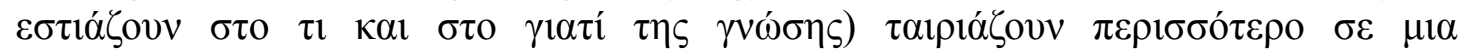

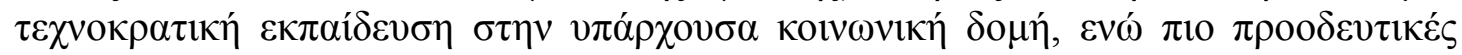

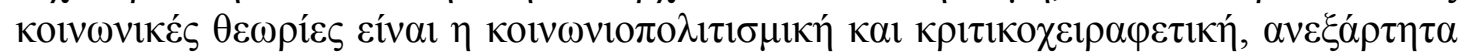

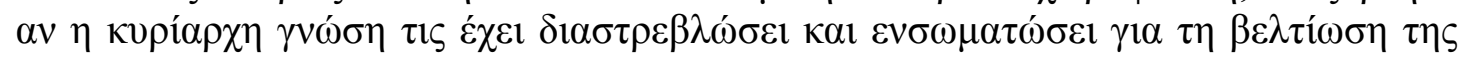

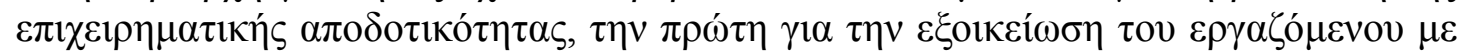

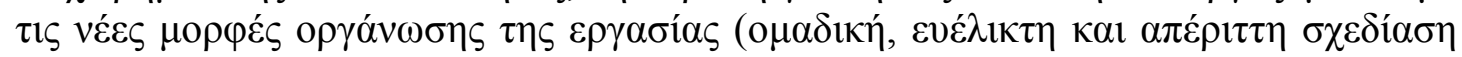

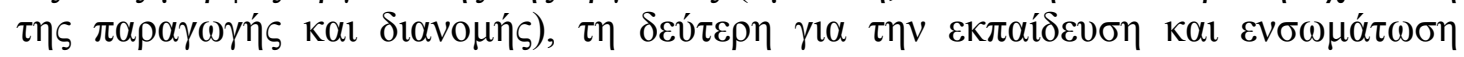

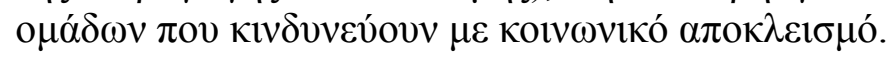

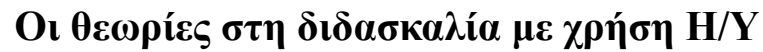

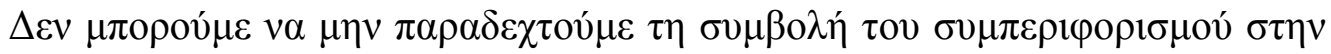

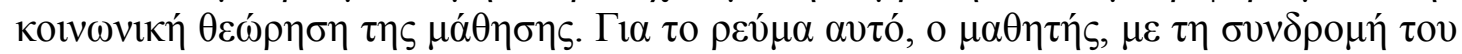

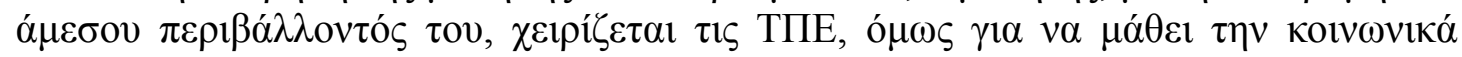

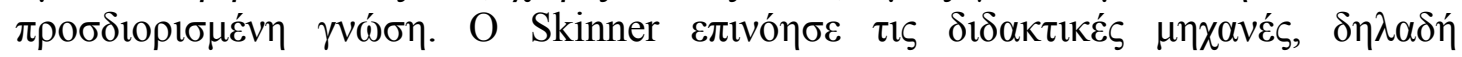

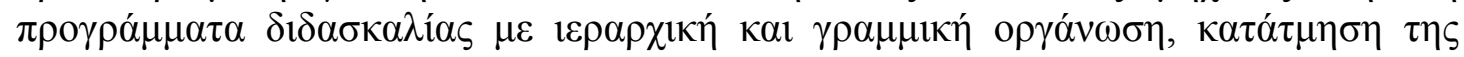

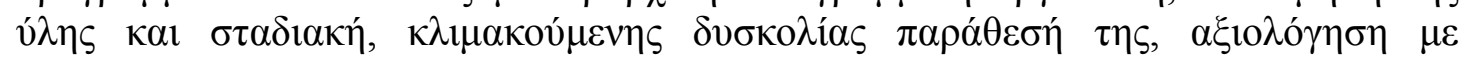

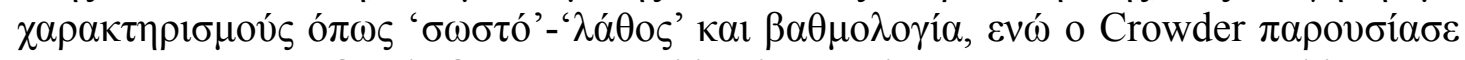

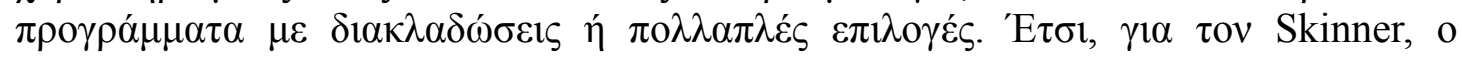

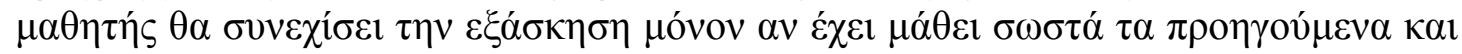

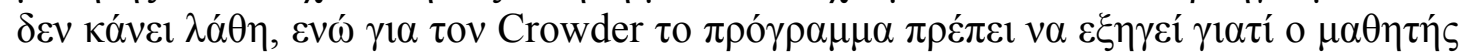

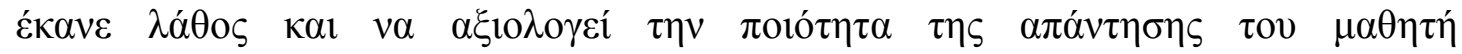

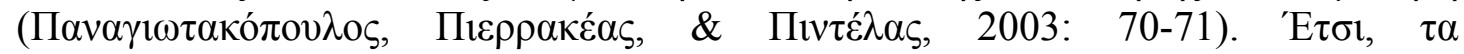

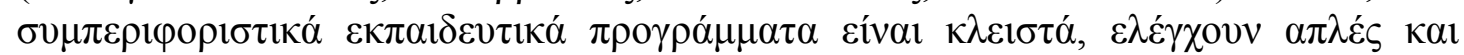

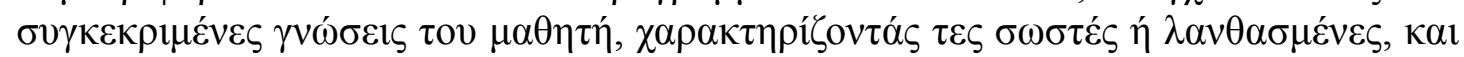

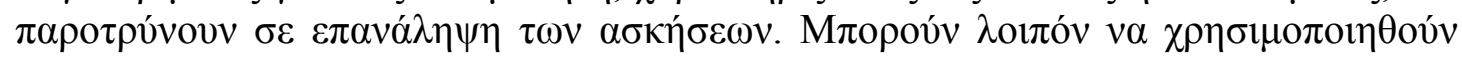

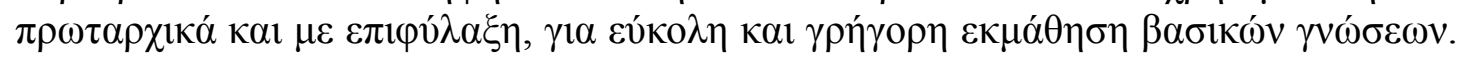

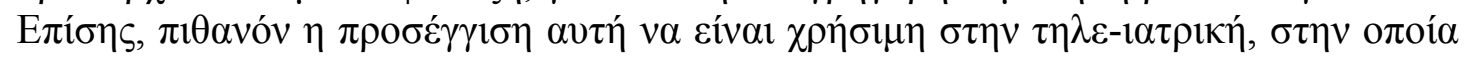

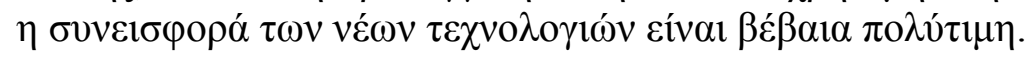

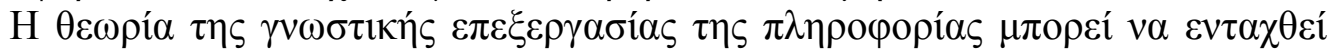

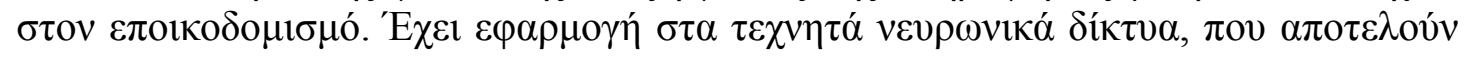




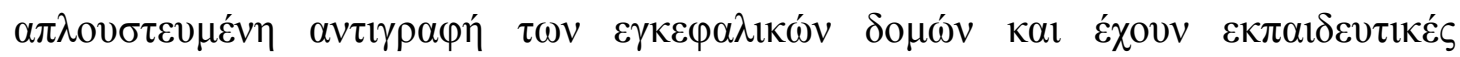

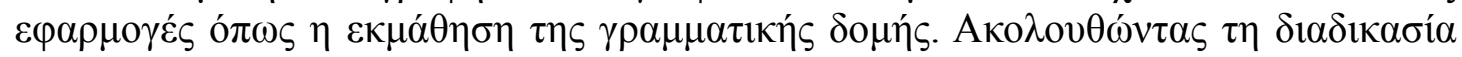

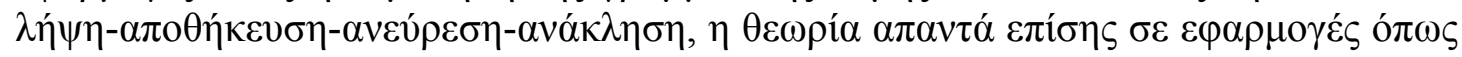

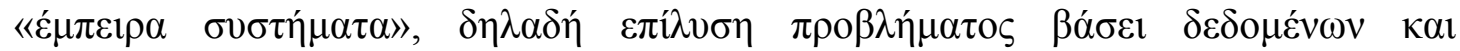

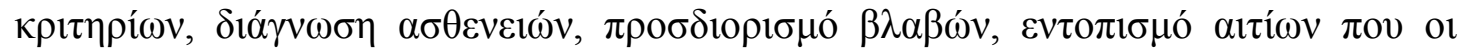

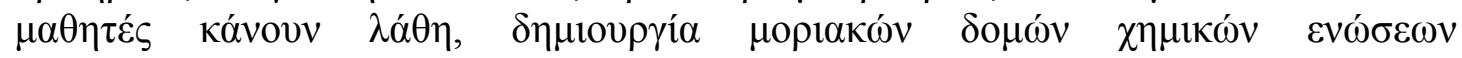

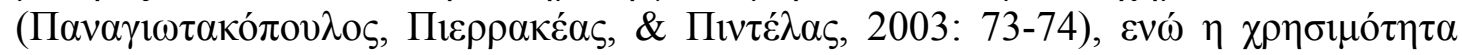

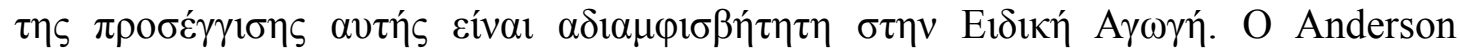

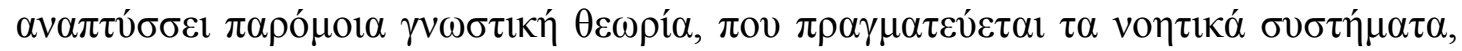

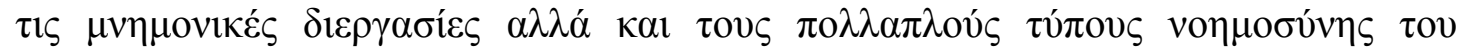

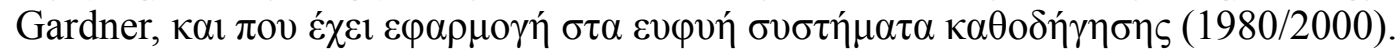

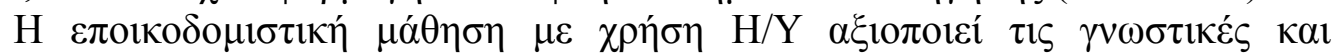

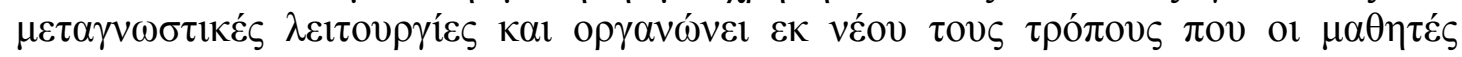

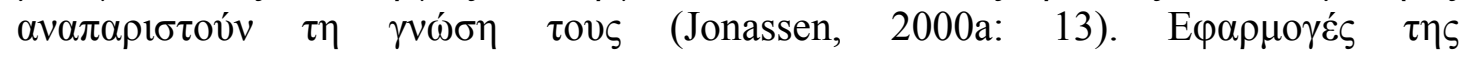

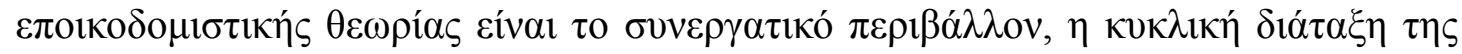

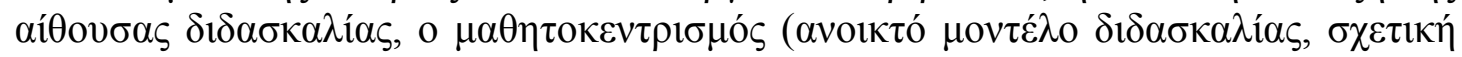

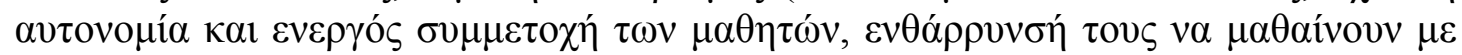

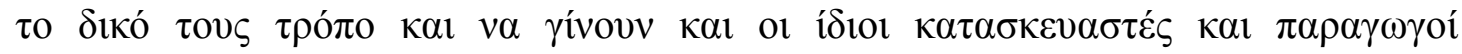

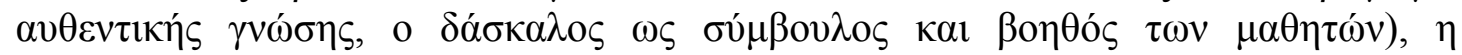

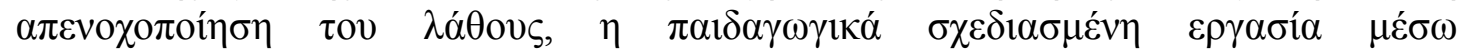

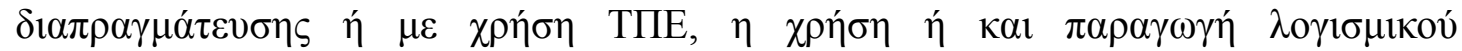

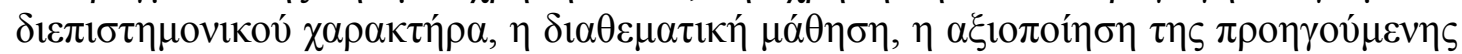

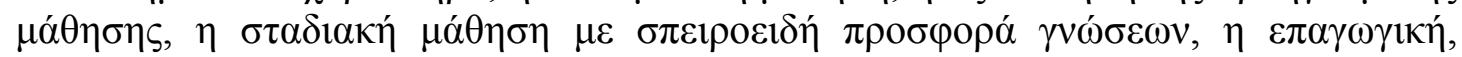

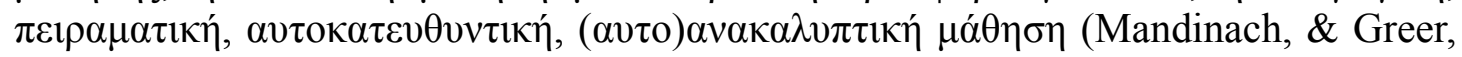
1992: 252. Jonassen, 1996: 13. Reusser, 1996: 87, 90. Davis, 1997: 179. Poole, 1998: 368. Kanuka, \& Anderson, 1999. Jonassen, 2000a: 12. Seufert, 2003: 229.

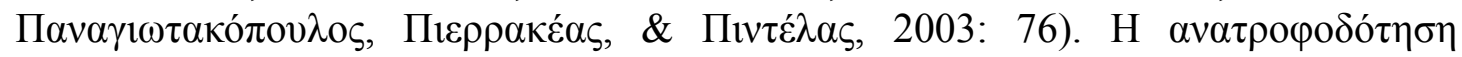

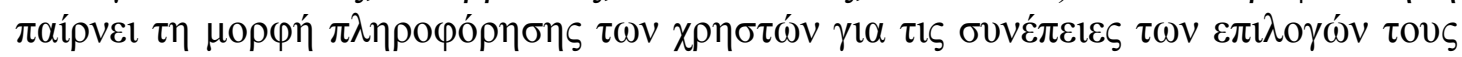

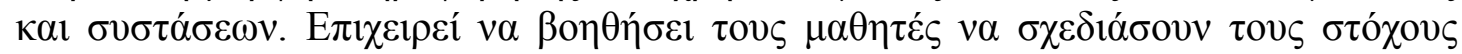

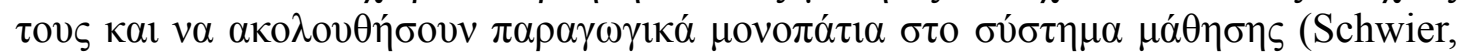
1995: 121).

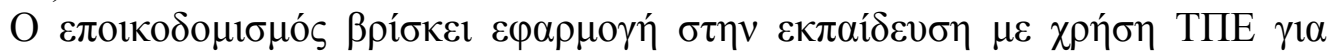

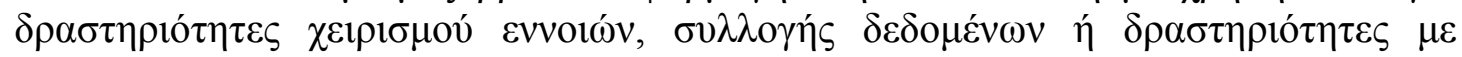

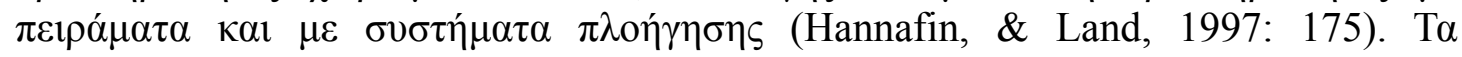

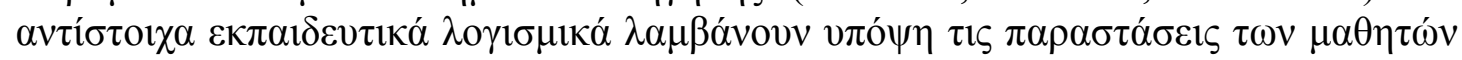

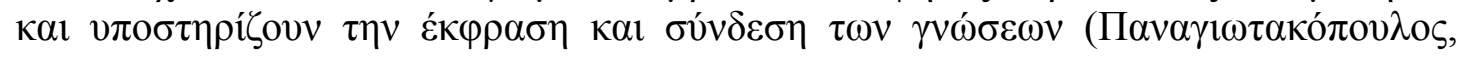

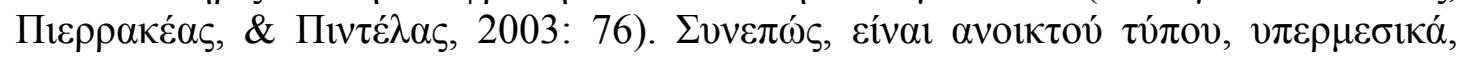

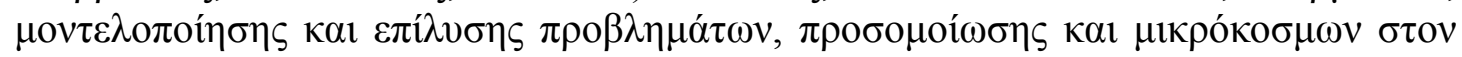

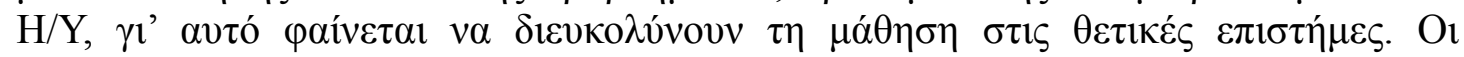

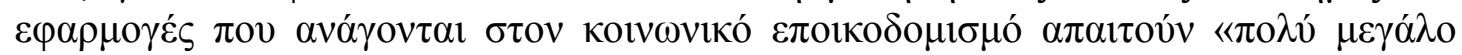

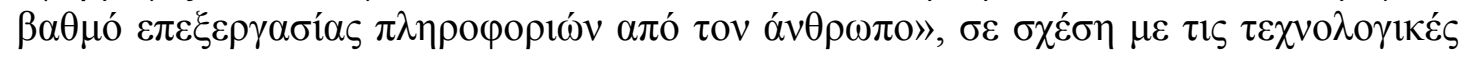

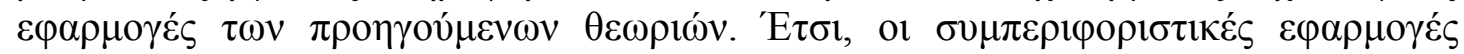

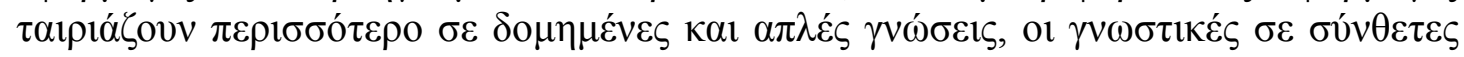

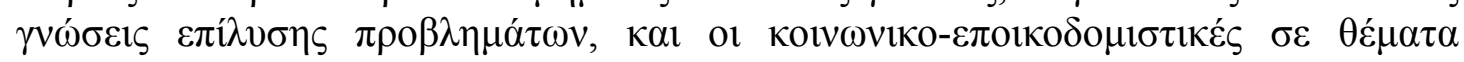

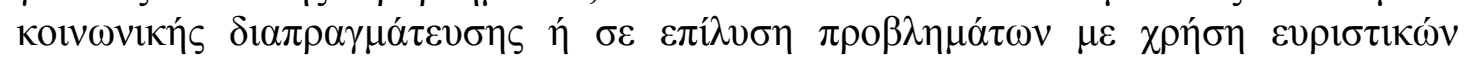

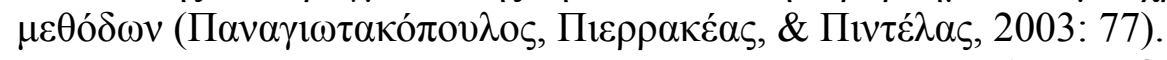

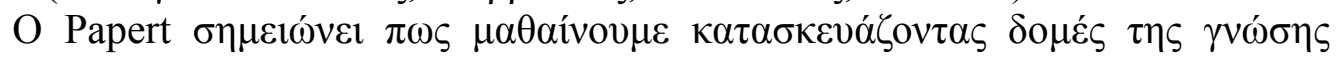

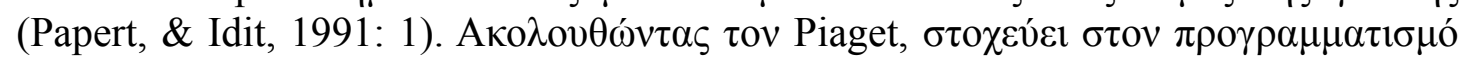

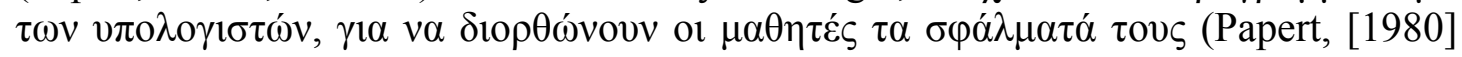

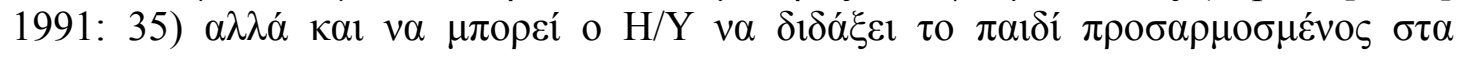




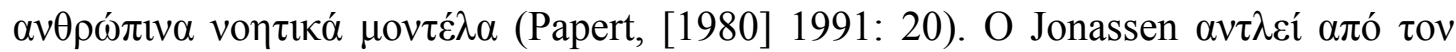

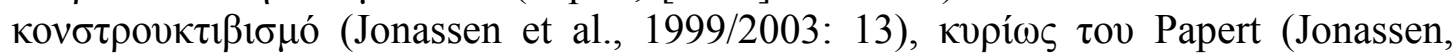

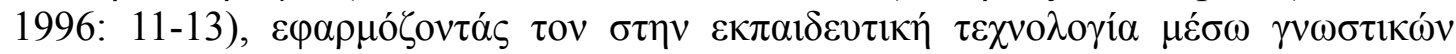

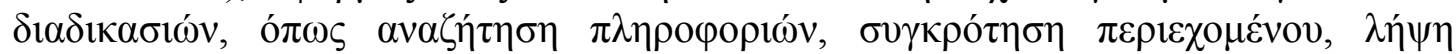

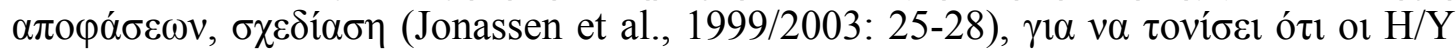

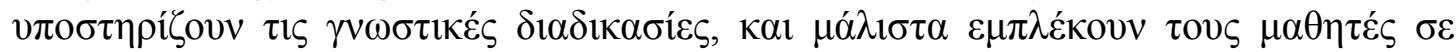

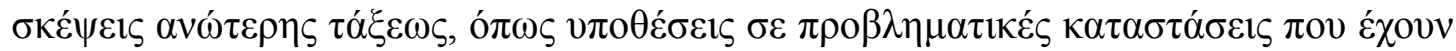

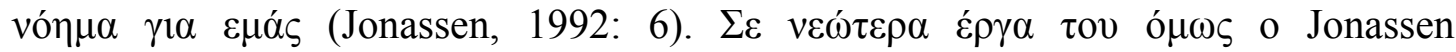

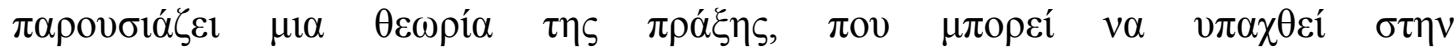

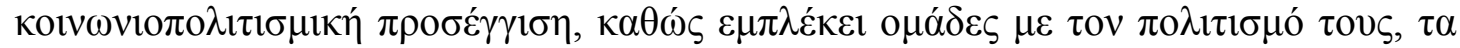
$\varepsilon \rho \gamma \alpha \lambda \varepsilon i ́ \alpha$

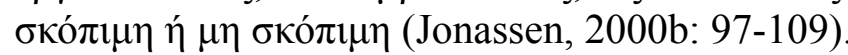

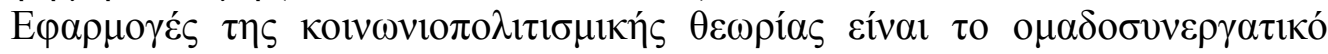

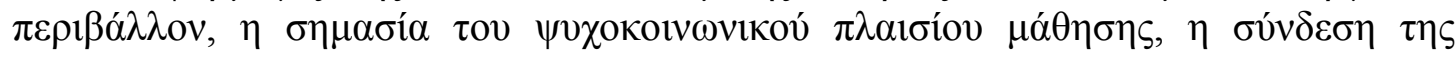

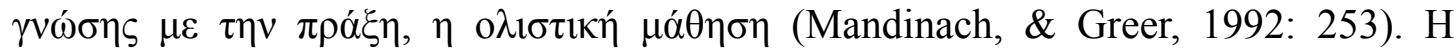

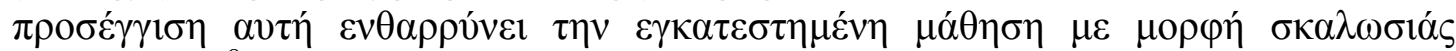

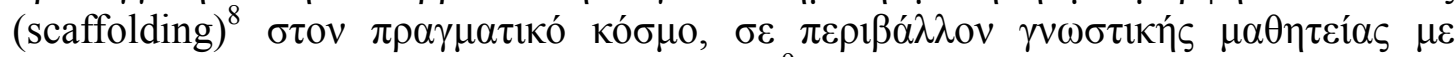

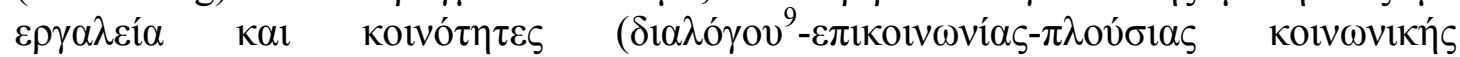

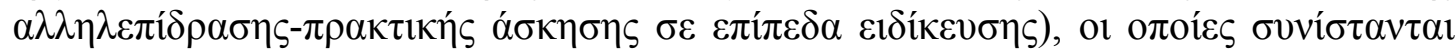

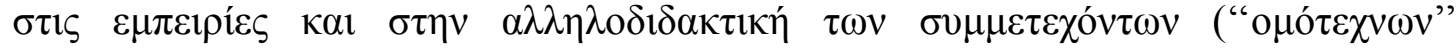

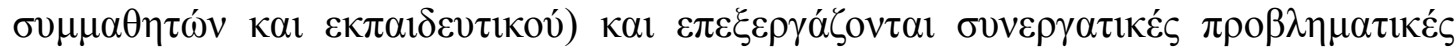

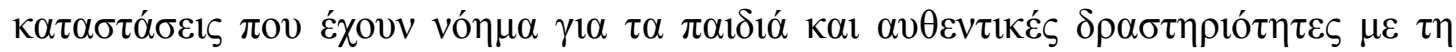

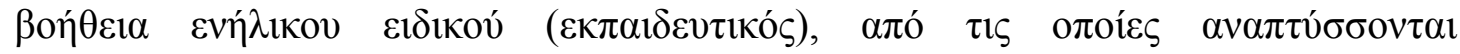

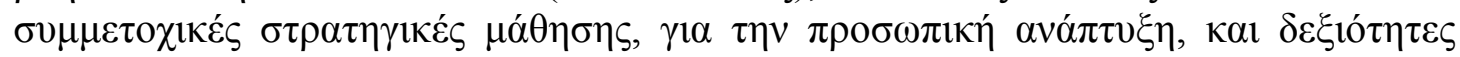

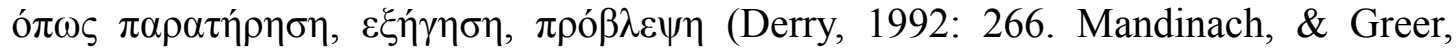
1992: 252. Pea, 1992: 315-316, 320. Scardamalia, \& Bereiter, 1992: 42. Schwier, 1995: 119. Bransford et al., 1996: 227. Dillenbourg, 1996: 170. Scrimshaw, 1997: 111. Crook, 1999: 109. Koivusaari, 1999: 312. Mercer, \& Wegerif, 1999: 79. Buysse,

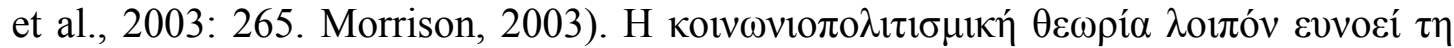

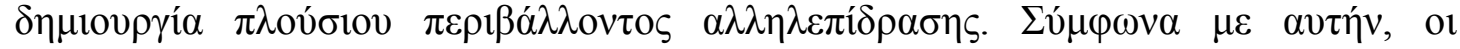

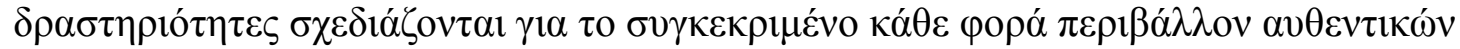

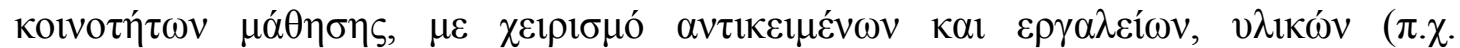

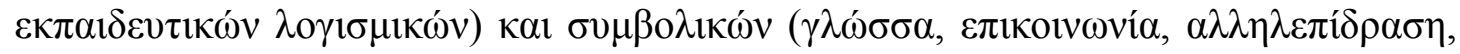

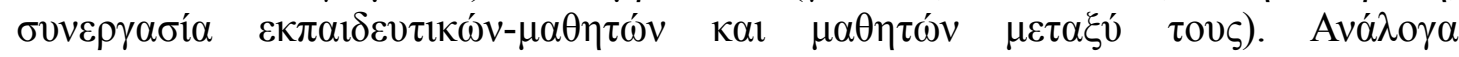

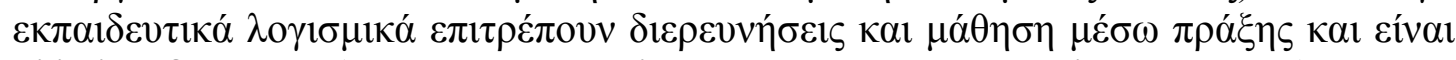

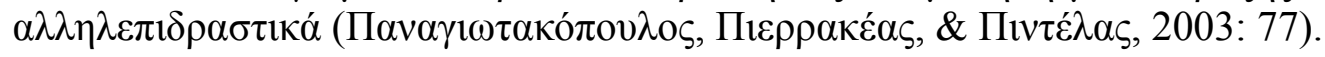

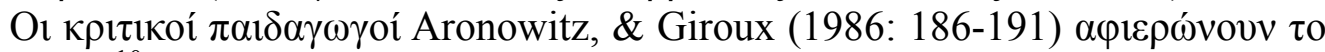

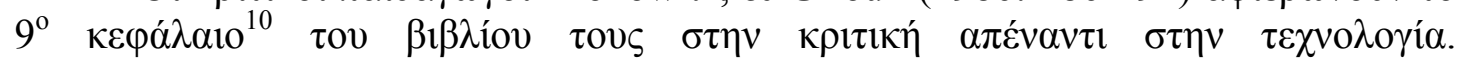

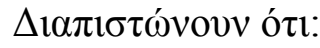

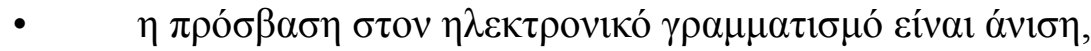

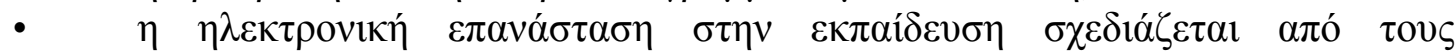

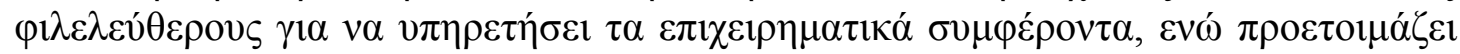

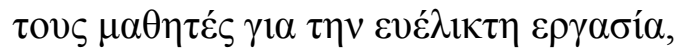

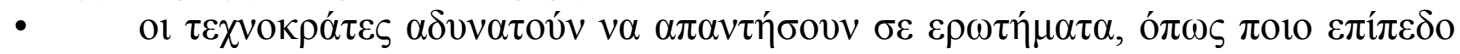

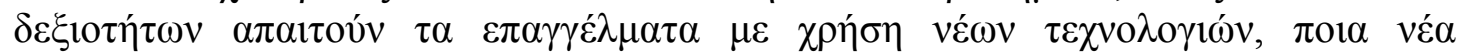

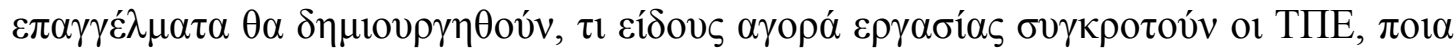

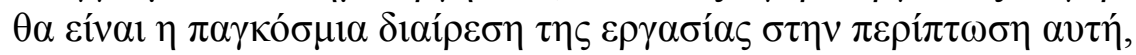

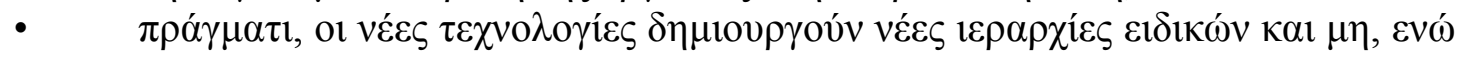

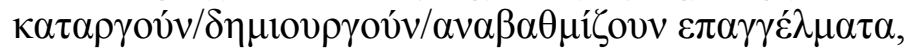




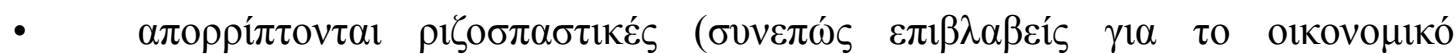

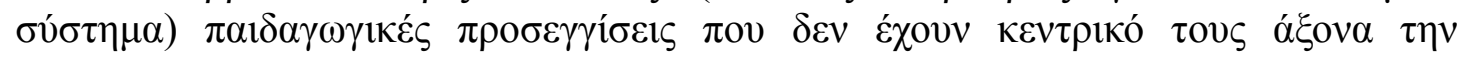

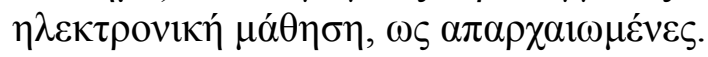

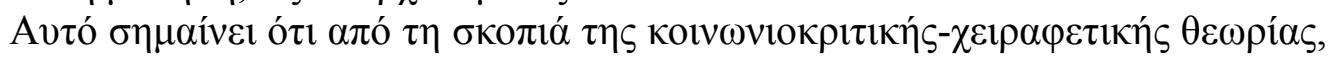

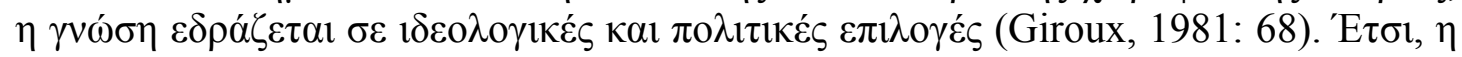

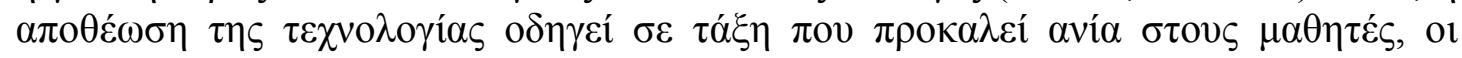

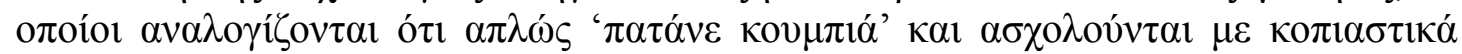

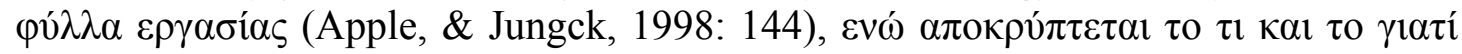

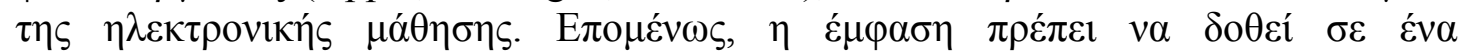

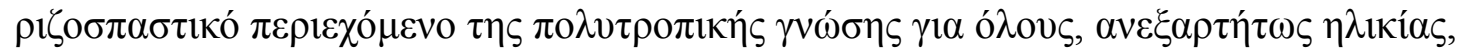

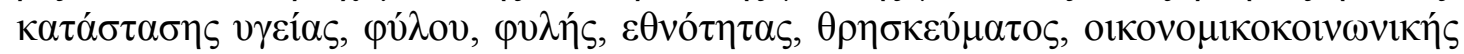

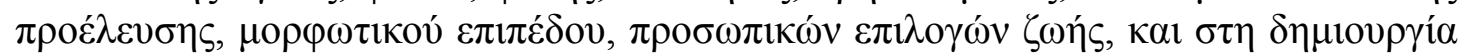

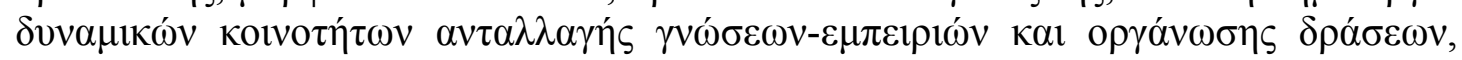

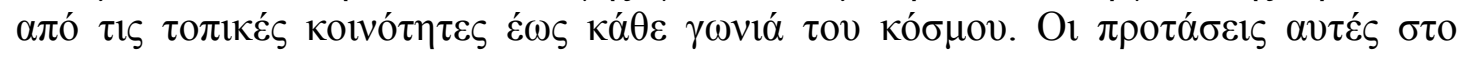

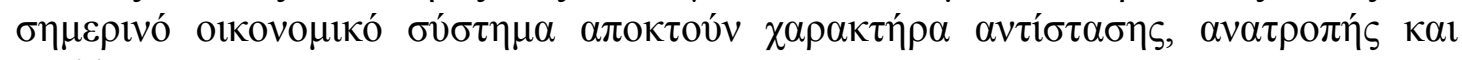

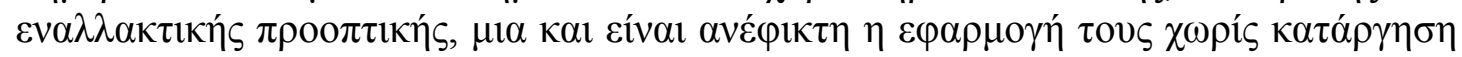

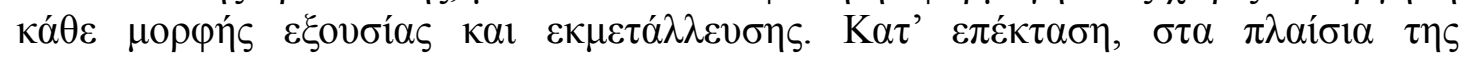

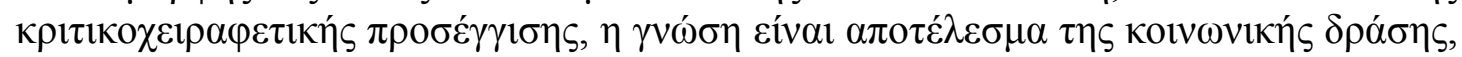

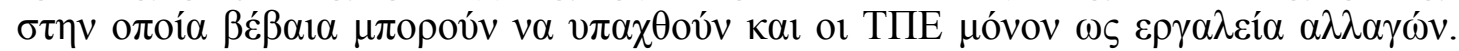

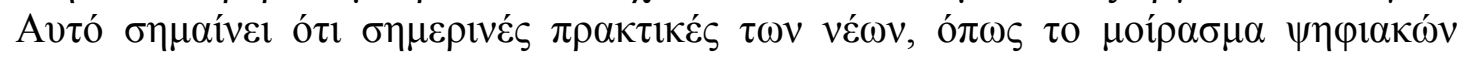

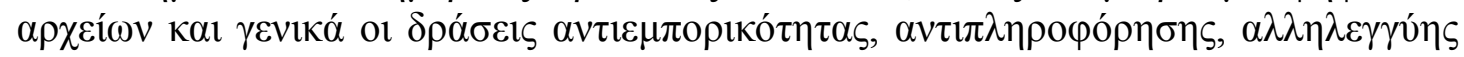

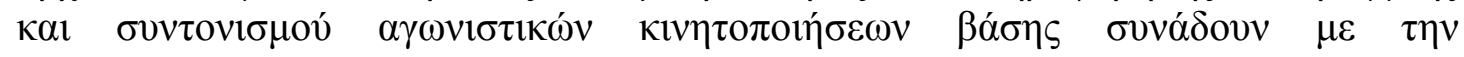

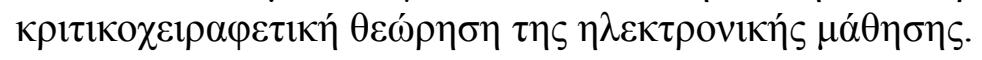

\section{$\Sigma \eta \mu \varepsilon เ \omega ́ \sigma \varepsilon เ \varsigma$}

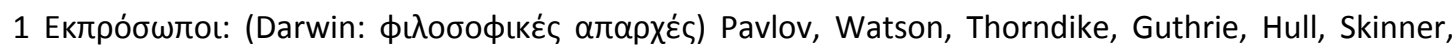

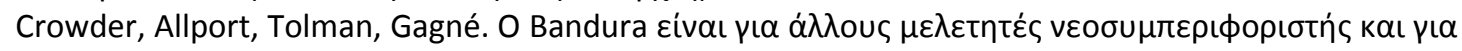

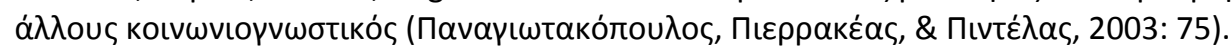

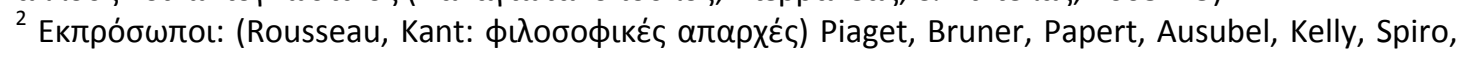
Jonassen, Schön, Glasersfeld, Barlett, Tulving, Loftus, Glynn \& Divesta, Pichert \& Anderson, Chi, Chiesi,

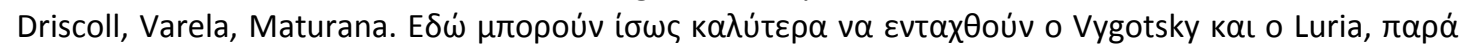

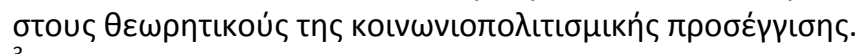

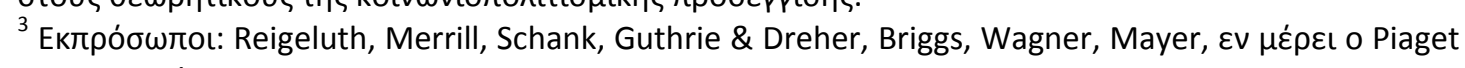
кaı o Gagné.

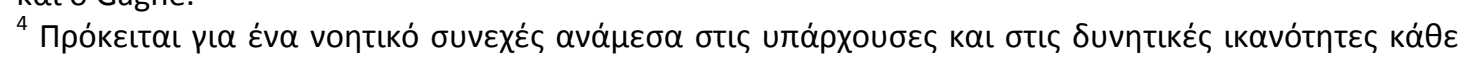
atónou (Jonassen, 2000: 13).

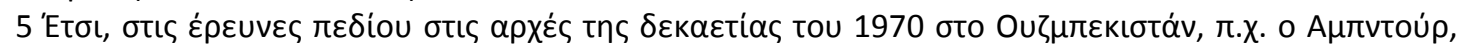

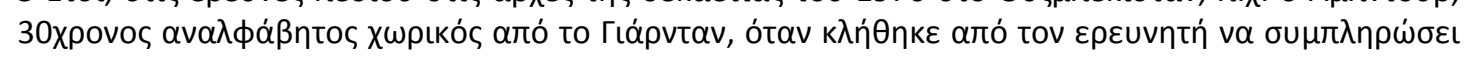

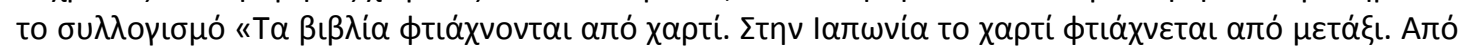

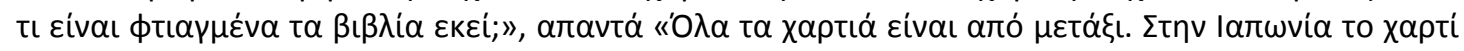

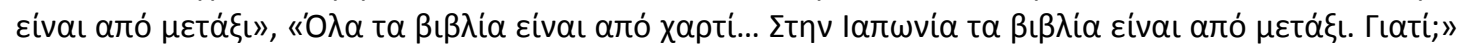

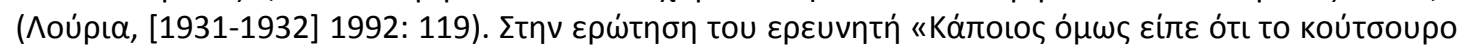

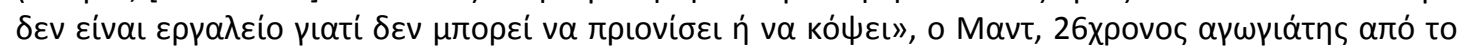

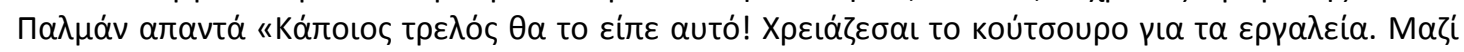

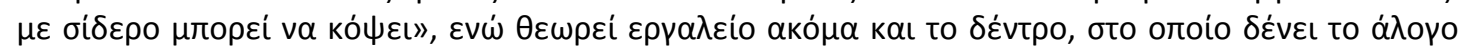

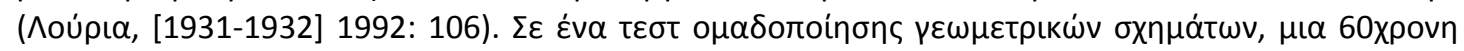

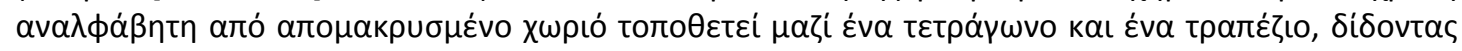

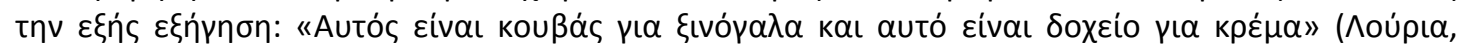

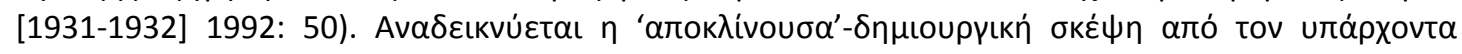

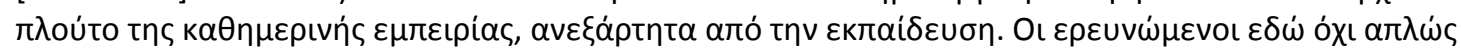

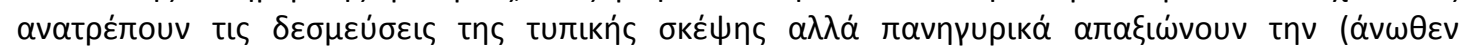

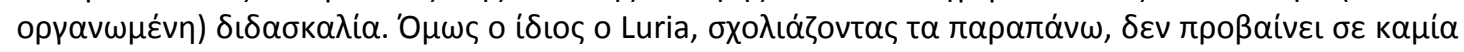




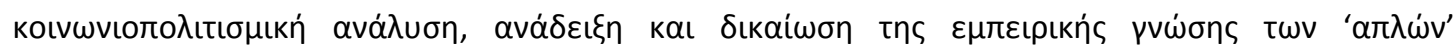

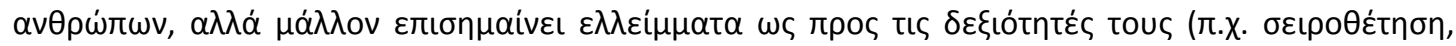

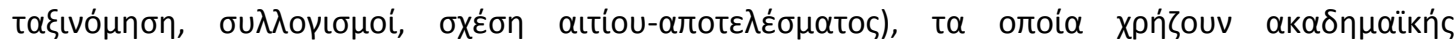
$\delta ı \delta \alpha \sigma \kappa \alpha \lambda i \alpha \varsigma !$

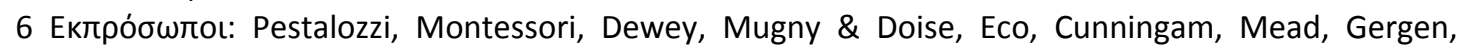
Barnes, Potter \& Wetherell, Sawicki, Parker, Derry.

${ }^{7}$ Eктро́бштол: Freire, Bourdieu \& Passeron, Bernstein, Giroux, Foucault, Derrida, Rorty, Adorno, Marcuse, Horkheimer, Habermas.

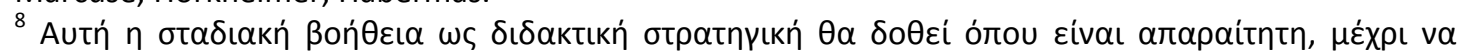

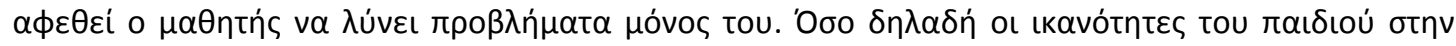

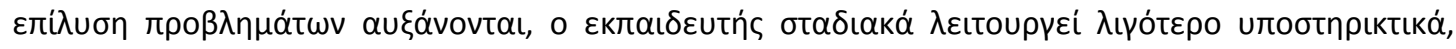

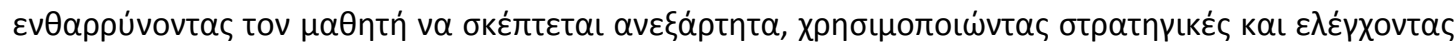

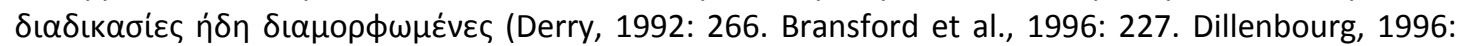
170).

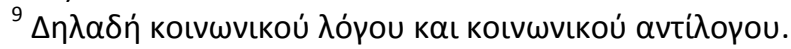

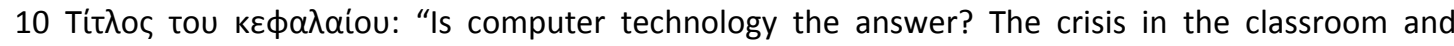
educational reform".

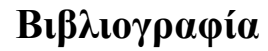

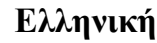

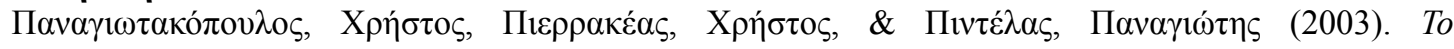

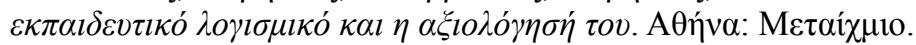

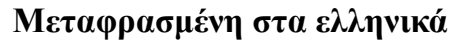

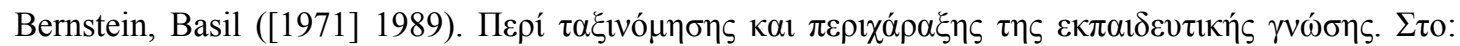

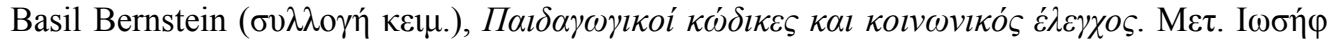

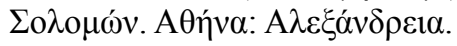

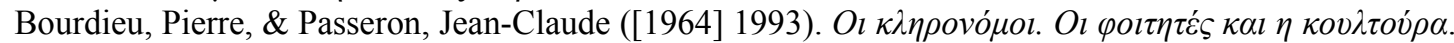

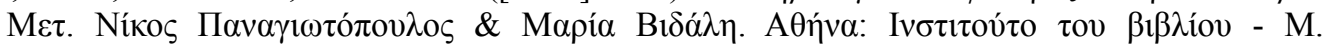
K $\alpha \rho \delta \alpha \mu i \tau \tau \sigma$.

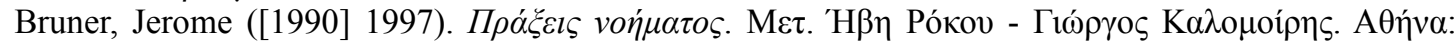

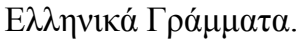

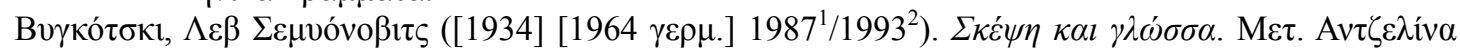

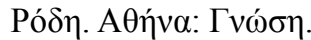

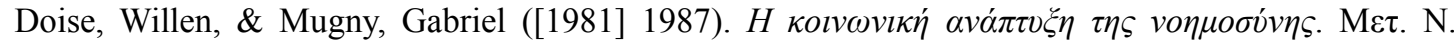

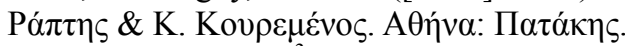

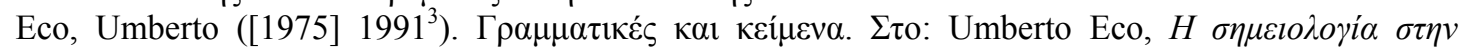

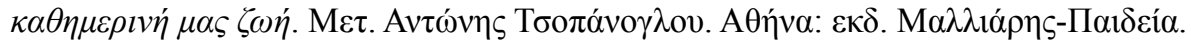

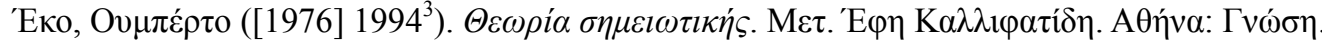

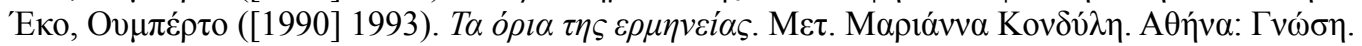

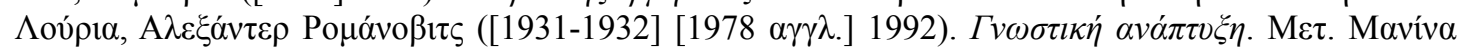

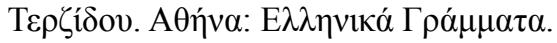

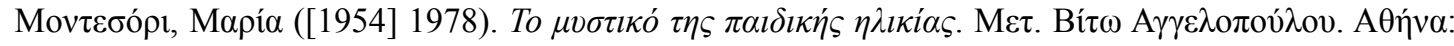
$\Delta$ íppos.

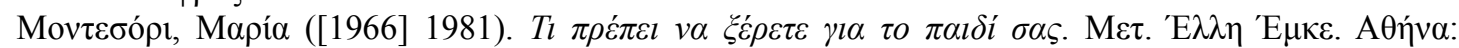

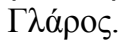

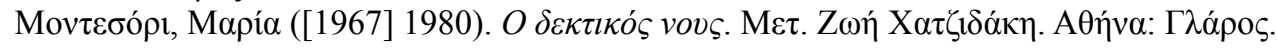

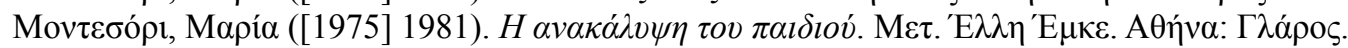

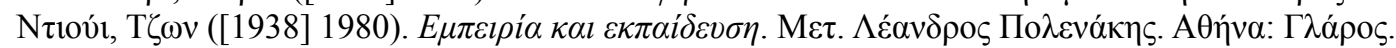

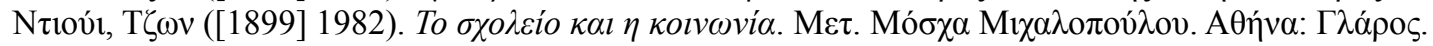

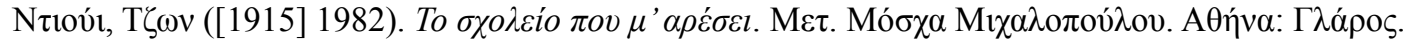

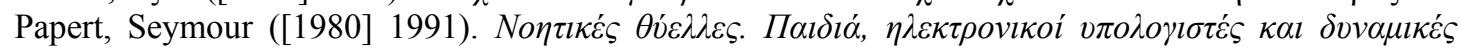

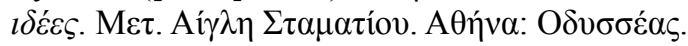

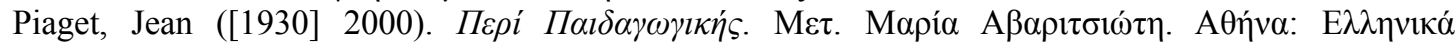
Гро́ $\mu \mu \alpha \tau \alpha$.

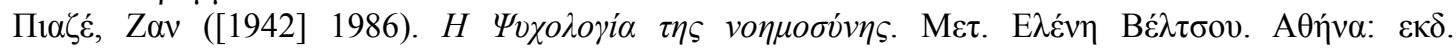




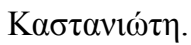

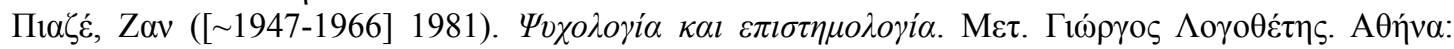

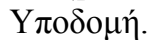

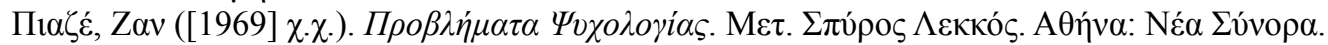

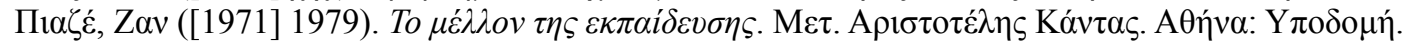

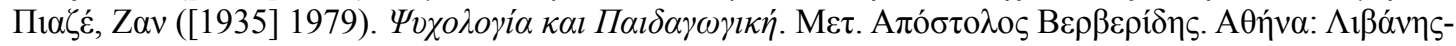

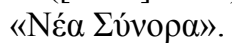

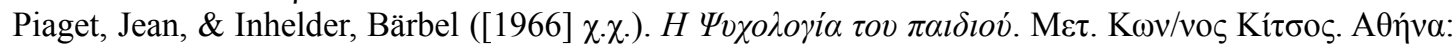

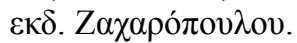

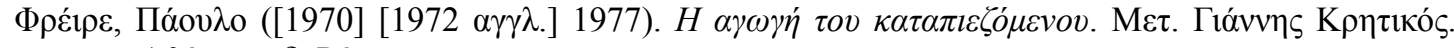

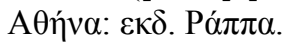

\section{$\Xi \varepsilon v o ́ \gamma \lambda \omega \sigma \sigma \eta$}

Aldrich, Frances, Rogers, Yvonne, \& Scaife, Mike (1998). Getting to grips with 'interactivity': helping teachers assess the educational value of cd-roms. British Journal of Educational Technology, 29(4).

Anderson, John R. $\left(1980 / 2000^{5}\right)$. Cognitive psychology and its implications. N.Y.: Worth Publishers.

Apple, Michael W., \& Jungck, Susan (1998). "You don't have to be a teacher to teach this unit": teaching, technology, and control in the classroom. In: Hank Bromley, \& Michael W. Apple (Eds.), Education, technology, power. Educational computing as a social practice. N.Y.: State University of New York Press.

Aronowitz, Stanley, \& Giroux, Henry A. (1986). Education under siege. The conservative, liberal, and radical debate over schooling. London \& Henley: Routledge \& Kegan Paul.

Atkinson, John W. (1958). Towards experimental analysis of human motivation in terms of motives, expectancies, and incentives. In: John W. Atkinson (Ed.), Motives in fantasy, action, and society. A method of assessment and study. N.Y.: D. van Nostrand company.

Atkinson, John W., \& Reitman, Walter R. ([1956] 1958). Performance as a function of motive strength and expectancy of goal-attainment. In: John W. Atkinson (Ed.), Motives in fantasy, action, and society. A method of assessment and study. N.Y.: D. van Nostrand company.

Bandura, Albert, \& Walters, Richard H. (1963/1967). Social learning and personality development. N.Y.: Holt, Rinehart, and Winston.

Bernstein, Basil (1971/1974). Class, codes and control. I. Theoretical studies towards a Sociology of Language. London: Routledge \& Kegan Paul.

Bernstein, Basil (1996/2000). Pedagogy, symbolic control and identity. Theory, research, critique. New York: Rowman \& Littlefield Publishers.

Bloom, Benjamin S. (1976/1982). Human characteristics and school learning. N.Y.: McGraw-Hill.

Bransford, John D., Miller Sharp, Diana, Vye, Nancy J., Goldman, Susan R., Hasselbring, Ted S., Goin, Laura, O’Banion, Keisha, Livernois, Jean, \& Saul, Elizabeth (1996). MOST environments for accelerating literacy development. In: Stella Vosniadou, Erik De Corte, Robert Glaser, \& Heinz Mandl (Eds.), International perspectives on the design of technology-supported learning environments. Mahwah, N.J.: Lawrence Erlbaum Associates.

Bruce, Bertram C., \& Hogan, Maureen P. (1998). The disappearance of technology: toward an ecological model of literacy. In: David Reinking, Michael C. McKenna, Linda D. Labbo, \& Ronald D. Kieffer (Eds.), Handbook of literacy and technology. Transformations in a posttypographic world. Mahwah-New Jersey: Lawrence Erlbaum Associates.

Bruner, Jerome S. (1960/1977). The process of education. Cambridge, Massachusetts: Harvard University Press.

Bruner, Jerome S. (1966). Toward a theory of instruction. Cambridge, Massachusetts: The Belknap Press of Harvard University Press.

Buysse, Virginia, Sparkman, Karen L., \& Wesley, Patricia W. (2003). Communities of practice: connecting what we know with what we do. Exceptional Children, 69(3).

Cantero Ortega, Manuel (2000). Computers in education: the near future. In: Manuel Ortega, \& José Bravo (Eds.), Computers and education in the $21^{\text {st }}$ century. Dordrecht: Kluwer Academic Publishers.

Collins, Allan (1996). Design issues for learning environments. In: Stella Vosniadou, Erik De Corte, Robert Glaser, \& Heinz Mandl (Eds.), International perspectives on the design of technologysupported learning environments. Mahwah, N.J.: Lawrence Erlbaum Associates.

Collis, Betty, \& Margaryon, Anoush (2004). Applying activity theory to computer-supported collaborative learning and work-based activities in corporate settings. Educational Technology 
Research and Development, 52(4).

Crook, Charles (1996). Computers and the collaborative experience of learning. A psychological perspective. London, N.Y.: Routledge.

Crook, Charles (1999). Computers in the community of classrooms. In: Karen Littleton \& Paul Light (Eds.), Learning with computers. Analysing productive interaction. London, N.Y.: Routledge.

Davis, Niki (1997). Do electronic communications offer a new learning opportunity in education? In: Bridget Somekh, \& Niki Davis (Eds.), Using information technology effectively in teaching and learning. Studies in pre-service and in-service teacher education. London, N.Y.: Routledge.

Davis, Niki, Desforges, Charles, Jessel, John, Somekh, Bridget, Taylor, Chris, \& Vaughan, Gay (1997). Can quality in learning be enhanced through the use of IT? In: Bridget Somekh, \& Niki Davis (Eds.), Using information technology effectively in teaching and learning. Studies in preservice and in-service teacher education. London, N.Y.: Routledge.

Derry, Sharon J. (1992). Metacognitive models of learning and instructional systems design. In: Marlene Jones, \& Philip H. Winne (Eds.), Adaptive learning environments. Foundations and frontiers. Berlin: Springer-Verlag.

Dewey, John (1916/1966). Democracy and education. An introduction to the philosophy of education. N.Y. \& London: The Free Press.

Dillenbourg, Pierre (1996). Distributing cognition over humans and machines. In: Stella Vosniadou, Erik De Corte, Robert Glaser, \& Heinz Mandl (Eds.), International perspectives on the design of technology-supported learning environments. Mahwah, N.J.: Lawrence Erlbaum Associates.

Driscoll, Marcy Perkins (1994/2005³). Psychology of learning for instruction. Boston: Pearson.

Filonov, G.N. (1994). Anton Makarenko. International Bureau of Education, 14(1-2). Paris: UNESCO. Retrieved 25-5-2010 from http://www.ibe.unesco.org/publications/ThinkersPdf/makarene.pdf.

Gagné, Robert Mills (1977/1984 $)$. The conditions of learning and theory of instruction. N.Y.: Holt, Rinehart \& Winston.

Gagné, Robert Mills, Briggs, Leslie J., \& Wager, Walter W. (1974/1988³). Principles of instructional design. N.Y.: Holt, Rinehart \& Winston.

Gagné, Robert Mills, \& Driscoll, Marcy Perkins (1988/1989²). Essentials of learning for instruction. Englewood Cliffs, N.J.: Prentice Hall.

Giroux, Henry A. (1981). Ideology, culture, and the process of schooling. Philadelphia: Temple University Press.

Giroux, Henry A. (1987/2001). Literacy and the pedagogy of political empowerment. In: Paulo Freire, \& Donaldo Macedo, Literacy. Reading the word and the world. London: Routledge.

Guthrie, John T., \& Dreher, Mariam Jean (1990). Literacy as search: explorations via computer. In: Don Nix, \& Rand Spiro (Eds.), Cognition, education, and multimedia: exploring ideas in high technology. Hillsdale, N.J.: Lawrence Erlbaum Associates.

Hannafin, Michael J., \& Land, Susan M. (1997). The foundations and assumptions of technologyenhanced student-centered learning environments. Instructional Science, 25.

Jarvis, Peter (2005). Human learning: the interrelationship of the individual and the social structures. In: Peter Jarvis, \& Stella Parker (Eds.), Human learning. An holistic approach. London \& N.Y.: Routledge.

Jarvis, Peter, Holford, John, \& Griffin, Colin (1998/1999). The theory and practice of learning. London: Kogan Page.

Jonassen, David H. (1992). What are the cognitive tools? In: Piet A.M. Kommers, David H. Jonassen, \& J. Terry Mayes (Eds.), Cognitive tools for learning. Heidelberg: Springer-Verlag.

Jonassen, David H. (1996). Computers in the classroom. Mindtools for critical thinking. Englewood Cliffs: Prentice Hall.

Jonassen, David H. (2000ª). Computers as mindtools for schools. Engaging critical thinking. Upper Saddle River: Merrill Prentice Hall.

Jonassen, David H. (2000b). Revisiting activity theory as a framework for designing student-centered learning environments. In: David H. Jonassen, \& Susan Land (Eds.), Theoretical foundations of learning environments. Mahwah, N.J.: Lawrence Erlbaum Associates.

Jonassen, David H., Howland, Jane, Moore, Joi, \& Marra, Rose M. (1999/2003²). Learning to solve problems with technology. A constructivist perspective. Upper Saddle River, N.J.: MerrillPrentice Hall.

Kahn, Peter H., \& Friedman, Batya (1998). Control and power in educational computing. In: Hank Bromley, \& Michael W. Apple (Eds.), Education, technology, power. Educational computing as a social practice. N.Y.: State University of New York Press. 
Kanuka, Heather, \& Anderson, Terry (1999). Using constructivism in technology-mediated learning: constructing order out of the chaos in the literature. Radical Pedagogy, 1(2).

Katz, Sandra (1993). The role of the tutor in computer-based collaborative learning situations. In: Susanne P. Lajoie \& Sharon Derry (Eds.), Computers as cognitive tools. N.J.: Lawrence Erlbaum Associates.

Kelly, George A. (1991). The psychology of personal constructs, vol. 1: a theory of personality. London \& N.Y.: Routledge.

Kern, Richard, \& Warschauer, Mark (2000). Introduction. Theory and practice of network-based language teaching. In: Mark Warschauer, \& Richard Kern (Eds.), Network-based language teaching: concepts and practice. Cambridge: Cambridge University Press.

Koivusaari, Ritva (1999). Cognitive strategies and computer-supported learning environments. Educational Psychology, 19(3).

Leont'ef, Alexei Nikolaevich (1977). Activity and consciousness. Retrieved 25-5-2010 from http://www.marxists.org/archive/leontev/works/1977/leon1977.htm.

Leont'ef, Alexei Nikolaevich (1978). Activity, consciousness, and personality. Retrieved 25-5-2010 from http://www.marxists.org/archive/leontev/works/1978/index.htm.

Leont'ef, Alexei Nikolaevich (1979). On Vygotsky's creative development. Retrieved 25-5-2010 from http://www.marxists.org/archive/leontev/works/1979/vygotsky.htm.

Makarenko, Anton Semyonovich (1955a). The Road to Life (An Epic of Education), vol. 1. Retrieved 25-5-2010

from

http://www.marxists.org/reference/archive/makarenko/works/road1/index.html.

Makarenko, Anton Semyonovich (1955b). The Road to Life (An Epic of Education), vol. 2. Retrieved 25-5-2010

from http://www.marxists.org/reference/archive/makarenko/works/road2/index.html.

Mandinach, Ellen, \& Greer, Jim (1992). Models of students' metacognition, motivation, and learning strategies. In: Marlene Jones, \& Philip H. Winne (Eds.), Adaptive learning environments. Foundations and frontiers. Berlin: Springer-Verlag.

Maslow, Abraham H. (1943). A Theory of Human Motivation. Psychological Review, 50(4). Retrieved 25-5-2010 from http://psychclassics.yorku.ca/Maslow/motivation.htm.

Mayer, Richard E. (2005). Cognitive theory of multimedia learning. In: Richard E. Mayer (Ed.), The Cambridge handbook of multimedia learning. Cambridge: Cambridge University Press.

McClelland, David C. ([1951] 1958). The importance of early learning in the formation of motives. In: John W. Atkinson (Ed.), Motives in fantasy, action, and society. A method of assessment and study. N.Y.: D. van Nostrand company.

Mead, George Herbert (1934/1965). On social psychology. Chicago \& London: Phoenix Books.

Mercer, Neil, \& Wegerif, Rupert (1999). Is 'exploratory talk' productive talk? In: Karen Littleton, \& Paul Light (Eds.), Learning with computers. Analysing productive interaction. London, N.Y.: Routledge.

Morrison, Dirk (2003). Using activity theory to design constructivist online learning environments for higher order thinking: a retrospective analysis. Canadian Journal of Learning and Technology, 29(3).

Myers, Jamie, Hammett, Roberta, \& McKillop, Ann Margaret (1998). Opportunities for critical literacy and pedagogy in student-authored hypermedia. In: David Reinking, Michael C. McKenna, Linda D. Labbo, \& Ronald D. Kieffer (Eds.), Handbook of literacy and technology. Transformations in a post-typographic world. Mahwah-New Jersey: Lawrence Erlbaum Associates.

Papert, Seymour (2000). Computers and computer cultures. In: Roy Pea (Ed.), Technology and learning. San Francisco: Jossey-Bass.

Papert, Seymour, \& Idit, Harel (1991). Situating constructionism. In: Seymour Papert (Ed.), Constructionism. Norwood, N.J.: Ablex Publishing Corporation.

Pea, Roy D. (1992). Augmenting the discourse of learning with computer-based learning environments. In: Erik De Corte, Marcia C. Linn, Heinz Mandl, \& Lieven Verschaffel (Eds.), Computerbased learning environments and problem solving. Heidelberg: Springer-Verlag.

Perraton, Hilary (1995). A practical agenda for theorists of distance education. In: Fred Lockwood (Ed.), Open and distance learning today. London \& N.Y.: Routledge.

Piaget, Jean ([1923] 1926/1989). The language and thought of the child. Translated by Marjorie \& Ruth Gabain. London: Routledge.

Piaget, Jean ([1926] 1989). The child's conception of the world. Translated by Joan \& Andrew Tomlinson. New Jersey: Rowman \& Littlefield Publishers.

Piaget, Jean (1951/1962). Play, dreams and imitation in childhood. Translated by C. Gattegno, \& F.M. 
Hodgson. New York \& London: W.W. Norton \& Company.

Poole, Bernard J. $\left(1998^{2}\right)$. Education for an information age. Teaching in the computerized classroom. Boston: WCB McGraw-Hill.

Reusser, Kurt (1996). From cognitive modeling to the design of pedagogical tools. In: Stella Vosniadou, Erik De Corte, Robert Glaser, \& Heinz Mandl (Eds.), International perspectives on the design of technology-supported learning environments. Mahwah, N.J.: Lawrence Erlbaum Associates.

Rogers, Carl R. (1969/1983). Freedom to learn for the 80s. Columbus: Charles E. Merrill Publishing Company.

Roy, Marguerite, \& Chi, Michelene T.H. (2005). The self-explanation principle in multimedia learning. In: Richard E. Mayer (Ed.), The Cambridge handbook of multimedia learning. Cambridge: Cambridge University Press.

Scardamalia, Marlene, \& Bereiter, Carl (1992). An architecture for collaborative knowledge building. In: Erik De Corte, Marcia C. Linn, Heinz Mandl, \& Lieven Verschaffel (Eds.), Computerbased learning environments and problem solving. Heidelberg: Springer-Verlag.

Schwier, Richard A. $\left(1995^{2}\right)$. Issues in emerging interactive technologies. In: Gary J. Anglin (Ed.), Instructional technology. Past, present, and future. Englewood: Libraries Unlimited.

Scrimshaw, Peter (1997). Computers and the teacher's role. In: Bridget Somekh, \& Niki Davis (Eds.), Using information technology effectively in teaching and learning. Studies in pre-service and in-service teacher education. London, N.Y.: Routledge.

Seufert, Tina (2003). Supporting coherence formation in learning from multiple presentations. Learning and Instruction, 13.

Shuell, Thomas J. (1992). Designing instructional computing systems for meaningful learning. In: Marlene Jones, \& Philip H. Winne (Eds.), Adaptive learning environments. Foundations and frontiers. Berlin: Springer-Verlag.

Skinner, B.F. (1953). Science and human behavior. New York - London: The Free Press \& Collier Macmillan Publishers.

Skinner, B.F. (1957). Verbal behavior. New Jersey: Prentice Hall.

Snelting, Gregor (1998). Paul Feyerabend and software technology. International Journal on Software Tools for Technology Transfer, 2.

Spiro, Rand J., \& Jehng, Jihn-Chang (1990). Cognitive flexibility and hypertext: theory and technology for the nonlinear and multidimensional traversal of complex subject matter. In: Don Nix, \& Rand Spiro (Eds.), Cognition, education, and multimedia: exploring ideas in high technology. Hillsdale, N.J.: Lawrence Erlbaum Associates.

Streibel, Michael J. $\left(1995^{2}\right)$. Instructional plans and situated learning. The challenge of Suchman's theory of situated action for instructional designers and instructional systems. In: Gary J. Anglin (Ed.), Instructional technology. Past, present, and future. Englewood: Libraries Unlimited.

Vygotsky, Lev Semyonovich ([ 1917-1923] 1978). Mind in society. The development of higher psychological processes. Translated by Martin Lopez-Morillas. Massachusetts: Harvard University Press.

Weiner, Bernard (2006). Social motivation, justice, and the moral emotions. An attributional approach. Mahwah, N.J.: Lawrence Erlbaum Associates.

Williams, Richard P., \& Hoover, Dean (1991). Matching computer software to learning theory in the reading classroom. The Clearing House, 64(5).

Wilson, Brent, \& Lowry, May (2000). Constructivist learning on the web. In: Elizabeth J. Burge (Ed.), The strategic use of learning technologies. San Francisco: Jossey-Bass. 


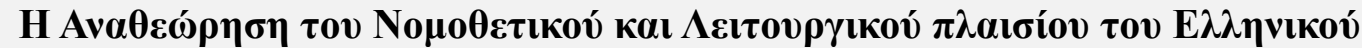

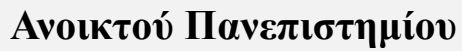 \\ The Review of the Legislative and Operational framework of the Greek Open University
}

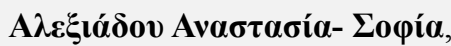

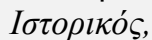

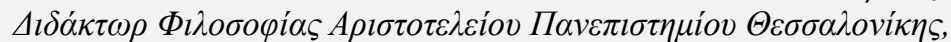

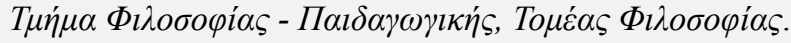 \\ sead@otenet.gr
}

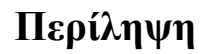

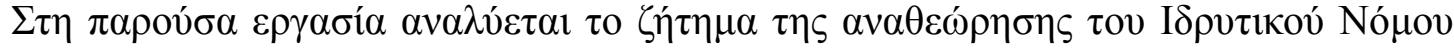

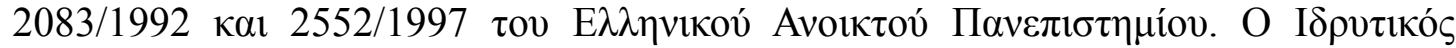

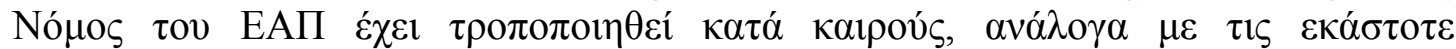

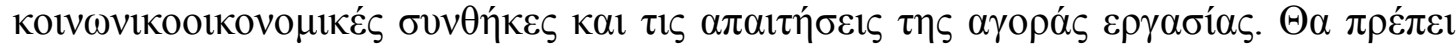

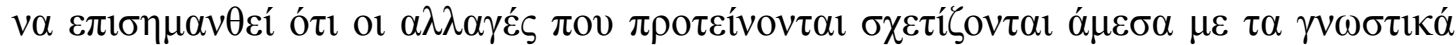

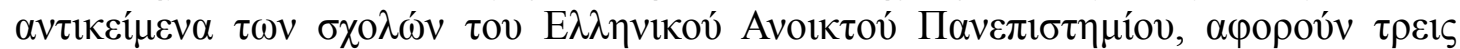

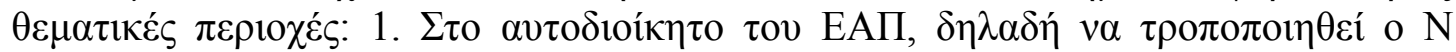

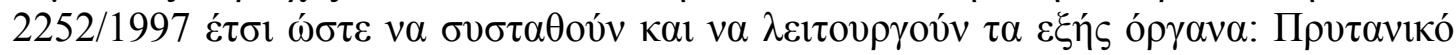

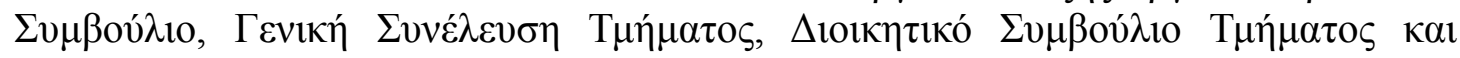

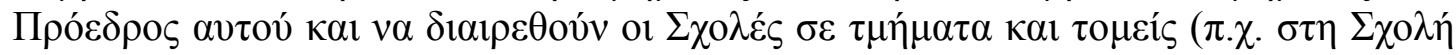

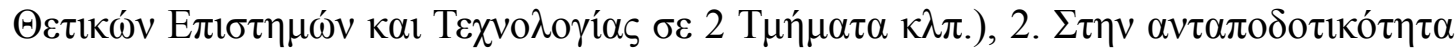

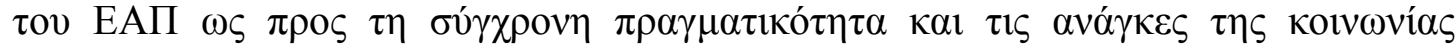

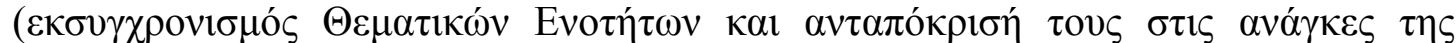

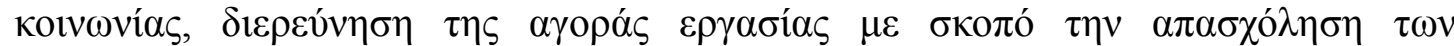

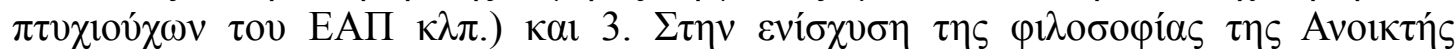

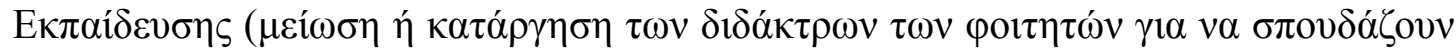

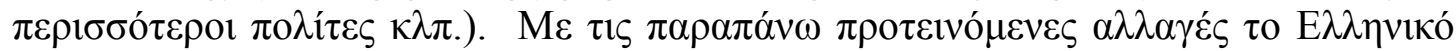

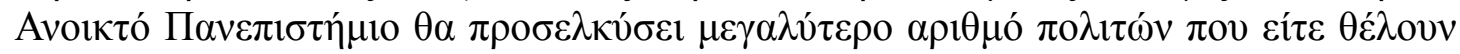

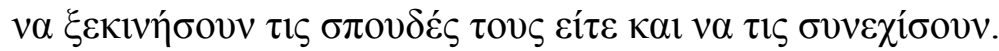

\begin{abstract}
This paper elaborates on the revision of the Founding Act 2083/1992 and 2552/1997 of the Greek Open University. The Founding Act of the Greek Open University has changed over time, depending on the socio-economic conditions and labor market requirements. It should be noted that the proposed changes are directly related to the subjects of the faculties of the Greek Open University and are focused in three areas: 1. The self-administered of the Greek Open University, which means to modify Law 2252/1997, in order to set up and operate the following bodies: Rector's Council, General Assembly Department, Board and Chairman of that Department and to divide Schools into departments and sectors (eg. School of Science and Technology in 2 Departments, etc.) 2. The modernization of the Greek Open University to the needs and demands of society (making subjects contemporary, in order to respond to the needs of society, exploring the labor market for the employment of graduates of the Greek Open University, etc.) and 3. Support of the philosophy of Open Education (reduction or abolition of tuition fees for students so that more people can study etc.). With these proposed changes the Greek Open University will attract more people who either wish to start their studies or they wish to continue them.
\end{abstract}




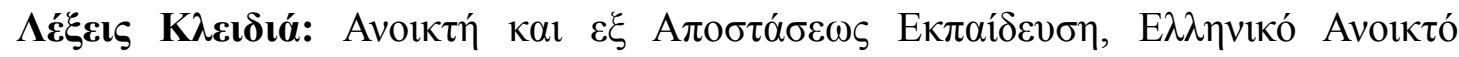

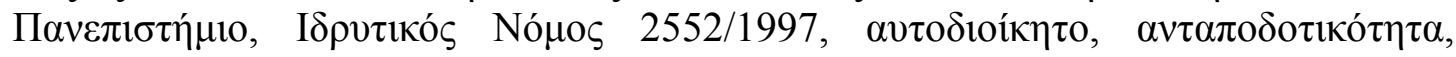

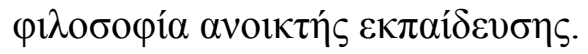

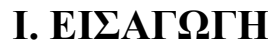

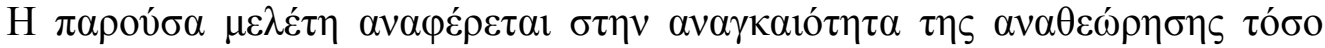

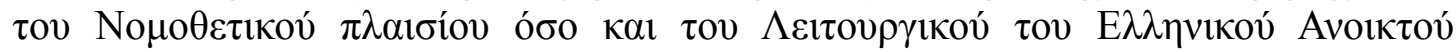

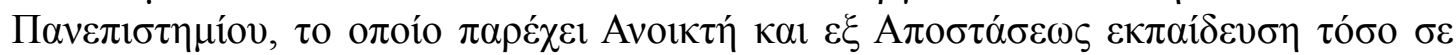

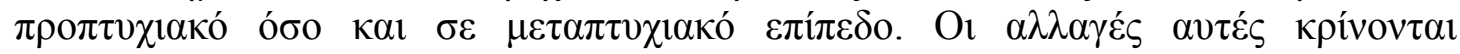

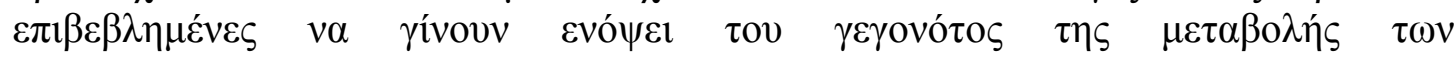

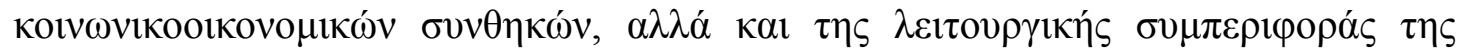

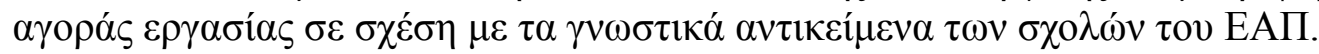

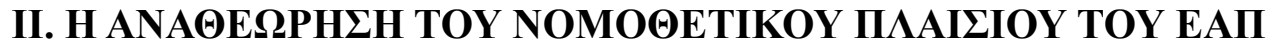

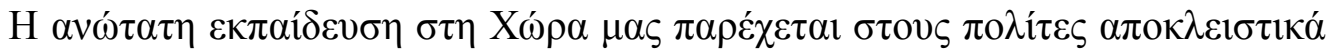

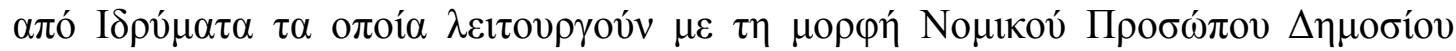

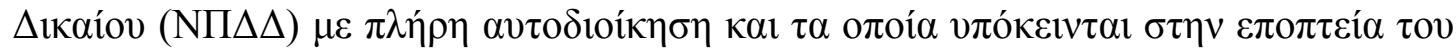

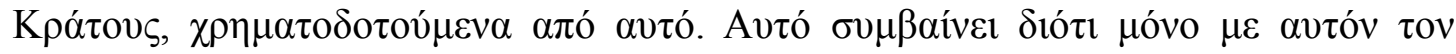

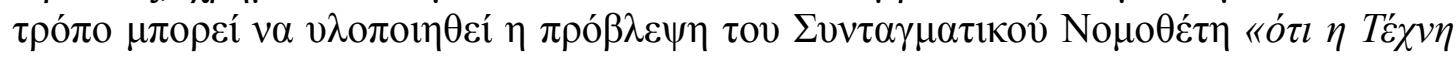

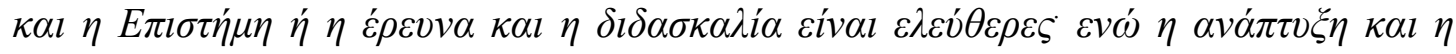

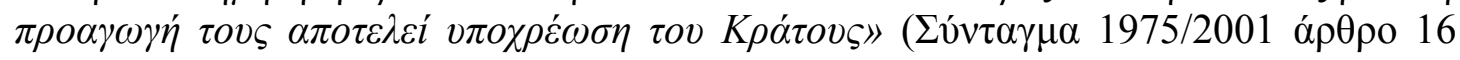

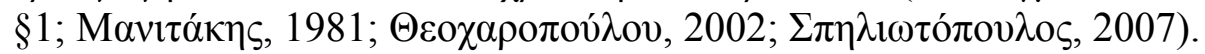

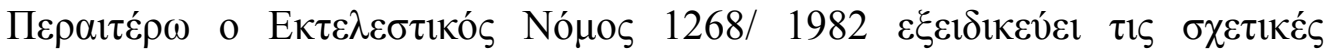

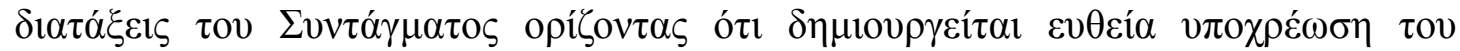

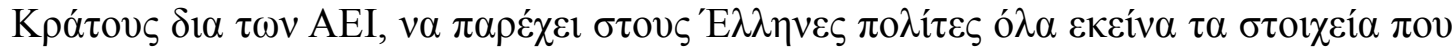

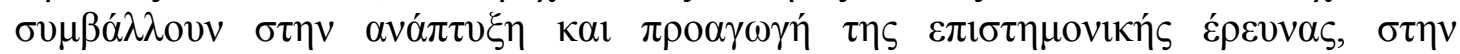

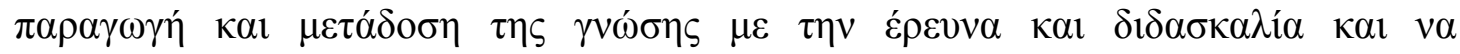

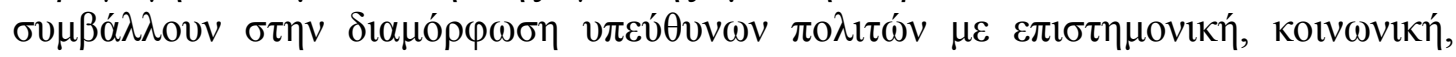

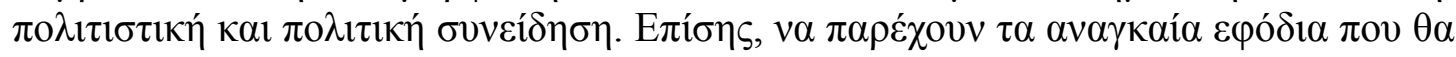

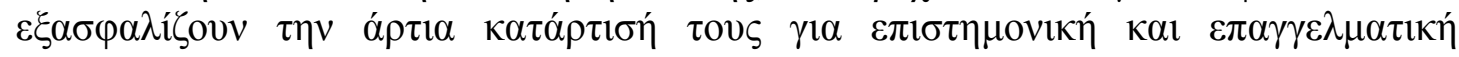

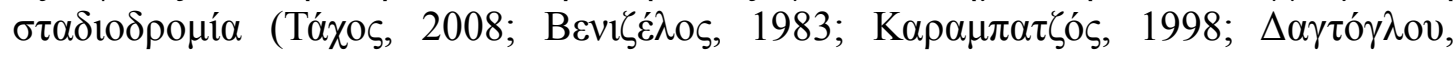
2004).

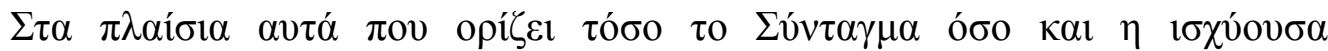

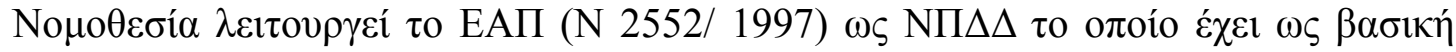

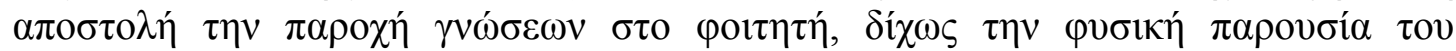

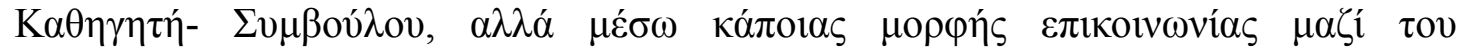

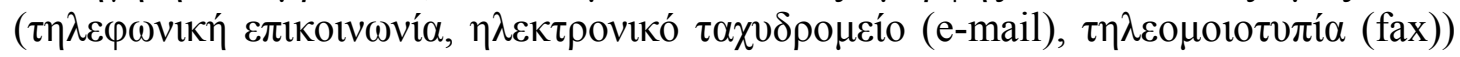

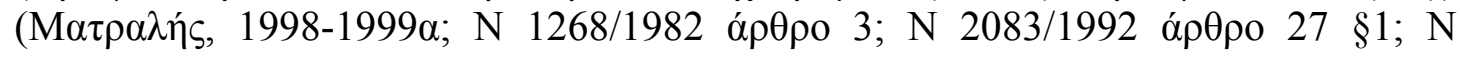

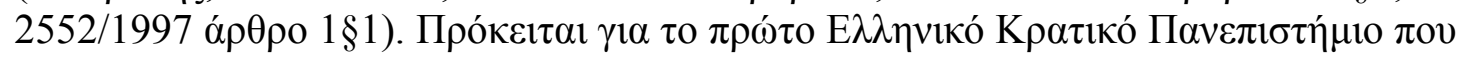

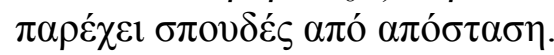

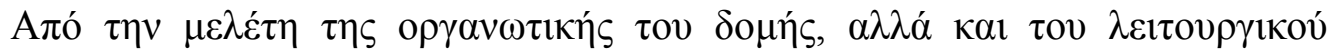

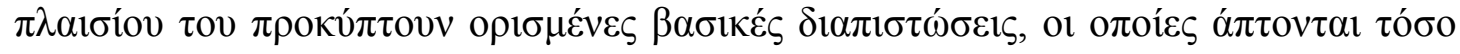




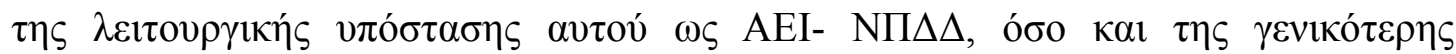

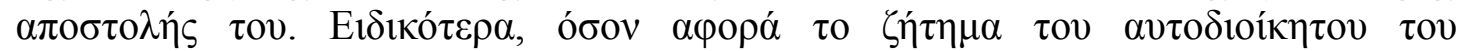

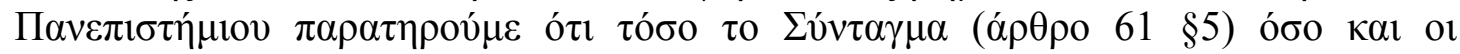

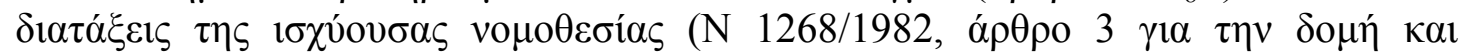

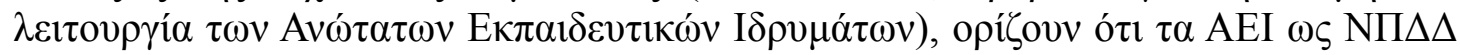

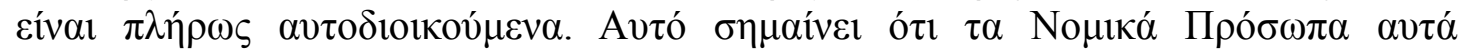

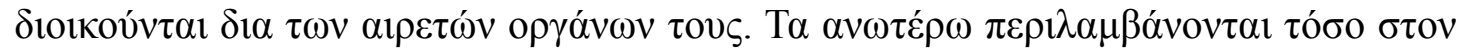

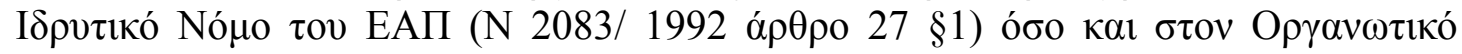

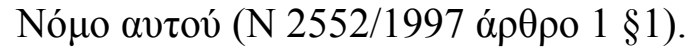

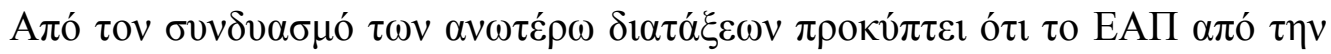

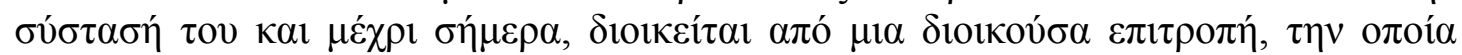

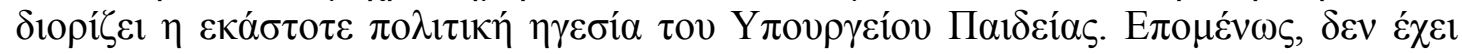

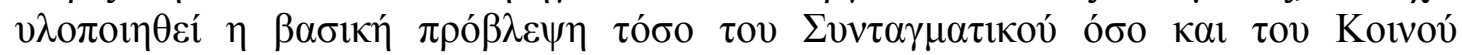

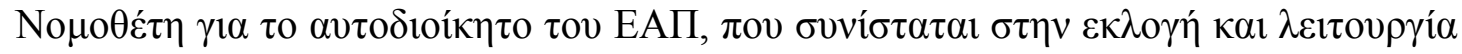

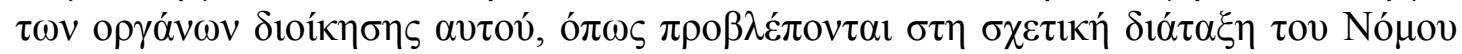

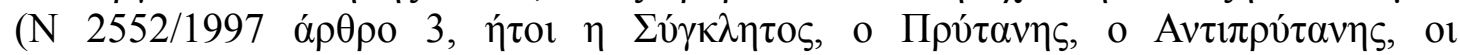

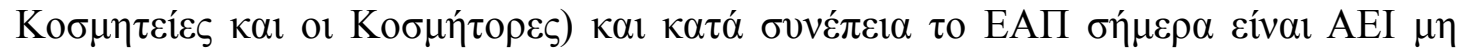

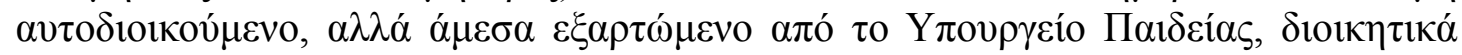

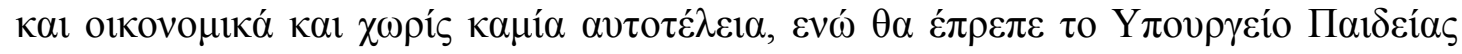

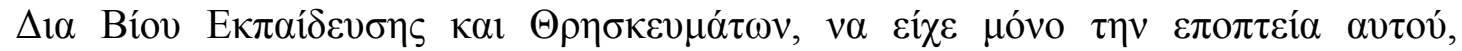

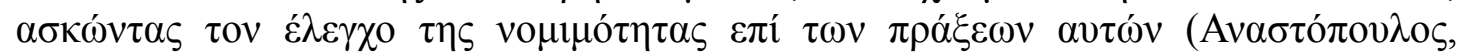

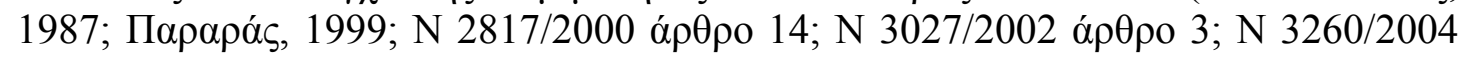
$\alpha ́ \rho \theta \rho o$ 13).

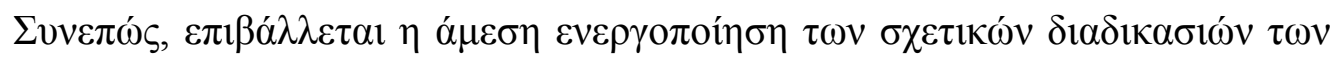

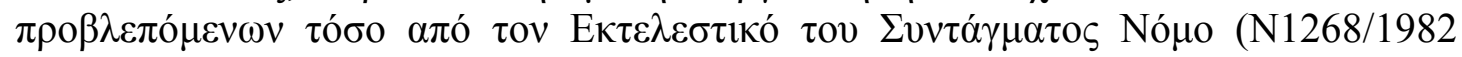

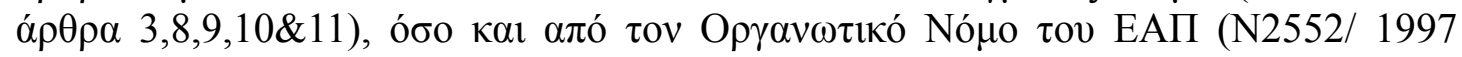

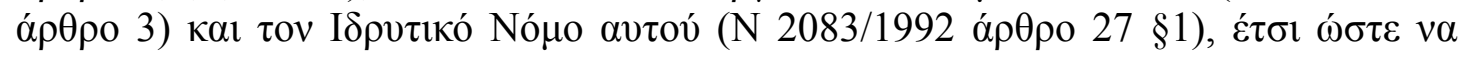

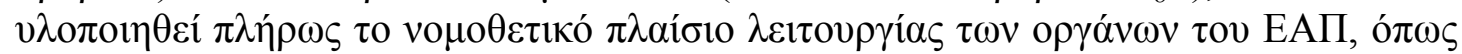

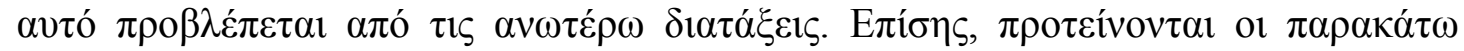

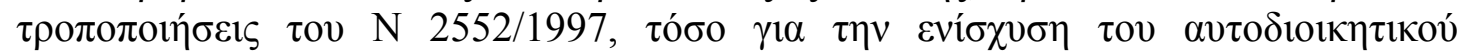

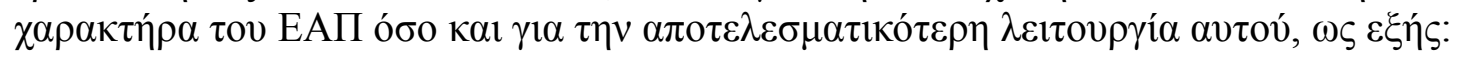

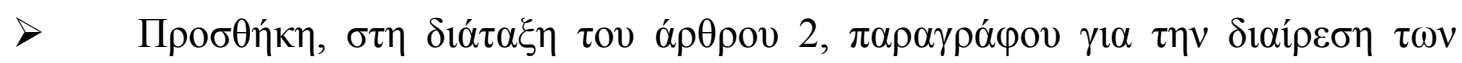

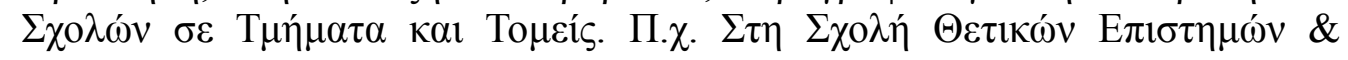

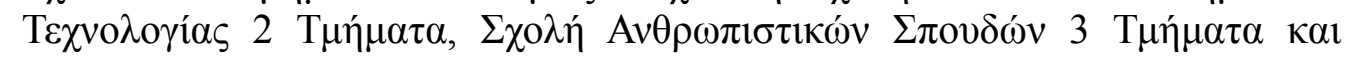

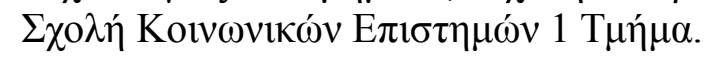

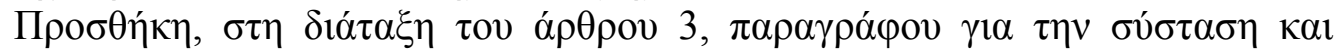

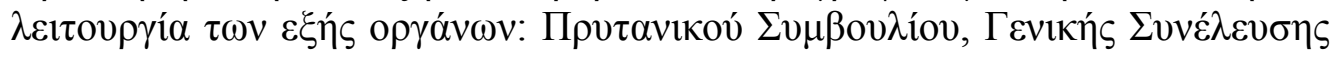

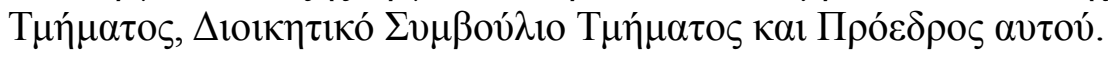

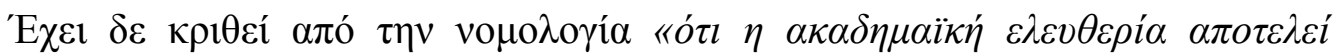

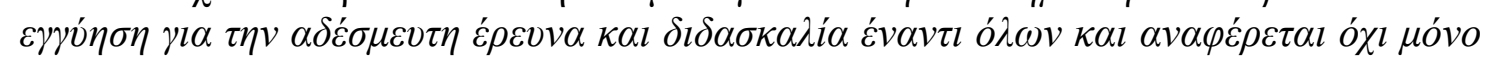

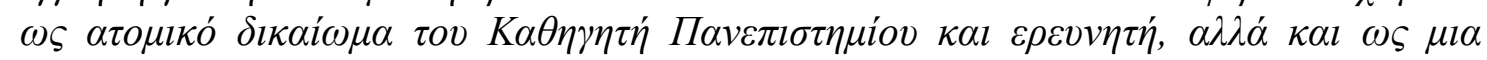

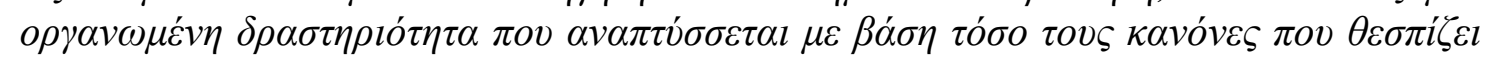

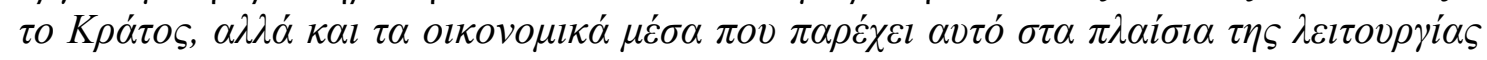

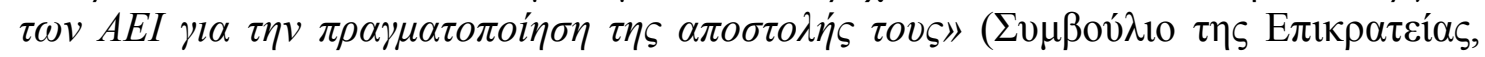
4009/2009). 


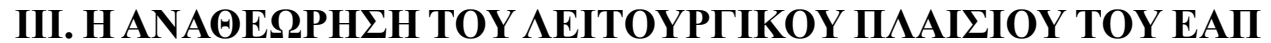

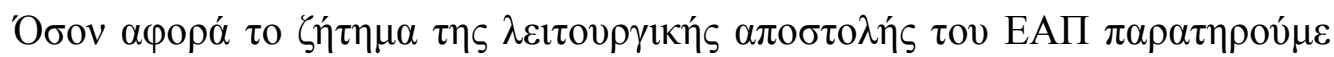

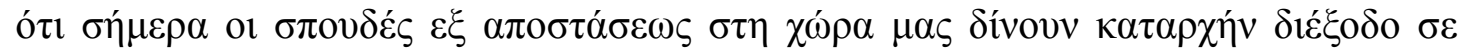

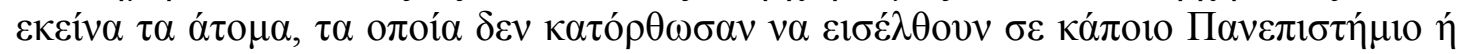

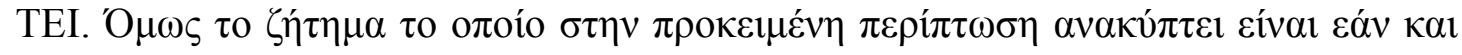

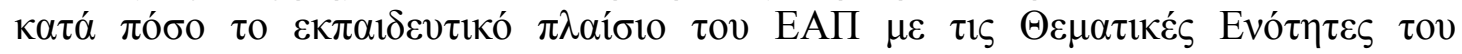

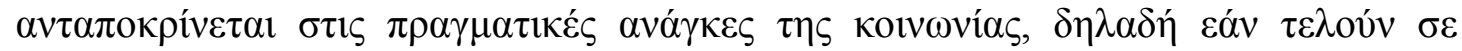

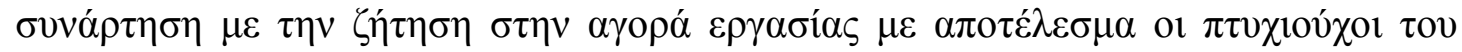

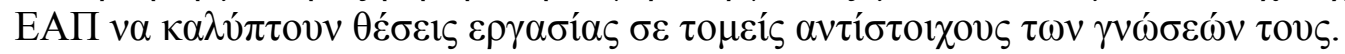

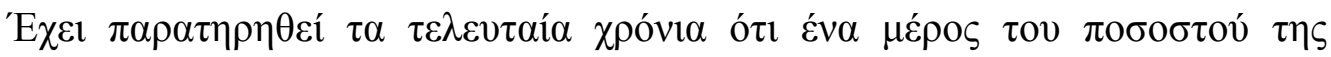

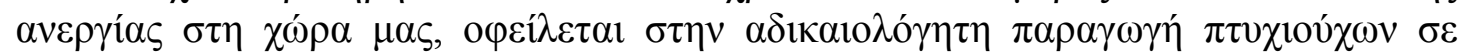

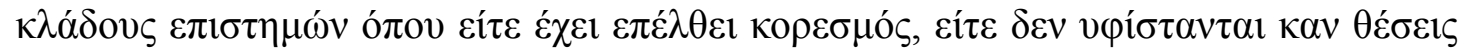

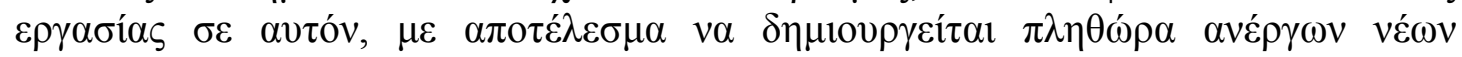

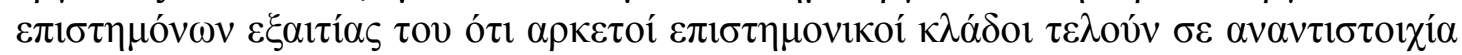

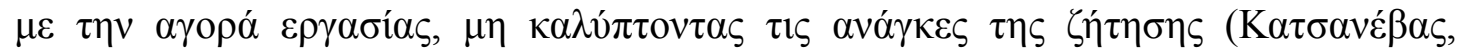

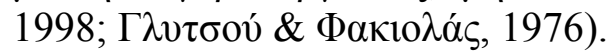

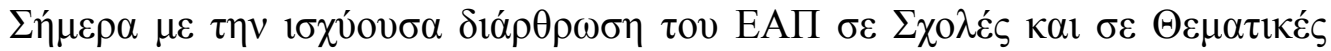

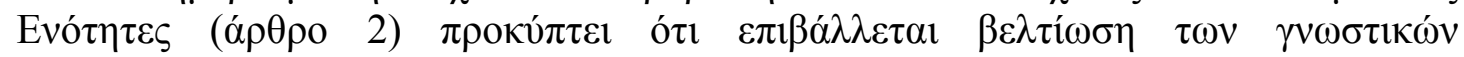

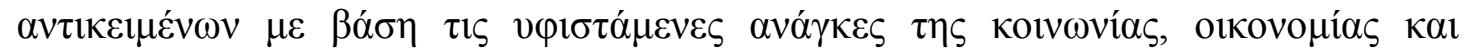

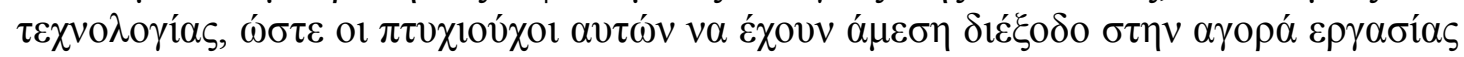

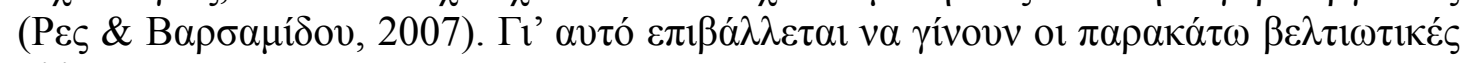

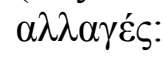

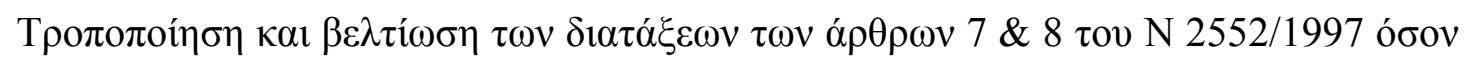

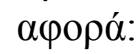

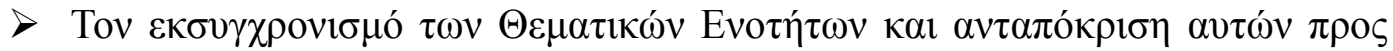

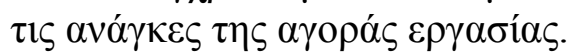

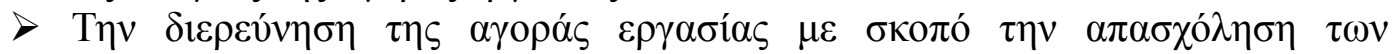

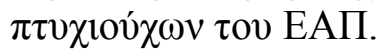

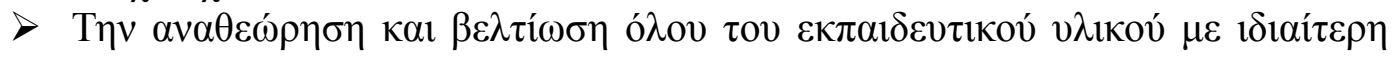

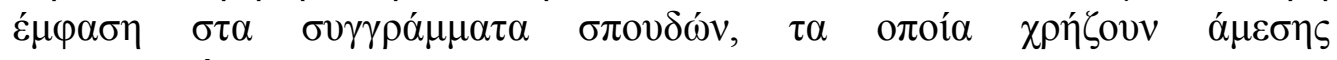

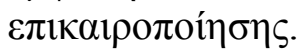

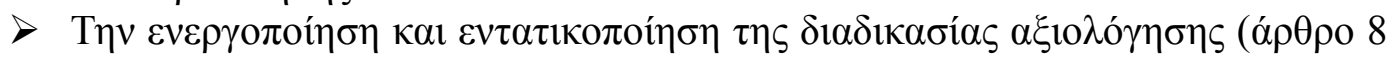

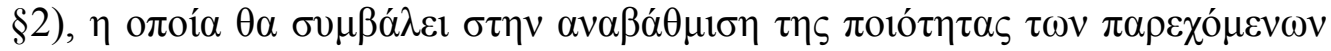

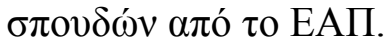

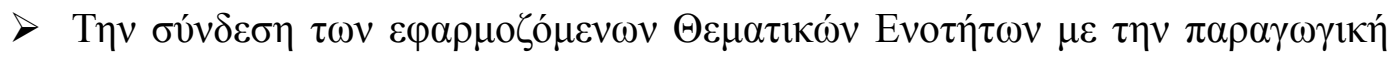

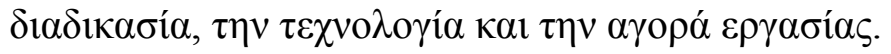

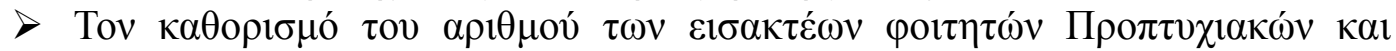

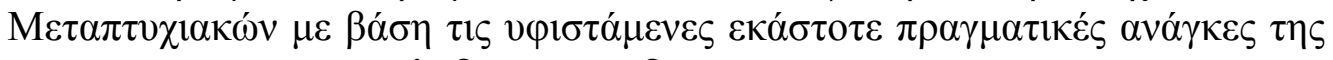

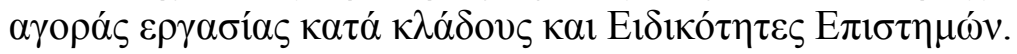

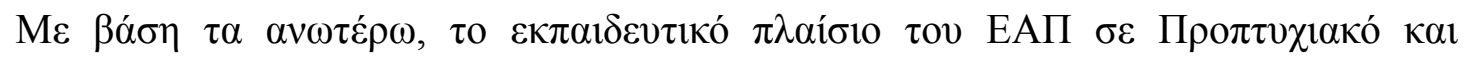

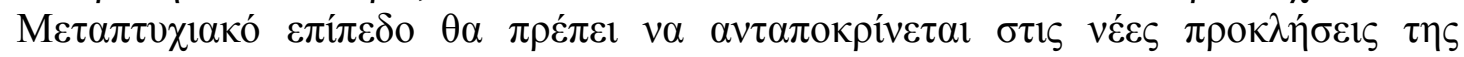

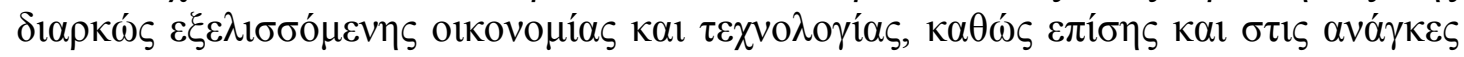

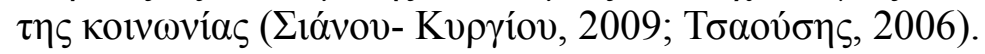




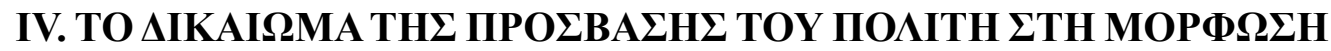

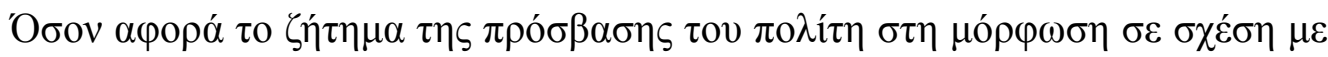

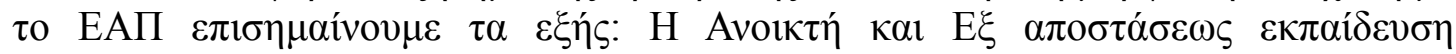

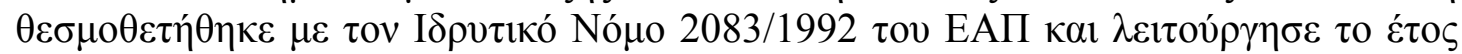

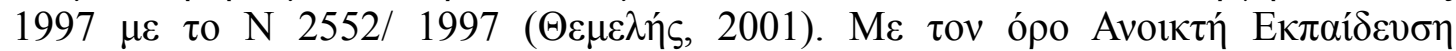

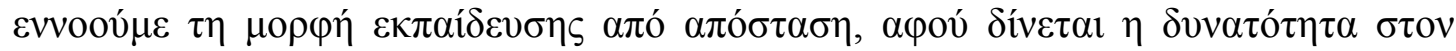

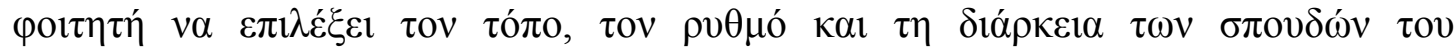

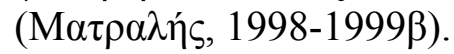

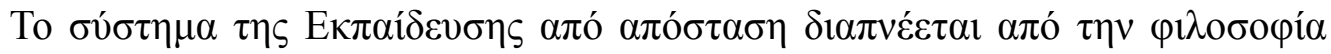

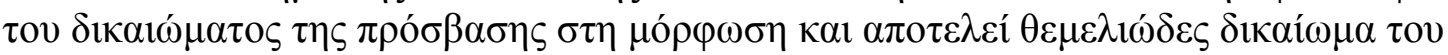

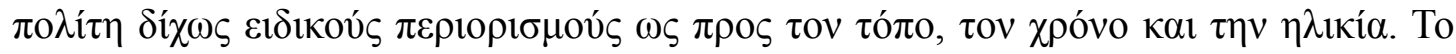

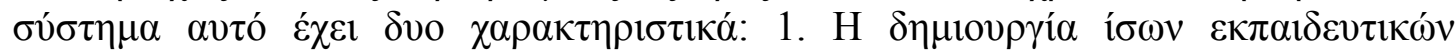

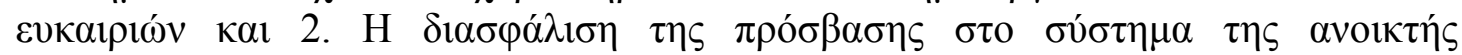

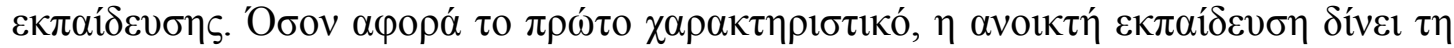

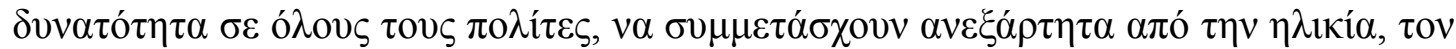

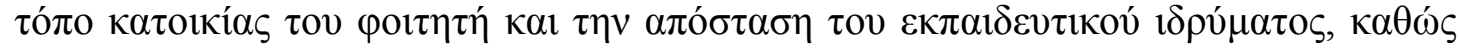

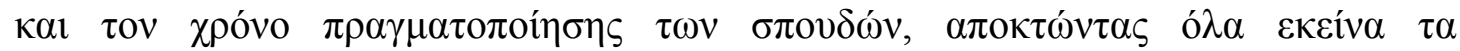

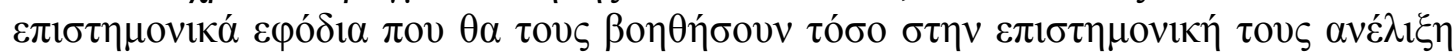

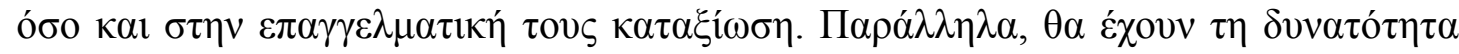

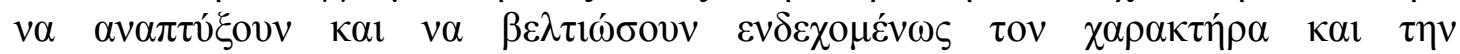

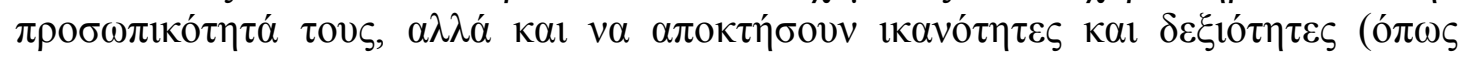

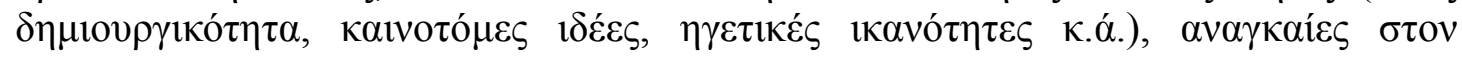

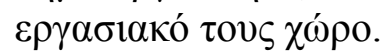

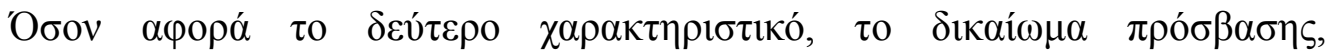

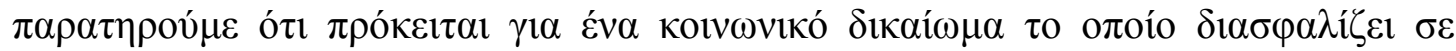

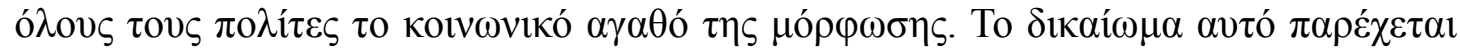

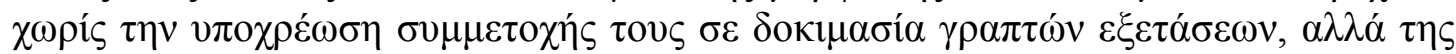

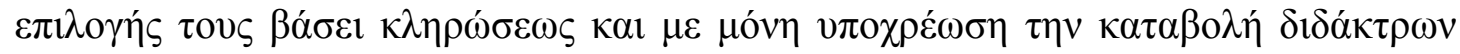

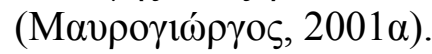

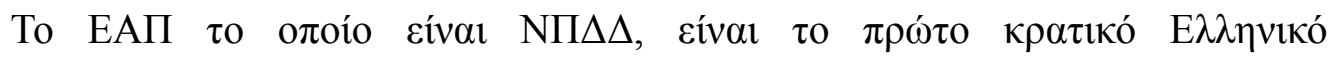

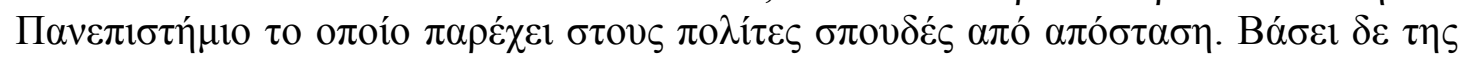

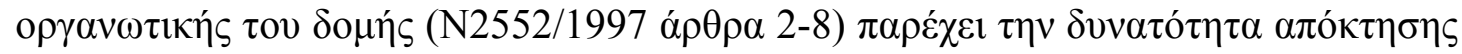

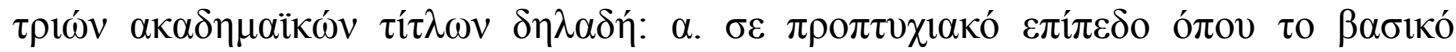

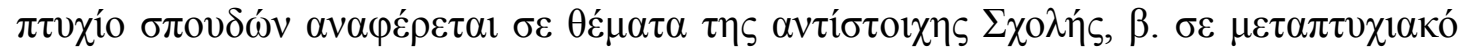

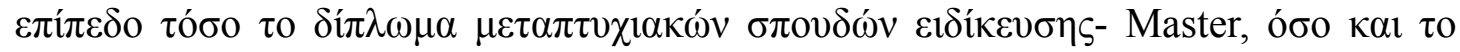

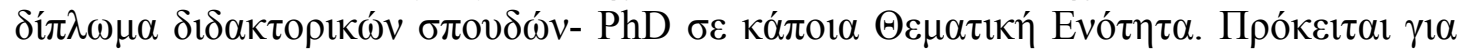

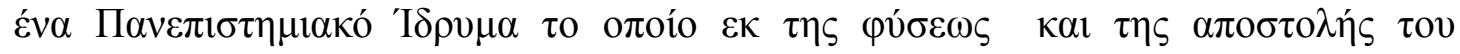

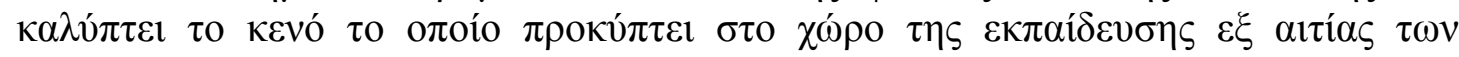

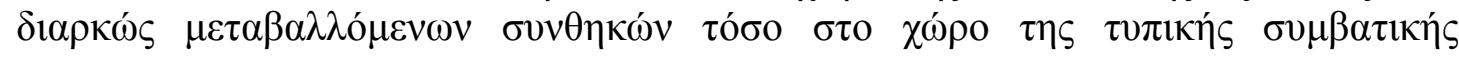

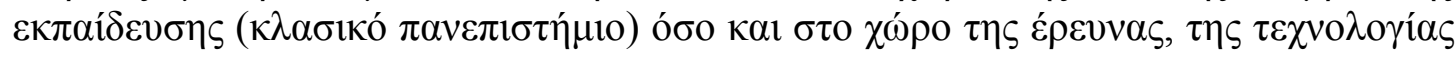

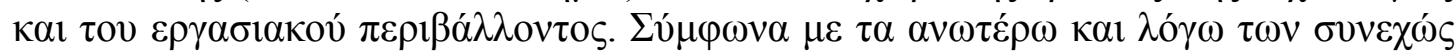

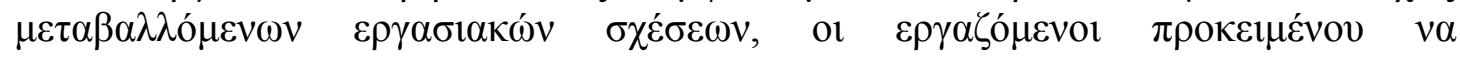

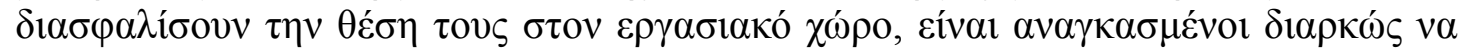

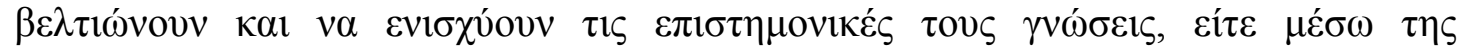




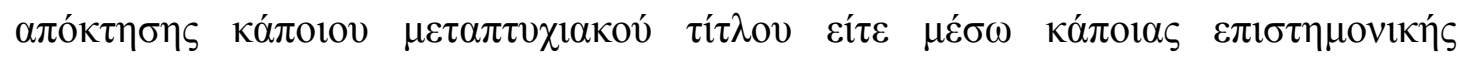

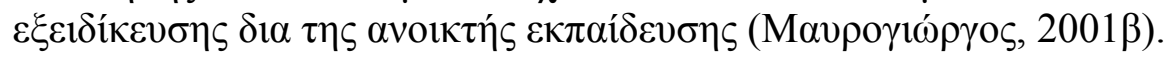

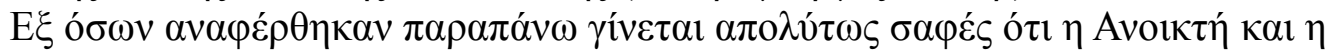

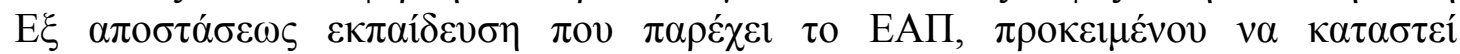

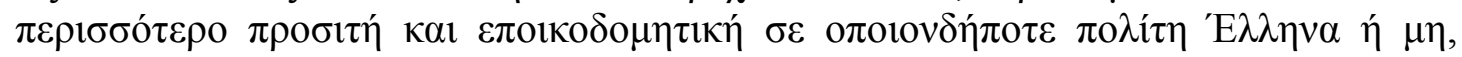

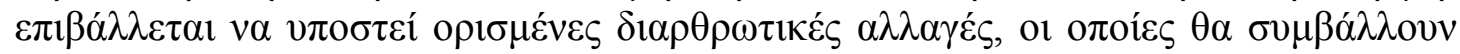

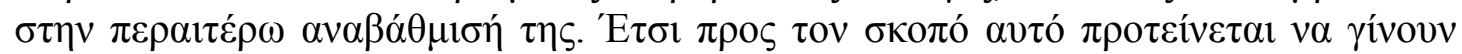

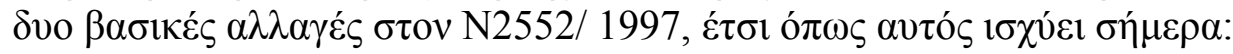

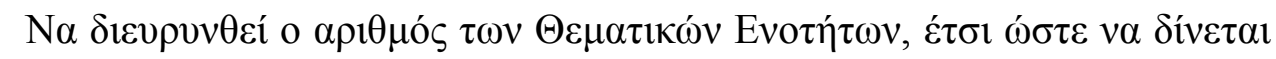

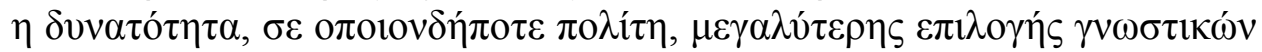

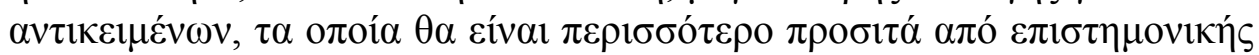

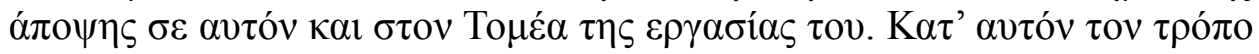

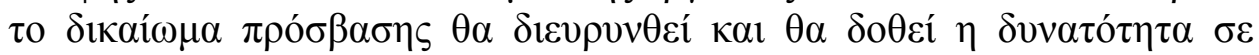

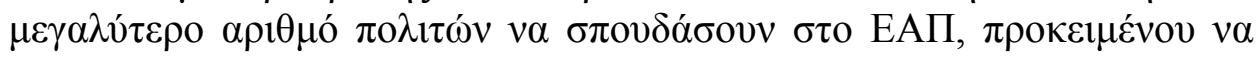

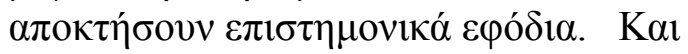

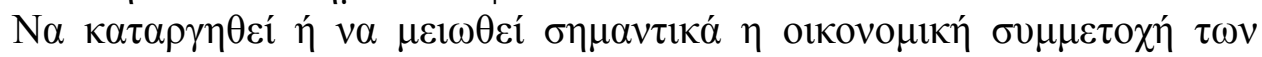

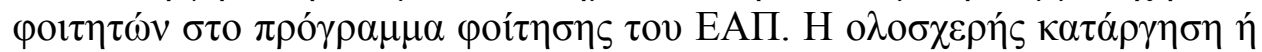

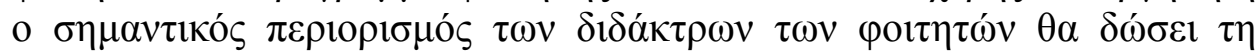

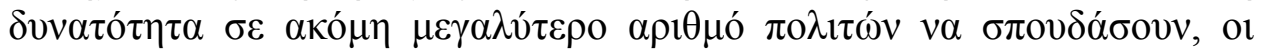

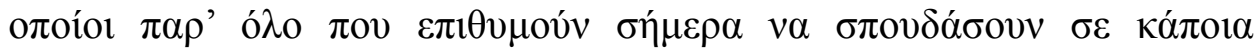

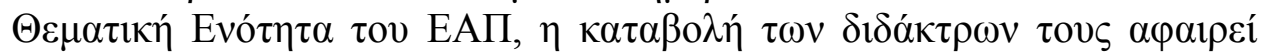

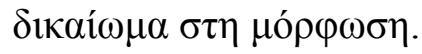

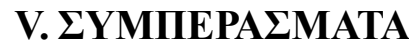

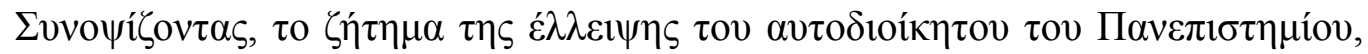

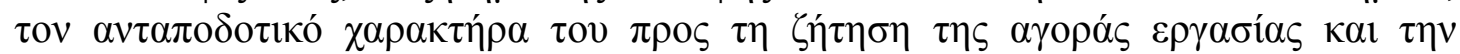

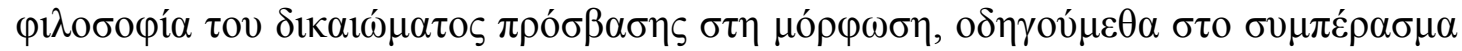

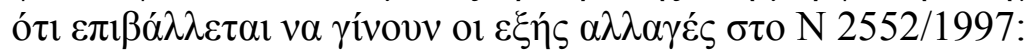

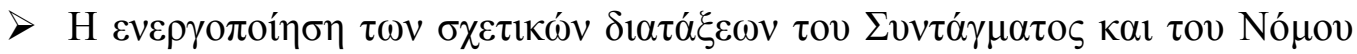

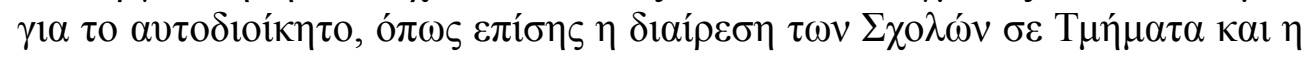

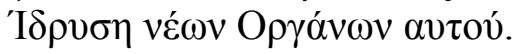

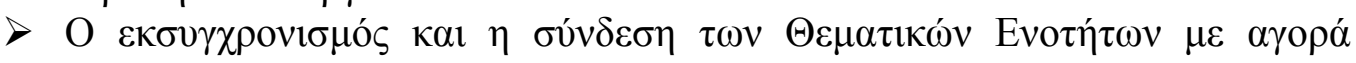

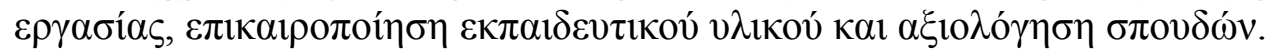

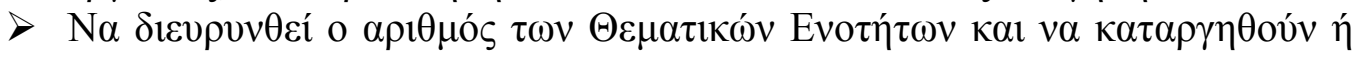

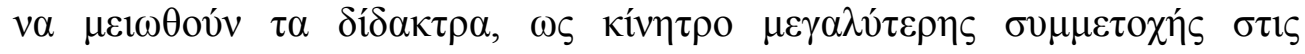
$\sigma \pi \mathrm{ov \delta \varepsilon ́s.}$

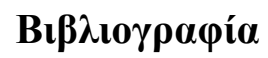

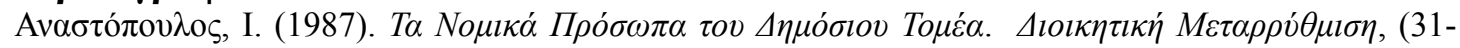
32), 107-110.

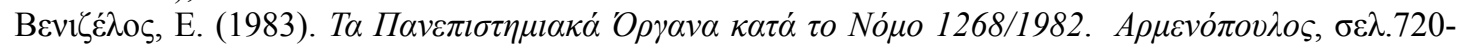
732.

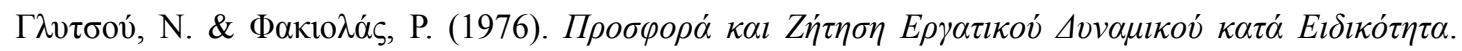




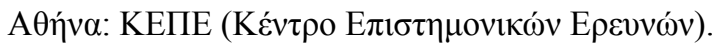

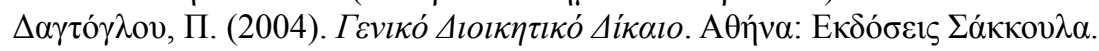

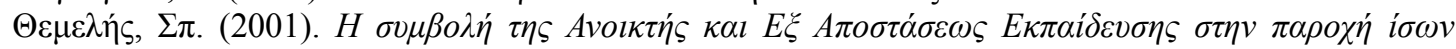

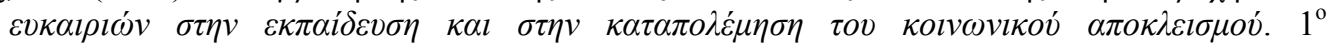

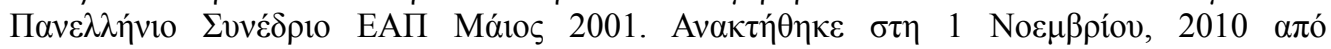
http://www.eap.gr/news/EXAGGELIA_SYNEDRIOU/synedrio/html/sect2/100.htm.

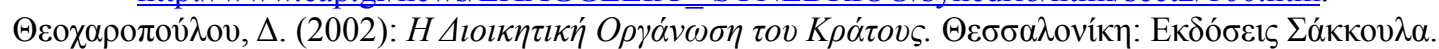

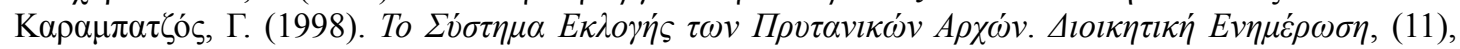
103-109.

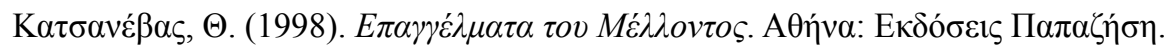

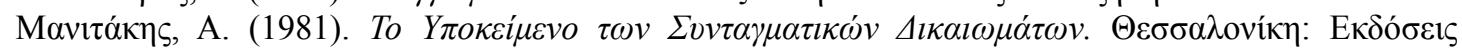

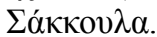

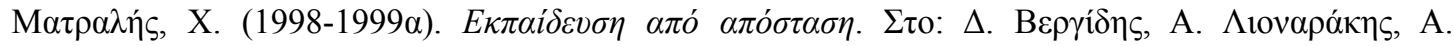

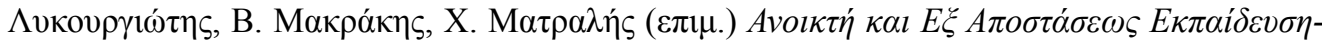

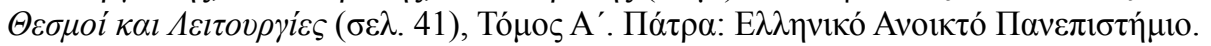

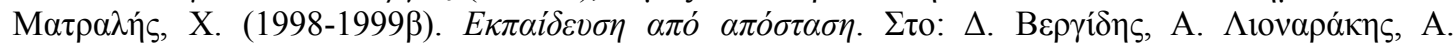

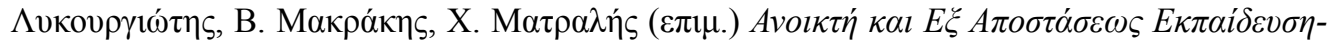

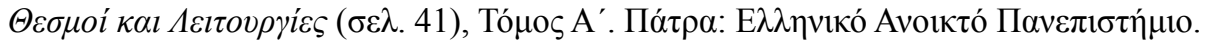

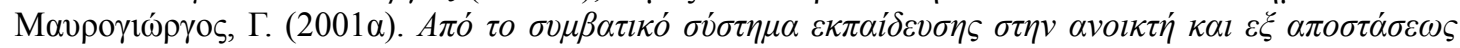

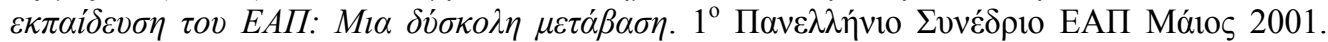

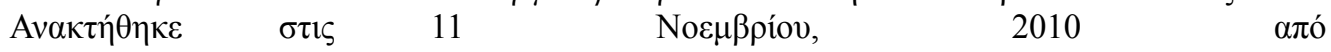
http://www.eap.gr/news/EXAGGELIA_SYNEDRIOU/synedrio/html/sect2/143.htm

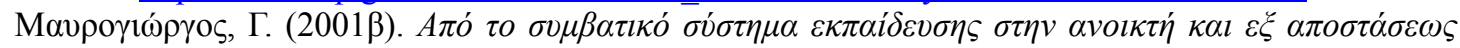

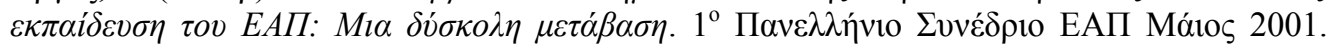

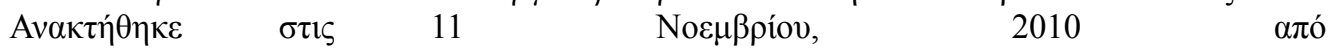
http://www.eap.gr/news/EXAGGELIA_SYNEDRIOU/synedrio/html/sect2/143.htm

N 1268/ 1982, ́́ $\rho \theta \rho \alpha 3,8,9,10 \& 11$.

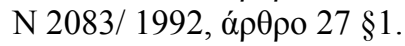

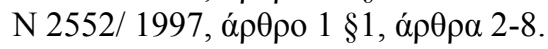

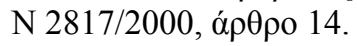

N 3027/2002, ́́ $\rho \theta \rho o ~ 3$.

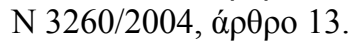

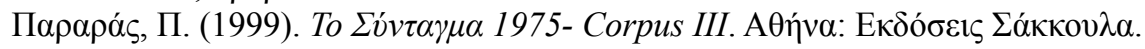

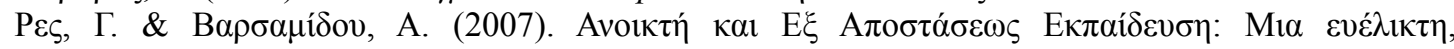

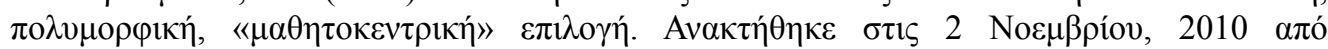
www.eduportal.gr/modules $/ \mathrm{php}$ ?name $=$ News\&file $=$ article \&sid $=175$

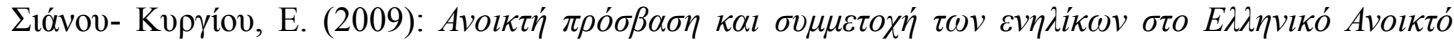

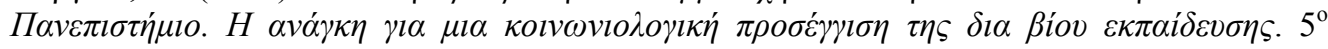

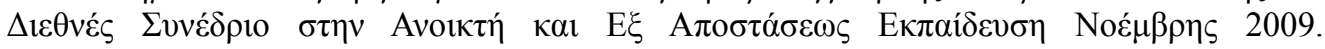

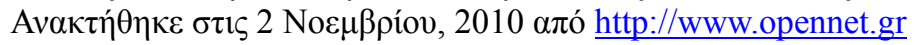

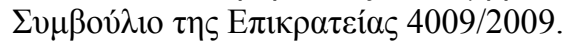

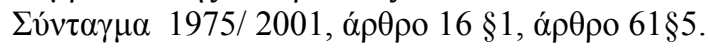

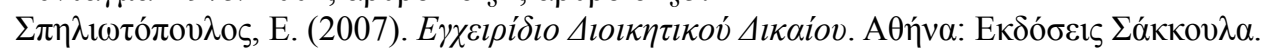

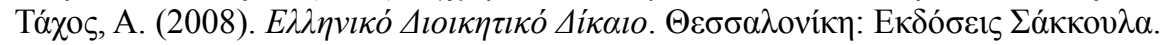

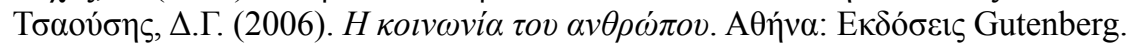

